\title{
Multikriterielle Bewertung von Technologien zur Bereitstellung von Strom und Wärme
}

\author{
Dissertation \\ zur Erlangung des wirtschaftswissenschaftlichen Doktorgrades \\ der Wirtschaftswissenschaftlichen Fakultät der Universität Göttingen
}

\author{
vorgelegt von \\ Julia Oberschmidt \\ aus Bruchsal
}

Göttingen, 2010 
Erstgutachterin:

Zweitgutachter:

Drittgutachter:

Tag der mündlichen Prüfung: $\quad$ 26.08.2010
Prof. Dr. Jutta Geldermann

Prof. Dr. Matthias Schumann

Prof. Dr. Martin Wietschel 


„Es sind nicht unsere Fähigkeiten, die zeigen, wer wir wirklich sind, es sind unsere Entscheidungen“

Joanne K. Rowling 



\section{Danksagung}

Zum Gelingen dieser Arbeit haben zahlreiche Personen beigetragen, die mich sowohl in fachlicher wie auch in persönlicher Hinsicht unterstützt haben. Mein besonderer Dank gilt Frau Prof. Dr. Jutta Geldermann für die Betreuung dieser Arbeit und das Vertrauen, das sie mir entgegengebracht hat. Die konstruktiven Diskussionen, die wir während meiner Promotionszeit führen konnten, haben wesentliche Impulse und Anregungen geliefert, die entscheidend zu meiner Motivation und letztlich zum erfolgreichen Abschluss der Arbeit beigetragen haben. Herrn Prof. Dr. Matthias Schumann sowie Herrn Prof. Dr. Martin Wietschel danke ich für die Bereitschaft zur Übernahme der Zweitgutachten.

Diese Arbeit ist während meiner Zeit als wissenschaftliche Mitarbeiterin im Competence Center für Energiepolitik und Energiesysteme (CCE) des Fraunhofer Instituts für System- und Innovationforschung ISI entstanden. Herrn Dr. Peter Radgen danke ich für die Möglichkeit, im Rahmen der Projektarbeit am ISI das Thema der multikriteriellen Analyse zu vertiefen und weitere Erfahrungen in energiepolitischen und -technischen Themenbereichen zu sammeln. Besonderer Dank gilt zudem Dr. Marian Klobasa, der mir durch seine konstruktiven Anmerkungen und die persönliche Unterstützung eine große Hilfe war. Danken möchte ich auch meinen Kollegen Anne Held, Dr. Christiane Klobasa, Vicki Duscha, Fabio Genoese und Jan Steinbach für fachliche Tipps und persönlichen Beistand. Den Assistentinnen des CCE, Bärbel Katz und Renate Schmitz, gilt mein Dank für die Hilfestellung bei organisatorischen Belangen sowie Brigitte Kallfaß für die Endkorrektur meiner Arbeit. Den wissenschaftlichen Hilfskräften Matthias Wolf, Maximilian Fütterer und Lucas Lürich danke ich für ihre gute Mitarbeit.

Herzlichen Dank möchte ich auch den Mitarbeitern am Lehrstuhl für Produktion und Logistik der Georg-August-Universität Göttingen aussprechen. Durch ihre offene Art und große Hilfsbereitschaft haben sie mir das Leben als externe Doktorandin wesentlich erleichtert und darüber hinaus zu einem gelungenen Prüfungstag beigetragen. Allen anderen Kollegen, die durch ein gutes Arbeitsklima und die kollegiale Zusammenarbeit zum Gelingen der Arbeit beigetragen haben, sei ebenfalls an dieser Stelle gedankt. Vielen Dank schließlich an meine Eltern Brigitte und Norbert Oberschmidt sowie meine Geschwister Judith, Anna und David, die immer hinter mir stehen. Ebenso danke ich meinem Freund Nils Eckhoff für den Rückhalt und die Unterstützung, mit der er mir stets zur Seite steht. 



\section{Inhalt}

Tabellen

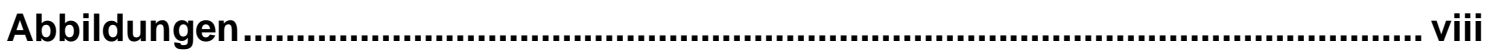

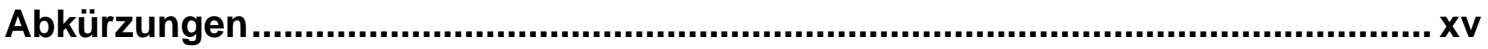

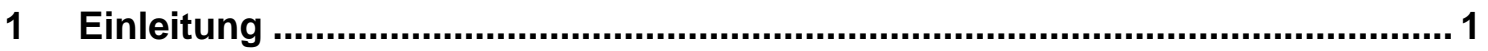

2 Rahmenbedingungen für Entscheidungen im Energiebereich ....................... 4

$2.1 \quad$ Historische Entwicklung der Energieversorgung .............................. 4

2.2 Energiebedarf und Nachfrage nach Energieträgern ......................... 7

2.3 Politische und gesetzliche Rahmenbedingungen............................ 11

2.3.1 Liberalisierung der Energiemärkte ............................................ 12

2.3.2 Klimapolitik und Handel mit Emissions-Zertifikaten.......................... 13

2.3.3 Erneuerbare Energien und Kraft-Wärme-Kopplung ......................... 16

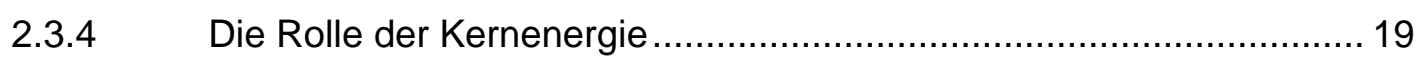

2.4 Notwendigkeit eines wissenschaftlichen Analyse-Rahmens für Energietechnologien ........................................................ 20

3 Technologien zur Bereitstellung von Strom und Wärme................................. 24

3.1 Bereitstellung von Elektrizität und Kraft-Wärme-Kopplung ................ 24

3.1.1 Thermische Kraftwerke ......................................................... 24

3.1.2 Erneuerbare Energien zur Stromerzeugung .................................... 29

3.1.3 Alternative Dampfkraftprozesse ................................................... 37

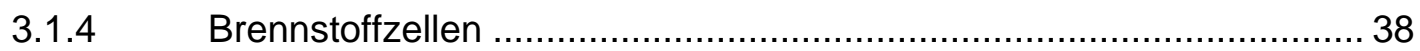

3.1.5 Weitere Technologien und Trends in der Stromerzeugung................ 39

3.1.6 Speicherung von Elektrizität.................................................. 41

3.1.7 Übertragung von Elektrizität ...................................................... 45 
3.2 Bereitstellung von Nutzwärme ….......................................... 46

3.2.1 Wärmebereitstellung auf Basis fossiler Primärenergien..................... 46

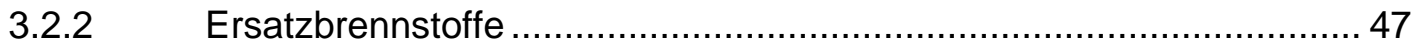

3.2.3 Wärmebereitstellung aus erneuerbaren Energien........................... 47

3.2.4 Speicherung von Wärme ...................................................... 51

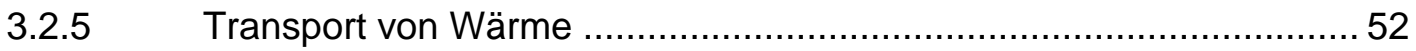

3.3 Auswahl von Energietechnologien ................................................. 53

4 Multikriterielle Entscheidungsunterstützung im Energiebereich................... 55

4.1 Grundlagen von Multi-Attribut-Methoden ..................................... 58

4.2 Anwendung von MADM-Methoden im Energiebereich ......................61 61

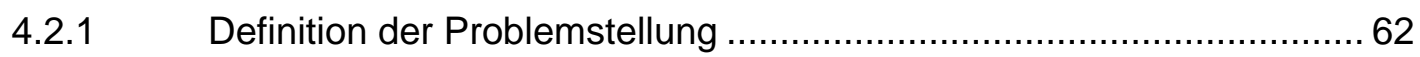

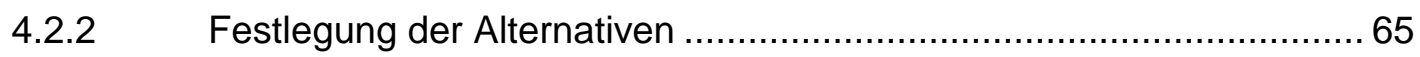

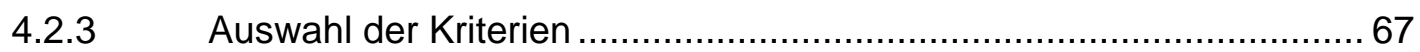

4.2.4 Bestimmung der Kriterien-Ausprägungen ....................................... 69

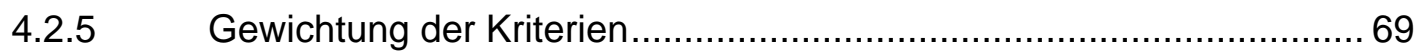

4.2.6 Aggregation zur Gesamtbewertung ............................................. 73

4.2.7 Sensitivitätsanalysen und weitere Methoden zur

Berücksichtigung von Unsicherheiten ......................................... 77

4.2.8 Einbeziehung von Stakeholdern und Entscheidungsfindung in

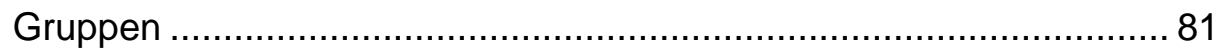

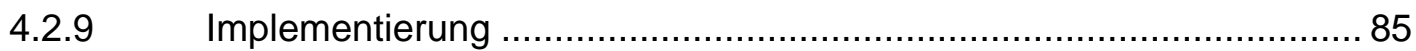

4.3 Schlussfolgerungen aus der Literaturanalyse ................................ 86

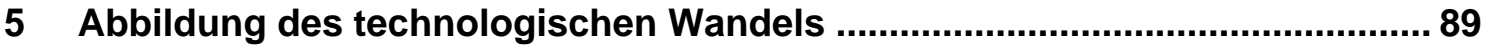

$5.1 \quad$ Das Konzept des Technologiezyklus .......................................... 90

5.2 Entwicklung von Energietechnologien im Zeitverlauf ........................96

5.2.1 Installierte Kraftwerksleistung in Europa ........................................... 96

5.2.2 Technologiezyklen von Optionen zur Wärmebereitstellung ............. 110

5.2.3 Indikatoren-basierte Zuordnung ............................................. 115

5.3 Zusammenfassung der Technologiezyklus-Analyse ....................... 121 
6 Konzeption eines multikriteriellen

Entscheidungsunterstützungssystems................................................... 122

6.1 Definition der Problemstellung .................................................. 122

6.2 Vorauswahl der Alternativen ................................................... 122

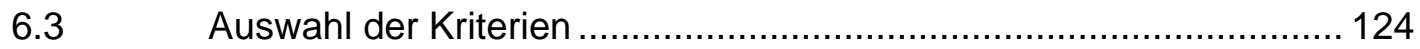

6.4 Bestimmung der Kriterien-Ausprägungen ................................ 126

6.5 Methoden zur Kriterien-Gewichtung .................................... 127

6.6 Wahl der Aggregationsmethode ................................................. 129

6.7 Berücksichtigung von Unsicherheiten ....................................... 135

6.8 Einbindung von Stakeholdern ................................................ 138

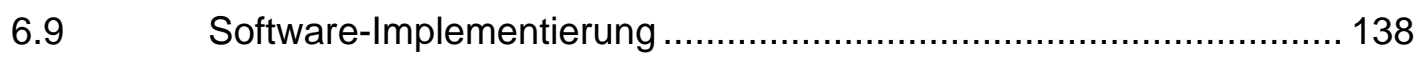

6.9.1 Datenbank Multidimensionale Technikbewertung.......................... 139

6.9.2 Excel-Tool MAESTRO ….................................................... 143

$7 \quad$ Bewertung von Energietechnologien .......................................................... 146

7.1 Bewertung alternativer Energieversorgungskonzepte für

Bioenergiedörfer.................................................................. 146

7.1.1 Definition der Problemstellung .............................................. 146

7.1.2 Vorauswahl der Alternativen ............................................... 148

7.1.3 Auswahl der Kriterien und Bestimmung der Ausprägungen.............. 149

7.1.4 Gewichtungen je Technologiezyklus-Phase ............................... 173

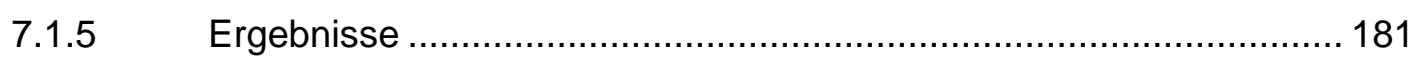

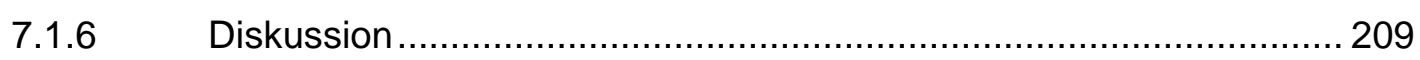

7.2 Vergleich von Stromspeichertechnologien ................................ 210

7.2.1 Vorbereitung der Entscheidungsmatrix ..................................... 212

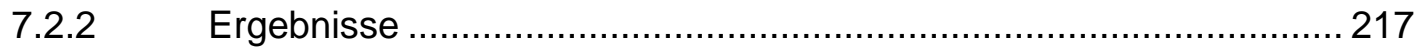

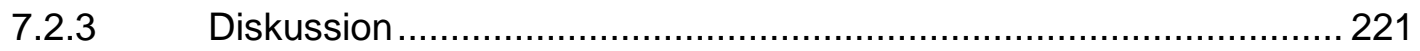

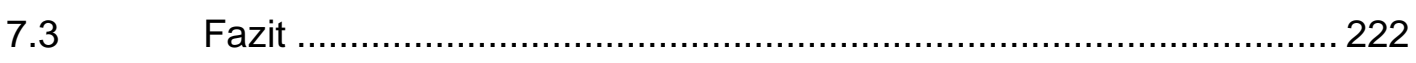

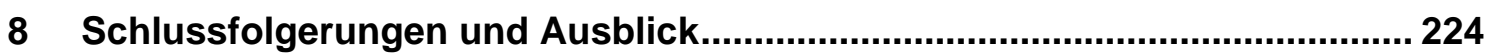




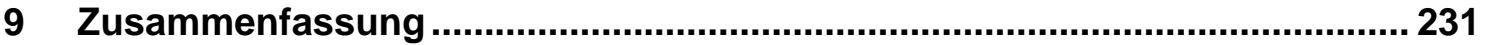

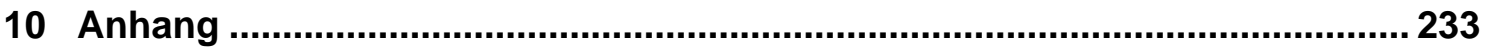

$10.1 \quad$ Datensätze in der Kraftwerksdatenbank ...................................... 233

10.2 Darstellung der Entwicklung der Verwendung unterschiedlicher Energieträgertypen in Europa ............................ 236

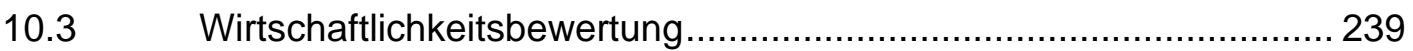

10.3.1 Generelle Vorgehensweise …............................................. 239

10.3.2 Anwendung im Fallbeispiel Bioenergiedörfer ................................ 245

10.4 Stabilitätsintervalle der Kriterien-Gewichtungen............................. 265

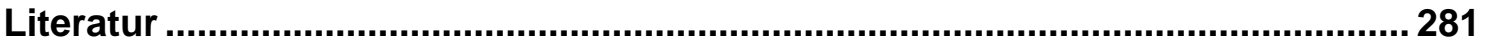




\section{Tabellen}

Tabelle 4.1: $\quad$ Abgrenzung von MADM- und MODM-Problemen

Tabelle 4.2: $\quad$ Abgrenzung von MADM-Methoden der amerikanischen und der europäischen Denkrichtungen

Tabelle 4.3: $\quad$ Abgrenzung von MADM-Anwendungen im Energiebereich nach dem Untersuchungsgegenstand

Tabelle 4.4: $\quad$ Berücksichtigung von Akteuren in multikriteriellen

Entscheidungsprozessen im Energiebereich

Tabelle 5.1: $\quad$ Zuordnung von Energietechnologien zu ihrem aktuellen

Entwicklungsstadium unterschiedenen nach dem verwendeten Energieträger („FUEL“ bzw. „FUELTYPE“;

Stand: 2007).

Tabelle 5.2: $\quad$ Zuordnung Energietechnologien zu ihrem aktuellen

Entwicklungsstadium unterschieden nach der Art der

Nutzung („UTYPE“; Stand: 2007).

Tabelle 5.3: $\quad$ Wärmeerzeugung aus erneuerbaren Energien in Europa

Tabelle 5.4: $\quad$ Entwicklung der installierten Leistung von Anlagen zur

Wärmebereitstellung aus erneuerbaren Energien in Europa ....... 115

Tabelle 5.5: $\quad$ Zuordnung von Optionen zur Wärmebereitstellung zu ihrem aktuellen Entwicklungsstadium (Stand: 2007)

Tabelle 5.6: $\quad$ Ergebnisse der Indikatoren-basierten Analyse hinsichtlich des Entwicklungsstandes von Brennstoffzellen, insbesondere der PEMFC.

Tabelle 6.1: $\quad$ Anwendung von PROMETHEE im Energiebereich 130

Tabelle 6.2:

Anwendung von PROMETHEE in anderen Bereichen

Tabelle 7.1:

Spezifische Kosten der Strombereitstellung in $\mathrm{Ct} / \mathrm{kWh}_{\mathrm{el}}$

Tabelle 7.2: $\quad$ Spezifische Kosten der Wärmebereitstellung in $\mathrm{Ct} / \mathrm{kWh}_{\text {th }}$

Tabelle 7.3:

Statische Reichweite und Importquote von Energieträgern 165

Tabelle 7.4:

Herfindahl-Index und Indikator zur Bewertung politischer und wirtschaftlicher Risiken in den Förderländern

Tabelle 7.5: $\quad$ Ausprägungen der Kriterien zur Bewertung der Versorgungssicherheit je Alternative. 
Tabelle 7.6: $\quad$ Kriterien zur Bewertung sozialer Aspekte der

Energiebereitstellung 170

Tabelle 7.7: $\quad$ Bewertung sozialer Aspekte durch Experten in

Deutschland

Tabelle 7.8: $\quad$ Einschätzung der sozialen Akzeptanz von

Energietechnologien durch Experten aus der

Energiewirtschaft. 173

Tabelle 7.9: $\quad$ Zuordnung der Alternativen zu Technologiezyklus-Phasen......... 174

Tabelle 7.10: $\quad$ Priorisierung von Umweltauswirkungen ................................... 176

Tabelle 7.11: $\quad$ Gewichtung der Kriterien zur Bewertung sozialer Aspekte .......... 177

Tabelle 7.12: $\quad$ Präferenzfunktionen und Schwellenwerte ................................... 180

Tabelle 7.13: $\quad$ Anteile der Technologien am zu bewertenden

Versorgungskonzept für die Stromerzeugung....

Tabelle 7.14: $\quad$ Anteile der Technologien am zu bewertenden

Versorgungskonzept für die Wärmebereitstellung ...................... 192

Tabelle 7.15: $\quad$ Stabilitätsintervalle Strom, Szenario 0 (ohne Förderung) ............. 198

Tabelle 7.16: $\quad$ Basisdaten der Speichertechnologien........................................ 213

Tabelle 7.17: $\quad$ Erlösmöglichkeiten durch Stromzwischenspeicherung je

Szenario in Euro/Jahr........................................................... 216

Tabelle 7.18: $\quad$ Festlegung der Präferenzfunktionen in PROMETHEE zur

Bewertung von Speichertechnologien.

Tabelle 7.19: $\quad$ Stabilitätsintervalle der Kriterien-Gewichtungen zur

Bewertung von Speichertechnologien

Tabelle 10.1: $\quad$ Energieträger und deren Nutzung zur Stromerzeugung in Europa (Anzahl der bei Platts 2007b registrierten

Kraftwerkseinheiten)

Tabelle 10.2: $\quad$ Basisannahmen zur Bewertung der Wirtschaftlichkeit unterschiedlicher Heizungssysteme.

Tabelle 10.3: Jahresnutzungsgrade der Komponenten von Systemen zur Hausenergieversorgung (Heizung und Warmwasser)

Tabelle 10.4: $\quad$ Verhältniszahlen zur Bestimmung des Hilfsenergiebedarfs und der mittleren Lagermenge

Tabelle 10.5: $\quad$ Annahmen zu Investitionen für konventionelle HeizölKessel 
Tabelle 10.6: $\quad$ Fixe Betriebskosten für Heizenergieanlagen zur

Einzelobjektversorgung 248

Tabelle 10.7: $\quad$ Aufteilung der Investitionen und fixen Betriebskosten für das Nahwärmenetz auf Biogas-BHKW und Heizwerk 253

Tabelle 10.8: $\quad$ Fixe Betriebskosten für das Heizwerk 255

Tabelle 10.9: $\quad$ Energieinhalt von Holzhackschnitzeln (HHS) (Basisdaten 2006) 256

Tabelle 10.10: $\quad$ Aufteilung der Investitionen und fixen Betriebskosten für das Nahwärmenetz auf Erdgas-BHKW und Heizwerk . 257

Tabelle 10.11: $\quad$ Aufteilung der Investitionen und fixen Betriebskosten für das Nahwärmenetz auf Erdgas-BHKW und Heizwerk .... 260

Tabelle 10.12: Investitionen und fixe Betriebskosten für Windkraftanlagen 261

Tabelle 10.13: Annahmen zu Investitionen für Erdgas-Brennwertkessel 262

Tabelle 10.14: Annahmen zu Investitionen für Sole-Wasser-Wärmepumpe 263

Tabelle 10.15: $\quad$ Annahmen zu Investitionen für Sole-Wasser-Wärmepumpe ....... 264

Tabelle 10.16: $\quad$ Stabilitätsintervalle Strom, Szenario 0 (mit Förderung)................. 266

Tabelle 10.17: $\quad$ Stabilitätsintervalle Strom, Szenario 1 (ohne Förderung) ............. 267

Tabelle 10.18: $\quad$ Stabilitätsintervalle Strom, Szenario 1 (mit Förderung)................. 268

Tabelle 10.19: $\quad$ Stabilitätsintervalle Strom, Szenario 2 (ohne Förderung) ............. 269

Tabelle 10.20: Stabilitätsintervalle Strom, Szenario 2 (mit Förderung)................ 270

Tabelle 10.21: $\quad$ Stabilitätsintervalle Strom, Szenario 3 (ohne Förderung) ............ 271

Tabelle 10.22: $\quad$ Stabilitätsintervalle Strom, Szenario 3 (mit Förderung)................ 272

Tabelle 10.23: $\quad$ Stabilitätsintervalle Wärme, Szenario 0 (ohne Förderung) ........... 273

Tabelle 10.24: $\quad$ Stabilitätsintervalle Wärme, Szenario 0 (mit Förderung) ................ 274

Tabelle 10.25: $\quad$ Stabilitätsintervalle Wärme, Szenario 1 (ohne Förderung) ........... 275

Tabelle 10.26: Stabilitätsintervalle Wärme, Szenario 1 (mit Förderung).............. 276

Tabelle 10.27: Stabilitätsintervalle Wärme, Szenario 2 (ohne Förderung) …....... 277

Tabelle 10.28: $\quad$ Stabilitätsintervalle Wärme, Szenario 2 (mit Förderung)............... 278

Tabelle 10.29: $\quad$ Stabilitätsintervalle Wärme, Szenario 3 (ohne Förderung) ........... 279

Tabelle 10.30: $\quad$ Stabilitätsintervalle Wärme, Szenario 3 (mit Förderung) ................ 280 


\section{Abbildungen}

Abbildung 2.1: $\quad$ Primärenergieverbrauch in Deutschland nach

Energieträgern

Abbildung 2.2: $\quad$ Statische Reichweite nuklearer und fossiler

Primärenergieträger

Abbildung 2.3: $\quad$ Anteil der Nettoimporte an den in Deutschland eingesetzten Primärenergieträgern

Abbildung 2.4: Grenzübergangspreise für fossile Energierohstoffe

Abbildung 2.5: $\quad$ Entwicklung des Weltprimärenergieverbrauchs im

Referenz-Szenario der IEA und der Weltbevölkerung

Abbildung 2.6: $\quad$ Endenergieverbrauch nach Sektoren und

Anwendungsbereichen in Deutschland im Jahr 2007

Abbildung 2.7: $\quad$ Beiträge der Sektoren zu unterschiedlichen Emissionsarten

in Deutschland im Jahr 2007

Abbildung 2.8: $\quad$ Anteile der Energieträger an der Stromerzeugung in

Deutschland im Jahr 2008 (gesamt: 639 TWh).

Abbildung 2.9: $\quad$ Anteile der Energieträger an der Wärmeversorgung in

Deutschland im Jahr 2008 (gesamt: 1.554 TWh).

Abbildung 3.1: $\quad$ Strukturentwicklung von Wärmeerzeugern in Deutschland (neu installierte Anlagen)

Abbildung 4.1: $\quad$ Schritte im Rahmen eines multikriteriellen

Entscheidungsunterstützungsprozesses.

Abbildung 5.1: Idealtypischer Lebenszyklus einer Technologie.....

Abbildung 5.2: Anteil der Rotordurchmessergruppen von

Windkraftanlagen an der jährlich in Deutschland neu

installierten Leistung

Abbildung 5.3: $\quad$ Schematische Darstellung eines modernen Technikzyklus-

Modells

Abbildung 5.4: Integrierter F\&E- und Marktzyklus einer Technologie

(schematische Darstellung).

Abbildung 5.5: $\quad$ Nutzung von Kohle-; Erdgas-, Öl- und Kernkraftwerken

sowie Wasser- und Windkraftanalagen zur

Stromerzeugung in Europa 
Abbildung 5.6: Nutzung von Hochofengas, Siedlungsabfällen, Holzbrennstoffen, LNG, Biomasse und Solarstrahlung zur Stromerzeugung. 101

Abbildung 5.7: Nutzung von Torf, Ölschiefer, Deponiegas und Biogas zur Stromerzeugung in Europa ....

Abbildung 5.8: Nutzung von Gasöl, Geothermie sowie RaffinerieRückständen und -Abgasen zur Stromerzeugung 103

Abbildung 5.9: Nutzung von Kohlevergasung, Erdölkoks, Kerosin, Schwarzlauge und Klärgas zur Stromerzeugung.... 104

Abbildung 5.10: Nutzung von Grubengas, Abfällen aus der Papierindustrie, Gichtgas und flüssigen Biokraftstoffen zur Stromerzeugung ....... 104

Abbildung 5.11: Installierte Leistung an GuD-Kraftwerken, Wasser-, und Dampfturbinen. 107

Abbildung 5.12: Installierte Leistung von Gas- und Windturbinen sowie Verbrennungsmotoren 108

Abbildung 5.13: Installierte Leistung von Offshore-Windturbinen 109

Abbildung 5.14: Installierte Leistung von ORC-Anlagen, Brennstoffzellen, Dampfkolbenmotoren und Turboexpandern

Abbildung 5.15: Brennstoffeinsatz in Heizkraftwerken und Fernheizwerken in Deutschland

Abbildung 5.16: Einsatz konventioneller Öl- und Gaskessel, von Thermen und Gas-Brennwertkesseln zur Wärmebereitstellung in Deutschland

Abbildung 5.17: Einsatz von fester Biomasse, von Wärmepumpen und ÖlBrennwertkesseln zur Wärmebereitstellung in Deutschland

Abbildung 6.1: Experteneinschätzung zukünftiger Potenziale von Technologien im Hinblick auf deren Beitrag zur Energieversorgung in Deutschland bis zum Jahr 2030

Abbildung 6.2: $\quad$ Relevanz von Kriterien zur Bewertung von Energietechnologien basierend auf einer Befragung von Entscheidungsträgern in der Energiewirtschaft.

Abbildung 6.3: Kriterien zur Bewertung von Energietechnologien 126

Abbildung 6.4: Nutzung von Web-HIPRE zur Gewichtung von Kriterien in Abhängigkeit von Technologiezyklus-Phasen. 
Abbildung 6.5: Verallgemeinerte Präferenzfunktionen zur Anwendung von

PROMETHEE

Abbildung 6.6: Entwicklung der Energieträgerpreise und der $\mathrm{CO}_{2-}$

Zertifikatspreise in Deutschland je Szenario

Abbildung 6.7: $\quad$ Spezifische $\mathrm{CO}_{2}$-Emissionen der Stromerzeugung in

Deutschland je Szenario

Abbildung 6.8: Konzeption des Entscheidungsunterstützungssystems

MAESTRO

Abbildung 6.9: $\quad$ Grundfunktionen der Datenbank „Multidimensionale

Technikbewertung“.....

Abbildung 6.10: $\quad$ Startformular zur Nutzung der Datenbank

„Multidimensionale Technikbewertung“.....

Abbildung 6.11: Formular zur Auswahl von Technologiedatensätzen

Abbildung 6.12: $\quad$ Gewichtung von Kriterien in den drei

Bewertungsdimensionen Technik, Ökonomie und Umwelt

Abbildung 6.13: Eingabe von Daten im Excel-Tool MAESTRO (fiktives

Beispiel)

Abbildung 7.1: $\quad$ Aktuelles Versorgungskonzept im Bioenergiedorf Jühnde

Abbildung 7.2: $\quad$ Aufteilung der spezifischen Gesamtkosten der

Strombereitstellung auf Investitionen, fixe und variable

Betriebskosten

Abbildung 7.3: $\quad$ Aufteilung der spezifischen Gesamtkosten der

Wärmebereitstellung auf Investitionen, fixe und variable

Betriebskosten

Abbildung 7.4: $\quad$ Ökobilanzierung der Optionen zur Strombereitstellung:

Ökotoxizität und Humantoxizität.

Abbildung 7.5: Ökobilanzierung der Optionen zur Strombereitstellung:

Treibhauseffekt, Landschaftsinanspruchnahme, Sommersmog und Erschöpfung abiotischer Ressourcen

Abbildung 7.6: Ökobilanzierung der Optionen zur Strombereitstellung:

Ozonabbau, Versauerung, Eutrophierung und Kumulierter

Energieaufwand 160

Abbildung 7.7: Ökobilanzierung der Optionen zur Wärmebereitstellung:

Ökotoxizität und Humantoxizität. 
Abbildung 7.8: Ökobilanzierung der Optionen zur Wärmebereitstellung:

Treibhauseffekt, Landschaftsinanspruchnahme, Sommersmog und Erschöpfung abiotischer Ressourcen

Abbildung 7.9: $\quad$ Ökobilanzierung der Optionen zur Wärmebereitstellung:

Ozonabbau, Versauerung, Eutrophierung und Kumulierter

Energieaufwand

Abbildung 7.10: Gewichtung der Bewertungsdimensionen in Abhängigkeit der Technologiezyklus-Phasen (Stromerzeugung)

Abbildung 7.11: Gewichtung der Bewertungsdimensionen in Abhängigkeit der Technologiezyklus-Phasen (Wärmebereitstellung). 176

Abbildung 7.12: Hierarchische Problemstrukturierung zur Ermittlung der Kriterien-Gewichtungen der untersten Ebene in Abhängigkeit der Technologiezyklus-Phasen (Alternativen zur Stromerzeugung)

Abbildung 7.13: Relative Stärke und relative Schwäche der Alternativen zur Stromerzeugung in Abhängigkeit der Szenarien.

Abbildung 7.14: Relative Stärke und relative Schwäche der Alternativen zur Wärmebereitstellung in Abhängigkeit der Szenarien

Abbildung 7.15: Analyse der Ergebnisse nach Bewertungsdimensionen

(Stromerzeugung)

Abbildung 7.16: Analyse der Ergebnisse nach Bewertungsdimensionen

(Wärmebereitstellung).

Abbildung 7.17: Rangfolge der Optionen zur Strombereitstellung nach

PROMETHEE I ohne Berücksichtigung von

Förderbedingungen

Abbildung 7.18: Rangfolge der Optionen zur Strombereitstellung nach

PROMETHEE I unter Berücksichtigung von

Förderbedingungen 188

Abbildung 7.19: Rangfolge der Optionen zur Strombereitstellung nach PROMETHEE II

Abbildung 7.20: Rangfolge der Optionen zur Wärmebereitstellung nach PROMETHEE I

Abbildung 7.21: Rangfolge der Optionen zur Wärmebereitstellung nach PROMETHEE II (alle Szenarien) ............................................... 190

Abbildung 7.22: Bewertung von Energieversorgungskonzepten 191 
Abbildung 7.23: Rangfolge der Versorgungskonzepte nach PROMETHEE I

ohne Berücksichtigung von Förderbedingungen.

Abbildung 7.24: Rangfolge der Versorgungskonzepte nach PROMETHEE I

unter Berücksichtigung von Förderbedingungen

Abbildung 7.25: Rangfolge der Versorgungskonzepte nach PROMETHEE II ....... 196

Abbildung 7.26: Besonderheiten der Sensitivitätsanalyse bei Anwendung des modifizierten PROMETHEE-Ansatzes

Abbildung 7.27: $\quad$ Ergebnisse der Optionen zur Stromerzeugung in

Abhängigkeit unterschiedlicher Technologiezyklus-Phasen

(Szenario 0, ohne Förderung)

Abbildung 7.28: Ergebnisse der Optionen zur Wärmebereitstellung in

Abhängigkeit unterschiedlicher Technologiezyklus-Phasen

(Szenario 0, ohne Förderung) 201

Abbildung 7.29: Ergebnisse der Optionen zur Wärmebereitstellung in

Abhängigkeit unterschiedlicher Technologiezyklus-Phasen

(Detailansicht)

Abbildung 7.30: Sensitivitätsanalyse hinsichtlich der Gewichtung des

Kriteriums „Treibhauspotenzial“ (GWP, U3) für Optionen

zur Stromerzeugung (Szenario 1, mit Förderung)

Abbildung 7.31: Sensitivitätsanalyse hinsichtlich der Gewichtung des

Kriteriums „Kumulierter Energieaufwand“ (KEA, U10) für

Optionen zur Stromerzeugung (Szenario 1, mit Förderung)

Abbildung 7.32: Sensitivitätsanalyse hinsichtlich der Gewichtung des

Kriteriums „Landschaftsinanspruchnahme" (LAND, U2) für

Optionen zur Wärmebereitstellung (Szenario 0, ohne

Förderung)

Abbildung 7.33: $\quad$ Sensitivitätsanalyse hinsichtlich der Gewichtung des

Kriteriums „Lokale Verfügbarkeit" (V3) für Optionen zur

Wärmebereitstellung (Szenario 0, ohne Förderung)

Abbildung 7.34: Sensitivitätsanalyse hinsichtlich der Gewichtung des

Kriteriums „Herfindahl-Index" (Konzentration der

Förderung, V5) für Optionen zur Wärmebereitstellung

(Szenario 0, ohne Förderung)

Abbildung 7.35: Sensitivitätsanalyse hinsichtlich der Gewichtung des

Kriteriums „Importquote“ (V2) für Optionen zur

Wärmebereitstellung (Szenario 0, mit Förderung) 206 
Abbildung 7.36: Sensitivitätsanalyse hinsichtlich der Gewichtung des Kriteriums „Eutrophierung“ (EP, U9) für Optionen zur Wärmebereitstellung (Szenario 0, ohne Förderung) 207

Abbildung 7.37: Sensitivitätsanalyse hinsichtlich der Gewichtung des Kriteriums „Süßwasser-Ökotoxizität“ (FAETP, U6.1) für Optionen zur Wärmebereitstellung (Szenario 3, mit Förderung) 208

Abbildung 7.38: Kriterien-Hierarchie zur Bewertung von Technologien zur Stromspeicherung

Abbildung 7.39: Gewichtung der übergeordneten Kriterien zur Bewertung der Speichertechnologien in Abhängigkeit der Technologiezyklusphasen.

Abbildung 7.40: $\quad$ Mittelwerte der aus der Expertenbefragung ermittelten

Gewichtungen 215

Abbildung 7.41: Bewertung der Speichertechnologien in Abhängigkeit der Szenarien

Abbildung 7.42: Analyse der Ergebnisse nach Bewertungsdimensionen (Szenario 1)

Abbildung 7.43: $\quad$ Rangfolge der Speichertechnologien nach PROMETHEE I und II (alle Szenarien)

Abbildung 7.44: Ergebnisse Stromspeichertechnologien in Abhängigkeit unterschiedlicher Technologiezyklus-Phasen (Szenario 1)

Abbildung 10.1: $\quad$ Nutzung von Braun- und Steinkohle, konventionellen Wasserkraftwerken, Schwerölen und Druckwasserreaktoren zur Stromerzeugung in Europa

Abbildung 10.2: Nutzung von Siedewasserreaktoren, horizontalen Windturbinen, Pumpspeichern, gasgekühlten Reaktoren und Leichtölen zur Stromerzeugung in Europa 236

Abbildung 10.3: Nutzung verschiedener Kernkraftwerkstypen sowie der geothermischen Stromerzeugung nach dem Trockendampf-Prinzip, der Photovoltaik und solarthermischer Kraftwerke zur Stromerzeugung in Europa.

Abbildung 10.4: Nutzung von Druckluftspeichern, kohlehaltigen Reststoffen, Stroh, des geothermischen Flash-Prinzip, Geflügelmist, landwirtschaftlicher Abfälle, Ersatzbrennstoffen sowie 
Wellen- und Gezeitenkraftwerken zur Stromerzeugung in Europa.

Abbildung 10.5: Nutzung vertikaler Windturbinen, von VOC-Gasen und Dämpfen, medizinischen Abfällen, Reishülsen, Palmöl, Fleisch- und Knochenmehl sowie der Geothermie nach dem binären Prinzip zur Stromerzeugung in Europa 238

Abbildung 10.6: Nutzung sonstiger Energieträger zur Stromerzeugung in Europa 


\section{Abkürzungen}

\begin{tabular}{|c|c|}
\hline AA-CAES & Advanced Adiabatic CAES \\
\hline AFC & Alkaline Fuel Cell (Alkalische Brennstoffzelle ) \\
\hline AHP & Analytischer-Hierarchie-Prozess \\
\hline ANP & Analytischer-Netzwerk-Prozess \\
\hline AtG & Atomgesetz \\
\hline BHKW & Blockheizkraftwerk \\
\hline BMBF & Bundesministerium für Bildung und Forschung \\
\hline BMU & Bundesministerium für Umwelt, Naturschutz und Reaktorsicherheit \\
\hline BMWi & Bundesministerium für Wirtschaft und Technologie \\
\hline BNetzA & Bundesnetzagentur \\
\hline BWE & Bundesverband Windenergie \\
\hline CAES & Compressed Air Energy Storage \\
\hline $\operatorname{ccs}$ & Carbon Capture and Storage $\left(\mathrm{CO}_{2}\right.$-Abscheidung und Speicherung $)$ \\
\hline CdTe & Cadmium-Tellurid \\
\hline $\mathrm{CIS}$ & Kupfer-Indium-Diselenid $\left(\mathrm{CulnSe}_{2}\right)$ \\
\hline COP & Conference of the Parties \\
\hline DMFC & Direct Methanol Fuel Cell (Direktmethanol-Brennstoffzelle) \\
\hline DSO & Distribution System Operator (Verteilungsnetzbetreiber) \\
\hline DSS & Decision Support System \\
\hline EAT & Equivalent Attribute Technique \\
\hline EEG & Erneuerbare-Energien-Gesetz \\
\hline EEWärmeG & Erneuerbare-Wärme-Gesetz \\
\hline el & elektrisch \\
\hline ELECTRE & Elimination Et Choix Traduisant la Réalité \\
\hline EnWG & Energiewirtschaftsgesetz \\
\hline EPO & Europäisches Patentamt \\
\hline EPR & European Pressurized Water Reactor (Europäischer Druckwasserreaktor) \\
\hline ETS & Emission Trading System (Emissionshandelssystem) \\
\hline EU & Europäische Union \\
\hline
\end{tabular}




\begin{tabular}{|c|c|}
\hline EUS & Entscheidungsunterstützungssystem \\
\hline $\mathrm{F} \& \mathrm{E}$ & Forschung und Entwicklung \\
\hline GHD & Gewerbe, Handel, Dienstleistungen \\
\hline GuD & Kombinierter Gas- und Dampfturbinenprozess \\
\hline GW & Gigawatt \\
\hline HAT & Humid Air Turbine \\
\hline HGÜ & Hochspannungs-Gleichstrom-Übertragung \\
\hline $\mathrm{HHS}$ & Holzhackschnitzel \\
\hline HS & Hoch-/Höchstspannung \\
\hline $\mathrm{Hz}$ & Hertz \\
\hline IEKP & Integriertes Energie- und Klimaprogramm \\
\hline IGCC & $\begin{array}{l}\text { Integrated Gasification Combined Cycle (Kraftwerksprozess mit integrierter } \\
\text { Kohlevergasung) }\end{array}$ \\
\hline IRIS & $\begin{array}{l}\text { Interactive Robustness Analysis and Parameters Interference for Multicriteria } \\
\text { Sorting Problems }\end{array}$ \\
\hline KHKW & Heizkraftwerk \\
\hline $\mathrm{kV}$ & Kilovolt \\
\hline $\mathrm{kW}$ & Kilowatt \\
\hline $\mathrm{kW}_{\mathrm{p}}$ & Kilowatt Peak (Spitzenleistung) \\
\hline KWK & Kraft-Wärme-Kopplung \\
\hline KWKK & Kraft-Wärme-Kälte-Kopplung \\
\hline LA & Lead Acid (Blei-Säure) \\
\hline LiB & Lithium-Ionen-Batterie \\
\hline LiS & Lithium-Schwefel \\
\hline LNG & Liquefied Natural Gas \\
\hline MACBETH & Measuring Attractiveness by a Categorial Based Evaluation Technique \\
\hline MADM & Multi Attribute Decision Making \\
\hline MAESTRO & Multi Criteria Assesment of Energy Supply Technologies - Research \& Outlook \\
\hline MAUT & Multi Attribute Utility Theory \\
\hline MAVT & Multi Attribute Value Theory \\
\hline MCDA & Multi Criteria Decision Aid \\
\hline MCFC & Molten Carbonate Fuel Cell (Schmelzkarbonat-Brennstoffzelle) \\
\hline
\end{tabular}




\begin{tabular}{|c|c|}
\hline MENA & Middle East \& North Africa \\
\hline MODM & Multi Objective Decision Making \\
\hline MS & Mittelspannung \\
\hline MTB & Multidimensionale Technikbewertung \\
\hline MW & Megawatt \\
\hline NAIADE & Novel Approach to Imprecise Assessment and Decision Environments \\
\hline $\mathrm{NaNiCl}$ & Natrium-Nickel-Chlorid \\
\hline NAP & Nationaler Allokationsplan \\
\hline NaS & Natrium-Schwefel \\
\hline NS & Niederspannung \\
\hline NT & Niedertemperatur (Heizkessel) \\
\hline ORC & Organic Rankine Cycle \\
\hline PAFC & Phosphoric Acid Fuel Cell (Phosphorsäure-Brennstoffzelle) \\
\hline PAIRS & Preference Assessment by Imprecise Ratio Statements method \\
\hline PCA & Principal Component Analysis (Hauptkomponentenanalyse) \\
\hline PCS & Phase Change Slurries \\
\hline PEFC & PEM Fuel Cell (Polymer-Elektrolyt-Membran-Brennstoffzelle) \\
\hline PEM & Polymer Electrolyte Membrane \\
\hline PEMFC & vgl. PEFC \\
\hline PROMETHEE & Preference Ranking Organisation Method for Enrichment Evaluations \\
\hline PV & Photovoltaik \\
\hline RFB & Redox-Flow-Batterie \\
\hline SAW & Simple Additive Weighting \\
\hline SMART & Simple Multi-Attribute Rating Technique \\
\hline SMARTER & SMART Exploiting Ranks \\
\hline SMES & Superconducting Magnetic Electricity Storage \\
\hline SOFC & Solid Oxide Fuel Cell (Fest-Oxid-Brennstoffzelle) \\
\hline SuperCaps & Super Capacitors (Doppelschichtkondensatoren) \\
\hline th & thermisch \\
\hline TSO & Transmission System Operator (Übertragungsnetzbetreiber) \\
\hline TWh & Terawatt-Stunden \\
\hline
\end{tabular}


UCTE Union pour la Coordination du transport de l'Electricité (Union für die Koordination des Transportes elektrischer Energie)

UN United Nations

UNFCCC United Nations Framework Convention on Climate Change

WBGU Wissenschaftlicher Beirat der Bundesregierung Globale Umweltveränderungen

Web-HIPRE Hierarchical Preference analysis on the World Wide Web

WEC World Energy Council

WKA Windkraftanlage 


\section{$1 \quad$ Einleitung}

Die bedarfsgerechte Bereitstellung von Energie ist Grundlage für das Zusammenleben in modernen Industrie-, Informations- und Dienstleistungsgesellschaften und entscheidet mit über Wachstum und Erfolg der Industriestaaten und Schwellenländer. Als hoch entwickeltes Industrieland ist Deutschland auf eine wirtschaftliche, umweltverträgliche und zuverlässige Energieversorgung angewiesen, um Wohlstand, Beschäftigung und wirtschaftliches Wachstum heute und in Zukunft zu gewährleisten. Die derzeitige Energieversorgung in Deutschland beruht auf den fossilen Energieträgern Braun- und Steinkohle sowie Erdgas und Mineralöl, auf der Kernkraft und zu einem kleineren Anteil auf erneuerbaren Energien wie Wasserkraft, Windkraft, Solarenergie, Biomasse und Geothermie. Vor dem Hintergrund des Klimawandels, der zunehmenden Verknappung fossiler Primärenergieträger und zunehmender Importanteile der in Deutschland genutzten fossilen Energieträger sowie der Liberalisierung der Energiemärkte stehen Energiewirtschaft und Politik vor der Herausforderung, den zukünftigen Energieversorgungsmix nachhaltig zu gestalten.

Um die Energieversorgungssicherheit für heutige und zukünftige Generationen zu gewährleisten und gleichzeitig die Treibhausgasemissionen zu reduzieren, sind Systemund Produktinnovationen auf Basis neuer Technologien erforderlich, die zum einen die Energieeffizienz in der Erzeugung erhöhen und den Energiebedarf auf Seiten der Nachfrage reduzieren. Zum anderen ist eine konsequente Erschließung erneuerbarer Energiequellen notwendig. Dazu bedarf es Investitionen in Forschung und Entwicklung, um neue, effiziente Technologien für Erschließung, Übertragung, Speicherung, Umwandlung und Nutzung von Energie zur erproben und zur Anwendung zu bringen (PEPP 2009). Dabei kann weder ein einzelner Energieträger noch eine einzelne Technologie alleine den Anforderungen an eine wirtschaftliche, umweltverträgliche und zuverlässige Energieversorgung gerecht werden. Vielmehr müssen die Möglichkeiten und Vorteile mehrerer Energieträger und Technologien in Form eines adäquaten Energiemix parallel genutzt werden, um das Gesamtsystem bestmöglich gestalten zu können (BMWi 2008; Praetorius et al. 2009).

Andererseits können aufgrund begrenzter Forschungs- und Entwicklungsbudgets nicht alle Technologiepfade uneingeschränkt verfolgt werden. Im Hinblick auf die Allokation von Forschungsmitteln müssen folglich Entscheidungsträger in Energiepolitik und Energiewirtschaft aus der Vielzahl technischer Alternativen zur Strom- und Wärmeversorgung diejenigen ausgewählen, welche den Anforderungen an die Energieversorgung heute und in Zukunft am besten gerecht werden. Einige dieser Alternativen zeichnen sich zumindest bei heutigen Rahmenbedingungen als besonders wirtschaftlich aus, jedoch könnten die mit der Nutzung der gleichen Technologie verbundenen 
Umweltauswirkungen mittel- und langfristig nicht vertretbar sein. Andere, besonders umweltfreundliche Technologien könnten hingegen Nachteile hinsichtlich der Versorgungssicherheit aufweisen. Die Realisierung einer alternativen Technologie könnte wiederum durch Akzeptanz-Probleme in Politik und Gesellschaft erschwert werden. Daher stellt sich die Frage, welche Energietechnologien im Hinblick auf eine nachhaltige Energieversorgung eingesetzt, entwickelt und gefördert werden sollten. Dabei ist insbesondere von Interesse, wie Entscheidungen hinsichtlich der zukünftigen Energieversorgung vor dem Hintergrund teilweise konfliktärer Zielsetzungen in effektiver und nachvollziehbarer Weise unterstützt werden können. Dieser Fragestellung wird in der vorliegenden Arbeit nachgegangen, indem geeignte Methoden zur Entscheidungsunterstützung identifiziert, bestehende Ansätze weiterentwickelt und die Weiterentwicklungen im Rahmen von Fallbeispielen aus der Praxis getestet werden.

Die Relevanz der Fragestellung wird in Kapitel 2 im Kontext wichtiger Rahmenbedingungen für den Energiebereich erläutert. Dazu zählen die Entwicklung von Energienachfrage und -angebot, Entwicklungen auf den Rohstoffmärkten für Energieträger sowie politische und gesetzliche Rahmenbedingungen. Vor dem Hintergrund der beschriebenen Entwicklungstendenzen sind Entscheidungen hinsichtlich der Umgestaltung von Energieversorgungssystemen zu treffen, welche die Strukturen in der Stromund Wärmeversorgung gegebenenfalls über Jahrzehnte festlegen. Eine zukunftsorientierte Entscheidungsunterstützung ist dabei insbesondere aufgrund des hohen Kapitalbedarfs und der langen Lebensdauer von Energietechnologien notwendig. Wie in Kapitel 3 gezeigt wird, steht eine Vielzahl an Energietechnologien zur Bereitstellung von Strom und Wärme zur Verfügung oder befindet sich in Entwicklung. Die wichtigsten Technologiepfade, die heute zur Energieversorgung beitragen bzw. in Zukunft dazu beitragen können, werden dargestellt. Dabei wird deutlich, dass jede der Optionen sowohl Stärken als auch Schwächen aufweist, wobei nicht unmittelbar ersichtlich ist, welche der Technologien unter Beachtung multipler Zielsetzungen zu bevorzugen ist. Somit wird der Bedarf einer multikriteriellen Entscheidungsunterstützung deutlich, bei der mehrere Kriterien simultan berücksichtigt werden können. Vor diesem Hintergrund werden in Kapitel 4 Anwendungen multikriterieller Methoden im Energiebereich vorgestellt und hinsichtlich spezifischer Merkmale analysiert. Diese Analyse zeigt, dass multikriterielle Ansätze für Problemstellungen im Energiebereich bereits etabliert sind und eine solide methodische Basis zur Verfügung steht. Jedoch zählt zu den Schwachstellen bekannter Anwendungen, dass der technologische Wandel bisher nur unzureichend berücksichtigt wird.

Ein Schwerpunkt der vorliegenden Arbeit liegt daher darauf, eine adäquate Berücksichtigung der Veränderung von Energietechnologien im Zeitverlauf im Rahmen der multikriteriellen Entscheidungsunterstützung zu integrieren. Daher werden in Kapitel 5 Me- 
thoden zur Abbildung des technologischen Wandels aufgezeigt und deren Anwendung im Kontext der multikriteriellen Bewertung von Energietechnologien überprüft. Darauf aufbauend wird eine multikriterielle Methode vorgeschlagen, die einen systematischen Vergleich etablierter und innovativer Technologien in Verbindung mit der Berücksichtigung unterschiedlicher Technologiezyklus-Phasen erlaubt. Die Implementierung der Methode in einem Entscheidungsunterstützungssystem wird in Kapitel 6 erläutert. Dieses System besteht aus Komponenten zur Datenverarbeitung und -verwaltung, zur Gewichtung von Kriterien in hierarchisch strukturierten Entscheidungsunterstützungssystemen sowie zur Aggregation der Daten mit Hilfe multikriterieller Bewertungsalgorithmen. Schließlich wird der vorgeschlagene Analyserahmen in Kapitel 7 auf praktische Fallbeispiele aus dem Energiebereich angewendet. Dabei werden zum einen Technologien zur Bereitstellung von Strom und Wärme in Zusammenhang mit einer konkreten Versorgungsaufgabe am Beispiel eines Bioenergiedorfes auf Basis des entwickelten Entscheidungsunterstützungssystems einander gegenübergestellt. Zum anderen wird im Hinblick auf mögliche zukünftige Anwendungsbereiche bzw. Forschungsund Entwicklungsarbeiten ein Vergleich von Stromspeichertechnologien, die sich noch in früheren Technologiezyklus-Phasen befinden, durchgeführt. Abschließend werden Vor- und Nachteile des Analyserahmens sowie Anknüpfungspunkte für weiteren Forschungsbedarf erläutert. 


\section{Rahmenbedingungen für Entscheidungen im Energiebereich}

Zur Abgrenzung des Untersuchungsrahmens werden die energiewirtschaftlichen Rahmenbedingungen mit einem Fokus auf Deutschland dargestellt. Letztlich kann die Energieversorgung jedoch nicht losgelöst von internationalen Zusammenhängen betrachtet werden, da zum einen der Markt für Energietechnologien wie auch für viele Energieträger international ist. Zum anderen sind die Auswirkungen der Energiebereitstellungen häufig globaler Natur und können nicht durch geopolitische Grenzen getrennt werden. Daher werden auch internationale Aspekte der Energieversorgung beleuchtet.

\subsection{Historische Entwicklung der Energieversorgung}

Die heutige Zusammensetzung des Kraftwerksparks wird durch ökonomische, ökologische, regionalwirtschaftliche, soziale und energiepolitische Einflussfaktoren, die teilweise weit zurück in der Vergangenheit liegen, bestimmt (BMWi 2009a). Vor Beginn der industriellen Revolution im 18.Jahrhundert wurde der Energiebedarf nahezu ausschließlich durch erneuerbare Energien gedeckt, beispielsweise Wind- und Wasserkraft oder Biomasse für Heizzwecke (Tester et al. 2005). Noch gegen Ende des 18. Jahrhunderts war Brennholz einer der wichtigsten Energieträger mit strategischer Bedeutung für die Entwicklung der Gesellschaft (Kaltschmitt et al. 2009). Kohlevorkommen wurden in Europa erst erschlossen, als Engpässe in der Holzversorgung auftraten. Bereits damals wurde somit deutlich, dass die örtliche Verfügbarkeit des Brennstoffes ein wesentliches Entscheidungskriterium bei der Auswahl von Energieträgern darstellt. Während Steinkohle zunächst vorwiegend zu Heizzwecken in Haushalten verwendet wurde, verlagerte sich der Schwerpunkt der Anwendung um das Jahr 1840 hin zur industriellen Produktion, beispielsweise zum Einsatz in Eisenhütten. Heute wird der Großteil der weltweit geförderten Kohle in Kraftwerken zur Stromerzeugung eingesetzt (Quaschning 2008).

Die ersten Kraftwerke gingen in den 1880er Jahren in Europa und in den USA in Betrieb (Crastan 2007; Ruttan 2001). Auch in Deutschland wurden mit Kohle befeuerte Dampfmaschinen erstmals Ende des 19. Jahrhunderts zur Stromerzeugung eingesetzt. Eine funktionsfähige Gasturbine wurde erstmals in Frankreich zu Beginn des 20. Jahrhunderts hergestellt. Der erzeugte Strom wurde zunächst lediglich zur Beleuchtung von Straßen und öffentlichen Gebäuden sowie für industrielle Zwecke verwendet. Aufgrund der Erschließung neuer Anwendungsmöglichkeiten für Elektrizität, sinkender Preise für die mit der Anwendung verbundenen Technologien (zum Beispiel für die Glühlampe) sowie sinkender Strompreise nahm die Nachfrage im Privatbereich sowie in Industrie 
und Gewerbe zu, und die bestehenden Kraftwerkskapazitäten mussten erweitert werden (Heuck et al. 2007; Praetorius et al. 2009; Tester et al. 2005). Dadurch wird deutlich, dass die Nachfrage nach bzw. die Bereitstellung von Energie wesentlich durch die damit verbundenen Kosten beeinflusst werden.

Die steigende Stromnachfrage führte zur Notwendigkeit, leistungsfähigere Erzeugungsanlagen zu errichten. Dabei kamen immer weniger Standorte im Stadtgebiet in Frage, zum einen aufgrund des teuren Transports und der Lagerung der Brennstoffe sowie des Bedarfs an erheblichen Wassermengen und zum anderen vor dem Hintergrund von Beschwerden der Anwohner infolge von Rauch, Staub und Lärm. Folglich wirkten sich sowohl ökologische wie auch technische Faktoren auf die weitere Ausgestaltung des Energiesystems auf. Mit der Einführung der Drehstrom-Fernübertragung Anfang der 1890er Jahre stand die Technik zur wirtschaftlichen Übertragung von den Randgebieten in die Stadt zur Verfügung (Heuck et al. 2007; Ruttan 2001). Der Drehstrom wurde zu Umformern in der Stadt übertragen und dort in das Gleichstromnetz eingespeist. Frühe Mikro-Netze wurden nach und nach mit anderen Erzeugungs- und Angebotsinseln verknüpft. Die bis heute dominierende Netzstruktur, die durch zentrale Großkraftwerke und lange Übertragungswege gekennzeichnet ist, wurde später durch große durch den Staat geschützte Firmen aufgebaut. Vom Beginn bis zur zweiten Hälfte des 20. Jahrhunderts blieben diese Strukturen nahezu unverändert. Lediglich die Größe und die thermische Effizienz des Dampfprozesses veränderten sich, da seit den 1950er Jahren durch Fortschritte in der Materialforschung höhere Temperaturen und Prozessdrücke und somit höhere Wirkungsgrade erreicht werden konnten (Dittmann 2009; Praetorius et al. 2009).

Der Schwerpunkt von Innovationen in der Stromwirtschaft lag lange Zeit auf der Einführung neuer, großer und kapitalintensiver Technologien wie beispielsweise der Nuklearenergie. In den 1970er und 1980 Jahren führten die Ölpreiskrisen zu einer Begünstigung von Kernkraftwerken sowie von Stein- und Braunkohlekraftwerken. Dabei blieb die Nachhaltigkeit als Entscheidungskriterium bei der Gestaltung von Energiesystemen weitgehend unberücksichtigt. Nur vereinzelt wurden Umweltprobleme thematisiert, beispielsweise in Zusammenhang mit der Gesundheitsgefährdung durch lokale Luftverschmutzung, saurem Regen, Risiken nuklearer Katastrophenfälle oder Landschaftszerstörung. Aufgrund der Großfeuerungsanlagenverordnung (Bundesregierung 2004a) wurden in den 1980er Jahren Maßnahmen zur Luftreinhaltung, insbesondere zur Entschwefelung und Entstickung der Rauchgase in Kohlekraftwerken, realisiert (Mühlstein und Schirmer 2008; Oeding und Oswald 2004). In den letzten Jahrzehnten hat sich die Wahrnehmung von Umweltproblemen verschärft. Einerseits wird weiterhin durch Primär- und Sekundärmaßnahmen in fossilen Kraftwerken eine Reduzierung der Emissionen angestrebt (z.B. Entschwefelung, Entstickung, Entstaubung, Entaschung, $\mathrm{CO}_{2}$ - 
Abscheidung). Andererseits werden Anstrengungen unternommen, um die Marktdurchdringung erneuerbarer Energien im Vergleich zu fossilen und nuklearen Energieträgern zu erhöhen (Bundesregierung 2009c; Europäische Kommission 2006; Kahle 2009; Praetorius et al. 2009; Ruttan 2001; Konstantin 2009; vgl. auch Abschnitt, 2.3.3, S. 16). Während in den Anfängen der Stromversorgung Elektrizität noch als Luxusgut betrachtet wurde, ist heute das Leben in modernen Industriegesellschaften ohne elektrischen Strom kaum noch denkbar. Die Elektrizitätsversorgung wird als grundlegende Voraussetzung für die Funktionsfähigkeit und Entwicklung der Gesellschaft angesehen (Europäische Kommission 2006; Kahle 2009; Moriarty und Honnery 2009). Dabei wird es nahezu als selbstverständlich erachtet, dass Strom jederzeit an (fast) jedem Ort und zu moderaten Preisen zur Verfügung steht.

Neben der Stromerzeugung resultiert ein Großteil der Energienachfrage aus dem Raum- und Nutzwärmebedarf. Auf dem Heizungsmarkt und in der Industrie wurden bis Mitte der 1980er Jahre vorwiegend (schweres) Heizöl und Kohle als Brennstoffe eingesetzt. Mit der Einführung der Großfeuerungsanlagenverordnung (Bundesregierung 2004a) und der TA Luft (BMU 2002) waren die Anlagenbetreiber zur Einhaltung erhöhter Umweltanforderungen verpflichtet. Daher waren Investitionen in technische Maßnahmen zu Anpassung bestehender Anlagen oder die Umstellung auf den umweltfreundlicheren Energieträger Erdgas erforderlich. In der Folge wurden Erdgasleitungen ausgebaut, und auch der Marktanteil von Erdgas zur Beheizung von Wohngebäuden stieg erheblich an (Konstantin 2009). Somit wirkten sich gesetzlich verankerte Umweltkriterien auf die Umgestaltung des Versorgungssystems aus. Neben verringerten Umweltauswirkungen spielt auch das für die Nutzer von Erdgasheizungen und anderen modernen Systemen zur Beheizung und Klimatisierung erhöhte Komfort-Niveau im Vergleich zu Kohleöfen eine Rolle (Hartmann und Schwarzburger 2009). Heute deckt Erdgas nahezu die Hälfte des Wärmebedarfs in Deutschland (vgl. auch Abbildung 2.9, S. 17). Weitere aktuelle Entwicklungen auf dem Wärmemarkt betreffen die Ziele, die im Erneuerbare-Energien-Wärme-Gesetz (EEWärmeG; vgl. Bundesregierung 2008b) und im Kraft-Wärme-Kopplungsgesetz (KWKG; vgl. Bundesregierung 2002a; Bundesregierung 2008a) zum Ausbau der Kraft-Wärme-Kopplung sowie zum Anteil der erneuerbaren Energien an der Wärmeversorgung festgelegt sind. Zudem ist die zukünftige Entwicklung der Nachfrage zu beachten sowie deren zeitliche und räumliche Verteilung, die stark durch demografische Entwicklungen geprägt wird.

Aufgrund aktueller Entwicklungen steht die Energieversorgung heute vor neuen Herausforderungen. Dabei spielen die Entwicklung des regionalen und globalen Energiebedarfs, die Verfügbarkeit von Energieträgern, Umweltaspekte, insbesondere der Klimawandel, sowie gesetzliche Rahmenbedingungen eine bedeutende Rolle. Diesbezüglich werden im Folgenden die wichtigsten Entwicklungstendenzen aufgezeigt. 


\subsection{Energiebedarf und Nachfrage nach Energieträgern}

Gemessen am Energiebedarf und an der Stromerzeugung stellt Deutschland einen der größten Energiemärkte weltweit dar (Kahle 2009; Schiffer 2008). Bis Ende der 1970er Jahre nahm die Energienachfrage stetig zu, und eine enge Kopplung von Wirtschaftswachstum und Energiebedarf galt weitgehend als unvermeidbar. Bemühungen zur Einsparung von Energie sowie zur Nutzung erneuerbarer Energieträger wurden erst infolge der Ölkrisen in den 1970er Jahren unternommen. Aufgrund sinkender Ölpreise in den Folgejahren wurden diese Ansätze zunächst jedoch wieder zurückgedrängt. Erst seit Anfang der 1980er Jahre, als die Energienachfrage trotz anhaltenden Wirtschaftswachstums stagnierte, setzte sich die Erkenntnis durch, dass steigender Wohlstand nicht zwangsläufig mit einem höheren Energieverbrauch einhergeht (Europäische Kommission 2006; Krewitt et al. 2009; Quaschning 2008). Infolge der Wiedervereinigung sank Anfang der 1990er Jahre der Gesamtenergiebedarf Deutschlands insbesondere aufgrund der Stilllegung großer Wirtschaftsbereiche in der ehemaligen DDR (Quaschning 2007). Seit einigen Jahren ist die Energienachfrage in Deutschland nahezu stabil und hat sich weitgehend vom Wirtschaftswachstum entkoppelt (Abbildung 2.1). Jedoch zeichnet sich trotz vermehrter Anstrengungen auf dem Gebiet regenerativer Primärenergienutzung keine signifikante Senkung des Bedarfs an fossilen und nuklearen Energieträgern ab.

Abbildung 2.1: Primärenergieverbrauch in Deutschland nach Energieträgern

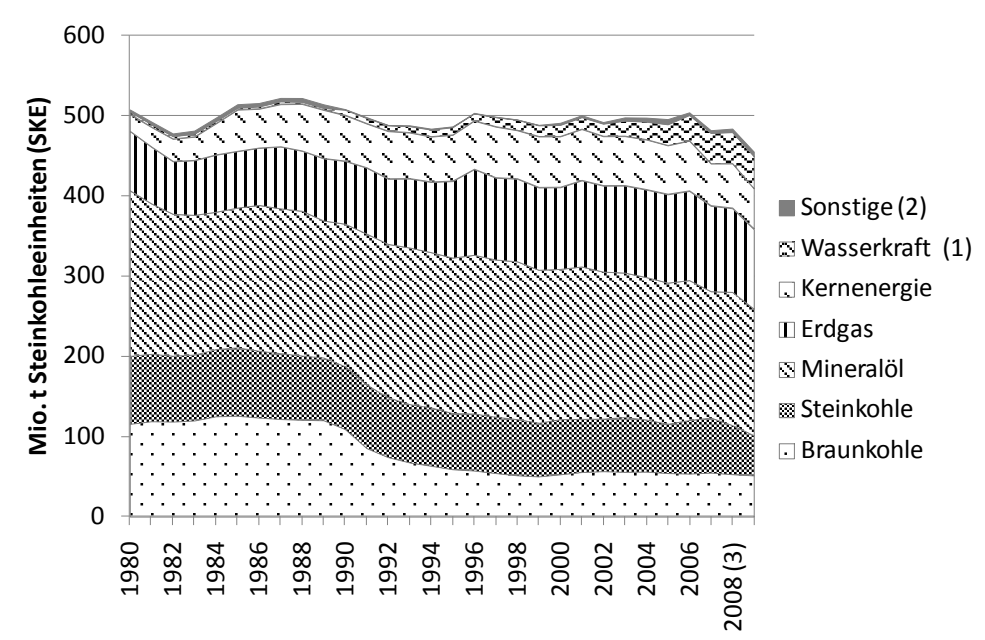

Anmerkungen: (1) Einschließlich Außenhandelssaldo Strom; ab 1995: einschließlich Windenergie und Photovoltaik. (2) Bis 1989: Brennholz, Brenntorf, Klärschlamm, Müll und sonstige Gase; ab 1990: Grubengas, nicht-erneuerbare Abfälle und Abwärme, Pumperzeugung Wasser sowie Stromaußenhandelssaldo. (3) Vorläufige Werte (2007-2009).

Quelle: Basierend auf AGEB (2010). 
Neben den mit dem Abbau und der Verwendung fossiler und nuklearer Energieträger verbundenen negativen Umwelt- und Gesundheitsauswirkungen scheint der hohe Bedarf an fossilen und nuklearen Primärenergieträgern insbesondere vor dem Hintergrund der begrenzten Reichweite dieser Rohstoffe problematisch (Abbildung 2.2). Die Situation wird darüber hinaus erschwert durch die hohe Importabhängigkeit Deutschlands bei fossilen und nuklearen Energieträgern (Abbildung 2.3). Zudem ist davon auszugehen, dass insbesondere die zukünftige Öl- und Gasversorgung in zunehmendem Maße von einer kleiner werdenden Anzahl politisch und ökonomisch instabilen Förderregionen abhängen wird (Cramer et al. 2009; IEA 2009c; Kahle 2009). Folglich werden Probleme bezüglich der Sicherheit der Versorgung mit diesen Energieträgern wahrscheinlicher. Der Bedarf an Braunkohle wird zwar vollständig aus heimischen Reserven gedeckt, die eine hohe Reichweite aufweisen. Jedoch ist der Einsatz dieses Energieträgers vor dem Hintergrund des Klimawandels (vgl. Abschnitt 2.3.2, S. 13) insbesondere aufgrund des hohen spezifischen $\mathrm{CO}_{2}$-Gehaltes kritisch zu hinterfragen. Zudem sind, mit Ausnahme der Braunkohle, tendenziell steigende Preise fossiler Energieträger sowie eine zunehmende Volatilität der Preisentwicklungen zu beobachten (Abbildung 2.4). Setzt sich dieser Trend in Zukunft fort, ist auch die Wirtschaftlichkeit der Bereitstellung von Strom und Wärme auf Basis dieser Energieträger in zunehmendem Maße mit Unsicherheiten behaftet (BMWi 2009a; Rempel et al. 2007).

Abbildung 2.2: $\quad$ Statische Reichweite nuklearer und fossiler Primärenergieträger

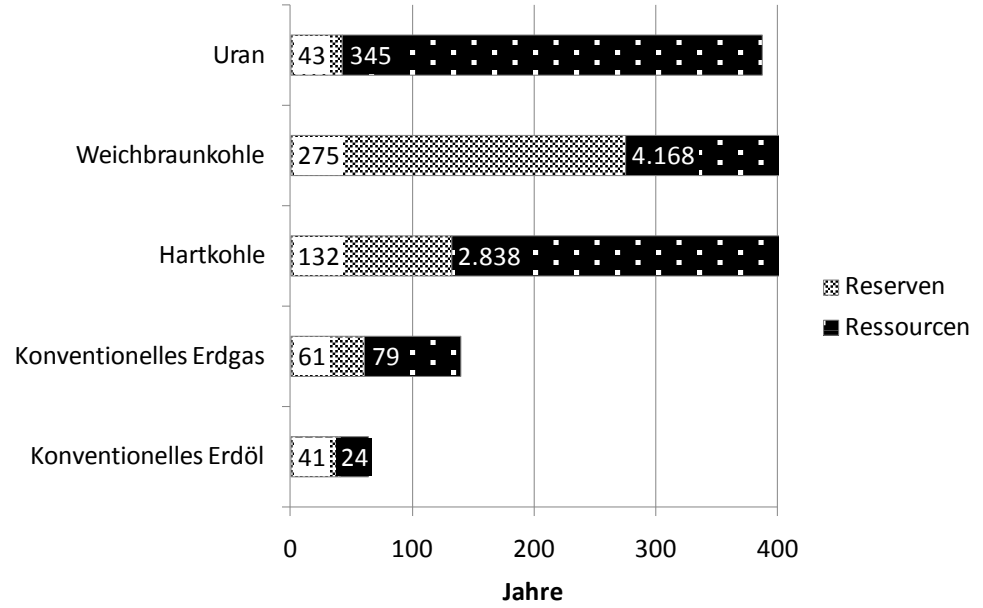

Anmerkungen: Die Darstellung basiert auf Angaben zu den weltweiten Fördermengen 2007 und den bis Ende 2007 festgestellten Reserven (das heißt den nachgewiesenen und wirtschaftlich gewinnbaren Vorkommen) bzw. Ressourcen (das heißt den zusätzlich nachgewiesenen, aber zurzeit nicht wirtschaftlich gewinnbaren Vorkommen)

Quelle: Basierend auf Cramer et al. (2009). 
Abbildung 2.3: Anteil der Nettoimporte an den in Deutschland eingesetzten Primärenergieträgern

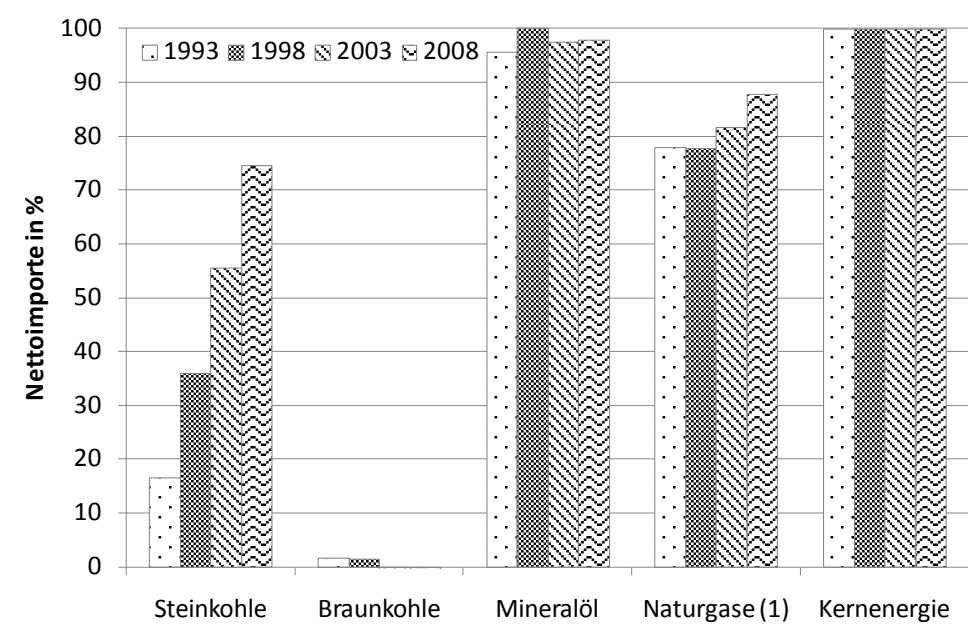

Anmerkungen: (1) Einschließlich Erdgas, Erdölgas und Grubengas.

Quelle: Basierend auf BMWi (2010).

Abbildung 2.4: Grenzübergangspreise für fossile Energierohstoffe

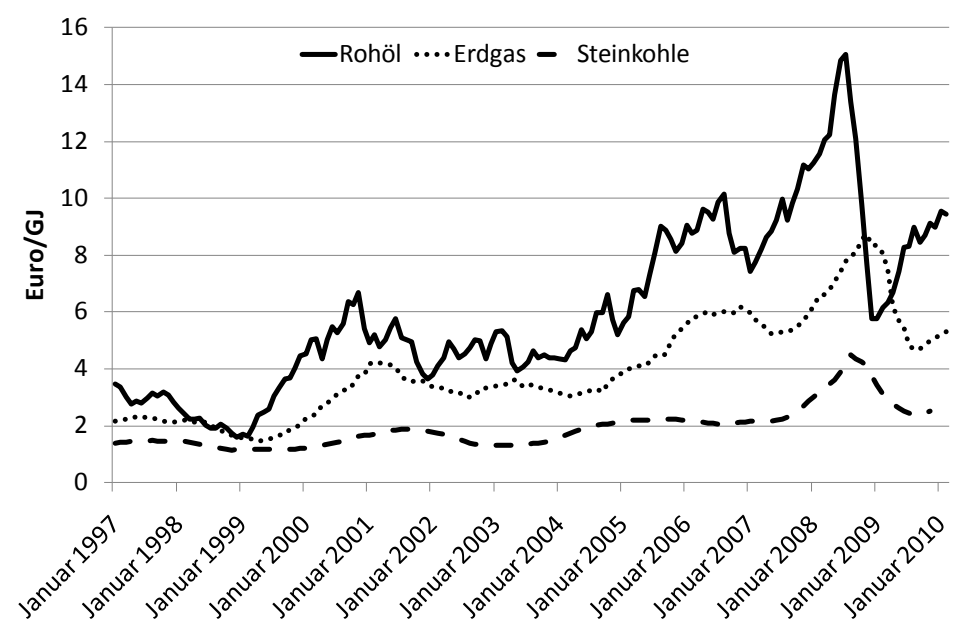

Anmerkungen: Basierend auf monatlichen Angaben für Rohöl und Erdgas bzw. Quartalswerten für Steinkohle. Preise von Erdgas beziehen sich auf den oberen Heizwert des Energieträgers. Quelle: Basierend auf BAFA (2009a); BAFA (2009b); BMWi und BAFA (2009).

Obwohl die Problematik der Energieversorgung der Weltbevölkerung unter energetischen, ökonomischen und ökologischen Gesichtspunkten zunehmend ins Blickfeld der Öffentlichkeit rückt, führt dies bisher nicht zu einer Senkung des weltweiten Primärenergieverbrauchs. Auch in den nächsten Jahrzehnten rechnen nahezu alle vorliegenden globalen Szenarien mit einem weiteren Anstieg des Primärenergieverbrauchs, vor allem bei den fossilen Energieträgern (Moriarty und Honnery 2009; vgl. zum Beispiel Abbildung 2.5). Die prognostizierten Nachfragesteigerungen sind in erster Linie durch 
das erwartete Anwachsen der Weltbevölkerung sowie den zunehmenden Lebensstandard und das Wirtschaftswachstum in den aufstrebenden Entwicklungs- und Schwellenländern, wie insbesondere China und Indien, zu begründen (Kahle 2009; UNPD 2009). Damit einhergehend ist mit einem weiteren Wachstum des Energiebedarfs pro Kopf zu rechnen.

Abbildung 2.5: Entwicklung des Weltprimärenergieverbrauchs im ReferenzSzenario der IEA und der Weltbevölkerung

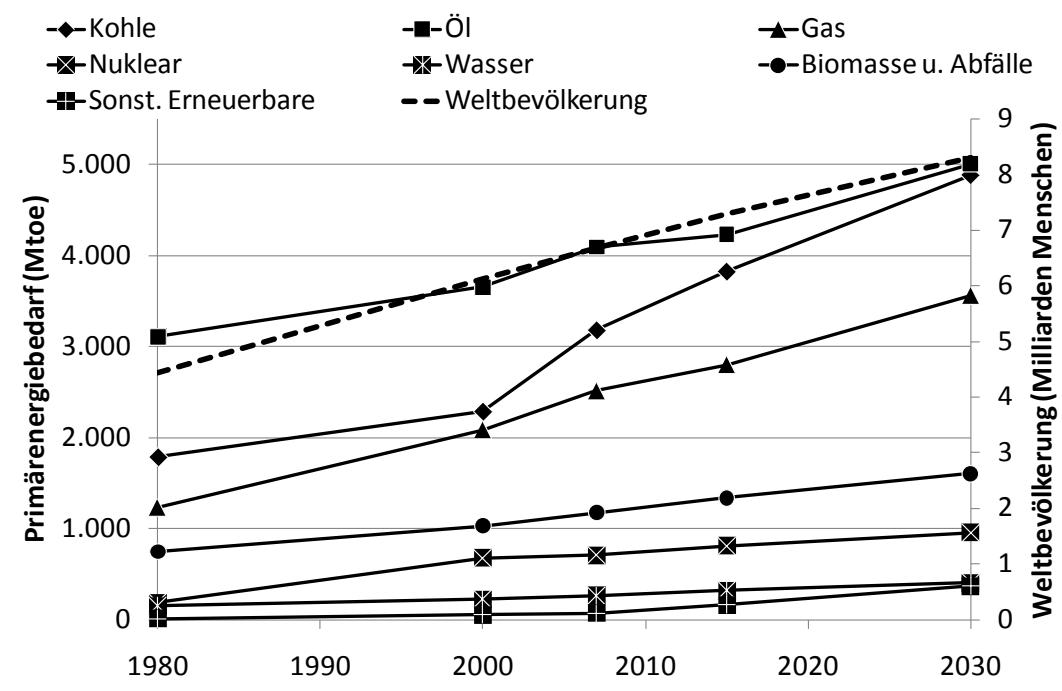

Quelle: Basierend auf IEA (2009c); UNPD (2009).

Da ein Großteil der Primärenergieträger zur Deckung des Bedarfs an Strom und Wärme in der Industrie, im Bereich Gewerbe, Handel und Dienstleistungen sowie in Privathaushalten eingesetzt wird (Abbildung 2.6), können Maßnahmen für eine nachhaltigere Energieversorgung, neben dem Verkehrssektor, vor allem in diesen Sektoren ansetzen. Der größte Anteil des Endenergiebedarfs ist auf den Wärmebereich zurückzuführen, wo hohe Potenziale zur Energieeinsparung und zur effizienteren Energienutzung liegen (BMU 2009c). Der Anteil von Strom am Endenergiebedarf scheint zwar relativ gering, jedoch ist nahezu die Hälfte der energiebedingten Treibhausgasemissionen auf diesen Strombedarf zurückzuführen (BMU 2009d). Maßnahmen zur Reduzierung des Endenergiebedarfs und zur Minderung der Umweltauswirkungen können einerseits auf Seiten der Nachfrage von Strom und Wärme umgesetzt werden. Beispielsweise können in der industriellen Produktion und in Haushaltsgeräten hocheffiziente Technologien eingesetzt werden. Andererseits kann auf der Angebotsseite der Bedarf an Primärenergieträgern sowie die durch die Bereitstellung von Strom und Wärme verursachten Umweltauswirkungen reduziert und die Versorgungssicherheit erhöht werden. So tragen zum Beispiel Wirkungsgradsteigerungen in fossil befeuerten Kraftwerken sowohl zu Einsparungen bei den Brennstoffkosten als auch zu einem geringeren 
Schadstoffausstoß bei. Weiterhin werden bei geringerem Brennstoffbedarf Abhängigkeiten von importierten Brennstoffen reduziert. Ebenso kann ein verstärkter Einsatz erneuerbarer Energien die Versorgungssicherheit und das Umweltschutzniveau erhöhen sowie Importabhängigkeiten und Risiken hinsichtlich der Brennstoffpreise reduzieren. Die vorliegende Arbeit konzentriert sich darauf, welche Maßnahmen auf der Angebotsseite der Bereitstellung von Strom und Wärme zu einer effizienteren, umweltfreundlichen und sicheren Energieversorgung beitragen können. Dies betrifft vor allem Technologien zur Umwandlung von Primär- bzw. Sekundärenergieträgern in Nutzbzw. Endenergie in Form von Strom und Wärme. Inwieweit die politischen Rahmenbedingungen in diesem Zusammenhang von Bedeutung sind, wird im Folgenden skizziert.

Abbildung 2.6: Endenergieverbrauch nach Sektoren und Anwendungsbereichen in Deutschland im Jahr 2007

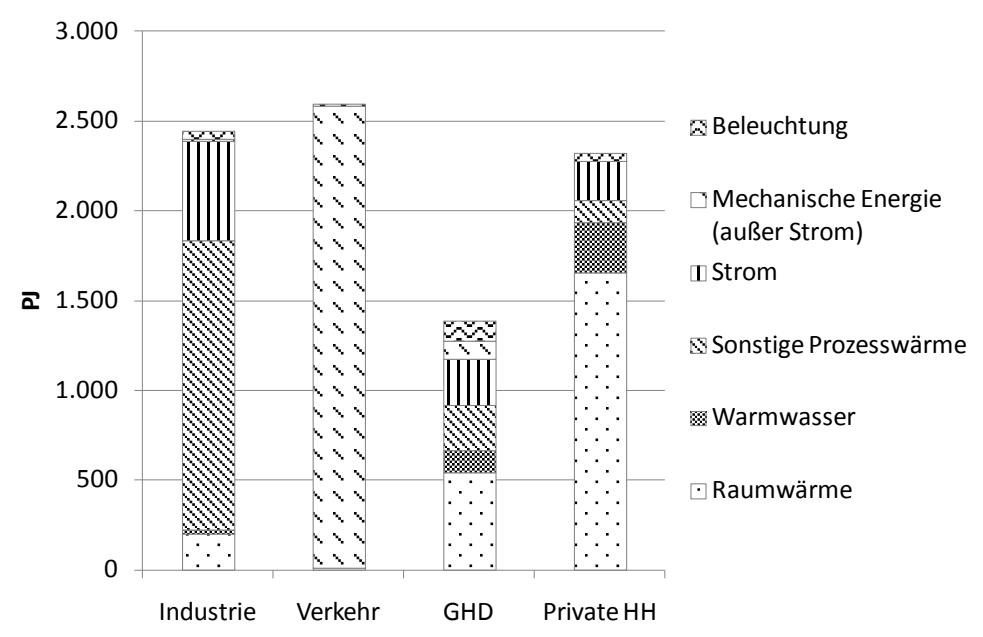

Quelle: Basierend auf BMWi (2010).

\subsection{Politische und gesetzliche Rahmenbedingungen}

Im Energiebereich spielen nationale, europäische und internationale Bestimmungen eine Rolle. Dabei werden rechtliche Rahmenbedingungen für den Energiesektor in Deutschland in zunehmendem Maße durch Vorgaben auf EU-Ebene determiniert. Die wesentlichen Schwerpunkte der europäischen Energiepolitik zielen ab auf eine sichere, umweltschonende und kostengünstige Energieversorgung, die auf einem Wettbewerbsmarkt basiert (Konstantin 2009). Zu den wichtigsten politischen Entwicklungen zählen die Liberalisierung der Energiemärkte, Maßnahmen vor dem Hintergrund des Klimawandels, der Beschluss zum Kernenergieausstieg sowie die Förderung des Ausbaus erneuerbarer Energien und der Kraft-Wärme-Kopplung. 


\subsubsection{Liberalisierung der Energiemärkte}

Die europäischen Richtlinien zur Liberalisierung des Elektrizitäts- und des Gasbinnenmarktes (Europäisches Parlament und Rat der Europäischen Union 2003a; Europäisches Parlament und Rat der Europäischen Union 2003b) wurden in Deutschland durch das Gesetz über die Elektrizitäts- und Gasversorgung (Energiewirtschaftsgesetz - EnWG) umgesetzt. Dieses wurde im zweiten Gesetz zur Neuregelung des Energiewirtschaftsrechts vom 7. Juli 2005 beschlossen (Bundesregierung 2005). Durch das EnWG in Verbindung mit mehreren Verordnungen wurden die Energiemärkte für den Wettbewerb freigegeben, um freien Handel zu ermöglichen. Gemäß $§ 1$ Abs. 1 EnWG liegt der Zweck des Gesetzes darin, „eine möglichst sichere, preisgünstige, verbraucherfreundliche, effiziente und umweltfreundliche leitungsgebundene Versorgung der Allgemeinheit mit Elektrizität und Gas" zu gewährleisten. Folglich sollten sich Entscheidungen im Energiebereich auf mehrere Kriterien stützen, die diesen vielfältigen Aspekten Rechnung tragen. Um den Zweck des EnWG umzusetzen, zielt die Liberalisierung darauf $a b$, dass die Verbraucher ihren Versorger frei wählen können, dass freier Zugang zu den Übertragungs- und Verteilnetzen besteht und dass die Bereiche Erzeugung, Netz und Handel soweit wie möglich voneinander getrennt organisiert sind („Unbundling"). Die Bundesnetzagentur (BNetzA) wurde als Regulierungsbehörde ins Leben gerufen, um die Einhaltung der Wettbewerbsbedingungen zu überwachen (Konstantin 2009).

Trotz des Inkrafttretens der entsprechenden Gesetze hat sich noch kein ausreichender Wettbewerb auf den Energiemärkten eingestellt. Die Europäische Kommission kritisiert insbesondere, dass viele Märkte noch immer von einigen wenigen Unternehmen dominiert werden und vorwiegend national orientiert sind, sowie den unzureichenden Verbundgrad zwischen den Mitgliedstaaten (Europäische Kommission 2006). Dennoch haben sich das Entscheidungsverhalten der Akteure sowie deren Netzwerke im liberalisierten Strommarkt bereits geändert. Neue Akteure wie beispielsweise Stromhändler oder kleinere Versorgungsunternehmen treten in den Markt ein und verschwinden zum Teil wieder. Auch auf dem Gasmarkt tritt eine zunehmende Anzahl an Wettbewerbern auf. Denn ähnlich wie auf den Strommärkten wird auch auf den Märkten für Erdgas eine Liberalisierung angestrebt, indem Produktion, Netzbetrieb und Handel voneinander getrennt werden und freier Netzzugang für alle Marktteilnehmer ermöglicht wird. Somit soll freier Wettbewerb nicht nur zwischen Erdgas und konkurrierenden Energieträgern, sondern auch zwischen Erdgasanbietern gewährleistet werden (Konstantin 2009). Während sich die Versorgungsunternehmen aufgrund des intensivierten Wettbewerbs mit neuen Risiken und Chancen konfrontiert sehen, steht den Konsumenten ein erweitertes Angebot durch eine wachsende Zahl von Anbieten zur Verfügung (Praetorius et al. 2009). Werden die politischen Maßnahmen zur Durchsetzung des 
europäischen Elektrizitäts- und Erdgasbinnenmarktes intensiviert, ist eine Verstärkung dieser Tendenzen zu erwarten.

\subsubsection{Klimapolitik und Handel mit Emissions-Zertifikaten}

Eine Motivation zur Umgestaltung der Energieversorgung ergibt sich nicht nur aus ökonomischen Gründen, sondern auch aufgrund der mit der Energiebereitstellung verbundenen Umwelt- und Gesundheitsauswirkungen. Diese können den Lebensstandard in Industrie-, Entwicklungs- und Schwellenländern langfristig erheblich gefährden (vgl. zum Beispiel Tester et al. 2005). Etwa zwei Drittel der weltweiten Treibhausgasemissionen sind durch den Energiebedarf in unterschiedlichen Sektoren bedingt. Daher kommt der Energiebereitstellung und -nutzung speziell vor dem Hintergrund des Klimawandels und der damit verbundenen politischen Maßnahmen eine besondere Bedeutung zu (IEA 2009b). Dabei wird die globale Erderwärmung vor allem auf die $\mathrm{CO}_{2}$ Konzentration in der Erdatmosphäre zurückgeführt (neben anderen Treibhausgasen wie zum Beispiel $\mathrm{CH}_{4}$ oder $\mathrm{N}_{2} \mathrm{O}$ ). Diese ist seit Ende des 19. Jahrhunderts, als fossile Energien im Rahmen der Industrialisierung in zunehmendem Maße Verwendung fanden, deutlich angestiegen (Tester et al. 2005). Ein Großteil der $\mathrm{CO}_{2}$ - und anderer Emissionen ist auch in Deutschland auf den Energiebedarf zurückzuführen (Abbildung 2.7).

Abbildung 2.7: Beiträge der Sektoren zu unterschiedlichen Emissionsarten in Deutschland im Jahr 2007

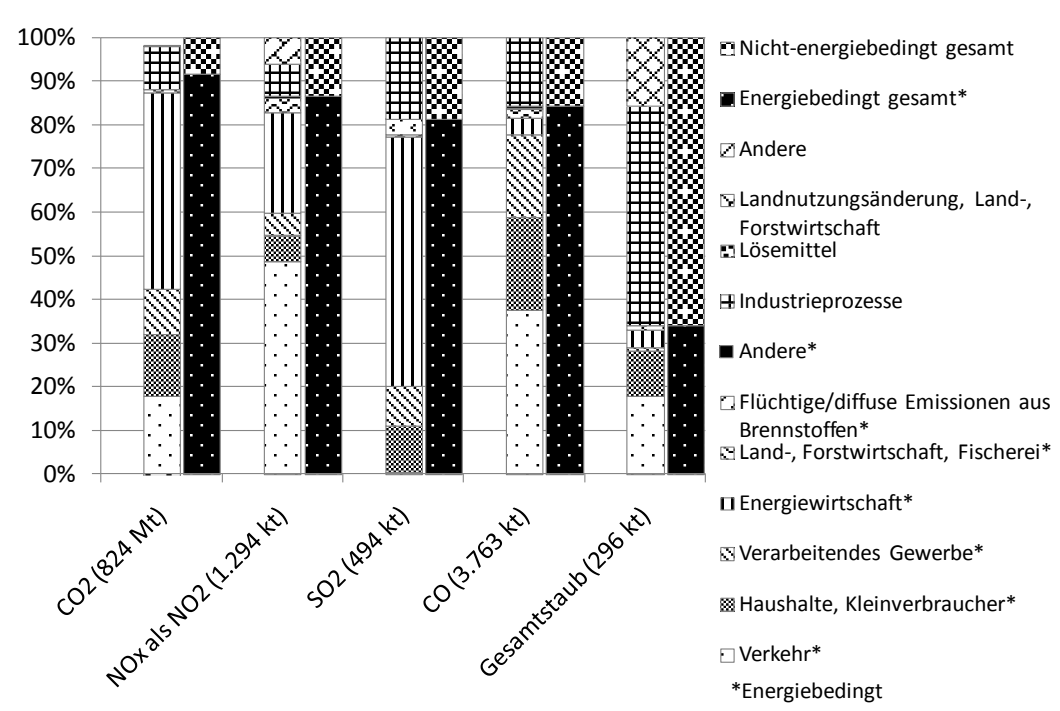

Anmerkungen: Für $\mathrm{CO}_{2}$-Emissionen sind $<100 \%$ ausgewiesen, da durch Landnutzungsänderung und Forstwirtschaft $\mathrm{CO}_{2}$ aus der Atmosphäre aufgenommen wird, welches in der Statistik als negative Emissionen ausgewiesen ist.

Quelle: Basierend auf BMWi (2010). 
In der Wissenschaft besteht weitgehend Einigkeit darüber, dass die globale Erderwärmung auf $2{ }^{\circ} \mathrm{C}$ begrenzt werden muss, um gravierende und irreversible Schäden durch den Klimawandel zu vermeiden. Schätzungen zufolge ist eine Stabilisierung der Konzentration von Treibhausgasemissionen in der Atmosphäre bei etwa $450 \mathrm{ppm}$ an $\mathrm{CO}_{2}$ Äquivalenten erforderlich, damit eine Temperaturerhöhung um maximal $2^{\circ} \mathrm{C}$ wahrscheinlich ist (den Elzen und Meinshausen 2006; Meinshausen 2006). Um dieses Ziel zu verwirklichen, müssen die Industrie- und Schwellenländer bis zum Jahr 2020 ihre Treibhausgasemissionen um 25 bis $40 \%$ bzw. bis zum Jahr 2050 um 80 bis $95 \%$ unter das Niveau von 1990 senken (IPCC 2007a).

Vor dem Hintergrund der befürchteten wirtschaftlichen und sozialen Folgen des Klimawandels wurden verpflichtende internationale Abkommen zur Begrenzung der Treibhausgasemissionen erarbeitet. Bereits im Jahr 1992 wurde auf dem Weltgipfel für Umwelt und Klima in Rio de Janeiro die Klimarahmenkonvention (United Nations Framework Convention on Climate Change - UNFCCC; vgl. UN 1992) beschlossen. Darin wurde erstmals im Rahmen einer internationalen Vereinbarung der Klimawandel als ernst zu nehmendes Problem diskutiert. Die unterzeichnenden Staaten haben sich dazu verpflichtet, Maßnahmen zur Senkung der Treibhausgasemissionen zu ergreifen und regelmäßig darüber Bericht zu erstatten. Im Rahmen der Weltklimakonferenz im japanischen Kyoto wurde im Jahr 1997 das Kyoto-Protokoll als Zusatzprotokoll zur Klimarahmenkonvention beschlossen. Darin verpflichten sich eine Reihe von Industrieländern im Zeitraum von 2008 bis 2012 ihre Treibhausgasemissionen im Schnitt um $5,2 \%$ im Vergleich zu 1990 zu senken (UN 1998). Im Juli 2009 sprachen sich auf dem G8-Gipfel in L'Aquila die führenden Staaten zu einer Vereinbarung für den Zeitraum nach 2012 aus und bekannten sich zum $2{ }^{\circ} \mathrm{C}$-Ziel und der damit verbundenen notwendigen weltweiten Reduzierung der Treibhausgasemissionen um $50 \%$ im Vergleich zum Jahr 1990 (G8 2009). Auf der 15. COP (Conference of the Parties) in Kopenhagen (7. bis 18. Dezember 2009) sollten die Verhandlungen über Emissionsminderungsziele für den Zeitraum nach 2012 abgeschlossen werden. Jedoch konnten sich die Vertragsparteien nicht auf verbindliche Ziele zur Emissionsminderung einigen. Im „Copenhagen Accord“ wird jedoch zumindest das Ziel bekräftigt, die mittlere globale Temperaturerhöhung durch entsprechende Maßnahmen auf höchstens $2{ }^{\circ} \mathrm{C} z u$ begrenzen (UN 2009).

Unabhängig von übergeordneten internationalen Vereinbarungen hat sich die EU verpflichtet, ihre Emissionen bis 2020 um 20 \% im Vergleich zu 1990 zu reduzieren. Sofern ein internationales Nachfolgeabkommen unter Beteiligung aller Industrieländer und fortgeschrittener Entwicklungsländer mit vergleichbaren Minderungsanstrengungen zustande kommt, hat sich die EU darüber hinaus bereit erklärt, dieses Ziel auf $30 \% \mathrm{zu}$ erhöhen (Europäische Kommission 2009; Europäischer Rat 2007). Die deutsche Bun- 
desregierung hat zudem im Koalitionsvertrag von Oktober 2009 ihr Ziel bekräftigt, bis 2020 die Treibhausgasemissionen in Deutschland um 40 \% unter das Niveau von 1990 zu senken (Bundesregierung 2009c).

Von Bedeutung für die Energiewirtschaft in Zusammenhang mit den internationalen Klimaabkommen und den damit verbundenen nationalen Verpflichtungen ist insbesondere der internationale Handel mit Emissionszertifikaten, der im Kyoto-Protokoll als flexibler Mechanismus zur Erreichung der verpflichtenden Emissionsminderung festgelegt ist (UN 1998). Um den internationalen Emissionshandel vorzubereiten, wurde im Jahr 2005 das europäische Emissionshandelssystem (engl. EU Emissions Trading Scheme - EU-ETS) eingeführt (Europäisches Parlament und Rat der Europäischen Union 2003c; Europäisches Parlament und Rat der Europäischen Union 2009). Derzeit läuft bereits die zweite Handelsperiode von 2008 bis 2012, entsprechend der ersten Verpflichtungsperiode des Kyoto-Protokolls. Die dritte und vierte Handelsperiode sind für 2013 bis 2020 bzw. 2021 bis 2028 geplant (Konstantin 2009).

In den ersten zwei Handelsperioden wurde die europäische Richtlinie zum Emissionshandel auf nationaler Ebene insbesondere durch die Nationalen Allokationspläne (NAP) umgesetzt. Diese setzten das nationale Emissionsbudget für $\mathrm{CO}_{2}$ für die Emissionshandelssektoren fest und teilen den emissionshandelspflichtigen Anlagen 1 eine gewisse Menge an Emissionsberechtigungen (EB bzw. EUA - EU Emission Allowance) zu (BMU 2006). Erhält ein Unternehmen weniger Emissionszertifikate als es benötigt, müssen entweder die Emissionen reduziert oder zusätzliche Zertifikate käuflich erworben werden. Somit zielt der Emissionshandel darauf ab, bei einer festen Gesamtmenge an Emissionen Anreize für Emissionsminderungen dort zu setzen, wo sie besonders günstig sind. Dies kann nach Praetorius et al. (2009) im Kraftwerksbereich beispielsweise durch Änderungen hin zu dezentraleren Strukturen und den Einsatz hocheffizienter Technologien erreicht werden. Die Novellierung der Emissionshandelsrichtlinie für die Handelsperiode 2013 bis 2020 sieht unter anderem eine Einbeziehung weiterer Treibhausgase zusätzlich zu $\mathrm{CO}_{2}$, die Berücksichtigung von CCSAnlagen, eine europaweite Allokation der Zertifikate sowie eine vollständige Versteigerung der Zertifikate im Stromsektor ab 2013 vor (Europäisches Parlament und Rat der Europäischen Union 2009).

Um unter Beachtung der Zielgrößen zur Senkung der Treibhausgasemissionen auch in Zukunft Wirtschaftswachstum, Sicherheit und Komfort sicherzustellen, sind politische Maßnahmen und technische Innovationen in allen Sektoren erforderlich, insbesondere

1 Dies betrifft in der laufenden Handelsperiode von 2008 bis 2012 Anlagen zur Energieerzeugung, Erdgasverdichter sowie Anlagen in der energieintensiven Industrie. 
im Energiebereich. Dabei tragen in den meisten Szenarien zur Stabilisierung der $\mathrm{CO}_{2}$ Emissionen die Sektoren Energiebereitstellung und -nutzung sowie industrielle Prozesse mit 60 bis $80 \%$ zur Emissionsminderung bei (IPCC 2007b). Deutschland versteht sich in diesem Zusammenhang als Impuls- und Innovationsgeber mit dem Ziel das Umweltschutzniveau durch neue Technologien zu verbessern (Bundesregierung 2009c). Ausgehend von den internationalen Vereinbarungen hat die Bundesregierung in Ergänzung zum europäischen Handel mit Emissionszertifikaten Ende 2007 das so genannten Energie- und Klimaprogramm (IEKP) beschlossen (auch bekannt als "Meseberg-Programm“, vgl. BMWi und BMU 2007; Bundesregierung 2007). Dieses beschreibt sektor- und technologiespezifische Maßnahmen, die eine moderne, sichere und klimaverträgliche Energieversorgung in Deutschland ermöglichen soll. Bei Umsetzung des Meseberg-Programms können die Treibhausgasemissionen um $34 \%$ gesenkt werden. Um das $40 \%$-Ziel zu erreichen, schlagen Jochem et al. (2008) weitere Maßnahmen vor. Neben dem Privat- und Gewerbebereich, dem Verkehrssektor, der Industrie sowie sektorübergreifenden Maßnahmen spielt hierbei auch der Energieumwandlungssektor eine bedeutende Rolle. Dies betrifft vor allem den Ausbau der KraftWärme-Kopplung sowie der erneuerbaren Energien im Strom- und Wärmebereich. Diese Maßnahmen werden im Wesentlichen durch technologische Optionen zur Gestaltung der Energieversorgung konkretisiert. Des Weiteren hat die Bundesregierung die Erarbeitung eines energiepolitischen Gesamtkonzeptes bis Herbst 2010 angekündigt (Bundesregierung 2009c; Bundesregierung 2010). Um beurteilen zu können, inwiefern die verfügbaren Optionen zu einer sicheren, umweltfreundlichen und wirtschaftlichen Energieversorgung beitragen, ist ein wissenschaftlicher Ansatz erforderlich, der die Berücksichtigung mehrerer Zielsetzungen erlaubt.

\subsubsection{Erneuerbare Energien und Kraft-Wärme-Kopplung}

Die Erhöhung des Anteils erneuerbarer Energien und der Ausbau der Kraft-WärmeKopplung sind wesentliche Eckpunkte des integrierten Energie- und Klimaprogramms (IEKP) der Bundesregierung (Bundesregierung 2007). Auf EU-Ebene ist die Förderung erneuerbarer Energien zur Stromerzeugung in der Richtline 2001/77/EG geregelt (Europäisches Parlament und Rat der Europäischen Union 2003a). Europäische Regelungen zur Kraft-Wärme-Kopplung sind in der Richtlinie 2004/8/EG zu finden (Europäisches Parlament und Rat der Europäischen Union 2004). Bereits vor der europäischen Richtlinie zur Förderung erneuerbarer Energien trat in Deutschland im Jahr 1991 das Stromeinspeisungsgesetz (Bundesregierung 1990) und im Jahr 2000 die erste Fassung des Erneuerbare-Energien-Gesetzes (EEG) in Kraft (Bundesregierung 2000). Im Jahr 2004 wurde die erste Novelle des EEG beschlossen (Bundesregierung 2004b). Das darin festgelegte Ziel, den Anteil erneuerbarer Energien an der Stromver- 
sorgung auf mindestens 12,5 \% im Jahr 2010 zu erhöhen, wurde bereits im Jahr 2007 übertroffen, als die erneuerbaren Energien mit über $13 \%$ zur Bruttostromerzeugung beitrugen (BMWi 2010).

Abbildung 2.8: Anteile der Energieträger an der Stromerzeugung in Deutschland im Jahr 2008 (gesamt: 639 TWh)

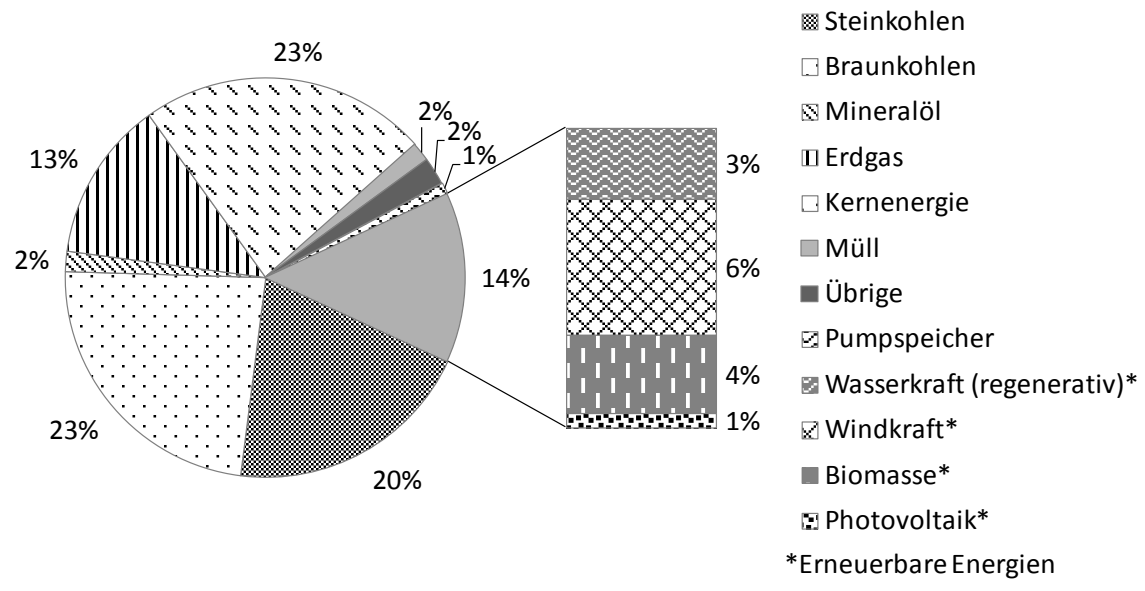

Quelle: Basierend auf BMWi (2010).

Abbildung 2.9: Anteile der Energieträger an der Wärmeversorgung in Deutschland im Jahr 2008 (gesamt: 1.554 TWh)

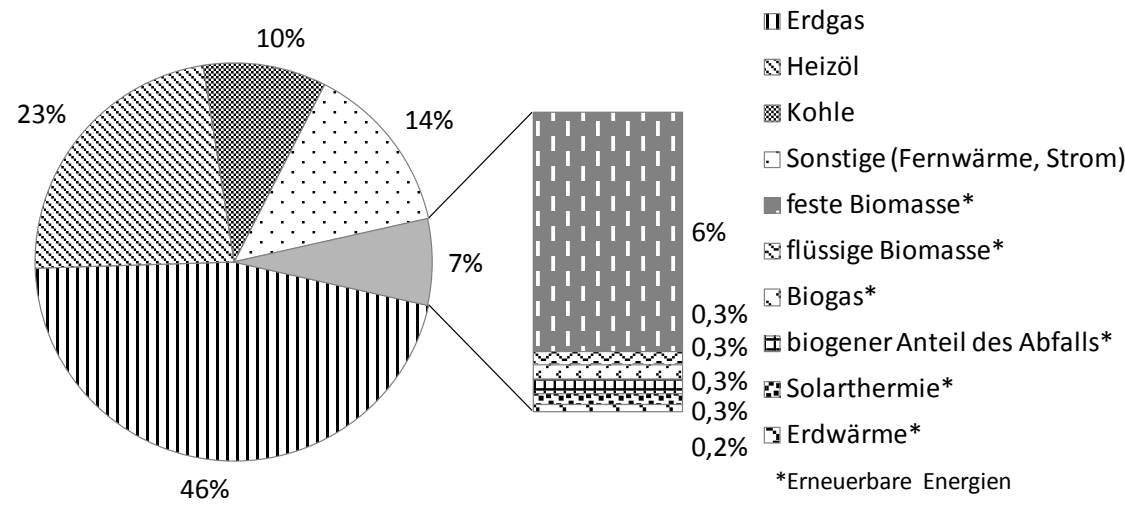

Quelle: Basierend auf BMU (2009c).

Im Jahr 2008 lag der Anteil erneuerbarer Energien an der Stromerzeugung bei ca. $14 \%$ und an der Wärmeerzeugung bei ca. $7 \%$ (vgl. Abbildung 2.8 bzw. Abbildung 2.9). Bis 2020 soll der Anteil an der Stromversorgung auf mindestens $20 \%$ erhöht werden (vgl. Art. 1 Abs. 2, EEG2004). Im Rahmen des integrierten Energie- und Klimaprogramms der Bunderegierung wurde das EEG zum zweiten Mal novelliert und ist in seiner aktuellen Fassung am 1. Januar 2009 in Kraft getreten (Bundesregierung 
2008c). Darin wurde die Zielvorgabe auf einen Anteil von $30 \%$ an der Stromversorgung erhöht (vgl. §1 Abs. 2 EEG2008).

Für Strom aus Anlagen, die mit erneuerbaren Energien betrieben werden, sind durch das EEG feste Einspeisevergütungen garantiert. Diese gelten vom Jahr der Inbetriebnahme der Anlage für 20 Jahre und beziehen sich auf die in die öffentlichen Netze eingespeisten Strommengen. Darüber hinaus sieht das EEG2008 in bestimmten Fällen auch dann eine Vergütung vor, wenn der erzeugte Strom vor Ort genutzt und nicht ins Netz eingespeist wird. Je später eine Anlage in Betrieb genommen wird, desto geringer sind die Einspeisevergütungen, das heißt von Jahr zu Jahr sinken die Fördersätze für Neuanlagen degressiv. Dies wird begründet durch technischen Fortschritt und die Verringerung von Herstellkosten der Technologien aufgrund von Skaleneffekten. Neben der festen Einspeisevergütung legt das EEG Boni fest, die für die Nutzung von Wärme aus KWK-Anlagen auf Basis von Biomasse oder Geothermie gewährt werden. Mit dem Ziel, den Anteil erneuerbarer Energien auch in der Wärmebereitstellung zu erhöhen, schreibt zudem das Erneuerbare-Wärme-Gesetz (EEWärmeG) vor, dass in Neubauten der Wärmeenergiebedarf zumindest teilweise durch erneuerbare Energien gedeckt werden muss (Bundesregierung 2008b). Ziel ist laut §1 Abs. 1 „den Anteil erneuerbarer Energien am Endenergieverbrauch für Wärme (Raum-, Kühl- und Prozesswärme sowie Warmwasser) bis zum Jahr 2020 auf 14 Prozent zu erhöhen“. Des Weiteren werden Wärmeerzeugungsanlagen nach der Maßgabe des Marktanreizprogramms gefördert (BMU 2009e). Neben Erzeugungsanlagen zur Umwandlung erneuerbarer Energieträger in Nutzwärme ist darin auch die Förderung für Nahwärmenetze, WärmeübergabeStationen, Wärmespeicher sowie für die Aufbereitung und den Transport von Biogas geregelt.

Weitgehend unabhängig vom Energieträger soll darüber hinaus der weitere Ausbau der Kraft-Wärme-Kopplung (KWK) durch das KWK-Gesetz vorangetrieben werden, um höhere Brennstoffnutzungsgrade zu erzielen (Bundesregierung 2002a; Bundesregierung 2008a). Darin ist die Förderung des Ausbaus von KWK-Anlagen sowie des Ausund Neubaus von Wärmenetzen geregelt. Zusätzlich zu den im KWKG festgelegten Fördermaßnahmen werden Steuererleichterungen für KWK-Anlagen mit einem Gesamtwirkungsgrad von mehr als $70 \%$ gewährt (Bundesregierung 2009a). Die Fördermaßnahmen zielen darauf ab, bis 2020 den Anteil der Stromerzeugung aus KWK auf $25 \%$ und den Anteil am Wärmebedarf auf $14 \%$ zu erhöhen, die Einführung der Brennstoffzelle zu unterstützen sowie den Neu- und Ausbau von Wärmenetzen zu fördern (Konstantin 2009; Suttor 2009). Nach Schätzungen des VDE betrug im Jahr 2007 der Anteil der KWK an der Stromerzeugung ca. $9 \%$ und der Anteil am Wärmemarkt ca. $8 \%$ (Botzian et al. 2009). Nach Suttor (2009) liegt der Anteil der KWK-Stromerzeugung derzeit bei $12 \%$ der gesamten Stromerzeugung. 


\subsubsection{Die Rolle der Kernenergie}

Im Jahr 2008 trug die Kernenergie in Deutschland mit 11,6 \% (451 TWh) zum Primärenergieverbrauch und 23,3 \% (149 TWh) zur Bruttostromerzeugung bei (BMWi 2010). Der Anteil an der installierten Bruttostromerzeugungskapazität in Deutschland betrug 15,5 \% (21,3 GW) im Jahr 2007. Nachdem in den 1950er und 1960er Jahren zunächst eine Euphorie über die Möglichkeiten der Kernenergienutzung zu beobachten war, führten insbesondere die Nuklearunfälle in Three Mile Island (USA) im Jahr 1979 und in Tschernobyl (Ukraine) im Jahr 1986 zu einer abnehmenden Akzeptanz von Kernkraftwerken in der Bevölkerung (Konstantin 2009; Oeding und Oswald 2004). Infolge der abnehmenden Akzeptanz aufgrund des Gefahrenpotenzials der Kernkraftnutzung sowie ungelöster Fragen der Endlagerung radioaktiver Abfälle wurde im Jahr 2000 zwischen der deutschen Bundesregierung und den betroffenen Energieversorgungsunternehmen eine Befristung der Nutzung bestehender Kernkraftwerke vereinbart (Bundesregierung und Energieversorgungsunternehmen 2000). Im April 2002 wurde der Kernenergieausstieg durch die Novelle des Atomgesetzes (AtG) in Deutschland beschlossen (Bundesregierung 2002b; Konstantin 2009). Die Vereinbarung legt fest, dass keine neuen Kernkraftwerke gebaut werden dürfen und bestehende Anlagen bis 2022 sukzessive außer Betrieb genommen werden.

Werden die vorhandenen Kernkraftkapazitäten wie geplant stillgelegt, muss der bisher durch die Kernenergie gedeckte Bedarf auf Basis anderer Technologien erbracht bzw. vermieden werden. Aufgrund der hohen inländischen Vorkommen werden neben den erneuerbaren Energien und Energieeffizienzmaßnahmen dabei häufig Braunkohlekraftwerke ins Feld geführt. Diese weisen jedoch aufgrund des hohen spezifischen $\mathrm{CO}_{2}$-Faktors des Brennstoffes höhere Treibhausgasemissionen im Betrieb auf, was vor allem vor dem Hintergrund des Klimawandels als kritisch anzusehen ist (vgl. Abschnitt 2.3.2, S. 13). Technologien zur Abscheidung und Speicherung des $\mathrm{CO}_{2}$ befinden sich noch im Entwicklungsstadium (vgl. Abschnitt 3.1.1.1, S. 25). Andererseits wird an erneuerbaren Energien als Alternative zur Kernkraft kritisiert, dass diese vor allem aufgrund der fluktuierenden Einspeisung zumindest kurzfristig Grundlastkraftwerke nicht in ausreichendem Umfang ersetzen können. Folglich wird der Ausstieg aus der Kernenergie weiterhin kontrovers diskutiert. Im Koalitionsvertrag bezeichnet die Bundesregierung die Kernenergie als eine Brückentechnologie, die so lange Einsatz finden soll, „bis sie durch erneuerbare Energien verlässlich ersetzt werden kann“ (Bundesregierung 2009c). Entgegen den Vereinbarungen zum Kernenergieausstieg wird eingeräumt, dass die Laufzeit deutscher Kernkraftwerke verlängert werden kann, sofern diese strengen deutschen und internationalen Sicherheitsstandards genügen. Dies ermöglicht den Kraftwerksbetreibern einen Weiterbetrieb ihrer Anlagen, gegebenenfalls unter der Bedingung diese zu modernisieren, um die geforderten Standards erfüllen zu kön- 
nen. Der Neubau von Kernkraftwerken bleibt weiterhin verboten (Bundesregierung 2009c). Konkrete Vereinbarungen mit den Betreibern zu den Voraussetzungen für eine Laufzeitverlängerung liegen jedoch zum Zeitpunkt der Erstellung dieser Arbeit noch nicht vor. Als Argumente für eine Laufzeitverlängerung werden Beschäftigungseffekte sowie positive Auswirkungen auf den Strompreis angeführt (BMWi 2009b; Lindenberger et al. 2005).

Den Befürwortern für einen „Ausstieg aus dem Ausstieg“ sind mehrere Argumente entgegen zu halten. Zunächst sind die Gründe für den ursprünglich beschlossenen Ausstieg weiterhin gültig. Die Endlagerung radioaktiver Abfälle konnte bisher nicht abschließend geklärt werden (Müller-Lyda et al. 2008). Bezüglich des mit der Kernenergienutzung verbundenen Gefahrenpotenzials versprechen Reaktortypen der so genannten dritten Generation zwar verbesserte Sicherheitskonzepte. Jedoch zeigt beispielsweise der laufende Neubau eines EPR (European Pressurized Water Reactor) in Olkiluoto (Finnland), dass ein solches Projekt mit erheblichen finanziellen und technischen Unwägbarkeiten verbunden ist (Konstantin 2009; Schneider et al. 2009). Darüber hinaus ist bei einem Weiterbetrieb von Altanlagen eher mit einer Zunahme der Störanfälligkeit zu rechnen. Zudem kann sich der Weiterbetrieb abgeschriebener Altanlagen negativ auf die Wettbewerbssituation auf dem deutschen Strommarkt auswirken (BMU 2009b). Des Weiteren können auch durch den weiteren Ausbau der erneuerbaren Energien und die Technologieführerschaft Deutschlands und Europas in diesem Bereich Beschäftigungseffekte erzielt werden (Europäische Kommission 2006; Staiß et al. 2006).

Letztlich wird der zukünftige Anteil der Kernenergie an der Stromversorgung von politischen Entscheidungen abhängen, da davon auszugehen ist, dass die großen Stromkonzerne ihre abgeschriebenen Altanlagen weiter zur billigen Stromproduktion nutzen werden, solange dies rechtlich möglich ist. Nur wenn die erforderlichen Investitionen zur Modernisierung so hoch sind, dass sie sich nicht in einem angemessenen Zeitrahmen amortisieren, ist damit zu rechnen, dass Kernkraftwerke auch ohne einen politisch beschlossenen Kernenergieausstieg vom Netz gehen. In jedem Fall müssen im Hinblick auf die Gestaltung der zukünftigen Energieversorgung die Stärken und Schwächen der Kernenergie sorgfältig gegeneinander abgewogen werden sowie in Relation zu den verfügbaren Alternativen betrachtet werden.

\subsection{Notwendigkeit eines wissenschaftlichen Analyse- Rahmens für Energietechnologien}

Unter Beachtung der beschriebenen Entwicklungstendenzen auf den Energiemärkten scheint die Umstrukturierung der Systeme zur Bereitstellung von Strom und Wärme 
unumgänglich. Daraus resultieren neue Herausforderungen für Entscheidungsträger in der Energiewirtschaft und -politik:

- Vor dem Hintergrund der Ressourcenverknappung, der Konzentration der Förderländer sowie der steigenden Volatilität der Brennstoffpreisentwicklungen ist die Energiebereitstellung auf Basis konventioneller, fossiler Technologien in zunehmendem Maße mit Unsicherheiten behaftet.

- Die aufgrund des Wachstums in Entwicklungs- und Schwellenländern weltweit steigende Energienachfrage führt zu intensiverem Wettbewerb um die geografisch ungleich verteilten Ressourcen.

- Infolge der Liberalisierung der Energiemärkte müssen Investitionsentscheidungen unter Beachtung einer zunehmenden Anzahl an Wettbewerbern getroffen werden.

- Aufgrund des Klimawandels und der damit verbundenen politischen Maßnahmen ergeben sich ungünstigere Voraussetzungen für konventionelle, fossile Technologien.

- Die politischen Rahmenbedingungen begünstigen den Ausbau der Kraft-WärmeKopplung und der erneuerbaren Energien.

- Die Kernenergie ist als Option zur Stromerzeugung stark umstritten. Bei Stilllegung von Kraftwerkskapazitäten gemäß dem Beschluss zum Kernenergieausstieg kommt es zu einem hohen Ersatzbedarf.

Nach dem Referenzszenario der IEA liegt die Stromnachfrage in Europa im Jahr 2030 bei 3.855 TWh. Dabei ergibt sich infolge der Altersstruktur des Kraftwerksparks in Europa ein Ersatz- und Neubaubedarf in Höhe von etwa $700 \mathrm{GW}$ installierter Stromerzeugungsleistung bis zum Jahr 2020 (IEA 2009c; vgl. auch Europäische Kommission 2006). In Deutschland besteht aufgrund altersbedingter Stilllegungen und unter Annahme der Beibehaltung der ursprünglichen Beschlüsse zum Kernenergieausstieg nach Erdmenger (2006) ein Ersatzbedarf von ca. 50 GW installierter Kraftwerksleistung bis 2020 (vgl. auch CONSENTEC et al. 2008; Kahle 2009). Bis 2015 ist nach Schätzungen von (CONSENTEC et al. 2008) ein Zubau von ca. $11 \mathrm{GW}$ an fossilthermischen Kraftwerken wahrscheinlich. Somit sind dringend Entscheidungen mit langfristiger Wirkung hinsichtlich der zukünftigen Stromversorgung zu treffen. Diese Entscheidungen können weitreichende Konsequenzen nach sich ziehen. Denn zum einen sind Investitionen in der Energiewirtschaft mit einem hohen Kapitalbedarf verbunden. Zum anderen legen diese Entscheidungen Strukturen in der Strom- und Wärmeversorgung gegebenenfalls über Jahrzehnte fest. Diese Faktoren können ein Hemmnis für die Umgestaltung des Energiesystems darstellen.

Auch auf dem Wärmemarkt rechnen Experten mit wesentlichen Veränderungen in den nächsten Jahren hinsichtlich Höhe und Verteilung des Wärmebedarfs. Diese sind ins- 
besondere auf demografische Entwicklungen wie Bevölkerungsrückgang und Zersiedelung zurückzuführen sowie auf Effizienzsteigerungen auf der Nachfrageseite (zum Beispiel verbesserte Dämmung von Gebäuden) und neue Technologien zur Bereitstellung von Nutzwärme (vgl. auch Lutsch et al. 2004). Zur Erreichung der Ziele der Bundesregierung, den Anteil der Kraft-Wärme-Kopplung an der Stromerzeugung bis 2020 auf $25 \%$ und den Anteil erneuerbarer Energien am Wärmebedarf auf $14 \%$ zu erhöhen, sind technische Maßnahmen bezüglich der Erzeugung und Verteilung von Wärme erforderlich. Beispielsweise könnte das Angebot an leitungsgebundener Wärme steigen, wenn im Rahmen der Erneuerung des Kraftwerksparks auch die Kraft-WärmeKopplung weiter ausgebaut wird. Daher ist auch bei den existierenden Wärmeanlagen und -netzen mit erheblichem Veränderungsdruck zu rechnen (Botzian et al. 2009; Strohschein et al. 2007).

Die aufgezeigten Abhängigkeiten verdeutlichen, dass Alternativen zu den etablierten Energietechnologien auf Basis fossiler und nuklearer Energieträger erforderlich sind, um die Importabhängigkeit Deutschlands zu reduzieren und damit die Versorgungssicherheit zu stärken sowie die durch die Energiebereitstellung verursachten negativen Umwelt- und Gesundheitsauswirkungen zu verringern (vgl. auch Tester et al. 2005). Das Energiewirtschaftsgesetz fordert „eine möglichst sichere, preisgünstige, verbraucherfreundliche, effiziente und umweltfreundliche leitungsgebundene Versorgung der Allgemeinheit mit Elektrizität und Gas" (§ 1 Abs. 1 EnWG). Ebenso zielen die wesentlichen Schwerpunkte der europäischen Energiepolitik auf eine sichere, umweltschonende und kostengünstige Energieversorgung ab (Europäische Kommission 2006). Folglich sind vor dem Hintergrund teilweise konfliktärer Zielsetzungen Entscheidungen zu treffen, welche die Auswahl von Energietechnologien im Hinblick auf die zukünftige Aus- und Umgestaltung des Energiesystems sowie zukünftige Investitionen in Forschung und Entwicklung betreffen (vgl. zum Beispiel Diakoulaki et al. 2005; Hobbs und Meier 2003). Die anstehenden Entscheidungen hinsichtlich der Deckung des Energiebedarfs heute und in Zukunft sollten in diesem Zusammenhang als Chance zur Umgestaltung hin zu einem nachhaltigeren Energiesystem gesehen werden (IEA 2009a; Krewitt et al. 2009). Möglichkeiten hierzu liegen beispielsweise in der Steigerung der Effizienz des bestehenden Kraftwerksparks, der Umsetzung sekundärer Maßnahmen zur Reduzierung des Schadstoffausstoßes, in der verstärkten Nutzung erneuerbarer Energien und der Kraft-Wärme-Kopplung sowie in der Einführung neuer Technologien (Bartels et al. 2008). Um das Ziel eines ausgewogenen Energiemixes zu realisieren, müssen dabei die Stärken und Schwächen der verfügbaren oder in Entwicklung befindlichen Technologien einander gegenübergestellt werden, um nachvollziehbare und transparente Entscheidungen auf einer sachlich-wissenschaftlichen Basis treffen zu 
können (vgl. zum Beispiel Tester et al. 2005). Daraus leiten sich als zentrale Fragestellungen dieser Arbeit ab:

- In welche Energietechnologien sollte in Zukunft investiert werden bzw. welche Energietechnologien sollten zukünftig verstärkt genutzt, weiterentwickelt, erforscht und gefördert werden?

- Wie können diesbezügliche Entscheidungen unter Berücksichtigung teilweise konfliktärer Zielsetzungen wie Versorgungssicherheit, Wirtschaftlichkeit und Umweltschutz in transparenter und nachvollziehbarer Weise unterstützt werden?

Eine Vielzahl an Energietechnologien zur Bereitstellung von Strom und Wärme steht zur Verfügung oder befindet sich in Entwicklung bzw. Erprobung. Weitgehend unumstritten ist, dass keine der technischen Optionen unter Berücksichtigung aller Zielsetzungen als optimal anzusehen ist. Um dennoch nachvollziehbare Entscheidungen treffen zu können und Entscheidungsträger in transparenter Weise zu unterstützen, bieten sich multikriterielle Methoden zur Entscheidungsunterstützung an. Diese ermöglichen die simultane Verarbeitung mehrerer Zielgrößen unter Berücksichtigung der Präferenzen der Entscheidungsträger und wurden bereits in unterschiedlichen Problemstellungen im Energiebereich eingesetzt (vgl. zum Beispiel Løken 2007; Wang et al. 2009b sowie Kapitel 4, S. 55 ff.). Daher soll die Eignung dieser Methoden zur Bearbeitung der Fragestellung im Folgenden genauer untersucht werden. Zuvor wird in Kapitel 3 zunächst geklärt, welche Optionen zur Bereitstellung von Strom und Wärme, das heißt welche Energietechnologien heute und perspektivisch zur Verfügung stehen, um den Gegenstandsbereich der Untersuchung genauer abzugrenzen. 


\section{$3 \quad$ Technologien zur Bereitstellung von Strom und Wärme}

In dieser Arbeit werden Energietechnologien untersucht, die zur Deckung des Bedarfs an Strom und Wärme dienen. Im Unterschied zur Technik sind unter Technologien abgegrenzte Ausschnitte naturwissenschaftlicher Grundlagen zu verstehen, die zur Wirklichkeitsgestaltung und Problemlösung eingesetzt werden (Perl 2007). In diesem Sinne werden Technologien als Teilgebiete der Technik verstanden. Der Begriff Technik ist nach Perl (2007) weiter gefasst und bezeichnet alle Prozesse und Ausrüstungen, die dazu dienen, die Natur für den Menschen nutzbar zu machen (zum Beispiel Elektrotechnik). In diesem Kapitel werden die wichtigsten der bekannten Energietechnologien zur Bereitstellung von Strom und/oder Wärme skizziert. Es werden sowohl bereits eingesetzte als auch neue, teilweise noch nicht auf dem Markt verfügbare Technologien dargestellt. Dabei besteht das Ziel darin, die vielfältigen technischen Optionen zur Strom- und Wärmeversorgung sowie deren Vor- und Nachteile aufzuzeigen.

\subsection{Bereitstellung von Elektrizität und Kraft-Wärme- Kopplung}

Elektrizität ist ein unsichtbares und indirektes Konsumgut, das zur Bereitstellung von Energiedienstleistungen für Industrie, Gewerbe, öffentliche Einrichtungen und Privathaushalte dient, beispielsweise in Form von Beleuchtung, Kraft und Wärme. Die technische Infrastruktur von Elektrizitätssystemen setzt sich zusammen aus Komponenten zur Erzeugung, Übertragung und Verteilung von Elektrizität (Praetorius et al. 2009). Heutzutage wird elektrische Energie zum Großteil in fossilen oder nuklearen Dampfkraftwerken sowie zu einem kleineren Anteil in Wasserkraftanlagen erzeugt (Heuck et al. 2007). Erneuerbare Energien wie zum Beispiel Wind-, Solar- oder geothermische Kraftwerke weisen derzeit nur einen relativ geringen aber deutlich steigenden Anteil an der Stromerzeugung auf (vgl. Abbildung 2.8, S.17). In Deutschland wie auch in anderen Ländern werden jedoch seit einigen Jahren Anstrengungen unternommen, diesen Anteil weiter zu erhöhen (Oeding und Oswald 2004).

\subsubsection{Thermische Kraftwerke}

In thermischen Kraftwerken (auch Dampf-, Wärmekraftwerke) wird durch Zuführung von Wärme, in der Regel aus der Verbrennung eines Primärenergieträgers, Wasserdampf erzeugt. Dieser wird zur Erzeugung mechanischer Arbeit in einer Dampfturbine entspannt, die wiederum einen Generator zur Stromerzeugung antreibt. Dampfturbinen werden ab einer Leistung von ca. $2 \mathrm{MW}$ eingesetzt. Als Brennstoffe finden in erster Linie Erdgas sowie Braun- und Steinkohle in zentralen Großkraftwerken Verwendung. 
Auch Kernkraftwerke zählen zu den Wärmekraftwerken. In Kondensationskraftwerken wird der Dampf in der Turbine bis auf den Kondensatordruck (Unterdruck) entspannt und auf Kondensattemperatur abgekühlt. Die entstehende mechanische Arbeit wird zur Stromerzeugung in einem Generator genutzt. Nach der Turbine kondensiert der Dampf bei konstantem Druck und konstanter Temperatur im Kondensator, wobei die Kondensationswärme abgeführt werden muss. Anschließend wird das Kondensat zur Erhöhung des Drucks auf Kesseldruck durch die Speisewasserpumpe geleitet. Häufig wird Anzapfdampf aus der Turbine zur Vorwärmung des Speisewassers vor dem Kessel entnommen. Dadurch wird zwar die von der Turbine abgegebene mechanische Arbeit verringert, jedoch erhöht sich der thermische Wirkungsgrad des Gesamtprozesses (Schwab 2009).

Durch Optimierung der Prozessführung und der Prozessparameter wie Frischdampfdruck und Temperatur können Wirkungsgradsteigerungen erzielt werden. Die maximal erreichbaren Wirkungsgrade sind dabei eng mit den Belastungsgrenzen der verwendeten Materialien verknüpft (Kaltschmitt et al. 2009; Oeding und Oswald 2004). In Industrie- oder Heizkraftwerken (HKW) werden höhere Brennstoffnutzungsgrade erzielt, indem Dampf entnommen und für industrielle Prozesse oder zu Heizzwecken genutzt wird (Kraft-Wärme-Kopplung - KWK). KWK-Betrieb ist jedoch nur dann sinnvoll, wenn ein entsprechender Wärmebedarf in hinreichend räumlicher Nähe vorhanden ist (Oeding und Oswald 2004; Suttor 2009). In technischer Hinsicht werden EntnahmeKondensations- und (Entnahme-) Gegendruck-Turbinen zur Wärmeauskopplung unterschieden. Durch die Entnahme von Dampf an der Turbine sinkt zwar der elektrische Wirkungsgrad, der Brennstoffnutzungsgrad wird dennoch insgesamt erhöht. Vorteilhaft gegenüber Gegendruck-Anlagen ist das variable Verhältnis von erzeugtem Strom und entnommener Wärme in Entnahme-Kondensations-Turbinen. In Gegendruck-Anlagen sind zwar höhere Brennstoffnutzungsgrade möglich, jedoch ist das Verhältnis der erzeugten Strom- und Wärmemengen eng gekoppelt. Dabei ist die Turbine so ausgelegt, dass das Temperatur- und Druckniveau am Ende ausreichend hoch für die vorgesehene Wärmeanwendung sind. Folglich ist der Einsatz von Gegendruckturbinen vor allem dann sinnvoll, wenn der Wärmebedarf über das Jahr weitgehend gleichbleibend ist, beispielsweise für industrielle Anwendungen. Vorteilhaft gegenüber EntnahmeKondensations-Anlagen sind geringere Investitionen, da keine Kondensations- und Rückkühlungseinrichtungen erforderlich sind (Kaltschmitt et al. 2009; Schwab 2009; Strohschein et al. 2007).

\subsubsection{Kohlekraftwerke}

Kohle wird heutzutage in erster Linie zur Stromerzeugung in Dampfkraftwerken verwendet. Da die weltweiten Vorkommen hoch und geografisch relativ breit verteilt sind, 
gilt Kohle als wichtiges Element der Versorgungssicherheit (Cramer et al. 2009). Jedoch sind vor allem aufgrund relativ hoher spezifischer $\mathrm{CO}_{2}$-Emissionen negative Umweltauswirkungen mit der Stromerzeugung in Kohlekraftwerken verbunden. Erwartete Wirkungsgradsteigerungen können diese Auswirkungen mindern, bedürfen jedoch des Einsatzes geeigneter Materialien, die höheren Prozessparametern (Temperatur, Druck) standhalten (Heuck et al. 2007; Oeding und Oswald 2004). Dies ist wiederum verbunden mit höheren Investitionen. Grundsätzlich weisen Steinkohle- im Vergleich zu Braunkohle-Kraftwerken höhere Wirkungsgrade auf. Dies liegt vor allem daran, dass die eingesetzte Rohbraunkohle einen hohen Wassergehalt aufweist. Die bei der Verbrennung frei werdende Energie muss teilweise zur Verdampfung dieses Wasseranteils eingesetzt werden, was sich negativ auf den Gesamtwirkungsgrad des Prozesses auswirkt (Mühlstein und Schirmer 2008). Zudem sind die spezifischen $\mathrm{CO}_{2}$-Emissionen aus Steinkohle-Kraftwerken niedriger. Jedoch sind die heimischen Reserven wesentlich geringer und aufwändiger zu fördern als die der Braunkohle, so dass in Deutschland inzwischen eine hohe Importabhängigkeit bei Steinkohle besteht (vgl. auch Abbildung 2.3, S. 9). Darüber hinaus führt die auslaufende Subventionierung in zunehmendem Maße zu ungünstigeren wirtschaftlichen Rahmenbedingungen für den Steinkohlebergbau in Deutschland (Cramer et al. 2009; Konstantin 2009).

Um negative Umwelteffekte der Stromerzeugung in Kohlekraftwerken zu mindern und Umweltvorschriften zu genügen, sind in modernen Anlagen Technologien zur Rauchgasentstaubung, -entschwefelung (REA) und Entstickung (DeNO ${ }_{x}$ ) integriert (Heuck et al. 2007; Oeding und Oswald 2004). Darüber hinaus haben in den letzten Jahren die Möglichkeiten zur integrierten oder nachgeschalteten $\mathrm{CO}_{2}$-Abscheidung und -Speicherung (Carbon Capture and Storage - CCS) zunehmende Aufmerksamkeit erfahren. Die Technologien zur Abscheidung vor, nach oder während der Kohleverbrennung werden zur Anwendung in Großkraftwerken noch erprobt. Ziel ist eine emissionsarme Nutzung des Energieträgers Kohle, indem das Entweichen des $\mathrm{CO}_{2}$ in die Atmosphäre vermieden wird (Cramer et al. 2009; Konstantin 2009). Jedoch sind zahlreiche Fragen hinsichtlich Technik, Wirtschaftlichkeit, Umweltauswirkungen, Sicherheit und gesetzlicher Rahmenbedingungen noch nicht abschließend geklärt. Weiterhin sind die Auswirkungen auf Kosten und Wirkungsgrade der Stromerzeugung zu beachten, die der potenziellen Verbesserung der Umweltauswirkungen entgegen stehen (Praetorius und Schumacher 2009).

\subsubsection{Gaskraftwerke}

In der Brennkammer einer Gasturbinenanlage wird komprimierte Luft mit dem gasförmigen oder flüssigen Brennstoff gemischt, gezündet und verbrannt. Durch Entspannung des entstehenden Luft-Verbrennungsgas-Gemisches im nachfolgenden Turbi- 
nenteil wird mechanische Energie erzeugt, die zum Antrieb von Verdichter und Generator dient. Als Brennstoff kommt meist Erdgas, in einigen Fällen auch Heizöl zum Einsatz (Crastan 2009; Heuck et al. 2007). Erdgas weist grundsätzlich geringere spezifische $\mathrm{CO}_{2}$-Emisisonen als Kohle auf (Cramer et al. 2009). Die Investitionen sind im Vergleich zu Kohlekraftwerken geringer, da weder Entstickung noch Entschwefelung oder Entstaubung der Abgase notwendig ist. Andererseits sind die Betriebskosten aufgrund höherer Brennstoffkosten und geringerer Wirkungsgrade höher. Weitere Vorteile der Gaskraftwerke liegen in der relativ freien Standortwahl, den Möglichkeiten zur Fernsteuerung, der Schnellstartfähigkeit sowie der Anpassungsfähigkeit an die bestehenden Versorgungsnetze. Somit sind Gaskraftwerke flexibel als Mittel- oder Spitzenlastkraftwerke einsetzbar (Heuck et al. 2007; Oeding und Oswald 2004). Nachteilig wirken sich schlechte Teillastwirkungsgrade und der starke Einfluss der Umgebungsbedingungen (Eigenschaften der Luft) aus (Konstantin 2009). Wie auch bei den Kohlekraftwerken werden Steigerungen der elektrischen Wirkungsgrade über eine Verbesserung der Prozessführung und Erhöhung der Prozessparameter angestrebt. Dazu sind wiederum eine Verbesserung der eingesetzten Werkstoffe und entsprechend höhere Investitionen erforderlich (Frost \& Sullivan 2005; Oeding und Oswald 2004).

Die Brennstoffnutzungsgrade von Gasturbinen-Anlagen können erhöht werden, indem die Energie der Abgase mit Temperaturen von 400 bis $600 \mathrm{C}$ über einen Abhitzekessel genutzt wird. Im KWK-Betrieb kann die Abwärme als industrielle Wärme oder Fernwärme genutzt werden. Eine andere Möglichkeit besteht in der Nutzung der hohen Abgastemperaturen zur Dampferzeugung in einem kombinierten Gas- und Dampfturbinenprozess (GuD). Dabei steigen zwar die Anfangsinvestitionen im Vergleich zur einfachen Gasturbine bzw. Dampfturbine, andererseits ist die Energieausbeute höher (Crastan 2009; Konstantin 2009; Oeding und Oswald 2004; Suttor 2009). Bisher werden GuD-Anlagen in der Regel mit Erdgas als Brennstoff betrieben (Heuck et al. 2007). In den 1990er Jahren wurden erstmals GuD-Anlagen mit integrierter Kohlevergasung (IGCC - Integrated Gasification Combined Cycle) in Spanien (Puertollano) und in den Niederlanden (Buggenum) in Demonstrationsanlagen erprobt. Dieses Verfahren ermöglicht auch eine Abtrennung des $\mathrm{CO}_{2}$ aus dem auf Kohle basierenden Synthesegas vor dessen Verbrennung. Effizienz- und Kostenvorteilen im Vergleich zu anderen Verfahren der $\mathrm{CO}_{2}$-Abtrennung stehen Probleme hinsichtlich Betriebssicherheit und Verfügbarkeit aufgrund der komplexen Anlagentechnik gegenüber (Mühlstein und Schirmer 2008).

Andere Weiterentwicklungen des Gasturbinen-Prozesses zielen beispielsweise auf eine vorübergehende Leistungssteigerung durch Dampfeindüsung in die Brennkammer ab (Crastan 2009). Beim Cheng-ISTIG-Prozess (Steam Injection Gas Turbine) wird dabei mit der Abwärme der Gasturbinen-Anlage Dampf in einem Abhitzekessel er- 
zeugt. Dieser wird teilweise oder vollständig in die Brennkammer eingedüst, um den Massenstrom in der Turbine zu erhöhen und so die Leistung zu steigern. Dadurch wird die Flexibilität von KWK-Anlagen erhöht, da Strom- und Nutzwärmeangebot voneinander entkoppelt werden. Vorteilhaft gegenüber GuD-Anlagen sind geringere realisierbare Anlagengrößen. Von Nachteil sind der erhöhte Wasserbedarf und geringere Wirkungsgrade. Höhere Wirkungsgrade werden vom HAT-Prozess (Humid Air Turbine) erwartet, bei dem im Rahmen des Gasturbinen-Prozesses der verdichteten Luft Dampf mittels Verdunstung zugeführt wird. Verschiedene technische Schwierigkeiten haben jedoch bisher eine weite Verbreitung dieser Technologie verhindert (Bartels et al. 2008; Carapellucci 2009; Frost \& Sullivan 2005).

\subsubsection{Kernkraftwerke}

In Kernkraftwerken wird anstatt des konventionellen Dampferzeugers in fossilthermischen Kraftwerken die bei der Spaltung schwerer Atomkerne entstehende Wärme genutzt. Diese wird durch Gas, Wasser oder flüssiges Metall abgeführt und übertragen. Von den bekannten Reaktortypen werden bisher in der Regel Leichtwasserreaktoren mit Wasser als Moderator und Kühlmittel eingesetzt. Diese sind weiter zu unterscheiden in Siede- und Druckwasserreaktoren, wobei letztere in Europa bevorzugt eingesetzt werden. In Druckwasserreaktoren wird die im Reaktor erzeugte Wärme mit Wasser als Wärmeträgermedium abgeführt und über einen Wärmetauscher an einen Sekundärkreislauf übertragen. Im Sekundärkreislauf wird in einem konventionellen Dampfkraftprozess Strom erzeugt (Heuck et al. 2007). Da Dampfdruck und Dampftemperatur prozessbedingt relativ niedrig bleiben müssen und aus Sicherheitsgründen auf eine Dampfüberhitzung verzichtet wird, können in Kernkraftwerken nur geringere Wirkungsgrade (ca. 30 \%) als in fossil befeuerten Anlagen erzielt werden (Crastan 2009; Oeding und Oswald 2004). Im Vergleich zu Kohlekraftwerken werden häufig die geringen $\mathrm{CO}_{2}$-Emissionen der Stromerzeugung in Kernkraftwerken hervorgehoben. Dem stehen jedoch ungelöste Probleme in Verbindung mit der Entsorgung ausgebrannter Brennelemente gegenüber, die im Vergleich zur Kohleverbrennung ungleich schwieriger, kostenintensiver und für die Umwelt risikoreicher ist. Darüber hinaus bestehen im Zusammenhang mit dem sicheren Einschluss des radioaktiven Materials schwer quantifizierbare Risiken auch während des Betriebs (Oeding und Oswald 2004; Tromm und Bahm 2007). Das Sicherheitsrisiko des Druckwasserreaktors soll durch Weiterentwicklungen in Form des EPR (European Pressurized Water Reactor) gesenkt werden (Heuck et al. 2007; vgl. auch Abschnitt 2.3.4).

Enorme Potenziale zur Stromerzeugung bei minimalen Umweltauswirkungen und hoher Ressourceneffizienz erhofft man sich von der Kernfusion. Diese beruht nicht wie in konventionellen Kernkraftwerken auf der Spaltung schwerer Atomkerne, sondern auf 
der Fusion leichter Atomkerne und der daraus resultierenden Freisetzung von Kernbindungsenergie. Die erforderlichen Technologien werden in einem internationalen Forschungsprogramm erforscht, sind jedoch noch weit von einer kommerziellen Umsetzung entfernt (Crastan 2009; Schwab 2009; Tester et al. 2005).

\subsubsection{Erneuerbare Energien zur Stromerzeugung}

Als erneuerbar oder regenerativ werden Energiequellen bezeichnet, „die nach menschlichen Maßstäben unerschöpflich sind“ (Neupert 2007). Aufgrund klimatischer und geografischer Bedingungen sind nach Kaltschmitt et al. (2009) im deutschsprachigen Raum in erster Linie Solarstrahlung, Windenergie, Wasserkraft, Erdwärme und Biomasse als regenerative Energieträger nutzbar. Ein wesentlicher Vorteil im Vergleich zu fossilen und nuklearen Energieträgern besteht darin, dass die Ressourcen grundsätzlich keiner zeitlichen und mengenmäßigen Begrenzung unterliegen. Jedoch ist das wirtschaftlich realisierbare Potenzial erneuerbarer Energien aufgrund verschiedener Faktoren eingeschränkt (WBGU 2009).

\subsubsection{Wasserkraft}

Wasserkraftwerke wandeln die potenzielle Energie oder Strömungsenergie von Wasser in elektrische Energie um. Die Nutzung der Wasserkraft an Land durch Speicherkraftwerke und Lauf- bzw. Flusskraftwerke ist technologisch auf einem sehr hohen Stand. In Speicherkraftwerken (Talsperren) werden große Höhenunterschiede durch Errichtung eines Staudamms genutzt. Über Druckrohrleitungen wird Wasser vom oberen ins untere Becken durch Turbinen geleitet, die Generatoren antreiben. Wird in Schwachlastzeiten zusätzlich Wasser vom unteren ins obere Becken gepumpt, spricht man von Pumpspeicherkraftwerken. Auch Pumpspeicherkraftwerke ohne natürlichen Zulauf sind üblich (reine Speicheranlagen, vgl. Deane et al. 2010; Quaschning 2008). Speicherkraftwerke zeichnen sich durch hohe Wirkungsgrade und ihre Schnellstartfähigkeit positiv aus (Ibrahim et al. 2008). In Laufwasser- oder Flusskraftwerken wird die Strömung in einem Kanal oder Fluss angestaut. Das abfließende Wasser wird durch eine Turbine geleitet, die über einen elektrischen Generator Grundlaststrom erzeugt (Neupert 2007). Sowohl für Speicher- als auch Laufwasserkraftwerke ist das Potenzial in Deutschland weitgehend erschlossen, so dass durch Modernisierung und Reaktivierung von Altanlagen nur in begrenztem Maße Ertragssteigerungen erreicht werden können (Quaschning 2008). Eine weitere Möglichkeit liegt in der Aufrüstung von Speicher- zu Pumpspeicherkraftwerken. Der abnehmenden Verfügbarkeit geeigneter Standorte sind der technische Fortschritt sowie Entwicklungen beim sparsamen Einsatz von Materialien und Energie gegenüberzustellen, so dass Standortnachteile teilweise kompensiert werden können. Zwar sind die direkten Emissionen während des 
Betriebs von Wasserkraftwerken minimal, jedoch sind Umweltauswirkungen hinsichtlich hydrologischer Veränderungen in der Umgebung der Kraftwerke zu beachten. Je nach Charakteristika des Standortes können der bauliche Aufwand und dementsprechend auch die Umweltbelastungen variieren (Bauer et al. 2007).

Technologien zur Nutzung von Meeres- bzw. Gezeitenströmungen befinden sich noch im Anfangsstadium der Entwicklung (Mueller und Wallace 2008; Neupert 2007). Gezeitenkraftwerke nutzen den Höhenunterschied (Tidenhub) des Meeresspiegels zwischen Ebbe und Flut. Die Zu- und Abströmung des Meerwassers wird dabei über Turbinen zur Stromerzeugung genutzt. Diese sind in Staumauern installiert, welche in Meeresbuchten errichtet werden. Nachteilig sind der hohe Platzbedarf, erhebliche Umweltauswirkungen auf Ökosysteme und hohe Kosten. Aufgrund des relativ geringen Tidenhubs an deutschen Küsten gibt es im Inland keine geeigneten Standorte. Für Wellenkraftwerke sind Standorte in Küstennähe mit geringen Wassertiefen geeignet, die in Deutschland nur begrenzt verfügbar sind. Unterschieden werden Schwimmerund Kammer- sowie TapChan-Systeme (Tapered Channel). Eine weite Verbreitung diese Anlagen wurde bisher vor allem wegen stark variierender und anspruchsvoller Bedingungen auf See nicht realisiert. Das technische Prinzip eines Meeresströmungskraftwerkes ähnelt dem einer Windkraftanlage, wobei die Rotorblätter unter Wasser durch die Meeresströmung bewegt werden. Vorteilhaft im Vergleich zu Windkraftanlagen sind die besser vorhersehbare und kontinuierliche Strömung sowie die höhere Dichte des Strömungsmediums Wasser (Quaschning 2008). Nachteile betreffen die Kosten und Risiken in Verbindung mit der Errichtung auf See sowie der Übertragung von Elektrizität an Land (Blunden und Bahaj 2006).

In einem Osmosekraftwerk (Salzgradientenkraftwerk) werden zwei Salzlösungen unterschiedlicher Konzentration über eine semipermeable Membran in Kontakt gebracht. Nur das Wasser (Lösungsmittel), nicht jedoch die gelösten Salze können die Membran passieren. Daher kann ein Druckausgleich nur dadurch erreicht werden, dass Wasser von der niedriger in die höher konzentrierte Lösung übertritt. Der so entstehende Überdruck kann zum Antrieb einer Turbine und damit zur Stromerzeugung genutzt werden. Geeignete Standorte sind begrenzt, da diese an Flussmündungen liegen, wo Süß- und Salzwasser in ausreichenden Größenordnungen natürlich vorhanden sind und aufeinander treffen. Der erste Prototyp eines Osmosekraftwerks ging im Jahr 2009 in Tofte (Norwegen) in Betrieb. Der erfolgreiche Einsatz der Technologie in Zukunft wird wesentlich durch die Entwicklung geeigneter Membranen und mögliche Kostensenkungen bestimmt (O.V. 2009; Skilhagen et al. 2008). 


\subsubsection{Windkraftanlagen}

Eine Windkraftanlage (WKA) wandelt die kinetische Energie des Windes in elektrische Energie um, indem die Bewegungsenergie der Windströmung auf die Rotorblätter wirkt und den Rotor in eine Drehbewegung versetzt. Gängig sind heutzutage Turmkonstruktionen mit radialen Drei-Blatt-Rotoren an Land (Onshore) (Crastan 2009; Heuck et al. 2007). Diese gelten als weitgehend ausgereifte Technologie und sind bei günstigen Standortbedingungen bereits heute wettbewerbsfähig. WKA mit vertikaler Rotationsachse konnten sich aufgrund geringerer Leistungsbeiwerte und Nachteile hinsichtlich der Wirtschaftlichkeit nicht durchsetzen. Technische Weiterentwicklungen in den letzten Jahren konzentrieren sich auf höhere Anlagen mit größerem Rotordurchmesser und höherer Leistung (Eurobserv'er 2009; Heuck et al. 2007; Neupert 2007; vgl. auch Abbildung 5.2, S. 92). Zunehmendes Interesse ist in den letzten Jahren zudem hinsichtlich Kleinwindanlagen für private und gewerbliche Nutzer zu beobachten, die teilweise noch im Entwicklungsstadium stehen und hohe Kosten aufweisen (Köpke 2009). Als Nachteile der Windenergie werden neben den diskontinuierlichen und nicht exakt vorhersehbaren Windverhältnissen häufig der hohe Flächenbedarf sowie als negativ empfundene Auswirkungen auf das Landschaftsbild genannt. Das Onshore-Potenzial in Deutschland ist bereits zu großen Teilen ausgeschöpft. In den nächsten Jahren werden vor allem im Offshore-Bereich sowie im Repowering 2 Zuwächse erwartet. Dem höheren und konstanteren Windangebot auf See steht dabei ein deutlich höherer konstruktiver Aufwand, besondere Ansprüche an den Korrosionsschutz und damit höhere Investitionen sowie ein höherer Wartungsaufwand im Vergleich zu Onshore-Anlagen gegenüber (BMU 2009a; Neupert 2007). Darüber hinaus sind teilweise neue technische Lösungen hinsichtlich Leitung (vgl. Abschnitt 3.1.7) und gegebenenfalls Speicherung (vgl. Abschnitt 3.1.6) von Bedeutung. Denn Angebot und Nachfrage werden in zunehmenden Maße zeitlich und räumlich voneinander abweichen, wenn große Offshore-Kapazitäten installiert werden (Jansen et al. 2005).

\subsubsection{Biomasse zur Stromerzeugung}

Zur Biomasse zählen organische Stoffe wie Energiepflanzen, Resthölzer aus Durchforstungen oder Sägewerken, landwirtschaftliche Reststoffe, beispielsweise Stroh, sowie Zoomasse, tierische Exkremente und Klärschlamm. Entsprechend der vielfältigen Ausgangsstoffe steht eine Vielzahl an Umwandlungsverfahren zur Verfügung, um Biomasse in Form fester, flüssiger oder gasförmiger Energieträger zur Bereitstellung von End-

2 Unter Repowering ist der Ersatz veralteter Windräder durch neue Anlagen mit höherer Leistung und Verfügbarkeit zu verstehen, um das Potenzial günstiger Standorte besser nutzen zu können (Neupert 2007). 
energie nutzbar zu machen. Häufig dienen dabei vorgelagerte Veredelungsprozesse der Aufwertung und besseren Handhabbarkeit der eingesetzten Biomassen. Im Allgemeinen ist die Nutzung von Biomasse zur Stromerzeugung mit thermo-chemischen, physikalisch-chemischen oder biochemischen Umwandlungsschritten verbunden. Die dabei anfallende Wärme kann bei entsprechendem Bedarf ebenfalls genutzt werden (Kraft-Wärme-Kopplung - KWK) (Heuck et al. 2007; Kaltschmitt et al. 2009; Kaltschmitt und Thrän 2007; Ruppert et al. 2008).

Biogene Festbrennstoffe (zum Beispiel Hackschnitzel, Holzpellets, Getreide, Stroh, Miscanthus, Ersatzbrennstoffe) können zur Stromerzeugung in (Heiz-)Kraftwerken auf Basis konventioneller Dampfprozesse eingesetzt werden. Dabei sind Anlagen ab einer Leistung von etwa 2 MW Stand der Technik. Im Kilowatt-Bereich ist die Anwendung fester Biomasse zur Stromerzeugung hingegen noch im Entwicklungsstadium (Kaltschmitt und Thrän 2007). Weiterhin ist die Mitverbrennung biogener Festbrennstoffe in konventionellen Kohlekraftwerken möglich. Dadurch wird die Abhängigkeit von fossilen Brennstoffen reduziert. Der verwendbare Biomassestrom ist jedoch begrenzt, einerseits durch die vor Ort verfügbare Biomasse und andererseits durch Anforderungen des Kraftwerksprozesses, die eingehalten werden müssen, um Betriebsstörungen und Schäden zu vermeiden. Noch im Forschungsstadium befinden sich Motoren zur Stromerzeugung, die mit einem Luft-Holzstaub-Gemisch befeuert werden. Des Weiteren ist die Nutzung von Holzstaub und stückiger fester Biomasse in indirekt gefeuerten Gasturbinenprozessen möglich. Nachteilig wirken sich hier insbesondere aggressive Bestandteile im Abgas bzw. Verbrennungsgas aus, die zu technischen Problemen führen können. Im Vergleich zu flüssigen und gasförmigen Brennstoffen ist der Wirkungsgrad der Strom- oder Wärmeerzeugung aus fester Biomasse in der Regel geringer, da diese bei geringeren Temperaturen betrieben werden muss, um Probleme wie Verschlackung und Korrosion zu vermeiden. Andererseits sind zur Nutzung fester Biomasse häufig weniger vorgelagerte Arbeitsschritte erforderlich (Kaltschmitt et al. 2009).

Flüssige Bioenergieträger wie Pflanzenöl können in stationären (Klein-)Anwendungen eingesetzt werden, beispielsweise in angepassten Blockheizkraftwerken (BHKW) mit Verbrennungsmotoren zur gekoppelten Strom- und Wärmeerzeugung (siehe auch Abschnitt 3.1.5). Diese Anwendungen stehen in Konkurrenz zur Nutzung als Treibstoff im Verkehrssektor. Teilweise gilt dies auch für Biogase, die durch bio-chemische oder thermo-chemische Verfahren gewonnen werden. Nach entsprechender Aufbereitung kann Biogas in Gasmotoren, Gasturbinen oder Brennstoffzellen zur Stromerzeugung bzw. zur gekoppelten Strom- und Wärmeerzeugung in BHKWs verwendet werden. Stand der Technik bei der biochemischen Biogaserzeugung sind Anlagen mit einer elektrischen Leistung vom zweistelligen Kilowatt-Bereich bis zum unteren MegawattBereich. Einsatzstoffe sind in diesem Fall meist organische Rückstände, Nebenproduk- 
te und Gülle oder Energiepflanzen wie etwa Maissilage. Anlagen zur Stromerzeugung mit Biogasen auf Basis thermo-chemischer Prozesse existieren bisher nur als Demonstrations- und Pilotanlagen. Den Nachteilen aufgrund eines hohen technischen Aufwands und damit verbundenen hohen Kosten sind hohe erreichbare elektrische Wirkungsgrade entgegen zu halten (Kaltschmitt und Thrän 2007).

Die Vorteilhaftigkeit der Nutzung von Biomasse ist wesentlich durch die regionale Verfügbarkeit geeigneter Einsatzstoffe bestimmt. In der Regel gilt Biomasse als klimaneutral, da das bei der Verbrennung emittierte $\mathrm{CO}_{2}$ zuvor beim Wachstum der Pflanzen aus der Atmosphäre gebunden wird. Andererseits sind insbesondere bei Festbrennstoffen höhere Staubemissionen im Vergleich zu fossilen Feuerungen zur beachten. Primärund Sekundärmaßnahmen dienen der Minimierung der Staubemissionen. Der wesentliche Vorteil im Vergleich zu anderen erneuerbaren Energieträgern wie Wind und Sonne liegt in der Speicherbarkeit und Lagerfähigkeit (Kaltschmitt et al. 2009). Weiterhin weist Biomasse im Vergleich zu fossilen Brennstoffen Vorteile hinsichtlich Krisensicherheit und Sozialverträglichkeit auf, beispielsweise durch Schaffung von Arbeitsplätzen im ländlichen Raum. Von Nachteil kann die unzureichend definierte Qualität der Energieträger sowie teilweise höhere Kosten im Vergleich zu fossilen Brennstoffen sein (Härdtlein und Eltrop 2004). Des Weiteren ist die Konkurrenz zur Nahrungsmittelproduktion sowie zu anderen Landnutzungsmöglichkeiten zu beachten (WBGU 2009). Aufgrund der Brennstofflogistik sind Anlagen auf Basis von Biomasse häufig kleiner als mit fossilen Brennstoffen betriebene Kraftwerke. Infolgedessen sind Maßnahmen zur Wirkungsgradsteigerung häufig nicht wirtschaftlich umsetzbar (Kaltschmitt et al. 2009).

\subsubsection{Photovoltaik}

Unter Photovoltaik (PV) versteht man die Umwandlung von (direkter und diffuser) Solarstrahlung in elektrische Energie auf Basis des photoelektrischen Effektes. Die Energiewandlung findet in Solarzellen statt, die aus mehreren Schichten halbleitender Materialien bestehen und zu Solarmodulen verbunden werden. Der erzeugte Gleichstrom kann direkt zum Betrieb elektrischer Geräte genutzt, in Batterien gespeichert oder mit Hilfe eines Wechselrichters in Wechselstrom umgewandelt und in das öffentliche Stromnetz eingespeist werden. PV-Anlagen verursachen keine direkten Emissionen im Betrieb. Da sie keine beweglichen Teile benötigen, sind sie praktisch verschleißfrei und arbeiten lautlos. Den sehr geringen Betriebskosten stehen hohe Investitionen gegenüber. Je nach Art und Qualität des eingesetzten Halbleiter-Materials variieren die Wirkungsgrade. Neben Verlusten im Wechselrichter begrenzen Reflexionsverluste, Leckströme, Widerstände und Rekombination erzeugter Ladungsträger die erreichbaren Wirkungsgrade. Durch Anlagen, bei denen ein System zur Nachführung entsprechend dem Einfallswinkel der direkten Sonneneinstrahlung integriert ist, kann die Energieaus- 
beute in der Regel erhöht werden. Dem stehen jedoch höhere Investitionen sowie Verluste durch Nebenantriebe gegenüber. Neben den hohen Investitionen können sich der relativ hohe Flächenbedarf sowie das diskontinuierliche Angebot der Solarstrahlung als nachteilig auswirken (Quaschning 2007; Quaschning 2008; Watter 2009; Wittwer 2007). Während es sich bei den meisten der in Deutschland installierten Anlagen um Aufdachanlagen handelt (Reichmuth et al. 2006), kann als Beispiel einer FreiflächenAnlage der Solarpark Walldorf an der Bundesautobahn A5 genannt werden. Dort sind auf einer Fläche von $40.000 \mathrm{~m}^{2}$ insgesamt 1,08 $\mathrm{MW}_{\mathrm{p}}$ installiert, die theoretische den Bedarf von etwa 350 Haushalten decken können (Stadtwerke Walldorf GmbH 2010).

Derzeit wird vorwiegend Silizium als Halbleiter-Material in PV-Zellen eingesetzt. Monokristalline Silizium-Zellen weisen höhere Wirkungsgrade auf als Zellen auf Basis von polykristallinem Silizium. Andererseits sind zur Herstellung des monokristallinen Materials mehr Verarbeitungsschritte notwendig und damit die Kosten höher. Vorteile hinsichtlich Materialeinsatz und Herstellungskosten weisen Dünnschichtzellen auf, die in zunehmendem Maße Anwendung finden. Diese können aus amorphem Silizium oder anderen Materialien (zum Beispiel Cadmiumtellurid - CdTe oder Kupfer-IndiumDiselenid - CulnSe ${ }_{2}$ bzw. CIS) bestehen. Jedoch erweisen sich geringere Wirkungsgrade teilweise toxische Materialien als nachteilig (Quaschning 2007; Wietschel et al. 2010). In Entwicklung befinden sich organische bzw. Polymer-Solarzellen auf Basis von Kunststoffen oder anderen organischen Materialien. Hierbei werden insbesondere Vorteile hinsichtlich Herstellkosten sowie Material- und Energieeinsatz bei der Produktion im Vergleich zu konventionellen PV-Zellen erwartet (Powell et al. 2009; Wittwer 2007).

Eine weitere vergleichsweise junge Technologie ist die konzentrierende PV. Dabei werden konzentrierende Linsen oder Spiegel eingesetzt, um einstrahlendes Licht zu bündeln und auf kleine Silizium-Solarzellen zu fokussieren. Dadurch kann die benötigte Halbleiterfläche und der Materialbedarf reduziert werden, bei gleichzeitiger Erhöhung des Wirkungsgrades. Die Wirtschaftlichkeit solcher Anlagen wird wesentlich durch den nutzbaren Anteil der direkten Solarstrahlung bestimmt. Daher müssen konzentrierende PV-Module mit Systemen zur Nachführung ausgestattet werden. Neben den langfristig zu erwartenden Kosten für die eingesetzten Nachführungssysteme sind für einen wettbewerbsfähigen Einsatz in Zukunft die Kosten der optischen Systeme entscheidend (Gombert et al. 2009; Quaschning 2008; Wietschel et al. 2010; Wittwer 2007).

\subsubsection{Solarthermische Kraftwerke}

In solarthermischen Kraftwerken wird direkte Sonnenstrahlung mit Hilfe von Spiegeln oder Linsen optisch auf einen Absorber konzentriert. Dadurch wird ein Medium erhitzt, 
welches, in der Regel über einen Sekundärkreislauf, Dampf erzeugt. Dieser wird zur Stromerzeugung in einer konventionellen Dampfturbine genutzt. Unterschiedliche technische Konzepte werden angewendet oder befinden sich in der Entwicklung (Parabolrinnen, Fresnel-Kollektoren, Solarturm, Dish-Stirling-Anlage bzw. Parabolspiegelsystem, Aufwindkraftwerk) (Heuck et al. 2007; Quaschning 2008; Wietschel et al. 2010; Wittwer 2007). Im Vergleich zur Photovoltaik als Alternative zur Nutzung der Solarstrahlung liegen hier Vorteile insbesondere in den Möglichkeiten zur Wärmespeicherung bzw. zum alternativen Betrieb des Dampfkraftprozesses mit einem Brenner. Beispielsweise können solarthermische Anlagen mit fossilen Kraftwerken zu Hybridkraftwerken kombiniert werden, um eine kontinuierliche Bereitstellung zu gewährleisten. Nachteilig sind der Flächenbedarf sowie die teilweise sehr hohen spezifischen Kosten zur Errichtung der notwendigen Anlagen und Infrastrukturen. Geeignete Standorte befinden sich in südlichen Ländern, an denen ausreichend direkte Solarstrahlung vorhanden ist (Bartels et al. 2008; Wietschel et al. 2010).

Die begrenzte Solarstrahlung in Deutschland rechtfertigt in der Regel nicht die hohen Investitionen für die Errichtung eines solarthermischen Kraftwerkes. Als Versuchs- und Demonstrationsanlage mit dem Ziel, solarthermische Kraftwerkstechnologien in Deutschland weiterzuentwickeln, wurde im Jahr 2009 der Solarturm Jülich in Betrieb genommen (Hartz und Koll 2008; Stadtwerke Jülich $\mathrm{GmbH}$ 2007). Weiterhin werden Konzepte diskutiert, die den Transport von Strom, der in solarthermischen Kraftwerken in sonnenreichen Regionen erzeugt wird, über weite Strecken nach Europa vorsehen (Czisch 2005). Für Deutschland sind dabei insbesondere Möglichkeiten zum Technologie-Export und Strom-Import von Interesse. Vor diesem Hintergrund wurde im Jahr 2009 die DESERTEC Industrie-Initiative (Dii) von mehreren deutschen Unternehmen im Energie- und Finanzsektor ins Leben gerufen. Ziel ist, bis zum Jahr $205015 \%$ des europäischen Bedarfs durch Strom zu decken, der in solarthermischen Kraftwerken in der MENA-Region (Middle East \& North Africa) erzeugt wird und durch Hochspannungs-Gleichstrom-Übertragung (HGÜ) nach Europa übertragen wird (DESERTEC Foundation 2009; Scholvin 2009).

\subsubsection{Geothermische Stromerzeugung und KWK}

Grundsätzlich wird unterschieden in oberflächennahe (bis ca. $400 \mathrm{~m}$ ) und tiefe (ca. 1.000 bis $3.000 \mathrm{~m}$ ) Geothermie. Oberflächennahe Geothermie wird in der Regel direkt zum Heizen oder Kühlen genutzt (vgl. Abschnitt 3.2.3.2). Eine Ausnahme stellen geothermischer Anomalien mit mehreren $100{ }^{\circ} \mathrm{C}$ dar, die zum Beispiel in Island vorkommen, und auch zur Stromerzeugung dienen können (Neupert 2007). Heißes Wasser bzw. Dampf aus tiefen Erdschichten kann entweder direkt für Heizzwecke eingesetzt werden oder über einen Wärmetauscher zur Dampf- und anschließenden Stromerzeu- 
gung genutzt werden. Dabei können hydrothermale Lagerstätten durch Förderung des natürlich vorhandenen heißen Wassers bzw. Dampfes an die Oberfläche über Bohrungen erschlossen werden. Wenn kein Thermalwasser vorhanden ist, kann das Hot-Dry-Rock-Verfahren (HDR; auch: Hot-Fractured-Rock - HFR) eingesetzt werden. Dabei wird Wasser von der Oberfläche über Bohrungen in tiefe (trockene) Gesteinsschichten geleitet und der Druck so lange erhöht, bis sich Risse bilden. Über Injektionssonden wird kaltes Wasser in die Tiefe geleitet, das sich in den Rissen verteilt, aufheizt und schließlich über eine Fördersonde wieder an die Oberfläche gelangt. Diese Technologie soll beispielsweise in einem Forschungskraftwerk mit $1 \mathrm{MW}_{\mathrm{el}}$ bei GroßSchönebeck in Brandenburg angewendet werden (Heuck et al. 2007). Eine weitere Möglichkeit der Erschließung tiefer Erdwärmequellen liegt in der Nutzung tiefer Erdwärmesonden. Dabei wird ein Wärmeträgermedium (zum Beispiel Wasser) über ein äußeres Stahlrohr in die Tiefe gepumpt. Das so aufgeheizte Medium gelangt durch ein isoliertes Förderrohr in der Mitte der Bohrung wieder an die Oberfläche, wo die Wärme genutzt werden kann. Diese Technologie ist jedoch aus energetischen und wirtschaftlichen Gründen nur in Ausnahmefällen sinnvoll (Neupert 2007; Strohschein et al. 2007; Wietschel et al. 2010). Für alle Verfahren gilt, dass aufgrund der hohen Erschließungskosten die Stromerzeugung in der Regel nur dann wirtschaftlich ist, wenn gleichzeitig eine Absatzmöglichkeit für die zur Verfügung stehende Wärme, beispielsweise als Heizwärme über Nah- oder Fernwärmenetze, besteht (Heuck et al. 2007).

Steht dampfförmiges Tiefenthermalwasser bei hohen Drücken und Temperaturen (ab ca. $150^{\circ} \mathrm{C}$ ) zur Verfügung, kann der Dampf direkt in einer Turbine zur Stromerzeugung genutzt werden. Nachteilig erweisen sich dabei oft stark korrosive Bestandteile des Dampfes. Bei nicht ausreichenden Dampfvorkommen werden so genannte FlashProzesse verwendet. Hierbei wird heißes, unter Druck stehendes Tiefenthermalwasser teilentspannt. Der dadurch erhöhte Dampfanteil kann abgetrennt und in einer Dampfturbine zur Stromerzeugung genutzt werden. Steht Erdwärme bei geringeren Temperaturen zur Verfügung (ab etwa $80^{\circ} \mathrm{C}$ ), können Dampfkraftprozesse angewendet werden, die Arbeitsmedien mit einem tieferen Siede- und Kondensationspunkt als Wasser verwenden (ORC, Kalina; vgl. Abschnitt 3.1.3). Grundsätzlich erweist sich im Vergleich zu anderen erneuerbaren Energien das kontinuierliche und gleichmäßige Angebot an Erdwärme als Vorteil. Bei verschiedenen Projekten sind jedoch Probleme in Zusammenhang mit der Stabilität des Untergrundes und Erdbeben in der Umgebung von Bohrungen aufgetreten. Des Weiteren besteht zusätzlicher Forschungsbedarf hinsichtlich der Vorhersage der Ergiebigkeit sowie zur Steigerung der Ausbeute geothermischer Energiequellen (Neupert 2007; Quaschning 2008; Tester et al. 2005; Wietschel et al. 2010). 
In Deutschland werden zur Strom- und Wärmeerzeugung aus Erdwärme derzeit Reservoirs mit Bohrtiefen von 2 bis $4,5 \mathrm{~km}$ erschlossen (tiefe Geothermie). Diese liefern Temperaturen von etwa 100 bis $150^{\circ} \mathrm{C}$ (Strohschein et al. 2007). Das erste geothermische Kraftwerk in Deutschland ging im Jahr 2003 in Neustadt-Glewe in Betrieb. Genutzt wird $98{ }^{\circ} \mathrm{C}$ heißes Wasser aus einer Bohrung mit einer Tiefe von 2.250 Metern. Die Anlage dient jedoch in erster Linie der Fernwärmeversorgung. Die volle elektrische Leistung von $210 \mathrm{~kW}_{\text {el }}$ wird nur in den Sommermonaten erreicht, wenn der Wärmebedarf gering ist (Heuck et al. 2007).

\subsubsection{Alternative Dampfkraftprozesse}

Neben der konventionellen Dampfturbine stehen weitere Technologien zur Verfügung, die zur Erzeugung von Strom in einem Dampfkraftprozess eingesetzt werden können. Kleindampfturbinen werden im Leistungsbereich von $150 \mathrm{~kW}$ bis $5 \mathrm{MW}$ angewendet. Diese sind mit einem Dampferzeugungsmodul gekoppelt, in dem unterschiedliche Brennstoffe eingesetzt werden können (Biomasse, Erdöl, Erdgas, Kohle). In der Regel werden Kleindampfturbinen im Gegendruckbetrieb als wärmegeführte KWK-Anlagen betrieben. Die elektrischen Wirkungsgrade erreichen, bezogen auf den eingesetzten Brennstoff, weniger als $20 \%$. Gesamtwirkungsgrade von mehr als $60 \%$ werden ausgewiesen, die im Teillastbetrieb auf etwa bis zu 35 bis $45 \%$ sinken (Bartels et al. 2008).

Anstelle von Dampfturbinen können Dampfmotoren eingesetzt werden, die als technisch ausgereifte Produkte im Leistungsbereich von $50 \mathrm{~kW}$ bis $2 \mathrm{MW}$ am Markt verfügbar sind. Bei Dampfkolbenmotoren bewegt unter Druck stehender Dampf unmittelbar einen Kolben in einem Zylinder, so dass thermische in mechanische Energie umgewandelt wird. Über eine Pleuelstange wird die an den Kolben übertragene Energie an die Arbeitswelle und letztlich an den Generator weitergegeben (Kaltschmitt et al. 2009). Da die Verbrennung außerhalb des Kolbens im separaten Dampferzeuger erfolgt, ist der Brennstoff prinzipiell frei wählbar. Vorteilhaft sind weiterhin die relativ stabilen Wirkungsgrade im Teillastbereich. Jedoch sind Dampfkolbenmotoren wartungsintensiv und weisen einen hohen Lärmpegel im Betrieb auf (Bartels et al. 2008). In einem Dampfschraubenmotor werden zwei ineinander greifende, gegenläufige Schraubenrotoren durch die Expansion von Dampf im Hohlraum, der sich um die Rotoren befindet, angetrieben. Von Vorteil im Vergleich zu anderen Expansionsmaschinen ist die hohe zulässige Dampfnässe, so dass auch Wärmeströme mit relativ geringem Temperaturniveau genutzt werden können. Dampfschraubenmotoren sind für Anlagen mit 150 $\mathrm{kW}_{\mathrm{el}}$ bis $1 \mathrm{MW}_{\mathrm{el}}$ geeignet und gelten als effizient und kostengünstig (Kaltschmitt et al. 2009; Suttor 2009). 
Um Wärmequellen mit niederen Temperaturen zur Stromerzeugung nutzen zu können, wurden Dampfkraftprozesse entwickelt, die im Gegensatz zu konventionellen WasserDampfkraftwerken Arbeitsmedien mit tieferem Siedepunkt verwenden. Beim Organic Rankine Cycle (ORC) werden organische Arbeitsmedien eingesetzt. Damit können Wärmequellen mit Temperaturniveaus ab etwa 70 bis $100^{\circ} \mathrm{C}$ zur Stromerzeugung genutzt werden, beispielsweise Abwärme aus Produktionsprozessen, Erdwärme, solare Wärme oder Wärme aus der thermo-chemischen Behandlung von Biomasse. Vor allem zur geothermischen Stromerzeugung findet der ORC-Prozess bereits weltweit Einsatz. Auch die Nachschaltung in einem „Bottoming Cycle“, beispielsweise zur Nutzung der Abgaswärme in BHKWs, ist gebräuchlich. Aufgrund hoher Kosten und geringer elektrischer Wirkungsgrade empfiehlt sich der Einsatz des ORC-Prozesses jedoch nur, wenn das Temperaturniveau der Wärmequelle keine andere Technologie zulässt. Andererseits sinkt auch der Wirkungsgrad mit der Temperatur der verwendeten Wärmequelle. Nachteilig sind auch die Eigenschaften einiger der verwendeten Arbeitsmittel. So sind beispielsweise Toluol, Pentan und Propan explosiv bzw. entflammbar und Fluorkohlenwasserstoffe klimaschädlich. Umweltfreundlichere Arbeitsmedien werden erprobt und sind teilweise bereits kommerziell verfügbar (Kaltschmitt et al. 2009). Höhere Wirkungsgrade bei noch niedrigen Temperaturen verspricht der Kalina-Prozess mit besseren thermodynamischen Prozesseigenschaften. Hier kommt ein Zweistoffgemisch, beispielsweise Wasser und Ammoniak, als Arbeitsmittel zum Einsatz (Quaschning 2008). Nachteilig im Vergleich zum ORC-Prozess sind größere benötigte Wärmetauscherflächen, eine höhere Anlagenkomplexität und damit verbunden höhere Kosten (Neupert 2007).

\subsubsection{Brennstoffzellen}

Bereits seit mehreren Jahrzehnten werden Bemühungen unternommen, die Kommerzialisierung von Brennstoffzellen zur stationären und mobilen Anwendung voranzutreiben (Blesl et al. 2006; Oertel 1997). Dabei handelt es sich um elektrochemische Wandler, in denen die chemisch gebundene Energie des zugeführten Brennstoffs (Erdgas, Biogas, Wasserstoff) direkt in elektrische Arbeit und Wärme umgewandelt wird, ohne den Zwischenschritt der Wärmeerzeugung. Durch die räumliche Trennung einer anodischen bzw. kathodischen Teilreaktion und den getrennten Transport der Ionen durch einen Elektrolyten bzw. der Elektronen durch einen externen Stromkreis entsteht eine Gleichstromquelle. Den hohen erzielbaren Wirkungsgraden, die zudem bei Teillast kaum absinken, und relativ geringen negativen Umweltauswirkungen stehen hohe Investitionen sowie eine begrenzte Lebensdauer gegenüber. Daher kam es bisher nicht zu einer weiten Verbreitung der Brennstoffzelle (Bartels et al. 2008; Suttor 2009). Sechs Typen von Brennstoffzellen können voneinander abgegrenzt werden, die sich 
durch die verwendete Membran, den Elektrolyten und die Betriebstemperatur unterscheiden (Pehnt 2002; Winkler 2002):

- AFC (Alkalische Brennstoffzelle; engl. Alkaline Fuel Cell),

- PEFC (auch: PEMFC, Polymer-Elektrolyt-Membran-Brennstoffzelle; engl. Polymer Electrolyte Membrane Fuel Cell),

- DMFC (Direktmethanol-Brennstoffzelle; engl. Direct Methanol Fuel Cell),

- PAFC (Phosphorsäure-Brennstoffzelle; engl. Phosphoric Acid Fuel Cell),

- MCFC (Schmelzkarbonat-Brennstoffzelle; engl. Molten Carbonate Fuel Cell),

- SOFC (Fest-Oxid-Brennstoffzelle; engl. Solid Oxide Fuel Cell), die auch in Kombination mit Gasturbinen betrieben wird.

Für den Einsatz in der Energiewirtschaft sind nach Suttor (2009) die PAFC, PEFC, MCFC und SOFC geeignet, wobei sich die meisten Systeme noch im Forschungs- und Entwicklungsstadium befinden. Dabei werden vor allem der PEFC und der SOCF hohe Entwicklungspotenziale zugeschrieben. Die MCFC ist mit einer elektrischen Leistung von $250 \mathrm{~kW}_{\mathrm{el}}$ bereits kommerziell verfügbar.

\subsubsection{Weitere Technologien und Trends in der Stromerzeugung}

Der traditionellen Stromversorgung mit zentralisierten Großkraftwerken steht insbesondere ein Trend zur Dezentralisierung gegenüber. Durch die Nutzung lokal verfügbarer erneuerbarer Energieträger (zum Beispiel Biomasse) besteht die Möglichkeit, die Energieversorgung umweltfreundlicher zu gestalten, Abhängigkeiten von wenigen großen Energieversorgern zu reduzieren und somit den Wettbewerb zu stärken. Dabei spielen verschiedene Technologien eine Rolle, die vor allem in kleineren Leistungsbereichen zum Einsatz kommen. Häufig werden dabei unter dem Begriff Blockheizkraftwerke (BHKW) mehrere Technologien zusammengefasst, die der dezentralen Bereitstellung von Strom und Wärme in Kraft-Wärme-Kopplung dienen. In vielen Fällen liegt dabei ein Schwerpunkt auf der Nutzung erneuerbarer (zum Beispiel BiogasBHKW) oder innovativer (zum Beispiel Brennstoffzelle) Technologien. In einer engeren Definition bezeichnen Blockheizkraftwerke kleinere Module im Leistungsbereich von etwa $5 \mathrm{~kW}_{\mathrm{el}}$ bis $20 \mathrm{MW}_{\mathrm{el}}$ mit Verbrennungsmotoren (Otto, Diesel) und einem Generator zur Stromerzeugung. Dabei können unterschiedliche flüssige und gasförmige Brennstoffe zum Einsatz kommen. Kleinere Module mit wenigen Kilowatt elektrischer Leistung (etwa 1 bis $5 \mathrm{~kW}_{\mathrm{el}}$ ) werden auch als Mini-BHKWs bezeichnet und kommen bevorzugt zur Versorgung größerer Einzelobjekte wie etwa Hotels, Krankenhäuser und Wohnanlagen zum Einsatz (Suttor 2009). Praetorius et al. (2009) fassen Klein-KWKAnlagen von bis zu $15 \mathrm{~kW}_{\text {el }}$ unter dem Begriff Mikro-KWK zusammen. Klein-BHKWs 
erreichen elektrische Wirkungsgrade von $28 \%$, größere Anlagen bis über $40 \%$ bei Gesamtwirkungsgrade von bis zu $90 \%$. Bei hohen Volllaststunden ist trotz höherer Investitionen im Vergleich zu Heizkesseln eine wirtschaftliche Nutzung von BHKWs im KWK-Betrieb möglich (Bartels et al. 2008; Suttor 2009).

Im Leistungsbereich von 1 bis $40 \mathrm{~kW}_{\mathrm{el}}$ sind Stirling-Motoren (auch: Heißgas-, Expansionsmotor) verfügbar. In diesen Anlagen wird durch Energiezufuhr aus einer externen Quelle ein eingeschlossenes Arbeitsgas (zum Beispiel Luft, Helium) komprimiert und expandiert. Über Kolben und Pleuelstangen wird die Energie auf eine Rotationswelle übertragen, die mit einem Generator verbunden werden kann. Vorteilhaft ist insbesondere die freie Wählbarkeit der externen Wärmequelle, beispielsweise Solarenergie, Biomasse oder industrielle Abwärme. Folglich können auch Brennstoffe mit relativ geringer Qualität verwendet werden. Weitere Vorteile liegen im leiseren und wartungsärmeren Betrieb im Vergleich zu Verbrennungsmotoren sowie im wartungsarmen Betrieb aufgrund weniger bewegter Teile. Problematisch ist hingegen die zuverlässige Abdichtung des Arbeitsgases. Die Komponenten zur Wärmeübertragung sind zudem hohen thermischen Belastungen und gegebenenfalls korrosiven Bestandteilen aus Verbrennungsgasen ausgesetzt. Weiterhin sind die hohen Temperaturen der Verbrennungsgase hinsichtlich der Betriebssicherheit problematisch. Darüber hinaus sind die erreichbaren Wirkungsgrade, die mit der Temperatur der Wärmequelle sinken, relativ gering (Kaltschmitt et al. 2009; Suttor 2009). Bei elektrischen Wirkungsgraden von 10 bis 30 \% können Gesamtwirkungsgrade von bis zu 85 \% erreicht werden, wobei das Teillastverhalten im Vergleich zu anderen KWK-Technologien als weniger gut einzustufen ist (Suttor 2009). Hohe Investitionen stellen ein weiteres Hemmnis für die Markteinführung dar (Bartels et al. 2008).

Neben der Nutzung unterschiedlicher Energiequellen in Wärme-Kraft-Prozessen liegen weitere Möglichkeiten der Stromerzeugung in physikalischen Effekten oder elektrochemischen Prozessen. Beispielsweise besteht das Prinzip der Thermoelektrik darin, dass aufgrund von Temperaturdifferenzen zwischen den Enden elektrischer Leiter ein Stromfluss entsteht (Seebeck-Effekt). Ebenso kann auf Basis thermoelektrischer Materialien aus elektrischer Energie Wärme (bzw. Kälte) erzeugt werden (Peltier-Effekt). Mögliche Vorteile liegen in der Wartungsfreiheit und Zuverlässigkeit, jedoch kommt die Thermoelektrik bisher nur in Nischenanwendungen zum Einsatz. Ein Hauptanwendungsbereich liegt derzeit bei der thermoelektrischen Kühlung. Zur Stromerzeugung sind prinzipiell thermoelektrische Generatoren mit Wirkungsgraden von 3 bis $8 \%$ realisierbar. Entsprechende Anlagenkonzepte sind jedoch derzeit noch im Versuchs- oder Demonstrationsstadium. Die Anwendungspotenziale hängen von den verfügbaren Temperaturniveaus, -differenzen und Wärmedichten ab und müssen noch näher untersucht werden (Bartels et al. 2008; Wietschel et al. 2010). 


\subsubsection{Speicherung von Elektrizität}

Die Stromnachfrage kann erheblichen tageszeitlichen, wöchentlichen wie auch saisonalen Schwankungen unterliegen, an die das Angebot angepasst werden muss. Stromspeicher erfüllen in diesem Zusammenhang wichtige Funktionen in Versorgungsnetzen, insbesondere um deren Stabilität und Zuverlässigkeit zu gewährleisten. Der fortschreitende Ausbau von Technologien basierend auf regenerativen Energiequellen, wie etwa Wind, Solarstrahlung und Wasserströmungen, führt zu einer stärkeren, nicht exakt vorhersehbaren Fluktuation der in das Versorgungsnetz eingespeisten Strommengen. Daher fallen Angebot und Nachfrage in zeitlicher wie räumlicher Hinsicht in zunehmendem Maße auseinander, und es ist damit zu rechnen, dass Stromspeicher in den nächsten Jahren an Bedeutung gewinnen. Auch der Trend hin zur Dezentralisierung in Verbindung mit erneuerbaren Energien und KWK verstärkt tendenziell den Bedarf an Speichern, da in dezentralen Netzen weniger Kapazitäten zur Verfügung stehen, die sich gegenseitig ausgleichen können (Baker 2008; Bouffard und Kirschen 2008; Bünger et al. 2009; Gatzen 2008; Tester et al. 2005). Als Alternativen zu stationären Stromspeichern bzw. parallel realisierbare Optionen seien an dieser Stelle Strategien des Netzausbaus sowie nachfrageseitige Lastmanagement-Maßnahmen genannt, die ebenfalls zu einem überregionalen Ausgleich von Angebot und Nachfrage beitragen können (Bünger et al. 2009; Klobasa 2009). Auch die Elektromobilität wird verstärkt diskutiert, wobei zukünftig eingesetzte Elektrofahrzeuge als Pufferspeicher im Stromversorgungssystem dienen und gleichzeitig Vorteile im Verkehrssektor (Senkung der $\mathrm{CO}_{2}$-Emissionen, Reduzierung der Importabhängigkeit) realisiert werden können (Bundesregierung 2009b).

Hinsichtlich des Prinzips der Speicherung ist in elektrochemische, elektromagnetische, kinetische, potenzielle und thermische Speicher zu unterscheiden (Baker 2008; Gatzen 2008). Thermische Speicher werden im Rahmen der Technologien zur Bereitstellung von Wärme in Abschnitt 3.2.4 diskutiert, können aber auch im Kontext der Strombereitstellung von Bedeutung sein, beispielsweise zur Speicherung von Solarwärme in solarthermischen Kraftwerken (vgl. Abschnitt 3.1.2.5) oder der Verdichtungswärme in adiabaten Druckluftspeicher-Kraftwerken (vgl. Abschnitt 3.1.6.1). Die Anwendungsbereiche der unterschiedlichen Stromspeicher-Technologien sind durch physikalische, technische und ökonomische Restriktionen begrenzt. Traditionell werden bei Spitzenlasten Pumpspeicher und Schnellstart-fähige fossile Kraftwerke (vor allem flexible Gasturbinen) sowie in dezentralen Netzen Diesel-Generatoren eingesetzt (Tester et al. 2005). Im Vergleich zu fossilen Spitzenlastkraftwerken oder Generatoren bieten Speicher das Potenzial eines $\mathrm{CO}_{2}$-ärmeren Betriebs (Baker 2008). Aufgrund hoher Kosten und teilweise geringer Wirkungsgrade kommen Stromspeicher derzeit jedoch kaum als Alternative zu Spitzenlastkraftwerken zum Einsatz (Gatzen 2008). 


\subsubsection{Mechanische Speicher}

Zu den mechanischen Speichern zählen Schwungräder, Pump- und Druckluftspeicher, die in den Ingenieurwissenschaften schon lange etabliert sind (Baker 2008). Pumpspeicher speichern die Energie in potenzieller Form und sind zur Speicherung über längere Zeiträume geeignet. Die installierte Leistung der in Deutschland installierten Pumpspeicherkraftwerke liegt zwischen wenigen MW bis etwa $1 \mathrm{GW}$ bei Gesamtwirkungsgraden von 75 bis $80 \%$ und Anfahrzeiten im Sekunden- bis Minutenbereich. Das zusätzliche Potenzial zur Ausweitung der Pumpspeicherkapazitäten ist vor allem durch geografische Gegebenheiten stark begrenzt (Bünger et al. 2009; Gatzen 2008; Oertel 2008; vgl. Abschnitt 3.1.2.1). Druckluftspeicherkraftwerke (Compressed Air Energy Storage - CAES) sind prinzipiell als Tages- oder Wochenspeicher einsetzbar, weisen jedoch hohe Kosten auf. Weltweit sind zwei Anlagen installiert (Huntdorf in Deutschland, $321 \mathrm{MW}$; McIntosh in Alabama, USA, $110 \mathrm{MW}$ ). Dort wird Luft mit einem Kompressor in Schwachlastzeiten komprimiert und in einer geeigneten Untergrundformation gespeichert. Durch Entspannung der Luft in einer Gasturbine wird Strom erzeugt (Bünger et al. 2009; Oeding und Oswald 2004; Oertel 2008). Dabei ist eine Zusatzfeuerung (in der Regel mit Erdgas) zur Erwärmung der kalten, komprimierten Luft erforderlich. Zukünftige Entwicklungen zielen auf adiabate Systeme ab (Advanced Adiabatic AA-CAES), die im Idealfall keine Zusatzfeuerung benötigen. Dabei wird die bei der Kompression entstehende Wärme in einem thermischen Speicher zwischengespeichert und zur Erwärmung der Druckluft bei der Stromerzeugung genutzt. Der dadurch erreichbaren Erhöhung des Gesamtwirkungsgrades stehen höhere Kosten gegenüber. Diese Technologie wird im Rahmen des EU-Projektes „AA-CAES“ erforscht (Jakiel et al. 2007; Zunft et al. 2006). Weiterhin befinden sich kleine Druckluftsysteme in Entwicklung, die keine Untergrundformationen als Speicherraum nutzen, sondern zum Beispiel Stahl-Drucktanks. Dadurch soll die Druckluftspeicherung auch an solchen Standorten ermöglicht werden, an denen keine geeigneten Untergrundformationen existieren (Baker 2008; Oertel 2008).

Schwungräder speichern kinetische Energie in einer rotierenden Masse, wobei die Umwandlung von bzw. in Elektrizität über einen Motor bzw. Generator erfolgt. Sie dienen zur kurzfristigen Überbrückung von Störungen des elektrischen Netzes, um eine zuverlässige und kontinuierliche Stromversorgung von sensiblen Produktionsprozessen sicherzustellen. Im Vergleich zu großen Blei-Säure-Batterien zeichnen sie sich durch höhere Zyklenfestigkeit und geringere negative Umweltauswirkungen aus. Darüber hinaus sind die Betriebskosten gering und es können hohe Leistungen abgedeckt werden, die innerhalb weniger Millisekunden aufgenommen oder abgegeben werden können. Zwar liegen die Wirkungsgrade bei Kurzzeitspeicherung bei 90 bis $95 \%$. Aufgrund von Standzeitverlusten von bis zu $20 \%$ je Stunde erscheinen jedoch Anwen- 
dungen, in denen Speicherzeiträume über mehrere Minuten und Stunden überbrückt werden müssen, wenig sinnvoll (Baker 2008; Bünger et al. 2009; Gatzen 2008; Oertel 2008).

\subsubsection{Elektrochemische Speicher}

Zu den elektrochemischen Speichersystemen zählen klassische Batterien (zum Beispiel Blei-Säure-Batterien, engl. Lead Acid - LA), Wasserstoffspeicher sowie so genannte Fluss-Zellen bzw. Redox-Flow-Batterien (RFB). Bei LA-Batterien handelt es sich um eine reife und etablierte Technologie. Den niedrigen Anfangsinvestitionen und Vorteilen hinsichtlich Selbstentladung stehen eine relativ geringe Zyklenfestigkeit und Energiedichte gegenüber (Oertel 2008; Wietschel et al. 2010). Lithium-IonenBatterien (LiB) haben sich aufgrund der hohen Energiedichte vor allem zur Anwendung in kleineren Geräten etabliert. Im Rahmen der Innovationsallianz "Lithium Ionen Batterie LIB 2015“ werden Forschungs- und Entwicklungsaktivitäten in der Industrie insbesondere im Hinblick auf Anwendungen in Elektrofahrzeugen forciert (BMBF 2010; Bundesregierung 2009b). Nachteile betreffen die Robustheit der Batterien sowie leicht brennbare Komponenten. Die Möglichkeiten zur Anwendung in größeren Leistungsbereichen sind derzeit noch begrenzt. Eine bessere Leistungsfähigkeit versprechen Lithium-Schwefel-Systeme (LiS). Diese werden zurzeit allerdings nur von einer kleinen Anzahl an Forschungseinrichtungen untersucht. Natrium-Schwefel-Batterien (NaS, auch: ZEBRA) werden bei hohen Temperaturen von etwa 270 bis $350{ }^{\circ} \mathrm{C}$ betrieben und können unter günstigen Bedingungen Wirkungsgrade von 70 bis $80 \%$ erzielen. Ein Hemmnis für Anwendungen im großen Maßstab stellen jedoch die hohen Kosten dar. Auch bei Natrium-Nickel-Chlorid-Batterien ( $\mathrm{NaNiCl}$ ) handelt es sich um Hochtemperatursysteme, deren Entwicklung sich für Anwendungen im Stromsektor noch in den Anfängen befindet. Im Vergleich zu NaS-Batterien werden Vorteile hinsichtlich der Sicherheit, der höheren Zellspannung und der Widerstandsfähigkeit bei Über- oder Unterladung erwartet (Baker 2008; Gatzen 2008; Oertel 2008; Wietschel et al. 2010).

Im Gegensatz zu konventionellen Batterie-Systemen wird die Energie in Redox-FlussBatterien (RFB) in einem flüssigen Elektrolyten (zum Beispiel Vanadium, Zink-Bromid, Polysulfid-Bromid oder Zink-Cerium) in externen Tanks gespeichert. Die Speicherkapazität wird durch das Tankvolumen bestimmt und kann somit unabhängig von der Leistungseinheit skaliert werden. Im Vergleich zu konventionellen Bleibatterien ist die höhere Zyklenfestigkeit hervorzuheben. Der zukünftige Markterfolg der RFB hängt von Entwicklungen hinsichtlich der Elektroden, Elektrolyte, Membranen, Dichtungen und der Fertigungstechnologie ab. Um auf Basis der RFB eine wettbewerbsfähige Alternative zur konventionellen elektrochemischen Speicherung für Anwendungen mit Spei- 
cherkapazitäten von mehreren Stunden zu schaffen, müssen die Kosten noch erheblich gesenkt werden (Baker 2008; Gatzen 2008).

Auch die Erzeugung von Wasserstoff, dessen Speicherung und Rückverstromung stellt eine Möglichkeit zur elektrochemischen Stromspeicherung dar. Dabei sind unterschiedliche Varianten denkbar. Beispielsweise kann mit Wind- oder Solarstrom über Elektrolyse Wasserstoff erzeugt, in gasförmiger oder flüssiger Form gespeichert und wiederum in Wasserstoff-Gasturbinen oder Brennstoffzellen zur Strom- und Wärmeerzeugung eingesetzt werden. Jedoch müssen insbesondere die Kosten der Brennstoffzelle und anderer Technologien zur Erzeugung und Nutzung des Wasserstoffs reduziert werden, um eine weitere Markdurchdringung voranzutreiben. Zudem ist der geringe Gesamtwirkungsgrad (von der Erzeugung von Wasserstoff mit Strom über die Speicherung und die Rückumwandlung in Strom etwa $30 \%$ ) und die geringe technische Lebensdauer von Technologien wie etwa der Brennstoffzelle problematisch (Bünger et al. 2009; Edwards et al. 2008; Gatzen 2008).

\subsubsection{Elektromagnetische Speicher}

Elektromagnetische Speicherung erfolgt in SMES (Superconducting Magnetic Electricity Storage) und Doppelschichtkondensatoren (SuperCaps - Super Capacitors). SuperCaps nutzen elektrostatische Wechselwirkungen für den Elektronenaustausch. Dem geringen Wartungs- und Instandhaltungsaufwand, der hohen Zyklenfestigkeit und Beständigkeit bei niedrigen Temperaturen stehen Selbstentladungen bei Temperaturen über $85^{\circ} \mathrm{C}$ und geringe Energiedichten als Nachteile gegenüber. Mittelfristig wird nicht erwartet, dass SuperCaps für größere stationäre Anwendungen im Megawatt-Bereich und zur Speicherung über längere Zeiträume eine Rolle spielen. Denn zum einen ist der Vorteil hoher Zyklenfestigkeit bei längeren Speicherzeiträumen von untergeordneter Bedeutung und zum anderen ist die Selbstentladung relativ hoch. SMES bestehen aus einer supraleitenden Spule und einer zusätzlich notwendigen Kühlvorrichtung. Die Elektrizität wird bei minimaler Selbstentladung in einem magnetischen Feld gespeichert, das durch den elektrischen Strom erzeugt wird, der durch die Spule fließt. Zwar werden Wirkungsgrade von $99 \%$ ausgewiesen, die jedoch nur unter Vernachlässigung des Kühlbedarfs gültig sind. Über kurze Zeiträume können Leistungen von bis zu $3 \mathrm{MW}$ innerhalb von Millisekunden bereitgestellt werden. Verfügbar sind derzeit Systeme mit 1 bis $10 \mathrm{~kW}$, die im Qualitätsmanagement und für die unterbrechungsfreie Stromversorgung eingesetzt werden. Eine stationäre Anwendung in größerem Maßstab erscheint eher unwahrscheinlich (Bünger et al. 2009; Gatzen 2008). 


\subsection{7 Übertragung von Elektrizität}

Das Stromnetz ist das wesentliche Verbindungselement zwischen der Erzeugungsund der Verbraucherseite (Praetorius et al. 2009). Von den Übertragungs- bzw. Transportnetzen mit weiträumigen Leitungen und hohen Spannungen werden die Verteilungsnetze niedriger Spannung in der Nähe der Verbraucher abgegrenzt. Kleinere Verbraucher wie Haushalte sind normalerweise an das Niederspannungsnetz (NS: $400 \mathrm{~V}$ für Drehstrom-Verbraucher), größere Industrie-Verbraucher an das Mittelspannungsnetz (MS: 10 bzw. 20 kV) oder Hochspannungsnetz (HS: 110 kV) angeschlossen (Verteilungsnetze). Großkraftwerke mit mehr als $700 \mathrm{MW}$ sind an das übergeordnete Höchstspannungsnetz mit $220 \mathrm{kV}$ bzw. $380 \mathrm{kV}$ angeschlossen (Transport/Übertragungsnetz). In der Regel wird elektrische Energie als Dreileiter-Drehstrom mit $50 \mathrm{~Hz}$ (Europa) oder $60 \mathrm{~Hz}$ (Nordamerika) bereitgestellt. Nur in Sonderfällen werden andere Stromarten verwendet, beispielsweise bei der Hochspannungs-GleichstromÜbertragung (HGÜ). Aus wirtschaftlichen oder technischen Gründen kann es zweckmäßig bzw. notwendig sein, HGÜ einzusetzen, wenn zum Beispiel weite Entfernungen überbrückt und/oder Wasser durchquert werden muss, um den Strom vom Erzeuger zum Nutzer zu bringen (Crastan 2007; Heuck et al. 2007; Oeding und Oswald 2004; Wietschel et al. 2010). Der Neubau von Höchstspannungsleitungen könnte zu einem weitflächigen Ausgleich von Angebot und Nachfrage in Stromversorgungssystemen beitragen (vgl. auch Abschnitt 3.1.6). Jedoch erschweren aufwändige Genehmigungsverfahren und Akzeptanzprobleme in der Bevölkerung die Umsetzung entsprechender Konzepte (Bünger et al. 2009). Aktuelle Entwicklungstendenzen bezüglich Technologien für Übertragungs- und Verteilungsnetze werden bei Wietschel et al. (2010) beschrieben.

Für die Verteilung von Strom in Deutschland sind Übertragungsnetzbetreiber (ÜNB bei $>110$ kV; engl:: Transmission System Operator - TSO) und Verteilungsnetzbetreiber (VNB bei $\leq 110$ kV; engl.: Distribution System Operator - DSO) verantwortlich. Dabei handelt es sich um acht große Verbundunternehmen sowie etwa 500 meist kommunale Energieversorgungsunternehmen. Die Verbundunternehmen sind zudem für den Stromaustausch mit dem Ausland über Koppelstellen zwischen den Übertragungsnetzen der einzelnen Länder verantwortlich. Die europäischen Stromnetze der meisten Länder sind im UCTE-Netz (Union pour la Coordination du transport de l'Electricité Union für die Koordination des Transportes elektrischer Energie) miteinander verbunden (Crastan 2007; Heuck et al. 2007). 


\subsection{Bereitstellung von Nutzwärme}

Der Wärmemarkt ist stark heterogen und unterliegt dynamischen Veränderungen, so dass die Beschaffung konsistenter Daten und das Aufzeigen von Entwicklungstendenzen problematisch ist. Die Akteure reichen von einzelnen Haushalten mit eigener Wärmeversorgung bis zu Unternehmen, die größere Gebiete mit Fernwärme versorgen. Nahezu 50 \% des deutschen Wärmemarktes werden durch die Gasversorgungsunternehmen bestimmt. Diese beliefern private Haushalte, Industrieunternehmen sowie den GHD-Sektor (Gewerbe, Handel, Dienstleistungen). Zwischen Erdgasimporteuren und -händlern herrscht bisher nur wenig Wettbewerb (Strohschein et al. 2007). Andererseits wurden in den letzten Jahren in zunehmendem Maße Konkurrenztechnologien vor allem auf Basis erneuerbarer Energien auf dem Wärmemarkt eingeführt (zum Beispiel Heizkessel mit Holzpellets, Wärmepumpen oder Solarkollektoren). Auch die Kombination von Brennwertgeräten mit Solarkollektoren, Mikro-KWK, Brennstoffzellen und Biogas-Anwendungen erfahren zunehmendes Interesse auf dem Wärmemarkt. Während die Verteilung und Nutzung von Gas sowie der Fernwärme an ein Netz gebunden sind, können Heizöl, Kohle und Biomasse leitungsunabhängig bereitgestellt werden. Vorteilhaft aus Sicht der Wärmekunden ist die bedarfsgerechte und komfortable Bereitstellung von Gas und Fernwärme, so dass keine Lagerung des Brennstoffes vor Ort (wie beispielsweise bei Verwendung von Heizöl) notwendig ist (Strohschein et al. 2007). Im Folgenden werden die wichtigsten Technologien zur Wärmebereitstellung skizziert. Alternativen zur Wärmebereitstellung aus Kraft-Wärme-Kopplung werden in Zusammenhang mit der Stromerzeugung diskutiert (Abschnitt 3.1).

\subsubsection{Wärmebereitstellung auf Basis fossiler Primärenergien}

In privaten Haushalten und im Gewerbe erfolgt die Wärmeversorgung in den meisten Fällen über Gas- oder Ölheizkessel. Bei Neuinstallationen kommt überwiegend die Niedertemperatur- oder die Brennwerttechnologie zum Einsatz. In Niedertemperaturkesseln können Energieverluste reduziert werden, indem die Vorlauftemperatur dem aktuellen Wärmebedarf angepasst wird. Durch die Brennwerttechnologie ist es möglich höhere Brennstoffnutzungsgrade zu erzielen, indem die Kondensationswärme des Abgases genutzt wird. Gas-Brennwertkessel gelten als ausgereifte Technologie und finden bei etwas mehr als der Hälfte neu installierter Anlagen Anwendung. Öl-Brennwertgeräte finden bisher wenig Verbreitung (vgl. Abbildung 3.1; Strohschein et al. 2007). Aufgrund des Reifegrades der Brennwerttechnologie scheinen Optimierungspotenziale eher begrenzt (Bartels et al. 2008). Erdgas bietet gegenüber Erdöl aufgrund des geringeren spezifischen $\mathrm{CO}_{2}$-Emissionsfaktors Vorteile hinsichtlich der Umweltauswirkungen. Zunehmend werden auch Erdgas-Brennwertkessel in Verbindung mit Solarkollektoren (vgl. Abschnitt 3.2.3.4) nachgefragt, um weitere Vorteile hinsichtlich der Um- 
weltauswirkungen zu erzielen. Der wesentliche Nachteil der fossil basierten Feuerungen im Vergleich zu erneuerbaren Technologien liegt in der Abhängigkeit von den erschöpfbaren Ressourcen der Brennstoffe. Die variablen Betriebskosten (Brennstoffkosten) sind von den Preisentwicklungen auf den Rohstoffmärkten für Energieträger abhängig.

Abbildung 3.1: Strukturentwicklung von Wärmeerzeugern in Deutschland (neu installierte Anlagen)

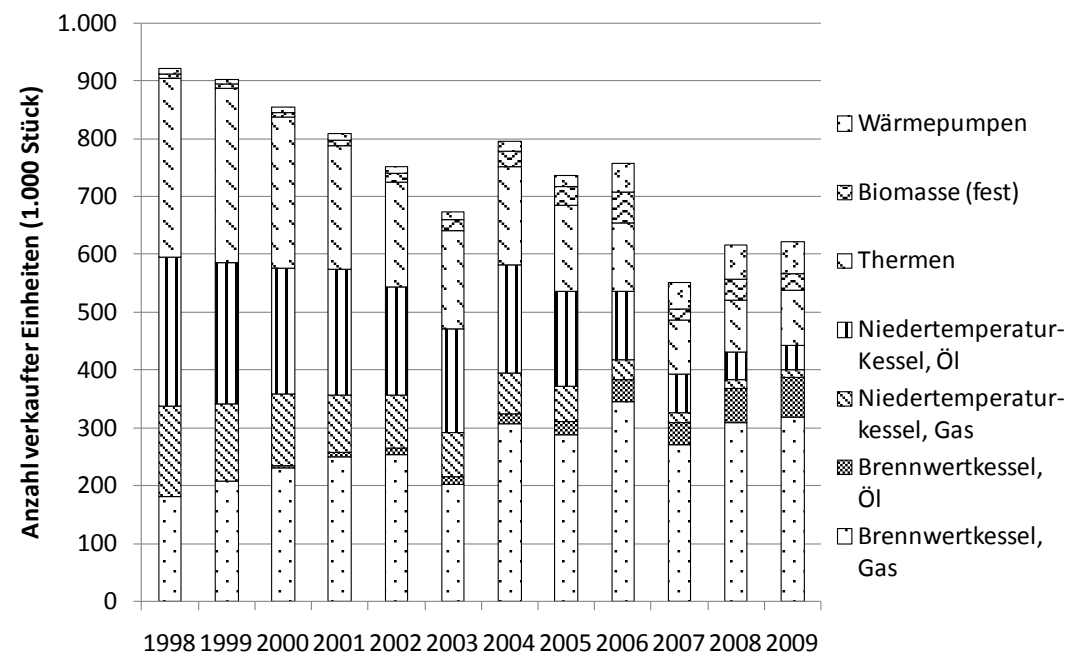

Quelle: Basierend auf Daten des BDH (Bundesindustrieverband Deutschland Haus-, Energieund Umwelttechnik e.V.; http://www.bdh-koeln.de/)

\subsubsection{Ersatzbrennstoffe}

In Hausmüllverbrennungsanlagen wird der Teil der Siedlungsabfälle thermisch umgesetzt, der nicht anderweitig verwertet werden kann. Die energetische Nutzung der Abfälle trägt zum Klimaschutz bei, da in der Regel mehr als die Hälfte biogenen Ursprungs und somit $\mathrm{CO}_{2}$-neutral ist. Aus dem Energiegehalt der Rest-Siedlungsabfälle wird Strom, Dampf und Heißwasser erzeugt. Die Wärme wird meist als Fernwärme oder Prozessdampf verwendet. In Deutschland könnten weitere Potenziale zur Wärmenutzung durch Anbindung neuer Abnehmer und erhöhte Einspeisung in bestehende Wärmenetze realisiert werden (Strohschein et al. 2007).

\subsubsection{Wärmebereitstellung aus erneuerbaren Energien}

Derzeit trägt unter den erneuerbaren Energien vorwiegend Biomasse zur Wärmebereitstellung bei (vgl. Abbildung 2.9, S. 17). Weitere Möglichkeiten zur Wärmebereitstellung aus erneuerbaren Energien bestehen aufgrund der vorhandenen Potenziale an Solarwärme und Erdwärme. 


\subsubsection{Biomasse zur Wärmebereitstellung}

Zur Wärmebereitstellung aus Biomasse finden vorwiegend biogene Festbrennstoffe und in kleinerem Umfang Biogas Anwendung (vgl. auch Abschnitt 3.1.2.3). Neben der Nutzung von Biogas zur Wärmebereitstellung aus KWK-Anlagen kann mittels Wärmepumpen auch die Abwärme genutzt werden, die beim aeroben Abbau der Biomasse (Kompostierung) zur Biogaserzeugung entsteht. Brennholz kann aus Restholz (Wald-, Industrierestholz, Altholz etc.) oder Energiepflanzen aus Kurzumtriebsplantagen (Weide, Pappel) gewonnen werden. Daraus können Stückgutbrennstoffe, beispielsweise in Form von Holzscheiten, oder Schüttgutbrennstoffe, zum Beispiel Hackschnitzel oder Pellets, bereitgestellt werden. In Einzelöfen, Zentralheizungen und Heizwerken finden Pellets, Holzhackschnitzel oder Scheitholz als Brennstoff Anwendung. Altholz, Waldrestholz und Pflanzenreststoffe werden vorwiegend in Heizkraftwerken mit 5 bis $20 \mathrm{MW}$ elektrischer Leistung zur gekoppelten Strom- und Wärmeerzeugung eingesetzt Dabei liegt die Obergrenze der EEG-Förderung für Biomasse-Anlagen bei einer installierten Leistung von $20 \mathrm{MW}$, wohingegen für größere Anlagen keine Einspeisevergütung vorgesehen ist (vgl. §27 EEG2008, Bundesregierung 2008c). Der als klimaneutral angesehenen Verbrennung der Biomasse stehen höhere Staubemissionen im Vergleich zu Öl- und Gaskesseln entgegen. Die Optimierung des Brennstoff-Luft-Verhältnisses mit Hilfe einer entsprechenden Regelungstechnik kann zur Minimierung dieser Emissionen beitragen. Reichen derartige Primärmaßnahmen nicht aus, sind Sekundärmaßnahmen wie eine nachgeschaltete Staubabscheidung erforderlich, um Emissionsgrenzwerte einhalten zu können. In zunehmendem Maße finden auch Holzfeuerungen in Kombination mit Solarkollektoren zur Brauch- und Heizwassererwärmung Verwendung. Im weiteren Sinne wird zur Biomassenutzung darüber hinaus die Nutzung von Deponie- und Klärgas sowie die Verwertung organischer Biomasseabfälle gezählt (FNR 2005; Kaltschmitt et al. 2009; Strohschein et al. 2007).

$\mathrm{Zu}$ den gebräuchlichen handbeschickten Holzfeuerungen zählen offene und geschlossene Kamine, Zimmer-, Kamin- und Speicheröfen, Küchenherde, Pellet-Öfen sowie Stückholzkessel. Zur Erhöhung des Bedienkomforts und der Vergleichmäßigung der Verbrennungsbedingungen wurden automatisch beschickte Feuerungen entwickelt. Darin werden Pellets, Hackgut, Rinden, Halmgut und staubförmige Biomassen eingesetzt. Feuerungstechnisch sind Festbett-, Wirbelschicht- und Flugstromverbrennung zu unterscheiden (Kaltschmitt et al. 2009). Anlagen, die mit Holzpellets befeuert werden, bieten insbesondere aufgrund der homogenen Zusammensetzung des Brennstoffes Vorteile hinsichtlich der Umweltauswirkungen im Vergleich zu anderen HolzBrennstoffen. Bedingt durch vorgelagerte Bearbeitungsschritte sind jedoch die Preise für Pellets im Vergleich zu anderen Holzbrennstoffen in der Regel höher. Grundsätzlich kann Biomasse in größeren Anlagen zwar effizienter in Wärme (und/oder Strom) um- 
gewandelt werden. Dem stehen jedoch Verluste bei der Verteilung der Wärme und ein höherer Aufwand bei der Beschaffung der Brennstoffe gegenüber (Strohschein et al. 2007). Weitere Stärken und Schwächen von Biomasse im Vergleich zu anderen Energieträgern wurden bereits in Zusammenhang mit Stromerzeugung und KWK diskutiert (Abschnitt 3.1.2.3).

\subsubsection{Geothermische Wärmenutzung}

Technologien zur Erschließung und Nutzung von Erdwärme in KWK-Anlagen sind in Abschnitt 3.1.2.6 beschrieben. Aus ökonomischen und ökologischen Gründen ist in vielen Fällen die direkte Wärmenutzung geothermischer Niedertemperatur-Quellen der Nutzung zur Stromerzeugung vorzuziehen. Andererseits setzt die Wärmenutzung einen entsprechenden Bedarf in hinreichender räumlicher Nähe voraus. In Deutschland wird sowohl oberflächennahe als auch tiefe Geothermie zum Heizen oder zur Warmwasserbereitung und bei ausreichend hohen verfügbaren Temperaturen auch als Prozesswärme genutzt. Um oberflächennahe Erdwärme mit geringen Temperaturnniveaus aus Tiefen bis etwa 400 Metern zum Heizen, Kühlen oder zur Warmwasserbereitung zu nutzen, können beispielsweise Erdwärmesonden oder Wärmepumpen (vgl. Abschnitt 3.2.3.3) eingesetzt werden (Neupert 2007; Quaschning 2008; Strohschein et al. 2007). Da Tiefenbohrungen mit erheblichen Kosten und Risiken verbunden sind, ist für deren wirtschaftliche Nutzung zur Wärmebereitstellung eine relativ große benötigte Heizleistung erforderlich. Daher ist der Anschluss mehrerer Wärmekunden über ein Nah- oder Fernwärmenetz sinnvoll. Für gewöhnlich wird zusätzlich ein Reserveoder Spitzenlastkessel installiert, der auch die Verfügbarkeit im Falle eines Ausfalls des geothermischen Prozesses oder bei Wartungsarbeiten sichert (Quaschning 2008). So ist zum Beispiel im Geothermie-Kraftwerk in Neustadt-Glewe eine zusätzliche Gasfeuerung zur Sicherung der Wärmebereitstellung installiert (Heuck et al. 2007).

\subsubsection{Wärmepumpen}

Wärmepumpen heben auf Basis von Kreisprozessen Wärme von einem niedrigen auf ein höheres nutzbares Temperaturniveau an. Dabei kann die Eingangswärme aus unterschiedlichen Quellen (zum Beispiel Erdwärme, Abwärme, Abwasser, Umgebungsluft) stammen. Durch Zuführung einer Antriebsenergie (elektrische Energie, Gas) durchläuft das Arbeitsmedium die Anlagenkomponenten Verdampfer, Verdichter, Wärmetauscher und Expansionsventil und gibt dabei Wärme ab bzw. nimmt diese auf (Hartmann und Schwarzburger 2009; Neupert 2007; Strohschein et al. 2007). In technischer Hinsicht sind Kompressions-, Absorptions- und Adsorptions-Wärmepumpen zu unterscheiden (Quaschning 2008). Nach der Wärmequelle kann in Luft-/Luft-, Luft/Wasser-, Wasser/Wasser- und Sole/Wasser-Wärmepumpen unterschieden wer- 
den (Hartmann und Schwarzburger 2009). Die Marktdurchdringung von Wärmepumpen wird begünstigt, wenn die Preise fossiler Primärenergieträger, die in Konkurrenztechnologien (vor allem Gas-Brennwertkessel) genutzt werden, steigen. Zudem ist die Erhöhung der Jahresarbeitszahl3 im Teillastbereich und eine Verbesserung der Regelbarkeit wünschenswert (Bartels et al. 2008). Beim derzeitigen Strommix sind elektrische Wärmepumpen aus Umweltsicht nur geringfügig besser zu bewerten als Gasbrennwertkessel. Dies könnte sich ändern, wenn der Antriebsstrom vorwiegend aus erneuerbaren Energien stammt. Obwohl Gasmotor-Wärmepumpen wesentlich günstigere Eigenschaften hinsichtlich der Treibhausgasemissionen aufweisen, haben diese bisher nur einen sehr kleinen Anteil am deutschen Wärmemarkt. Thermisch angetriebene Sorptions-Wärmepumpen weisen Vorteile hinsichtlich Wartung und Lärmemissionen im Betrieb auf. Weiterhin ermöglichen sie die effiziente Nutzung von Abwärme, ohne dass halogenierte Kältemittel erforderlich sind (Strohschein et al. 2007).

\subsubsection{Solarkollektoren}

Mit Hilfe von Sonnenkollektoren wird diffuse und direkte Solarstrahlung zur Gewinnung von Nutzwärme verwendet. Die Strahlung erhitzt einen Metall- oder Kunststoffabsorber, durch den ein Wärmeträgermedium fließt. Dieses transportiert die erzeugte Wärme zum Nutzer oder zu einem Speicher (Wittwer 2007). Absorber in Form von schwarzen Kunststoffmatten finden in der Regel zur Beheizung des Wassers in Freibädern Anwendung. Flachkollektoren, deren Absorber durch eine ebene Glasscheibe abgedeckt ist, sind am weitesten verbreitet zur Brauchwassererwärmung und Heizungsunterstützung in Ein- oder Zweifamilienhäusern. Ein kleinerer Anteil der solaren Wärmebereitstellung wird durch Vakuumröhrenkollektoren abgedeckt. Diese können zwar höhere Erträge erzielen, weisen jedoch im Vergleich zur Flachkollektoren höhere Systemkosten auf. Beide Arten von Anlagen sind als weitgehend ausgereifte Produkte verfügbar, werden für Gewöhnlich auf Hausdächern montiert und sind zur Beheizung von Räumen und Brauchwasser geeignet. Etwa 80 \% der derzeit installierten Solarkollektoren dienen der Bereitstellung von Warmwasser. Dabei wird in der Regel ein Brauchwasserspeicher zum Ausgleich von Angebot und Nachfrage eingesetzt. Die übrigen $20 \%$ werden zur kombinierten Heizungsunterstützung und Warmwasserbereitung verwendet. In diesem Fall ist neben dem Brauchwasserspeicher auch ein Pufferspeicher für die Heizungsanlage erforderlich. Häufig ist zusätzlich zu den Solarkollekt-

3 Die Jahresarbeitszahl einer Wärmepumpe beschreibt das Verhältnis der abgegebenen thermischen Leistung zu zugeführten elektrischen Leistung, die an einer realen Anlage im Betrieb gemessen werden. Die Jahresarbeitszahl trifft somit analog dem Wirkungsgrad bei anderen Erzeugungsanlagen eine Aussage über die Effizienz der Energiebereitstellung (Hartmann und Schwarzburger 2009). 
oren auch ein Gasbrennwertkessel oder eine alternative Technologie installiert, um eine bedarfsgerechte Wärmebereitstellung sicherzustellen. Während sich die meisten derzeit installierten Anlagen auf Ein- oder Zweifamilienhäusern befinden, wird in Modellvorhaben untersucht, inwieweit eine Nutzung von Solarkollektoren in Verbindung mit Langzeitwärmespeichern und Nahwärmenetzen in Gemeinschaftsanlagen möglich ist (Quaschning 2008; Strohschein et al. 2007).

\subsubsection{Speicherung von Wärme}

Wärmespeicher (thermische Speicher) dienen zur Aufrechterhaltung einer konstanten Versorgungstemperatur, deren schnellen Verfügbarkeit beim Verbraucher sowie zur Vergleichmäßigung der Fördermengen in Rohrleitungssystemen und damit zur Erhöhung der Lebensdauer von Anlagenkomponenten. Weiterhin wird eine Kompensation von Lastspitzen bei gleichzeitiger Minimierung der installierten Erzeugerleistung (Investition) und der Brennstoffkosten angestrebt. Neben dem verwendeten Speichermedium ist die konstruktive Ausführung der Speicher, zum Beispiel gute Isolation, für einen thermodynamisch effizienten Betrieb von besonderer Bedeutung. Bei wenigen Belade- $I$ Entladezyklen und langen Standzeiten können Wärmeverluste an die Umgebung und zunehmende Durchmischung des Speichermediums eine größere Rolle bei der optimalen Auslegung eines Wärmespeicher spielen (Bartels et al. 2008; Tester et al. 2005).

Nach dem physikalischen Prinzip der Speicherung kann in sensible (fühlbare), latente und chemische Wärmespeicher unterschieden werden. Derzeit dominieren sensible Wärmespeicher, die durch eine Temperaturänderung des Speichermediums im Rahmen Aufnahme bzw. Abgabe der Wärme gekennzeichnet sind. Insbesondere finden etablierte Warm- oder Kühlwassertanks Anwendung. Auch Gestein ist ein gebräuchliches Wärmespeichermedium. Nachteile liegen in großen Volumina bzw. großen Temperaturdifferenzen, die zur Speicherung großer Wärmemengen erforderlich sind. Dennoch sind aufgrund geringer Kosten und minimaler Umweltauswirkungen insbesondere Warmwasserspeicher weit verbreitet. Vorteile der Latentwärmespeicher liegen in den großen Energiemengen, die bereits bei geringen Temperaturänderungen gespeichert werden können. Dabei wird thermische Energie durch die Phasenumwandlung (zum Beispiel Schmelze, Verdampfung) aufgenommen bzw. abgegeben. Folglich ist die Speicherkapazität im Wesentlichen durch den Temperaturbereich der Phasenumwandlung begrenzt. Eis- und Dampfspeicher sind kommerziell verfügbar, während andere Materialien (zum Beispiel Salzschmelzen, Phase Change Slurries - PCS) noch erprobt bzw. für bestimmte Temperaturbereiche entwickelt werden. Die chemische Wärmespeicherung beruht auf reversiblen chemischen Reaktionen wie etwa der Adsorption. Der wesentliche Vorteil im Vergleich zu latenten und sensiblen Speichern liegt darin, dass in der Regel keine Selbstentladung auftritt. Aufgrund der technischen Komplexi- 
tät, hoher Kosten und geringer Wärmeleistung sind diese Wärmespeicher bisher jedoch nicht verbreitet (Baker 2008; FIZ 2005; Wietschel et al. 2010).

\subsubsection{Transport von Wärme}

Wärme kann in Form von Warm- bzw. Heißwasser oder Dampf in Rohrleitungen vom Erzeuger zum Verbraucher transportiert werden. Derzeit werden ca. $14 \%$ der Wohngebäude in Deutschland mit auf diesem Wege bereit gestellter Nah- oder Fernwärme beheizt. Dabei ist der Ausbau existierender Nah- und Fernwärmenetze insbesondere vor dem Hintergrund der angestrebten Erhöhung des Anteils von Strom aus KWKAnlagen von Bedeutung (Wietschel et al. 2010). Der Bau von Nah- und Fernwärmenetzen ist in der Regel mit hohen Investitionen verbunden, die sich über lange Zeiträume amortisieren müssen. Andererseits können Primär- bzw. Sekundärenergieträger in größeren Heizwerken häufig effizienter in Nutzwärme umgewandelt werden im Vergleich zu Kleinanlagen. Neben technischen Eigenschaften der Übertragungsrohre (zum Beispiel Isolierung) ist die Effizienz der Wärmeverteilung in Nah- und Fernwärmenetzen von den zu überbrückenden Entfernungen im Verhältnis zur Anzahl und dem Bedarf der zu versorgenden Kunden abhängig. Dadurch wird auch wesentlich die Wirtschaftlichkeit der Wärmeversorgung bestimmt. Beispielsweise kann ein Rückgang im Wärmebedarf durch bessere Isolierungen im Gebäudebestand zu einer geringeren Auslastung bestehender Leitungssysteme führen und dadurch die spezifischen Kosten der Wärmeversorgung erhöhen (Strohschein et al. 2007). Somit sind die Erfolgsaussichten einer Investition in Nah- und Fernwärmenetze wesentlich durch zukünftige Entwicklungen hinsichtlich Höhe und Verteilung des Wärmebedarfs bestimmt, die nicht mit Sicherheit vorhersehbar sind. Beim Einsatz dezentraler Technologien in einzelnen Gebäuden oder Wohnungen besteht hingegen eine höhere Flexibilität, um auf Änderungen in der Nachfrage zu reagieren.

Im weiteren Sinne kann auch die Übertragung von Erdgas in Erdgasnetzen zur Wärmeübertragung gezählt werden. Dabei kann auch Biogas ins Erdgas-Netz eingespeist werden, sofern dieses auf Erdgas-Qualität aufbereitet wird (Kaltschmitt et al. 2009). Weiterhin werden mobile Wärmespeicherkonzepte diskutiert, um Wärmeangebot und Wärmenachfrage zusammenzuführen, wenn ein Nahwärmenetz nicht realisiert werden kann. Dabei wird die in einem Trägermedium sensibel, latent oder chemisch gespeicherte Wärme mit LKWs zu den Verbrauchern transportiert. Ob sich der Zusatzaufwand für den Transport per LKW wirtschaftlich und energetisch lohnt, ist im Einzelfall zu überprüfen (Cavelius et al. 2007). 


\subsection{Auswahl von Energietechnologien}

Die vorangegangenen Ausführungen verdeutlichen, dass eine große Vielfalt an Energietechnologien zur Verfügung steht, die für die Erzeugung, Speicherung und Übertragung von Strom und Wärme angewendet werden oder in Entwicklung sind. Es steht außer Frage, dass auch in Zukunft alle Komponenten der Strom- und Wärmebereitstellung von der Erzeugung über Speicherung und Verteilung der Produkte benötigt werden. Zusätzlicher Bedarf an Technologien ergibt sich zum einen aus der Notwendigkeit, sich den verändernden Strukturen in der Strom- und Wärmenachfrage anzupassen. Dies ist beispielsweise aufgrund steigender Nachfrage oder demografischer Veränderungen erforderlich. Zum anderen besteht Ersatzbedarf bei Technologien, die ihre Nutzungsdauer überschritten haben. Auch ist offensichtlich, dass keine einzelne Technologie den Anforderungen einer wirtschaftlichen, umweltfreundlichen und sicheren Energieversorgung gerecht werden kann. Jede der beschriebenen Technologien weist Stärken und Schwächen auf, wobei nicht nur Technologie-spezifische Charakteristika von Bedeutung sind, sondern ebenso die Rahmenbedingungen, unter denen diese eingesetzt werden. Daher müssen die Stärken und Schwächen der Technologien sorgfältig gegeneinander abgewogen und bewertet werden, wenn Entscheidungen im Hinblick auf die Energieversorgung zu treffen sind. Auch sind aufgrund der vielfältigen Funktionen und Zielgruppen der Strom- und Wärmeversorgung in der Regel mehrere komplementäre Technologien erforderlich.

Dabei ist auch zu beachten, dass einige Energietechnologien bereits weit verbreitet und technologisch ausgreift sind. Da bereits Erfahrungen mit dem Einsatz dieser Technologien vorliegen, können mögliche positive und negative Auswirkungen, die damit verbunden sind, hinreichend genau beschrieben werden. Jedoch sind bei etablierten Technologien häufig keine fundamentalen Verbesserungen mehr zu erwarten, beispielsweise hinsichtlich der Erhöhung des Wirkungsgrades, Verlängerung der Lebensdauer und Verringerung der Kosten. Andererseits sind innovative Technologien zu berücksichtigen, die noch in einem frühen Entwicklungsstadium oder am Anfang der Markteinführung stehen. In diesen Fällen ist die erwartete Leistungserbringung häufig mit erheblichen Unsicherheiten behaftet. Beispielsweise können aufgrund mangelnder Erfahrungen im Dauerbetrieb häufig nur unzuverlässige Aussagen über die tatsächliche technische Lebensdauer und den Wartungsaufwand getroffen werden. In der Regel erhoffen sich jedoch Entwickler und Anwender von innovativen Technologien Vorteile gegenüber etablierten Alternativen, auch wenn diese in einem frühen Entwicklungsstadium unter Umständen noch nicht realisiert werden können.

Aus den zum Teil gegensätzlichen Eigenschaften verfügbarer oder in Entwicklung befindlicher Technologien zur Strom- und Wärmeversorgung ergibt sich der Bedarf eines 
systematischen Vergleichs der Optionen, um Entscheidungen in Zusammenhang mit der Auswahl von Energietechnologien auf einer wissenschaftlich Basis treffen zu können. Dabei wird aufgrund der weitreichenden gesamtwirtschaftlichen Bedeutung der Energieversorgung besondere Transparenz im Entscheidungsprozess gefordert. Dies betrifft Privatinvestoren, die Entscheidungen über zukünftig einzusetzende oder zu entwickelnde Technologien treffen müssen, ebenso wie staatliche Akteure, die im Rahmen der Allokation von Forschungsmitteln vor dem Hintergrund begrenzter Budgets Prioritäten setzen müssen.

Folglich ist eine wissenschaftlich fundierte Entscheidungsunterstützung erforderlich, die es ermöglicht, Energietechnologien unter dem Blickwinkel multipler Zielsetzungen wie Wirtschaftlichkeit, Versorgungssicherheit und Umweltschutz, systematisch einander gegenüberzustellen. Somit bieten sich multikriterielle Methoden der Entscheidungsunterstützung an, da diese die simultane Berücksichtigung mehrerer Kriterien beim Vergleich mehrerer Handlungsoptionen erlauben. Daher werden im folgenden Kapitel Grundlagen dieser Methoden erläutert und deren bisherige Anwendungen im Energiebereich zusammengefasst. Dabei werden Schwachstellen bestehender multikriterieller Bewertungsansätze identifiziert, insbesondere bezüglich der Berücksichtigung des technologischen Wandels. Diese Schwachstellen sollen durch methodische Weiterentwicklungen, die in den folgenden Kapiteln erläutert werden, behoben werden. 


\section{$4 \quad$ Multikriterielle Entscheidungsunterstützung im Energiebereich}

Multikriterielle Methoden wurden entwickelt, um die Nachteile klassischer Optimierungsmodelle zu überwinden. Letztere sind in der Regel alleine auf eine ökonomische Zielgröße wie zum Beispiel die Kostenminimierung unter Berücksichtigung von Randbedingungen ausgerichtet. Jedoch stellen Entscheidungen basierend auf einer einzelnen Zielgröße in der Praxis eher eine Ausnahme dar, da meist eine Vielzahl unterschiedlicher Sichtweisen bzw. Kriterien eine Rolle spielen. Wie die Ausführungen in den vorhergehenden Kapiteln verdeutlichen, gilt dies insbesondere für den Energiebereich. Weiterhin werden bei der Ermittlung eines Optimums im Sinne des klassischen Operations Research eine Reihe von Annahmen getroffen, die in der Realität häufig nicht praktikabel sind. Dies betrifft beispielsweise die vollständige Vergleichbarkeit der Alternativen, die Transitivität von Präferenzvorstellungen4 und eindeutig quantifizierbare Daten als Grundlage der Bewertung. Zudem bedingt die zunehmende Einbeziehung der verschiedensten Interessengruppen in betriebliche und politische Entscheidungsprozesse, dass zusätzliche, häufig konfliktäre Maßstäbe und subjektive Wertungen eine Rolle spielen (Beccali et al. 1998; Roy 1980; Zimmermann, Gutsche 1991).

Wenn neben den direkten Kosten weitere Zielgrößen berücksichtigt werden sollen, bieten sich anstelle multikriterieller Methoden auch traditionelle makroökonomische Nutzenmodelle an. Diese streben eine Einbeziehung von Externalitäten bei der Bewertung von Alternativen an. Dabei erfolgt eine Monetarisierung aller in die Bewertung einbezogenen Aspekte, wie beispielsweise Umweltschutz und menschliche Gesundheit, die nicht direkt am Markt über einen Preis gehandelt werden (für Anwendungen im Energiebereich siehe zum Beispiel Bickel und Friedrich 2005; Buchanan 1990; Friedrich und Voss 1993; Hohmeyer 1992). Diese Vorgehensweise bietet den Vorteil, dass die monetären Werte im Ergebnis leicht verständlich und gut vergleichbar sind. Zur Ableitung monetärer Bewertungsgrößen stehen mehrere Methoden zur Verfügung, die jedoch mit verschiedenen praktischen und methodischen Schwierigkeiten verbunden sind (vgl. zum Beispiel Kim 2007; Mirasgedis und Diakoulaki 1997). Des Weiteren erscheint die Monetarisierung einiger Bewertungsgrößen (zum Beispiel Anzahl von Todes- oder Krankheitsfällen) aus ethischer Sicht fragwürdig (Cavallaro 2009; Goletsis et al. 2003; Siskos und Hubert 1983). Multikriterielle Methoden überwinden einige der Nachteile sowohl traditioneller makroökonomischer Nutzenmodelle als auch klassi-

4 Unter Transitivität ist folgende Annahme zu verstehen: Wenn ein Entscheidungsträger die Option A der Option B vorzieht und die Option B der Option C, so zieht er auch A der Option $C$ vor: $A \succ B$ und $B \succ C \Rightarrow A \succ C$ (vgl. zum Beispiel Zimmermann, Gutsche 1991). 
scher Optimierungsmodelle, indem einerseits Schwierigkeiten, die mit der Monetarisierung von Bewertungsgrößen verbunden sind, vermieden werden (Mirasgedis und Diakoulaki 1997) und andererseits mehrere Aspekte unter Einbeziehung qualitativer und quantitativer Informationen parallel berücksichtigt werden können (Bana e Costa et al. 1997; Roy 2005). Folglich scheinen sie besser geeignet, um Optionen zur Energiebereitstellung unter Berücksichtigung multipler Zielsetzungen zu bewerten. So weisen zum Beispiel Greening und Bernow (2004) auf das Potenzial multikriterieller Methoden zur Unterstützung bei der Entscheidungsfindung in der Energie- und Umweltpolitik hin. Daher werden in diesem Kapitel multikriterielle Methoden im Kontext bereits vorliegender Anwendungen im Energiebereich dargestellt, um deren Eignung für die vorliegende Fragestellung zu überprüfen.

Grundsätzlich zeichnen sich multikriterielle Problemstellungen dadurch aus, dass eine diskrete oder kontinuierliche Menge an Optionen (auch bezeichnet als Alternativen5, Lösungen, Handlungsmöglichkeiten, Aktionen) basierend auf mindestens zwei Kriterien für oder von mindestens einem Entscheidungsträger analysiert wird. Das Ziel besteht meist darin, zwischen den zur Verfügung stehenden Optionen zu wählen, eine Rangfolge zu bilden oder die Optionen in bestimmte Kategorien einzuordnen (Figueira et al. 2005a). Die vielfältigen Lösungsansätze unterscheiden sich hinsichtlich des Rechenaufwandes sowie der Art und Menge der benötigten Informationen (Zimmermann, Gutsche 1991). Seit den 1960er Jahren wurden viele theoretische und angewandte Veröffentlichungen zur multikriteriellen Entscheidungsunterstützung publiziert (Bana e Costa et al. 1997; Roy 2005). Dies gilt insbesondere auch für Anwendungen im Energie- und Umweltbereich (Pohekar und Ramachandran 2004; Wang et al. 2009b; Zhou et al. 2006). Dabei sind Multi-Attribut-Methoden (Multi Attribute Decision Making - MADM) von den Multi-Objective-Methoden (Multi Objective Decision Making -MODM) zu unterscheiden (Tabelle 4.1). Letztere zielen darauf ab, aus der stetigen Menge der Aktionen (Lösungsraum) unter Berücksichtigung von Randbedingungen eine im Sinne des Entscheidungsträgers optimale Lösung zu berechnen. MODM-Probleme sind als mathematisches Programmierungsmodell bzw. Vektoroptimierungsmodell mit mehreren gleichzeitig zu optimierenden Zielfunktionen formulierbar. Folglich stützen sich Lösungsansätze vorwiegend auf Methoden des Linearen Programmierens (Figueira et al. 2005a; Zimmermann, Gutsche 1991).

5 Im engeren Sinn bezeichnen Alternativen sich gegenseitig ausschließende Optionen. Im weiteren Sinn kann der Begriff Alternativen auch zur Bezeichnung von Handlungsmöglichkeiten verwendet werden, die parallel realisierbar sind. 
Tabelle 4.1: Abgrenzung von MADM- und MODM-Problemen

\begin{tabular}{l|l}
\hline & $\begin{array}{l}\text { Multi-Attribut-Methoden (Multi Attribute } \\
\text { Decision Making - MADM) }\end{array}$
\end{tabular}

Im Gegensatz dazu zielen MADM-Methoden darauf ab, eine Menge bereits bekannter Alternativen auf Basis der simultanen Betrachtung mehrerer Kriterien zu bewerten. Die Kriterien können dabei in unterschiedlichen Einheiten bzw. auf unterschiedlichen Skalenniveaus ausgedrückt werden (Belton und Stewart 2002; Figueira et al. 2005b; Zimmermann, Gutsche 1991). Daher sind im Kontext der vorliegenden Problemstellung insbesondere MADM-Methoden geeignet, da der Fokus auf der vergleichenden Bewertung etablierter und innovativer Energietechnologien liegt, das heißt die Alternativen können vorab klar voneinander abgegrenzt und definiert werden. Diese sollen hinsichtlich ihrer Eigenschaften bezüglich Wirtschaftlichkeit, Versorgungssicherheit und Umweltschutz betrachtet werden. Der Beitrag einzelner Energietechnologien zur Erreichung dieser Ziele kann mit Hilfe mehrerer Kriterien auf unterschiedlichen Skalenniveaus gemessen werden. Somit sind die notwendigen Voraussetzungen zur Anwendung von MADM-Methoden gegeben. Die Anwendung von MODM-Methoden scheint hingegen besser geeignet zur Systemoptimierung, wenn etwa aus einer nahezu unendlichen Anzahl möglicher Kombinationen von Energietechnologien das optimale System ermittelt werden soll. Andererseits ziehen zum Beispiel auch Capros et al. (1988) zur Bewertung von Energieversorgungsoptionen eine MADM-Methode den MODM-Ansätzen vor, da sie es in den meisten Fällen für möglich halten, durch eine vorläufige Analyse die Anzahl der realistischen Optionen auf ein endliches, überschaubares Maß zu reduzieren. Darüber hinaus sehen Diakoulaki et al. (2005) einen Nachteil 
der MODM-Ansätze darin, dass die Anzahl der Zielfunktionen und Restriktionen, die integriert werden können, aufgrund von Modellierungs-Beschränkungen in der Regel begrenzt ist.

Aus den genannten Gründen beschränkt sich die folgende Betrachtung der in Frage kommenden multikriteriellen Methoden auf die Multi-Attribut-Ansätze. Im nächsten Abschnitt werden zunächst Grundlagen von MADM-Methoden zusammengefasst. Daran anschließend werden bestehende Ansätze in Zusammenhang mit der Bewertung von Optionen zur Energiebereitstellung diskutiert. Darauf aufbauend werden geeignete Methoden für ein multikriterielles Entscheidungsunterstützungssystem (EUS) im Energiebereich ausgewählt und Aspekte identifiziert, die bei Anwendung der gängigen Verfahren bisher nur unzureichend Berücksichtigung finden. Diese Analyse dient als Ausgangspunkt für die Entwicklung eines EUS zur multikriteriellen Bewertung von Optionen zur Bereitstellung von Strom und Wärme, das einige der identifizierten Schwachstellen überwinden soll.

\subsection{Grundlagen von Multi-Attribut-Methoden}

Das Ziel der MADM-Verfahren besteht in der Regel darin, diejenigen Alternativen zu identifizieren, die den Präferenzen des (der) Entscheidungsträger(s) am besten gerecht werden. Dabei wird jede Alternative zunächst hinsichtlich einzelner Kriterien beurteilt, welche die Ziele des Entscheidungsträgers widerspiegeln. Weiterhin wird in Form von Gewichten die relative Bedeutung der Kriterien berücksichtigt, welche die subjektiven Wertvorstellungen eines oder mehrerer Entscheidungsträger ausdrücken. Verschiedene mathematische Aggregationsmodelle können auf Basis dieser Informationen für eine Gesamtbewertung je Alternative angewendet werden (Beccali et al. 1998; Zimmermann, Gutsche 1991). Zu unterscheiden sind MADM-Methoden der amerikanischen Schule einerseits und der französischen oder europäischen Schule andererseits (Tabelle 4.2; vgl. auch Geldermann und Rentz 2000; Geldermann et al. 2002b). 6

6 Nach Belton und Stewart (2002) können darüber hinaus solche Modelle unterschieden werden, deren Bewertung sich an einem bestimmten Ziel- bzw. Referenzpunkt oder an einem Anspruchsniveau orientiert (z.B. Compromise Programming, Reference Point Approach). Diese Modelle erfordern jedoch in der Regel, dass die Kriterien-Ausprägungen auf einem kardinalen Skalenniveau vorliegen (vgl. Belton und Stewart 2002, S. 104). Daher wird diese Methoden-Gruppe nicht weiter in Betracht gezogen, da diese Eigenschaft die Flexibilität der Entscheidungsunterstützung erheblich einschränken würde. 
Tabelle 4.2: $\quad$ Abgrenzung von MADM-Methoden der amerikanischen und der europäischen Denkrichtungen

\begin{tabular}{l|l|l}
\hline & Amerikanische Schule & Europäische/französische Schule \\
\hline $\begin{array}{l}\text { Grundsätzliche } \\
\text { Annahmen }\end{array}$ & $\begin{array}{l}\text { Der Entscheidungsträger ist in der } \\
\text { Lage, seinen Präferenzen in eindeu- } \\
\text { tiger Weise Ausdruck zu verleihen }\end{array}$ & $\begin{array}{l}\text { Dem Entscheidungsträger sind seine Präfe- } \\
\text { renzen nicht vollständig bewusst bzw. er kann } \\
\text { diese nicht in eindeutiger Weise ausdrücken }\end{array}$ \\
\hline $\begin{array}{l}\text { Offenlegung und Interpretation der } \\
\text { Präferenzvorstellungen }\end{array}$ & $\begin{array}{l}\text { Verarbeitung widersprüchlicher Informationen; } \\
\text { Aufzeigen der Konsequenzen unterschiedli- } \\
\text { cher Kriterien-Gewichtungen }\end{array}$ \\
& $\begin{array}{l}\text { Klassische MADM-Verfahren, zum } \\
\text { Beispiel } \\
\text { - Nutzwertanalyse } \\
\text { AHPIANP (Analytischer- } \\
\text { Hierarchie/Netzwerk-Prozess) }\end{array}$ & $\begin{array}{l}\text { Outranking-Verfahren (Entscheidungstechno- } \\
\text { logien), zum Beispiel } \\
\text { - ELECTRE (Elimination Et Choix Traduisant } \\
\text { la Réalité) } \\
\text { PROMETHEE (Preference Ranking Organi- } \\
\text { sation Method for Enrichment Evaluations) }\end{array}$ \\
\hline
\end{tabular}

Ausgangspunkt der aus der amerikanischen Schule hervorgegangenen Methoden ist in der Regel die klassische Annahme in der Ökonomie, dass Präferenzen durch eine Nutzenfunktion abgebildet werden können, die jeder Option einen numerischen Wert zuordnet. Dieses Modell geht darüber hinaus häufig davon aus, dass sich der Gesamtnutzen einer Option aus der Summe der Teilnutzen bezüglich unterschiedlicher Kriterien ergibt. Jedoch spiegeln diese Annahmen nur unter sehr restriktiven Bedingungen die Realität wieder (Brans und Mareschal 2005; Figueira et al. 2005a). Zu den dennoch häufig verwendeten Verfahren der amerikanischen Schule zählen die Nutzwertanalyse (MAUT/MAVT - Multi Attribute Utility/Value Theory; vgl. Dyer 2005; Siskos 2005; Zimmermann, Gutsche 1991) sowie der Analytische Hierarchie/Netzwerk-Prozess (AHPIANP, vgl. zum Beispiel Saaty 2005; Saaty und Vargas 2006). Die nutzentheoretisch basierten Methoden sind grundsätzlich dadurch charakterisiert, dass der Entscheidungsträger eine genaue Vorstellung über den Nutzen der Kriterien-Ausprägungen hat und in der Lage ist, Kriterien-Gewichtungen entsprechend seiner Präferenzen in eindeutiger Weise auszudrücken. Das Ziel der Entscheidungsunterstützung besteht darin, die Präferenzen der Entscheidungsträger in transparenter Weise offenzulegen und zu interpretieren. Nachteile der amerikanischen Ansätze liegen beispielsweise im hohen Aggregationsniveau der Ergebnisse und dem damit verbundenen Informationsverlust. Weiterhin können sich gute und schlechte Kriterien vollständig gegeneinander kompensieren (vgl. zum Beispiel Geldermann und Rentz 2000). Aufgrund der leichten Nachvollziehbarkeit und Umsetzbarkeit kommen diese Methoden dennoch häufig zur Anwendung.

Mit dem Ziel, Nachteile der durch die amerikanische Schule geprägten Ansätze zu überwinden, wurden die so genannten Outranking-Methoden der französischen Schule entwickelt (im Deutschen auch als entscheidungstechnologische Ansätze oder 
Prävalenz-Verfahren bezeichnet; vgl. Zimmermann, Gutsche 1991; Geldermann et al. 2002b). Dabei vertritt die französische Schule die Auffassung, dass sich der (die) Entscheidungsträger seiner (ihrer) Präferenzen nicht vollständig bewusst ist (sind). Daher ist eine Entscheidungsunterstützung erforderlich, die auch widersprüchliche Informationen verarbeiten kann, um die Entscheidungssituation zu strukturieren und die Konsequenzen unterschiedlicher Kriterien-Gewichtungen aufzuzeigen (Geldermann und Rentz 2001; Lootsma 1996). Outranking-Methoden weisen im Vergleich zu klassischen MADM-Verfahren Vorteile auf, da sie Möglichkeiten bieten, eine vollständige Kompensation von Kriterien-Ausprägungen zu vermeiden. Beispielsweise können Regeln implementiert werden, die gewährleisten, dass eine schlechte Ausprägung in einem Kriterium nur dann durch eine gute Ausprägung in einem anderen Kriterium kompensiert werden kann, wenn die Differenzen zwischen den Kriterien-Ausprägungen bestimmte Schwellenwerte nicht über- bzw. unterschreiten (French et al. 2009; Geldermann und Rentz 2000).

Wenn die vorliegenden Informationen nicht ausreichend sind, um eine eindeutige Rangfolge der Optionen abzuleiten, bieten Outranking-Methoden die Möglichkeit, neben einer Unterscheidung in strikte Präferenz (Alternative $A$ ist der Alternative B auf jeden Fall vorzuziehen) und Indifferenz (zwei Alternativen werden als gleichwertig angesehen) auch schwache Präferenzen und Unvergleichbarkeiten darzustellen (Rogers und Bruen 1998; Roy 1980; Topcu und Ulengin 2004). Des Weiteren bestehen Möglichkeiten zur Berücksichtigung von Unsicherheiten sowohl bezüglich der KriterienAusprägungen wie auch hinsichtlich menschlicher Präferenzvorstellungen. Darüber hinaus werden im Vergleich zu den nutzentheoretischen Ansätzen in der Regel weniger Informationen vom Entscheidungsträger benötigt, wobei quantitative und qualitative Kriterien simultan einbezogen werden können (Araz und Ozkarahan 2007; Geldermann et al. 2000; Haralambopoulos und Polatidis 2003; Løken 2007; Ren et al. 2009; Salo und Hämäläinen 1995; Xu 2005). Im Vergleich zum weit verbreiteten AHP bzw. ANP der amerikanischen Denkrichtung müssen bei Anwendung von Outranking-Ansätzen weniger paarweise Vergleiche durch den Entscheidungsträger durchgeführt werden, und die Vergleiche sind nicht auf Saaty's neunstufige Skala begrenzt (Albadvi et al. 2007; Anand und Kodali 2008; Dagdeviren 2008).

Zu den bekanntesten Outranking-Ansätzen zählen die Methoden der Familie ELECTRE (Elimination Et Choix Traduisant la Réalité), die von Roy entwickelt wurden (zum Beispiel Roy 1980; Roy 1996), sowie PROMETHEE (Preference Ranking Organisation Method for Enrichment Evaluations), die von Brans entwickelt wurden (zum Beispiel Brans et al. 1986, Brans und Mareschal 2005). Neben den etablierten Outranking-Verfahren und auch nutzentheoretischen Ansätzen sind in den letzten Jahren 
zahlreiche methodische Neu- und Weiterentwicklungen zu beobachten, die zum Beispiel bei Figueira et al. (2005a) vorgestellt werden.

MADM-Methoden aller Denkrichtungen kommen in einem breiten Spektrum von Problemstellungen im Energiebereich zum Einsatz. Dabei ist keine Methode einer anderen grundsätzlich vorzuziehen. Vielmehr sollten bei der Auswahl einer geeigneten Methode die Spezifika der jeweiligen Problemstellung sowie die Vorlieben der am Entscheidungsprozess beteiligten Personen berücksichtigt werden. Weiterhin kann die parallele Anwendung unterschiedlicher Methoden sinnvoll sein, um die Stabilität der Ergebnisse zu überprüfen (Al Shemmeri et al. 1997; Løken 2007; Polatidis et al. 2006). Im Folgenden werden bisherige Anwendungen von MADM-Methoden zur Bewertung von Optionen der Bereitstellung von Strom und Nutzwärme analysiert.

\subsection{Anwendung von MADM-Methoden im Energiebereich}

Einen aktuellen Überblick über Methoden, die für Multi-Attribut-Probleme bezüglich einer nachhaltigen Planung im Energiebereich eingesetzt werden, geben Wang et al. (2009b). Zhou et al. (2006) evaluieren Publikationen zur Entscheidungsunterstützung im Energie- und Umweltbereich, die zwischen 1975 und 2004 in Fachzeitschriften veröffentlicht wurden. Anwendungen multikriterieller Methoden im Energiebereich werden bei Diakoulaki et al. (2005), Løken (2007) sowie Pohekar und Ramachandran (2004) zusammengefasst. Die Anwendungen reichen von taktischen über operative bis hin zu strategischen Entscheidungen, beziehen sich auf unterschiedliche räumliche und zeitliche Dimensionen sowie auf unterschiedliche Abgrenzungen hinsichtlich der untersuchten Optionen. Im Rahmen der vorliegenden Arbeit sind insbesondere solche Anwendungen von Interesse, in denen Energietechnologien zur Bereitstellung von Strom und/oder Wärme als Optionen untersucht werden. Zur Analyse der entsprechenden Veröffentlichungen systematisiert das in Abbildung 4.1 dargestellte Schema die wichtigsten Schritte im Rahmen eines multikriteriellen Entscheidungsprozesses. Jedoch sei an dieser Stelle darauf hingewiesen, dass sich die dargestellten Schritte nicht immer eindeutig voneinander abgrenzen lassen.

Nach der Definition der Problemstellung (vgl. Abschnitt 4.2.1) erfolgen in der Regel die Festlegung der Alternativen (vgl. Abschnitt 4.2.2) und die Auswahl der Kriterien (vgl. Abschnitt 4.2.3). Daran anschließend werden die Kriterien-Ausprägungen je Alternative bestimmt (vgl. Abschnitt 4.2.4) und die relative Bedeutung der Kriterien in Form von Gewichten festgelegt (vgl. Abschnitt 4.2.5). Diese Informationen werden mit Hilfe eines Algorithmus aggregiert (vgl. Abschnitt 4.2.6), um beispielsweise eine Rangfolge oder Kategorisierung der Alternativen abzuleiten. Anschließend kann die Stabilität der Ergebnisse durch Sensitivitätsanalysen überprüft werden. Andere Methoden zur Berück- 
sichtigung von Unsicherheiten können bereits in vorangegangenen Schritten des Entscheidungsprozesses ansetzen (vgl. Abschnitt 4.2.7). Darüber hinaus können Methoden für Gruppenentscheidungsprozesse an unterschiedlichen Stufen ansetzen, um mehrere Stakeholder in den Entscheidungsprozess einzubeziehen (vgl. Abschnitt 4.2.8). Die Umsetzung des gesamten Prozesses kann durch Implementierung in einem Entscheidungsunterstützungssystem erfolgen (vgl. Abschnitt 4.2.9). Das Ziel besteht in der Regel letztlich darin Handlungsempfehlungen abzuleiten.

Abbildung 4.1: $\quad$ Schritte im Rahmen eines multikriteriellen Entscheidungsunterstützungsprozesses

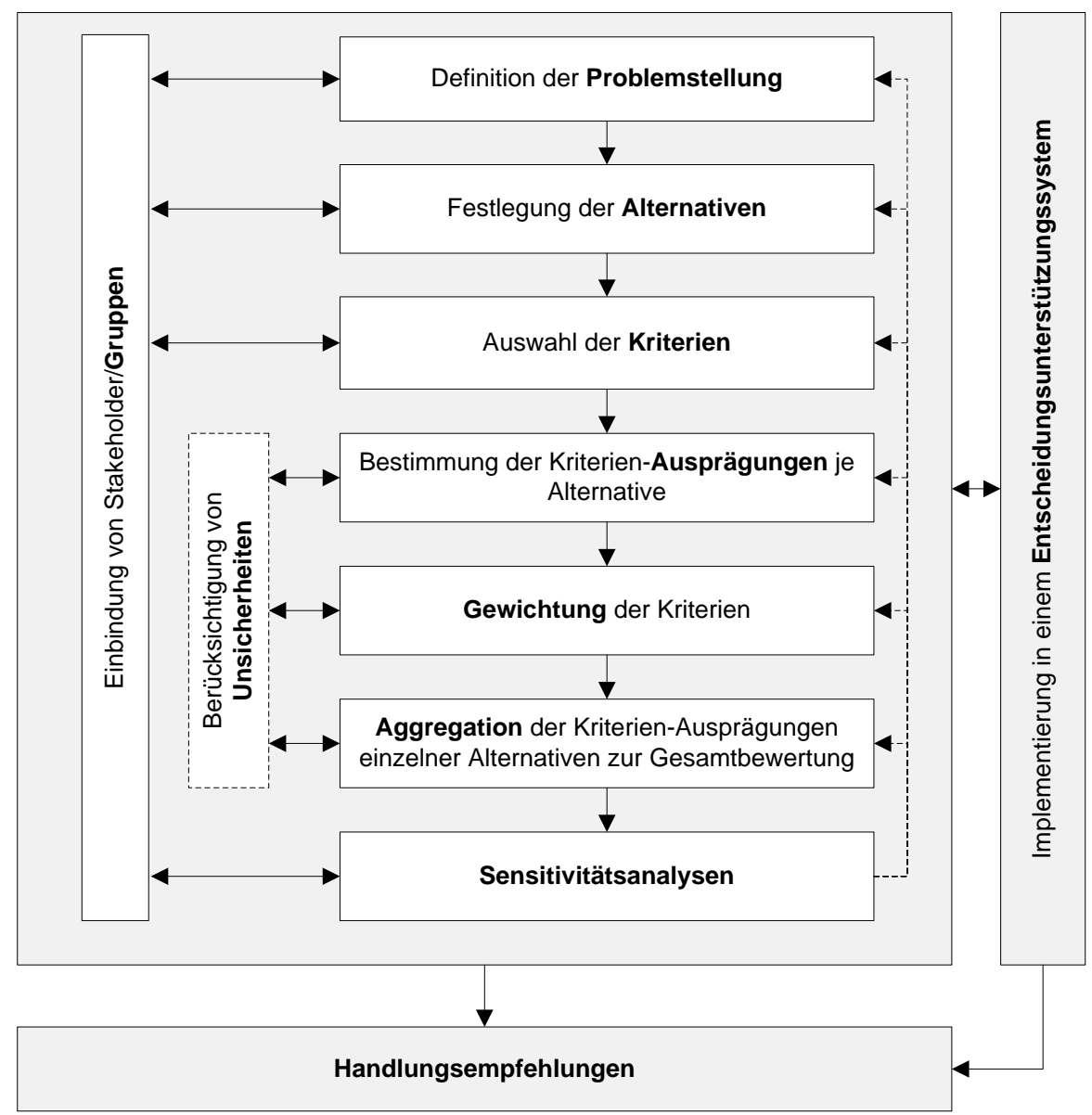

\subsubsection{Definition der Problemstellung}

Problemstellungen hinsichtlich der Strom- und Wärmebereitstellung, die durch MADMMethoden untersucht werden, können nach dem Untersuchungsgegenstand unterschieden werden (Tabelle 4.3). Die untersuchten Optionen reichen von konkreten Projektvorschlägen über unterschiedliche Technologien oder Technologiegruppen bis hin zu lokalen, regionalen oder nationalen Szenarien zur Energieversorgung. Die in Tabel- 
le 4.3 zusammengefassten Veröffentlichungen dienen als Ausgangspunkt für die folgenden Untersuchungen im Hinblick auf die Eignung spezifischer MADM-Methoden für die vorliegende Problemstellung. Eine Durchsicht der genannten Veröffentlichungen lässt erste Schlussfolgerungen in Bezug auf die Abgrenzung eines geeigneten Untersuchungsgegenstandes zu:

- Szenarien bieten den Vorteil, dass Wechselwirkungen zwischen einzelnen Technologien im Energiesystem implizit berücksichtigt werden können. Zudem trägt eine Analyse von Szenarien der Tatsache Rechnung, dass eine einzelne Technologie den Anforderungen an die Energieversorgung nicht alleine gerecht werden kann. Andererseits stellt die Modellierung von Szenarien einen zusätzlichen Aufwand im Entscheidungsprozess dar. Weiterhin wird die Abschätzung der Auswirkungen von Szenarien in Form von Kriterien-Ausprägungen erschwert. Dies gilt für quantitative Modellierungen wie auch für qualitative Einschätzungen durch Experten. Rückschlüsse auf den Entwicklungsstand und die Entwicklungsbedarfe einzelner Technologien, die Teil der Szenarien sind, sind nur bedingt möglich.

- Eine Unterscheidung der Optionen nach Energieträgern, die für unterschiedliche Technologien eingesetzt werden können, erschwert ebenfalls Rückschlüsse auf Technologien und deren Entwicklungspotenziale.

- Werden Technologien, die sich gegebenenfalls aus mehreren Komponenten zusammensetzen, als Einzeloptionen untersucht, besteht die Gefahr, dass Wechselwirkungen im Energiesystem vernachlässigt werden. Andererseits scheint die Abschätzung der Kriterien-Ausprägungen im Vergleich zu Szenarien einfacher möglich. Zudem besteht die Möglichkeit, Entwicklungspotenziale der Technologien in transparenter Weise abzubilden. Weiterhin ist es denkbar, Wechselwirkungen im Energiesystem beispielsweise über zusätzliche Kriterien oder Unsicherheiten in den Kriterien-Ausprägungen abzubilden. 
Tabelle 4.3: $\quad$ Abgrenzung von MADM-Anwendungen im Energiebereich nach dem Untersuchungsgegenstand

\begin{tabular}{|c|c|}
\hline Gegenstand der Untersuchung & Quellen \\
\hline \multicolumn{2}{|l|}{ Szenarien } \\
\hline Stromversorgung auf nationaler Ebene & $\begin{array}{l}\text { Diakoulaki und Karangelis 2007; Haldi et al. 2002; Heinrich } \\
\text { et al. 2007; Karger und Hennings 2009; Lootsma et al. } \\
\text { 1990a; Stagl } 2006\end{array}$ \\
\hline $\begin{array}{l}\text { Strom- und Wärmeversorgung auf nationa- } \\
\text { ler Ebene }\end{array}$ & $\begin{array}{l}\text { Georgopoulou et al. 1998; Hyde et al. 2003; Jones et al. } \\
\text { 1990; Kowalski et al. 2009; Liposcak et al. 2006; Madlener et } \\
\text { al. 2007; Papadaki et al. } 2003\end{array}$ \\
\hline $\begin{array}{l}\text { Stromversorgung auf regionaler und loka- } \\
\text { ler Ebene }\end{array}$ & $\begin{array}{l}\text { Andrews 2003; Buehring et al. 1978; Phdungsilp 2010; } \\
\text { Shackley und McLachlan } 2006\end{array}$ \\
\hline $\begin{array}{l}\text { Strom- und Wärmeversorgung auf regio- } \\
\text { naler und lokaler Ebene }\end{array}$ & $\begin{array}{l}\text { Assimakopoulos et al. 1991; Browne et al. 2010; } \\
\text { Georgopoulou et al. 1997; Jovanovic et al. 2009; Kowalski et } \\
\text { al. 2009; Løken et al. 2009; Papadopoulos und Karagianni- } \\
\text { dis 2008; Renn } 2003\end{array}$ \\
\hline \multicolumn{2}{|c|}{ Unterscheidung von Optionen nach Energieträgen } \\
\hline $\begin{array}{l}\text { Optionen zur Stromerzeugung unter Be- } \\
\text { rücksichtigung fossiler, erneuerbarer und } \\
\text { nuklearer Energieträger, ohne eine Ab- } \\
\text { grenzung spezifischer Technologien }\end{array}$ & $\begin{array}{l}\text { Akash et al. 1999; Chatzimouratidis und Pilavachi 2007; } \\
\text { Chatzimouratidis und Pilavachi 2008; Chatzimouratidis und } \\
\text { Pilavachi 2009; Gholamnezhad und Saaty 1982; Köne und } \\
\text { Büke 2007; Mamlook et al. 2001a; Mamlook 2006; Topcu } \\
\text { und Ulengin 2004; Ulutas } 2005\end{array}$ \\
\hline \multicolumn{2}{|c|}{ Technologien auf Basis unterschiedlicher Energieträger } \\
\hline $\begin{array}{l}\text { Stromerzeugung aus fossilen, erneuerba- } \\
\text { ren und nuklearen Energieträgern }\end{array}$ & $\begin{array}{l}\text { Afgan und Carvalho 2002; Nwofor et al. 2007; Roth et al. } \\
\text { 2009; Siskos und Hubert } 1983\end{array}$ \\
\hline $\begin{array}{l}\text { Stromerzeugung aus fossilen und nuklea- } \\
\text { ren Energieträgern }\end{array}$ & $\begin{array}{l}\text { Güngör und Arikan 2000; Hämäläinen 1990; Hämäläinen } \\
\text { und Karjalainen 1992; Hämäläinen und Seppäläinen 1986; } \\
\text { Keeney und Sicherman } 1983\end{array}$ \\
\hline $\begin{array}{l}\text { Stromerzeugung aus fossilen und erneu- } \\
\text { erbaren Energieträgern }\end{array}$ & $\begin{array}{l}\text { Afgan et al. 2000; Afgan und Carvalho 2008; Doukas et al. } \\
\text { 2006; Doukas et al. 2007; Kablan 1997; Kagazyo et al. 1997; } \\
\text { Karakosta et al. 2009; Lhendup 2008; Ramanathan und } \\
\text { Ganesh 1995; Zangeneh et al. } 2009\end{array}$ \\
\hline $\begin{array}{l}\text { Stromerzeugung aus Wasserstoff in Ver- } \\
\text { bindung mit bzw. im Vergleich zu anderen } \\
\text { Energieträgern }\end{array}$ & $\begin{array}{l}\text { Afgan et al. 2007c; Afgan und Carvalho 2004; Pilavachi et al. } \\
2009\end{array}$ \\
\hline $\begin{array}{l}\text { Stromerzeugung aus fossilen und erneu- } \\
\text { erbaren Energieträgern unter Berücksich- } \\
\text { tigung von KWK-Optionen }\end{array}$ & Afgan et al. 2007a; Begic und Afgan 2007 \\
\hline $\begin{array}{l}\text { Bereitstellung von Strom und Wärme aus } \\
\text { verschiedenen Energieträgern, teilweise } \\
\text { unter Berücksichtigung von KWK-Optionen }\end{array}$ & $\begin{array}{l}\text { Georgopoulou et al. 2003; Schulz und Stehfest 1984; Tzeng } \\
\text { et al. } 1992\end{array}$ \\
\hline \multicolumn{2}{|l|}{ Technologien auf Basis fossiler Energieträger } \\
\hline $\begin{array}{l}\text { Fossil-thermische Kraftwerke, teilweise } \\
\text { unter Berücksichtigung von KWK }\end{array}$ & $\begin{array}{l}\text { Afgan et al. 2007b; Basson und Petrie 2007; Daim et al. } \\
\text { 2009; Dinca et al. 2007; Liang et al. } 2006\end{array}$ \\
\hline $\begin{array}{l}\text { KWK-Technologien auf Basis fossiler } \\
\text { Energieträger }\end{array}$ & Jiang-Jiang et al. 2010; Pilavachi et al. 2006; Ren et al. 2009 \\
\hline
\end{tabular}


Tabelle 4.3 (Fortsetzung)

\begin{tabular}{|c|c|}
\hline Gegenstand der Untersuchung & Quellen \\
\hline \multicolumn{2}{|l|}{ Technologien auf Basis erneuerbarer Energieträger } \\
\hline $\begin{array}{l}\text { Stromerzeugung aus verschiedenen erneuerbaren Ener- } \\
\text { gieträgern, meist unter Berücksichtigung regionaler Gege- } \\
\text { benheiten }\end{array}$ & $\begin{array}{l}\text { Burton und Hubacek 2007; Cherni et al. } \\
\text { 2007; Kaminaris et al. 2006; Madlener und } \\
\text { Stagl 2005; van Alphen et al. } 2007\end{array}$ \\
\hline $\begin{array}{l}\text { Bereitstellung von Strom und Wärme aus verschiedenen } \\
\text { erneuerbaren Energieträgern, meist unter Berücksichtigung } \\
\text { regionaler Gegebenheiten }\end{array}$ & $\begin{array}{l}\text { Beccali et al. 1998; Beccali et al. 2003; } \\
\text { Kahraman et al. 2009; Nigim et al. 2004; } \\
\text { Terrados et al. 2009; Tsoutsos et al. } 2009\end{array}$ \\
\hline $\begin{array}{l}\text { Bioenergie-Projekte (Prozessketten oder Anlagen zur } \\
\text { Energiebereitstellung) }\end{array}$ & $\begin{array}{l}\text { Buchholz et al. 2009; Hanegraaf et al. 1998; } \\
\text { Madlener et al. 2009; Recchia et al. } 2010\end{array}$ \\
\hline Wasserkraftprojekte an unterschiedlichen Standorten & $\begin{array}{l}\text { Brans et al. 1986; de Almeida et al. 2005; } \\
\text { Wenstöp und Carlsen } 1988\end{array}$ \\
\hline Windkraftprojekte & $\begin{array}{l}\text { Cavallaro und Ciraolo 2005; Daim et al. } \\
\text { 2009; Polatidis und Haralambopoulos } 2007\end{array}$ \\
\hline Geothermische Stromerzeugung und Wärmebereitstellung & $\begin{array}{l}\text { Capros et al. 1988; Goumas und Lygerou } \\
\text { 2000; Goumas et al. 1999; Haralambopou- } \\
\text { los und Polatidis } 2003\end{array}$ \\
\hline Konzentrierende solartehrmische Kraftwerke & Cavallaro 2009 \\
\hline $\begin{array}{l}\text { Andere Optionen zur Bereitstellung von Strom oder Wärme } \\
\text { (u.a.) aus Solarstrahlung }\end{array}$ & Mamlook et al. 2001b \\
\hline \multicolumn{2}{|l|}{ Weitere Anwendungsbereiche } \\
\hline Bereitstellung von Raumwärme und/oder Warmwasser & $\begin{array}{l}\text { Alanne et al. 2007; Erdogmus et al. 2006; } \\
\text { Ghafghazi et al. 2009; Jaber et al. 2008; } \\
\text { Mohsen und Akash 1997; Mróz und Thiel } \\
\text { 2005; Thiel und Mróz } 2001\end{array}$ \\
\hline $\begin{array}{l}\text { Priorisierung von F\&E-Themen oder Projekten im Energie- } \\
\text { bereich, meist im Hinblick auf die Allokation von For- } \\
\text { schungsmitteln }\end{array}$ & $\begin{array}{l}\text { Gholamnezhad 1983; Goletsis et al. 2003; } \\
\text { Lee und Hwang 2010; Lootsma et al. 1986; } \\
\text { Lootsma et al. 1990b; Son und Min } 1998\end{array}$ \\
\hline $\begin{array}{l}\text { Planung im Energiesektor, in der Regel im Rahmen der } \\
\text { Kapazitätserweiterung von Stromversorgungssystemen, } \\
\text { unter Berücksichtigung angebots- und nachfrageseitiger } \\
\text { Maßnahmen }\end{array}$ & $\begin{array}{l}\text { Hanson et al. 1991; Rahman und Frair } \\
\text { 1984; Voropai und Ivanova } 2002\end{array}$ \\
\hline
\end{tabular}

\subsubsection{Festlegung der Alternativen}

In vielen Anwendungsfällen stützt sich die Auswahl der Alternativen auf die regional verfügbaren Potenziale unterschiedlicher Energieträger (zum Beispiel Ghafghazi et al. 2009; Gholamnezhad und Saaty 1982; Kahraman et al. 2009; Mamlook 2006; Nigim et al. 2004; Nwofor et al. 2007; Papadopoulos und Karagiannidis 2008; Tsoutsos et al. 2009; Ulutas 2005). In diesem Zusammenhang können auch GIS-Systeme hilfreich sein (vgl. zum Beispiel Recchia et al. 2010). Aus technologischer Sicht beschränkt sich die Vorauswahl der Alternativen häufig auf die bereits eingesetzten, verfügbaren bzw. technisch machbaren Alternativen (zum Beispiel Chatzimouratidis und Pilavachi 2008; Goumas et al. 1999; Kablan 1997; Lootsma et al. 1990a; Mróz und Thiel 2005; Thiel und Mróz 2001). Einige Veröffentlichungen legen dabei vorab einen Schwerpunkt auf 
bestimmte Technologien, zum Beispiel Gasturbinen (Afgan et al. 2007b), Technologien zur dezentralen Erzeugung (Lhendup 2008), Wasserstoff-Systeme (Afgan et al. 2007c; Afgan und Carvalho 2004), Hybrid-Systeme (Afgan und Carvalho 2008), Solarenergie (Mamlook et al. 2001b). Gegebenenfalls wird Bezug genommen auf die realistisch verfügbaren Optionen unter Berücksichtigung zukünftiger Entwicklungspotenziale (zum Beispiel Roth et al. 2009). In eher zukunftsorientierten Anwendungen kann ein Überprüfung neuerer Entwicklungen und Aussichten im Energiebereich bzw. Trends für die zukünftige Energieversorgung als Ausgangspunkt für die Vorauswahl der Alternativen dienen (Browne et al. 2010; Doukas et al. 2007; Tzeng et al. 1992). Generell kann eine Literaturanalyse zur Identifikation und Auswahl von Alternativen beitragen (zum Beispiel Doukas et al. 2006; Karakosta et al. 2009; Lee und Hwang 2010). (Lootsma et al. 1986; Lootsma et al. 1990b) berufen sich bei der Auswahl der Optionen auf Forschungsprogramme bzw. vorliegende Klassifizierungen zu F\&E im Energiebereich. In einigen Fällen liegen die Alternativen in Form von Projektvorschlägen bereits vor und werden daher nicht durch den Analysten oder die Stakeholder bestimmt (de Almeida et al. 2005; Goletsis et al. 2003; Liang et al. 2006; Wenstöp und Carlsen 1988).

Eine SWOT-Analyse7 der bestehenden regionalen Energieversorgung führen (Terrados et al. 2009) durch, um darauf aufbauend Alternativen zu generieren. (Schulz und Stehfest 1984) leiten die möglichen Optionen aus den Bedarfen ab, die mit unterschiedlichen Energieträgern zu decken sind. Auch bei (Jaber et al. 2008; Mohsen und Akash 1997; Ramanathan und Ganesh 1995) stützt sich die Auswahl der Alternativen auf eine Erhebung der zu deckenden Bedarfe. Bei (Cherni et al. 2007) dient eine Befragung von Haushalten, Stakeholdern und lokalen Führungspersönlichkeiten zur Identifikation der lokalen Anforderungen und Potenziale als Ausgangspunkt für die Vorauswahl der Alternativen. In einigen Fällen werden Experten zur Auswahl der Alternativen konsultiert (zum Beispiel Daim et al. 2009). (Lee und Hwang 2010) nutzen neben einer Literaturanalyse Interviews mit Experten aus der Industrie und aus akademischen Einrichtungen. Interviews bzw. Diskussionen mit unterschiedlichen Akteuren nutzen auch (Georgopoulou et al. 1997), um relevante Alternativen ausgehend von den verfügbaren Potenzialen erneuerbarer Energien abzuleiten. (Haralambopoulos und Polatidis 2003) leiten die Alternativen in Beratung mit den lokalen Behörden ab.

Sollen Szenarien zur Energieversorgung Gegenstand der Untersuchung sein, kann beispielsweise auf bereits vorliegende Szenarien aus früheren Veröffentlichungen zu-

7 Im Rahmen einer SWOT-Analyse werden die Faktoren, die auf eine bestimmte Situation positiv bzw. negativ wirken, unterschieden nach internen Faktoren (Stärken und Schwächen - Strengths and Weaknesses) und externen Faktoren (Chancen und Risiken - $\underline{\text { Op}}$ portunities and Threats) analysiert (vgl. zum Beispiel Markovska et al. 2009) 
rückgegriffen werden (zum Beispiel Jones et al. 1990; Liposcak et al. 2006; Shackley und McLachlan 2006). Andererseits können Szenarien im Rahmen von Workshops in Zusammenarbeit mit Stakeholdern entwickelt werden (zum Beispiel Haldi et al. 2002; Karger und Hennings 2009; Kowalski et al. 2009; Madlener et al. 2007; Renn 2003; Stagl 2006). Die Erzeugung und quantitative Berechnung der Szenarien wird in vielen Fällen durch Modellrechnungen unterstützt (Andrews 2003; Assimakopoulos et al. 1991; Buehring et al. 1978; Heinrich et al. 2007; Jovanovic et al. 2009; Løken et al. 2009; Phdungsilp 2010; van Alphen et al. 2007).

\subsubsection{Auswahl der Kriterien}

Die Kriterien zur Bewertung von Energiebereitstellungs-Optionen werden in der Regel ausgehend von den Zielen der Entscheidungsträger festgelegt (zum Beispiel Beccali et al. 2003; Diakoulaki und Karangelis 2007; Goletsis et al. 2003; Wenstöp und Carlsen 1988). Literaturanalysen bzw. Analysen bereits vorliegender Studien und Forschungsarbeiten dienen häufig als Ausgangspunkt zur Erstellung einer Liste von Kriterien (Dinca et al. 2007; Ghafghazi et al. 2009; Haldi et al. 2002; Kahraman et al. 2009; Topcu und Ulengin 2004; Tzeng et al. 1992). Beispielsweise erstellen (Jones et al. 1990) aus einer Analyse aktueller Veröffentlichungen eine Liste mit Kriterien, aus denen die Stakeholder diejenigen auswählen können, die ihnen am wichtigsten erscheinen. Zudem können Kriterien durch die Stakeholder modifiziert oder ergänzt werden. In anderen Veröffentlichungen finden Nachhaltigkeitsindikatoren aus vorliegenden Studien Anwendung (Afgan und Carvalho 2008; Phdungsilp 2010). In vielen Fällen dienen unterschiedliche Dimensionen der Nachhaltigkeit als Ausgangspunkt, denen jeweils mehrere Kriterien zugeordnet werden (zum Beispiel Afgan et al. 2007a; Doukas et al. 2006; Doukas et al. 2007; Karakosta et al. 2009; Köne und Büke 2007; Liposcak et al. 2006; Madlener et al. 2007; Papadaki et al. 2003).

Häufig wird ein Entscheidungsbaum („value tree“) bzw. eine Hierarchie zur Strukturierung der relevanten Kriterien genutzt (zum Beispiel Alanne et al. 2007; Hämäläinen und Karjalainen 1992; Kagazyo et al. 1997; Keeney und Sicherman 1983; Renn 2003; Siskos und Hubert 1983). Zum Beispiel entwickeln (Karger und Hennings 2009) einen Entscheidungsbaum auf Basis von Interviews mit Experten. In einem diskursiven Prozess entwickelt (Renn 2003) im Rahmen von mehreren Meetings und Interviews individuelle und Gruppen-Entscheidungsbäume. Die hierarchische Strukturierung der Kriterien und Alternativen ist ein besonderes Merkmal des AHP (zum Beispiel Assimakopoulos et al. 1991; Gholamnezhad und Saaty 1982; Lee und Hwang 2010; Zangeneh et al. 2009), kann aber auch bei Anwendung anderer Aggregationsmethoden eingesetzt werden. Im Rahmen des ANP wird die hierarchische Struktur des AHP zu einer Netzwerk-Struktur erweitert. In einigen Fällen werden getrennte Hierarchien 
für Nutzen („,benefits“) und Kosten („costs“) unterschieden, um abschließend das Nutzen-Kosten-Verhältnis („B/C-ratio“) je Alternative abbilden zu können (zum Beispiel Jaber et al. 2008; Mamlook et al. 2001a; Mamlook 2006; Mamlook et al. 2001b; Mohsen und Akash 1997).

Durch Beratungen, Diskussionen, Interviews und Workshops können Kriterien in Zusammenarbeit mit Experten und Stakeholdern erarbeitet werden (zum Beispiel Buchholz et al. 2009; Erdogmus et al. 2006; Georgopoulou et al. 1997; Haldi et al. 2002; Hanson et al. 1991; Lootsma et al. 1986; Nigim et al. 2004; Schulz und Stehfest 1984). Zum Beispiel wendet Stagl (2006) im Rahmen von Workshops ein Brainstorming an. Andrews (2003) nutzt zusätzlich zu offenen Diskussionen vertrauliche Fragebögen, um Aspekte zu erfassen, die in der Gruppe gegebenenfalls nicht genannt werden. Goletsis et al. (2003) wenden einen der Delphi-Methode8 ähnlichen Ansatz an, um eine Kriterien-Liste in Zusammenarbeit mit den Entscheidungsträgern zu erstellen. Ein DelphiAnsatz ist auch Bestandteil der Kriterien-Bestimmung bei Roth et al. (2009).

Weitere Ansätze zur Unterstützung bei der Auswahl von Kriterien stützen sich beispielsweise auf die Identifikation kritischer Parameter bzw. Hemmnisse (Lootsma et al. 1986; Lootsma et al. 1990b; Tsoutsos et al. 2009), auf die Vor- und Nachteile der zuvor festgelegten Alternativen (de Almeida et al. 2005), auf Anforderungen aus Gesetzestexten und Richtlinien (Son und Min 1998), auf eine Dekompositions-Analyse (Polatidis und Haralambopoulos 2007), auf die fünf Attribute von Innovationen nach Rogers (van Alphen et al. 2007) oder auf die fünf Arten des Kapitals (Ressourcen) nach dem so genannten SL-Ansatz („Sustainable Livelihoods“; vgl. Cherni et al. 2007). Zumindest die beiden zuletzt genannten Ansätze scheinen dabei jedoch nur im speziellen Anwendungskontext nützlich zu sein. In einigen Fällen ist die Auswahl der Kriterien durch die (Nicht-)Verfügbarkeit von quantitativen und/oder qualitativen Daten eingeschränkt (Cavallaro 2009; Cavallaro und Ciraolo 2005; Chatzimouratidis und Pilavachi 2008; Goletsis et al. 2003; Lootsma et al. 1990a; Tsoutsos et al. 2009).

Für die vorliegende Problemstellung bietet sich eine Zusammenstellung der in den analysierten Veröffentlichungen verwendeten Kriterien als Ausgangspunkt an, um eine Liste möglicherweise relevanter Kriterien zu erstellen. Darauf aufbauend können die als bedeutend angesehenen Kriterien ausgewählt, gegebenenfalls ergänzt, modifiziert und in das zu entwickelnde EUS integriert werden. Zur Überprüfung der Vollständigkeit

8 Bei der Delphi-Methode handelt es sich um eine spezielle Form der Expertenbefragung, bei der untereinander anonyme Experten in mehreren Runden auf Basis eines Fragebogens befragt werden. Das Ziel besteht in der Regel darin, einen Konsens zwischen den Experten bezüglich der zugrunde liegenden Fragestellung zu erzielen. Häufig wird die Delphi-Methode für Prognose-Zwecke eingesetzt (Götze und Rudolph 1994). 
des Kriterien-Sets dienen zusätzlich Ergebnisse aus einer Befragung energiewirtschaftlicher Entscheidungsträger (vgl. Kapitel 6).

\subsubsection{Bestimmung der Kriterien-Ausprägungen}

Zur Bestimmung der Kriterien-Ausprägungen können quantitative Methoden aus unterschiedlichen Fachgebieten hinzugezogen werden, wie zum Beispiel Methoden der Investitionsrechnung (zum Beispiel Doukas et al. 2006; Goletsis et al. 2003; Papadopoulos und Karagiannidis 2008), ökobilanzielle Methoden (zum Beispiel Basson und Petrie 2007; Roth et al. 2009) oder thermodynamische Analysen (zum Beispiel Thiel und Mróz 2001). Weiterhin können Ergebnisse aus Energiemodellen oder SzenarienRechnungen als Eingangsgrößen der multikriteriellen Bewertung dienen (zum Beispiel Assimakopoulos et al. 1991; Jovanovic et al. 2009; Løken et al. 2009; Phdungsilp 2010). Andererseits erlauben multikriterielle Methoden auch die Berücksichtigung von Informationen qualitativer Art. Zum Beispiel können qualitative Ausprägungen auf einer Ordinal-Skala von gut $(=1)$ über mittel $(=2)$ bis schlecht $(=3)$ eingestuft werden (zum Beispiel Beccali et al. 2003; Karakosta et al. 2009; Madlener und Stagl 2005). Auch die Beurteilung von Alternativen hinsichtlich einzelner Kriterien durch linguistische oder quantitative Variablen und deren Transformation in Fuzzy-Zahlen ist gebräuchlich (zum Beispiel Beccali et al. 1998; Doukas et al. 2007; Goumas und Lygerou 2000; Güngör und Arikan 2000). Dabei können auch paarweise Vergleiche, beispielsweise auf Basis des AHPIANP, zur Beurteilung von Alternativen hinsichtlich einzelner Kriterien im Vergleich zueinander dienen (zum Beispiel Akash et al. 1999; Chatzimouratidis und Pilavachi 2009; Erdogmus et al. 2006; Karger und Hennings 2009).

\subsubsection{Gewichtung der Kriterien}

Die Gewichtung der Kriterien soll die subjektiven Wertvorstellungen des (der) Entscheidungsträger(s) entsprechend seines persönlichen Zielsystems widerspiegeln. Daher ist bei der Interpretation und Auswertung der Gewichtungen besondere Vorsicht geboten. Die einfachste Methode zur Gewichtung von Kriterien besteht darin, zumindest anfänglich allen Kriterien die gleiche Bedeutung beizumessen und diese gegebenenfalls im Rahmen einer nachgelagerten Sensitivitätsanalyse zu variieren (zum Beispiel Basson und Petrie 2007; Papadaki et al. 2003; Recchia et al. 2010; Topcu und Ulengin 2004; van Alphen et al. 2007; Wenstöp und Carlsen 1988). Eine einfache Alternative besteht in der direkten Vergabe von Gewichten durch die Entscheidungsträger, um die relative Bedeutung der Kriterien zueinander zu bewerten, beispielsweise durch die Verteilung von 100 Punkten auf die Kriterien (Renn 2003; Shackley und McLachlan 2006) die Vergabe von Punkten von 1 bis 10 (Burton und Hubacek 2007; Lhendup 2008) oder durch eine Einordnung auf einer Skala von 0 bis 1 (Papadopoulos 
und Karagiannidis 2008). Eine weitere Möglichkeit besteht in der Vorab-Definition von Kategorien unterschiedlicher Priorität (etwa 1 = geringe Bedeutung, 2 = mittlere Bedeutung, 3 = hohe Bedeutung), denen die Kriterien zugeordnet werden (Beccali et al. 1998; Dinca et al. 2007; Erdogmus et al. 2006; Güngör und Arikan 2000). Bei der Anwendung von SMART (Simple Multi-Attribute Rating Technique), werden zunächst dem wichtigsten Kriterium 100 Punkte zugeordnet und anschließend die anderen Kriterien in Bezug dazu mit weniger Punkten eingestuft (zum Beispiel Jones et al. 1990).

In nutzentheoretisch basierten Modellen werden die Gewichtungen klassischerweise basierend auf Trade-off-Analysen mit den Entscheidungsträgern abgeleitet (zum Beispiel Buehring et al. 1978; Hämäläinen und Karjalainen 1992; Hanson et al. 1991; Heinrich et al. 2007; Keeney und Sicherman 1983; Schulz und Stehfest 1984; Voropai und Ivanova 2002). Bei Anwendung der Swing-Technik (zum Beispiel Løken et al. 2009) wird zur Bestimmung der Kriterien-Gewichtung zunächst der „Swing“, das heißt die Differenz zwischen der besten und der schlechtesten Ausprägung eines jeden Kriteriums bestimmt. Das Kriterium, dessen Swing für den Entscheidungsträger den Gesamtnutzen einer Alternative am meisten erhöhen würde, erhält die höchste Gewichtung. Die anderen Kriterien werden in Relation dazu ebenfalls in Abhängigkeit der Auswirkungen ihres Swings auf den Gesamtnutzen bestimmt (vgl. auch Belton und Stewart 2002; French et al. 2009). Goletsis et al. (2003) greifen auf eine Methode zurück, die dem Swing-Ansatz ähnlich ist und auf der „Personal Construct“-Theorie nach Kelly beruht. Dabei wird jedem Kriterium ein bipolares „Konstrukt“ basierend auf dessen zwei extremen Ausprägungen zugeordnet (zum Beispiel geringe Kosten, hohe Kosten). Dann werden paarweise Vergleiche dieser Konstrukte von jeweils zwei Kriterien durchgeführt. Dabei muss der Entscheidungsträger angeben, in welchem der beiden Konstrukte er eher eine Änderung von der guten hin zur schlechten Ausprägung akzeptieren würde. Daraus werden schließlich Kriterien-Gewichtungen abgeleitet. Diese Methoden erfordern einen erheblichen Informations-Input von Seiten des Entscheidungsträgers und gehen darüber hinaus davon aus, dass dieser seine Präferenzen eindeutig ausdrücken und den Nutzenunterschied auf Basis von Unterschieden in den Kriterien-Ausprägungen einschätzen kann.

Als Alternative zu Trade-off-Analysen bieten sich paarweise Vergleiche auf unterschiedlichen Skalen zur Ableitung von Kriterien-Gewichtungen an. Die entsprechenden Methoden sind aufgrund ihrer intuitiven Verständlichkeit und Nachvollziehbarkeit auch im Energiebereich weit verbreitet. Besonderer Beliebtheit erfreut sich hierbei die Anwendung des AHP, wobei die Kriterien basierend auf der neunstufigen Skala nach Saaty (von 1 = "gleiche Bedeutung" bis 9 = „extrem viel höhere Bedeutung") verglichen werden und anschließend mit Hilfe von Saaty's Eigenvektor-Methode die KriterienGewichtungen berechnet werden (zum Beispiel Akash et al. 1999; Chatzimouratidis 
und Pilavachi 2009; Gholamnezhad 1983; Hämäläinen 1990; Kablan 1997; Kagazyo et al. 1997; Karger und Hennings 2009; Lee und Hwang 2010; Mohsen und Akash 1997; Nigim et al. 2004; Rahman und Frair 1984; Ramanathan und Ganesh 1995; Ren et al. 2009; Son und Min 1998; Tzeng et al. 1992; Zangeneh et al. 2009). Nachteile liegen im Aufwand zur Durchführung der paarweisen Vergleiche, der durch die Anzahl der zugrunde gelegten Kriterien (und gegebenenfalls Alternativen) bestimmt wird. Des Weiteren können Inkonsistenzen in den paarweisen Vergleichen auftreten, so dass der als Gewichtungsvektor berechnete Eigenvektor nur unter bestimmten Bedingungen akzeptabel ist (Belton und Stewart 2002). Zudem wird die Rationalität der Neun-PunkteRatio-Skala in Verbindung mit einer reziproken Vergleichsmatrix angezweifelt. Denn für die Transformation verbaler Werturteile in Zahlen existiert keine allgemeingültige Skala. Prinzipiell können auch andere Skalen mit abweichender Spannbreite zugrunde gelegt werden, die unter Umständen zu anderen Ergebnissen führen (Belton und Stewart 2002; Geldermann 2005; Lootsma 1980; Geldermann et al. 2002b).

Verschiedene Ergänzungen oder Erweiterungen zur Gewichtung der Kriterien mittels AHP wurden vorgeschlagen, beispielsweise indem Vergleiche durch Experten auf Basis linguistischer Variablen in Fuzzy-Zahlen transformiert werden (zum Beispiel Kahraman et al. 2009) oder durch eine Kombination des AHP mit einer Delphi-Befragung (Liang et al. 2006). Der ANP stellt eine Erweiterung des hierarchisch strukturierten AHP zur Netzwerk-Struktur dar und erlaubt, zusätzliche Feedback-Schleifen und Interdependenzen bei den paarweisen Vergleichen zu berücksichtigen. Diese Möglichkeit nutzen im Energiebereich beispielsweise Erdogmus et al. (2006), Köne und Büke (2007), Önüt et al. (2008) sowie Ulutas (2005). Anwendungen, die ebenfalls paarweise Vergleiche nutzen, die jedoch auf anderen Skalen und/oder anderen Transformationen als der AHPIANP nach Saaty beruhen, sind beispielsweise zu finden bei Lootsma et al. (1986; 1990a; 1990b) und Mamlook (2006).

Andere Ansätze zur Ableitung von Gewichtungen basieren auf einer festzulegenden Rangfolge der Kriterien (zum Beispiel Daim et al. 2009). Dabei wird im Energiebereich recht häufig die Methode nach Simos bzw. Modifikationen dieser Methode eingesetzt (zum Beispiel Beccali et al. 2003; Cavallaro 2009; Georgopoulou et al. 1997; Georgopoulou et al. 1998; Haldi et al. 2002; Kowalski et al. 2009; Madlener et al. 2007; Tsoutsos et al. 2009). Nach Polatidis und Haralambopoulos (2008) weist diese Technik Vorteile auf, da sie weniger willkürlich als die direkte Gewichtung und einfacher als die meisten anderen indirekten Methoden ist. Zudem ist sie leicht verständlich und lässt den Entscheidungsträgern ausreichend Freiraum, um ihren Präferenzen Ausdruck zu verleihen. Die Methode basiert auf einer Rangfolge der Kriterien, die mit Hilfe von Karten mit den Namen der Kriterien ermittelt wird. Dabei sind auch doppelt belegte Rangplätze zulässig, um Indifferenzen auszudrücken, sowie leere Karten, um Unterschiede 
in der relativen Bedeutung abbilden zu können. Aus der Anzahl der Kriterien und der Nummerierung der Rangplätze wird schließlich die Gewichtung bestimmt. Die Methode kann auch zur Bewertung von Alternativen hinsichtlich einzelner Kriterien angewendet werden (Figueira und Roy 2002; Pictet und Bollinger 2005). In ähnlicher Weise werden die Gewichtungen im Rahmen der Erwartungswertmethode (Expected Value Method) berechnet (zum Beispiel Ghafghazi et al. 2009). Andere Autoren leiten zulässige Intervalle von Kriterien-Gewichtungen ab, die einer durch den Entscheidungsträger festzulegenden Rangfolge der Kriterien gerecht werden (zum Beispiel Afgan und Carvalho 2008; Begic und Afgan 2007; Jovanovic et al. 2009; Liposcak et al. 2006; Pilavachi et al. 2006).

Im Gegensatz zu den bisher beschriebenen Methoden, welche in erster Linie die subjektiven Präferenzen der Entscheidungsträger widerspiegeln, grenzen Wang et al. (2009a) „objektive“ Methoden ab, die alleine auf der Spannbreite der Kriterien-Ausprägungen beruhen. So kommt zum Beispiel bei Jiang-Jiang et al. (2010) im Rahmen der Bewertung von KWKK-Systemen (Kraft-Wärme-Kälte-Kopplung) die so genannte Entropie-Methode zur Anwendung, wobei einem Kriterium ein umso höheres Gewicht zugeordnet wird, je größer die Unterschiede in den Ausprägungen dieses Kriteriums sind. Jedoch wird einer der Vorteile von MADM-Methoden gerade darin gesehen, dass subjektive Wertvorstellungen explizit und in transparenter Weise berücksichtigt werden können (vgl. zum Beispiel Alanne et al. 2007; Cavallaro 2009; Lee und Hwang 2010; Løken et al. 2009; Madlener et al. 2009; Önüt et al. 2008; Papadopoulos und Karagiannidis 2008; Roth et al. 2009). Bei Anwendung „objektiver" Gewichtungsmethoden werden diese Möglichkeiten eingeschränkt.

Dem Nutzer des im Rahmen der vorliegenden Arbeit zu entwickelnden EUS sollte eine oder mehrere Methode(n) angeboten werden, die es ihm ermöglichen, seine Wertvorstellungen in Form von Gewichten auf einer ausreichend differenzierten Skala auszudrücken. Dafür bieten sich zum Beispiel die direkte Gewichtung auf einer offenen Skala, der AHP oder die Methode nach Simos an. Noch wichtiger als die Wahl einer geeigneten Methode zur Gewichtung scheint jedoch das Aufzeigen der Auswirkungen unterschiedlicher Kriterien-Gewichtungen auf das Ergebnis im Rahmen von Sensitivitätsanalysen (vgl. auch Abschnitt 4.2.7). Weiterhin sollte die Möglichkeit zur wiederholten Durchführung von Bewertungen mit variierenden Gewichtungen bestehen, damit der Entscheidungsträger seine individuellen Wertangaben gegebenenfalls im Rahmen einer Feedback-Schleife anpassen kann. 


\subsubsection{Aggregation zur Gesamtbewertung}

Entsprechend der Differenzierung in Tabelle 4.2 wird in nutzentheoretisch basierte Modelle und Outranking-Ansätze unterschieden, um Anwendungen multikriterieller Aggregationsmethoden im Energiebereich darzustellen.

\subsubsection{Anwendung nutzentheoretisch basierter Modelle}

Eines der am häufigsten angewendeten Nutzenwertmodelle stellt die SAW-Technik dar (Simple Additive Weighting). Dabei wird der Gesamtnutzen einer Alternative über die Summe der Teilnutzen ermittelt. Letztere werden häufig in Form von KriterienAusprägungen dargestellt, die zwischen 0 (= schlechteste Ausprägung) und 1 (= beste Ausprägung) normiert werden, um eine Addition der in unterschiedlichen Einheiten vorliegenden Werte zu ermöglichen (für Anwendungen im Energiebereich siehe Basson und Petrie 2007; de Almeida et al. 2005; Hämäläinen und Karjalainen 1992; Hanson et al. 1991; Heinrich et al. 2007; Lhendup 2008; Renn 2003; Roth et al. 2009; Shackley und McLachlan 2006; van Alphen et al. 2007; Voropai und Ivanova 2002). Eine Alternative hierzu stellt die multiplikative Nutzenfunktion dar, die zum Beispiel bei Schulz und Stehfest (1984) sowie Wenstöp und Carlsen (1988) zur Bewertung von Optionen für die Energiebereitstellung eingesetzt wird.

Im englischen Sprachgebrauch werden im Allgemeinen nutzentheoretische MAUTModelle (Multi Attribute Utility Theory) von MAVT-Modellen (Multi Attribute Value Theory) abgegrenzt. Erstere sind durch die Berücksichtigung von Unsicherheiten in Form von Risiken gekennzeichnet (vgl. auch Dyer 2005). Keeney und Sicherman (1983) wenden zum Beispiel eine additive Nutzenfunktion zur Bewertung von Alternativen im Energiebereich unter Berücksichtigung der Eintritts-Wahrscheinlichkeiten unterschiedlicher Kriterien-Ausprägungen an. Dabei wird die Gültigkeit der additiven Nutzenfunktion im spezifischen Anwendungsfall ausführlich begründet (ebenso bei Buehring et al. (1978)). Als Erweiterung zur klassischen additiven Nutzenfunktion schlagen Løken et al. (2009) eine Äquivalenz-Technik vor („Equivalent Attribute Technique“ - EAT). Die Auswahl einer additiven Nutzenfunktion als Basis wird durch die in diesem Rahmen zur Verfügung stehenden Möglichkeiten zur Berücksichtigung von Unsicherheiten begründet sowie aufgrund der Nachvollziehbarkeit der Aggregationsmethode. Die Darstellung der Ergebnisse als Äquivalenz-Werte bezüglich eines der verwendeten Attribute soll die Verständlichkeit der Ergebnisse für den Entscheidungsträger erhöhen. Eine additive Nutzenfunktion ist auch Ausgangsbasis für die Bewertung von Energieversorgungssystemen bei Alanne et al. (2007) mit Hilfe der Methode PAIRS (Preference Assessment by Imprecise Ratio Statements method), wobei Unsicherheiten in Form von Intervallen berücksichtigt werden. Eine ähnliche Methode dient zur Berechnung des Nach- 
haltigkeitsindikators („General Index of Sustainability“) bei Afgan und Co-Autoren (Afgan et al. 2000; 2007a; 2007b; 2007c; Afgan und Carvalho 2002; 2004; 2008; Begic und Afgan 2007; Jovanovic et al. 2009; Liposcak et al. 2006; Pilavachi et al. 2006; vgl. Abschnitt 4.2.5).

Zu den nutzentheoretischen Methoden zählen auch Modelle auf Basis paarweiser Vergleiche, wobei insbesondere der AHP im Energiebereich weit verbreitet ist (für die methodischen Grundlagen siehe zum Beispiel Saaty 1980; Saaty 2005; Saaty und Vargas 2006). Ausgangspunkt des AHP ist eine hierarchische Strukturierung des Entscheidungsproblems. In einigen Fällen werden mehrere Hierarchien, zum Beispiel hinsichtlich der Nutzen und Kosten der Alternativen, unterschieden und aus den Teilergebnissen Nutzen-Kosten-Verhältnisse abgeleitet (zum Beispiel Akash et al. 1999; Jaber et al. 2008; Mohsen und Akash 1997). Als Vorteil des AHP wird angeführt, dass dieser eine Vereinfachung komplexer Probleme durch systematische Strukturierung in kleinere Teilprobleme erlaubt (Chatzimouratidis und Pilavachi 2008; Lee und Hwang 2010; Liang et al. 2006; Pilavachi et al. 2009; Zangeneh et al. 2009). Weiterhin gilt der Prozess als einfach und intuitiv verständlich (Gholamnezhad 1983; Mohsen und Akash 1997; Rahman und Frair 1984; Ren et al. 2009). Dabei können quantitative, qualitative bzw. schwer quantifizierbare und unsichere Informationen simultan verarbeitet werden (Gholamnezhad und Saaty 1982; Kagazyo et al. 1997; Rahman und Frair 1984; Ren et al. 2009; Son und Min 1998). Mehrere Autoren verweisen zudem auf die Effektivität und Flexibilität des Ansatzes im Vergleich zu anderen MCDA-Methoden (zum Beispiel Daim et al. 2009; Jaber et al. 2008; Liang et al. 2006; Ren et al. 2009; Zangeneh et al. 2009). Nachteile des AHP werden im Kontext der Anwendung zur Ableitung von Kriterien-Gewichtungen diskutiert (vgl. Abschnitt 4.2.5). Darüber hinaus ist an dieser Stelle auf das Problem der Rangumkehr („Rank Reversal“) hinzuweisen. Dies bedeutet, dass sich bei Anwendung des AHP die Rangfolge der ursprünglich betrachteten Alternativen ändern kann, wenn dem Entscheidungsmodell Alternativen hinzugefügt bzw. Alternativen gestrichen werden, bei gleichzeitiger Beibehaltung aller anderen Bedingungen (French et al. 2009).

Verschiedene Erweiterungen und Ergänzungen zum AHP werden im Kontext von Anwendungen im Energiebereich vorgeschlagen, um den Prozess zu verbessern und den spezifischen Anforderungen anzupassen (zum Beispiel Kahraman et al. 2009; Karger und Hennings 2009; Liang et al. 2006; Ramanathan und Ganesh 1995). Eine bekannte Erweiterung des AHP stellt der ANP dar, der häufig zur vergleichenden Bewertung der Nutzen („Benefits“), Chancen („Opportunities“), Kosten („Costs“) und Risiken („Risks“) 
der Alternativen eingesetzt wird (BOCR-Analyse). 9 Dabei werden Sub-Netzwerke für diese Komponenten gebildet und die Ergebnisse der Sub-Netzwerke in unterschiedlicher Weise miteinander verknüpft (im Energiebereich zum Beispiel angewendet von Erdogmus et al. (2006), Köne und Büke (2007), Önüt et al. (2008) sowie Ulutas (2005)). Die Gründe zur Anwendung des ANP entsprechen weitgehend den Vorteilen des AHP. Dass beim ANP darüber hinaus Wechselbeziehungen und FeedbackSchleifen zulässig sind, wird als zusätzlicher Vorteil hinsichtlich der Flexibilität der Methode angesehen (siehe auch Hämäläinen und Seppäläinen 1986).

Neben der neunstufigen Skala des AHP/ANP und der damit verbundenen Saaty'schen Eigenvektormethode finden auch andere Skalen und Berechnungsalgorithmen auf $\mathrm{Ba}-$ sis paarweiser Vergleiche zur multikriteriellen Entscheidungsunterstützung im Energiebereich Anwendung (zum Beispiel Lootsma et al. 1986; Lootsma et al. 1990a; Mamlook 2006). Lootsma et al. (1990b) merken in diesem Zusammenhang an, dass paarweise Vergleiche und Auswertungen auf Basis der neunstufigen Skala des AHP die Präferenzen der Entscheidungsträger nicht unbedingt korrekt widerspiegeln, während die von innen verwendete geometrische Progression auf psycho-physischen Argumenten beruht. Als Nachteil ist allen Modellen, die auf paarweisen Vergleichen beruhen, gemein, dass unter Umständen eine hohe Anzahl an Vergleichen durch den Entscheidungsträger durchgeführt werden muss.

Vereinzelt finden andere nutzentheoretisch basierte Methoden Anwendung zur Bewertung von Energiebereitstellungs-Optionen. Zum Beispiel nutzen Burton und Hubacek (2007) das Verfahren MACBETH (Measuring Attractiveness by a Categorial Based Evaluation Technique), da mit dieser Methode schwer zu quantifizierende Kriterien auf einfache Weise gehandhabt werden können, wobei auch qualitative Daten als Eingabewerte zulässig sind (vgl. auch Bana e Costa 2005).

\subsubsection{Anwendung von Outranking-Ansätzen}

Outranking-Verfahren basieren auf paarweisen Vergleichen, die im Rahmen eines mehr oder weniger automatisierten Berechnungsprogrammes durchgeführt werden. Im Vergleich zu nutzentheoretisch basierten Methoden wird in der Regel weniger Informations-Input vom Entscheidungsträger benötigt (vgl. zum Beispiel Kowalski et al. 2009). Dabei finden im Energiebereich insbesondere ELECTRE- und PROMETHEE-Metho-

9 Im Gegensatz zur SWOT-Analyse (vgl. Fn. 7, Abschnitt 4.2.2), die in interne und externe Faktoren unterscheidet, grenzt Saaty sichere und unsichere Aspekte voneinander ab. Dabei werden sichere positive bzw. negative Aspekte als Nutzen („benefits“) bzw. Kosten („costs“) bezeichnet, während Chancen („opportunities“) und Risiken („risks“) die unsicheren Aspekte beschreiben (vgl. Saaty und Vargas 2006). 
den Anwendung. Nach Georgopoulou et al. (1997) handelt es sich bei ELECTRE (vgl. Roy 1980; Roy 1996) um eine im Umwelt- und Energiebereich weit verbreitete Methode, die auch Unvergleichbarkeiten und Intransitivitäten verarbeiten kann. Als Begründung zur Anwendung von ELECTRE-Methoden im Energiebereich anstelle nutzentheoretisch basierter Modelle wird darüber hinaus häufig angeführt, dass Unsicherheiten in Kriterien-Ausprägungen wie auch in menschlichen Präferenzvorstellungen adäquat abgebildet werden können (zum Beispiel Karakosta et al. 2009; Madlener et al. 2009; Papadopoulos und Karagiannidis 2008; Siskos und Hubert 1983). So können auch Indifferenz und schwache Präferenzen berücksichtigt werden (Georgopoulou et al. 2003; Mróz und Thiel 2005; Thiel und Mróz 2001). Daher ziehen auch Capros et al. (1988) ELECTRE einer Kosten-Nutzen-Analyse vor, da die Outranking-Methode als flexibler, realistischer und effizienter angesehen wird, um eine gute KompromissLösungen zu erzielen. Beccali et al. (2003) heben zudem hervor, dass bei Anwendung von ELECTRE einer vollständigen Kompensation guter und schlechter KriterienAusprägungen vorgebeugt wird. Darüber hinaus können auch qualitative Wertungen bzw. linguistische Variablen als Eingangsgrößen dienen (Beccali et al. 1998).

PROMETHEE-Methoden bieten ähnliche Möglichkeiten wie ELECTRE zur Berücksichtigung von Unsicherheiten in den Präferenzvorstellungen und Input-Daten (Brans et al. 1986) und weisen daher ebenfalls die bereits genannten Vorteile im Vergleich zu nutzentheoretischen Modellen auf (vgl. zum Beispiel Georgopoulou et al. 1998; Madlener et al. 2007; Ren et al. 2009). Die Literaturanalyse von Behzadian et al. (2010) vermittelt einen Überblick über das breite Spektrum von Anwendungen dieser Methoden-Familie in unterschiedlichen Bereichen, einschließlich des Energiesektors. Im Vergleich zu ELECTRE wird PROMETHEE in einigen Fällen vorgezogen, da die zugrunde liegenden Berechnungs-Algorithmen als transparenter, leichter verständlich und einfacher umsetzbar gelten (zum Beispiel Hyde et al. 2003; Polatidis und Haralambopoulos 2007; Topcu und Ulengin 2004). Dies ist insbesondere in Gruppenentscheidungsprozessen von Vorteil, da die Ergebnisse aus Sicht der Entscheidungsträger leichter nachvollziehbar und somit glaubwürdiger sind (Diakoulaki und Karangelis 2007; Doukas et al. 2006; Georgopoulou et al. 1998; Goumas et al. 1999; Haldi et al. 2002; Haralambopoulos und Polatidis 2003). Weiterhin bietet PROMETHEE Möglichkeiten zur klaren Visualisierung der Ergebnisse (Polatidis und Haralambopoulos 2007). Zudem weisen Brans et al. (1986) sowie Topcu und Ulengin (2004) auf die Stabilität von Ergebnissen basierend auf PROMETHEE hin. Goletsis et al. (2003) wählen eine Kombination von ELECTRE- und PROMETHEE-Methoden, um die Vorteile der beiden am weitesten verbreiteten Outranking-Ansätze bei der Bewertung von Energieprojekten zu vereinen. Um die Möglichkeiten zur Berücksichtigung von Unsicherheiten bei der Bewertung von Energieprojekten weiter zu verbessern, wenden Goumas und Lygerou 
(2000) sowie Papadaki et al. (2003) einen fuzzifizierten PROMETHEE-Ansatz an. Als Alternativen zu den weit verbreiteten PROMETHEE- und ELECTRE-Ansätzen wird zur Bewertung von Optionen zur Energiebereitstellung vereinzelt NAIADE (Novel Approach to Imprecise Assessment and Decision Environments) vorgeschlagen (vgl. Browne et al. 2010; Cavallaro und Ciraolo 2005; Dinca et al. 2007).

Die Aggregationsmethoden, die im Kontext der vorliegenden Arbeit zur Bewertung von Energietechnologien verwendet werden, sollten einerseits leicht verständlich für die Entscheidungsträger sein und andererseits ausreichende Möglichkeiten zur Berücksichtigung vager Präferenzvorstellungen und Werturteile bieten. Weiterhin sind Möglichkeiten zur transparenten grafischen Darstellung der Ergebnisse und der Sensitivitäten wünschenswert. Im Hinblick auf die Verständlichkeit und Nachvollziehbarkeit scheint sich insbesondere die additive Nutzenfunktion anzubieten. Diese bringt jedoch Schwierigkeiten hinsichtlich der Normierung einzelner Bewertungsgrößen und der Kompensation schlechter und guter Kriterien-Ausprägungen mit sich. OutrankingAnsätze weisen demgegenüber Vorteile auf, beispielsweise in Zusammenhang mit der Berücksichtigung vager Präferenzvorstellungen. Dabei wird bei der Wahl zwischen den am weitesten verbreiteten Outranking-Methoden PROMETHEE häufig ELECTRE vorgezogen, da erstere vorteilhaft hinsichtlich Transparenz und Verständlichkeit sind. Bei einer parallelen Implementierung mehrerer Methoden in einem EUS kann der Entscheidungsträger selbst wählen, welche Methode zur Abbildung seiner Präferenzvorstellungen am besten geeignet ist. Darüber hinaus können Ergebnisse auf Basis unterschiedlicher Methoden verglichen werden. Dabei sollte jedoch bei der Entwicklung des EUS zwischen dem Aufwand der Implementierung zusätzlicher Methoden einerseits und dem damit verbundenen Zusatznutzen andererseits abgewogen werden.

\subsubsection{Sensitivitätsanalysen und weitere Methoden zur Berücksichtigung von Unsicherheiten}

Die am häufigsten angewendete Methodik zur Berücksichtigung von Unsicherheiten im Rahmen der multikriteriellen Bewertung von Optionen zur Energiebereitstellung ist die Sensitivitätsanalyse. In den meisten Fällen wird hierbei die Gewichtung der Kriterien variiert. Dabei werden entweder unterschiedliche Gewichtungssets einander gegenüber gestellt, die aus der Befragung einzelner Entscheidungsträger oder von AkteursGruppen hervorgegangen sind (Ghafghazi et al. 2009; Goletsis et al. 2003; Haldi et al. 2002; Hanson et al. 1991; Haralambopoulos und Polatidis 2003; Kagazyo et al. 1997; Kowalski et al. 2009; Løken et al. 2009; Madlener et al. 2007; Renn 2003; Shackley und McLachlan 2006; Tzeng et al. 1992; Wenstöp und Carlsen 1988), oder die Autoren legen ohne die Akteure direkt zu befragen „fiktive“ Gewichtungs-Szenarien zugrunde, die jeweils eine spezifische Sichtweise widerspiegeln sollen (Beccali et al. 1998; 
Beccali et al. 2003; Capros et al. 1988; Chatzimouratidis und Pilavachi 2009; de Almeida et al. 2005; Diakoulaki und Karangelis 2007; Doukas et al. 2006; Doukas et al. 2007; Gholamnezhad und Saaty 1982; Köne und Büke 2007; Papadopoulos und Karagiannidis 2008; Pilavachi et al. 2009; Ren et al. 2009; Siskos und Hubert 1983; Voropai und Ivanova 2002). In einigen Fällen werden einzelne oder mehrere Kriterien im Rahmen der Sensitivitätsanalyse vollständig ausgeschlossen oder ausgetauscht, um die Bewertung auf bestimmte Aspekte zu fokussieren (zum Beispiel Browne et al. 2010; Georgopoulou et al. 1997; Tsoutsos et al. 2009). Eine weitere Möglichkeit besteht in der Bestimmung von Stabilitätsintervallen der Kriterien-Gewichtungen, innerhalb derer sich die Rangfolge der bewertenden Optionen nicht verändert (Burton und Hubacek 2007; Cavallaro 2009; Topcu und Ulengin 2004; Mareschal 1988; Wolters und Mareschal 1995). Häufig wird in einer zweidimensionalen Grafik das Endergebnis in Abhängigkeit der Variation der Gewichtung jeweils eines Kriteriums zwischen 0 \% und $100 \%$ dargestellt, während die relative Gewichtung aller anderen Kriterien konstant bleibt. Jede Alternative wird somit durch eine Linie dargestellt, deren Steigung den Einfluss des jeweiligen Kriteriums auf das Ergebnis repräsentiert (Heinrich et al. 2007; Tsoutsos et al. 2009; Treitz 2006). Durch diese Darstellungsform werden Stabilitätsintervalle visualisiert und der Einfluss sich ändernder Kriterien-Gewichtungen auf die Rangfolge verdeutlicht. In interaktiven Software-Tools wird dem Anwender darüber hinaus die Möglichkeit geboten, die Gewichtung eines Kriteriums mit Hilfe eines Schiebereglers zu variieren, wobei die Änderungen der Gewichtungen der übrigen Kriterien und der Endergebnisse je Alternative in Form von Balkendiagrammen dargestellt werden (,Walking Weights“; vgl. Tsoutsos et al. 2009). Eine weitere Möglichkeit der Sensitivitätsanalyse liegt in der Bestimmung der minimal notwendigen Variation der Gewichtungen, damit eine bestimmte Alternative am besten bewertet wird (Geldermann et al. 2002b; Wolters und Mareschal 1995). Im Rahmen von PROMETHEE VI besteht darüber hinaus die Möglichkeit, den Einfluss auf das Ergebnis aufgrund der Variation von KriterienGewichtungen innerhalb definierter Intervalle in der so genannten GAIA-Ebene ${ }^{10} \mathrm{zu}$ visualisieren (Treitz 2006). Weitere Methoden der Sensitivitätsanalyse sind bei zum Beispiel bei Geldermann et al. (2003), Mareschal (1988), Treitz (2006), Wolters und Mareschal (1995) dargestellt.

Neben den Gewichtungen werden im Rahmen von Sensitivitätsanalysen bei Einsatz von Outranking-Methoden auch Schwellenwerte, beispielsweise für strikte Präferenz

10 Die GAIA-Ebene (Geometric Analysis for Interactive Aid) kann als Meta-Modell bezeichnet werden, in dem der k-dimensionale Raum, der durch die Einzelbewertungen auf Basis von k Kriterien aufgespannt wird, auf eine zweidimensionale Fläche projiziert wird, um ein übersichtliches Bild der Entscheidungssituation zu erhalten (Belton und Stewart 2002; Brans und Mareschal 2005; Vetschera 1994). 
und Indifferenz oder zur Abgrenzung zwischen Kategorien, variiert (Cavallaro und Ciraolo 2005; Dinca et al. 2007; Georgopoulou et al. 1997; Georgopoulou et al. 2003; Güngör und Arikan 2000; Mróz und Thiel 2005). Darüber hinaus kann bei PROMETHEE der Typ der zugrunde gelegten Präferenzfunktion geändert werden (Geldermann et al. 2002b; Treitz 2006). Bei Anwendung von MAVT/MAUT kann stattdessen die Form der Nutzen- bzw. Wertfunktionen (Krümmung, Ober- und Untergrenzen) variiert werden (Basson und Petrie 2007; Bertsch 2008; Hämäläinen und Karjalainen 1992; Wenstöp und Carlsen 1988). Auch Input-Daten in Form von Kriterien-Ausprägungen, Rahmendaten oder subjektive Bewertungen von Alternativen werden im Rahmen von Sensitivitätsanalysen verändert (Dinca et al. 2007; Haldi et al. 2002; Hämäläinen 1990; Hanegraaf et al. 1998; Jiang-Jiang et al. 2010; Keeney und Sicherman 1983). Schließlich kann auch die Gewichtungs- oder Aggregationsmethode selbst variiert oder geändert werden, um die Auswirkungen auf die Ergebnisse zu beobachten (Doukas et al. 2007; Erdogmus et al. 2006; Lootsma et al. 1986; Lootsma et al. 1990a; Lootsma et al. 1990b).

In einigen Fällen werden Wahrscheinlichkeiten hinsichtlich der Attribut-Ausprägungen und Randbedingungen zur Abbildung von Unsicherheiten eingesetzt (zum Beispiel Heinrich et al. 2007). Buehring et al. (1978) berücksichtigen die Wahrscheinlichkeiten unterschiedlicher Kriterien-Ausprägungen im Rahmen der Bestimmung von Nutzenfunktionen. Voropai und Ivanova (2002) sowie Løken et al. (2009) beziehen die Eintrittswahrscheinlichkeiten unterschiedlicher Rahmenbedingungen in ihre Betrachtungen ein. Durch Anwendung der Monte-Carlo-Technik können die Input-Daten in Form von Kriterien-Ausprägungen und/oder -Gewichtungen unter Berücksichtigung entsprechender Wahrscheinlichkeitsverteilungen variiert und die Auswirkungen auf das Ergebnis beobachtet werden (zum Beispiel Basson und Petrie 2007; Goumas et al. 1999; Hyde et al. 2003). Diese Technik setzt voraus, dass Wahrscheinlichkeitsverteilungen zu den Kriterien-Ausprägungen bzw. -Gewichtungen vorliegen bzw. aus den verfügbaren Daten abgeleitet werden können. Andernfalls sind die festzulegenden Eintrittswahrscheinlichkeiten mit einer hohen Subjektivität behaftet.

Um die Festlegung von Wahrscheinlichkeiten zu vermeiden und dennoch Unsicherheiten in den Kriterien-Ausprägungen bzw. Input-Daten zu berücksichtigen, werden häufig linguistische Variablen verwendet, die in Form von Fuzzy-Zahlen abgebildet werden (zum Beispiel Beccali et al. 1998; Doukas et al. 2007; Goumas und Lygerou 2000; Güngör und Arikan 2000; Kaminaris et al. 2006; Mamlook et al. 2001a; Mamlook et al. 2001b). In einigen Anwendungen kommt diese Methodik auch für die relative Gewichtung der Kriterien zur Anwendung (zum Beispiel Kahraman et al. 2009). Andererseits können Fuzzy-Zahlen auch auf Basis von Intervallen und Verteilungen verfügbarer quantitativer Daten definiert werden (zum Beispiel Siskos und Hubert 1983). Liang et 
al. (2006) wenden eine Delphi-Befragung an, um Zugehörigkeitsfunktionen der Kriterien-Ausprägungen in Form von Fuzzy-Zahlen zu bestimmen. Die Verwendung von Fuzzy-Zahlen setzt voraus, dass geeignete Informationen vorliegen, um die Zugehörigkeitsfunktionen zu bestimmen. Bei Anwendung linguistischer Variablen muss eine geeignete Skala zur Transformation in Fuzzy-Zahlen festgelegt werden.

In einigen wenigen Anwendungsfällen wird die Szenario-Technik zu Hilfe genommen, um denkbare zukünftige Rahmenbedingungen abzubilden und die Bewertung der Optionen in Abhängigkeit unterschiedlicher Zukunftsszenarien abzubilden (zum Beispiel Capros et al. 1988). Diese Vorgehensweise hat den Vorteil, dass ein „Raum“ möglicher Zukunftsentwicklungen aufgespannt wird, ohne dass Eintrittswahrscheinlichkeiten abgeschätzt werden müssen. Weiterhin können einerseits robuste Optionen identifiziert werden, die bei allen zugrunde gelegten Szenarien gute Ergebnisse aufweisen. Andererseits können solche Optionen identifiziert werden, deren Abschneiden zu einem hohen Maße von den zukünftigen Rahmenbedingungen abhängen und deren Einsatz mit einem höheren Risiko verbunden ist. Bei Lootsma et al. (1990a) und Zangeneh et al. (2009) werden paarweise Vergleiche vor dem Hintergrund unterschiedlicher Szenarien bzw. politischer Rahmenbedingungen durchgeführt. Dies setzt jedoch voraus, dass die Personen, welche die paarweisen Vergleiche durchführen, ausreichend Erfahrung und Vorstellungsvermögen besitzen, um die komplexen Wechselwirkungen zwischen unterschiedlichen Szenarien und Optionen abschätzen zu können.

Eine weitere Möglichkeit zur Berücksichtigung von Unsicherheiten besteht darin, dass eine Maßzahl für die Zuverlässigkeit der Input-Daten eingeführt wird. Zum Beispiel geben bei Karger und Hennings (2009) die Experten im Rahmen von AHP-Vergleichen an, wie hoch ihr Vertrauen in die eigene subjektive Einschätzung ist. Buchholz et al. (2009) nehmen ein Standard-Unsicherheits-Intervall von $10 \%$ für Kriterien-Ausprägungen und Gewichtungen an. Entsprechend der Methode PAIRS geben Alanne et al. (2007) Unsicherheiten in den Kriterien-Ausprägungen und -Gewichtungen sowie die daraus resultierenden Ergebnisse in Form von Intervallen an. Ähnlich gehen auch Afgan et al. (2000; 2007a; 2007b; 2007c), Afgan und Carvalho (2002; 2004; 2008), Begic und Afgan (2007), Jovanovic et al. (2009), Liposcak et al. (2006) sowie Pilavachi et al. (2006) vor.

Hinsichtlich der Fragestellung in der vorliegenden Arbeit sollten Möglichkeiten zur Durchführung von Sensitivitätsanalysen und deren grafische Darstellung, insbesondere in Zusammenhang mit den Kriterien-Gewichtungen, fester Bestandteil des zu entwickelnden EUS sein. Damit können die variierenden Sichtweisen mehrere Akteure (vgl. auch Abschnitt 4.2.8) adäquat beurteilt und die Transparenz und Nachvollziehbarkeit des Entscheidungsprozesses gefördert werden. Dabei stellt insbesondere die Ermitt- 
lung von Stabilitätsintervallen in Verbindung mit einer Darstellung der Endergebnisse in Abhängigkeit der Variation der Gewichtung einzelner Kriterien eine effektive Methode zur Untersuchung und Veranschaulichung der Stabilität einer Rangfolge dar. Weiterhin scheint eine Analyse von Unsicherheiten hinsichtlich wichtiger Randbedingungen in Form von Szenarien, zum Beispiel der Energieträgerpreise, eine sinnvolle Möglichkeit zur Bewertung der Robustheit unterschiedlicher Optionen. Bei Überlegungen zur Berücksichtigung weiterer Unsicherheiten, zum Beispiel auch in Form von Wahrscheinlichkeitsverteilungen oder Fuzzy-Zahlen, sollte grundsätzlich zwischen Komplexität und Transparenz bzw. Verständlichkeit der Ergebnisse abgewogen werden.

\subsubsection{Einbeziehung von Stakeholdern und Entscheidungs- findung in Gruppen}

Aufgrund der weitreichenden Konsequenzen von Entscheidungen im Energiebereich und des damit verbundenen großen Interesses der Öffentlichkeit ist die Einbeziehung unterschiedlicher Akteure in diesem Zusammenhang von besonderer Bedeutung. Während in einigen Veröffentlichungen die Autoren stellvertretend als Entscheidungsträger auftreten (vgl. zum Beispiel Gholamnezhad 1983; Phdungsilp 2010), teilweise mit Berufung auf eigene Erfahrungen im Energiebereich (vgl. zum Beispiel Gholamnezhad und Saaty 1982; Kagazyo et al. 1997), sind bei anderen Anwendungen explizit mehrere Akteure aus unterschiedlichen Bereichen involviert. Welche Akteure im Rahmen eines Entscheidungsprozesses einbezogen werden sollen und können, ist letztlich von der konkreten Problemstellung und den spezifischen Rahmenbedingungen abhängig. Dabei kann Tabelle 4.4 Anhaltspunkte zur Identifikation relevanter Akteure im Energiebereich liefern.

Die Einbindung der identifizierten Akteure kann zu mehreren Phasen im Entscheidungsprozess mit Hilfe unterschiedlicher Methoden erfolgen. Im Rahmen partizipativer Entscheidungsprozesse werden häufig Workshops und bilaterale Interviews mit mehreren Stakeholdern aus unterschiedlichen Bereichen durchgeführt, teilweise unterstützt durch Fragebögen (vgl. zum Beispiel Buchholz et al. 2009; Georgopoulou et al. 1997; Georgopoulou et al. 1998; Georgopoulou et al. 2003; Karakosta et al. 2009; Stagl 2006). So liegt ein besonderes Anliegen von Andrews (2003) darin, einen offenen Prozess zur Förderung der Kreativität und des Lernens durchzuführen, um letztlich einen Konsens zu erzielen. In einigen Fällen werden die Entscheidungsträger bereits bei der Definition der Problemstellung und der Rahmenbedingungen, der Auswahl der Alternativen und/oder der Erarbeitung der Kriterien einbezogen (zum Beispiel Georgopoulou et al. 1997; Jones et al. 1990). Alternativen in Form von Szenarien und Kriterien werden bei Haldi et al. (2002), Kowalski et al. (2009) und Madlener et al. (2007) gemeinsam mit den Stakeholdern im Rahmen von Workshops und Interviews erarbeitet. 
Tabelle 4.4: $\quad$ Berücksichtigung von Akteuren in multikriteriellen Entscheidungsprozessen im Energiebereich

\begin{tabular}{|c|c|}
\hline Akteursgruppe & Quellen \\
\hline $\begin{array}{l}\text { Energieversorgungsunternehmen, Anlagenbe- } \\
\text { treiber, Netzbetreiber }\end{array}$ & $\begin{array}{l}\text { z.B. Diakoulaki und Karangelis 2007; Doukas et al. } \\
\text { 2007; Heinrich et al. 2007; Roth et al. 2009; Shackley } \\
\text { und McLachlan } 2006\end{array}$ \\
\hline Verbände, Industrievertreter & $\begin{array}{l}\text { z.B. Andrews 2003; Georgopoulou et al. 2003; } \\
\text { Karakosta et al. 2009; Önüt et al. 2008; Ulutas } 2005\end{array}$ \\
\hline Gewerkschaften & Jones et al. 1990 \\
\hline $\begin{array}{l}\text { Repräsentanten der Verbraucher, zum Beispiel } \\
\text { aus Verbraucherorganisationen }\end{array}$ & $\begin{array}{l}\text { Burton und Hubacek 2007; Erdogmus et al. 2006; } \\
\text { Lootsma et al. 1986; Önüt et al. } 2008\end{array}$ \\
\hline Entwickler, Anlagenbauer & Assimakopoulos et al. 1991; Ghafghazi et al. 2009 \\
\hline $\begin{array}{l}\text { Repräsentanten der betroffenen Region oder } \\
\text { Gemeinde (Anwohner, Bürgermeister etc.) }\end{array}$ & $\begin{array}{l}\text { z.B. Ghafghazi et al. 2009; Haralambopoulos und } \\
\text { Polatidis 2003; Nigim et al. 2004; Polatidis und Hara- } \\
\text { lambopoulos 2007; Tsoutsos et al. } 2009\end{array}$ \\
\hline Finanzierungsinstitutionen, mögliche Investoren & $\begin{array}{l}\text { z.B. Doukas et al. 2007; Haralambopoulos und Polati- } \\
\text { dis 2003; Karakosta et al. 2009; Polatidis und Hara- } \\
\text { lambopoulos 2007; Tsoutsos et al. } 2009\end{array}$ \\
\hline Sonstige private Akteure & Papadopoulos und Karagiannidis 2008 \\
\hline $\begin{array}{l}\text { Regierungsvertreter, zum Beispiel aus For- } \\
\text { schungs-, Entwicklungs-, Wirtschafts-, Finanz- } \\
\text { Umwelt- oder Landwirtschaftsministerien }\end{array}$ & $\begin{array}{l}\text { z.B. Buchholz et al. 2009; Diakoulaki und Karangelis } \\
\text { 2007; Heinrich et al. 2007; Karakosta et al. 2009; Önüt } \\
\text { et al. 2008; Tsoutsos et al. } 2009\end{array}$ \\
\hline Vertreter lokale/regionaler Behörden & $\begin{array}{l}\text { z.B. Buchholz et al. 2009; Papadopoulos und Kara- } \\
\text { giannidis 2008; Polatidis und Haralambopoulos 2007; } \\
\text { Tsoutsos et al. } 2009\end{array}$ \\
\hline $\begin{array}{l}\text { Vertreter von Universitäten und andere For- } \\
\text { schungseinrichtungen }\end{array}$ & $\begin{array}{l}\text { z.B. Diakoulaki und Karangelis 2007; Doukas et al. } \\
\text { 2007; Georgopoulou et al. 2003; Haralambopoulos } \\
\text { und Polatidis 2003; Tsoutsos et al. 2009; Ulutas } 2005\end{array}$ \\
\hline $\begin{array}{l}\text { Experten bzw. Personen mit Know-how zur spe- } \\
\text { zifischen Entscheidungssituation }\end{array}$ & $\begin{array}{l}\text { z.B. Daim et al. 2009; Karger und Hennings 2009; Lee } \\
\text { und Hwang 2010; Løken et al. } 2009\end{array}$ \\
\hline $\begin{array}{l}\text { Umweltschutzorganisationen, Umweltaktivisten, } \\
\text { weitere Nicht-Regierungs-Organisationen }\end{array}$ & $\begin{array}{l}\text { z.B. Andrews 2003; Buchholz et al. 2009; Erdogmus } \\
\text { et al. 2006; Ghafghazi et al. 2009; Haralambopoulos } \\
\text { und Polatidis 2003; Karakosta et al. 2009; Polatidis } \\
\text { und Haralambopoulos 2007; Renn 2003; Shackley } \\
\text { und McLachlan 2006; Tsoutsos et al. 2009 }\end{array}$ \\
\hline Kirchen, Philosophen & Renn 2003 \\
\hline Sonstige Interessengruppen & $\begin{array}{l}\text { Haralambopoulos und Polatidis 2003; Jones et al. } \\
1990\end{array}$ \\
\hline
\end{tabular}

Um die Charakteristika der lokalen Anforderungen zu definieren, führen Cherni et al. (2007) Befragungen mit Haushalten, Stakeholdern und lokalen Führungspersönlichkeiten durch. Auch um die Kriterien-Ausprägungen zu bestimmen bzw. Alternativen hinsichtlich einzelner Kriterien auf unterschiedlichen Skalen zu bewerten (ordinal, kardinal, paarweise Vergleiche, linguistische Variablen, etc.), wird häufig auf das Know-how der Experten und anderer Akteure zurückgegriffen, insbesondere bei nicht direkt quantifizierbaren Kriterien (zum Beispiel Burton und Hubacek 2007; Doukas et al. 2007; 
Georgopoulou et al. 1997; Kahraman et al. 2009; Lootsma et al. 1990a; Madlener et al. 2009; Stagl 2006). Dies kann auch in Form eines Delphi-Prozesses umgesetzt werden (zum Beispiel Liang et al. 2006; Terrados et al. 2009).

In den meisten Fällen werden die Standpunkte der Entscheidungsträger durch Kriterien-Gewichtungen abgebildet, welche die subjektiven Präferenzen hinsichtlich unterschiedlicher Zielsetzungen widerspiegeln. Zu Aggregation individueller KriterienGewichtungen einzelner Entscheidungsträger oder Akteursgruppen dient häufig der (gewichtete oder ungewichtete) arithmetische Mittelwert (so zum Beispiel bei Doukas et al. 2006; Ramanathan und Ganesh 1995; Renn 2003). Auch der geometrische Mittelwert wird verwendet, insbesondere bei Anwendung des AHP (Hämäläinen 1990; Zangeneh et al. 2009). Lootsma et al. (1990a) stellen aggregierte Ergebnisse auf Basis des geometrischen und arithmetischen Mittelwerts einander gegenüber. Jedoch weisen Schulz und Stehfest (1984) darauf hin, dass eine Aggregation individueller Bewertungen nicht unbedingt förderlich zur Konfliktlösung ist, da hierdurch Informationen über individuelle Präferenzen verloren gehen (siehe auch Hämäläinen 1990). Folglich wird in einigen Fällen auf eine explizite Aggregation individueller Bewertungen verzichtet, und stattdessen werden Ergebnisse basierend auf verschiedenen individuellen Gewichtungen in einem offenen und transparenten Prozess einander gegenübergestellt (zum Beispiel Ghafghazi et al. 2009; Haralambopoulos und Polatidis 2003; Kowalski et al. 2009; Madlener et al. 2007). In einigen Fällen können die Gewichtungen nach einer Feedback-Schleife durch die Entscheidungsträger angepasst werden (zum Beispiel Ghafghazi et al. 2009). Dies erscheint insbesondere dann sinnvoll, wenn die Akteure zu Beginn des Entscheidungsprozesses noch nicht mit den zugrunde liegenden Methoden vertraut sind. Anstelle einer mathematischen Aggregation können Diskussionsprozesse dazu dienen, einen Konsens über die Gewichtungen zu erzielen (zum Beispiel de Almeida et al. 2005; Nigim et al. 2004). Bei Kowalski et al. (2009) dient neben einer Auswertung nach individuellen Gewichtungen eine so genannte „Silent Negotiation“ dazu, ein Gewichtungsset im Konsens mit allen Beteiligten abzuleiten. Hierbei handelt es sich im Wesentlichen um einen für Gruppen angepassten Simos-Prozess (vgl. Abschnitt 4.2.5), bei dem alle Teilnehmer in mehreren „stillen“ Runden die Gelegenheit haben, die Rangfolge der Karten, die Kriterien, Alternativen oder Abstandshalter repräsentieren, zu ändern. Wenn auf diese Weise kein Konsens über eine allgemein anerkannte Rangfolge erzielt werden kann, soll ein Moderator im Rahmen einer nachfolgenden Diskussion dabei helfen, einen Konsens herbeizuführen (Pictet und Bollinger 2005).

Eine weitere Möglichkeit zur Vermeidung einer vollständigen Aggregation individueller Präferenzen besteht in der Bildung von Clustern. So führen zum Beispiel Haldi et al. (2002) und Lootsma et al. (1986) eine Cluster-Analyse individueller Kriterien- 
Gewichtungen bzw. Bewertungen mit Hilfe einer Maßzahl basierend auf der „euklidischen Distanz" zwischen den Gewichtungsvektoren einzelner Stakeholder durch. Ebenso werden bei Karger und Hennings (2009) ausgehend von der Anwendung des AHP Cluster mit ähnlichen Bewertungen gebildet. Die Cluster-Analyse dient hier vor allem als Basis zur Identifikation von Uneinigkeiten und diesbezüglichen Gründen. Im Rahmen der Cluster-Analyse von Shackley und McLachlan (2006) geht es insbesondere darum, Strategien der Stakeholder bei der Vergabe der Gewichtungen und der Beurteilung der Alternativen zu analysieren. Bei Tsoutsos et al. (2009) werden Cluster im Rahmen eines partizipativen Entscheidungsprozesses genutzt, um die Akteure entsprechend ihrer Machtbefugnisse zu klassifizieren. In ähnlicher Weise werden bei Tzeng et al. (1992) Experten entsprechend ihrer Erfahrungen und ihres Wissensstandes in Gruppen eingeordnet und individuelle Bewertung in Abhängigkeit der so bestimmten Gruppen durchgeführt.

In einigen Fällen werden anstelle der Gewichtungen die Ergebnisse, die auf individuellen Gewichtungen basieren, aggregiert. Nwofor et al. (2007) bilden den Mittelwert und die Varianz aus den individuellen Bewertungen (direktes Scoring) einzelner Experten. Goumas et al. (1999) verwenden eine so genannte „Extended Contribution Rule“, wonach eine Option einer anderen vorzuziehen ist, wenn die Summe der Meinungen, die diese Aussage unterstützen, größer ist als die Summe der Standpunkte, die dieser Aussage widersprechen. Bei Goletsis et al. (2003) wird eine gewichtete Summe individueller Präferenzflüsse gebildet. Die den Beteiligten zugeordneten Gewichtungen basieren dabei auf deren Expertise und den zugrunde liegenden Machtverhältnissen. Der Rangkorrelationskoeffizient nach Spearman11 dient als Maßzahl für Abweichung der individuellen Ergebnisse vom so ermittelten Gruppenergebnis. Weiterhin werden Indizes zur Abbildung von Uneinigkeiten und Übereinstimmungen abgeleitet, um die Konsens-Findung zu unterstützen.

Auch das im Kontext der Fragestellung der vorliegenden Arbeit zu entwickelnde EUS sollte Möglichkeiten zur Berücksichtigung mehrerer Akteure bieten. Das Ziel besteht darin, einzelnen Entscheidungsträgern die Möglichkeit zu bieten, Alternativen und Kriterien auszuwählen, individuelle Kriterien-Gewichtungen einzugeben und zu analysieren. Weiterhin sollte die Darstellung aggregierter Gewichtungen mehrere Akteure sowie die Darstellung von Extrempositionen ermöglicht werden. Darüber hinaus dienen Ergebnisse einer Befragung von Entscheidungsträgern in der Energiewirtschaft, um ein

11 Der Spearman Rangkorrelationskoeffizient $r_{S}$ ist ein Maß dafür, inwieweit die Rangfolgen zweier Datenreihen übereinstimmen (in diesem Fall: Gewichtungsvektoren). Bei vollständiger Übereinstimmung der Rangfolge nimmt $r_{S}$ den Wert 1 an, bei maximal möglicher $A b-$ weichung -1 (Keller 2008; Madsen und Moeschberger 1983). 
möglichst breites Spektrum an Sichtweisen bei der Konzeption des EUS zu berücksichtigen.

\subsubsection{Implementierung}

Die Umsetzung der vielschichtigen Ansätze zur multikriteriellen Entscheidungsunterstützung erfordert die Anwendung geeigneter Software-Instrumente, um einen effektiven und transparenten Entscheidungsprozess zu gewährleisten. Dabei stehen unterschiedliche Software-Pakete zur Verfügung, die eine oder mehrere der zuvor dargestellten Methoden umsetzen. Einen Überblick über Software-Pakete zur Umsetzung multikriterieller Methoden stellen Weistroffer et al. (2005) sowie Belton und Stewart (2002) dar. Im Folgenden werden diejenigen Software-Systeme zusammengefasst, die für Problemstelllungen im Energiebereich innerhalb des letzten Jahrzehnts angewendet wurden. Eine Betrachtung älterer Anwendungen scheint vor dem Hintergrund der schnell fortschreitenden Entwicklungen bei Software-Systemen nicht sinnvoll. Die Anwendbarkeit der bekannten Software-Systeme für die vorliegende Problemstellung ist anschließend unter Berücksichtigung der identifizierten Schwachstellen bekannter Methoden zu klären.

Zur Umsetzung einer einfachen additiven Nutzwerfunktion reicht für gewöhnlich eine Kalkulationstabelle, zum Beispiel basierend auf MS Excel, aus (so zum Beispiel bei Lhendup 2008; Shackley und McLachlan 2006). Roth et al. (2009) setzen zur Anwendung der einfachen additiven Nutzwertanalyse ein im Rahmen des Projektes NEEDS12 entwickeltes Tool ein. Weitere Software-Pakete, die zum Einsatz der additiven Nutzwertanalyse im Energiebereich angewendet werden, schließen DEFINITE (van Alphen et al. 2007) und SOYUZ (Voropai und Ivanova 2002) ein. Basson und Petrie (2007) entwickeln ein Modell auf Basis von Analytica und Statistica, um eine MAVT-Analyse in Verbindung mit einer Hauptkomponenten-Analyse (PCA - Principal Component Analysis) umzusetzen. DecidelT dient der Anwendung einer MAUT-Methode und wurde im Energiebereich von Buchholz et al. (2009) getestet. Um eine additive Nutzwertfunktion (MAVT) mit Gewichtungen in Form von Intervallen umzusetzen, wenden Afgan et al. (2000; 2007a), Begic und Afgan (2007), Liposcak et al. (2006) sowie Pilavachi et al. (2006) das System ASPID (Analysis and Synthesis of Parameters under Information Deficiency) an. Alanne et al. (2007) setzt die Software WinPRE ein, um Optionen zur Energiebereitstellung auf Grundlage der Methode PAIRS unter Verwendung von Daten-Intervallen zu bewerten. Burton und Hubacek (2007) nutzen die Software MACBETH, um die gleichnamige Methode anzuwenden.

12 Vgl. http://www.needs-project.org/ (letzter Zugriff: 16.02.2010) 
Die kommerzielle Software Expert Choice wurde speziell zur Anwendung des AHP entwickelt und für Problemstellungen in Zusammenhang mit der Energiebereitstellung beispielsweise von Kahraman et al. (2009) und Zangeneh et al. (2009) angewendet. Daim et al. (2009) nutzen ein Open Source Online-Tool, um den AHP für Problemstellungen im Energiebereich anzuwenden. Die kommerziell erhältliche Software WebHIPRE implementiert neben dem AHP weitere MAVT-Methoden (SAW, SWING, SMART, SMARTER) und unterstützt zudem Kombinationen dieser Methoden sowie Sensitivitätsanalysen und Gruppenentscheidungen. Diese Software nutzen im Energiebereich Phdungsilp (2010). Der ANP ist im Software-Paket Super Decisions implementiert, die im Energiebereich zum Beispiel von Erdogmus et al. (2006), Köne und Büke (2007), Önüt et al. (2008) und Ulutas (2005) eingesetzt wird.

Eine Methode basierend auf ELECTRE TRI zum Zwecke der Kategorisierung von Alternativen zur Energiebereitstellung ist im System IRIS (Interactive Robustness Analysis and Parameters Interference for Multicriteria Sorting Problems) implementiert (Madlener et al. 2009). Papadopoulos und Karagiannidis (2008) implementieren ELECTRE III in MS Excel. Decision Lab ist eine kommerziell erhältliche Software, die der Implementierung von PROMETHEE dient und im Energiebereich zum Beispiel bei Buchholz et al. (2009), Ghafghazi et al. (2009), Kowalski et al. (2009), Madlener et al. (2007) und Madlener und Stagl (2005) eingesetzt wird. Im Rahmen des EU-Projektes MCDA-RES13 wird PROMETHEE auf einer Plattform zur Entscheidungsunterstützung für Erneuerbare-Energien-Projekte in Europa implementiert (Polatidis und Haralambopoulos 2007). Hyde et al. (2003) implementieren PROMETHEE in MS Excel in Verbindung mit dem Add-in @Risk, um Wahrscheinlichkeitsverteilungen der KriterienAusprägungen und -Gewichtungen berücksichtigen zu können, und wenden dieses System auf ein Fallbeispiel im Energiebereich an. Goletsis et al. (2003) nennen das von innen entwickelte EUS zur Implementierung einer aus ELECTRE und PROMETHEE kombinierten Outranking-Methode MURAME (Multicriteria Ranking Method). Die Outranking-Methode NAIADE ist in einer Software gleichen Namens implementiert, die im Energiebereich zum Beispiel von Buchholz et al. (2009) sowie Cavallaro und Ciraolo (2005) eingesetzt wird.

\subsection{Schlussfolgerungen aus der Literaturanalyse}

Die Literaturanalyse zeigt, dass multikriterielle Methoden seit den 1970er Jahren in einer wachsenden Anzahl an Problemstellungen in Zusammenhang mit der Energiebereitstellung eingesetzt werden. Insbesondere die zunehmende Wahrnehmung von

13 Vgl. http://www.exergia.net/mcda/MCDA-toolkit.html (letzter Zugriff: 16.02.2010) 
Umweltproblemen führt dazu, dass eine monokriterielle Optimierung, die ausschließlich auf ökonomische Bewertungsgrößen abzielt (zum Beispiel Minimierung der Kosten, der Investitionen oder Maximierung des Kapitalwertes), nicht mehr als ausreichend angesehen wird. Die analysierten Veröffentlichungen zeigen Wege auf, wie Umweltprobleme neben ökonomischen Zielgrößen und anderen Aspekten bei der Planung der Energiebereitstellung berücksichtigt werden können. Dabei steht aufgrund der weiten Verbreitung multikriterieller Ansätze im Energiebereich und auch in anderen Fachgebieten eine etablierte methodische Basis zur Verfügung, die in den vorangegangen Abschnitten systematisiert wurde und eine solide Grundlage für ein multikriterielles EUS bietet. Die direkte Auswahl und Übertragung einer bzw. mehrerer der bereits implementierten und verwendeten Methoden auf die vorliegende Problemstellung ist jedoch aus folgenden Gründen nicht ausreichend:

- Die meisten der beschriebenen Anwendungen sind auf spezifische Problemstellungen, beispielsweise eine bestimmte Region, zugeschnitten, so dass die Methoden und Ergebnisse nicht ohne weiteres auf ähnliche Problemstellungen übertragbar sind.

- Häufig sind die Ergebnisse nicht reproduzierbar, da spezifische Eingangsgrößen zugrunde liegen, die nicht immer verfügbar sind bzw. allgemeingültig angewendet werden können.

- Einige Zielgrößen, die bei einer nachhaltigkeitsorientierten Planung der Energieversorgung beachtet werden sollten, finden in bisherigen Anwendungen nur in eingeschränktem Maße Berücksichtigung, beispielsweise werden Aspekte der Versorgungssicherheit und der langfristigen Brennstoff-Verfügbarkeit in den vorliegenden Veröffentlichungen nur vereinzelt berücksichtigt.

- Nur in wenigen der betrachteten Veröffentlichungen zur multikriteriellen Bewertung von Energietechnologien wird die Entwicklung zukünftiger Rahmenbedingungen (zum Beispiel Entwicklung der Energieträgerpreise), die einen erheblichen Einfluss auf das (zukünftige) Abschneiden einer Alternative ausüben können, systematisch betrachtet.

- In den bekannten Anwendungen wird der Entwicklungsstand und zukünftige Entwicklungspotenziale der zu bewertenden Technologien nicht oder nur unzureichend in systematischer Weise betrachtet. Dies scheint jedoch insbesondere vor dem Hintergrund langfristiger Planungshorizonte im Energiebereich wünschenswert.

Insbesondere die unzureichende Berücksichtigung des technologischen Wandels wurde bereits von anderen Autoren beobachtet (zum Beispiel Greening und Bernow 2004). So heben auch Polatidis et al. (2006) hervor, dass Aspekte bezüglich möglicher zukünftiger Entwicklungen, die sowohl die Alternativen als auch die Kriterien und deren Bedeutung betreffen, in bisherigen Forschungsarbeiten in der Regel keine Beachtung finden und in keinem EUS implementiert wurden. Andererseits weisen Madlener und 
Stagl (2001) im Kontext der Analyse der unterschiedlichen Förderinstrumente für erneuerbare Energien darauf hin, „dass sich erneuerbare Energietechnologien in der Regel in unterschiedlichen Stadien der technologischen Entwicklung bzw. Marktfähigkeit befinden. Deshalb ist ein Wettbewerbssystem, das auf diesen Umstand keine Rücksicht nimmt, nicht besonders sinnvoll, weil es zu einer Diskriminierung der weniger entwickelten (und daher in der Regel weniger konkurrenzfähigen) Technologien kommen würde“. Folglich scheint eine adäquate Berücksichtigung des technologischen Wandels für ein multikriterielles EUS durchaus wünschenswert. Zwar werden in einigen bekannten Anwendungsfällen Kriterien wie "Technologische Reife" oder "Marktreife" berücksichtigt (so zum Beispiel Beccali et al. 2003; Cavallaro 2009; Doukas et al. 2007; Kaminaris et al. 2006; Terrados et al. 2009; Tsoutsos et al. 2009). Diese dienen jedoch eher als Proxy-Kriterium für die Zuverlässigkeit einer Technologie und weniger der Abbildung des Entwicklungsstandes oder zukünftiger Entwicklungspotenziale. Andere Autoren berücksichtigen zumindest zu einem gewissen Grad mögliche Entwicklungspotenziale hinsichtlich technischer Daten und zukünftiger Kostensenkungen (vgl. zum Beispiel Roth et al. 2009). Wie die folgenden Ausführungen zeigen sollen, scheint dies jedoch nicht ausreichend für eine Berücksichtigung des dynamischen Wandels, dem Energietechnologien unterliegen, im Rahmen der multikriteriellen Entscheidungsunterstützung.

Daher liegt der Fokus der vorliegenden Arbeit auf der Entwicklung von Methoden, die eine Einbeziehung des technischen Fortschritts im Rahmen der multikriteriellen Bewertung von Optionen zur Energiebereitstellung ermöglichen. Das Ziel besteht darin, ein multikriterielles EUS zu entwickeln, welches es ermöglicht, Technologien zur Energiebereitstellung unter Berücksichtigung des aktuellen Entwicklungsstandes und zukünftiger Entwicklungspotenziale systematisch zu analysieren. Dazu werden zunächst Methoden aufgezeigt, die für eine systematische Betrachtung des dynamischen technologischen Wandels geeignet scheinen. Diese werden im Hinblick auf die Eignung zur Integration in ein multikriterielles EUS geprüft. Anschließend wird ein EUS konzipiert, das neben der Berücksichtigung des technologischen Wandels weitere Schwachpunkte bekannter Anwendungen beheben soll, wie etwa die Berücksichtigung zusätzlicher Zielgrößen sowie sich ändernder Rahmenbedingungen in Form von Szenarien. 


\section{$5 \quad$ Abbildung des technologischen Wandels}

Technologien unterliegen im Allgemeinen dynamischen und komplexen Veränderungen im Zeitverlauf. Dies liegt begründet im permanenten Wandel der Rahmenbedingungen, in sich änderndem Konsumentenverhalten sowie im technischen Fortschritt und den hieraus resultierenden Verbesserungen hinsichtlich der Produkt-, Verfahrensund Fertigungstechnologien (Hoffmann 1972; Pfeiffer und Bischof 1981; Siegwart und Senti 1995; Utterback und Abernathy 1975). Folglich stehen für einen bestimmten Anwendungszweck und zu einem bestimmten Zeitpunkt in der Regel mehrere Technologien zur Auswahl, die sich in unterschiedlichen Entwicklungsstadien befinden. Dabei ist die Anwendung von innovativen Technologien (frühes Entwicklungsstadium) im Vergleich zu etablierten Alternativen (hoher Reifegrad) meist mit höheren Unsicherheiten und Risiken sowie höheren Kosten verbunden. Dies spricht zunächst für eine Entscheidung zugunsten der etablierten Technologie. Andererseits sind Bemühungen, neue Technologien zu erforschen, zu entwickeln, zu erproben und letztlich in den Markt einzuführen, gerade dadurch zu begründen, dass diese zumindest zukünftig, wenn ausstehende Forschungsfragen geklärt und die Anwendung erprobt ist, verbesserte Eigenschaften wie etwa ein höheres Leistungsniveau oder geringere Umweltauswirkungen aufweisen. Bei Energietechnologien können sich die erwarteten Vorteile beispielsweise auf einen höheren Wirkungsgrad oder geringere $\mathrm{CO}_{2}$-Emissionen im Betrieb beziehen. So erfahren Technologien auf Basis erneuerbarer Energieträger zunehmendes Interesse, obwohl diese relativ hohe Kosten aufweisen, da die Umweltauswirkungen in der Regel wesentlich geringer sind als bei der Stromerzeugung bzw. Wärmebereitstellung mit etablierten Kraftwerks- und Heizungstechnologien auf Basis fossiler Energieträger.

Insbesondere im Kontext der Priorisierung von Forschungsthemen und Entwicklungsaufgaben fällt somit die Entscheidung nicht zwangsläufig zugunsten besser bekannter und etablierter Technologien aus. Wenn etwa Steigerungen im Leistungsniveau der innovativen Technologie erwartet werden, die über das durch die etablierte Technologie erreichbare Niveau hinausgehen, kann die Entscheidung auch auf die zum Zeitpunkt der Entscheidung als „schlechter" eingestufte, innovative Technologie fallen (Perl 2007). Aus Sicht eines Entscheidungsträgers, der zwischen unterschiedlichen Technologien mit variierendem Entwicklungsstand wählen muss, bedeutet dies letztlich, dass bei der Bewertung von innovativen Technologien in einem frühen Entwicklungsstadium andere Maßstäbe angesetzt werden sollten als bei einer etablierten Technologie, die bereits einen hohen Reifegrad erreicht hat. Anders ausgedrückt ist es plausibel, je nach Entwicklungsstand einer Technologie den Bewertungsgrößen unterschiedliche Bedeutung beizumessen. Im Sinne einer multikriteriellen Entscheidungsunterstützung heißt dies, dass die Kriterien-Gewichtungen je nach aktuellem Entwicklungsstand der 
zu bewertenden Alternative anders ausfallen können. In der vorliegenden Arbeit soll daher eine Methode entwickelt und implementiert werden, die in dieser Weise eine Berücksichtigung des technologischen Wandels erlaubt.

Zwar weisen auch Polatidis et al. (2003) und Polatidis et al. (2006) auf die zeitliche Variabilität von Kriterien-Gewichtungen im Rahmen der multikriteriellen Entscheidungsunterstützung für die Energiesystemplanung hin. Jedoch schlagen Polatidis et al. (2003) ausgehend von der Theorie des Transformationsmanagements („transition management") vor, die Gewichtungen in Abhängigkeit der aktuellen Transformationsphase des Energiesystems festzulegen. Dieser Ansatz unterscheidet sich von der hier vorgeschlagenen Vorgehensweise, da nicht auf den individuellen Entwicklungsstand einzelner Technologien Bezug genommen wird. Vielmehr hängt die Gewichtung vom Transformationsstand des Energiesystems zum Zeitpunkt der Bewertung ab und gilt zu diesem Zeitpunkt gleichermaßen für alle Alternativen. Wird zu einem späteren Zeitpunkt (zum Beispiel 20 Jahre später) erneut eine vergleichende Bewertung von Energietechnologien durchgeführt, sollte entsprechend der von Polatidis et al. (2003) vorgeschlagenen Methodik die Gewichtung dem fortgeschrittenen Transformationsstand des Energiesystems angepasst werden. Wie die oben geführte Argumentation nahe legt, soll hier jedoch ein Ansatz verwendet werden, mit dessen Hilfe die KriterienGewichtungen dem Entwicklungsstand individueller Energietechnologien angepasst werden kann. Um diese Idee zu implementieren, wird ein Konzept benötigt, welches es erlaubt, Energietechnologien entsprechend ihres Entwicklungsstandes voneinander abzugrenzen. Dabei bieten sich bekannte Lebenszykluskonzepte an, die den Verlauf einer Produkt- oder Technologieentwicklung über die Zeit darstellen. 14

\subsection{Das Konzept des Technologiezyklus}

Einen Überblick über Modelle zur Abbildung der Entwicklung von Technologien vermittelt Zotter (2007). Das klassische Konzept des Produkt- bzw. Technologielebenszyklus stellt ein Instrument der Unternehmensplanung dar und wird sowohl zur Erklärung wie auch zur Prognose der Absatzentwicklung eingesetzt. Traditionell wird es verwendet, um Marketing-Strategien ausgehend von der Zuordnung eines Produktes zu dessen Position im Lebenszyklus zu entwickeln. Als ein Instrument im Rahmen der strategi-

14 Der Begriff des Lebenszyklus wird in der Literatur zweideutig verwendet. Im hier verwendeten Kontext ist unter dem Lebenszyklus eines Produktes bzw. einer Technologie dessen Marktdurchdringung von der Markteinführung bis zur Marktsättigung zu verstehen, die z.B. über die im Zeitverlauf verkaufte Menge des entsprechenden Produktes abgebildet werden kann. Andererseits wird im Rahmen ökobilanzieller Bewertungsmethoden unter dem Lebenszyklus die Gesamtheit der Phasen verstanden, die ein Produkt „von der Wiege bis zur Bahre" durchläuft, einschließlich Produktion, Verteilung, Nutzung und Entsorgung. 
schen Unternehmensplanung kann es die Aufbereitung von Informationen zur Gestaltung eines optimalen Produktionsprogrammes unterstützen. Die aktuellen und potenziellen Erfolgschancen einer Technologie können auf Basis des LebenszyklusKonzeptes beurteilt werden. Daraus lassen sich wiederum Schlüsse hinsichtlich notwendiger Forschungs- und Entwicklungsaktivitäten ziehen (Hoffmann 1972). Zahlreiche sich ähnelnde Darstellungen des klassischen Lebenszyklus von Produkten sind in der Literatur zu finden (vgl. zum Beispiel Hoffmann 1972; Pfeiffer und Bischof 1981; Siegwart und Senti 1995). In Anlehnung an die Ergebnisse der Diffusionsforschung von Rogers (vgl. Rogers 2003) wird in der Regel der Umsatz über die Zeit als Normalverteilung bzw. der kumulierte Umsatz als S-Kurve dargestellt (Hoffmann 1972; Siegwart und Senti 1995). Häufig wird die erste Phase als Markteinführung bezeichnet, gefolgt von Reife- und Wachstumsphase, die schließlich in die Sättigungsphase übergeht, an die sich in einigen Darstellungen als fünfte Phase die Degeneration oder Rückbildung anschließt. Dabei können je nach Produkt sowohl der Lebenszyklus als auch die einzelnen Phasen von unterschiedlicher Dauer sein (Götze und Rudolph 1994; Pfeiffer und Bischof 1981; Siegwart und Senti 1995). Die folgende Darstellung der idealtypischen Lebenszykluskurve einer Technologie erfolgt in Anlehnung an Weule (2002) und Perl (2007) (vgl. Abbildung 5.1).

Abbildung 5.1: Idealtypischer Lebenszyklus einer Technologie

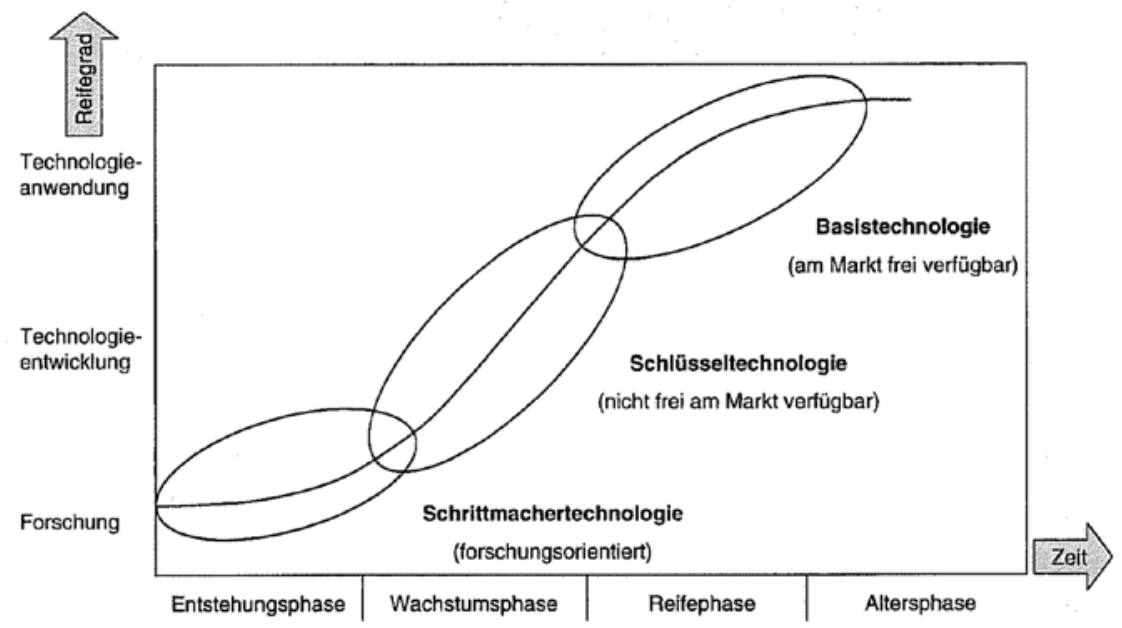

Quelle: Weule (2002); eine ähnliche Darstellung findet sich auch bei Zotter (2007).

Nach Weule (2002) beginnt der Lebenszyklus einer Technologie mit der Entstehungsphase, in der mögliche praktische Anwendungen allenfalls als Visionen vorliegen. Dabei sprechen andere Autoren auch von Zukunftstechnologien oder neuen Technologien, die aus Visionen über mögliche Zusammenhänge hervorgehen (vgl. Perl 2007). Die Potenziale sind in dieser Phase mit sehr hohen Unsicherheiten behaftet. Bei ausreichend hohen Erwartungen über zukünftig realisierbare Potenziale und Wettbe- 
werbsvorteile investieren Unternehmen und Forschungseinrichtungen in Weiterentwicklungen. Gegebenenfalls wird die Wachstumsphase erreicht, in der eine zunehmende Anzahl von Anwendungsmöglichkeiten realisiert wird. Dabei haben Zukunftstechnologien grundsätzlich das Potenzial, zu Schrittmachertechnologien zu werden, wobei weiterhin Unsicherheiten hinsichtlich technischer Leistungsfähigkeit, Realisierbarkeit und Wettbewerbsfähigkeit bestehen. Im Laufe der Wachstumsphase nehmen das verbleibende unerschlossene Marktpotenzial und der Zusatznutzen weiterer technischer Fortschritte ab. Von einer Schlüsseltechnologie ist die Rede, wenn bedeutende Wettbewerbsvorteile und Markterfolge mit der Technologie erzielt werden. Nach dem Übergang in die Reifephase sind Verbesserungen nur noch in geringerem Maße möglich. Charakteristisch für eine Basistechnologie ist hierbei, dass diese als allgemein gültiger Standard angesehen werden und eine Wettbewerbsdifferenzierung zwischen Anbietern der Technologie kaum noch möglich ist. Mit den weiter abnehmenden Verbesserungspotenzialen nähert sich die Technologie der Altersphase, die in der Regel durch eine fortschreitende Substitution der alten Technologie durch neuere Alternativen charakterisiert ist.

Abbildung 5.2: $\quad$ Anteil der Rotordurchmessergruppen von Windkraftanlagen an der jährlich in Deutschland neu installierten Leistung

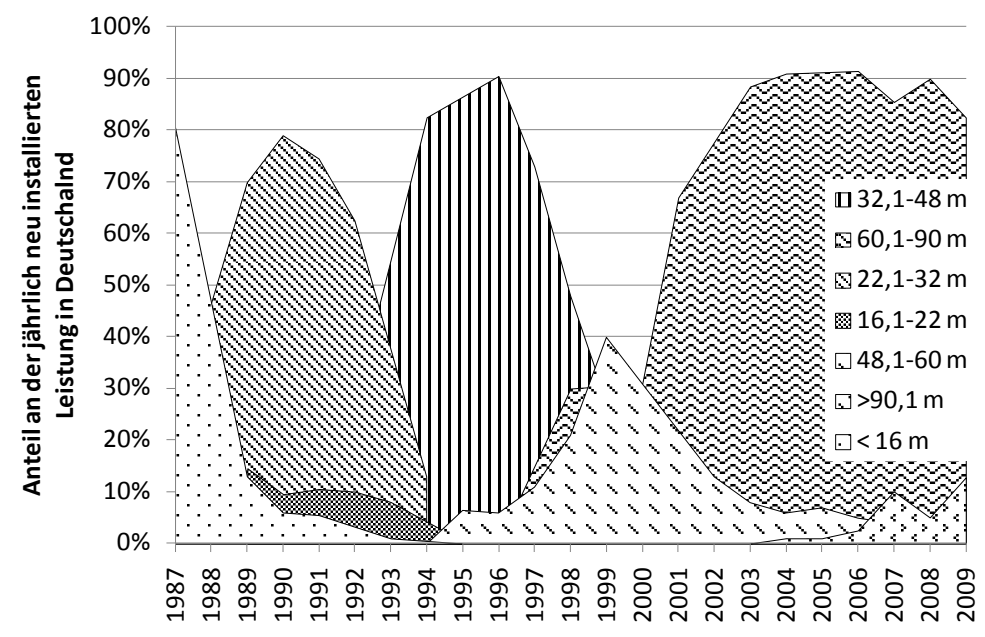

Quelle: Ender (2010).

Derartige Substitutionseffekte können am Beispiel der Weiterentwicklung von Windenergieanlagen aufgezeigt werden (Abbildung 5.2). Während Ende der 1980er Jahre noch der überwiegende Anteil der neu installierten Windenergieanlagen in Deutschland einen Rotordurchmesser von weniger als $32 \mathrm{~m}$ aufwies (Anlagen im Kilowatt-Bereich), wurden im Laufe der folgenden Jahre in zunehmendem Maße größere Anlagen realisiert. Folglich werden kleinere Anlagen durch größere Anlagen mit einem höheren Leistungsniveau ersetzt, sobald der technologische Fortschritt dies zulässt. In den letz- 
ten Jahren wurden in erster Linie Anlagen mit einem Rotordurchmesser von 60 bis $90 \mathrm{~m}$ neu installiert, was einer installierten Anlagenleistung von bis zu $3 \mathrm{MW}$ entspricht (vgl. auch BWE 2007). Zudem ist eine steigende Tendenz für die Rotorengruppe mit einem Durchmesser von mehr als 90 m erkennbar, was nach BWE (2007) einer installierten Leistung von 2 bis 5 MW entspricht.

Das dargestellte Modell des Lebenszyklus wird in der Literatur aus unterschiedlichen Gründen kritisiert. So stellt die S-förmige Kurve einen idealtypischen Verlauf dar, der nicht immer nachgewiesen werden kann und folglich nicht unbedingt zur Prognose zukünftiger Entwicklungen geeignet ist (Götze und Rudolph 1994; für eine ausführliche Diskussion siehe Hoffmann 1972). Dennoch kann zumindest annähernd bestimmt werden, in welcher Phase sich ein Produkt gegenwärtig befindet. Im Kontext der vorliegenden Anwendung ist das Konzept somit grundsätzlich geeignet, um unterschiedliche Phasen der Entwicklung von Energietechnologien voneinander abzugrenzen, ohne den Anspruch auf eine exakte Prognose der zukünftigen Entwicklung zu erheben. Weiterhin wird kritisiert, dass in den meisten Modellen des Lebenszyklus die Entstehungsphase von Produkten bzw. Technologien nur unzureichend abgebildet ist. Denn der Schwerpunkt der meisten Modelle liegt auf der Abbildung des Marktzyklus, das heißt auf dem Zeitraum, in dem sowohl ein kommerzielles Angebot als auch eine Nachfrage nach dem Produkt besteht (Hoffmann 1972; Pfeiffer und Bischof 1981). Daher schlagen mehrere Autoren Erweiterungen des klassischen Lebenszyklus vor, die in einem integrierten Konzept neben dem Marktzyklus auch dem vorgelagerten Entstehungszyklus stärker Beachtung schenken (zum Beispiel Denkena et al. 2002; Götze und Rudolph 1994; Pfeiffer und Bischof 1981; Zotter 2007). Das von Meyer-Krahmer und Dreher (2004) vorgeschlagene Modell eines modernen Technikzyklus fokussiert auf die Entstehungsphase von Technologien und integriert Theorien aus unterschiedlichen Fachgebieten. Dazu zählen erfahrungsbasierte Ansätze zur Technikentwicklung (wie zum Beispiel der oben dargestellte Ansatz von Weule (2002), Konzepte des hauptsächlich angelsächsisch geprägten Technologie- und Innovationsmanagement (zum Beispiel Tidd und Bessant 2009; Utterback und Abernathy 1975) sowie die Theorie der Innovationsökonomik, die sich beispielsweise mit der Messbarmachung des technischen Wandels beschäftigt (vgl. Grupp 1997). Das Modell von Meyer-Krahmer und Dreher (2004) (vgl. Abbildung 5.3) konzentriert sich auf die F\&E-Phase vor der Markteinführung und bietet somit eine Möglichkeit die Entstehungsphase differenziert abzubilden.

Nach Meyer-Krahmer und Dreher (2004) folgt auf die Entdeckung neuer technischer Prinzipien (Phase 1) eine Phase der Euphorie (Phase 2), in der eine breiter werdende Gemeinschaft von Wissenschaftlern die damit verbundenen Möglichkeiten erforscht. Mit fortschreitendem Wissensstand stellen sich in der Regel zumindest einige dieser Möglichkeiten als nicht realisierbar heraus, so dass es zu einer Phase der Ernüchte- 
rung kommt, wobei Forschungsaktivitäten eingeschränkt oder eingestellt werden (Phase 3). Zu einer Neuorientierung der Entwicklungen (Phase 4) tragen nur diejenigen Akteure bei, die den längsten Atem besitzen oder radikal neue Ansätze vertreten. Dabei kommt es zu dominanten Designs, die in der Phase des Aufstiegs (Phase 5), in der industrielle Durchbrüche erzielt werden, den Umgang mit der neuen Technik am Markt prägen. Durch eine zunehmende Verbreitung der Technologie kommt es zu Skaleneffekten, so dass in der Diffusionsphase (Phase 6) in zunehmendem Maße Anwendungsmöglichkeiten erschlossen werden können.

Abbildung 5.3: $\quad$ Schematische Darstellung eines modernen Technikzyklus-Modells

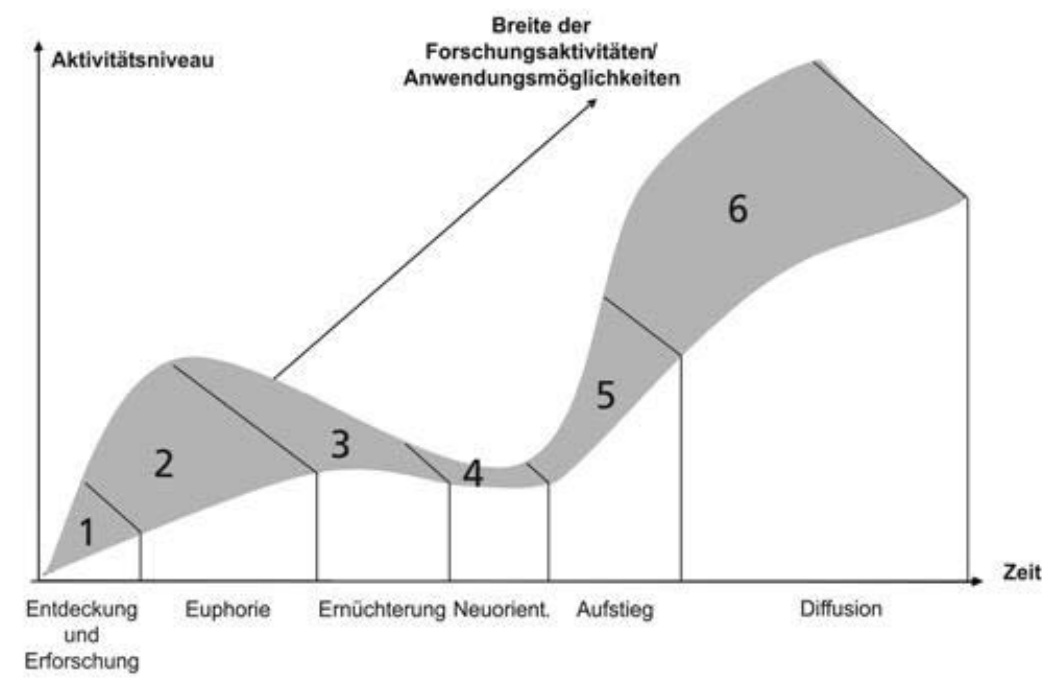

Quelle: Meyer-Krahmer und Dreher (2004).

Dieses Technologiezyklus-Modell wird von Jochem et al. (2009) im Hinblick auf die Prioritätensetzung in der Forschungsförderung im Energiebereich in Verbindung mit anderen Methoden angewendet, um dem technologischen Wandel von Energietechnologien Rechnung zu tragen. Eine direkte Verknüpfung mit einer multikriteriellen Bewertung erfolgt nicht. Vor dem Hintergrund der vorangegangenen Diskussion bietet dies jedoch eine vielversprechende Möglichkeit zur Berücksichtigung des technologischen Wandels von Energietechnologien bei der multikriteriellen Entscheidungsunterstützung. Daher dient das von Jochem et al. (2009) angewendete Technologiezyklus-Konzept in dieser Arbeit als Ausgangspunkt zur Integration des technologischen Wandels in ein multikriterielles EUS. Das Konzept wird um eine detailliertere Abbildung der Diffusionsphase ergänzt, die auf dem klassischen Modell des Lebenszyklus beruht (vgl. Abbildung 5.4). Dadurch wird im Hinblick auf das zu entwickelnde EUS die Möglichkeit geboten, sowohl Energietechnologien zu differenzieren, die sich noch in einem Forschungs- bzw. Entwicklungsstadium befinden, als auch solche, die bereits kommerziell am Markt verfügbar sind. 
Abbildung 5.4: Integrierter F\&E- und Marktzyklus einer Technologie (schematische Darstellung)

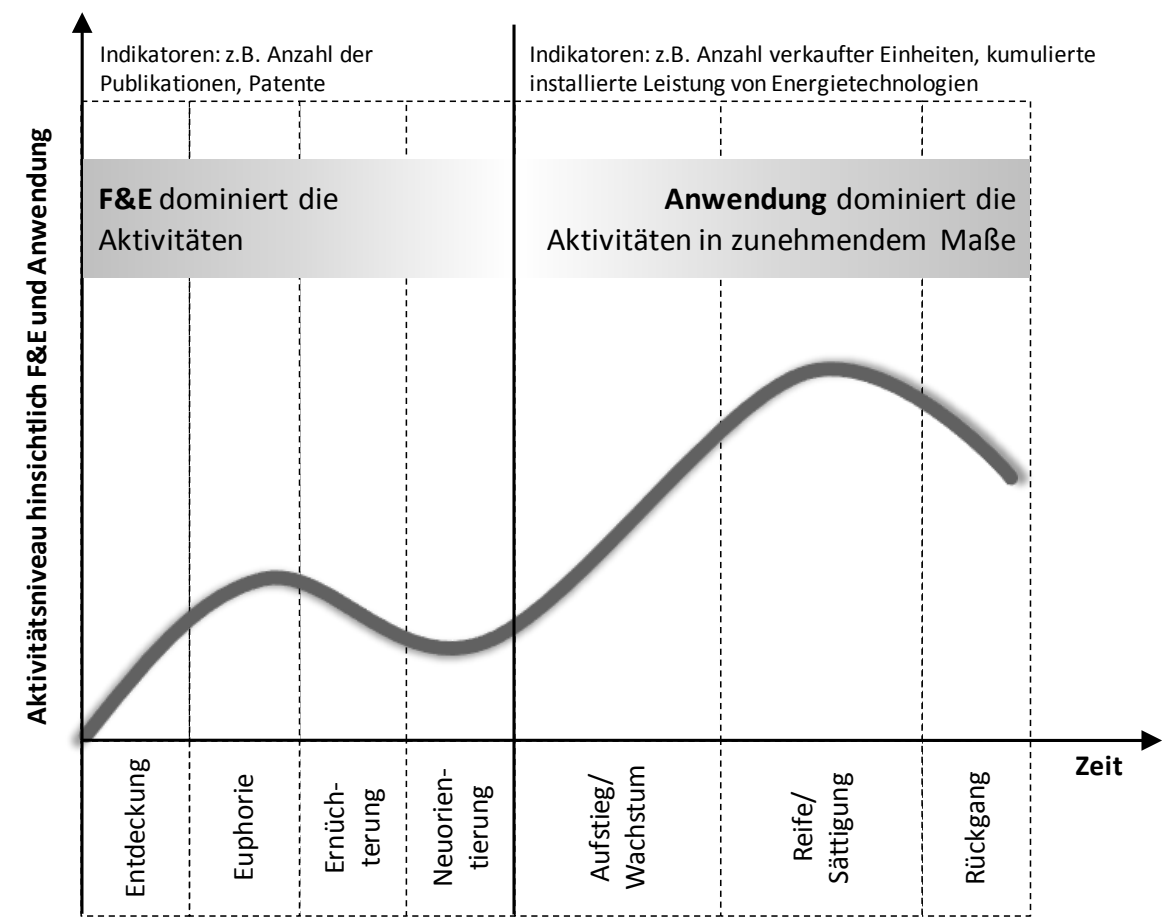

Quelle: Basiernd auf Grupp (1997); Meyer-Krahmer und Dreher (2004); Weule (2002).

Die Zuordnung von Energietechnologien zu den beschriebenen Phasen kann sich im Rahmen der multikriteriellen Entscheidungsunterstützung in unterschiedlicher Weise auf die Kriterien-Gewichtungen auswirken. Zum Beispiel kann den Kosten einer Energietechnologie in der Reifephase eine höhere Bedeutung zugeordnet werden als einer Energietechnologie in der Einführungsphase, in der zu erwartende Skaleneffekte noch nicht realisiert werden können. In ähnlicher Weise wird auch bei Anwendung des klassischen Produktlebenszykluskonzeptes im Marketing argumentiert, dass in der Sättigungsphase der Preispolitik als Wettbewerbsinstrument die höchste Bedeutung zukommen sollte, da Möglichkeiten der Marktausweitung, beispielsweise durch Qualitätsmaßnahmen, nur noch in begrenztem Maße möglich sind (Hoffmann 1972). In der Wachstumsphase sind hingegen Qualitätsmaßnahmen besser geeignet, um sich von der Konkurrenz abzuheben und Marktanteile zu gewinnen. Für Energietechnologien könnte hier analog argumentiert werden, dass Qualitätsmerkmalen, beispielsweise Kriterien zur Messung der Umweltauswirkungen, in früheren Phasen eine höhere Bedeutung beigemessen werden sollte. Dabei bietet das vorgestellte TechnologiezyklusKonzept die Grundlage zur differenzierten Abgrenzung von Entwicklungsstufen und damit zur Integration des dynamischen Wandels von Energietechnologien in ein EUS. Durch die Unterscheidung in sieben Phasen werden sowohl die Verbreitung am Markt (Marktzyklus) als auch vorgelagerte Entstehungsphasen der Technologien berücksich- 
tigt. Letztlich kann es im Rahmen der multikriteriellen Entscheidungsunterstützung den Entscheidungsträgern selbst überlassen bleiben, ob und inwieweit die KriterienGewichtungen in Abhängigkeit der Entwicklungsphasen determiniert werden. Bevor die Konzeption eines entsprechenden EUS in Kapitel 6 vorgestellt wird, wird im nächsten Abschnitt zunächst die Zuordnung von Energietechnologien zu den Entwicklungsphasen entsprechend Abbildung 5.4 überprüft.

\subsection{Entwicklung von Energietechnologien im Zeitverlauf}

Die Berücksichtigung von Gewichtungen in Abhängigkeit unterschiedlicher Entwicklungsphasen von Energietechnologien erfordert zunächst eine Zuordnung der Technologien zu den Phasen. Je nachdem, ob sich eine Technologie noch in einem frühen Stadium vor der breiten Markteinführung befindet oder bereits kommerziell am Markt verfügbar ist, dienen unterschiedliche Indikatoren zur Verortung im Technologiezyklus. Daher wird zur Darstellung der Entwicklung von Energietechnologien im Zeitverlauf und Zuordnung zu einer der Phasen in Abbildung 5.4 zunächst die kumulierte installierte Leistung von Energietechnologien zur Bereitstellung von Strom und Wärme dargestellt. Diese Messgröße erlaubt eine Einschätzung der Absatzentwicklung und technologischen Reife von Energietechnologien, die bereits am Markt verfügbar sind. Für die Zuordnung zu früheren Stadien im F\&E-Zyklus ist die installierte Kapazität als Messgröße jedoch nicht geeignet. Bei Jochem et al. (2009) werden im Rahmen von Fallbeispielen Energietechnologien zu den Phasen nach Meyer-Krahmer und Dreher (2004) durch Indikatoren-basierte Literatur-, Patent- und Marktanalysen sowie Interviews mit relevanten Akteuren zugeordnet. Diese Vorgehensweise wird beispielhaft dargestellt. Eine Anwendung dieser Methodik, die soweit wie möglich alle in Entwicklung befindlichen Energietechnologien erfasst, ist jedoch im Rahmen dieser Arbeit nicht zu leisten.

\subsubsection{Installierte Kraftwerksleistung in Europa}

Zur Analyse der installierten Kraftwerkskapazitäten steht eine umfangreiche Datenbank zur Verfügung, in der seit Ende des 19. Jahrhunderts installierte Kraftwerke erfasst sind (Platts 2007b). Die Datenbank enthält unterschiedliche Merkmale einzelner Kraftwerkseinheiten, beispielsweise das Jahr der Installation, den vorwiegend verwendeten Energieträger sowie das Jahr der Stilllegung. Aus den in dieser Form zur Verfügung stehenden Daten kann die in einem bestimmten Jahr installierte Leistung berechnet und im Zeitverlauf dargestellt werden. Dabei können einerseits einzelne Energieträger, die auf Basis mehrere Technologien zur Stromerzeugung genutzt werden können, betrachtet werden. Andererseits können einzelne Technologien betrachtet werden, die sich zur Nutzung unterschiedlicher Energieträger eignen, wobei gegebenenfalls An- 
passungen entsprechend der spezifischen Brennstoffeigenschaften erforderlich sind. Daher wird bei der Untersuchung der Technologiezyklen von Optionen zur Energiebereitstellung zum einen nach dem verwendeten Energieträger („FUEL“ bzw. „FUELTYPE“) und zum anderen nach der Art der Nutzung („UTYPE“) unterschieden. Einen Überblick über die in Platts (2007b) vorkommenden Energieträger („FUEL“), weiter unterschieden in Energieträger-Typen („FUELTYPE“), und die zu deren Umwandlung erfassten Stromerzeugungstechnologien („UTYPE“) vermittelt Tabelle 10.1 (Anhang 10.1).

Prinzipiell erfasst die Platts-Datenbank Kraftwerke aller Arten und Größen, die von Energieversorgern, Privatunternehmen sowie industriellen und gewerblichen Eigenerzeugern betrieben werden (vgl. Platts 2007a). Jedoch sind stationäre Kleinanlagen wie zum Beispiel Photovoltaik-Dachanlagen in Wohngebäuden oder Mikro-Windturbinen in der Regel nicht erfasst. Weiterhin wird eingeräumt, dass die Erfassung kleiner, dezentraler Anlagen unter Umständen nicht den gesamten Markt abdeckt. Dies betrifft insbesondere erneuerbare Energien, die vorwiegend dezentral eingesetzt werden. Daher werden ergänzende Informationen zur Entwicklung erneuerbarer Energien innerhalb der letzten 20 Jahre aus Held et al. (2010) hinzugezogen. Die entsprechenden Daten zeigen, dass die Anteile einiger erneuerbarer Energieträger an der Stromerzeugung, insbesondere Wind, Solarstrahlung und Biomasse, bei Platts (2007b) in der Regel unterschätzt werden.

\subsubsection{Technologiezyklen von Optionen zur Stromerzeugung unter- schieden nach dem Energieträger}

In den folgenden Abbildungen ist die Entwicklung der Verwendung unterschiedlicher Energieträger zur Stromerzeugung in Europa über die Zeit dargestellt. Die Darstellung beginnt dabei mit den gemessen an der kumulierten installierten Leistung am weitesten verbreiteten Energieträgern und wird fortgeführt bis zu den am wenigsten verbreiteten Energieträgern. Ergänzende Abbildungen zu den Energieträger-Typen finden sich im Anhang (vgl. Abschnitt 10.2). Bei den ältesten der in der Datenbank erfassten Kraftwerke handelt es sich um Wasserkraftanlagen (Abbildung 5.5). Die erste registrierte Wasserkraftanlage ging im Jahr 1882 in Betrieb. Seither ist die Nutzung der Wasserkraft gestiegen, wobei seit Ende des 19. Jahrhunderts bis heute ein deutlicher SKurvenverlauf erkennbar ist. Für die letzten Jahre deutet sich ein Maximum der Kurve bei etwa $188 \mathrm{GW}$ an. Dieser Verlauf wird im Wesentlichen durch konventionelle Wasserkraftwerke (Abbildung 10.1) und Pumpspeicher (Abbildung 10.2) bestimmt, die somit der Sättigungsphase zugeordnet werden. Wellen- und Gezeitenkraftwerke (Abbildung 10.4), von denen bisher nur eine geringe Anzahl installiert ist, sind hingegen einem frühen Entwicklungsstadium zuzuordnen. 
Abbildung 5.5: Nutzung von Kohle-; Erdgas-, Öl- und Kernkraftwerken sowie Wasser- und Windkraftanalagen zur Stromerzeugung in Europa

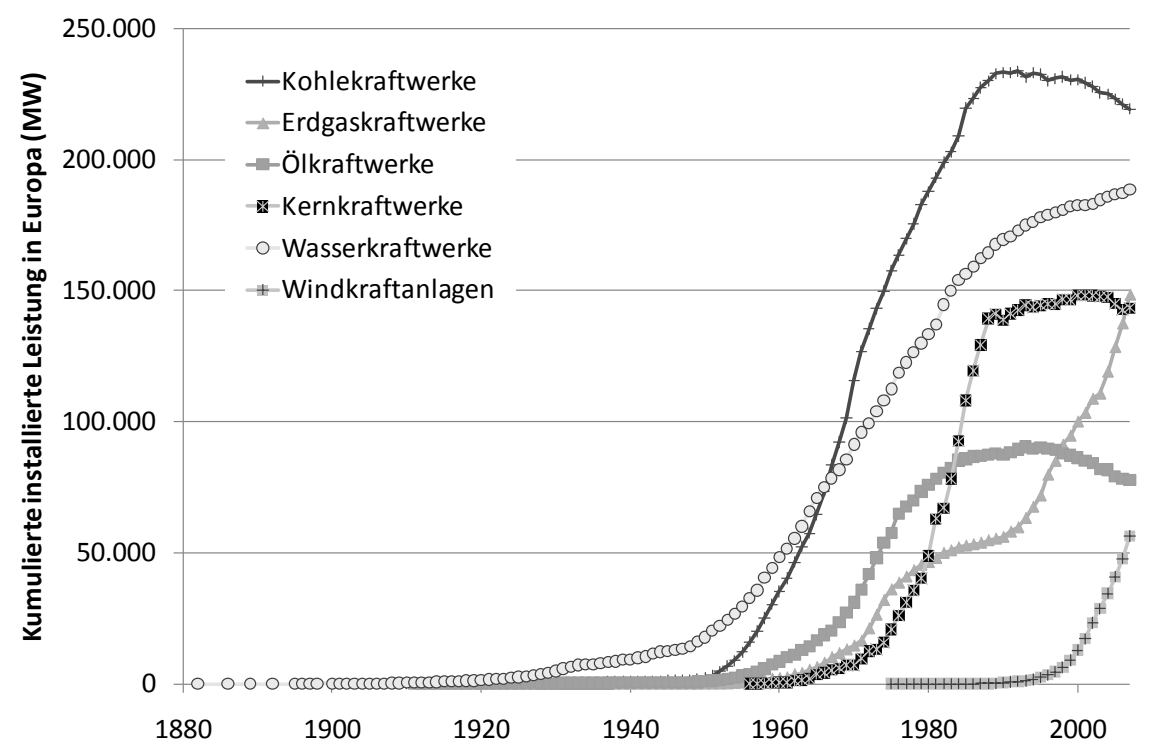

Quelle: Basierend auf Held et al. (2010); Platts (2007b).

Für Kohle-, Kern- und Ölkraftwerke zeigt Abbildung 5.5 einen ähnlichen Verlauf. Dabei deutet sich nach einem kontinuierlichen Anstieg der Kraftwerksleistung in den vorangegangenen Jahrzehnten seit Mitte bzw. Ende der 1990er Jahre ein Rückgang der installierten Leistung an. Die erste Inbetriebnahme eines Kohlekraftwerks in Europa ist im Jahr 1907 registriert. Nach einem steilen Anstieg zwischen 1960 und 1980 wird die bisher maximale installierte Leistung von ca. 234 GW im Jahr 1992 erreicht. Eine Analyse nach der Art der Kohle zeigt, dass sowohl die Kurve der Braun- als auch der Steinkohlekraftwerke parallel zu dieser Entwicklung verläuft, so dass beide Typen der Rückgangs-Phase zugeordnet werden (Abbildung 10.1, Anhang 10.2). 15 Kohlehaltige Reststoffe werden hingegen nur vereinzelt eingesetzt, so dass die entsprechenden Technologien als Nischentechnologie eingestuft werden (Abbildung 10.4, Anhang 10.2). Die ersten Ölkraftwerke gingen wenige Jahre nach den Kohlekraftwerken in Betrieb. Die installierte Leistung der Ölkraftwerke steigt jedoch weniger stark an und erreicht ein Maximum im Jahr 1993 mit ca. 90 GW. Bei einer Unterscheidung nach

15 An dieser Stelle sei darauf hingewiesen, dass bei einer weltweiten Betrachtung gegebenenfalls ein weiter steigender Trend für Kohlekraftwerke erkennbar wäre, da sich vor allem in China der Ausbau der Stromerzeugungskapazitäten vorwiegend auf Kohle stützt (vgl. IEA 2009c). Die Betrachtungen dieser Arbeit konzentrieren sich jedoch auf den Europäischen Markt. Des Weiteren könnte es zu einem Aufstieg von Kohlekraftwerkstechnologien mit $\mathrm{CO}_{2}$-Abscheidung und -Speicherung kommen, sofern die noch ausstehenden Forschungsfragen geklärt werden. Diese speziellen Kohlekraftwerkstechnologien werden jedoch separat betrachtet (vgl. Abschnitt 5.2.3.2). 
dem Brennstofftyp wird deutlich, dass Anlagen zur Stromerzeugung basierend auf Schwerölen eher der Rückgangs- und solche basierend auf Leichtölen eher der Reifephase zugeordnet werden können (Abbildung 10.1 und Abbildung 10.2, Anhang 10.2).

Kernkraftwerke gingen erstmals in den 1950er Jahren in Betrieb. Das Maximum der installierten Kernkraftwerksleistung liegt mit etwa $148 \mathrm{GW}$ zwischen dem maximalen Niveau der Kohle- und Ölkraftwerke und wird im Jahr 2000 erreicht. Somit werden Kernkraftwerke der Rückgangs-Phase zugeordnet. Diese Einordnung scheint insbesondere vor dem Hintergrund politischer Entscheidungen für alle unter „FUELTYPE“ aufgeführten Kernkraftwerkstypen gerechtfertigt (vgl. auch Abbildung 10.1 bis Abbildung 10.3, Anhang 10.2). Denn selbst wenn die Laufzeit der zurzeit installierten Anlagen verlängert wird, ist in Europa nur in einzelnen Ländern mit einem Zubau weiterer Kernkraftwerke zu rechnen.

Die Entwicklung der installierten Leistung von Gaskraftwerken weicht ab vom typischen S-Kurvenverlauf. Nach Inbetriebnahme der ersten Gaskraftwerke Ende der 1920er Jahre deutet sich Ende der 1980er Jahre eine Sättigungsphase an, jedoch sind seit Anfang der 1990er Jahre wieder stärkere Zuwachsraten zu beobachten. Wie die weitere Analyse zeigen wird, ist dies vor allem auf die zunehmende Nutzung von GuDKraftwerken zurückzuführen (vgl. auch Abbildung 5.11). Auch Druckluftspeicherkraftwerke stellen nach Platts (2007b) einen „FUELTYPE“ von Gaskraftwerken dar. Von diesen ist jedoch lediglich eine Anlage in Europa (Deutschland) installiert (vgl. auch Abschnitt 3.1.6.1 und Abbildung 10.4 im Anhang). Daher scheint es sich um eine Nischentechnologie bzw. um ein frühes Entwicklungsstadium zu handeln.

Weiterhin abgebildet in Abbildung 5.5 ist die installierte Leistung von Windkraftanlagen. Diese werden seit Mitte der 1970er Jahren in Europa zur Stromerzeugung genutzt und verzeichnen einen steilen Anstieg seit den 1990er Jahren bis auf über $56 \mathrm{GW}$ im Jahr 2007. Eine Sättigung des Marktes ist aufgrund der dargestellten Entwicklung nicht erkennbar. Folglich werden Windkraftanlagen der Wachstumsphase zugeordnet. Die bisherige Entwicklung wird dabei im Wesentlichen durch die Installation von horizontalen Windturbinen an Land bestimmt (vgl. auch Abbildung 5.12; Abbildung 10.2, Anhang 10.2). Bei vertikalen Windturbinen scheint es sich hingegen eher um eine Nischentechnologie zu handeln (Abbildung 10.5, Anhang 10.2). Offshore-Anlagen sind hingegen einem frühen Entwicklungsstadium bzw. der beginnenden Wachstumsphase zuzuordnen. Dies kann erklärt werden durch die bekannten Pläne zu deren Ausbau und aufgrund der in Platts (2007b) verzeichneten zunehmenden installierter Leistung dieser Anlagen in den letzten Jahren (vgl. auch Abbildung 5.13). 
Die Entwicklung der Nutzung weiterer Energieträger zur Stromerzeugung spielt sich, gemessen an der in Europa installierten Leistung, auf einem wesentlich niedrigeren Niveau ab als die bisher gezeigten. Dies kann einerseits durch eingeschränkte Verfügbarkeit von Rohstoffen erklärt werden. Andererseits kann ein frühes Entwicklungsstadium Grund für den geringen Anteil an der Stromerzeugung sein. Die Nutzung von Biomasse zur Stromerzeugung verzeichnet seit Ende der 1980er Jahre ein steiles Wachstum (Abbildung 5.6). Dieser Trend kann auch für die Biomasse-Typen („FUELTYPE“) Stroh und landwirtschaftliche Reststoffe bestätigt werden (Abbildung 10.4, Anhang 10.2). Bei den anderen Biomasse-Energieträgern16 scheint es sich aufgrund der geringen Anzahl bekannter Anlagen eher um Nischentechnologien zu handeln (Tabelle 10.1, Anhang 10.1, und Abbildung 10.5, Anhang 10.2). Daher werden Technologien zur Nutzung von Stroh und landwirtschaftlichen Reststoffen der Wachstumsphase zugeordnet und solche, in denen andere Biomassen eingesetzt werden, als Nischentechnologie eingestuft. Die Nutzung von Holzbrennstoffen und Siedlungsabfällen zur Stromerzeugung zeigt seit Ende der 1950er bzw. Anfang der 1960er Jahre tendenziell steigende Zuwachsraten. Ihr bisheriges Maximum erreicht die installierte Leistung von Anlagen zur Stromerzeugung aus Holzbrennstoffen im Jahr 2007 bei ca. 3,7 GW und die Stromerzeugung aus Siedlungsabfällen im Jahr 2006 bei ca. 5,8 GW. Unter den Siedlungsabfällen weisen insbesondere die Ersatzbrennstoffe eine steile Wachstumskurve innerhalb der letzten 20 bis 30 Jahre auf, die jedoch nur auf einer relativ geringen Anzahl installierter Anlagen basiert (vgl. Tabelle 10.1, Anhang 10.1, und Abbildung 10.4, Anhang 10.2). Die weiteren Energieträgertypen der Kategorie Siedlungsabfälle werden nur vereinzelt eingesetzt und somit als Nischentechnologien eingestuft. Aufgrund der in den letzten Jahren beobachteten tendenziell steigenden Zuwachsraten werden Technologien zur Nutzung von Holz- und Ersatzbrennstoffen der Wachstumsphase zugeordnet.

Die Inbetriebnahme der ersten Anlagen auf Basis von Solarstrahlung ist im Jahr 1982 registriert. Da sich die entsprechenden Technologien erst seit etwa 30 Jahren in Europa verbreiten und starke Zuwachsraten in den letzten Jahren aufweisen, werden diese der beginnenden Wachstumsphase zugeordnet. Bei Betrachtung der entsprechenden Energieträger-Typen wird deutlich, dass die Nutzung der Solarkraft bisher in erster Linie durch Photovoltaik-Anlagen erfolgt, die eine steile Wachstumskurve innerhalb der letzten 20 bis 30 Jahre aufweisen. Solarthermische Kraftwerke sind bisher nur vereinzelt installiert und werden daher anstelle der Wachstumsphase einem frühen Stadium zugeordnet (Abbildung 10.3, Anhang 10.2).

16 Reishülsen: vier Anlagen in Italien und eine in Spanien; Geflügelmist: vier Anlagen in Italien, drei in Großbritannien, eine in den Niederlanden. 
Abbildung 5.6: Nutzung von Hochofengas, Siedlungsabfällen, Holzbrennstoffen, LNG, Biomasse und Solarstrahlung zur Stromerzeugung

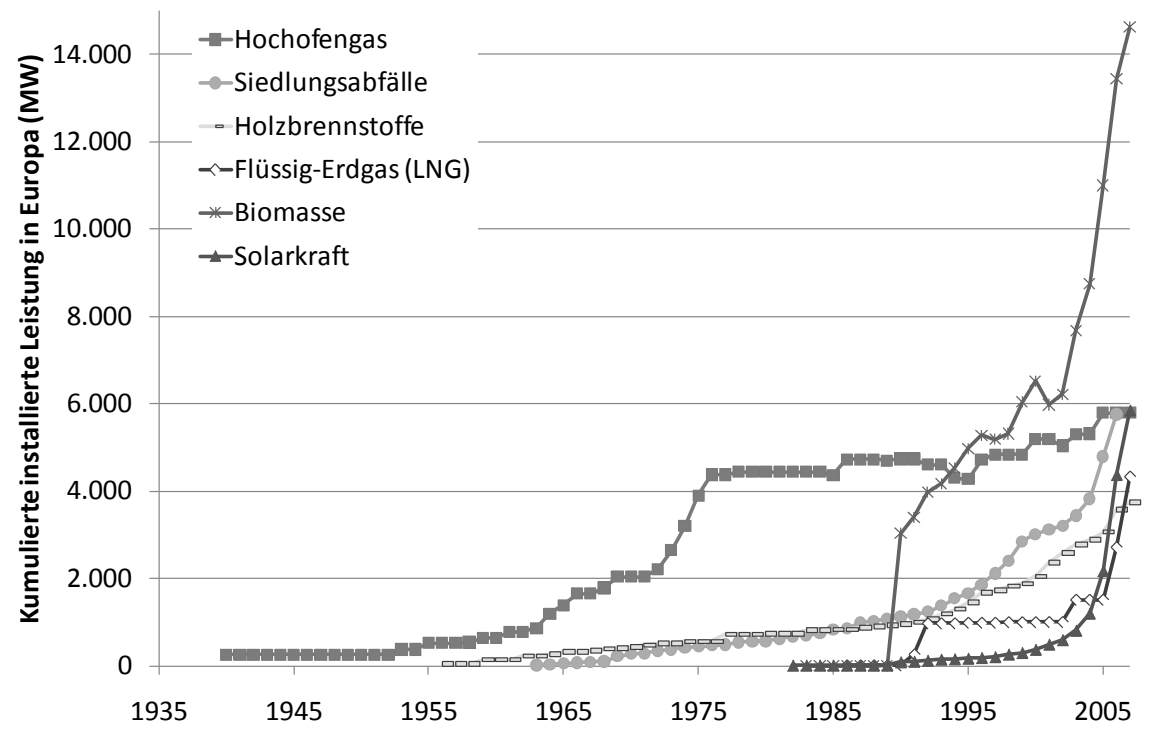

Quelle: Basierend auf Held et al. (2010); Platts (2007b).

Hochofengas wird seit 1940 zur Stromerzeugung mit Dampfturbinen eingesetzt. Die Nutzung scheint weniger durch technische Entwicklungen, sondern durch lokal verfügbare Potenziale getrieben zu sein, so dass eine Zuordnung zur Reifephase gerechtfertigt scheint. Weiterhin zeigt Abbildung 5.6 die installierte Leistung von Anlagen zur Nutzung von LNG (Liquefied Natural Gas). Dabei handelt es sich um einige wenige Gasturbinen- und GuD-Anlagen, die im Jahr 2007 insgesamt eine installierte Leistung von ca. 4,3 GW aufweisen. Aufgrund der noch sehr geringen Anzahl solcher Anlagen, in denen Flüssiggas als Brennstoff verwendet wird, scheint eine Zuordnung zu einem frühen Entwicklungsstadium angebracht.

Torf wird seit 1939 zur Stromerzeugung mit Dampfturbinen eingesetzt (Abbildung 5.7). Jedoch findet aus Gründen des Naturschutzes in Moorgebieten und aufgrund des hohen Wassergehaltes bzw. relativ geringen Heizwertes keine Nutzung in großem Maßstab statt. Die Verwendung beschränkt sich auf skandinavische Länder und Irland und erreicht ihr bisheriges Maximum mit ca. 1,6 GW installierter Leistung im Jahr 2002. Weitere Entwicklungspotenziale der verwendeten Technologie sind kaum zu erwarten, so dass eine Zuordnung zur Reifephase erfolgt. Der bisherige Maximalwert der installierten Leistung von Deponiegas wird im Jahr 2007 mit ca. 2 GW erreicht. Die relativ steile Wachstumskurve in den letzten Jahren legt eine Zuordnung zur beginnenden Wachstumsphase nahe. Ähnlich wie bei Torf ist jedoch davon auszugehen, dass das zukünftige Nutzungspotenzial im Wesentlichen durch lokal verfügbare Potenziale vorangetrieben wird. Die dargestellte Entwicklung der Nutzung von Ölschiefer deutet an, 
dass sich die entsprechenden Technologien in der Reifephase befinden. Die Nutzung von Biogas verzeichnet ein steiles Wachstum in den letzten Jahren, so dass eine Zuordnung zur beginnenden Aufstiegsphase angebracht scheint.

Abbildung 5.7: $\quad$ Nutzung von Torf, Ölschiefer, Deponiegas und Biogas zur Stromerzeugung in Europa

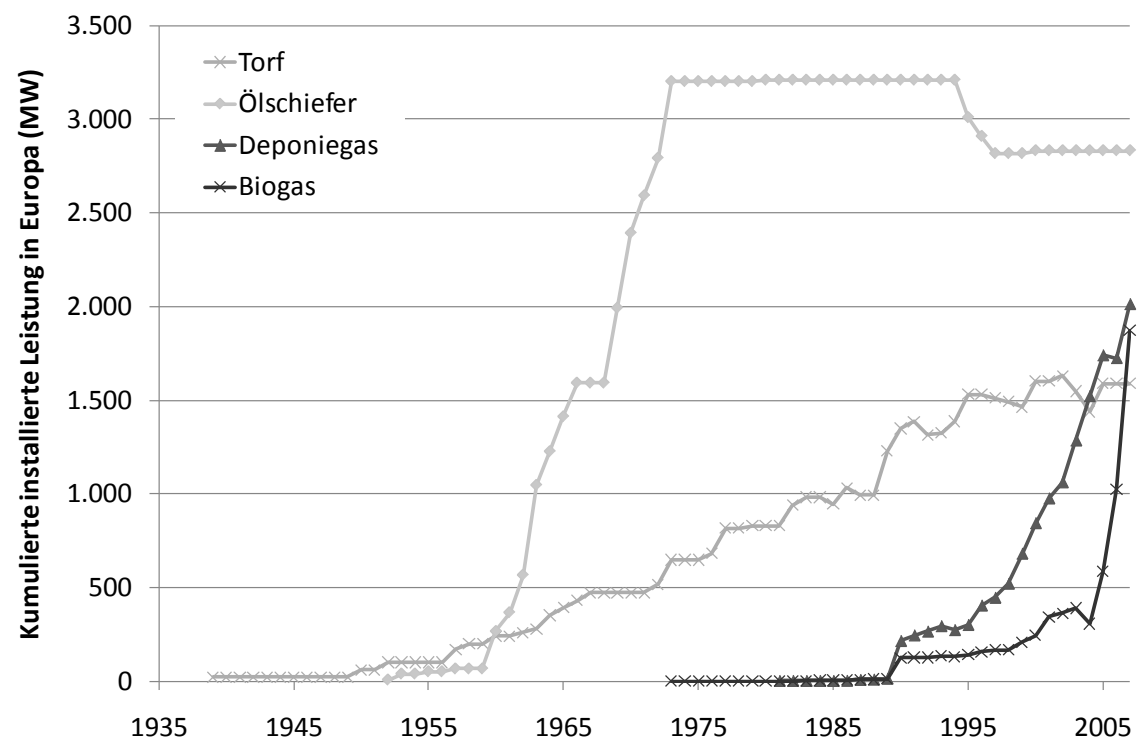

Quelle: Basierend auf Held et al. (2010); Platts (2007b.

Energieträger, die mit einer installierten Leistung zwischen $800 \mathrm{MW}$ und 1,5 GW zur europäischen Kraftwerkskapazität beitragen, sind in Abbildung 5.8 dargestellt. Geothermische Quellen werden bereits seit 1913 zur Stromerzeugung genutzt. Bis zum Jahr 2007 ist die installierte Leistung der entsprechenden Anlagen auf ihr bisheriges Maximum von ca. 1,3 GW gestiegen. Die dargestellte Kurve scheint das endgültige Maximum noch nicht erreicht zu haben, so dass sich die geothermische Stromerzeugung weiterhin in der Wachstumsphase zu befinden scheint. Bei einer Betrachtung unterschiedlicher Arten der geothermischen Stromerzeugung („FUELTYPE“) wird deutlich, dass Anlagen auf Basis des Trockendampf-Prinzips eher der Reifephase zuzuordnen sind, während sich das Flash-Prinzip in der Aufstiegsphase und das binäre Prinzip in einem frühen Entwicklungsstadium zu befinden scheint (Abbildung 10.3 bis Abbildung 10.5, Anhang 10.2).

Die Inbetriebnahme von Anlagen, die Abgase aus Raffinerien zur Stromerzeugung nutzen, ist erstmals in den 1930er Jahren erfasst. Seither ist die installierte Leistung nahezu kontinuierlich gestiegen, wobei sich in den letzten zehn bis zwanzig Jahren eine Sättigung bei etwa $890 \mathrm{MW}$ andeutet. Daher erfolgte eine Zuordnung zur Reifephase. Die Nutzung von Gasöl zur Stromerzeugung, die erstmals im Jahr 1961 erfasst 
ist, scheint sich noch in einer Phase des Aufstieges zu befinden und erreicht das bisherige Maximum im Jahr 2006 mit ca. 1,1 GW. Für die Nutzung von vergastem Rohöl bzw. anderen Raffinerie-Rückständen ist nur eine sehr geringe Anzahl von Anlagen erfasst, so dass eine Zuordnung zu einem bestimmten Entwicklungsstadium schwierig ist. Aus der geringen Anzahl der bisher in Betrieb gegangenen Anlagen kann auf ein frühes Entwicklungsstadium vor dem Marktaufstieg geschlossen werden. Allerdings ist zu erwarten, dass die weitere Verbreitung der Nutzung im Wesentlichen von dem Produktionsvolumen in Raffinerien und dem Vorliegen geeigneter Reststoffe abhängt und weniger durch technologische Entwicklungen vorangetrieben wird.

Abbildung 5.8: Nutzung von Gasöl, Geothermie sowie Raffinerie-Rückständen und -Abgasen zur Stromerzeugung

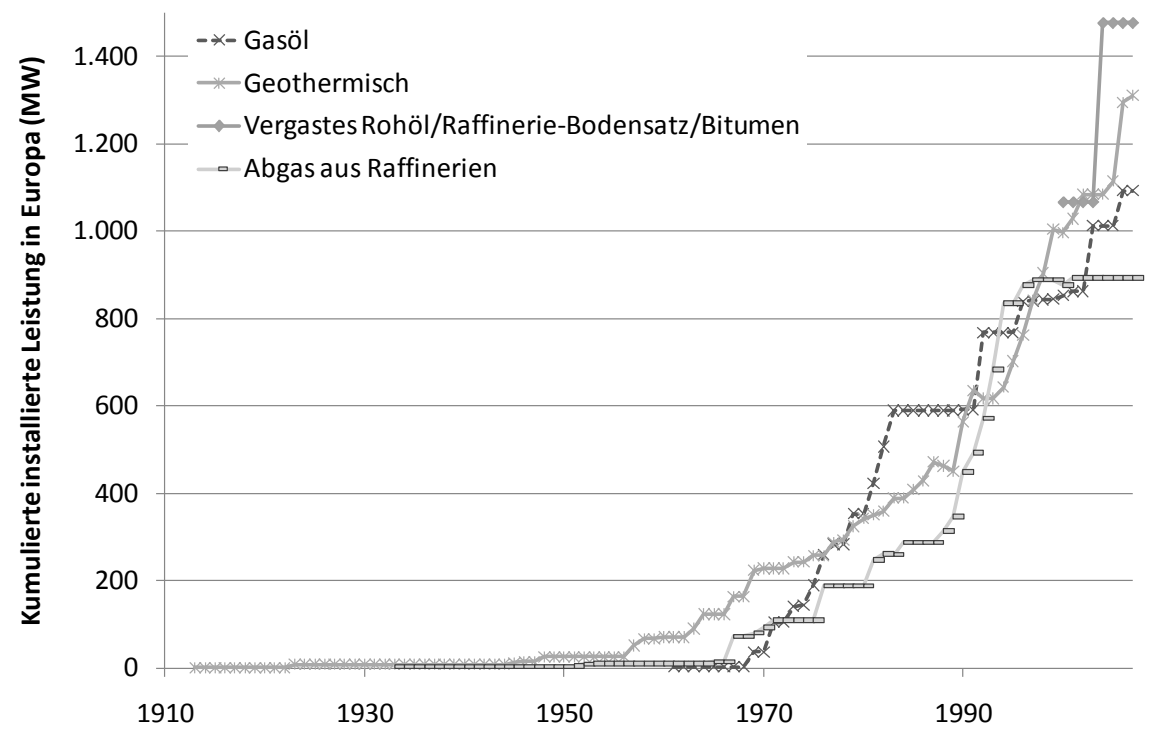

Quelle: Basierend auf Held et al. (2010); Platts (2007b).

Hinsichtlich der Nutzung von Synthesegasen aus Kohlevergasung, von Erdölkoks, Kerosin und Schwarzlauge kann aufgrund der bisher geringen Anzahl installierter Anlagen allenfalls darauf geschlossen werden, dass sich diese Optionen zur Stromerzeugung in einem frühen Entwicklungsstadium vor der Wachstumsphase befinden oder aber, dass es sich hierbei um Nischentechnologien handelt (vgl. Abbildung 5.9 und Tabelle 10.1, Anhang 10.1). Klärgas wird seit Mitte der 1980er Jahre zur Stromerzeugung verwendet und die installierte Leistung der entsprechenden Anlagen ist seither kontinuierlich gestiegen. In den letzten Jahren scheint sich aufgrund abnehmender Zuwachsraten eine Sättigung abzuzeichnen. Dabei könnte es sich jedoch auch um eine vorübergehende Erscheinung handeln, wie sie bereits für Ende der 1990er Jahre zu beobachten ist. 
Abbildung 5.9: Nutzung von Kohlevergasung, Erdölkoks, Kerosin, Schwarzlauge und Klärgas zur Stromerzeugung

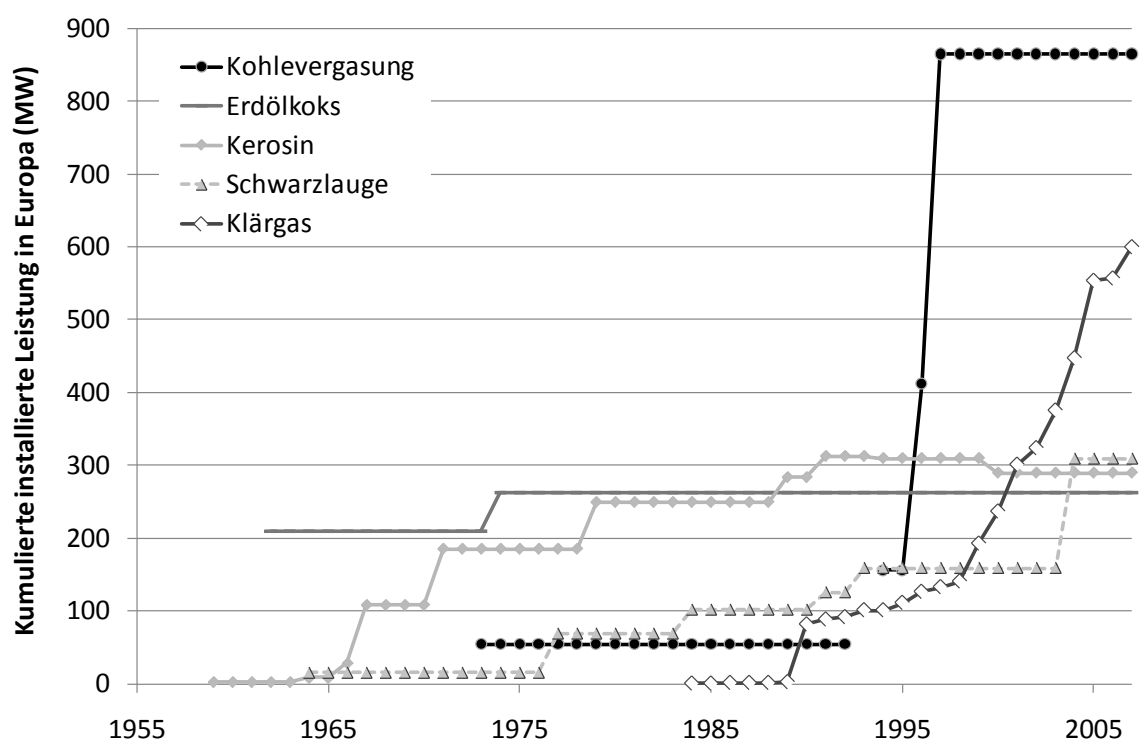

Quelle: Basierend auf Held et al. (2010); Platts (2007b).

Abbildung 5.10: Nutzung von Grubengas, Abfällen aus der Papierindustrie, Gichtgas und flüssigen Biokraftstoffen zur Stromerzeugung

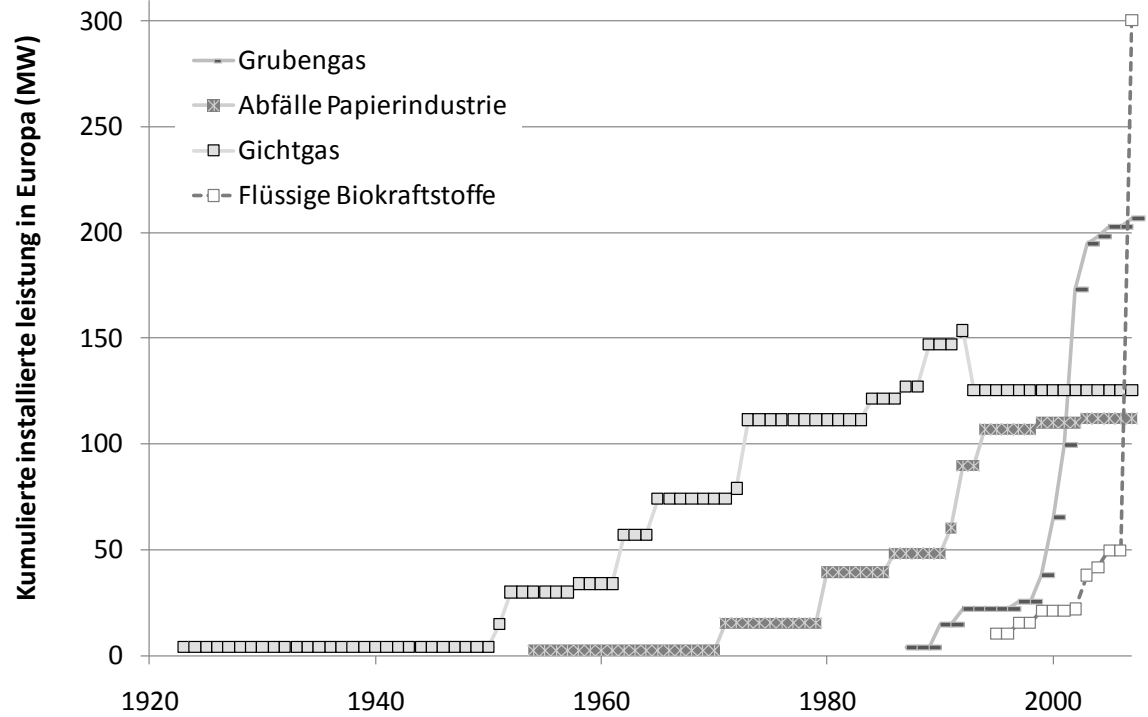

Quelle: Basierend auf Platts (2007b).

Die Nutzung von flüssigen Biokraftstoffen zeigt starke Zuwachsraten in den letzten Jahren (Abbildung 5.10). Entsprechende Anlagen wurden erstmals 1995 erfasst. Da sich diese somit erst seit weniger als 20 Jahren in Europa verbreiten und aufgrund der starken Zuwachsraten in den letzten Jahren, erfolgt eine Zuordnung zur Wachstums- 
phase. Ein ähnlicher Verlauf zeigt sich für die zwischen 1987 und 2007 aufgezeigte Entwicklung der Nutzung von Grubengas, wobei sich jedoch eine Sättigung in den letzten Jahren abzeichnet. Dabei ist zu erwarten, dass die Verwendung dieses Energieträgers stärker durch lokal verfügbare Potenziale als durch technologische Entwicklungen bestimmt ist. Gichtgas wird bereits seit den 1920er Jahren zur Stromerzeugung genutzt. Aufgrund der Kurve in Abbildung 5.10 ist in den letzten Jahrzehnten eine Sättigung bei einer installierten Leistung von weniger als 1,5 GW erkennbar. Dies gilt auch für Abfälle aus der Papierindustrie, wobei diese erst seit den 1950er Jahren zur Stromerzeugung genutzt werden.

Weitere Energieträger, die mit einer installierten Leistung von weniger als $100 \mathrm{MW} \mathrm{zu}$ den installierten Kraftwerkskapazitäten in Europa beitragen, sind in Abbildung 10.6 (Anhang 10.2) dargestellt. Dabei handelt es sich in der Regel um vereinzelte Anlagen, die seit den 1960er Jahren oder später in Betrieb gingen (vgl. auch Tabelle 10.1, Anhang 10.1). Folglich handelt es sich hierbei entweder um Nischentechnologien oder Technologien, die sich noch in einem frühen Entwicklungsstadium befinden. Die Zuordnung der nach den verwendeten Energieträgern unterschiedenen Technologien zu Entwicklungsphasen ist in Tabelle 5.1 zusammengefasst. Wenn auf Basis der vorangegangen Analyse keine eindeutige Zuordnung möglich ist, sind gegebenenfalls einem Energieträger zwei Entwicklungsstadien zugeordnet. Weiterhin werden Technologien, die nur zu einem sehr kleinen Anteil zur Stromversorgung beitragen bzw. von denen nur eine sehr geringe Anzahl an Anlagen installiert ist, der Kategorie „Frühes Stadium/Nische" zugeordnet. Das heißt bei diesen Technologien wird davon ausgegangen, dass sie sich in ihrer Entwicklung entweder in einer der Phasen vor dem Aufstieg befinden oder aber dass es sich um Nischentechnologien handelt. 
Tabelle 5.1: $\quad$ Zuordnung von Energietechnologien zu ihrem aktuellen Entwicklungsstadium, unterschieden nach dem verwendeten Energieträger („FUEL“ bzW. „FUELTYPE“; Stand: 2007)

\begin{tabular}{|c|c|c|c|c|c|c|c|}
\hline „FUEL“ bzw. „FUELTYPE“ (alphabetisch) & $\begin{array}{l}\text { Jahr der ersten } \\
\text { registrierten } \\
\text { Inbetriebnahme }\end{array}$ & $\begin{array}{l}\text { Jahr der bisher } \\
\text { maximal } \\
\text { installierten } \\
\text { Leistung }\end{array}$ & $\begin{array}{c}\text { Bisher maximale } \\
\text { installierte } \\
\text { Leistung (MW }\end{array}$ & $\begin{array}{c}\text { Frühes Stadium/ } \\
\text { Nische }\end{array}$ & $\begin{array}{c}\text { Aufstiegl } \\
\text { Wachstum }\end{array}$ & $\begin{array}{c}\text { Reifel } \\
\text { Sättigung }\end{array}$ & Rückgang \\
\hline Abfälle aus der Papierindustrie & 1954 & $2003-07$ & 112 & & & $\mathrm{x}$ & \\
\hline Abgase aus Raffinerien & 1933 & 2001-07 & 892 & & & $x$ & \\
\hline Biogas & 1973 & 2007 & 1.873 & & $x$ & & \\
\hline Biomasse & 1983 & 2007 & 14.614 & $\mathrm{x}$ & $\mathrm{x}$ & & \\
\hline Geflügelmist & 1992 & 2007 & 103 & $\mathrm{x}$ & & & \\
\hline Landwirtschaftliche Abfälle & 1992 & $2006-07$ & 156 & & $\mathrm{x}$ & & \\
\hline Reishülsen & 1983 & $1999-2007$ & 3 & $x$ & & & \\
\hline Stroh & 1989 & $2002-07$ & 171 & & $x$ & & \\
\hline Deponiegas & 1981 & 2007 & 2.012 & & $(\mathrm{X})$ & $x$ & \\
\hline Erdgaskraftwerke & 1928 & 2007 & 148.299 & $\mathrm{x}$ & $\mathrm{x}$ & $\mathrm{x}$ & \\
\hline Druckluftspeicher & 1978 & $1978-2007$ & 290 & $x$ & & & \\
\hline Erdölkoks & 1962 & 1974-2007 & 262 & $x$ & & & \\
\hline Flüssige Bioenergieträger & 1995 & 2007 & 300 & $x$ & $x$ & & \\
\hline Flüssig-Erdgas (LNG) & 1986 & 2007 & 4.322 & $\mathrm{x}$ & & & \\
\hline Gasöl & 1961 & $2006-07$ & 1.092 & & $\mathrm{x}$ & & \\
\hline Geothermie & 1913 & 2007 & 1.310 & $x$ & $x$ & $x$ & \\
\hline Binäres Prinzip & 1994 & 2007 & 31 & $\mathrm{x}$ & & & \\
\hline Flash-Prinzip & 1969 & $2006-07$ & 182 & & $x$ & & \\
\hline Trockendampf-Prinzip & 1913 & $2002-07$ & 792 & & & $x$ & \\
\hline Gichtgas & 1923 & 1992 & 154 & & & $x$ & \\
\hline Grubengas & 1987 & 2007 & 207 & & $x$ & $x$ & \\
\hline Hochofengas & 1940 & $2005-07$ & 5.794 & & & $x$ & \\
\hline Holzbrennstoffe & 1956 & 2007 & 3.748 & & $x$ & & \\
\hline Kernkraftwerke (Uran) & 1956 & $2000-01$ & 148.217 & & & & $\mathrm{x}$ \\
\hline Kerosin & 1959 & $1991-93$ & 312 & $x$ & & & \\
\hline Klärgas & 1984 & 2007 & 599 & & $x$ & $x$ & \\
\hline Kohlekraftwerke & 1907 & 1992 & 233.642 & $x$ & & & $x$ \\
\hline Braunkohle & 1921 & 2000 & 79.142 & & & & $x$ \\
\hline Kohlehaltige Reststoffe & 1965 & 1993-2007 & 235 & $\mathrm{x}$ & & & \\
\hline Steinkohle & 1907 & 1990 & 142.608 & & & & $x$ \\
\hline Kohlevergasung & 1973 & $1997-2007$ & 865 & $x$ & & & \\
\hline Ölkraftwerke & 1911 & 1993 & 90.340 & & & $x$ & $x$ \\
\hline Leichtöle & 1932 & 1998 & 12.932 & & & $x$ & \\
\hline Schweröle & 1929 & 1993 & 66.772 & & & & $x$ \\
\hline Ölschiefer & 1952 & $1980-94$ & 3.208 & & $(X)$ & $x$ & \\
\hline Schwarzlauge & 1964 & $2004-07$ & 309 & $x$ & & & \\
\hline Siedlungsabfälle & 1963 & 2006 & 5.755 & $x$ & $\mathrm{x}$ & & \\
\hline Ersatzbrennstoffe & 1992 & 1999-2007 & 241 & & $x$ & & \\
\hline Solarkraft & 1982 & 2007 & 5.833 & $x$ & $x$ & & \\
\hline Photovoltaik & 1982 & 2007 & 4.689 & & $\mathrm{x}$ & & \\
\hline Solarthermische Kraftwerke & 1982 & $2006-07$ & 1.144 & $x$ & $(\mathrm{X})$ & & \\
\hline Sonstige Energieträger (vgl. Abbildung 10.6) & & & & $x$ & & & \\
\hline Torf & 1939 & 2002 & 1.630 & & & $x$ & \\
\hline Vergastes Rohö//Raffinerie-Bodensatz/Bitumen & 2000 & $2004-07$ & 1.477 & $x$ & & & \\
\hline Wasserkraft & 1882 & 2007 & 188.385 & $\mathrm{x}$ & & $\mathrm{x}$ & \\
\hline Konventionell & 1882 & 2007 & 148.998 & & & $x$ & \\
\hline Pumpspeicher & 1920 & $2005-07$ & 39.143 & & & $x$ & \\
\hline Wellen- und Gezeitenkraft & 1990 & $1990-2007$ & 240 & $x$ & & & \\
\hline Windkraft & 1975 & 2007 & 56.347 & $x$ & $x$ & & \\
\hline Horizontal & 1975 & 2007 & 37.534 & & $\mathrm{x}$ & & \\
\hline Vertikal & 1990 & $1990-2007$ & 1 & $x$ & & & \\
\hline
\end{tabular}

Anmerkungen: Die aktuell als zutreffend erachtete Entwicklungsphase ist durch „X“ gekennzeichnet. Bei nicht eindeutiger Zuordnung ist die als weniger wahrscheinlich angesehene Phase durch „(X)“ gekennzeichnet. 


\subsubsection{Technologiezyklen von Optionen zur Stromerzeugung nach der Art der Nutzung}

Ein weiteres in der Datenbank nach Platts (2007b) erfasstes Merkmal ist die Art der Nutzung („UTYPE“), wonach den einzelnen Datensätzen spezifische Technologien zugeordnet werden. Wie bereits erläutert, kann eine Technologie zur Nutzung unterschiedlicher Energieträger eingesetzt werden (vgl. auch Tabelle 10.1). Abbildung 5.11 zeigt, dass die am weitesten verbreitete Technologie die Dampfturbine ist. Der Dampf kann hierbei auf Basis unterschiedlicher Energieträger erzeugt werden. Abbildung 5.11 legt eine Zuordnung der Dampfturbine zur Rückgangs-Phase nahe. Jedoch sollte bei der Zuordnung der jeweils verwendete Energieträger berücksichtigt werden, dass zur Dampferzeugung und Nutzung in einer Dampfturbine je nach Brennstoff zum Teil erhebliche Unterschiede in der Auslegung beachtet werden müssen (so zum Beispiel beim Einsatz von Siedlungsabfällen, vgl. Platts (2007a)). Die Entwicklung der installierten Leistung von Wasserturbinen stimmt weitgehend mit der Entwicklung der Nutzung von Wasser zur Stromerzeugung überein (vgl. Abbildung 5.5 und Abbildung 5.11), da es sich hierbei um speziell für diese Energieträger entwickelte Technologien handelt. Des Weiteren verdeutlicht Abbildung 5.11, dass die Nutzung von GuD-Kraftwerken in den letzten Jahren steigende Zuwachsraten aufweist. Daher werden diese der Wachstumsphase zugeordnet.

Abbildung 5.11: Installierte Leistung an GuD-Kraftwerken, Wasser-, und Dampfturbinen

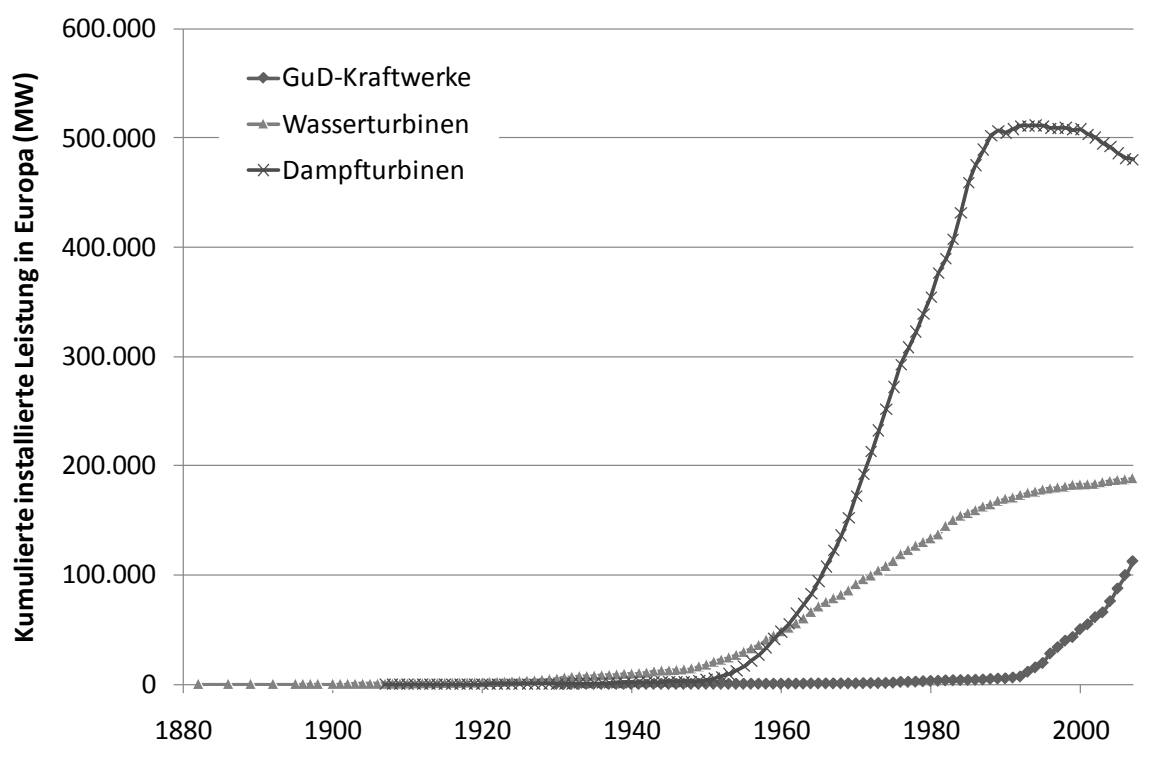

Anmerkungen: GuD = Kombinierter Gas- und Dampfturbinenprozess.

Quelle: Basierend auf Platts (2007b). 
Auch die Verwendung konventioneller Gasturbinen scheint weiter zuzunehmen (vgl. Abbildung 5.12). Da jedoch inzwischen der Einsatz von GuD-Anlagen überwiegt, die einen besseren Brennstoffnutzungsgrad erzielen, ist davon auszugehen, dass konventionelle Gasturbinen bald ein Stadium der Sättigung erreichen. Daher scheint eine Zuordnung der Gasturbinen-Technologie zur Reifephase gerechtfertigt. Wie auch bei der Wasserkraft stimmt die Entwicklung des Einsatzes von Windturbinen mit der in Abbildung 5.5 dargestellten Kurve für die Windkraftnutzung überein, da es sich hierbei um eine speziell zur Nutzung von Windströmungen entwickelte Technologie handelt. Die Nutzung von Verbrennungsmotoren zur Stromerzeugung weist eine lange Tradition auf, wobei unterschiedliche gasförmige und flüssige Energieträger zum Einsatz kommen. Aufgrund der in Abbildung 5.12 dargestellten Entwicklung erfolgt eine Zuordnung zur Reifephase, jedoch sollten gegebenenfalls Unterschiede in Abhängigkeit des verwendeten Energieträgers beachtet werden.

Abbildung 5.12: Installierte Leistung von Gas- und Windturbinen sowie Verbrennungsmotoren

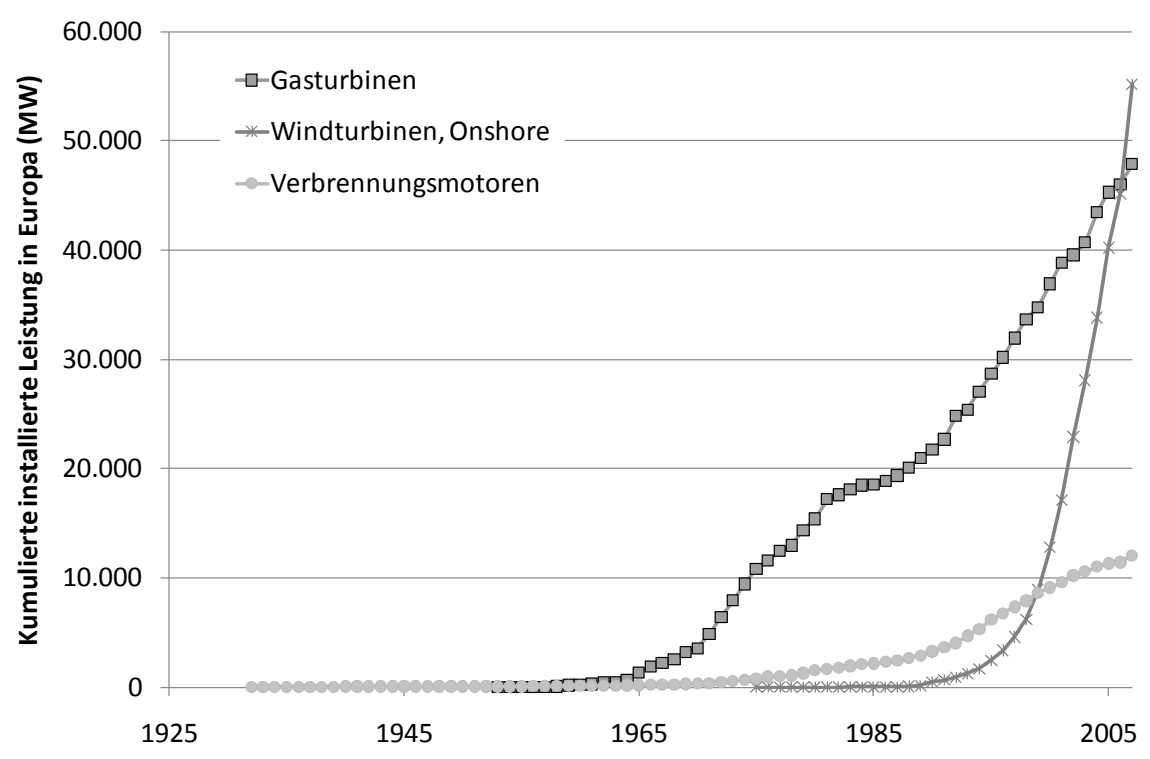

Quelle: Basierend auf Held et al. (2010); Platts (2007b).

Einen starken Zuwachs verzeichnet in den letzten Jahren die installierte Leistung von Offshore-Windenergieanlagen (vgl. Abbildung 5.13). Im Gegensatz zu den OnshoreWindenergieanlagen werden diese einem frühen Entwicklungsstadium bzw. der beginnenden Aufstiegsphase zugeordnet, da zusätzliche technologische Herausforderungen hinsichtlich der Installation auf hoher See bestehen. Die Entwicklung von ORCAnlagen (Abbildung 5.14) weist in den letzten Jahren steigende Zuwachsraten auf, was auf einen beginnenden Aufstieg der Technologie hinweisen könnte. Dabei werden sowohl geothermische Wärmequellen als auch Biogase zur Stromerzeugung in ORC- 
Prozessen genutzt. Bei den bisher am wenigsten verbreiteten „Nutzungstypen“, die in der Datenbank erfasst sind, handelt es sich um Brennstoffzellen, Dampfkolbenmotoren und Turboexpander. In Brennstoffzellen und Turboexpandern kommt bisher vorwiegend Erdgas als Brennstoff zum Einsatz. Dampfkolbenmotoren werden in erster Linie auf Basis von Hochofengas, Solarwärme oder Holzbrennstoffen betrieben (vgl. auch Tabelle 10.1). Diese Technologien kommen in eher kleinen Leistungsbereichen zum Einsatz und sind einem frühen Entwicklungsstadium zuzuordnen. Die Zuordnung unterschiedlicher „Nutzungstypen“ zu Entwicklungsstadien ist in Tabelle 5.2 zusammengefasst.

Abbildung 5.13: Installierte Leistung von Offshore-Windturbinen

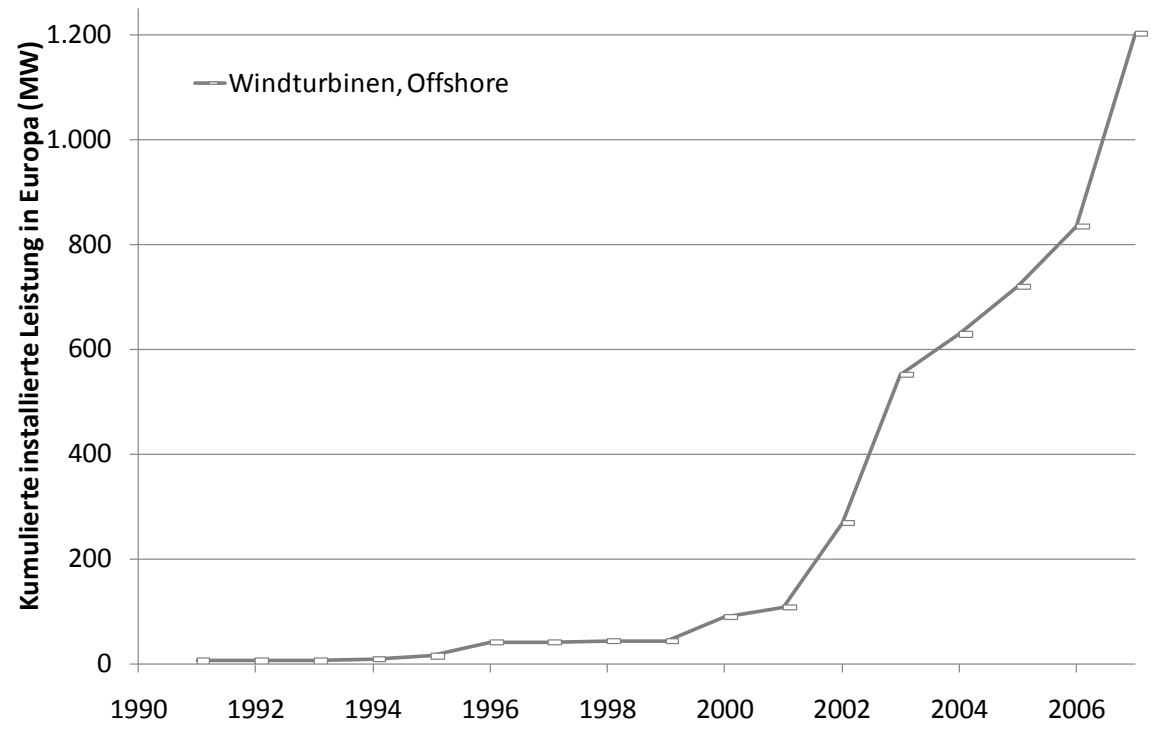

Quelle: Basierend auf Held et al. (2010); Platts (2007b). 
Abbildung 5.14: Installierte Leistung von ORC-Anlagen, Brennstoffzellen, Dampfkolbenmotoren und Turboexpandern

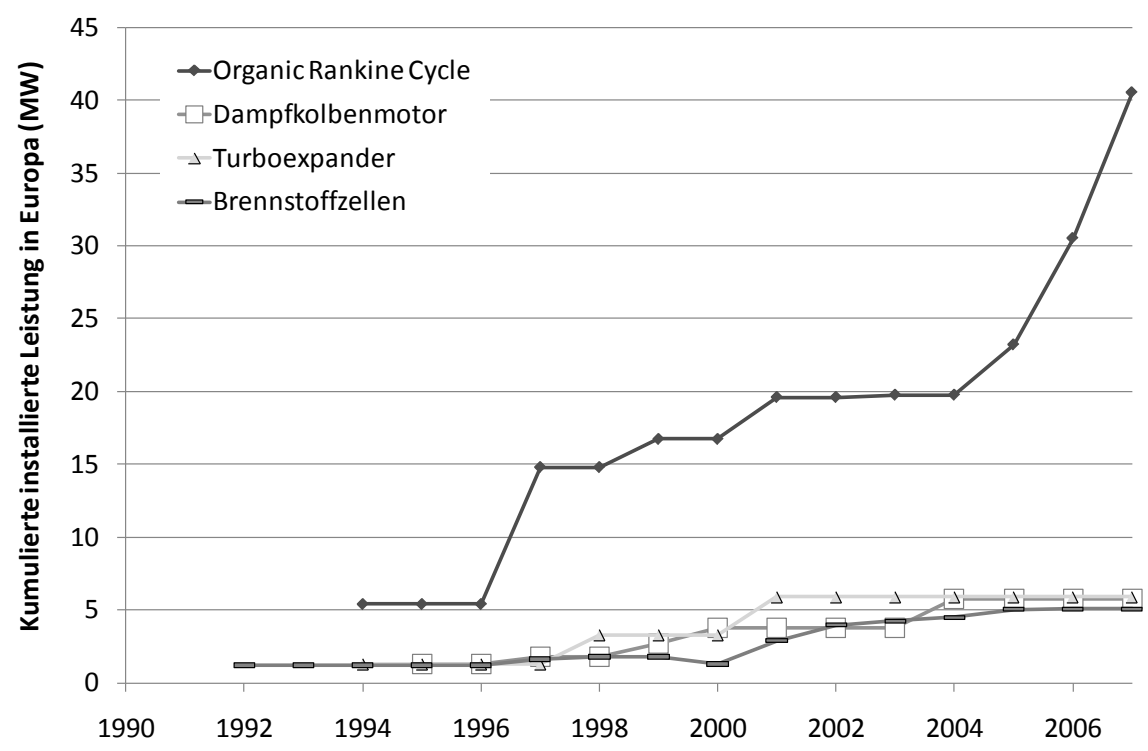

Quelle: Basierend auf Platts (2007b).

Tabelle 5.2: $\quad$ Zuordnung Energietechnologien zu ihrem aktuellen Entwicklungsstadium, unterschieden nach der Art der Nutzung („UTYPE“; Stand: 2007)

\begin{tabular}{|c|c|c|c|c|c|c|c|}
\hline „UTYPE“ (alphabetisch) & $\begin{array}{c}\text { Jahr der ersten } \\
\text { registrierten } \\
\text { Inbetriebnahme }\end{array}$ & $\begin{array}{c}\text { Jahr der bisher } \\
\text { maximal } \\
\text { installierten } \\
\text { Leistung } \\
\end{array}$ & $\begin{array}{c}\text { Bisher maximale } \\
\text { installierte } \\
\text { Leistung }\left(\mathrm{MW}_{\mathrm{el}}\right) \\
\end{array}$ & \begin{tabular}{|c|} 
Frühes Stadium $/$ \\
Nische
\end{tabular} & $\begin{array}{c}\text { Aufstieg/ } \\
\text { Wachstum }\end{array}$ & $\begin{array}{c}\text { Reifel } \\
\text { Sättigung }\end{array}$ & Rückgang \\
\hline Brennstoffzellen & 1992 & $2006-07$ & 5 & $\mathrm{x}$ & & & \\
\hline Dampfkolbenmotoren & 1995 & 2007 & 6 & $\mathrm{x}$ & & & \\
\hline Dampfturbinen & 1907 & 1994 & 511.371 & & & & $(\mathrm{X})^{\star}$ \\
\hline Gasturbinen & 1953 & 2007 & 47.896 & & $(\mathrm{X})$ & $x$ & \\
\hline GuD-Kraftwerke & 1930 & 2007 & 112.158 & & $\mathrm{x}$ & & \\
\hline ORC-Anlagen (Organic Rankine Cycle) & 1994 & 2007 & 41 & $\mathrm{x}$ & $(\mathrm{X})$ & & \\
\hline Turboexpander & 1994 & 2001-07 & 6 & $\mathrm{x}$ & & & \\
\hline Verbrennungsmotoren & 1932 & 2007 & 12.052 & & & $(\mathrm{X})^{*}$ & \\
\hline Wasserturbinen & 1882 & 2007 & 188.326 & & & $\mathrm{x}$ & \\
\hline Windturbinen, Offshore & 1992 & 2007 & 1.200 & $\mathrm{x}$ & $(\mathrm{X})$ & & \\
\hline Windturbinen, Onshore & 1976 & 2007 & 55.213 & & $\mathrm{x}$ & & \\
\hline
\end{tabular}

Anmerkungen: Die aktuell als zutreffend erachtete Entwicklungsphase ist durch „X“ gekennzeichnet. Bei nicht eindeutiger Zuordnung ist die als weniger wahrscheinlich angesehene Phase durch „(X)“ gekennzeichnet. Bei Kennzeichnung $(X)^{\star}$ sollte der jeweilig verwendete Energieträger bei der Zuordnung berücksichtigt werden. GuD = Kombinierter Gas- und Dampfturbinenprozess.

\subsubsection{Technologiezyklen von Optionen zur Wärmebereitstellung}

Im Gegensatz zu den Kraftwerkstechnologien steht für Technologien zur Bereitstellung von Nutzwärme keine vergleichbare Datensammlung zur Verfügung, die eine langfristige Abbildung von deren Entwicklung über die Zeit ermöglicht. Daher muss auf kurzfristigere Darstellungen mit relativ geringem Detaillierungsgrad zurückgegriffen werden. 
Bei BMWi (2010) ist die Verwendung unterschiedlicher Brennstoffe in Heizkraftwerken und Fernheizwerken (im Folgenden unter dem Begriff „Heizwerke“ zusammengefasst) in Deutschland seit dem Jahr 1990 bis 2006 erfasst (Abbildung 5.16). Für Braunkohle ist über diesen Betrachtungszeitraum ein deutlich fallender Trend zu beobachten. Auch hinsichtlich der Verwendung von Mineralöl ist ein abnehmender Trend zu beobachten. Daher werden Heizwerke, die mit Braunkohle oder Öl befeuert werden, der Rückgangs-Phase zugeordnet. Die Bereitstellung von Nutzwärme aus Steinkohlen scheint sich in den letzten beiden Jahrzehnten auf einem Niveau zwischen 100 und 150 PJ zu stabilisieren, so dass eine Zuordnung zur Reifephase nahe liegt. Tendenziell steigend stellt sich die Nutzung von Erdgas zur Wärmebereitstellung in Heizwerken dar, so dass sich diese Technologien noch in der Wachstumsphase zu befinden scheinen. Auch die Verwendung von Müll und sonstigen Brennstoffen in Heizwerken hat zwischen 1990 und 2006 zugenommen, so dass ebenfalls eine Zuordnung zur Wachstumsphase erfolgt. Insgesamt sind die in Abbildung 5.15 dargestellten Entwicklungstrends mit Vorsicht zu interpretieren, da es sich um einen relativ kurzen Betrachtungszeitraum handelt, so dass langfristige Trends die dargestellten kurzfristigen Entwicklungen überlagern könnten.

Abbildung 5.15: Brennstoffeinsatz in Heizkraftwerken und Fernheizwerken in Deutschland

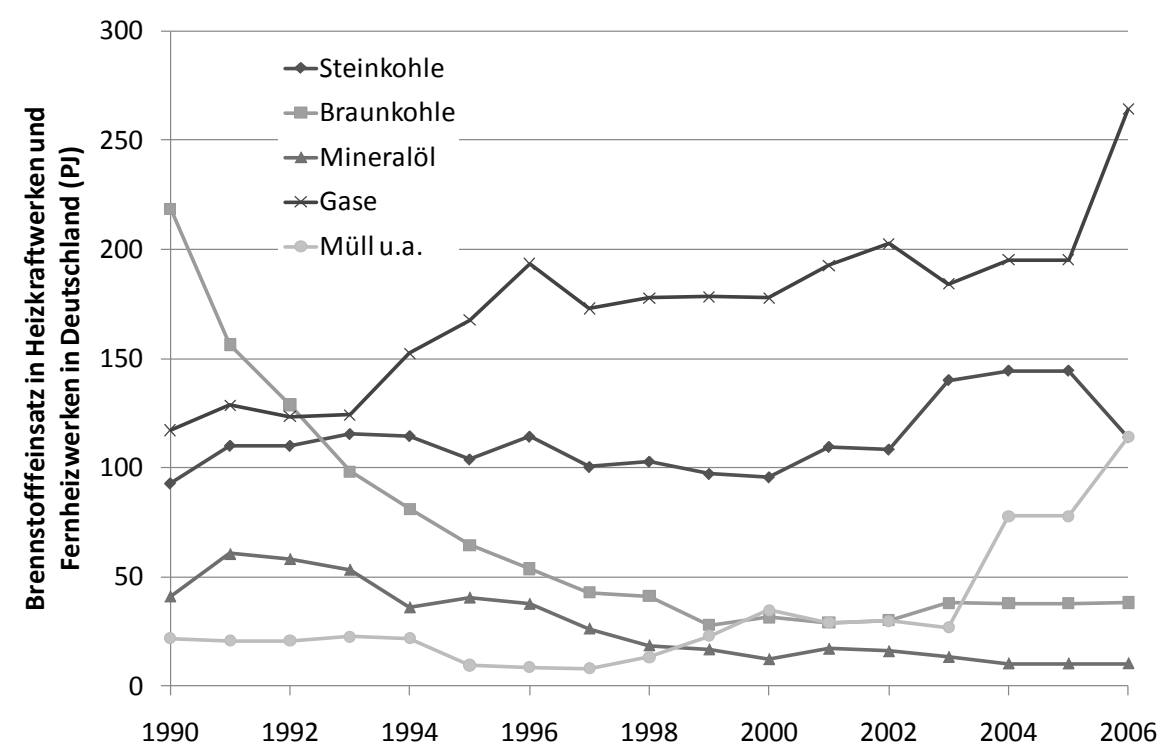

Quelle: Basierend auf BMWi (2010).

Eine etwas langfristigere Betrachtung findet sich bei (BDEW 2009). In dieser Veröffentlichung ist die Entwicklung der Beheizungsstruktur des Wohnungsbestandes in Deutschland von 1975 bis 2008 unterschieden nach Energieträgern dargestellt. Dabei ist ein deutlicher Rückgang des Anteils der Kohle/Koks- und Heizöl-basierten Techno- 
logien zur Wärmebereitstellung sichtbar. Auch für Strom-basierte Technologien ist tendenziell ein Rückgang des Anteils an der Wärmeversorgung zu erkennen. Der Anteil der Fernwärme ist hingegen seit Ende der 1970er Jahre tendenziell gestiegen. Sehr stark gestiegen ist in den letzten 30 Jahren der Anteil Gas-basierter Technologien zur Wärmebereitstellung. Seit Mitte der 1990er Jahre übersteigt der Anteil Gas-basierter Technologien die Nutzung von Heizöl. Die daraus ableitbaren Schlussfolgerungen decken sich weitgehend mit der Interpretation von Abbildung 5.15.

Abbildung 5.16: Einsatz konventioneller Öl- und Gaskessel, von Thermen und GasBrennwertkesseln zur Wärmebereitstellung in Deutschland

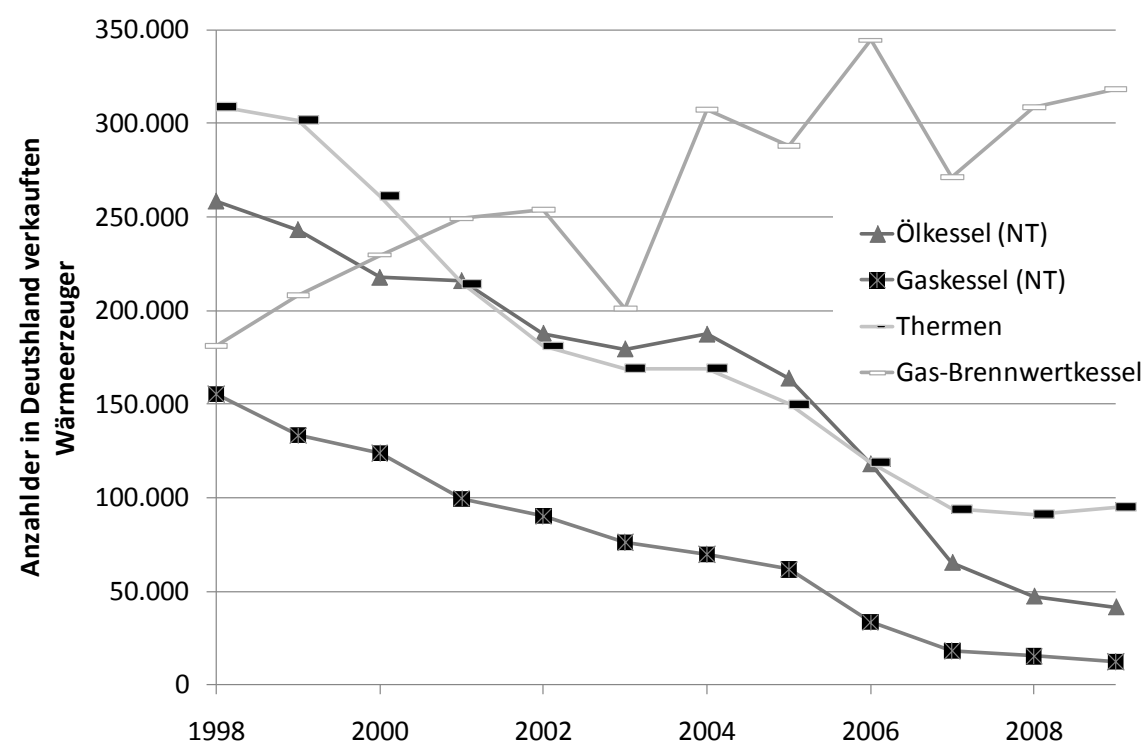

Anmerkungen: NT = Nieder-Temperatur; unter Thermen werden im Allgemeinen Etagenheizungen und Durchlauferhitzer verstanden.

Quelle: Basierend auf Daten des BDH (Bundesindustrieverband Deutschland Haus-, Energieund Umwelttechnik e.V.; http://www.bdh-koeln.de/)

Hinsichtlich des Einsatzes unterschiedlicher Wärmeerzeuger-Technologien in Deutschland stehen für einen begrenzten Zeitraum Daten des BDH über die jährlichen Verkaufszahlen zur Verfügung (vgl. Abbildung 5.16 und Abbildung 5.17). Deutlich erkennbar sind die rückläufigen Verkaufszahlen für konventionelle Niedertemperatur-Kessel auf Basis von Öl oder Gas sowie von Thermen (Etagenheizungen bzw. Durchlauferhitzer). Daher werden diese Technologien der Rückgangs-Phase zugeordnet. Die Anzahl der verkauften Gas-Brennwertkessel ist hingegen in den letzten Jahren tendenziell gestiegen, scheint sich jedoch auf einem relativ hohen Niveau einzupendeln. Andererseits könnte den dargestellten kurzfristigen Schwankungen auch ein langfristig eher steigender Trend zugrunde liegen. Daher können Gas-Brennwertkessel der Wachstums- oder der Reifephase zugeordnet werden. Auch die Anzahl der verkauften Öl- 
Brennwertkessel ist in den letzten zehn Jahren nahezu kontinuierlich gestiegen (vgl. Abbildung 5.17). Dies legt eine Zuordnung von Brennwertkesseln zur Wachstumsphase nahe. Die Verkaufszahlen für Wärmpumpen und Heizungsanlagen auf Basis fester Biomasse sind ebenfalls in den letzten Jahren gestiegen. Somit werden diese Technologien der Phase des Aufstiegs zugeordnet.

Abbildung 5.17: Einsatz von fester Biomasse, von Wärmepumpen und ÖlBrennwertkesseln zur Wärmebereitstellung in Deutschland

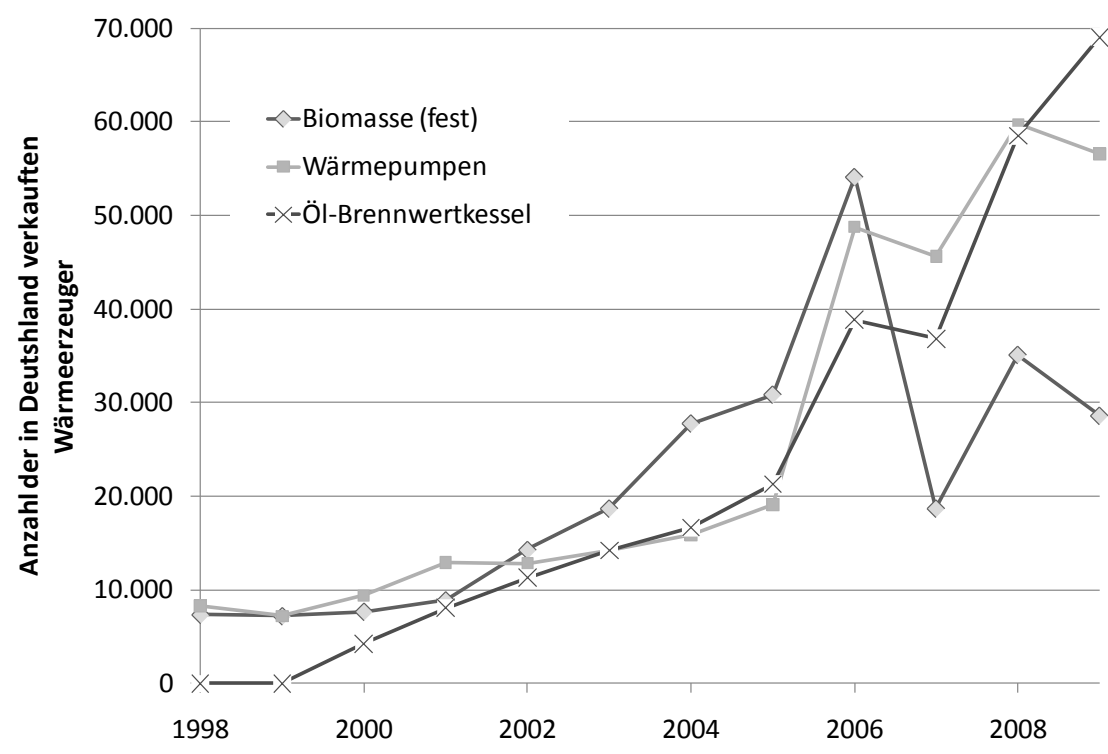

Quelle: Basierend auf Daten des BDH (Bundesindustrieverband Deutschland Haus-, Energieund Umwelttechnik e.V.; http://www.bdh-koeln.de/)

Neben den Verkaufszahlen aus der BHD-Statistik kann weiterhin auf Basis der Datenbank von Held et al. (2010) die Verwendung erneuerbarer Energieträger zur Bereitstellung von Wärme in Europa seit 1990 abgebildet werden (vgl. Tabelle 5.3 und Tabelle 5.4). Da für einige Optionen nur die erzeugte Wärmemenge, nicht aber die installierte Leistung zur Verfügung steht, erfolgt eine Darstellung beider Kenngrößen. Zwar ist die erzeugte Wärmemenge durch den Wirkungsgrad und die Verfügbarkeit der Anlagen beeinflusst, dennoch ist davon auszugehen, dass beide Kenngrößen den gleichen Trend aufweisen. Der bereits in Abbildung 5.17 aufgezeigte wachsende Trend für den Einsatz von Biomasse-Anlagen zur Wärmebereitstellung wird auch auf Basis der Daten zur Wärmeerzeugung aus Held et al. (2010) bestätigt. Dabei kommt insbesondere Holz als Brennstoff zum Einsatz. Die Bereitstellung von Wärme aus KWK-Anlagen mit Biomasse, Bioabfällen oder Siedlungsabfällen ist in den letzten 20 Jahren nahezu kontinuierlich gestiegen. Insbesondere in den letzten 10 Jahren sind steigende Zuwachsraten zu beobachten. Dies deutet auf die Phase des Aufstieges hin. 
Wie bereits für die geothermische Stromerzeugung beobachtet, nimmt auch die Bereitstellung von Wärme auf Basis von Geothermie zu. Dabei kommen insbesondere Wärmepumpen in zunehmendem Maße zum Einsatz. Diese scheinen sich somit in einer Phase des Aufstieges zu befinden, wie schon in Abbildung 5.17 beobachtet. Auch die installierte Wärmeleistung von KWK-Anlagen basierend auf Biogas hat in den letzten 20 Jahren kontinuierlich zugenommen, so dass eine Einordnung in die Wachstumsphase erfolgt. Unter den Biogasen hat sich dabei in den letzten Jahren insbesondere das auf Basis landwirtschaftlicher Stoffströme erzeugte Biogas durchgesetzt. Die in Tabelle 5.3 und Tabelle 5.4 dargestellten Daten legen somit eine Zuordnung sämtlicher Wärmebereitstellungsoptionen auf Basis erneuerbarer Energieträger zur Wachstumsphase nahe. Eine Zusammenfassung der Schlussfolgerungen hinsichtlich des aktuellen Entwicklungsstadiums von Optionen zur Wärmebereitstellung zeigt Tabelle 5.5.

Tabelle 5.3: $\quad$ Wärmeerzeugung aus erneuerbaren Energien in Europa

\begin{tabular}{|c|c|c|c|c|c|c|c|c|c|c|}
\hline & Einheit & 1991 & 1993 & 1995 & 1997 & 1999 & 2001 & 2003 & 2005 & 2007 \\
\hline Holz (Biomasse) & $\mathrm{TWh}_{\mathrm{th}}$ & 545 & 572 & 581 & 632 & 627 & 622 & 657 & 671 & 689 \\
\hline $\begin{array}{l}\text { Bioma } \\
\text { Wärm } \\
\end{array}$ & $\mathrm{Wh}_{\text {th }}$ & 23,4 & 32,9 & 36,3 & 44,1 & 51,7 & 53,9 & 66,6 & 80,6 & 92,5 \\
\hline e (ohne Bioabfälle) & $\mathrm{TWh}_{\mathrm{th}}$ & 8,4 & 9,6 & 12,1 & 13,4 & 14,7 & 16,5 & 24,2 & 32,4 & 43,1 \\
\hline Geothermisch & $\mathrm{TWh}_{\mathrm{th}}$ & 15,6 & 16,7 & 16,7 & 16,1 & 20,3 & 27,0 & 30,8 & 37,7 & 35,8 \\
\hline davon mit Wär & $\mathrm{TWh}_{\mathrm{th}}$ & & & & & 2,1 & 4,8 & 5,9 & 10,1 & 12,8 \\
\hline Solarwärme & $\mathrm{TWh}_{\text {th }}$ & 2,5 & 3,5 & 5,0 & 6,1 & 7,3 & 9,0 & 10,7 & 12,6 & 16,1 \\
\hline Siedlungsabfälle & $\mathrm{TWh}_{\mathrm{th}}$ & 0,72 & 0,68 & 0,76 & 0,45 & 1,96 & 8,04 & 9,71 & 12,9 & 15,8 \\
\hline Siedlungsabfälle - & $\mathrm{TWh}_{\mathrm{th}}$ & 4,7 & 5,0 & 5,8 & 7,0 & 8,4 & 9,9 & 12,1 & 17,4 & \\
\hline Bioabfälle - KWK & $\mathrm{TWh}_{\mathrm{th}}$ & 2,8 & 2,9 & 3,4 & 3,8 & 5,5 & 5,8 & 6,8 & 9,5 & 11,1 \\
\hline Biogas & $\mathrm{TWh}_{\mathrm{th}}$ & 4,8 & 3,8 & 4,3 & 4,8 & 5,1 & 7,8 & 4,5 & 4,8 & 5,1 \\
\hline Biogas - KWK & $\mathrm{TWh}_{\mathrm{th}}$ & 0,36 & 0,32 & 0,53 & 0,70 & 0,80 & 1,48 & 2,06 & 3,07 & 5,69 \\
\hline davon Deponiegas & $\mathrm{TWh}_{\mathrm{th}}$ & 0,11 & 0,10 & 0,17 & 0,24 & 0,28 & 0,50 & 0,69 & 1,06 & 1,09 \\
\hline davon Klärgas & $\mathrm{TWh}_{\mathrm{th}}$ & 0,09 & 0,09 & 0,16 & 0,22 & 0,21 & 0,39 & 0,54 & 0,84 & 0,84 \\
\hline $\begin{array}{l}\text { davon aus landwirtsc } \\
\text { lichen Stoffströmen }\end{array}$ & $\mathrm{TWh}_{\mathrm{th}}$ & 0,15 & 0,12 & 0,19 & 0,24 & 0,32 & 0,58 & 0,83 & 1,17 & 3,76 \\
\hline
\end{tabular}

Quelle: Basierend auf Held et al. (2010). 
Tabelle 5.4: $\quad$ Entwicklung der installierten Leistung von Anlagen zur Wärmebereitstellung aus erneuerbaren Energien in Europa

\begin{tabular}{l|l|r|r|r|r|r|r|r|r|r}
\hline & Einheit & $\mathbf{1 9 9 1}$ & $\mathbf{1 9 9 3}$ & $\mathbf{1 9 9 5}$ & $\mathbf{1 9 9 7}$ & $\mathbf{1 9 9 9}$ & $\mathbf{2 0 0 1}$ & $\mathbf{2 0 0 3}$ & $\mathbf{2 0 0 5}$ & $\mathbf{2 0 0 7}$ \\
\hline $\begin{array}{l}\text { Biomasse (ohne Bioabfälle) } \\
\text { - KWK }\end{array}$ & $\mathrm{MW}_{\text {th }}$ & $\mathbf{7 . 1 9 0}$ & 7.922 & 9.432 & 9.943 & 12.412 & 11.893 & 14.854 & 20.714 & 26.394 \\
\hline Siedlungsabfälle - KWK & $\mathrm{MW}_{\text {th }}$ & 3.304 & 4.532 & 4.767 & 5.305 & 5.896 & 6.825 & 9.038 & 13.158 & \\
\hline \begin{tabular}{l} 
Geothermisch \\
\hline davon mit Wärmepumpe
\end{tabular} & $\mathrm{MW}_{\text {th }}$ & & & & 763 & 2.083 & 4.628 & 5.800 & 8.653 & 11.223 \\
\hline Bioabfälle - KWK & $\mathrm{MW}_{\text {th }}$ & 2.526 & 3.245 & 3.383 & 3.787 & 4.211 & 4.795 & 6.598 & 9.588 & 9.160 \\
\hline Biogas - KWK & $\mathrm{MW}_{\text {th }}$ & 533 & 564 & 592 & 657 & 888 & 1.471 & 1.681 & 2.713 & 4.866 \\
\hline $\begin{array}{l}\text { davon aus landwirtschaft- } \\
\text { lichen Stoffströmen }\end{array}$ & $\mathrm{MW}_{\text {th }}$ & 230 & 236 & 242 & 256 & 331 & 574 & 633 & 992 & 3.129 \\
\hline davon Deponiegas & $\mathrm{MW}_{\text {th }}$ & 169 & 183 & 192 & 230 & 316 & 509 & 597 & 961 & 1.002 \\
\hline davon Klärgas & $\mathrm{MW}_{\text {th }}$ & 134 & 145 & 158 & 171 & 242 & 388 & 451 & 760 & 735 \\
\hline
\end{tabular}

Quelle: Basierend auf Held et al. (2010).

Tabelle 5.5: $\quad$ Zuordnung von Optionen zur Wärmebereitstellung zu ihrem aktuellen Entwicklungsstadium (Stand: 2007)

\begin{tabular}{|c|c|c|c|c|}
\hline Energieträger bzw. Technologie (alphabetisch) & $\begin{array}{c}\text { Frühes Stadium } / \\
\text { Nische }\end{array}$ & $\begin{array}{c}\text { Aufstiegl } \\
\text { Wachstum }\end{array}$ & $\begin{array}{c}\text { Reifel } \\
\text { Sättigung }\end{array}$ & Rückgang \\
\hline \multicolumn{5}{|l|}{ Heizkraftwerke und Fernheizwerke nach (BMWi 2010) } \\
\hline Braunkohle & & & & $\mathrm{x}$ \\
\hline Gase & & $\mathrm{x}$ & & \\
\hline Mineralöl & & & & $\mathrm{x}$ \\
\hline Müll u.a. & & $\mathrm{x}$ & & \\
\hline Steinkohle & & & $\mathrm{x}$ & \\
\hline \multicolumn{5}{|l|}{ Wärmeerzeuger nach BDH } \\
\hline Biomasse (fest) & & $\mathrm{x}$ & & \\
\hline Brennwertkessel, Gas & & & $\mathrm{x}$ & $(\mathrm{X})$ \\
\hline Brennwertkessel, Öl & & & $\mathrm{x}$ & \\
\hline Niedertemperaturkessel (Gas, Öl) & & & & $\mathrm{x}$ \\
\hline Thermen & & & & $\mathrm{x}$ \\
\hline Wärmepumpen & & $\mathrm{x}$ & & \\
\hline $\begin{array}{l}\text { Erneuerbare Energien nach (Held et al. 2010; vgl. Tabelle } 5.4 \text { und Tabelle } \\
\text { 5.3) }\end{array}$ & & $\mathrm{x}$ & & \\
\hline
\end{tabular}

Anmerkungen: Die aktuell als zutreffend erachtete Entwicklungsphase ist durch „X“ gekennzeichnet. Bei nicht eindeutiger Zuordnung ist die als weniger wahrscheinlich angesehene Phase durch „(X)“ gekennzeichnet.

\subsubsection{Indikatoren-basierte Zuordnung}

In diesem Abschnitt wird beispielhaft aufgezeigt, wie Technologien den Phasen nach Meyer-Krahmer und Dreher (2004) zugeordnet werden können. Die folgende Darstellung basiert dabei weitgehend auf den von Jochem et al. (2009) durchgeführten Arbeiten, deren übergeordnetes Ziel darin besteht, die Effizienz der Verwendung von Forschungsmitteln im Energiebereich zu erhöhen und die Prioritätensetzung zu unterstützen. Die Autoren erheben jedoch keinen Anspruch auf eine vergleichende Bewertung einzelner Projektvorschläge. Vielmehr werden techno-ökonomische Bewertungen, Technologiezyklus- und Innovationssystem-Analysen miteinander kombiniert, um davon ausgehende Empfehlungen hinsichtlich geeigneter Förderinstrumente für bestimm- 
te Technologien oder Technologie-Gruppen abzuleiten. Im Rahmen der vorliegenden Problemstellung ist insbesondere von Interesse, wie Jochem et al. (2009) Technologien den Phasen im Modell nach Meyer-Krahmer und Dreher (2004) zuordnen, um davon ausgehend Kriterien-Gewichtungen in Abhängigkeit des Entwicklungsstandes einzelner Alternativen anwenden zu können. Demnach basiert die Zuordnung der Technologien auf bibliometrischen, patent- und diffusionsanalytischen Indikatoren. Während Patent-Indikatoren vor allem erfinderische Tätigkeiten, also eher marktorientierte Forschung widerspiegeln, dienen Indikatoren auf Basis von Veröffentlichungen eher der Abbildung wissenschaftlicher Forschungsaktivitäten, die in der Regel stärker Grundlagen-orientiert sind. Die verwendete Methodik wird im Folgenden anhand der von Jochem et al. (2009) durchgeführten Fallbeispiele für die Technologien Brennstoffzelle und der $\mathrm{CO}_{2}$-freie Kraftwerke dargestellt.

\subsubsection{Brennstoffzellen-Technologien}

Es existieren mehrere Typen von Brennstoffzellen, die sich teilweise in unterschiedlichen Entwicklungsstadien befinden (vgl. Abschnitt 3.1.5). Brennstoffzellen in MikroKWK-Anwendungen zeichnen sich vor allem durch eine hohe Stromkennzahl, einen hohen Gesamtwirkungsgrad sowie geringe Emissionen im Betrieb aus. Die bei Jochem et al. (2009) genauer untersuchten Typen umfassen die PEMFC (Polymer Electrolyte Membrane Fuel Cell), MCFC (Molten Carbonate Fuel Cell) und SOFC (Solid Oxide Fuel Cell), die bereits alle zumindest als Prototypen verfügbar sind. Wesentliche Herausforderungen im Hinblick auf eine breite Diffusion der Technologien am Markt betreffen die hohen Anfangsinvestitionen sowie die geringe Lebensdauer. Dabei wird die PEMFC als die aussichtsreichste Variante erachtet und daher von Jochem et al. (2009) detaillierter untersucht.

Ausgangspunkt der Zuordnung zu den Technologiezyklus-Phasen bei Jochem et al. (2009) ist eine Matrix, in der die charakteristischen Ausprägungen der verwendeten Indikatoren je Technologiezyklus-Phase hinterlegt sind (Tabelle 5.6). Um eine Technologie einer Phase zuzuordnen, werden zunächst deren Ausprägungen je Indikator bestimmt, etwa durch Analysen basierend auf Datenbanken von Patentämtern. Auf dieser Grundlage werden die Felder in der Matrix mit den für die betrachtete Technologie zutreffenden Merkmals-Ausprägungen je Indikator markiert. In Tabelle 5.6 sind die zutreffenden Felder für die Brennstoffzelle schwarz hinterlegt. Grau hinterlegte Indikatoren signalisieren, dass diese für Brennstoffzellen nicht bestimmt werden konnten. Die Analyse der Patente zeigt einen steigenden Trend für Brennstoffzellen im Vergleich zur Gesamtzahl der beim Europäischen Patentamt (EPO) eingereichten Patente. Für die PEMFC wird auf dieser Basis ein besonders hohes Aktivitätsniveau festgestellt, wobei auch die Anzahl der Veröffentlichungen weiter ansteigt. Dies deutet darauf hin, dass 
viele wissenschaftliche Fragestellungen weiterhin ungelöst sind, oder aber, dass in der nahen Zukunft technische Verbesserungen basierend auf neuen Forschungsergebnissen zu erwarten sind. Ähnliche Schlussfolgerungen werden auch aus der Patentanalyse für die SOFC abgeleitet, während für die MCFC aufgrund insgesamt sehr geringer Patenzahlen keine aussagekräftigen Folgerungen möglich sind.

Die Marktanalyse für Brennstoffzellen zeigt, dass weltweit bereits einige Pilot- und Demonstrationsprojekte in unterschiedlichen Anwendungsbereichen realisiert wurden. Insbesondere die Anzahl der installierten PEMFCs ist seit Ende der 1980er Jahre bis zum Ende des Betrachtungszeitraums kontinuierlich gestiegen. Die Menge der Anbieter der entsprechenden Systeme ist seit den 1990er Jahren stark gewachsen. Dabei ist der deutsche Markt rückblickend durch mehrere Wellen verfrühter Euphorie charakterisiert. Diese sind durch sehr ehrgeizige Vorhersagen gekennzeichnet, welche jedoch nicht realisiert werden konnten. In Interviews mit relevanten Akteuren zeichnete sich ab, dass inzwischen ein realistischeres Bild hinsichtlich der zukünftigen Entwicklung von Brennstoffzellen vorherrscht. Dabei werden Potenziale vor allem in wachsenden Nischenmärkten gesehen. Ein Massenmarkt mit gefestigten Strukturen existiert für Brennstoffzellen hingegen noch nicht. In vielen Bereichen ist die Entwicklung weniger durch unabhängige Märkte, sondern vielmehr durch eine relativ kleine Anzahl an Demonstrationsprojekten gekennzeichnet. Markennamen kommen bisher nur in sehr geringem Umfang vor, was ebenfalls darauf hinweist, dass sich noch kein breiter Markt gebildet hat. Insgesamt schlussfolgern Jochem et al. (2009) aus der IndikatorenAnalyse für Brennstoffzellen, dass diese sich weiterhin in einer (späten) Phase der Euphorie befinden. 


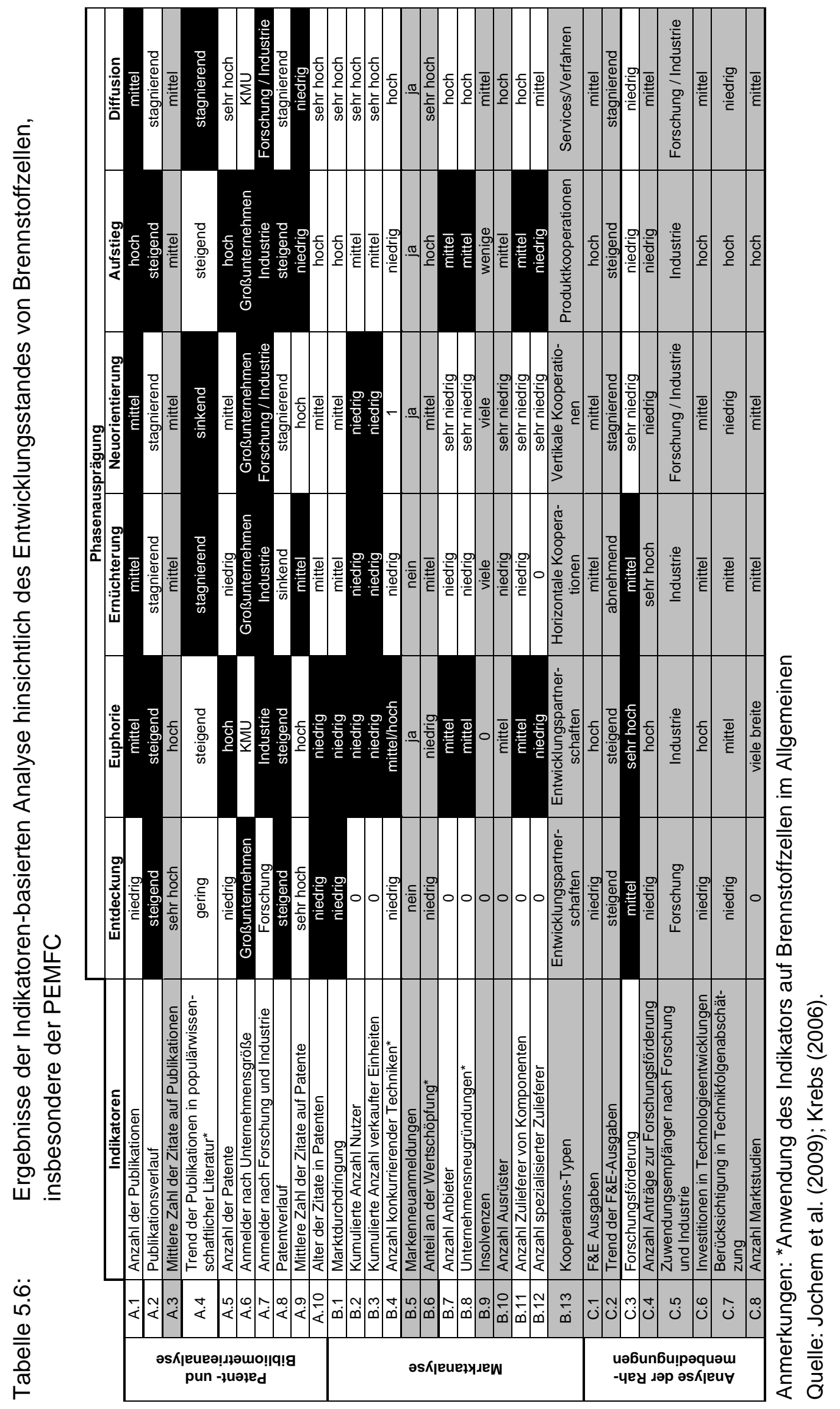




\subsubsection{2 $\mathrm{CO}_{2}$-freie Kraftwerke}

In den letzten Jahren haben Technologien zur Abscheidung und Speicherung von $\mathrm{CO}_{2}$ (CCS) zunehmendes Interesse erfahren, da diese eine umweltfreundlichere Nutzung des Energieträgers Kohle versprechen (vgl. auch Abschnitt 3.1.1.1). Ein Vorteil der Technologie im Vergleich zu anderen Optionen $\mathrm{CO}_{2}$-armer Stromerzeugung wird darin gesehen, dass bei einer Implementierung die bestehenden Strukturen der Stromversorgung weitgehend beibehalten werden können (Jochem et al. 2009). Prinzipiell wird CCS als realisierbar angesehen, jedoch sind weiterhin Pilot- und Demonstrationsprojekte in Verbindung mit Grundlagenforschung erforderlich. Denn sowohl die technische Machbarkeit als auch die ökologischen Auswirkungen der großtechnischen Anwendung sind noch ungewiss. Von diesen Faktoren ist wiederum die wirtschaftliche Machbarkeit betroffen. Auch wenn CCS nicht als revolutionär neue Option gilt, befindet sich die Technologie dennoch in einem sehr frühen Entwicklungsstadium. Dabei ist noch unklar, welches der in Entwicklung befindlichen technologischen Konzepte sich zur Abscheidung und Speicherung von $\mathrm{CO}_{2}$ durchsetzen wird. Die größte Herausforderung besteht darin, den zusätzlichen Energiebedarf der Abscheidung auf ein akzeptables Niveau zu senken. Weiterhin muss die Zuverlässigkeit und Sicherheit der Abscheidung und Speicherung in technischer wie auch ökologischer Hinsicht unter Beweis gestellt werden.

Die Indikatoren, die in Abschnitt 5.2.3.1 für Brennstoffzellen dargestellt sind, konnten von Jochem et al. (2009) für CCS nur in sehr begrenztem Maße erhoben werden. Marktstudien, die sich speziell auf CCS beziehen, standen zumindest zum Zeitpunkt der Durchführung der Untersuchung nicht zur Verfügung. Markennamen werden bisher nicht mit CCS in Verbindung gebracht, jedoch ist diesbezüglich anzumerken, dass im Bereich der Kraftwerkstechnologien Markennamen grundsätzlich keine große Rolle spielen. Die Patenanalyse stellt sich für CCS aus folgenden Gründen als wenig praktikabel heraus (Jochem et al. 2009):

- Bei Anwendung von CCS findet keine radikale Änderung im Konzept der Energieumwandlung statt.

- CCS basiert auf konventionellen Technologien, die bereits in anderen Industriezweigen entwickelt wurden.

- F\&E-Arbeiten zur Abscheidung und die daraus resultierenden Patente können nicht immer von anderen Entwicklungsanstrengungen abgegrenzt werden (zum Beispiel IGCC). 
- $\mathrm{CO}_{2}$-freie Kraftwerke bestehen aus unterschiedlichen Technologien und diese wiederum aus mehreren technologischen Komponenten, so dass sehr viele Patentklassen betroffen sind.

- Diejenigen Patentklassen, in denen CCS-Technologien vorkommen, enthalten viele andere Technologien, die für CCS nicht relevant sind.

- Einige der Anmelder in den relevanten Patentklassen versuchen den tatsächlichen Zweck ihrer Entwicklung zu verschleiern.

Aus den oben genannten Gründen ist eine Analyse von CCS nach relevanten Patentklassen alleine nicht ausreichend, um aussagekräftige Ergebnisse zu erhalten. Vielmehr müssen durch Stichwortsuchen in Zusammenfassungen irrelevante Patente herausgefiltert werden. Diese Möglichkeit ist jedoch sehr aufwändig und wird von Jochem et al. (2009) nur beispielhaft dargestellt. Die Analyse von Publikationen zu CCS stellt sich nach Jochem et al. (2009) weniger problematisch dar. Nach einer bisher maximalen Anzahl an Veröffentlichungen zu CCS in der zweiten Hälfte der 1990er Jahre folgte ein kleiner Rückgang um das Jahr 2000. Durch die im Rahmen des Projektes interviewten Akteure wurde bisher jedoch keine Phase der Desillusionierung angedeutet. Die Anzahl der insgesamt identifizierten wissenschaftlichen Veröffentlichungen ist relativ gering. Jochem et al. (2009) weisen diesbezüglich darauf hin, dass durch Veröffentlichungen in international anerkannten wissenschaftlichen Zeitschriften nicht alle relevanten Publikationen zu CCS erfasst werden, da in diesem Bereich eine besondere Dynamik bei Konferenzbeiträgen zu beobachten ist. Eine Analyse der wichtigsten Konferenzbeiträge zeigt folglich auch ein insgesamt höheres Aktivitätsniveau.

Basierend auf Interviews wird bei Jochem et al. (2009) festgestellt, dass die Aktivitäten in Zusammenhang mit CCS seit dem Jahr 2005 kontinuierlich angestiegen sind, wobei die Diversifizierung der unterschiedlichen Ansätze und der industriellen Akteure weitgehend unverändert blieb. Dadurch, dass in den Jahren 2005 und 2006 mehrere Projekte zur großtechnischen Demonstration angekündigt wurden, beschleunigte sich die Entwicklungsgeschwindigkeit. Diese Ankündigungen stellen darüber hinaus erste Anzeichen für das Entstehen eines realen Marktes für CCS dar. Die Art der angekündigten Projekte lässt jedoch vermuten, dass es sich weiterhin um Prototypen handelt und ein nicht zu vernachlässigendes Restrisiko eines Scheiterns besteht. Darüber hinaus sind die meisten der Interview-Partner der Ansicht, dass $\mathrm{CO}_{2}$-freie Kraftwerke frühestens im Jahr 2020 kommerziell verfügbar sein werden. Daher schlagen Jochem et al. (2009) auf Basis der Interviews und der (wenigen) verfügbaren quantitativen Indikatoren eine Einordnung in Phase 2 (Euphorie) vor. 


\subsection{Zusammenfassung der Technologiezyklus-Analyse}

Um Kriterien-Gewichtungen im Rahmen der multikriteriellen Entscheidungsunterstützung an den Entwicklungsstand von Energietechnologien anpassen zu können, wurden basierend auf der vorangegangenen Analyse Energieträger bzw. Technologien zur Strom- bzw. Wärmebereitstellung den Phasen im Technologiezyklus nach Abbildung 5.4 zugeordnet. Der Entwicklungsverlauf verfügbarer Kraftwerkstypen konnte mit Hilfe von Platts (2007b) über einen langfristigen Zeitraum von mehr als 100 Jahren dargestellt werden. Dabei zeigt die Analyse für viele Optionen zur Stromerzeugung einen deutlichen S-Kurvenverlauf, insbesondere für solche, die bereits seit langer Zeit eingesetzt werden. Offensichtlich sind Schlussfolgerungen bezüglich des Entwicklungsstadiums vor allem für diejenigen Optionen schwierig, für die nur über einen geringen Zeitraum Daten zur Verfügung stehen bzw. von denen bisher nur eine geringe Anzahl installiert wurde. Der erste Fall betrifft insbesondere Optionen zur Wärmebereitstellung, die in der Literatur und in verfügbaren Datenbanken bisher weit weniger Beachtung finden als Optionen zur Stromerzeugung. Im zweiten Fall sind Sonderformen von Energieträgern betroffen oder aber neue Energietechnologien. Für letztere wird aufgezeigt, wie eine Einordnung in frühe Entwicklungsstadien auf der Grundlage Indikatorenbasierter Analysen und Interviews stattfinden kann. Grenzen dieser Methodik werden insbesondere am Beispiel der CCS-Technologie aufgezeigt.

In den meisten Fällen decken sich die Schlussfolgerungen aus der TechnologiezyklusAnalyse mit den Erwartungen, so dass die Notwendigkeit der durchgeführten Analyse gegebenenfalls fragwürdig scheint. Andererseits ist keine Arbeit bekannt, in der der Entwicklungsverlauf unterschiedlicher Optionen zur Energiebereitstellung systematisch einander gegenüber gestellt und analysiert und eine Zuordnung zu Phasen im Technologiezyklus vorgenommen wird. Mit der durchgeführten Analyse wird die notwendige Ausgangsbasis für Kriterien-Gewichtungen in Abhängigkeit unterschiedlicher technologischer Entwicklungsphasen geschaffen. Hinsichtlich der Abgrenzung von und Einordnung in unterschiedliche Phasen sind jedoch weitere Forschungsarbeiten erforderlich. Dabei sind von Fall zu Fall der Nutzen einer präzisen Einordnung einerseits und der damit verbunden Aufwand zur Daten-Ermittlung andererseits abzuwägen. Für die vorliegende Arbeit wird die Einordnung von Energietechnologien in Entwicklungsphasen, wie sie in diesem Kapitel durchgeführt wurde, als ausreichend angesehen. Im Rahmen einer multikriteriellen Analyse können dabei Sensitivitätsanalysen hinsichtlich der Zuordnung zu Entwicklungsphasen und der damit verbundenen Gewichtung der Kriterien dazu dienen, fehlgeleiteten Schlussfolgerungen vorzubeugen. Bevor die Anwendung der vorgeschlagenen Methode anhand von Fallbeispielen illustriert wird, erläutert das folgende Kapitel deren Implementierung in einem multikriteriellen Entscheidungsunterstützungssystem. 


\section{$6 \quad$ Konzeption eines multikriteriellen Entscheidungsunterstützungssystems}

Gemäß der in Kapitel 2.4 formulierten Fragestellung besteht das Ziel dieser Arbeit darin, Entscheidungen in Zusammenhang mit der Prioritätensetzung bei mehreren zur Wahl stehenden Energietechnologien zu unterstützen. Das zu entwickelnde Entscheidungsunterstützungssystem (EUS) zielt dabei nicht auf eine konkrete Versorgungsaufgabe ab, sondern soll durch ein ausreichendes Maß an Flexibilität die Anwendung in unterschiedlichen Problemstellungen im Kontext der Energieversorgung ermöglichen. Angestrebte Anwendungsbereiche schließen die Auswahl von Energietechnologien für eine konkrete Versorgungsaufgabe ein, aber zum Beispiel auch die Prioritätensetzung in der nationalen Forschungsförderung. Im Folgenden wird ein entsprechendes EUS entwickelt. Die Konzeption orientiert sich dabei an den in Abbildung 4.1 (S. 62) dargestellten Schritten, die von der Definition der Problemstellung bis zur Implementierung reichen.

\subsection{Definition der Problemstellung}

Die Anforderungen an das EUS werden durch die Problemstellungen determiniert, in deren Kontext es angewendet werden soll. Grundsätzlich besteht das Ziel dieser Arbeit darin, Energietechnologien im Hinblick auf zukünftige Investitionen und Forschungsaktivitäten zu bewerten (vgl. Kapitel 2.4). Diese Definition einer Problemstellung ist sehr breit gefasst und impliziert ein breites Anwendungsspektrum. Neben der aus diesem Grunde erforderlichen Flexibilität des EUS soll darüber hinaus die implementierte Bewertungsmethode dem Entwicklungsstand einzelner Technologien Rechnung tragen. Dazu ist eine Anwendung zu realisieren, die eine Zuordnung von Alternativen zu den Phasen im Technologiezyklus und die entsprechende Anpassung der KriterienGewichtungen erlaubt. Folglich wird das EUS so konzipiert, dass etablierte und/oder innovative Technologien zur Bereitstellung von Strom bzw. Wärme miteinander verglichen werden können. In nachgelagerten Schritten kann die Bewertung einer Kombination von Technologien im Sinne eines Versorgungssystems überprüft werden. Spezifische Problemstellungen werden im Rahmen der Anwendung des EUS auf Fallbeispiele in Kapitel 7 dargestellt.

\subsection{Vorauswahl der Alternativen}

Grundlage für eine Entscheidungsunterstützung ist eine Vorauswahl an zu bewertenden Alternativen. Diese Vorauswahl der zu bewertenden Energietechnologien wird durch den Untersuchungsrahmen und die konkrete Fragestellung eingegrenzt. In Abschnitt 4.2.2 werden Methoden zur Identifizierung relevanter Alternativen aufgezeigt. 
Beispielsweise können Befragungen von Experten im Energiebereich dazu beitragen, vorab solche Optionen auszuschließen, die im Hinblick auf die konkrete Problemstellung keine realistische Alternative darstellen bzw. deren praktische Umsetzbarkeit aufgrund technischer, wirtschaftlicher oder anderer Hemmnisse als höchst unwahrscheinlich angesehen wird.

Abbildung 6.1: Experteneinschätzung zukünftiger Potenziale von Technologien im Hinblick auf deren Beitrag zur Energieversorgung in Deutschland bis zum Jahr 2030

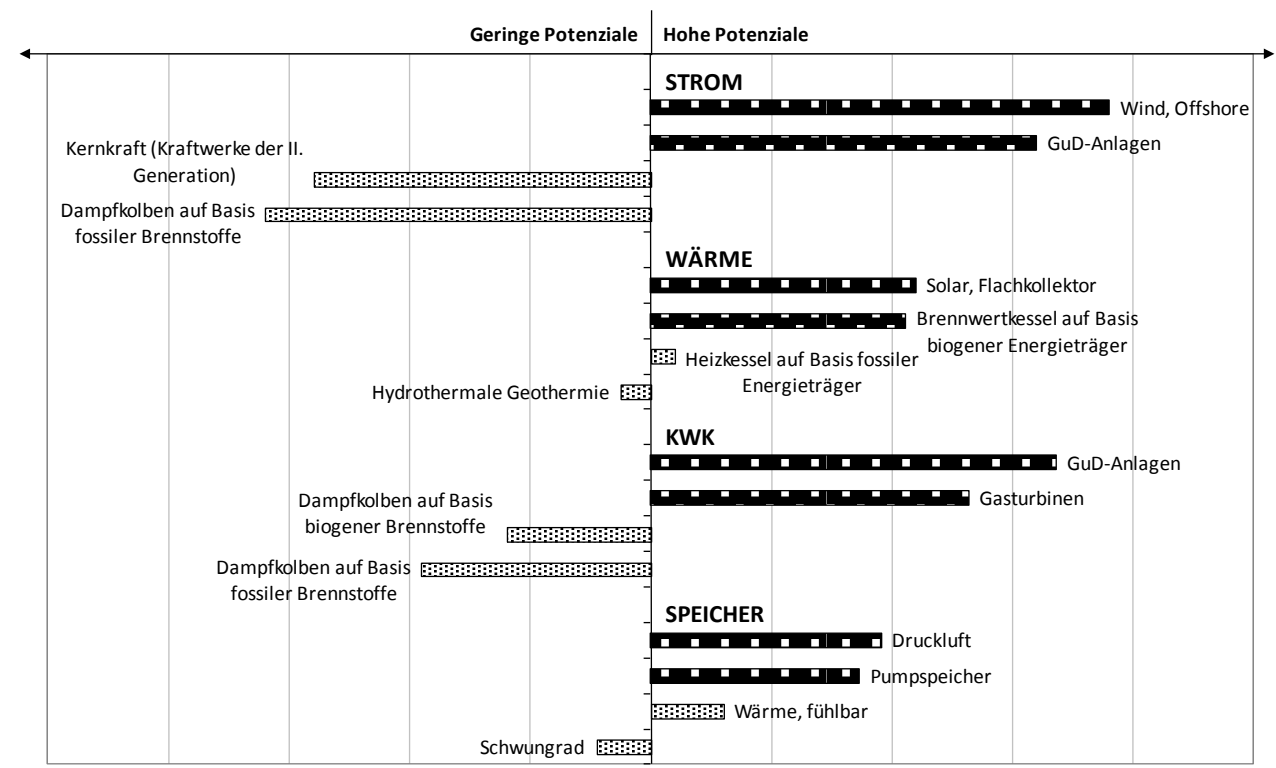

Anmerkung: Die Grafik zeigt für die Kategorien Strom, Wärme, KWK und Speicher jeweils die beiden Technologien, die im Mittel am besten bzw. am schlechtesten bewertet wurden. Im Fragebogen konnten die Befragten (Anzahl: 13) auf einer Skala in Anlehnung an Schulnoten ( 1 = sehr gut bis $6=$ ungenügend) ihre Einschätzung durch Ankreuzen angeben. Für eine anschauliche Darstellung wurden die Mittelwerte der Ergebnisse je Technologie zwischen -1 (entspricht Note 6) und 1 (entspricht Note 1) normiert.

Quelle: Bartels et al. (2008).

So werden zum Beispiel bei Bartels et al. (2008) basierend auf einer Befragung von Entscheidungsträgern in der Energiewirtschaft die Potenziale einiger Technologien für die zukünftige Energieversorgung in Deutschland als gering eingeschätzt (zum Beispiel Kernkraftwerke der zweiten Generation, Dampfkolben zur Strom- bzw. KWK-Erzeugung und geothermische Hydrothermal-Verfahren zur Wärmebereitstellung, vgl. Abbildung 6.1). Als besonders vielversprechend wird hingegen die Stromerzeugung in Offshore-Windenergieanlagen sowie die Strom- bzw. KWK-Erzeugung in GuD-Anlagen angesehen. Für die Bereitstellung von Nutzwärme wird insbesondere den Flachkollektoren zur Nutzung von Solarwärme und den Brennwertkesseln auf Basis biogener Brennstoffe ein relativ hohes Potenzial bescheinigt. Auch das Potenzial von Gasturbi- 
nen zur Nutzung im KWK-Betrieb wird weiterhin relativ hoch eingeschätzt. Basierend auf diesen Einschätzungen kann sich der weitere Entscheidungsprozess auf die vielversprechendsten Energietechnologien fokussieren. Jedoch sollte die Vorauswahl der Alternativen immer vor dem Hintergrund der spezifischen Problemstellung, den im konkreten Fall vorliegenden Potenzialen und Hemmnissen sowie den exogen vorgegebenen Rahmenbedingungen überprüft werden.

\subsection{Auswahl der Kriterien}

Der dritte Schritt umfasst die Auswahl der Kriterien zur Bewertung der ausgewählten Alternativen. Eine Vielzahl an Bewertungsgrößen ist denkbar, die in unterschiedlichen Entscheidungssituationen im Energiebereich eine Rolle spielen. Die Bewertungskriterien sollten dabei stets in Bezug zu den spezifischen Zielsetzungen der beteiligten Akteure definiert werden und hängen daher von der konkreten Problemstellung ab. Bei Bartels et al. (2008) werden auf Basis der Befragung von Experten im Energiebereich die Wirtschaftlichkeit, Umweltaspekte und Nachhaltigkeit sowie Aspekte der Versorgungssicherheit als Kriterien von besonderer Bedeutung für die multikriterielle Bewertung von Energietechnologien identifiziert (Abbildung 6.2).

Abbildung 6.2: Relevanz von Kriterien zur Bewertung von Energietechnologien basierend auf einer Befragung von Entscheidungsträgern in der Energiewirtschaft

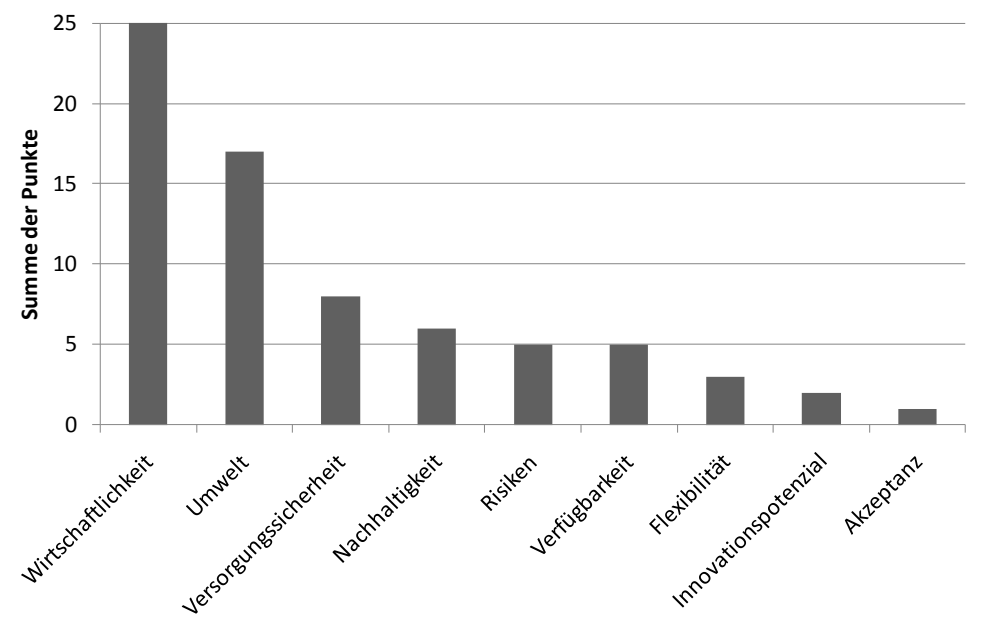

Anmerkung: Jedem Befragten war freigestellt, drei Kriterien in der Reihenfolge ihrer Relevanz zu nennen. In der Auswertung wurden dem erstgenannten Kriterium drei Punkte, dem zweitgenannten zwei und dem letztgenannten ein Punkt zugerechnet; Anzahl der Befragten: 13 Personen.

Quelle: Bartels et al. (2008). 
Eine Analyse der aus der Literatur bekannten Anwendungen multikriterieller Methoden im Energiebereich (vgl. Kapitel 4.2) erlaubt eine detailliertere Differenzierung und Konkretisierung von Kriterien zur Bewertung von Energietechnologien. In Abbildung 6.3 werden die in der Literatur genannten Kriterien in acht Gruppen zusammengefasst. In den meisten Anwendungsfällen schließt die multikriterielle Bewertung Aspekte der Wirtschaftlichkeit ein, indem Kosten oder betriebswirtschaftliche Kenngrößen analysiert werden. Auch Umweltauswirkungen finden in den meisten Veröffentlichungen Berücksichtigung, zum Beispiel indem die spezifischen Emissionen der Stromerzeugung oder ökobilanzielle Wirkungskategorien als Kriterien dienen. Die Versorgungssicherheit wird häufig mit der Verfügbarkeit der Energieträger auf lokaler bzw. globaler Ebene in Verbindung gebracht, zum Beispiel indem die Importquote je Energieträger als Kriterium dient. Zudem finden in diesem Zusammenhang auch solche Kriterien Anwendung, die ein Maß für die Zuverlässigkeit von Technologien hinsichtlich der Kontinuität und Vorhersehbarkeit der Leistungserbringung darstellen. Durch Kriterien, die sich auf kurzfristige Reaktionsgeschwindigkeiten von Technologien im System oder auf benötigte Zeiträume zur Realsierung bzw. Anpassung von Alternativen beziehen, wird Aspekten der Flexibilität Rechnung getragen. In einigen Veröffentlichungen werden soziale Aspekte berücksichtigt, beispielsweise indem Maßgrößen für die soziale Akzeptanz, Beschäftigungseffekte, Gesundheitsauswirkungen und Sicherheitsrisiken einbezogen werden. Aspekte der Kompatibilität, die sich auf die Passfähigkeit der Alternativen mit den vorherrschenden Rahmenbedingungen beziehen, können meist nur auf Basis einer qualitativen Einstufung berücksichtigt werden. Technologische Merkmale können als Kriterien dazu dienen, die grundsätzliche Eignung der Alternativen als Lösung für die konkrete Problemstellung zu beurteilen. Grundsätzlich ist zu beachten, dass eine eindeutige Abgrenzung der in Abbildung 6.3 dargestellten Kriterien-Gruppen nicht immer möglich ist und auch andere Arten der Kategorisierung denkbar sind. Beispielsweise können die Eigenschaften der Technologie Auswirkungen auf die Wirtschaftlichkeit oder auf die Versorgungssicherheit ausüben. Zudem ist eine weitere Untergliederung der zweiten dargestellten Ebene in zusätzliche Kriterien und TeilIndikatoren möglich. Eine uneingeschränkte Berücksichtigung aller in Abbildung 6.3 erfassten Kriterien ist nicht zielführend, da nicht in allen Problemstellungen die gleichen Zielsetzungen von Bedeutung sind. Des Weiteren müssen der Nutzen der Berücksichtigung zusätzlicher Kriterien einerseits und der Aufwand zur Datenerhebung andererseits gegeneinander abgewogen werden. Dabei kann Abbildung 6.3 Entscheidungsträger vor dem Hintergrund konkreter Problemstellungen im Energiebereich bei der Auswahl relevanter Kriterien unterstützen. 
Abbildung 6.3: Kriterien zur Bewertung von Energietechnologien

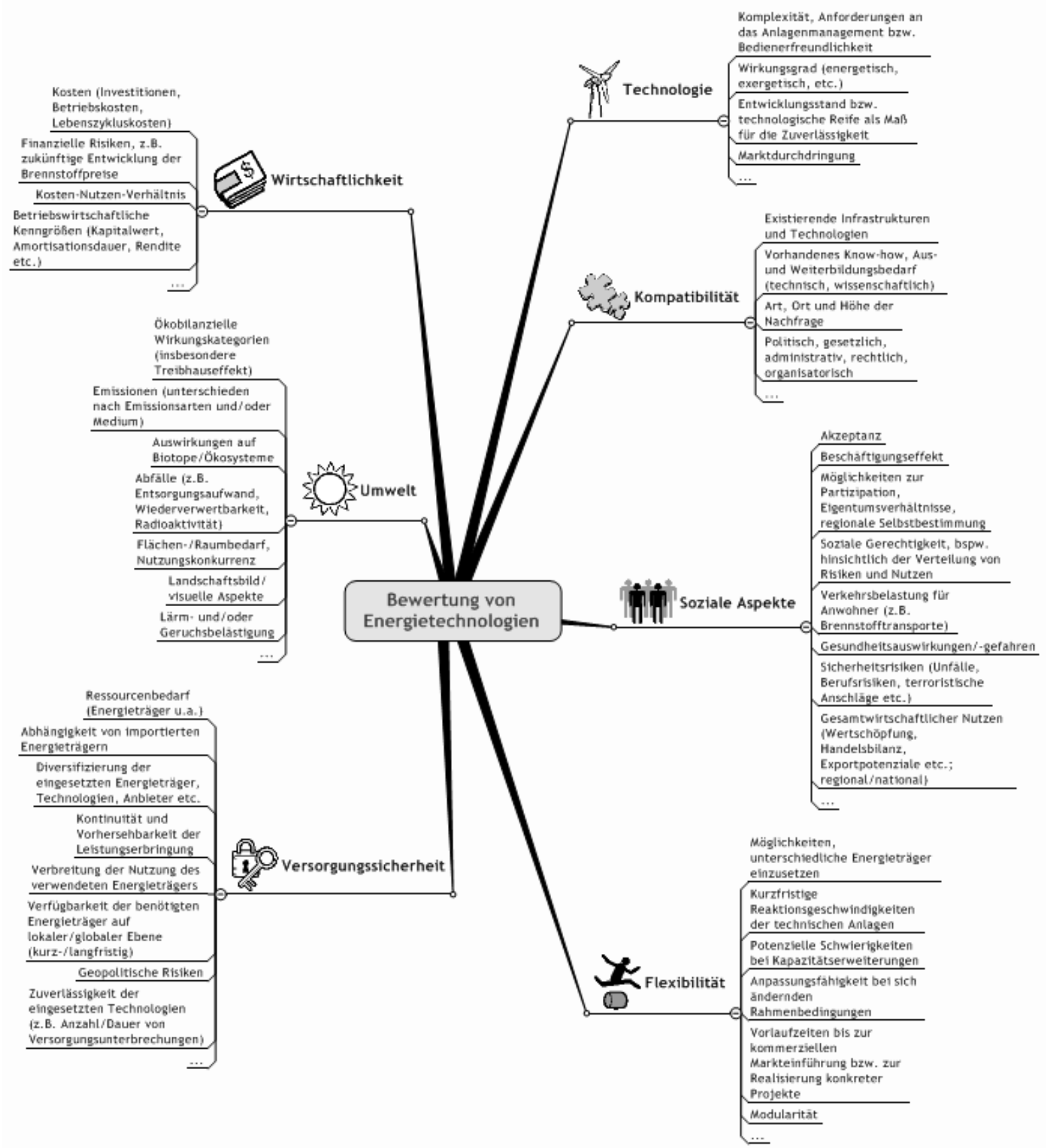

Anmerkungen: Die Abbildung erhebt keinen Anspruch auf Vollständigkeit. Die Darstellung impliziert keine Rangfolge oder Gewichtung der genannten Kriterien.

\subsection{Bestimmung der Kriterien-Ausprägungen}

Methoden zur Bestimmung von Kriterien-Ausprägungen bzw. zur Bewertung von Alternativen hinsichtlich einzelner Kriterien werden in Abschnitt 4.2.4 aufgezeigt. Um den Aufwand der Datenerhebung zu reduzieren, bieten sich grundsätzlich existierende Da- 
tenbanken an, welche die benötigten Informationen zur Verfügung stellen. Beispielsweise stellen Datenbanksysteme wie Ecoinvent (ecoinvent Centre 2007) und GEMIS (vgl. Fritsche und Schmidt 2008) umfangreiche Lebenszyklusinventare zur ökobilanziellen Bewertung bereit. Da jedoch für eine multikriterielle Bewertung in der Regel nicht nur Bewertungsgrößen einer sondern mehrerer Wissensdisziplinen und Fachgebiete eine Rolle spielen, ist es meist erforderlich, Daten aus unterschiedlichen Quellen (Datenbanken, Literatur, Experteneinschätzungen etc.) zusammenzuführen. Daher wurde im Rahmen der Dissertation als Grundlage für die multikriterielle Bewertung von Energietechnologien die Datenbank „Multidimensionale Technikbewertung“ (MTB) entwickelt, in der Daten zu Energietechnologien aus unterschiedlichen Quellen zusammengestellt sind. Die MTB-Datenbank enthält Datensätze zu einzelnen Energietechnologien sowie Informationen zu relevanten Rahmendaten wie zum Beispiel Brennstoffpreisen. Ein Großteil der Daten wurde im Rahmen des Forschungsprojektes „Multidimensionale Technikbewertung" ermittelt (vgl. Bartels et al. 2008). Mit Hilfe von Abfragen können die Ausprägungen eines vordefinierten Sets an Kriterien berechnet werden. Dabei finden insbesondere Aspekte der Wirtschaftlichkeit, Umweltauswirkungen und technologische Merkmale Berücksichtigung. Somit stehen für die implementierten Technologien und Kriterien die notwendigen Basis-Informationen zur Durchführung einer multikriteriellen Analyse bereit. Für spezifische Problemstellungen kann flexibel auf die Daten zugegriffen werden (vgl. auch Abschnitt 6.9).

\subsection{Methoden zur Kriterien-Gewichtung}

Sind für die einzelnen Kriterien die Ausprägungen bestimmt, müssen die Kriterien gegeneinander gewichtet werden. In Abschnitt 4.2.5 werden die im Energiebereich angewendeten Methoden zur Kriterien-Gewichtung vorgestellt. Im hier entwickelten EUS sollen dem Entscheidungsträger mehrere Methoden angeboten werden, die es ihm ermöglichen, seine subjektiven Wertvorstellungen auf einer ausreichend differenzierten Skala in Form von Kriterien-Gewichten auszudrücken und die Auswirkungen unterschiedlicher Gewichtungen zu untersuchen. Daher bietet sich eine Einbindung des Software-Tools Web-HIPRE (Hierarchical Preference analysis on the World Wide Web) an (vgl. auch Abschnitt 4.2.9). Dieses Tool wurde mit dem Ziel entwickelt, unterschiedliche Phasen im Entscheidungsprozess (Problemstrukturierung, Priorisierung, Ergebnis- und Sensitivitätsanalyse) durch moderne, interaktive Computersysteme zu unterstützen. Das Java-Applet implementiert die am weitesten verbreiteten Gewichtungsmethoden (AHP, SMART, SWING, SMARTER und Wertfunktionen). Sowohl die implementierten MAVT-Verfahren als auch der AHP basieren auf einem Entscheidungsbaum mit Zielen und Attributen (in der Terminologie der MAVT) bzw. einem hierarchisch strukturierten Entscheidungsmodell (in der Terminologie des AHP) (vgl. auch 
die Abschnitte 4.2.5 und 4.2.6.1). Grundsätzlich geht Web-HIPRE davon aus, dass eine additive Nutzenfunktion angewendet werden kann. Unabhängig von der Gewichtungsmethode werden die Ergebnisse der Bewertung zwischen 0 („am schlechtesten“) und 1 („am besten“) normiert. Gewichtungs-Faktoren werden im Sinne der nutzentheoretischen Modelle so interpretiert, dass sie die relative Bedeutung einer Änderung von der schlechtesten zur besten Kriterien-Ausprägung darstellen. Die Gewichte der Attribute können direkt durch Vergabe von Punkten abgeleitet werden. Weiterhin stehen grafische Benutzeroberflächen zur Anwendung von SMART, SWING, SMARTER und AHP zur Verfügung. Innerhalb einer Kriterien-Hierarchie können an unterschiedlichen Knoten-Punkten verschiedene Methoden angewendet werden, das heißt in einem Modell können mehrere Gewichtungsmethoden kombiniert werden. Auf der untersten Hierarchie-Ebene, welche in der Regel die zu bewertenden Alternativen darstellt, können direkt Werte zwischen 0 und 1 vergeben oder aber ausgehend von quantifizierten Kriterien-Ausprägungen Wertfunktionen zur Normierung auf Werte zwischen 0 und 1 definiert und variiert werden.

Abbildung 6.4: Nutzung von Web-HIPRE zur Gewichtung von Kriterien in Abhängigkeit von Technologiezyklus-Phasen

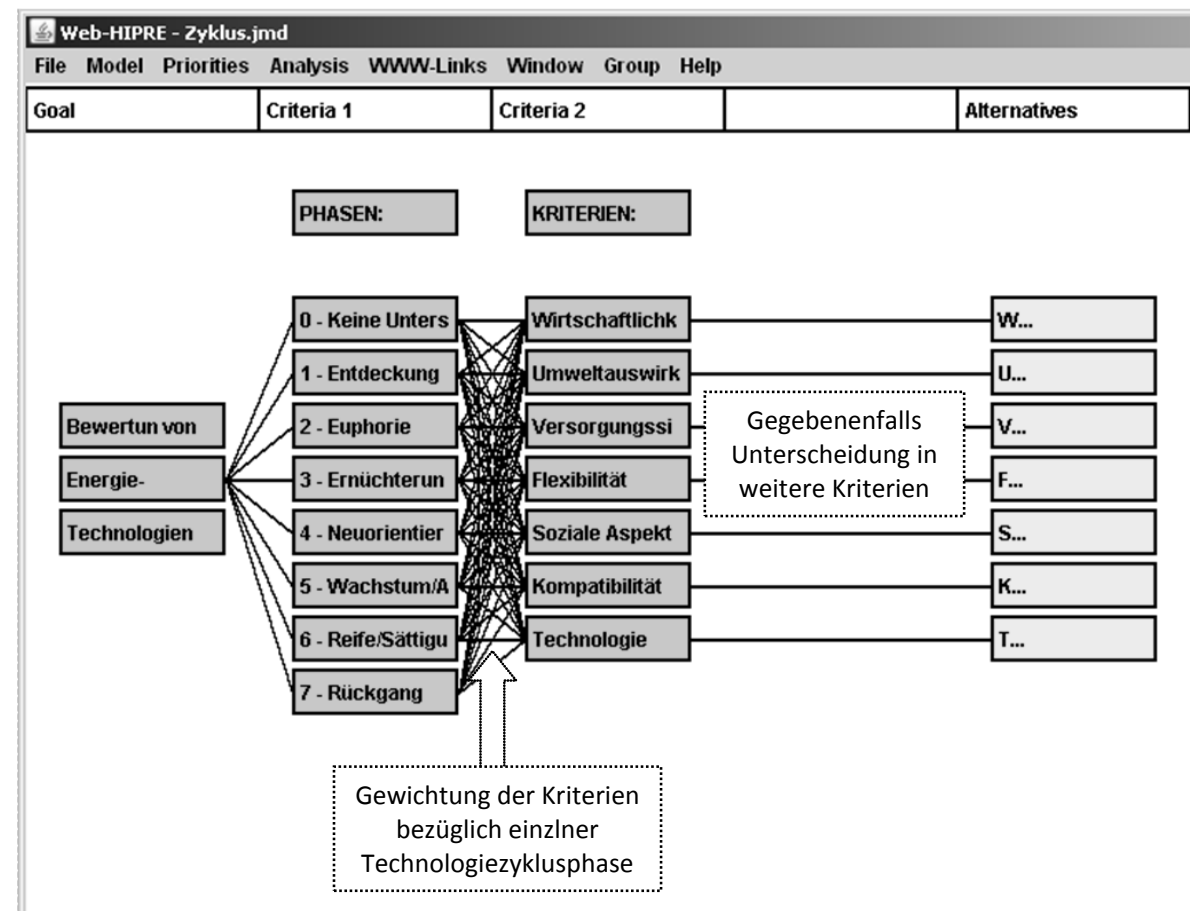

Quelle: Basierend auf Web-HIPRE Global Decision Support Version 1.22, Systems Analysis Laboratory, Helsinki University of Technology.

Dadurch dass mehrere Methoden in der gleichen Software implementiert sind, werden Vergleiche von Ergebnissen basierend auf unterschiedlichen Methoden erleichtert. Web-HIPRE ermöglicht weiterhin die Aggregation individueller Gewichtungen in einem 
Gruppen-Modell basierend auf dem gewichteten arithmetischen Mittelwert. Aufgrund grafischer Schnittstellen zeichnet sich Web-HIPRE durch eine hohe Nutzerfreundlichkeit aus (Mustajoki 2006; Mustajoki und Hämäläinen 2000). Für die vorliegende Arbeit wird Web-HIPRE zunächst genutzt, um einen Entscheidungsbaum unter Berücksichtigung unterschiedlicher Technologiezyklusphasen zu erstellen (Abbildung 6.4). Die Kriterien, die mit Bezug zu den unterschiedlichen Phasen zu gewichten sind, sowie die zu bewertenden Alternativen können dabei je nach Problemstellung variiert werden. Auf dieser Basis können die Kriterien-Gewichtungen je Technologiezyklus-Phase bestimmt werden.

\subsection{Wahl der Aggregationsmethode}

Auf Basis der in den vorangegangenen Schritten gesammelten Informationen kann die Entscheidungsmatrix erstellt werden, in der die Kriterien-Ausprägungen je Alternative erfasst sind. Diese dient in Verbindung mit den Kriterien-Gewichtungen als Ausgangspunkt für die Bewertung auf Basis einer oder mehrerer Aggregationsmethoden. Die in Abschnitt 6.5 vorgestellte Software Web-HIPRE ermöglicht die Durchführung einer additiven Nutzwertanalyse in Verbindung mit unterschiedlichen GewichtungsMethoden. Auch im Rahmen der MTB-Datenbank (vgl. Abschnitt 6.4) kann eine additive Nutzwertanalyse mit direkter Gewichtung der implementierten Kriterien durchgeführt werden (vgl. auch Abschnitt 6.9). Dabei können Gewichtungen, die in Web-HIPRE abgeleitet werden, als Eingabewerte für die MTB-Datenbank dienen. Ebenso können Technologiedaten aus der MTB-Datenbank als Inputdaten für Web-HIPRE verwendet werden. Aufgrund der leichten Verständlichkeit, Nachvollziehbarkeit und Umsetzbarkeit ist die in diesen Systemen implementierte additive Nutzwertanalyse in der Praxis weit verbreitet. Da Outranking-Methoden andere Vorteile aufweisen (vgl. Abschnitt 4.1), soll durch das in dieser Arbeit entwickelte EUS darüber hinaus die Möglichkeit bestehen, auch diese anzuwenden.

Sowohl ELECTRE als auch PROMETHEE und andere, weniger bekannte OutrankingAnsätze, wurden bereits in verschiedenen Problemstellungen im Energiebereich und in anderen Sektoren angewendet (für Anwendungen von ELECTRE im Energiebereich vgl. Beccali et al. 2003; Cloquell-Ballester et al. 2007; Georgopoulou et al. 2003; Karakosta et al. 2009; Papadopoulos und Karagiannidis 2008; Pires Neves et al. 2008; für Anwendungen von PROMETHEE siehe unten). In einigen Fällen werden mehrere Methoden miteinander kombiniert, um zusätzliche Vorteile zu erzielen (zum Beispiel Goletsis et al. 2003; Madlener et al. 2009). 
Tabelle 6.1: $\quad$ Anwendung von PROMETHEE im Energiebereich

\begin{tabular}{l|l}
\hline Anwendungsbereich & Quellen \\
\hline $\begin{array}{l}\text { Vergleich konzentrierender solarthermischer Kraft- } \\
\text { werkstechnologien }\end{array}$ & Cavallaro 2009 \\
\hline $\begin{array}{l}\text { Regionale Planung mit einem Fokus auf erneuerbare } \\
\text { Energien }\end{array}$ & $\begin{array}{l}\text { Cavallaro 2005; Polatidis und Haralambopoulos } \\
\text { 2007; Terrados et al. 2009; Tsoutsos et al. 2009 }\end{array}$ \\
\hline $\begin{array}{l}\text { Priorisierung von Energieprojekten im Rahmen des } \\
\text { CDM (Clean Development Mechanism) }\end{array}$ & Diakoulaki et al. 2007 \\
\hline $\begin{array}{l}\text { Analyse nationaler Energieszenarien in Griechenland } \\
\text { mit einem Fokus auf erneuerbare Energien }\end{array}$ & $\begin{array}{l}\text { Diakoulaki und Karangelis 2007; Georgopoulou } \\
\text { et al. 1998 }\end{array}$ \\
\hline Gestaltung energiepolitischer Instrumente & Doukas et al. 2006; Madlener und Stagl 2005 \\
\hline Bewertung von Optionen zur Wärmebereitstellung & Ghafghazi et al. 2009 \\
\hline $\begin{array}{l}\text { Priorisierung von Optionen zur Nutzung geothermi- } \\
\text { scher Energiequellen }\end{array}$ & $\begin{array}{l}\text { Goumas und Lygerou 2000; Goumas et al. } \\
\text { 1999; Haralambopoulos und Polatidis 2003 }\end{array}$ \\
\hline $\begin{array}{l}\text { Partizipative Analyse nationaler erneuerbarer Energie- } \\
\text { szenarien in Österreich }\end{array}$ & Kowalski et al. 2009; Madlener et al. 2007 \\
\hline Bewertung der Nutzung von Biomasse & Kumar et al. 2006 \\
\hline Standortwahl für Wasserkraftwerke & Mladineo et al. 1987 \\
\hline $\begin{array}{l}\text { Vergleich von Systemen zur Energiebereitstellung für } \\
\text { Kochstellen }\end{array}$ & Pohekar und Ramachandran 2004 \\
\hline Bewertung von Systemen zur Hausenergieversorgung & Ren et al. 2009 \\
\hline $\begin{array}{l}\text { Bewertung von Energietechnologien basierend auf } \\
\text { erneuerbaren, fossilen und nuklearen Energieträgern }\end{array}$ & Topcu und Ulengin 2004 \\
\hline reich & Tzeng et al. 1992 \\
\hline
\end{tabular}

In dieser Arbeit wird PROMETHEE bevorzugt, da diese Methoden Vorteile im Vergleich zu anderen Outranking-Ansätzen aufweisen. Diese betreffen den Aggregationsalgorithmus, der leicht nachzuvollziehen ist und mit einem vertretbaren Aufwand in eigene Berechnungsmodelle implementiert werden kann. Des Weiteren hebt Brans et al. (1986) die Stabilität der Ergebnisse im Vergleich zu ELECTRE hervor. Unscharfe Präferenzvorstellungen und Unsicherheiten in den Kriterien-Ausprägungen können durch verallgemeinerte Präferenzfunktionen und Schwellenwerte in einfacher Weise abgebildet werden. Durch Sensitivitätsanalysen kann der Einfluss der Anwendung unterschiedlicher Präferenzfunktionen und Kriterien-Gewichte analysiert sowie Stabilitätsintervalle bestimmt werden. In dieser Weise kann die Transparenz von Entscheidungsprozessen auf Basis von PROMETHEE gewährleistet und die Validierung der Ergebnisse erleichtert werden. Darüber hinaus eignet sich PROMETHEE auch zur Anwendung in Gruppen-Entscheidungen (Al Shemmeri et al. 1997; Løken 2007; Wang et al. 2009b). Dies wird insbesondere dadurch begründet, dass PROMETHEE im Vergleich zu anderen Outranking-Ansätzen als transparenter und leichter verständlich auch für diejenigen Entscheidungsträger angesehen wird, die nicht mit Multi-Attribut-Methoden vertraut sind (vgl. auch Buchholz et al. 2009; Løken 2007; Polatidis et al. 2006). Zudem 
wurde PROMETHEE bereits in verschiedenen Problemstellungen im Energiebereich und in anderen Sektoren erfolgreich eingesetzt (vgl. Tabelle 6.1 und Tabelle 6.2). Einen aktuellen Überblick über Anwendungen von PROMETHEE gibt Behzadian et al. (2010).

Tabelle 6.2: $\quad$ Anwendung von PROMETHEE in anderen Bereichen

\begin{tabular}{l|l}
\hline Anwendungsbereich & Quellen \\
\hline Auswahl von Optionen im Aktienhandel & Albadvi et al. 2007 \\
\hline Auswahl zwischen Fertigungssystemen & Anand und Kodali 2008 \\
\hline $\begin{array}{l}\text { Bewertung von Lieferanten und Outsourcing- } \\
\text { Entscheidungen }\end{array}$ & $\begin{array}{l}\text { Araz et al. 2007; Araz und Ozkarahan 2007; } \\
\text { Routroy und Kodali 2007; Wang und Yang 2007 }\end{array}$ \\
\hline Untersuchung chemometrischer Analyseergebnisse & $\begin{array}{l}\text { Ayoko et al. 2007; Carmody et al. 2007; Ni et al. } \\
\text { 2007; Purcell et al. 2007 }\end{array}$ \\
\hline Rangfolge von Transportfahrzeugen & $\begin{array}{l}\text { Beynon und Wells 2008; Safaei Mohamadabadi et } \\
\text { al. 2009 }\end{array}$ \\
\hline Präventive Wartungsplanung & Cavalcante und de Almeida 2007 \\
\hline Vergleich von Optionen zum Gewässer-Management & Chou et al. 2007; Hermans et al. 2007 \\
\hline Rangfolge von Biopolymeren & Cornelissen et al. 2009 \\
\hline Auswahl von Materialien und Anlagen & $\begin{array}{l}\text { Dagdeviren 2008; Tuzkaya et al. 2009; Zhu et al. } \\
\text { 2010 }\end{array}$ \\
\hline $\begin{array}{l}\text { Priorisierung von Forschungsgebieten im Bereiche } \\
\text { der Nanotechnologie }\end{array}$ & Ghazinoory et al. 2009 \\
\hline Bewertung von Optionen zum Abfallmanagement & $\begin{array}{l}\text { Kapepula et al. 2007; Mergias et al. 2007; Rousis } \\
\text { et al. 2008; Vego et al. 2008 }\end{array}$ \\
\hline $\begin{array}{l}\text { Entwicklung von Strategien für das Management von } \\
\text { Verlusten in Wassersystemen }\end{array}$ & Morais und de Almeida 2007 \\
\hline $\begin{array}{l}\text { Bewertung unterschiedlicher Landnutzungsmöglich- } \\
\text { keiten }\end{array}$ & Palma et al. 2007 \\
\hline Standortwahl für Recycling-Anlagen & Queiruga et al. 2008 \\
\hline Rangfolge von Alternativen in der Terminplanung & Roux et al. 2008 \\
\hline
\end{tabular}

Im Allgemeinen basieren Outranking-Ansätze auf paarweisen Vergleichen von Alternativen bezüglich mehrerer Kriterien (Brans et al. 1986; Brans und Mareschal 2005; Roy 1980; Roy und Bouyssou 1993). Im Gegensatz zum AHP/ANP muss der Entscheidungsträger jedoch nicht selbst die Vergleiche auf einer vorgegebenen Skala durchführen. Vielmehr werden diese in automatisierter Weise durchgeführt. Dabei können die Kriterien-Ausprägungen in unterschiedlichen Einheiten auf unterschiedlichen Skalenniveaus vorliegen. Basis der Bewertung ist die Entscheidungsmatrix, in der die KriterienAusprägungen je Alternative hinterlegt sind. Davon ausgehend werden die paarweisen Vergleiche hinsichtlich einzelner Kriterien durchgeführt. Bei Anwendung von PROMETHEE werden mit Hilfe von Präferenz-Funktionen und Kriterien-Gewichtungen die paarweisen Vergleichswerte aggregiert, um eine partielle oder vollständige Rangfolge der Alternativen abzuleiten. Die Gewichtungen spiegeln die relative Bedeutung 
der Kriterien untereinander wieder und werden normalerweise durch einen Gewichtungs-Vektor wiedergegeben. $\mathrm{Da}$ in der vorliegenden Arbeit jedoch Gewichtungen in Abhängigkeit unterschiedlicher Technologiezyklusphasen angewendet werden sollen, ist eine Modifikation des originären PROMETHEE-Algorithmus erforderlich. Anstelle eines Gewichtungs-Vektors wird eine Gewichtungs-Matrix angewendet, welche die Gewichtungen je Technologiezyklus-Phase enthält. Der modifizierte PROMETHEEAlgorithmus kann dann wie folgt beschrieben werden:

Es sei $A=\left\{a_{1}, \ldots, a_{i}, \ldots, a_{m}\right\}$ die diskrete Menge der betrachteten Alternativen und $C=\left\{c_{1}, \ldots, c_{j}, \ldots, c_{n}\right\}$ das ausgewählte Set an Kriterien. Nach der Bestimmung der Kriterien-Ausprägungen $f_{j}\left(a_{i}\right)$ für jede Alternative und jedes Kriterium, umfasst der Algorithmus zur Anwendung von PROMETHEE I und II basierend auf (Brans et al. 1986) folgende Schritte:

1. Für jedes Kriterium $c_{j}$ ist eine Präferenzfunktion $p_{j}$ festzulegen, die in Abhängigkeit der Differenz zwischen den Kriterien-Ausprägungen die Vorziehenswürdigkeit von Alternative $a_{i^{*}}$ gegenüber $a_{i}$ widerspiegelt:

$$
p_{j}\left(d_{j}\left(a_{i^{*}}, a_{i}\right)\right)=p_{j}\left(f_{j}\left(a_{i^{*}}\right)-f_{j}\left(a_{i}\right)\right) \text { mit } p_{j} \in[0 ; 1] \text {. }
$$

Unterschiedliche vordefinierte Präferenzfunktionen stehen zur Auswahl, die in der Regel ausreichen, um reale Präferenzvorstellungen abzubilden (vgl. Abbildung 6.5).

2. Bei Anwendung von PROMETHEE I und II wird ein Gewichtungs-Vektor $w^{T}=\left(w_{1}, \ldots, w_{j}, \ldots, w_{n}\right)$ durch den Entscheidungsträger bestimmt, wobei in der Regel gilt $\sum_{j=1}^{n} w_{j}=1$. Da jedoch Gewichtungen in Abhängigkeit der Technologiezyklusphasen angewendet werden sollen, ist stattdessen eine Gewichtungs-Matrix zu definieren, welche die Gewichtungen in Abhängigkeit der Phasen $t_{k}$ mit $k=1, \ldots, o$ enthält:

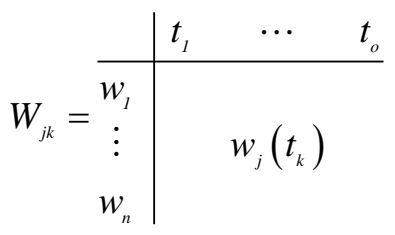

Weiterhin müssen die Alternativen den jeweils aktuellen Phasen im Technologiezyklus zugeordnet werden:

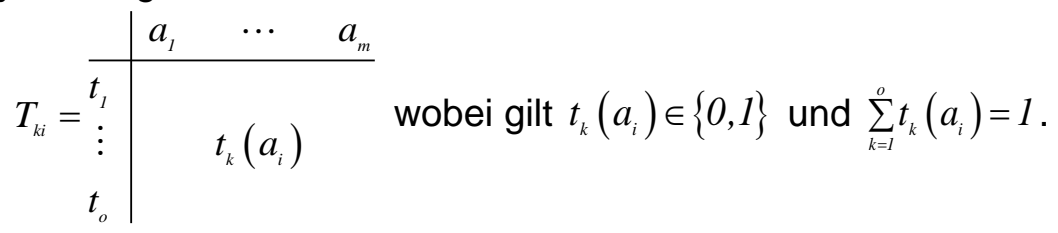

Indem die beiden Matrizen miteinander multipliziert werden, wird für jede Alternati- 
ve genau dasjenige Gewichtungs-Set angewendet, das der aktuellen Technologiezyklus-Phase entspricht:

$$
w_{j}\left(a_{i}\right)=W_{j k} \cdot T_{k i}=\left[w_{j}\left(t\left(a_{i}\right)\right)\right] .
$$

3. Als Maß für die relative Vorziehenswürdigkeit der Alternative $a_{i^{*}}$ im Vergleich zur Alternative $a_{i}$ unter Berücksichtigung aller Kriterien, wird der Präferenzindex $\pi$ unter Beachtung der Gewichtungen in Abhängigkeit der Technologiezyklusphasen berechnet:

$$
\pi\left(a_{i^{*}}, a_{i}\right)=\sum_{j=1}^{n} w_{j}\left(t\left(a_{i^{*}}\right)\right) \cdot p_{j}\left(a_{i^{*}}, a_{i}\right) .
$$

4. Um eine Alternative mit allen anderen Alternativen basierend auf allen Kriterien zu vergleichen, werden der Ausgangs- und der Eingangsfluss berechnet.

a. Der Ausgangsfluss ist ein Maß für die relative Stärke der Alternative $a_{i^{*}}$ im Vergleich zu allen anderen Alternativen:

$$
\phi^{+}\left(a_{i^{*}}\right)=\sum_{\substack{i=1 \\ i \neq i^{*}}}^{m} \pi\left(a_{i^{*}}, a_{i}\right)=\sum_{\substack{i=1 \\ i \neq \hbar^{*}}}^{m} \sum_{j=1}^{n} w_{j}\left(t\left(a_{i^{*}}\right)\right) \cdot p_{j}\left(a_{i^{*}}, a_{i}\right) \text {. }
$$

Basierend auf dieser Kennzahl können die folgende Präordnungen abgeleitet werden:

i. $\quad \phi^{+}\left(a_{i^{*}}\right)>\phi^{+}\left(a_{i}\right) \Rightarrow a_{i^{*}} P^{+} a_{i}$ ( $a_{i^{*}}$ wird gegenüber $a_{i}$ präferiert)

ii. $\phi^{+}\left(a_{i^{*}}\right)=\phi^{+}\left(a_{i}\right) \Rightarrow a_{i^{*}} I^{+} a_{i}$ ( $a_{i^{*}}$ und $a_{i}$ werden als gleichwertig angesehen)

b. Der Eingangsfluss ist ein Maß für die relative Schwäche von Alternative $a_{i^{*}}$ im Vergleich zu allen anderen Alternativen:

$$
\phi^{-}\left(a_{i^{*}}\right)=\sum_{\substack{i=1 \\ i \neq i^{*}}}^{m} \pi\left(a_{i}, a_{i^{*}}\right)=\sum_{\substack{i=1 \\ i \neq i^{*}}}^{m} \sum_{j=1}^{n} w_{j}\left(t\left(a_{i^{*}}\right)\right) \cdot p_{j}\left(a_{i}, a_{i^{*}}\right)
$$

Basierend auf dieser Kennzahl können die folgende Präordnungen abgeleitet werden:

i. $\quad \phi^{-}\left(a_{i^{*}}\right)<\phi^{-}\left(a_{i}\right) \Rightarrow a_{i^{*}} P^{-} a_{i}$ ( $a_{i^{*}}$ wird gegenüber $a_{i}$ präferiert)

ii. $\phi^{-}\left(a_{i^{*}}\right)=\phi^{-}\left(a_{i}\right) \Rightarrow a_{i^{*}} I^{-} a_{i}$ ( $a_{i^{*}}$ und $a_{i}$ sind werden als gleichwertig angesehen)

5. Nach PROMETHEE I kann durch Verschneidung der beiden Präordnungen aus Schritt 4 eine partielle Präordnung gebildet werden:

a. $a_{i *} P^{I} a_{i}$, wenn

i. $a_{i^{*}} P^{+} a_{i}$ und $a_{i^{*}} P^{-} a_{i}$ oder

ii. $a_{i^{*}} P^{+} a_{i}$ und $a_{i+} I^{-} a_{i}$ oder

iii. $a_{i^{*}} P^{-} a_{i}$ und $a_{i^{*}} I^{+} a_{i}$ oder

b. $a_{i^{*}} I^{I} a_{i}$, wenn $a_{i^{*}} I^{+} a_{i}$ und $a_{i^{*}} I^{-} a_{i}$

c. Andernfalls gilt $a_{i^{*}} R^{I} a_{i}$ ( $a_{i^{*}}$ und $a_{i}$ unvergleichbar). 
6. Eine vollständige Präordnung, durch die Unvergleichbarkeiten vermieden werden, kann nach PROMETHEE II auf Basis des Nettoflusses $\phi^{\text {net }}\left(a_{i^{*}}\right)=\phi^{+}\left(a_{i^{*}}\right)-\phi^{-}\left(a_{i^{*}}\right)$ je Alternative bestimmt werden:
a. $\phi^{\text {net }}\left(a_{i^{*}}\right)>\phi^{\text {net }}\left(a_{i}\right) \Rightarrow a_{i *} P^{I I} a_{i}$
b. $\quad \phi^{\text {net }}\left(a_{i^{*}}\right)=\phi^{\text {net }}\left(a_{i}\right) \Rightarrow a_{i^{*}} I^{I I} a_{i}$

Die Kriterien-Gewichtungen in Abhängigkeit der Technologiezyklusphasen haben in diesem modifizierten PROMETHEE-Algorithmus Auswirkungen auf die Berechnung der Outranking-Relation $\pi$ und somit auch auf die Maßgrößen für die relative Stärke $\phi^{+}$ und die relative Schwäche $\phi^{-}$, aus denen letztlich die Rangfolge der Alternativen nach PROMETHEE I und/oder II abgeleitet wird. Brans und Mareschal (2005) weisen darauf hin, dass die Berechnung des Netto-Flusses nach PROMETHEE II mit einem Informationsverlust im Vergleich zu PROMETHEE I einhergeht. Denn durch das höhere Aggregationsniveau können in ähnlicher Weise wie bei den MAVT/MAUT-Ansätzen Kompensationseffekte zwischen positiven und negativen Kriterien-Ausprägungen auftreten (vgl. auch Belton und Stewart 2002). Folglich wird empfohlen immer beide Varianten zu nutzen, da die vollständige Rangfolge nach PROMETHEE II nur dann unmissverständlich interpretiert werden kann, wenn auch die partielle Rangfolge nach PROMETHEE I bekannt ist. Daher werden im hier entwickelten EUS sowohl PROMETHEE I als auch PROMETHEE II implementiert.

Abbildung 6.5: Verallgemeinerte Präferenzfunktionen zur Anwendung von PROMETHEE

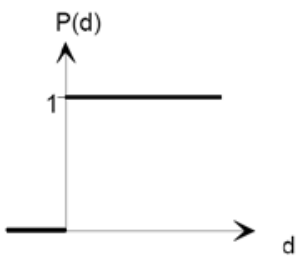

$$
\text { Typ } 1
$$

Gewöhnliches Kriterium

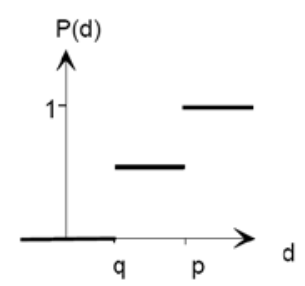

Typ 4

Stufenkriterium

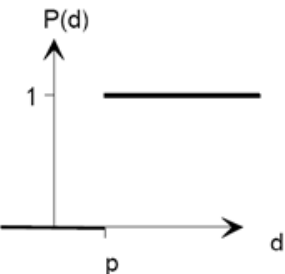

Typ 2

Quasikriterium

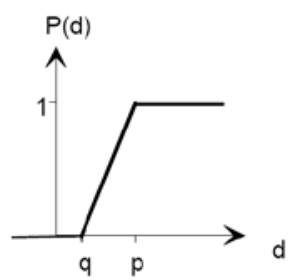

Typ 5

Kriterium mit linearer Präferenz und Indifferenzbereich
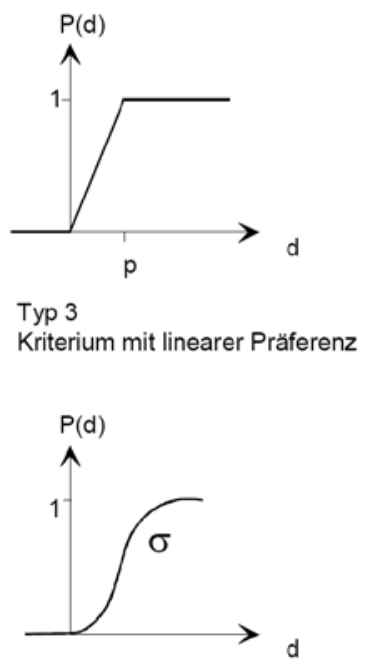

Typ 6

Gaußsches Kriterium

Quelle: Brans et al. (1986); Geldermann (2005) 
Im Hinblick auf die Auswahl einer geeigneten Präferenzfunktion (Schritt 1 des beschriebenen Algorithmus; Abbildung 6.5), sind Hinweise zu finden bei Brans et al. (1986), Routroy und Kodali (2007) sowie Anand und Kodali (2008). Typ 1 (Gewöhnliches Kriterium) unterscheidet lediglich in strikte Präferenz und Indifferenz, ohne Angabe eines Schwellenwertes. Dabei wird bei jedem noch so geringen Unterschied zwischen den Kriterien-Ausprägungen auf jeden Fall eine Alternative der anderen strikt vorgezogen. Im Gegensatz dazu wird bei Typ 2 (Quasikriterium) ein Schwellenwert für Indifferenz eingeführt. Unterschreitet die Differenz zwischen den KriterienAusprägungen diesen Schwellenwert, werden diese trotz unterschiedlicher Ausprägungen als indifferent angesehen. Das heißt Unterschiede, die kleiner sind als der Schwellenwert, werden nicht als relevant erachtet. Beim Typ 3 (Kriterium linearer Präferenz) wird darüber hinaus ein Bereich linear steigender, schwacher Präferenz eingeführt. Dies bedeutet, dass die Präferenz umso stärker ist, je näher die Differenz am Schwellenwert $p$ liegt, oberhalb dessen strikte Präferenz gilt. Auch beim Typ 4 (Stufenkriterium) gibt es einen Bereich schwacher Präferenz, der jedoch nicht monoton steigend sondern stufenweise definiert wird. Beim Typ 5 (Kriterium mit linearer Präferenz und Indifferenzbereich) handelt es sich um eine Kombination der Typen 2 und 3, das heißt es wird sowohl ein Bereich der Indifferenz als auch ein Bereich schwacher Präferenz definiert. Beim Typ 6 schließlich steigt die Präferenz monoton an, entsprechend einer Gauß'schen Normalverteilung. Die Typen 1, 2 und 4 werden meist für qualitative Kriterien zugrunde gelegt, während die Typen 3, 5 und 6 eher für quantitative Kriterien verwendet werden (Anand und Kodali 2008; Routroy und Kodali 2007). Besonders robuste Ergebnisse, die relativ stabil gegenüber kleinen Variationen in den Input-Daten sind, weisen Bewertungen auf Basis von Typ 6 auf (Brans et al. 1986; Queiruga et al. 2008). Hinsichtlich der Festlegung der Schwellenwerte weisen Tsoutsos et al. (2009) im Kontext einer Anwendung im Energiebereich darauf hin, dass der Schwellenwert für Indifferenz häufig mit Null gleichgesetzt oder bei 5 bis $15 \%$ der maximalen Differenz zwischen den Ausprägungen des betrachteten Kriteriums festgelegt wird. Für strikte Präferenz werden häufig Werte zwischen 10 und $30 \%$ der maximalen Differenz gewählt oder der Wert, welcher der maximalen Differenz geteilt durch die Anzahl der betrachteten Alternativen entspricht.

\subsection{Berücksichtigung von Unsicherheiten}

Unsicherheiten in den Wertvorstellungen der Entscheidungsträger sowie bezüglich der Kriterien-Ausprägungen werden bei Anwendung von PROMETHEE implizit durch die Auswahl geeigneter Präferenzfunktionen berücksichtigt. Weiterhin werden im hier entwickelten EUS Sensitivitätsanalysen einschließlich der Bestimmung von Stabilitätsintervallen der Gewichtungen implementiert (vgl. Abschnitt 6.9). Dabei sind insbesondere 
die unterschiedlichen Kriterien-Gewichtungen in Abhängigkeit der Lebenszyklusphasen zu beachten. Darüber hinaus werden Szenarien genutzt, um Unsicherheiten hinsichtlich der zukünftigen Entwicklung der Rahmenbedingungen abzubilden. Dabei werden drei Szenarien zugrunde gelegt, welche die wesentlichen Tendenzen in bekannten Studien zur zukünftigen Entwicklung der Energiemärkte widerspiegeln. Diese Vorgehensweise ermöglicht eine differenzierte Darstellung von Ergebnissen in Abhängigkeit denkbarer zukünftiger Entwicklungspfade sowie die Identifikation von Alternativen, die über alle Szenarien ein robustes Bewertungsergebnis aufweisen. Unterschieden werden in Anlehnung an Wietschel et al. (2010) folgenden Szenarien als Grundlage für die multikriterielle Bewertung:

1. Im moderaten Szenario (MOD) wird angenommen, dass die aktuellen politischen Ziele hinsichtlich des Anteils erneuerbarer Energien und der Reduzierung von Emissionen nach der Europäischen Kommission (2007) beibehalten und nicht weiter verschärft werden. In Anlehnung an Bartels et al. (2005) sowie EWI und EEFA (2007) wird angenommen, dass die Nachfrage bis 2050 nur langsam abnimmt. Die Preise fossiler Energieträger steigen in diesem Szenario nur geringfügig an.

2. Im Vergleich zum moderaten Szenario werden im Klimaschutz-Szenario (KLIM) ehrgeizigere Ziele bezüglich des Ausbaus erneuerbarer Energien und der Reduzierung von Treibhausgasemissionen unterstellt. Es wird angenommen, dass die Preise fossiler Energieträger im gleichen Maße ansteigen wie im moderaten Szenario, jedoch bewirken die verschärften Ziele höhere Preise für $\mathrm{CO}_{2}$-Zertifikate.

3. Das Szenario Ressourcenverknappung (RESS) ist insbesondere durch einen sehr starken Anstieg der Preise für fossile Energieträger gekennzeichnet. Es wird angenommen, dass die Stromnachfrage aufgrund von Einsparmaßnahmen stark abnimmt. Dieses Hochpreis-Szenario basiert auf Joest et al. (2009). Hinsichtlich des Ausbaus erneuerbarer Energien werden (absolut) die gleichen Ziele wie im moderaten Szenario unterstellt.

Die voneinander abweichenden Preisentwicklungen und Zusammensetzungen des Energiemixes je Szenario sind als Resultat aus der unterstellten Nachfrageentwicklung sowie aus den vorgegebenen Zielen hinsichtlich des Anteils erneuerbarer Energien und der Reduzierung der $\mathrm{CO}_{2}$-Emissionen zu verstehen. Die Entwicklung der Energieträgerpreise und der Preise für $\mathrm{CO}_{2}$-Zertifikate je Szenario sind in Abbildung $6.6 \mathrm{zu}$ sammengefasst. Deutlich wird die im Vergleich zum MOD- und KLIM-Szenario starke Steigerung der Energieträgerpreise im RESS-Szenario. Die höheren Preise für $\mathrm{CO}_{2}$ Zertifikate im KLIM- im Vergleich zum MOD-Szenario können auf die höheren Emissionsreduktionsziele zurückgeführt werden. In beiden Szenarien steigen die Preise für $\mathrm{CO}_{2}$-Zertifikate bis 2020 an und sinken anschließend aufgrund des zunehmenden Anteils erneuerbarer Energien und den höheren Wirkungsgraden neuer Kraftwerke. Auf- 
grund der zunehmenden Stromproduktion steigen die Preise ab 2030 wieder leicht an, sinken jedoch wieder leicht ab 2040 aufgrund des weiter steigenden Anteils erneuerbarer Energien. Im RESS-Szenario wird angenommen, dass der Preis für $\mathrm{CO}_{2}$-Zertifikate über den gesamten Betrachtungszeitraum bei 0 Euro liegt, da die Ziele zur Emissionsreduzierung durch den Rückgang in der Stromnachfrage und den hohen Anteil erneuerbarer Energien erreicht werden. Im Rahmen der multikriteriellen Analyse wird die Wirtschaftlichkeit von Energietechnologien in Abhängigkeit der so unterstellten Preisentwicklungen für Energieträger und $\mathrm{CO}_{2}$-Zertifikate bewertet. Durch Vergleich der Bewertung auf Basis der Szenarien MOD und KLIM kann zum einen untersucht werden, welchen Einfluss Unterschiede in den Preisen für $\mathrm{CO}_{2}$-Zertifikate auf das Bewertungsergebnis ausüben. Durch zusätzliche Berücksichtigung des Szenarios RESS können zum anderen die Auswirkungen extremer Steigerungen in den Brennstoffpreisen untersucht werden.

Abbildung 6.6: Entwicklung der Energieträgerpreise und der $\mathrm{CO}_{2}$-Zertifikatspreise in Deutschland je Szenario

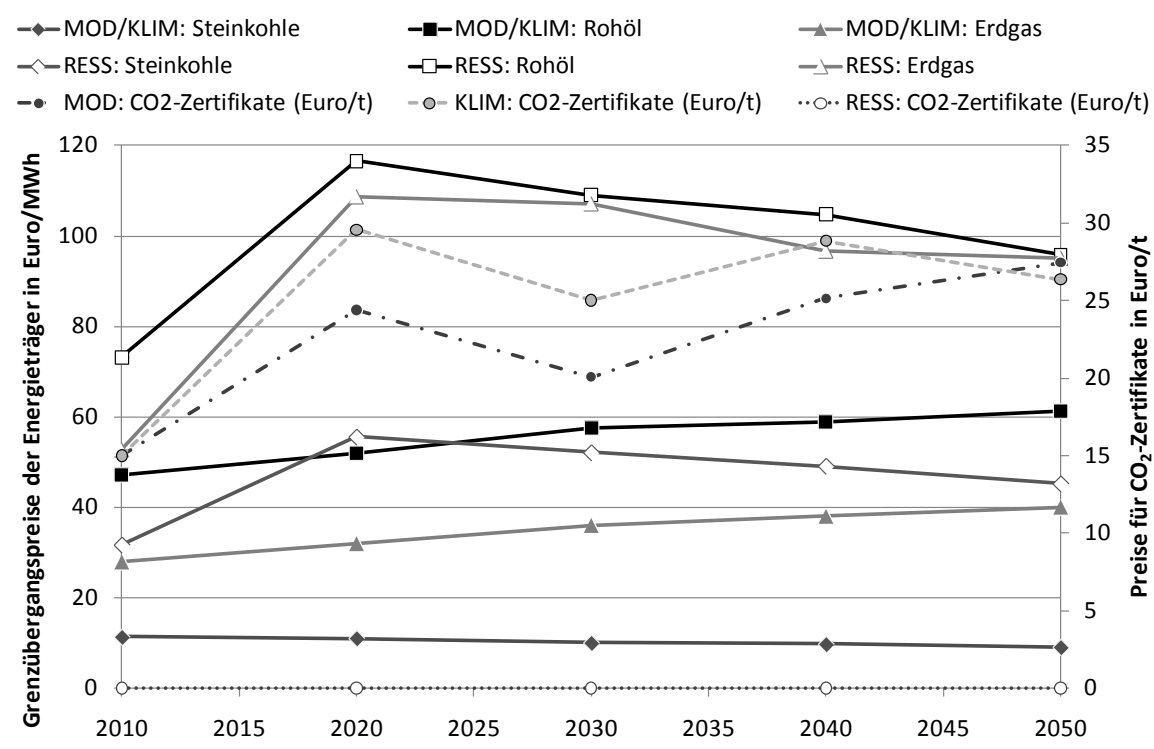

Anmerkungen: $\mathrm{MOD}=$ Moderates Szenario; $\mathrm{KLIM}=$ Klimaschutz-Szenario; RESS $=$ Ressourcenverknappungs-Szenario.

Quelle: In Anlehnung an Wietschel et al. (2010).

Weiterhin unterscheiden sich in Abhängigkeit des Energiemixes die spezifischen $\mathrm{CO}_{2}$ Emissionen der Stromerzeugung in den einzelnen Szenarien (Abbildung 6.7). Dies wird im Wesentlichen durch die unterschiedlichen Anteile erneuerbarer Energien begründet. Variierende $\mathrm{CO}_{2}$-Emissionen in der Stromerzeugung können sich im Rahmen der multikriteriellen Analyse von Energietechnologien zum Beispiel hinsichtlich des Beitra- 
ges zum Treibhauseffekt auswirken, wenn Strom einen wesentlichen Input-Faktor für einzelne Technologien darstellt.

Abbildung 6.7: $\quad$ Spezifische $\mathrm{CO}_{2}$-Emissionen der Stromerzeugung in Deutschland je Szenario

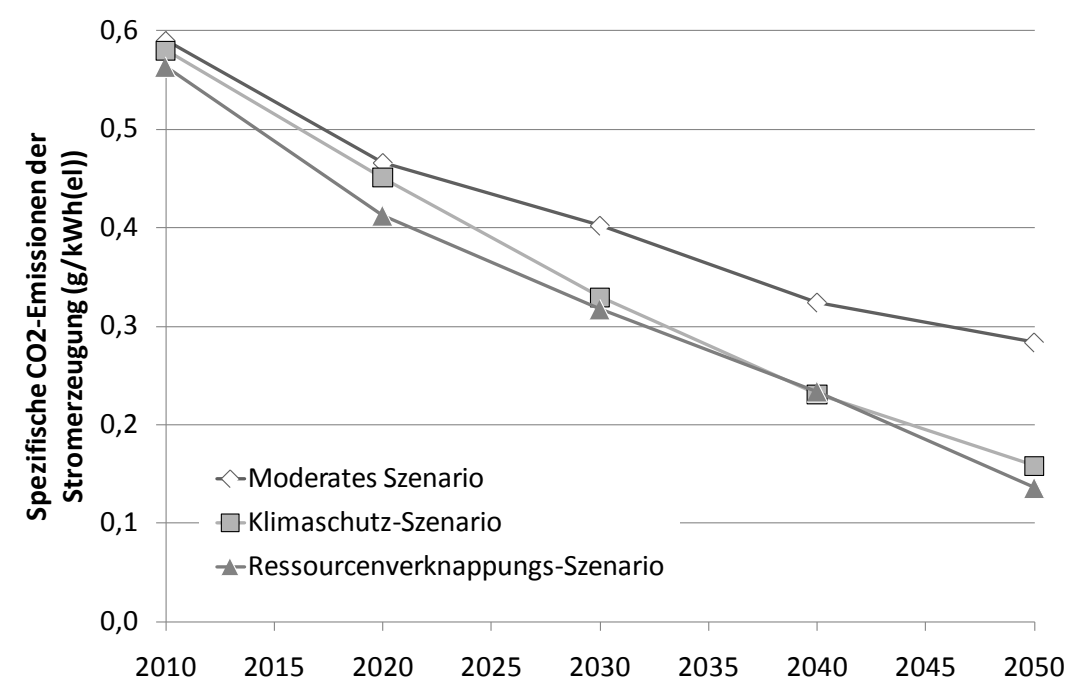

Quelle: In Anlehnung an Wietschel et al. (2010).

\subsection{Einbindung von Stakeholdern}

Bei der Entwicklung und Anwendung eines multikriteriellen EUS können Stakeholder, wie etwa Entscheidungsträger im Energiebereich, an unterschiedlichen Stellen eingebunden werden, beispielsweise bei der Wahl der zu implementierenden Kriterien und Aggregationsmethoden. Die Entscheidungsfindung in Gruppen stellt jedoch keinen Schwerpunkt dieser Arbeit dar. Dennoch ist die Einbindung von Stakeholdern und Gruppen bei der Nutzung des hier entwickelten EUS an unterschiedlichen Stellen möglich. Zum einen kann die Vorauswahl der Alternativen und Kriterien beispielsweise auf Basis von Workshops oder Befragungen zusammen mit Stakeholdern und Experten erarbeitet werden. Dies ist in den Abschnitten 6.2 und 0 beispielhaft dargestellt. Weiterhin ermöglicht Web-HIPRE die Kombination individueller Entscheidungsmodelle mit unterschiedlichen Wertvorstellungen und Gewichtungen zu einem Gruppen-Modell (vgl. Abschnitt 6.5). Weitere Möglichkeiten zur Konsensfindung in Gruppen werden in Abschnitt 4.2.8 diskutiert.

\subsection{Software-Implementierung}

Auf Basis der Konzeption in den vorangegangenen Abschnitten wird das EUS MAESTRO (Multi Criteria Assessment of Energy Supply Technologies - Research \& 
Outlook) entworfen. Dieses besteht aus drei Hauptkomponenten, die in unterschiedlichen Phasen im Verlauf des Entscheidungsprozesses zum Einsatz kommen und miteinander Daten austauschen (Abbildung 6.8). Die MTB-Datenbank dient vorwiegend der Datensammlung und -verarbeitung und kann zudem zur Durchführung einer additiven Nutzwertanalyse für eine Auswahl an Alternativen und Kriterien genutzt werden. Daten aus der MTB-Datenbank können als Input für die anderen Komponenten dienen. Das Software-Tool Web-HIPRE soll in dieser Anwendung vorwiegend zur Ableitung von Kriterien-Gewichtungen mit Bezug zu unterschiedlichen Technologiezyklusphasen dienen. Damit ermittelte Gewichtungen dienen als Input für die anderen Komponenten von MAESTRO. In einem Excel-Tool ist schließlich die in Abschnitt 6.6 beschriebene modifizierte PROMETHEE-Methode implementiert. Die MTB-Datenbank und das Excel-Tool werden im Folgenden genauer beschrieben, während die Funktionalitäten von WebHIPRE bereits in Abschnitt 6.5 erläutert wurden.

Abbildung 6.8: Konzeption des Entscheidungsunterstützungssystems MAESTRO

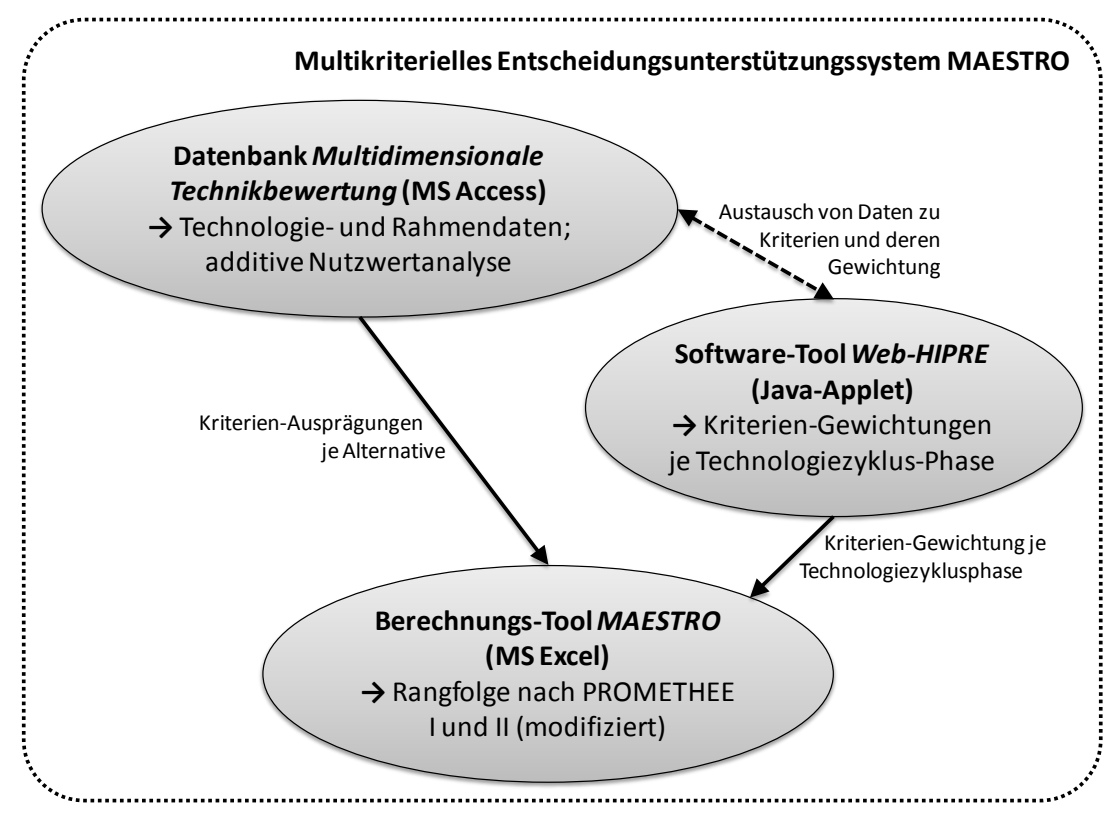

\subsubsection{Datenbank Multidimensionale Technikbewertung}

In der MTB-Datenbank, die auf Basis von MS Access entwickelt wurde, sind technische, ökonomische und ökologische Daten von Energietechnologien hinterlegt (zum Beispiel Wirkungsgrade, Investitionen, Betriebskosten, Emissionsfaktoren). Des Weiteren sind Daten zu Energieträgern erfasst, zum Beispiel zu den Preisen eingesetzter Brennstoffe oder zu deren Emissionsfaktoren. Zudem ist die Berechnung eines vordefinierten Sets an Kriterien und deren Normierung zur Durchführung einer additiven Nutzwertanalyse implementiert. Hierbei kann die Analyse auf Basis verschiedener Kri- 
terien und unterschiedlicher Gewichtungen, die vom Nutzer direkt gesetzt und geändert werden, durchgeführt werden. Die Datenbank dient somit sowohl der Datensammlung als auch der Durchführung einer multikriteriellen Analyse von Energietechnologien. Durch die Datenbankstruktur kann für die Analyse flexibel auf die Daten zugegriffen werden. Die Datenbank besteht aus Tabellen, Abfragen, Formularen und Berichten (Abbildung 6.9). Formulare dienen als intuitive Steuerwerkzeuge für den Benutzer, während die Verwaltung und Verarbeitung der Daten im Hintergrund auf Basis von Tabellen und Abfragen erfolgt. In Berichten werden Bewertungsergebnisse sowie Basisdaten für den Nutzer bereitgestellt.

Abbildung 6.9: Grundfunktionen der Datenbank „Multidimensionale Technikbewertung"

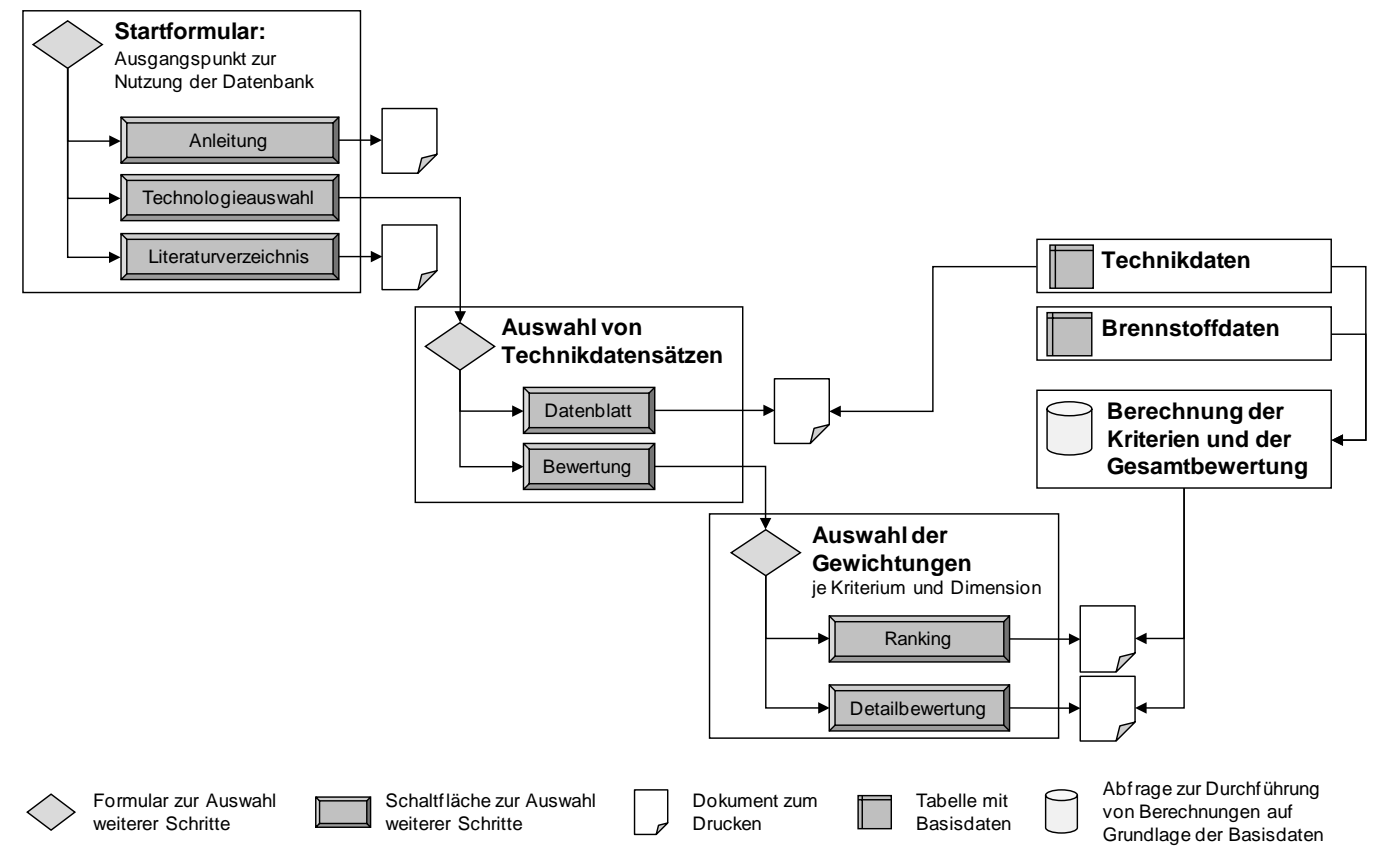

Beim Öffnen der Datenbank erscheint ein Startformular, über das eine Anleitung zu Nutzung sowie eine Literaturliste aller verwendeten Datenquellen abgerufen werden kann (Abbildung 6.10). Über die Schaltfläche „Technologieauswahl“ erfolgt die Weiterleitung zum Formular, das der Auswahl der zu bewertenden Technologien dient (Abbildung 6.11). Über dieses Formular kann der Nutzer im Bereich „Technologie“ eine Auswahl an zu bewertenden Alternativen treffen. Diese Auswahl kann eingegrenzt werden, indem die Suche im Bereich „Brennstoff“ auf bestimmte Energieträger eingeschränkt wird. Weiterhin können Filter gesetzt werden, welche die Auswahl zum Beispiel auf einen bestimmten Leistungsbereich oder hinsichtlich anderer Merkmale einschränken. Die Auswahl wird im Bereich „Suchergebnis“ angezeigt und kann an dieser Stelle gegebenenfalls weiter eingegrenzt werden. Für die ausgewählten Datensätze 
können Datenblätter mit den Basisdaten der Technologievarianten angezeigt und ausgedruckt werden (Schaltfläche „Datenblatt“).

Abbildung 6.10: Startformular zur Nutzung der Datenbank „Multidimensionale Technikbewertung"

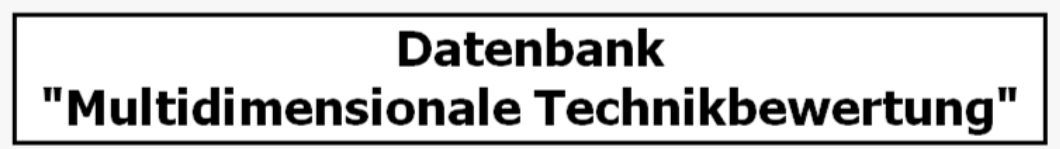

Bewertung von Techniken zur Bereitstellung von Strom und/oder Wärme

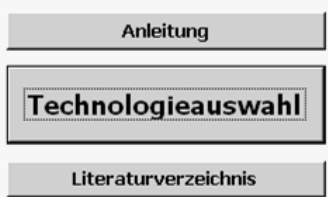

Abbildung 6.11: Formular zur Auswahl von Technologiedatensätzen

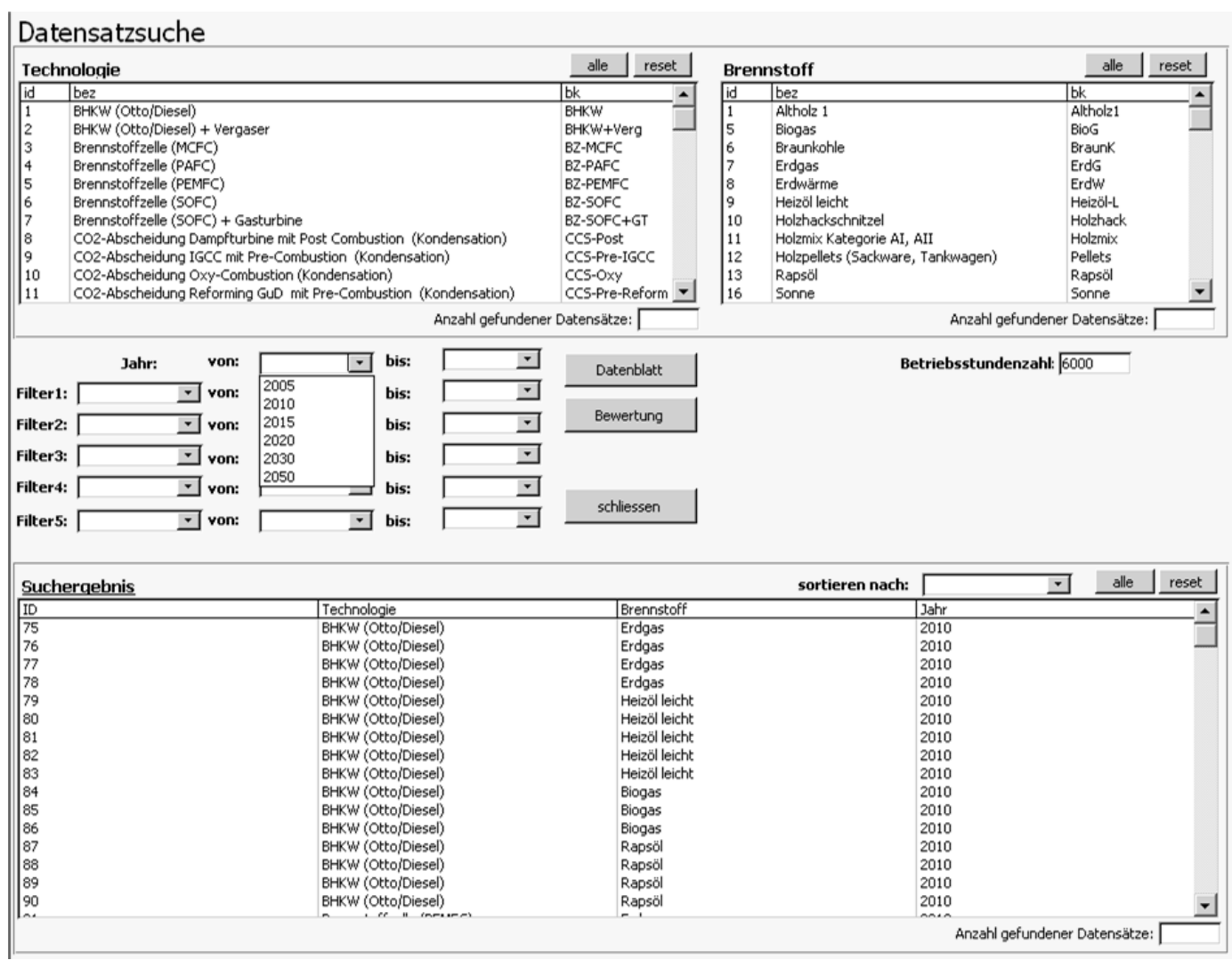

Über die Schaltfläche „Bewertung“ gelangt der Nutzer zu einem weiteren Formular, welches die Eingabe der Gewichtungen je Kriterium und Dimension erlaubt (Abbildung 6.12). Hier kann der Nutzer entsprechend seiner Wertvorstellungen die Gewichtung von Kriterien auf einer offenen Skala frei wählen oder die mit Hilfe von Web-HIPRE bestimmten Kriterien-Gewichtungen eingeben. 
Abbildung 6.12: Gewichtung von Kriterien in den drei Bewertungsdimensionen Technik, Ökonomie und Umwelt

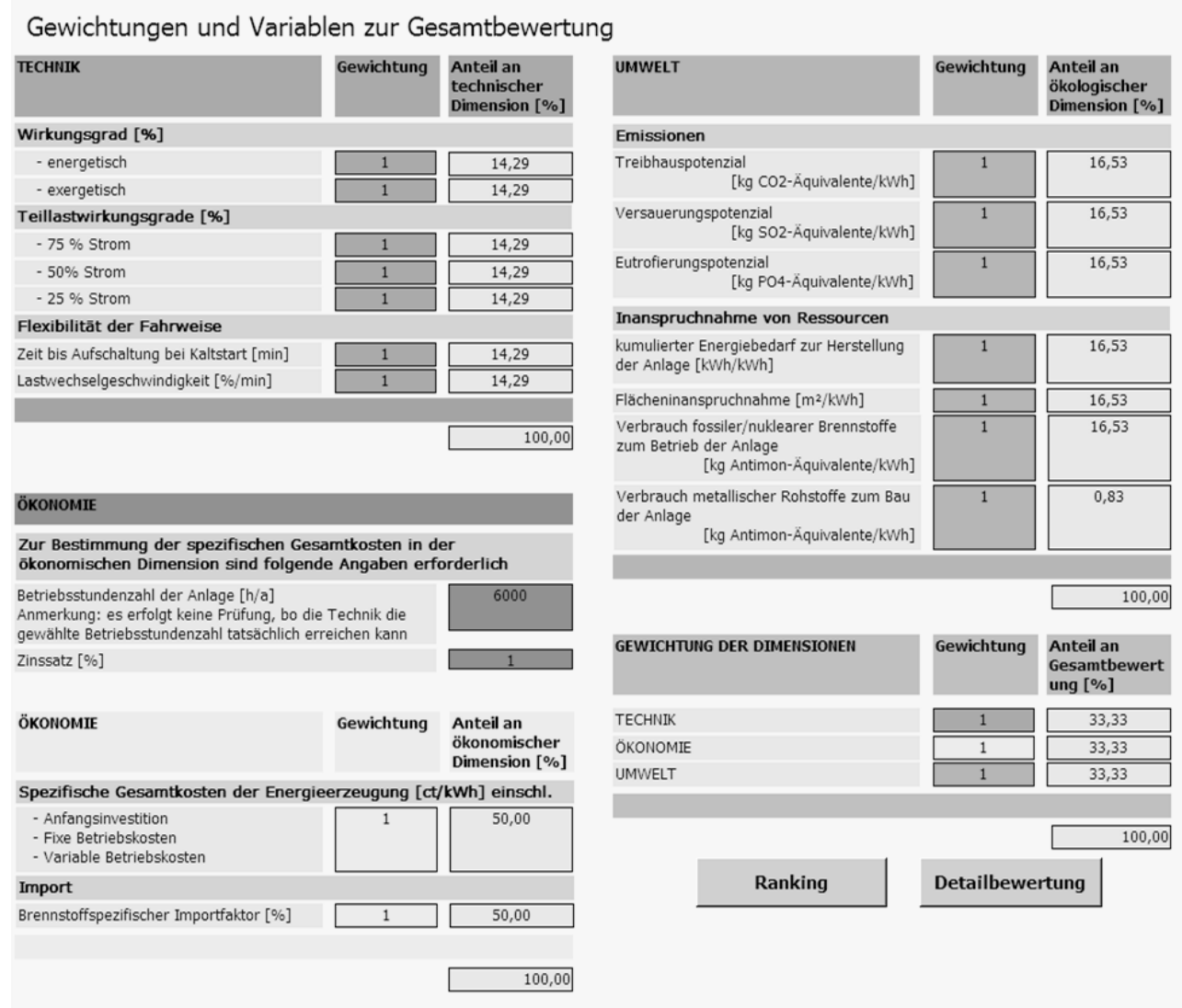

Bei der Durchführung der Analyse erfolgt eine automatische Normierung der vom Nutzer eingegebenen Zahlenwerte auf $100 \%$. Die an dieser Stelle implementierte Auswahl an Bewertungsgrößen basiert auf einer Kriterien-Hierarchie, die auf der Grundlage von Ergebnissen einer Befragung von Entscheidungsträgern in der Energiewirtschaft erarbeitet wurde (vgl. Bartels et al. 2008). Diese Hierarchie unterscheidet in die Dimensionen „Technik“, „Ökonomie“ und „Umwelt”, denen jeweils mehrere Kriterien zugeordnet sind. Dabei können zum einen die Dimensionen und zum anderen die Kriterien innerhalb einer Dimension gegeneinander gewichtet werden. Weiterhin kann der Nutzer die für die Wirtschaftlichkeitsberechnungen zugrunde gelegte Betriebsstundenzahl sowie den Zinssatz variieren. Auf Basis der in dieser Weise durch den Nutzer gewählten Parameter kann innerhalb der Datenbank eine additive Nutzwertanalyse durchgeführt werden. Die Ergebnisse können einerseits als detaillierter Bericht („Detailbewertung“) abgerufen werden, in dem für jede Alternative sämtliche KriterienAusprägungen und deren Normierung sowie das Ergebnis je Dimension zusammengefasst sind. Andererseits wird im Bericht „Ranking“ eine Rangfolge der gewählten Alternativen dargestellt, indem eine geordnete Liste von der „besten“ bis zur „schlechtesten“ 
Alternative erzeugt wird. Diese Rangfolge basiert auf den Gesamtergebnissen der Alternativen, die zwischen 0 („am schlechtesten“) und 1 („am besten) normiert sind.

\subsubsection{Excel-Tool MAESTRO}

Da der in Abschnitt 6.6 dargestellte modifizierte Algorithmus in existierenden SoftwareTools zur Anwendung von PROMETHEE nicht ohne Weiteres umgesetzt werden kann, wird das Excel-Tool MAESTRO entwickelt (vgl. Abbildung 6.13). Technologiedaten aus der MTB-Datenbank können in das Excel-Tool importiert werden, um die Entscheidungsmatrix als Ausgangsbasis der Bewertung zu erstellen. Dabei erlaubt das ExcelTool eine flexible Erweiterung der bewerteten Alternativen und der zugrunde gelegten Kriterien, indem der Entscheidungsmatrix weitere Spalten und Zeilen hinzugefügt werden. Weiterhin sind alle standardmäßig für PROMETHEE verwendeten Präferenzfunktionen hinterlegt, so dass flexibel zwischen den Funktionen 1 bis 6 gewählt werden kann (Abbildung 6.5). Dem Nutzer des Excel-Tools stehen Informationen zur Auswahl geeigneter Präferenzfunktionen im Tabellenblatt „Präferenzfunktion“ zur Verfügung. Die Zuordnung der Alternativen zu Technologiezyklus-Phasen erfolgt über eine Tabelle oberhalb der eigentlichen Entscheidungsmatrix. Für die Zuordnung kann auf die Informationen aus Kapitel 5.2 zurückgegriffen werden. Des Weiteren sind im Tabellenblatt „Technologiezyklus“ innerhalb des Excel-Tools die wichtigsten Merkmale der Phasen zusammengefasst. Wird eine Bewertung unabhängig von Technologiezyklusphasen bevorzugt, kann die Phase „Keine“ gewählt werden. Die relative Gewichtung der Kriterien in Abhängigkeit der Technologie-Zyklus-Phasen wird in der dafür vorgesehenen Matrix eingegeben und grafisch dargestellt. Wenn alle notwendigen Daten determiniert sind, kann die Auswertung über die Schaltfläche „Berechnung“ gestartet werden. Als Ergebnis werden der positive, der negative und der Netto-Fluss in tabellarischer und grafischer Darstellung ausgegeben. Weiterhin werden Stabilitätsintervalle für die Kriterien-Gewichtungen in Abhängigkeit der Technologiezyklusphasen berechnet. Darüber hinaus kann die Veränderung des Netto-Flusses bei Variation der Gewichtung jeweils eines Kriteriums zwischen $0 \%$ und $100 \%$ in grafischer Form dargestellt werden. Dadurch werden Stabilitätsintervalle und die sich in Abhängigkeit der Kriterien-Gewichtung ändernde Rangfolge der Alternativen visualisiert. Die Benutzeroberfläche des MAESTRO-Excel-Tools, die der Eingabe der für die Bewertung notwendigen Daten dient, ist in Abbildung 6.13 dargestellt. Kontrollfunktionen gewährleisten, dass die Eingabe ungültiger Werte vermieden wird. 


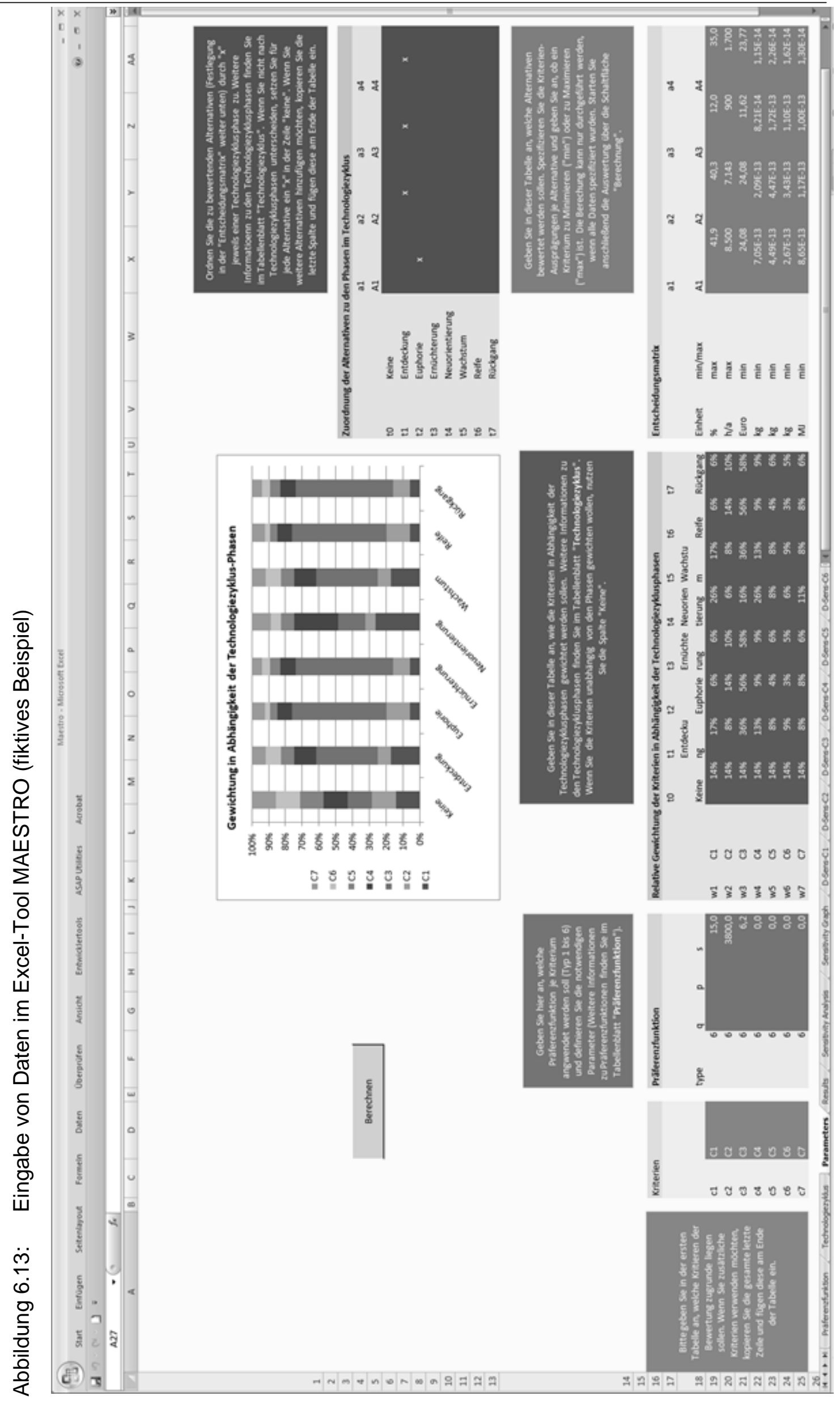


Somit wurde ein EUS entwickelt, das sowohl die Anwendung von Nutzenwertmodellen als auch Outranking-Ansätzen erlaubt. Durch die MTB-Datenbank werden Basisdaten zur Bewertung von Energietechnologien bereitgestellt. In Verbindung mit Web-HIPRE ist eine Anwendung unterschiedlicher Methoden zur Ableitung von Kriterien-Gewichtungen möglich. Sowohl die MTB-Datenbank als auch Web-HIPRE können zur Durchführung einer additiven Nutzwertanalyse eingesetzt werden. Das Excel-Tool MAESTRO dient darüber hinaus zur Bewertung von Alternativen auf Basis von PROMETHEE I und II unter Berücksichtigung von Kriterien-Gewichtungen in Abhängigkeit von Technologiezyklus-Phasen. Die Anwendung dieses EUS für Problemstellungen im Energiebereich wird im folgenden Kapitel durch mehrere Fallbeispiele demonstriert. 


\section{$7 \quad$ Bewertung von Energietechnologien}

Um das entwickelte Entscheidungsunterstützungssystem MAESTRO zu validieren, wird dieses im Folgenden auf konkrete Fragestellungen im Energiebereich angewendet. Im ersten Fallbeispiel wird MAESTRO zur Auswahl von Energietechnologien für die Strom- und Wärmeversorgung in so genannten Bioenergiedörfern eingesetzt. Im zweiten Fallbeispiel muss aus einer Vorauswahl von Stromspeichertechnologien eine Alternative ausgewählt werden, die weiterentwickelt werden soll. Das Ziel besteht darin, die Anwendung des EUS aufzuzeigen.

\subsection{Bewertung alternativer Energieversorgungskonzepte für Bioenergiedörfer}

Vor dem Hintergrund steigender Energiepreise, der Verknappung fossiler Energieträger und der zunehmenden Wahrnehmung von Umweltaspekten haben mehrere Gemeinden in Deutschland entschieden, ihre Energieversorgung eigenverantwortlich umzugestalten. Die Initiative „Bioenergiedörfer“ fokussiert dabei auf das Ziel einer regionalen Nachhaltigkeit, indem Energieversorgungskonzepte umgesetzt werden, die auf lokal verfügbaren Energieträgern basieren und unabhängig von den großen Energieversorgern organisiert werden. Somit gelten Bioenergiedörfer als „Baustein für eine zukunftsfähige Energieversorgung im ländlichen Raum“ (Ruppert et al. 2008). Im ersten Bioenergiedorf Jühnde wurde eine weitgehend autarke Strom- und Wärmeversorgung auf Basis regional verfügbarer Biomasse erfolgreich realisiert. Die Durchführung dieses Vorhabens gilt als Vorbild für andere Gemeinden und Regionen, in denen die regionale Energieversorgung in erster Linie auf die Nutzung erneuerbarer Energien, insbesondere Biomasse, umgestellt werden soll (Ahl et al. 2007; Karpenstein-Machan und Schmuck 2007; Ruppert et al. 2008). Zwar wurden für den Entscheidungsprozess in Jühnde keine multikriteriellen Methoden eingesetzt. Dies bietet sich jedoch für zukünftige Entscheidungsprozesse in Bioenergiedörfern an, um die verschiedenen Zieldimensionen der Nachhaltigkeit simultan zu berücksichtigen. Dabei bieten multikriterielle EUS die Möglichkeit, die zur Verfügung stehenden Optionen auf Basis mehrere Kriterien zur Abbildung der Nachhaltigkeits-Dimensionen systematisch einander gegenüberzustellen. Vor diesem Hintergrund wird im Folgenden die Anwendung des EUS MAESTRO zum Vergleich von Technologien zur Bereitstellung von Strom und Wärme für Bioenergiedörfer am Beispiel Jühnde aufgezeigt.

\subsubsection{Definition der Problemstellung}

Das erste Bioenergiedorf in Deutschland wurde initiiert durch Wissenschaftler der Universitäten Göttingen, Kassel und Berlin. Die Realisierung erfolgte unter aktiver Partizi- 
pation der Bevölkerung des Dorfes Jühnde im südlichen Niedersachsen, in dem insgesamt etwa 800 Einwohner leben. Vor der Umsetzung des Bioenergiedorf-Konzeptes im Jahr 2005 basierte die Energieversorgung in Jühnde in erster Linie auf der Stromversorgung durch die regionalen Energieversorgungsunternehmen und der Wärmeversorgung der Haushalte auf Basis konventioneller Heizöl-Kessel. Ausgehend von der regionalen Verfügbarkeit biogener Brennstoffe wurde ein alternatives Versorgungskonzept auf Basis von Biomasse entwickelt und umgesetzt (Abbildung 7.1).

Abbildung 7.1: $\quad$ Aktuelles Versorgungskonzept im Bioenergiedorf Jühnde

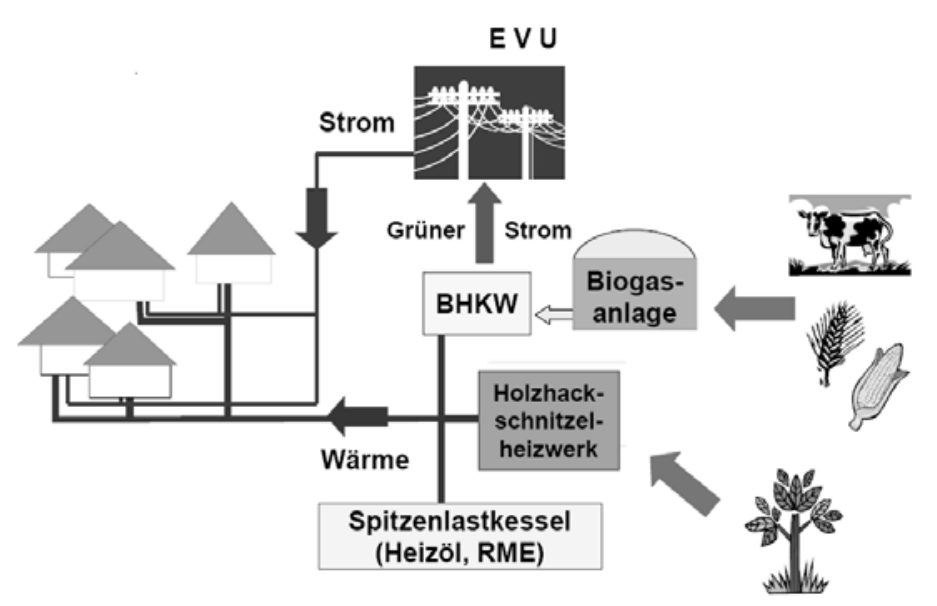

Anmerkungen: EVU = Energieversorgungsunternehmen; RME = Rapsölmethylsester (Biodiesel). Quelle: Ahl et al. (2007); Ruppert et al. (2008).

In einer Biogasanlage werden Gülle und andere Biomasse (Weizen, Roggen Triticale, Mais, Sonnenblumen, etc.) angeliefert und vergoren. Das so erzeugte Biogas wird in einem Blockheizkraftwerk zur gekoppelten Erzeugung von Strom und Wärme eingesetzt. Der erzeugte Strom wird in das Stromnetz des regionalen Energieversorgungsunternehmens eingespeist und nach EEG vergütet. Um die anfallende Wärme an die Haushalte zu verteilen, wurde ein Nahwärmenetz verlegt. Die zuvor in den Haushalten installierten Heizkessel wurden durch Wärmeübergabe-Stationen ersetzt. Für einen erhöhten Wärmebedarf im Winter ist neben dem BHKW ein Holzhackschnitzelheizwerk an das Nahwärmenetz angeschlossen. Im Fall, dass die Wärme aus der Biogasanlage und dem Holzhackschnitzelheizwerk nicht ausreicht, beispielsweise an sehr kalten Tagen oder bei Wartungsarbeiten an den Anlagen, steht ein Spitzenlastkessel zur Verfügung, der mit Heizöl oder Rapsölmethylester (RME) betrieben werden kann ((Ahl et al. 2007; Karpenstein-Machan und Schmuck 2007; Ruppert et al. 2008). Das EUS MAESTRO wird im Folgenden dazu angewendet, das in Jühnde realisierte BiomasseKonzept anderen denkbaren Alternativen gegenüberzustellen. 


\subsubsection{Vorauswahl der Alternativen}

Der Fokus des Versorgungskonzeptes in Jühnde liegt auf Biomasse, da deren Nutzung zur Energiebereitstellung keine negativen Umwelteffekte nach sich zieht und im betrachteten ländlichen Raum in Niedersachsen ausreichende Mengen zur Verfügung stehen (Eigner und Schmuck 2002). Generell sollte sich die Vorauswahl der Alternativen für eine dezentrale Energieversorgung auf die Potenziale der regional verfügbaren Energieträger stützen. Unter Berücksichtigung lokal verfügbarer Potenziale und nachhaltiger Landnutzungskonzepte wurde für Jühnde ein Versorgungskonzept basierend auf Biomasse entwickelt. Dieses kann als Vorbild für die Erarbeitung von Alternativen zur Biomasse-Nutzung in anderen Regionen dienen. Insbesondere in Gebieten, in denen die lokalen Biomasse-Potenziale nicht ausreichen, sind neben der BiomasseNutzung weitere Alternativen denkbar, die eine nachhaltige Energieversorgung versprechen (vgl. Kapitel 3). Beispielsweise können Windkraft- und/oder PhotovoltaikAnlagen zur Stromerzeugung und/oder Solarkollektoren zur Unterstützung der Wärmebereitstellung eingesetzt werden. Eine weitere Alternative zur Wärmebereitstellung besteht in der Nutzung moderner Erdgas-Brennwertkessel, die eine effiziente Nutzung des im Vergleich zum Heizöl $\mathrm{CO}_{2}$-ärmeren, fossilen Energieträgers versprechen. Denkbar ist ebenso die Installation eines Erdgas-basierten BHKWs anstelle der Biomasse-Anlage. Eine innovative Alternative stellen Brennstoffzellen dar. Weiterhin können als Alternative zur Bereitstellung von Nutzwärme Wärmepumpen oder HolzpelletsKessel in den einzelnen Haushalten installiert werden. Weitere lokal nutzbare Potenziale für Wasserkraft- oder geothermische Anlagen sind für Jühnde nicht bekannt. Da Jühnde nicht in Küstennähe liegt, können des Weiteren Optionen zur Nutzung von Meeresenergie und Offshore-Windenergieanlagen für diese Region ausgeschlossen werden. Aufgrund des kleinen Versorgunggebietes können zudem Technologien, die sich zur Anwendung in Großkraftwerken eigenen (zum Beispiel GuD-Anlagen), ausgeschlossen werden. Davon ausgehend werden für diese Fallstudie beispielhaft folgende Alternativen betrachtet:

A1. Als Referenz die Stromversorgung aus dem öffentlichen Versorgungsnetz, abgebildet durch den deutschen Strom-Mix; Wärmeversorgung basierend auf konventionellen Heizöl-Kesseln in einzelnen Haushalten.

A2. Das in Jühnde umgesetzte Biomasse-Konzept zur Bereitstellung von Strom und Wärme (vgl. Abschnitt 7.1.1).

A3. Anstelle des Biomasse-BHKWs in Nr. A2 Installation eines Erdgas-BHKWs; Holzhackschnitzel-Heizwerk und Spitzenlastkessel werden beibehalten.

A4. Anstelle des Biomasse-BHKWs in Nr. A2 Installation eines BrennstoffzellenBHKWs basierend auf Erdgas; Holzhackschnitzel-Heizwerk und Spitzenlastkessel werden beibehalten. 
A5. Stromversorgung basierend auf Windkraft- und Photovoltaik-Anlagen, wobei Abweichungen von Angebot und Nachfrage durch Strombezug aus dem öffentlichen Versorgungsnetz ausgeglichen werden; Wärmeversorgung auf Basis moderner Gas-Brennwertkessel, die in einzelnen Haushalten installiert werden.

A6. Wie Nr. A5, jedoch ohne Photovoltaik-Anlagen; Wärmeversorgung auf Basis von Solarkollektoren in Verbindung mit modernen Gas-Brennwertkesseln, die in einzelnen Haushalten installiert werden.

A7. Stromversorgung wie Nr. A5; Wärmeversorgung auf Basis von Wärmepumpen, die in einzelnen Haushalten installiert werden.

A8. Stromversorgung wie Nr. A5; Wärmeversorgung auf Basis von Holzpellets-Kesseln, die in einzelnen Haushalten installiert werden.

\subsubsection{Auswahl der Kriterien und Bestimmung der Ausprägungen}

Nach Karpenstein-Machan und Schmuck (2007) wurde das erste BioenergiedorfProjekt durch drei Gründe motiviert: die Verpflichtungen zur Reduzierung der Treibhausgasemissionen im Rahmen des Kyoto-Protokolls, Forschungsbedarf im Bereich der nachhaltigen Entwicklung sowie Bedarf an Lösungen zur Umsetzung umweltfreundlicher Lebensweisen in die Praxis. Folglich sollten die Kriterien zur Bewertung von Versorgungskonzepten für Bioenergiedörfer dem Ziel einer nachhaltigen Energieversorgung, insbesondere Umweltauswirkungen wie dem Treibhauseffekt, Rechnung tragen. Entsprechend dem so genannten Brundtland-Bericht (UN 1987) ist eine nachhaltige Entwicklung dadurch gekennzeichnet, dass heutige Bedürfnisse befriedigt werden, ohne die Möglichkeiten zur Befriedigung von Bedürfnissen zukünftiger Generationen einzuschränken. Die Operationalisierung des Nachhaltigkeitskonzeptes ist hierbei in der Regel durch die Integration der drei Dimensionen Ökonomie, Ökologie und Soziales gekennzeichnet (zum Beispiel Gallego Carrera und Mack 2010; Geldermann 2005; Strebel 2009). So weisen auch Karpenstein-Machan und Schmuck (2007) darauf hin, dass „,basierend auf den ökologischen, ökonomischen und sozialen Vorteilen des kommunalen Bioenergie-Konzeptes, das Leuchtturm-Projekt Jühnde als Modell für viele andere Dörfer in Deutschland dienen wird“. Darüber hinaus spielt im Energiebereich die Versorgungssicherheit eine bedeutende Rolle (Bundesregierung 2005; Europäische Kommission 2006). Diesbezüglich heben in Zusammenhang mit Bioenergiedörfern Eigner und Schmuck (2002), Karpenstein-Machan und Schmuck (2007) sowie Ruppert et al. (2008) die fortschreitende Verknappung fossiler Energieträger sowie Importabhängigkeiten hervor. Folglich sollen zur Bewertung von Technologien zur Bereitstellung von Strom und Wärme in Bioenergiedörfern folgende Aspekte Berücksichtigung finden: 
- Die Wirtschaftlichkeit der Energieversorgung basierend auf den Bereitstellungskosten, einschließlich Investitionen, fixe und variable Betriebskosten.

- Umweltauswirkungen aufgrund der Bereitstellung von Strom und Wärme.

- Der Beitrag der Optionen zur Versorgungssicherheit.

- Soziale Aspekte der Energiebereitstellung.

\subsubsection{Wirtschaftlichkeit}

Zur Beurteilung der absoluten und relativen Vorteilhaftigkeit von Projekten aus wirtschaftlicher Sicht stehen Methoden der Investitionsrechnung zur Verfügung. Dabei werden statische und dynamische Methoden unterschieden (vgl. Götze 2006; Wöhe und Döring 1996). In Energiewirtschaft und Industrie werden statische Methoden in der Regel nur zur Bewertung relativ kleiner Investitionsvorhaben angewendet, wenn es beispielsweise um Maßnahmen zur Einsparung von Energie und Kosten geht. Vorteile liegen im geringeren Rechenaufwand im Vergleich zu den dynamischen Methoden. Letztere basieren auf der Kapitalwertmethode (Net Present Value - NPV) und berücksichtigen die unterschiedlichen Zeitpunkte, zu denen die Zahlungen anfallen, durch Auf- bzw. Abzinsung der Zahlungsströme auf einen Bezugszeitpunkt. Dabei werden die Barwerte aller Einnahmen und Ausgaben über die Nutzungsdauer der betrachteten Investition bestimmt und summiert. Als Bezugszeitpunkt für die Barwerte wird in der Regel der Zeitpunkt der Inbetriebnahme der Anlage gewählt. Zahlungen, die früher bzw. später anfallen, sind mit dem Kalkulationszinssatz auf- bzw. abzuzinsen. Eine Investitionsmaßnahme ist im absoluten Sinn dann wirtschaftlich, wenn der Kapitalwert positiv ist. Beim Vergleich mehrerer Optionen ist diejenige Alternative wirtschaftlich am vorteilhaftesten, die den höchsten Kapitalwert aufweist (Konstantin 2009).

Für eine umfassende Wirtschaftlichkeitsanalyse auf Basis einer Vollkostenrechnung sind alle erwarteten Ausgaben und Einnahmen zu berücksichtigen, die über den gesamten Zeitraum der geplanten Nutzungsdauer anfallen. Bei Energieprojekten resultieren Einnahmen aus den Erlösen des Verkaufs der Energie. Neben der Anfangsinvestition entstehen während der Nutzung Ausgaben aufgrund von Betriebskosten, beispielsweise für die eingesetzten Primärenergieträger, Hilfs- und Betriebsstoffe sowie Personal und Instandhaltung. In der Praxis beschränkt sich der Vergleich mehrerer Optionen für ein bestimmtes Projekt in der Regel auf die Kostenseite, da dies für die Bestimmung der relativen Vorteilhaftigkeit aus wirtschaftlicher Sicht ausreicht (Konstantin 2009). Um mehrere Optionen mit variierenden Erzeugungsmengen und Nutzungsdauern einander gegenüberzustellen eignen sich die spezifischen Kosten je Produkteinheit als Vergleichsgröße. Für einen Vergleich von Optionen zur Strom- bzw. Wärmebereitstellung sind somit die Energiegestehungskosten ( $\mathrm{Ct} / \mathrm{kWh}_{\mathrm{el}} \mathrm{bzW}$. Ct/kWh $\left.\mathrm{h}_{\mathrm{th}}\right)$ 
ein geeignetes Wirtschaftlichkeitskriterium. Diese werden auf Basis der Annuitätenmethode als finanzmathematischer Mittelwert über die Nutzungsdauer ermittelt. Die Implementierung der hierzu notwendigen Rechenschritte in der MTB-Datenbank ist im Anhang 10.3.1 erläutert.

Bei KWK-Technologien erfolgt eine Zurechnung der Kosten zur Strom- bzw. Wärmebereitstellung basierend auf einem exergetischen Allokationsfaktor. Die Exergie beruht auf physikalischen Prinzipien und berücksichtigt die Qualität der energetischen Produkte (Briem et al. 2004). Die Exergie ist dabei umso höher je umfangreicher die Weiterverwendungsmöglichkeiten der energetischen Produkte sind. Somit weist ein exergetischer Allokationsfaktor sowohl gegenüber ökonomischen als auch gegenüber anderen mengenmäßigen Faktoren (zum Beispiel bezogen auf Energiegehalt, Gewicht) Vorteile auf. Letztere tragen den Produktqualitäten bzw. Wertigkeiten der erzeugten Produkte Strom und Wärme nicht in angemessener Weise Rechnung. Ökonomische Größen wie etwa Preise für Strom oder Nutzwärme unterliegen zum Teil starken Schwankungen und sind zudem von regionalen Rahmenbedingungen abhängig. Daher kommt im Fallbeispiel der exergetische Allokationsfaktor zur Anwendung. Da Strom prinzipiell in alle anderen Energieformen umgewandelt werden kann, entspricht der Exergiegehalt einer Kilowattstunde Strom auch dessen Energiegehalt. Bei Wärme hängt der Exergiegehalt von der Temperatur der abgegebenen Wärme ab. Je geringer die Temperatur ist desto geringer ist auch der Exergiegehalt im Vergleich zur entsprechenden Energiemenge (Szargut et al. 1988; WEC 1992).

Für einen möglichst objektiven Technologievergleich werden im Fallbeispiel zunächst die Kosten der Bereitstellung ohne Berücksichtigung möglicher Förderbeträge betrachtet. Dabei variieren die variablen Kosten der Bereitstellung je nach eingesetztem Energieträger in Abhängigkeit der zugrunde gelegten Szenarien zur Entwicklung der Energieträgerpreise (vgl. Abschnitt 6.7). In einer weiteren Variante werden bei der Wirtschaftlichkeitsbewertung der Alternativen zusätzlich die aus der öffentlichen Förderung bestimmter Technologien resultierenden Einzahlungen aus Sicht des Anlagenbetreibers berücksichtigt. Dabei finden sowohl Einspeisevergütungen nach EEG (Bundesregierung 2008c) und KWKG (Bundesregierung 2008a) als auch Investitionszuschüsse für Technologien zur Wärmebereitstellung basierend auf erneuerbaren Energien entsprechend dem Marktanreizprogramm (BMU 2009e) Berücksichtigung.

Die einzelnen Schritte zur Ermittlung der spezifischen Energiegestehungskosten für die Alternativen des Fallbeispiels sind im Anhang 10.3.2 erläutert. Tabelle 7.1 zeigt die spezifischen Gesamtkosten der Stromerzeugung je Technologie, die je nach Szenario variieren. Unter den betrachteten Alternativen weisen Windkraftanlagen (A5 bis A8: Wind) die geringsten spezifischen Gesamtkosten auf, die zudem über alle Szenarien 
konstant sind. Da die derzeitigen Einspeisevergütungssätze für Windstrom höher liegen als die ermittelten spezifischen Gesamtkosten, sind unter Beachtung der Förderbedingungen negative Kosten ausgewiesen. Die höchsten Kosten weist das Brennstoffzellen-BHKW (A4: BZ-BHKW) auf. Durch die Inanspruchnahme von Fördergeldern können die Kosten aus Sicht des Anlagenbetreibers reduziert werden, sind jedoch auch unter diesen Bedingungen in allen Szenarien am höchsten. Auch die spezifischen Gesamtkosten der Photovoltaik-Anlagen (A5, A7, A8: PV) sind sehr hoch, können aber durch die Inanspruchnahme von Einspeisevergütungen gemäß EEG deutlich reduziert werden. Dabei ist aus Sicht des Anlagenbetreibers das Fördermodell zum Eigenverbrauch des in PV-Anlagen erzeugten Stroms wirtschaftlich vorteilhafter als das Modell zur vollständigen Einspeisung der erzeugten Strommengen. Die Kosten der Stromerzeugung im Biogas-BHKW (A2: BioG-BHKW) liegen höher als im Erdgas-BHKW (A3: ErdG-BHKW). Im Ressourcen-Szenario (Szenario 3), in dem die Preise der fossilen Energieträger stark steigen, sind die Unterschiede zwischen diesen beiden Alternativen geringer. Bei Berücksichtigung der Förderbedingungen im Szenario 3 liegen die Kosten der Strombereitstellung im Biogas-BHKW unter denen des Erdgas-BHKW. Die Kosten der Strombereitstellung aus dem öffentlichen Versorgungsnetz (A1: Strommix D) liegen im mittleren Bereich der betrachteten Alternativen.

Tabelle 7.1: $\quad$ Spezifische Kosten der Strombereitstellung in $\mathrm{Ct} / \mathrm{kWh}_{\mathrm{el}}$

\begin{tabular}{l|l|r|r|r|r|r|r|r|r}
\hline \multirow{2}{*}{ Alternative } & \multirow{2}{*}{ Technologie } & \multicolumn{2}{|c|}{ Szenario 0 } & \multicolumn{2}{c|}{ Szenario 1 } & \multicolumn{2}{c|}{ Szenario 2 } & \multicolumn{2}{c}{ Szenario 3 } \\
\cline { 3 - 10 } & o. F. & m. F. & o. F. & m. F. & \multicolumn{1}{c}{ o. F. } & m. F. & \multicolumn{1}{c}{ o. F. } & m. F. \\
\hline A1 & Strommix D & 18,9 & 18,9 & 19,8 & 19,8 & 20,2 & 20,2 & 23,7 & 23,7 \\
\hline A2 & BioG-BHKW & 37,3 & 23,9 & 38,6 & 25,2 & 38,6 & 25,2 & 46,9 & 33,5 \\
\hline A3 & ErdG-BHKW & 14,2 & 13,6 & 18,4 & 17,8 & 18,4 & 17,8 & 36,5 & 35,8 \\
\hline A4 & BZ-BHKW & 56,3 & 51,7 & 57,8 & 53,2 & 57,8 & 53,2 & 62,0 & 57,4 \\
\hline A5-A8 & Wind & 7,6 & $-2,0$ & 7,6 & $-2,0$ & 7,6 & $-2,0$ & 7,6 & $-2,0$ \\
\cline { 2 - 9 } & PV & 42,6 & 3,1 & 42,6 & 3,1 & 42,6 & 3,1 & 42,6 & 3,1 \\
\cline { 2 - 9 } & PV (*) & 42,6 & 2,6 & 42,6 & 2,3 & 42,6 & 2,3 & 42,6 & 1,6 \\
\hline
\end{tabular}

Anmerkungen: o. F. = ohne Berücksichtigung öffentlicher Förderung; $m$. F. = unter Berücksichtigung aktueller Förderbedingungen; $\left(^{*}\right)$ Inanspruchnahme des Fördersatzes für Eigenverbrauch. Unter Szenario 0 sind die Kosten dargestellt, die sich bei konstanten Preisen der Energieträger ergeben.

Quellen: Vgl. Anhang 10.3.2; eigene Annahmen und Berechnungen. 
Abbildung 7.2: $\quad$ Aufteilung der spezifischen Gesamtkosten der Strombereitstellung auf Investitionen, fixe und variable Betriebskosten
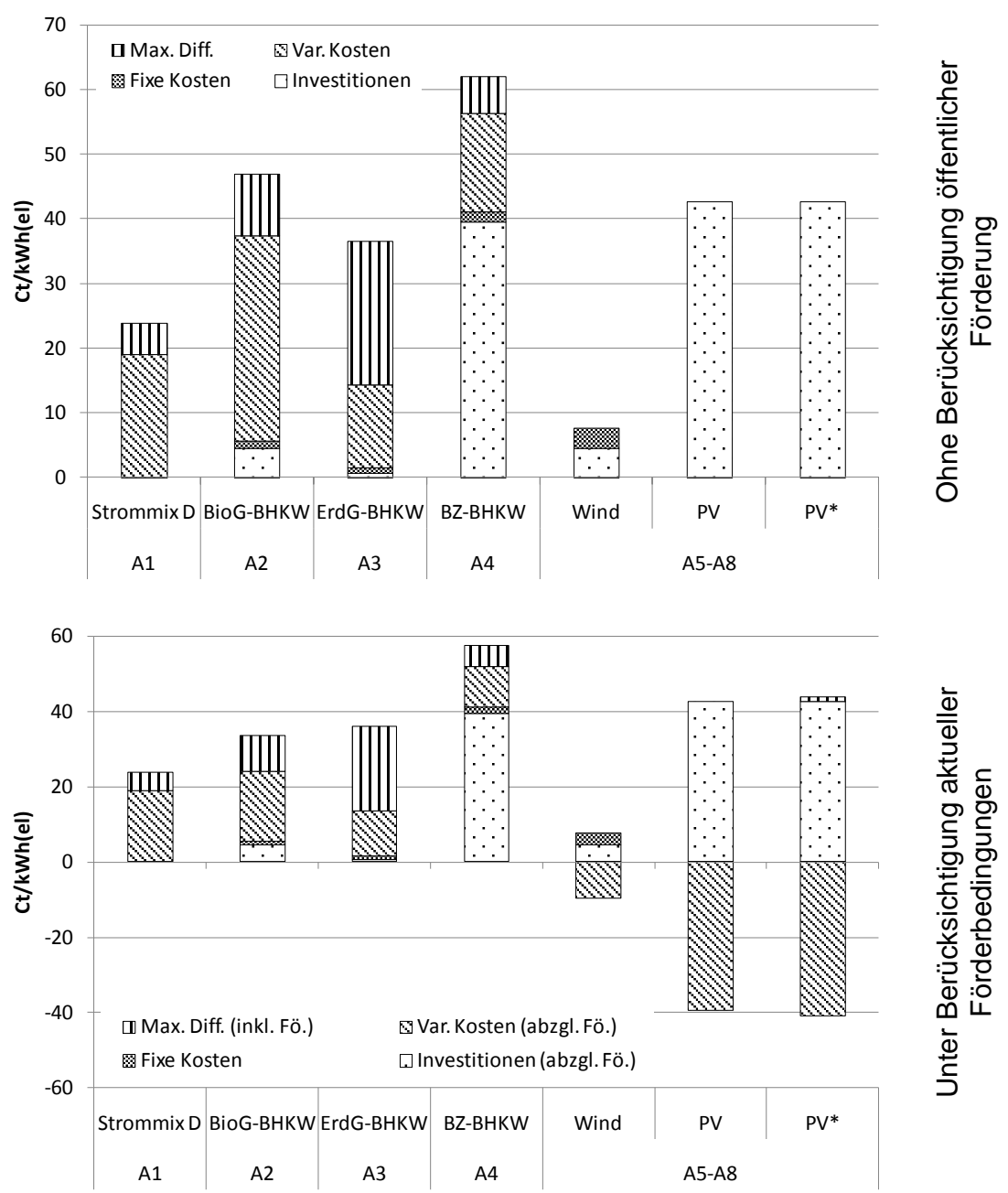

Anmerkungen: Max. Diff. = maximale Differenz zwischen den in Abhängigkeit der Szenarien berechneten variablen Kosten; Var. Kosten = Variable Kosten; Fö. = Förderung.

Quellen: Vgl. Anhang 10.3.2; eigene Annahmen und Berechnungen.

In Abbildung 7.2 wird deutlich, dass die Kosten bei den Alternativen 2 (Biogas-BHKW) und 3 (Erdgas-BHKW) im Wesentlichen durch die variablen Kosten bestimmt sind. Dies ist auf die Kosten der eingesetzten Energieträger zurückzuführen. Deren Entwicklung in Abhängigkeit der Szenarien übt hierbei einen starken Einfluss auf die Gesamtkosten dieser Alternativen aus. Im Gegensatz dazu sind die Kosten der PV- und Windkraftanlage (A5 bis A8) sowie der Brennstoffzelle (A4) vor allem durch die Investitionen bestimmt. Beim Brennstoffzellen-BHKW ist zudem der Anteil der variablen Kosten aufgrund des eingesetzten Energieträgers Erdgas von wesentlicher Bedeutung, wohingegen die spezifischen Kosten der Windkraft- und der PV-Anlage nicht von der Entwicklung der Energieträgerpreise beeinflusst werden. Des Weiteren verdeutlicht Abbildung 
7.2 (unten) die Relation der Fördersätze zu den tatsächlichen Kosten der Stromerzeugung in Wind- und PV-Anlagen.

Die spezifischen Gesamtkosten der Wärmebereitstellung in Abhängigkeit der Szenarien sind in Tabelle 7.2 zusammengefasst. Die geringsten Kosten der Wärmebereitstellung weist das Erdgas-BHKW auf (A3). Andererseits liegen die spezifischen Kosten der Wärmebereitstellung durch das Holzhackschnitzel-Heizwerk (HHS-HW) für diese Alternative am höchsten. Dies ist darauf zurückzuführen, dass in diesem Fall nur ein relativ geringer Anteil der Wärme über das Heizwerk bereit gestellt wird, so dass die Annuitäten auf eine vergleichsweise geringe Wärmemenge bezogen werden. Ist der Anteil der aus dem Heizwerk bereit gestellten Wärmemenge höher (A2 und A4), sinken die spezifischen Kosten der Wärmebereitstellung entsprechend.

Tabelle 7.2: $\quad$ Spezifische Kosten der Wärmebereitstellung in $\mathrm{Ct} / \mathrm{kWh}$ th

\begin{tabular}{|c|c|c|c|c|c|c|c|c|c|}
\hline \multirow[b]{2}{*}{ Alternative } & \multirow[b]{2}{*}{ Technologie } & \multicolumn{2}{|c|}{ Szenario 0} & \multicolumn{2}{|c|}{ Szenario 1} & \multicolumn{2}{|c|}{ Szenario 2} & \multicolumn{2}{|c|}{ Szenario 3} \\
\hline & & o. F. & m. F. & o. F. & m. F. & o. F. & m. F. & o. F. & m. F. \\
\hline A1 & HL-K & 14,5 & 14,5 & 16,0 & 16,0 & 16,1 & 16,1 & 20,2 & 20,2 \\
\hline \multirow[t]{2}{*}{ A2 } & BioG-BHKW & 9,6 & 6,7 & 9,8 & 6,9 & 9,8 & 6,9 & 11,4 & 8,5 \\
\hline & HHS-HW & 20,6 & 20,0 & 20,7 & 20,0 & 20,7 & 20,0 & 21,1 & 20,4 \\
\hline \multirow[t]{2}{*}{ A3 } & ErdG-BHKW & 6,4 & 5,7 & 7,2 & 6,5 & 7,2 & 6,5 & 10,5 & 9,8 \\
\hline & HHS-HW & 23,9 & 23,2 & 23,9 & 23,2 & 23,9 & 23,2 & 24,3 & 23,6 \\
\hline \multirow[t]{2}{*}{ A4 } & BZ-BHKW & 14,2 & 12,8 & 14,5 & 13,0 & 14,5 & 13,0 & 15,2 & 13,8 \\
\hline & HHS-HW & 12,0 & 11,4 & 12,0 & 11,4 & 12,0 & 11,4 & 12,4 & 11,8 \\
\hline A5 & ErdG-BWK & 11,8 & 11,8 & 12,8 & 12,8 & 12,8 & 12,8 & 17,1 & 17,1 \\
\hline A6 & Sol+ErdG-BWK & 14,1 & 13,6 & 15,0 & 14,4 & 15,0 & 14,4 & 18,6 & 18,1 \\
\hline A7 & WP & 14,3 & 12,9 & 14,8 & 13,3 & 14,8 & 13,4 & 16,0 & 14,5 \\
\hline A8 & Pellets-K & 19,6 & 18,7 & 19,8 & 18,9 & 19,8 & 18,9 & 21,2 & 20,3 \\
\hline
\end{tabular}

Anmerkungen: o. F. = ohne Berücksichtigung öffentlicher Förderung; m. F. = unter Berücksichtigung aktueller Förderbedingungen; $\left({ }^{*}\right)$ Inanspruchnahme des Fördersatzes für Eigenverbrauch. Unter Szenario 0 sind die Kosten dargestellt, die sich bei konstanten Preisen der Energieträger ergeben.

Quellen: Vgl. Anhang 10.3.2; eigene Annahmen und Berechnungen.

Der Einfluss der Szenarien wird insbesondere bei den mit fossilen Energieträgern befeuerten Technologien deutlich, bei denen die variablen Kosten zu einem wesentlichen Anteil die Gesamtkosten bestimmen (A1: Heizölkessel - HL-K, A3: Erdgas-BHKW, A5: Erdgas-Brennwertkessel - ErdG-BWK). Bei den mit Biomasse befeuerten Anlagen (A2: Biogas-BHKW, A8: Holzpellets-Heizung - Pellets-K) haben steigende Energieträgerpreise einen weniger starken Einfluss. Die Kosten der Wärmebereitstellung aus dem 
Biogas-BHKW (A2) sind nach dem Erdgas-BHKW (A3) am geringsten und weisen beim Ressourcen-Szenario (Szenario 3 ) nur noch eine sehr geringe Differenz zum Erdgas-BHKW auf. Unter Beachtung der aktuellen Förderkonditionen ist in diesem Szenario aus Sicht des Anlagenbetreibers das Biogas-BHKW wirtschaftlich vorteilhafter als das Erdgas-BHKW. Die geringeren Brennstoffkosten der solar unterstützten Erdgas-Heizung (A6: Sol+ErdG-BWK) können in keinem der Szenarien die höheren Investitionen im Vergleich zum Erdgas-Brennwertkessel (A5) kompensieren. Dies gilt auch unter Berücksichtigung eines Investitionszuschusses für Solarkollektoren. Die spezifischen Bereitstellungskosten der Wärmepumpe (A7: WP) liegen im Bereich der solar unterstützten Erdgas-Heizung, steigen jedoch weniger stark an in Abhängigkeit der Szenarien. Die spezifischen Bereitstellungskosten der Holzpellets-Heizung (A8: Pellets-K) liegen deutlich höher, sind andererseits aber auch weniger stark beeinflusst durch die unterschiedlichen Szenarien.

Bei allen Alternativen zur Wärmebereitstellung werden die spezifischen Gesamtkosten zu einem wesentlichen Anteil durch die Investitionen bestimmt (Abbildung 7.3). Beim Holzhackschnitzel-Heizwerk (A2 bis A4) wird zudem der hohe Anteil der fixen Betriebskosten deutlich. Wie zu erwarten, wird die Schwankungsbreite der Kosten in Abhängigkeit der Szenarien insbesondere bei den mit fossilen Energieträgern befeuerten Anlagen deutlich (A1: HL-K; A3: ErdG-BHKW, A5/A6: ErdG-BWK). Bei diesen Alternativen ist der Anteil der variablen Kosten aufgrund der eingesetzten Energieträger von erheblicher Bedeutung. Dies gilt auch für die Holzpellets-Heizung (A8) und das BiogasBHKW (A2). Bei letzterem übersteigt der Anteil der variablen Kosten an den spezifischen Gesamtkosten den der Investitionen. Bei Inanspruchnahme von Fördergeldern reduziert sich der Anteil der variablen Kosten bei dieser Alternative deutlich. 
Abbildung 7.3: $\quad$ Aufteilung der spezifischen Gesamtkosten der Wärmebereitstellung auf Investitionen, fixe und variable Betriebskosten
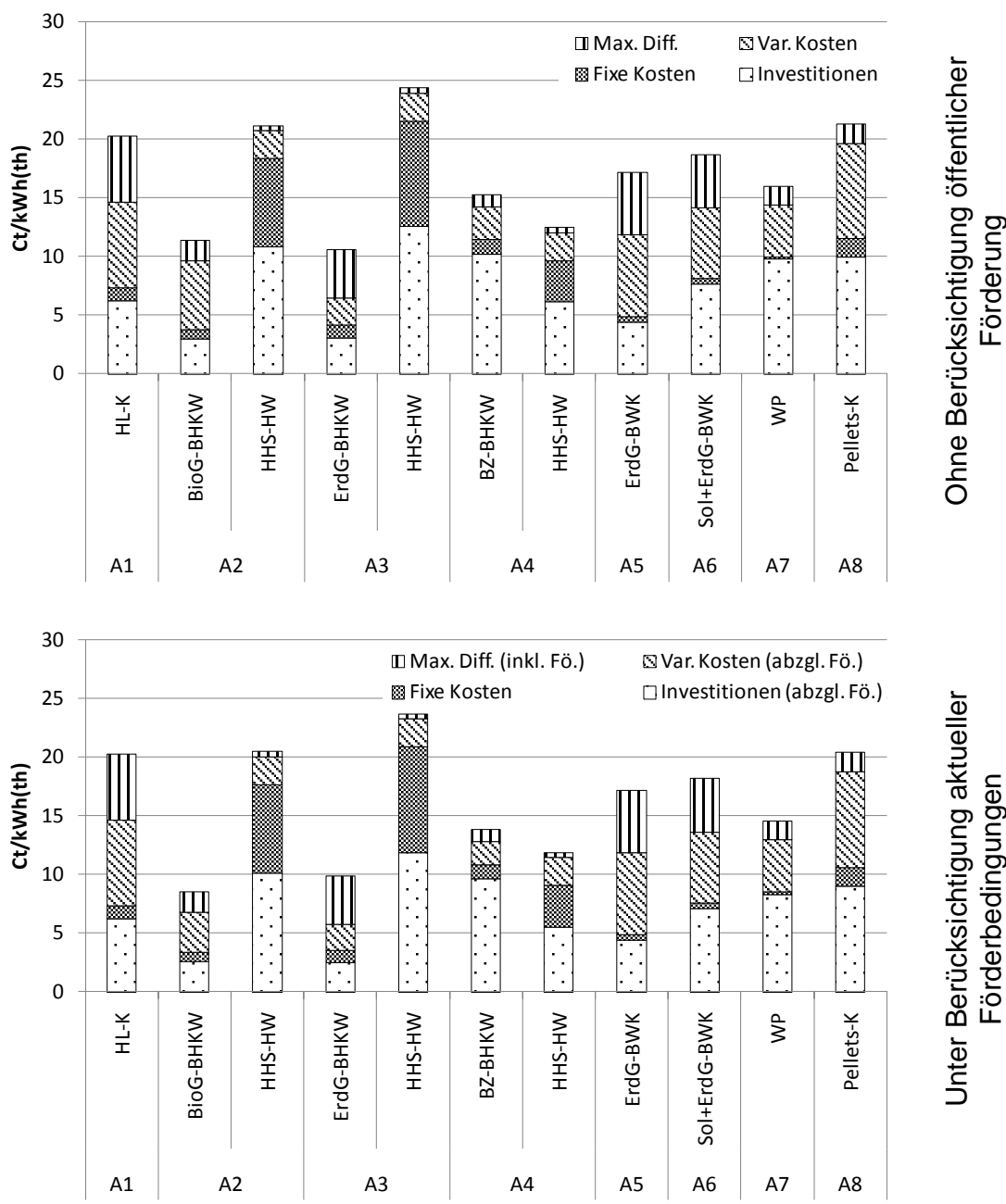

Anmerkungen: Max. Diff. = maximale Differenz zwischen den in Abhängigkeit der Szenarien berechneten variablen Kosten; Var. Kosten = Variable Kosten; Fö. = Förderung.

Quellen: Vgl. Anhang 10.3.2; eigene Annahmen und Berechnungen.

\subsubsection{Umweltauswirkungen}

Zur Abbildung von Umweltauswirkungen der Energiebereitstellung über deren gesamten Lebensweg (von der Herstellung über die Nutzung bis zur Entsorgung) bieten sich wissenschaftlich etablierte Methoden der Ökobilanzierung an. Im Allgemeinen kann bei Methoden zur Wirkungsabschätzung im Rahmen von Ökobilanzen in „Endpoint"- und „Midpoint“-Modelle unterschieden werden (vgl. Hillenbrand 2009). Endpoint-Modelle (schadensorientiert, „damage approach“) versuchen die Effekte bis ans Ende der Wirkungskette nachzuvollziehen (zum Beispiel Auswirkungen auf die menschliche Gesundheit). In Midpoint-Modellen werden die Effekte nur bis zu einem bestimmten Punkt 
der Wirkungskette nachvollzogen, an dem die Aggregation zu einem Wirkungsindikator möglich ist. Bisher kommen überwiegend Midpoint-Modelle zum Einsatz, da diese mit geringeren Unsicherheiten behaftet sind. Unter den Midpoint-Modellen wird für diese

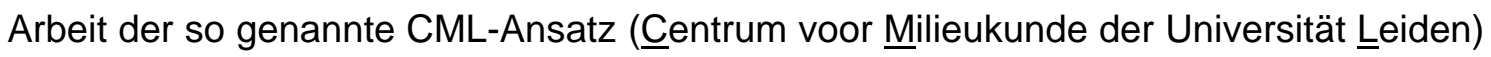
verwendet, da dieser weit verbreitet ist und sich durch ein hohes Maß an Konformität mit den Normen zur Ökobilanzierung auszeichnet (vgl. Hillenbrand 2009; für nähere Informationen zur CML-Methode vgl. Guinée et al. 2002; zur Implementierung in Ecoinvent vgl. Frischknecht et al. 2007a). Entsprechend den Empfehlungen bei Guinée et al. (2002) für eine vereinfachte Ökobilanzierung werden im Rahmen des Fallbeispiels folgende Wirkungskategorien berücksichtigt:

U1. Erschöpfung abiotischer Ressourcen (Abiotic Depletion Potential - ADP)

U2. Landschaftsinanspruchnahme (LAND)

U3. Klimawandel bzw. Treibhauspotenzial (Global Warming Potential - GWP)

U4. Stratosphärischer Ozonabbau (Ozone Depletion Potential - ODP)

U5. Humantoxozität (Human Toxicity Potential - HTP)

U6. Ökotoxizität (Ecotoxicity Potential - ETP)

U6.1 Süßwasser (Freshwater Aquatic Ecotoxicity Potential FAETP)

U6.2 Seewasser (Marine Aquatic Ecotoxicity Potential - MAETP)

U6.3 Boden (Terrestrial Ecotoxicity Potential - TAETP)

U7. Bildung von Photo-Oxidantien (Sommersmog; Photochemical Ozone Creation Potential - POCP)

U8. Versauerung (Acidification Potential - AP)

U9. Eutrophierung (Eutrophication Potential - EP)

Um darüber hinaus einen Überblick über den Gesamtenergieaufwand eines Prozesses zu vermitteln, wird zusätzlich der kumulierte Energieaufwand (KEA) nach VDI (1997) als Kriterium angewendet (U10). Auf die Anwendung weiterer Wirkungskategorien wird an dieser Stelle aus Gründen der Übersichtlichkeit verzichtet. Je nach spezifischem Anwendungskontext und Ziel der Untersuchung sollten in der Praxis für eine detaillierte Ökobilanzierung gegebenenfalls zusätzliche Wirkungskategorien Berücksichtigung finden. Dazu zählen beispielsweise Auswirkungen der Landschaftsinanspruchnahme auf Lebensunterstützende Systeme und auf die Biodiversität, sedimentäre Ökotoxizität, ionisierende Strahlung, Geruchs- und Lärmbelästigung sowie Abwärme (vgl. Guinée et al. 2002).

Die ökobilanziellen Wirkungskategorien werden mit Hilfe der Datenbank Ecoinvent v2.0 (ecoinvent Centre 2007) in Verbindung mit dem Stoffstrommodellierungs-Tool Umberto 
(Version 5.5; ifu und Ifeu 2008) für die einzelnen Technologien ermittelt. Bei Ecoinvent handelt es sich um eine weit verbreitete und anerkannte Datenbank für Lebenszyklusinventare (auch: Sachbilanz; engl.: Life Cycle Inventories - LCI). Diese beinhaltet unter anderem Datensätze, die sich auf Systeme zur Bereitstellung von Elektrizität und Nutzwärme auf Basis unterschiedlicher Energieträger und Technologien beziehen. Methodisch folgt der Aufbau von Ecoinvent den ISO-Standards zur Ökobilanzierung (ISO 2006), wobei der Fokus auf der Bereitstellung von LCls liegt. Dabei sind die Umweltauswirkungen unter Annahme des Normalbetriebs von Anlagen und Prozessen unter Berücksichtigung regelmäßig auftretender Störfälle abgebildet. Daten über Ausnahmeereignisse, zum Beispiel Unfälle in Kernkraftwerken wie in Tschernobyl, die zwar dramatische Auswirkungen haben können, aber nur selten tatsächlich auftreten, sind nicht enthalten. In der Regel stellen die auf einzelne Länder bezogenen Daten nationale Durchschnittswerte dar (Frischknecht et al. 2007b).

Umberto ermöglicht den Aufbau und die Bilanzierung von Stoffstromnetzen, wobei einzelne Knotenpunkte der Netzwerke durch Datensätze aus Ecoinvent, aber auch durch andere Datenquellen und eigene Angaben spezifiziert werden können. Um Daten hinsichtlich Umweltauswirkungen für die multikriterielle Bewertung im Rahmen dieser Arbeit bereitzustellen, sind für einzelne Optionen zur Strom- bzw. Wärmebereitstellung Stoffstromnetze in Umberto aufgebaut und spezifiziert. Auf dieser Basis werden ökobilanzielle Bewertungen nach der CML- und der KEA-Methode durchgeführt. Die Ergebnisse dieser Bewertungen sind für einzelne Optionen zur Strom- und Wärmebereitstellung in der MTB-Datenbank gespeichert und können je nach Bedarf abgerufen werden. Abbildung 7.4 bis Abbildung 7.9 zeigen Ergebnisse der Ökobilanzierung für die im Fallbeispiel betrachteten Optionen. Die Ergebnisse sind bezogen auf den Maximalwert der betrachteten Alternativen in der jeweiligen Wirkungskategorie dargestellt. Dies bedeutet, dass diejenige Option, bei der $100 \%$ angezeigt werden, in der entsprechenden Wirkungskategorie die höchsten Auswirkungen aufweist und damit in dieser Wirkungskategorie am schlechtesten abschneidet.

Unter den Optionen zur Strombereitstellung weist der Deutsche Strommix in sieben der zwölf Wirkungskategorien die höchsten Auswirkungen auf. Dies betrifft die Kategorien zur Bewertung der Öko- und Humantoxizität (MAETP, FAETP, TAETP, HTP; vgl. Abbildung 7.4), den Treibhauseffekt und die Erschöpfung abiotischer Ressourcen (GWP, ADP; vgl. Abbildung 7.5) sowie den kumulierten Energieaufwand (KEA; vgl. Abbildung 7.6). Hinsichtlich Landschaftsinanspruchnahme (LAND), Versauerung (AP) und Eutrophierung (EP) schneidet hingegen das Biogas-BHKW zur Stromerzeugung am schlechtesten ab (Abbildung 7.5 und Abbildung 7.6). In den anderen Wirkungskategorien werden andererseits die Auswirkungen der Stromerzeugung im Biogas-BHKW relativ gering bewertet (insbesondere MAETP, TAETP, ADP, ODP und KEA). 
Abbildung 7.4: $\quad$ Ökobilanzierung der Optionen zur Strombereitstellung: Ökotoxizität und Humantoxizität

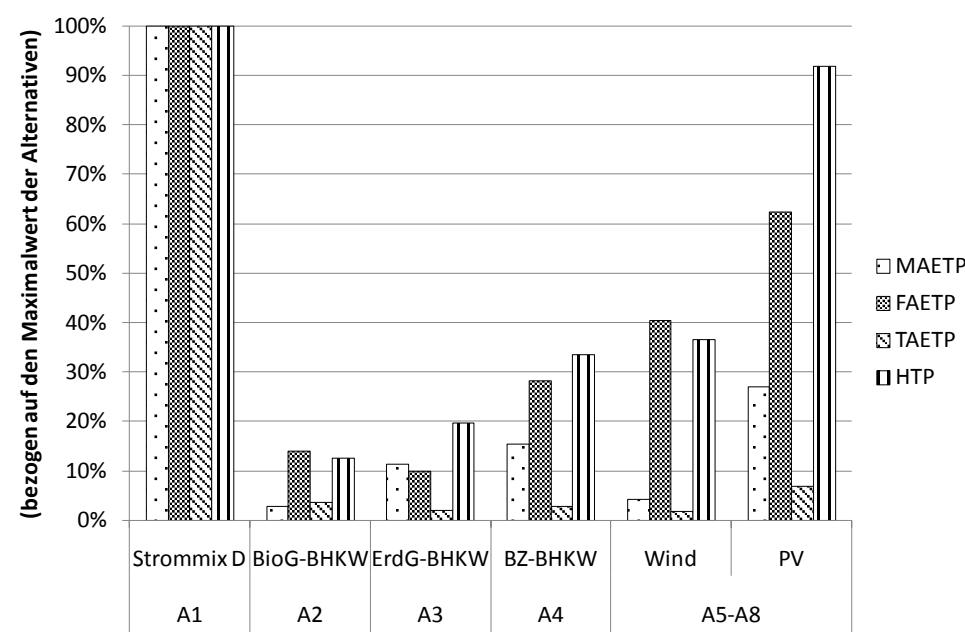

Quellen: ecoinvent Centre (2007); ifu und Ifeu (2008); eigene Annahmen und Berechnungen.

Abbildung 7.5: Ökobilanzierung der Optionen zur Strombereitstellung: Treibhauseffekt, Landschaftsinanspruchnahme, Sommersmog und Erschöpfung abiotischer Ressourcen

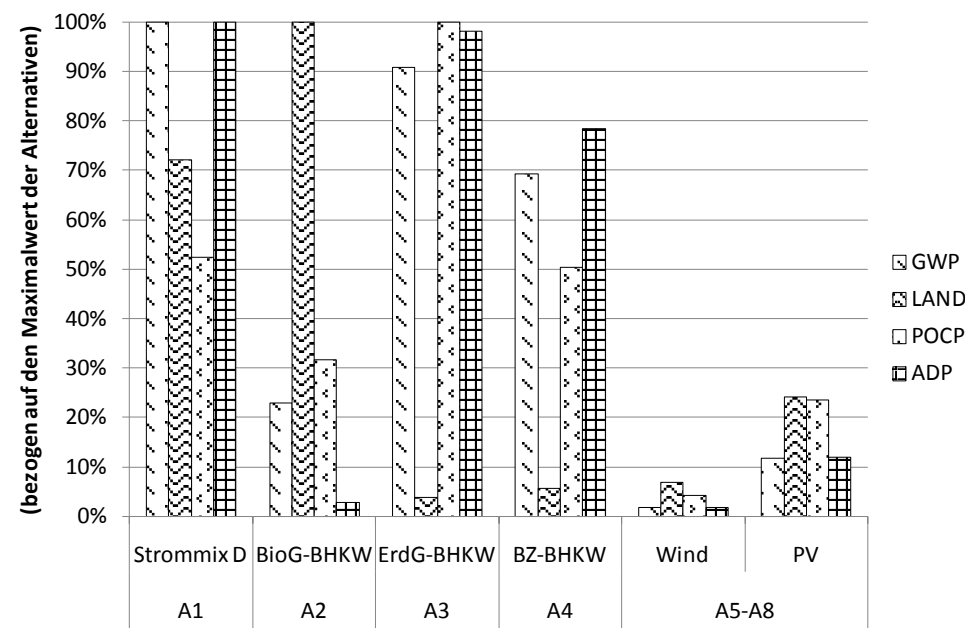

Quellen: ecoinvent Centre (2007); ifu und Ifeu (2008); eigene Annahmen und Berechnungen.

Bei Sommersmog (POCP) und Ozonabbau (ODP) erzielt das Erdgas-BHKW das schlechteste Ergebnis (Abbildung 7.5 und Abbildung 7.6). Dieses weist zudem bezüglich des Treibhauseffektes (GWP), der Erschöpfung abiotischer Ressourcen (ADP) und dem kumulierten Energieaufwand (KEA) vergleichsweise hohe Auswirkungen auf. Die Windkraftanlage zeigt im Vergleich sehr geringe Auswirkungen in den meisten Wirkungskategorien. Lediglich hinsichtlich FAETP und HTP sind die Auswirkungen relativ hoch. Ähnliches gilt für die PV-Anlage, wobei die Auswirkungen generell höher einzustufen sind als bei der Windkraftanlage. Die Umweltauswirkungen der Stromerzeugung 
im Brennstoffzellen-BHKW liegen im Allgemeinen im mittleren Bereich des Vergleichs. Deutlich höhere Auswirkungen im Vergleich zu den meisten anderen Alternativen sind bei der Brennstoffzelle in den Wirkungskategorien GWP, POCP und ADP (Abbildung 7.5) sowie ODP und KEA (Abbildung 7.6) zu beobachten.

Abbildung 7.6: Ökobilanzierung der Optionen zur Strombereitstellung: Ozonabbau, Versauerung, Eutrophierung und Kumulierter Energieaufwand

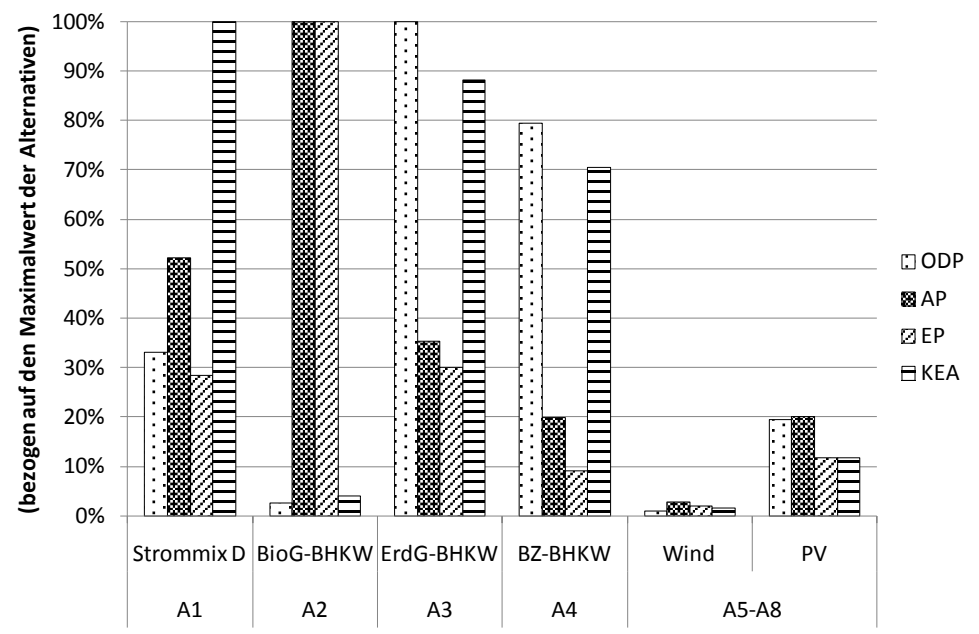

Quellen: ecoinvent Centre (2007); ifu und Ifeu (2008); eigene Annahmen und Berechnungen.

Beim Vergleich der Optionen zur Wärmebereitstellung (Abbildung 7.7 bis Abbildung 7.9) zählt in den meisten ökobilanziellen Wirkungskategorien das Biogas-BHKW zu den am besten bewerteten Optionen. Lediglich in den Wirkungskategorien Versauerung $(A P)$ und Eutrophierung (EP) sind deutlich höhere Auswirkungen im Vergleich zu einigen anderen Optionen zu beobachten (Abbildung 7.9). Die Maximalwerte in den Wirkungskategorien GWP, POCP (Abbildung 7.8) und KEA (Abbildung 7.9) resultieren beim Heizölkessel. Dieser weist zudem im Vergleich zu den anderen Alternativen sehr hohe Werte in den Wirkungskategorien ADP (Abbildung 7.8) und AP (Abbildung 7.9) auf. Die Unterschiede, die bei der Bewertung des Holzhackschnitzel-Heizwerks (inklusive Spitzenlastkessel) aufgrund unterschiedlicher Wärmeerzeugungsmengen zwischen den Alternativen A2, A3 und A4 resultieren, sind nicht signifikant. Die schlechteste Bewertung im Vergleich aller Alternativen weist dieses Heizwerk bezüglich Landschaftsinanspruchnahme (LAND) und Eutrophierung (EP) auf. Mit Ausnahme des Holzpellets-Heizkessels sind die Resultate aller anderen Alternativen in diesen Kategorien wesentlich geringer (Abbildung 7.9). Die Bewertungen des HolzhackschnitzelHeizwerks hinsichtlich HTP (Abbildung 7.7) und AP (Abbildung 7.9) liegen bei etwa $50 \%$ des Maximalwertes. In allen weiteren Wirkungskategorien wird das Heizwerk vergleichsweise niedrig eingestuft mit besonders geringen Ausprägungen $(<10 \%)$ hinsichtlich MAETP, GWP, ADP, ODP und KEA. 
Abbildung 7.7: $\quad$ Ökobilanzierung der Optionen zur Wärmebereitstellung: Ökotoxizität und Humantoxizität

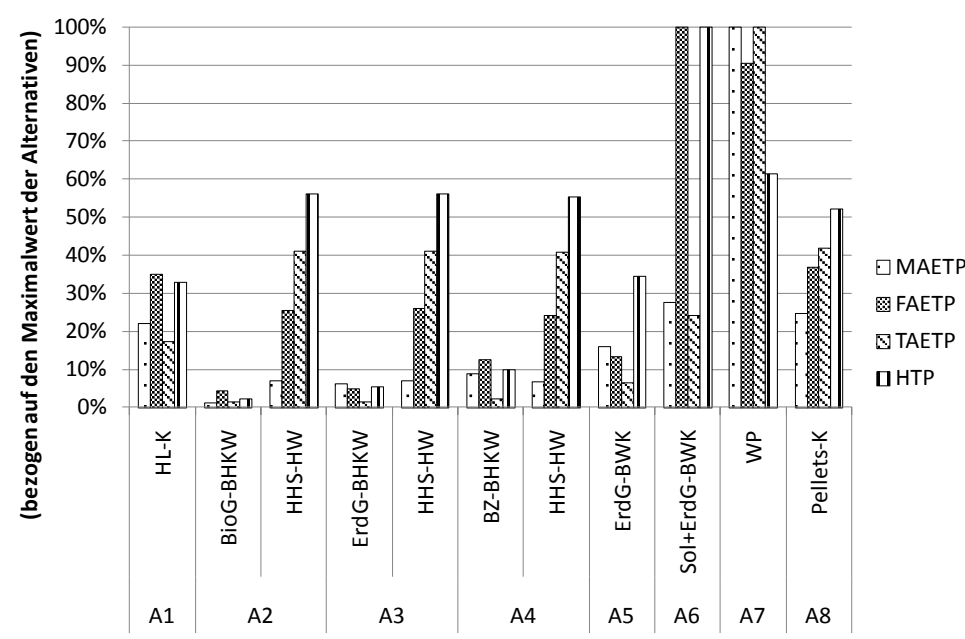

Quellen: ecoinvent Centre (2007); ifu und Ifeu (2008); eigene Annahmen und Berechnungen.

Abbildung 7.8: Ökobilanzierung der Optionen zur Wärmebereitstellung: Treibhauseffekt, Landschaftsinanspruchnahme, Sommersmog und Erschöpfung abiotischer Ressourcen

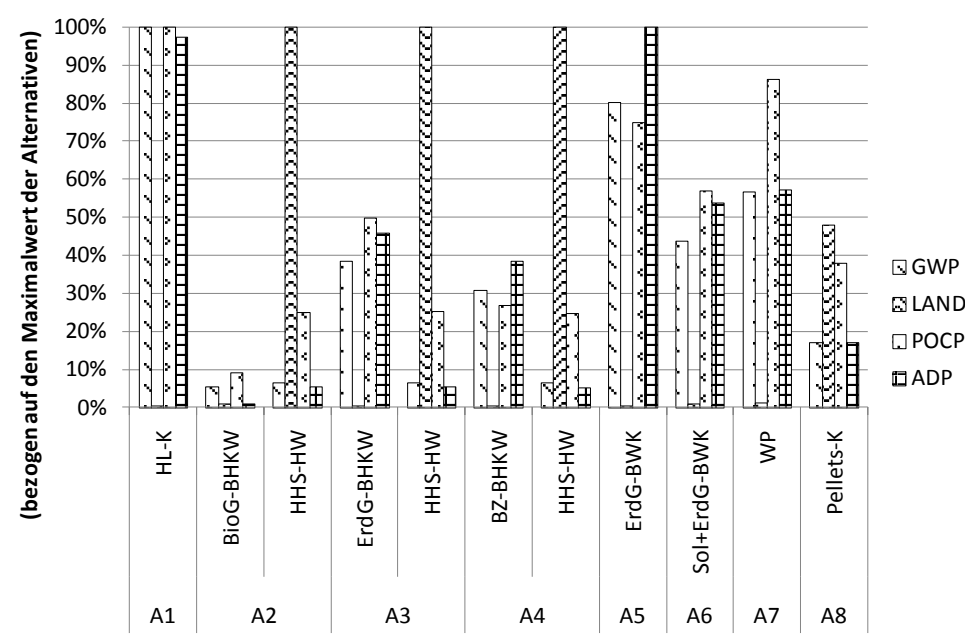

Quellen: ecoinvent Centre (2007); ifu und Ifeu (2008); eigene Annahmen und Berechnungen.

Die Wärmebereitstellung aus dem Erdgas- und dem Brennstoffzellen-BHKW schneidet hinsichtlich Öko- und Humantoxizität mit am besten ab, wobei das Erdgas-BHKW im Vergleich zur Brennstoffzelle etwas bessere Bewertungen aufweist (Abbildung 7.7). Die Bewertungen in den Kategorien GWP, POCP und ADP (Abbildung 7.8) sowie ODP, AP, EP und KEA (Abbildung 7.9) liegen hingegen für das Erdgas-BHKW etwas höher im Vergleich zur Brennstoffzelle. Der Erdgas-Brennwertkessel weist im Vergleich besonders hohe Auswirkungen hinsichtlich GWP, POCP und ADP (Abbildung 7.8) sowie KEA (Abbildung 7.9) auf. Bei der Erdgas-Heizung in Verbindung mit Solarkollektor 
können im Vergleich zum Erdgas-Brennwertkessel ohne solare Unterstützung, die Umweltauswirkungen über den gesamten Lebensweg in den Kategorien GWP, POCP und ADP (Abbildung 7.8) sowie ODP und KEA (Abbildung 7.9) reduziert werden. Jedoch weist die solar unterstützte Erdgas-Heizung die schlechtesten Ergebnisse in den Kategorien FAETP und HTP auf (Abbildung 7.6). Die Wärmepumpe schneidet in den Wirkungskategorien MAETP, TAETP, ODP und AP am schlechtesten ab. Auch die Umweltauswirkungen hinsichtlich FAETP, HTP, POCP und KEA sind bei der Wärmebereitstellung mit Wärmepumpe vergleichsweise hoch.

Abbildung 7.9: Ökobilanzierung der Optionen zur Wärmebereitstellung: Ozonabbau, Versauerung, Eutrophierung und kumulierter Energieaufwand

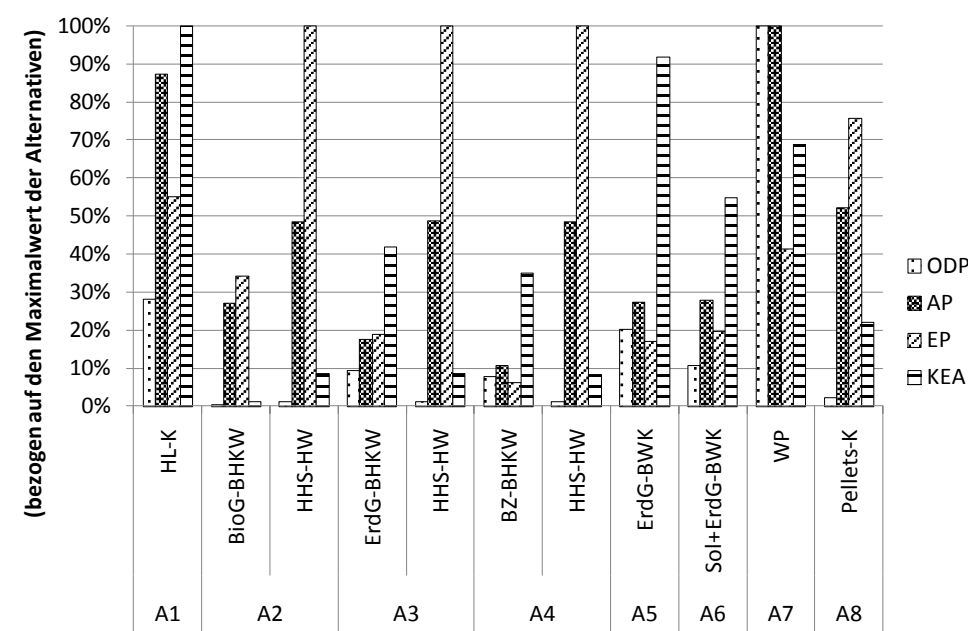

Quellen: ecoinvent Centre (2007); ifu und Ifeu (2008); eigene Annahmen und Berechnungen.

Die Ergebnisse der ökobilanziellen Bewertung zeigen, dass keine der Alternativen hinsichtlich der Umweltauswirkungen uneingeschränkt am besten abschneidet. Erst in Verbindung mit einer Priorisierung unterschiedlicher Umweltprobleme, wie sie im Rahmen der multikriteriellen Bewertung durch die Gewichtung der Wirkungskategorien erfolgt, kann ein Gesamtbild der relativen Vorziehenswürdigkeit vermittelt werden.

\subsubsection{Versorgungssicherheit}

Die Versorgungssicherheit wird nur in relativ wenigen Veröffentlichungen zur multikriteriellen Analyse von Optionen zur Energiebereitstellung mit messbaren Indikatoren bewertet. Lootsma et al. (1986) sowie Karger und Hennings (2009) nutzen paarweise Vergleiche von Experten, um unterschiedliche Aspekte der Versorgungssicherheit wie zum Beispiel strategische Risiken, Verfügbarkeit, Machbarkeit, Anpassungsfähigkeit, Diversifizierung, Anlagensicherheit und Zuverlässigkeit in die Bewertung einzubeziehen. Mehrere Autoren nehmen eine qualitative Einstufung des Beitrags unterschiedli- 
cher Alternativen zur Versorgungssicherheit vor (Browne et al. 2010; Kowalski et al. 2006; Madlener et al. 2007; Renn 2003; Shackley und McLachlan 2006; Stagl 2006). Schulz und Stehfest (1984) legen zur Bewertung der Versorgungssicherheit den Anteil der Importe der verwendeten Energieträger sowie die Kosten der Lagervorhaltung als Kriterien zugrunde.

Um aufwändige paarweise Vergleiche durch Experten und subjektiv belastete Werturteile zur Bestimmung der Kriterien-Ausprägungen zu vermeiden, sollen für dieses Fallbeispiel weitere Indikatoren zur Bewertung der Versorgungssicherheit hinzugezogen werden. Jedoch weisen Chester (2010), Löschel et al. (2010) sowie Frondel und Schmidt (2008) darauf hin, dass das Konzept der Energieversorgungssicherheit bisher nur unzureichend beschrieben ist und in der Literatur kaum etablierte Indikatoren zur Messung zur Verfügung stehen. Löschel et al. (2010) sowie Frondel und Schmidt (2008) schlagen Indikatoren zur Bewertung der Energieversorgungssicherheit auf nationaler Ebene vor. Weitere Ansätze zur Bewertung der Energieversorgungssicherheit werden beispielsweise bei Jansen und Seebregts (2010), Lefèvre (2010) sowie Markandya und Pemberton (2010) vorgestellt. Stirling (2010) diskutiert Aspekte der Versorgungssicherheit in Zusammenhang mit der Diversität von Energieportfolios. Jedoch zielt keine der genannten Publikationen auf die Bewertung des Beitrags einzelner Energietechnologien zur Versorgungssicherheit ab. Daher sind die darin beschriebenen Indikatoren nicht unmittelbar zur Bewertung von Energietechnologien geeignet. Basierend auf den vorliegenden Veröffentlichungen werden für das Fallbeispiel folgende Kriterien zur Bewertung der Versorgungssicherheit mit Bezug zu den betrachteten Technologien bzw. den je Technologie eingesetzten Energieträgern zugrunde gelegt:

- Energieträger-bezogene Indikatoren:

V1. Die statische Reichweite der Ressourcen (Jahre) als Maß für die globale Verfügbarkeit des je Technologie eingesetzten Energieträgers.

V2. Die Importquote des eingesetzten Energieträgers (Prozent) als Maß für die nationale Verfügbarkeit des je Technologie eingesetzten Energieträgers und die Abhängigkeit von anderen Ländern.

V3. Die lokale Verfügbarkeit des Energieträgers (ja/nein) zur Berücksichtigung der Abhängigkeit der Gemeinde von anderen Regionen.

V4. Die Möglichkeit zur Lagerung des eingesetzten Energieträgers (ja/nein), um eine Aussage über die Planbarkeit der Energiebereitstellung zu treffen.

V5. Der Herfindahl-Index $H$ zur Bewertung der Konzentration der Förderung (Frondel et al. 2007):

$H=\sum_{i} s_{i}^{2}$

mit $s_{i}$ : Anteil des Landes $i$ an der weltweiten Förderung. 
V6. Um den politischen und wirtschaftlichen Risiken in den Förderländern Rechnung zu tragen, wird ein konstruierter Risiko-Indikator je Energieträger bestimmt.

V7. Der Variationskoeffizient der Energieträgerpreise in der Vergangenheit, um eine Aussage über die finanziellen Risiken in Verbindung mit dem Einsatz des Energieträgers für eine bestimmte Technologie zu treffen.

- Technologie-bezogene Indikatoren:

V8. Der Nutzungsgrad je Technologie (Prozent) als Maß für die Effizienz des Einsatzes von Rohstoffen. Dabei trägt ein höherer Nutzungsgrad insofern zur Versorgungssicherheit bei, dass eine geringere Menge an Primär- bzw. Sekundärenergieträgern eingesetzt werden muss, um eine gegebene Menge an Nutzenergie bereit zu stellen.

V9.Zur Bewertung der Zuverlässigkeit der eingesetzten Technologie die jährliche Verfügbarkeit (Stunden je Jahr) unter Berücksichtigung von Wartungs- und/oder Instandsetzungsintervallen, Ausfallhäufigkeiten, Umweltbedingungen etc.

Die statische Reichweite wird für die Primärenergieträger bestimmt, auf denen die in der Regel als Sekundärenergieträger eingesetzten Brennstoffe basieren. Für Haushaltsstrom wird dieser Indikator auf Basis des gewichteten Mittels der statischen Reichweiten der zur Stromerzeugung eingesetzten Primärenergieträger bewertet (Tabelle 7.3). Die Reichweite der erneuerbaren Energieträger ist per definitionem nahezu unbegrenzt. Da die quantitative Abbildung der „Unendlichkeit“ problematisch ist, wird als Kriterium der Kehrwert der statischen Reichweite zugrunde gelegt. Je geringer dieser Wert ist, desto besser ist der jeweilige Energieträger hinsichtlich dieses Kriteriums zu bewerten. Den erneuerbaren Energieträgern kann dann ein Wert von Null zugeordnet werden, wenn vorausgesetzt wird, dass deren Nutzungsrate die Regenerationsrate nicht überschreitet. Da in Bioenergiedörfern nachhaltige Landnutzungskonzepte angestrebt werden, ist diese Annahme auch für die dort eingesetzte Biomasse plausibel. Für die solar unterstützte Erdgasheizung wird entsprechend dem zugrunde gelegten solaren Deckungsanteil von 13 \% ein gewichtetes Mittel der Energieträger Solarenergie und Erdgas gebildet. Die Importquote der erneuerbaren Energieträger wird mit Null bewertet. Diese Annahme ist auch für Biomasse gerechtfertigt, da ein Grundgedanke des Bioenergiedorf-Konzeptes der Einsatz regional verfügbarer Biomasse ist. Analog dem Vorgehen bei der statischen Reichweite werden für den Strommix und die solar unterstützte Erdgas-Heizung gewichtete Mittelwerte zugrunde gelegt (Tabelle 7.3). Lokal verfügbare Energieträger sind entsprechend dem Konzept der Bioenergiedörfer zu bevorzugen. Um dieser Tatsache Rechnung zu tragen, wird Energieträgern, die in der Region verfügbar sind, ein Wert von Eins und anderen Energieträgern der Wert Null zugeordnet. 
Tabelle 7.3: $\quad$ Statische Reichweite und Importquote von Energieträgern

\begin{tabular}{|c|c|c|c|c|}
\hline & & $\begin{array}{c}\text { Statische } \\
\text { Reichweite }\end{array}$ & $\begin{array}{l}\text { Kehrwert der stati- } \\
\text { schen Reichweite }\end{array}$ & Nettoimporte 2008 \\
\hline Energieträger & Anteil ${ }^{(1)}$ & (Jahre) & (1/Jahre) & $\%$ \\
\hline Steinkohle & $19,5 \%$ & $2.970^{(3)}$ & $3,37 \cdot 10^{-4}$ & 71,8 \\
\hline Braunkohle & $25,3 \%$ & $4.443^{(4)}$ & $2,25 \cdot 10^{-4}$ & $-1,4$ \\
\hline Übrige feste Brennstoffe & $5,6 \%$ & Unbekannt & --- & Unbekannt \\
\hline Heizöl & $1,6 \%$ & $64^{(5)}$ & $1,56 \cdot 10^{-2}$ & $97,7 \%^{(6)}$ \\
\hline Erdgas & $10,0 \%$ & $140^{(7)}$ & $7,14 \cdot 10^{-3}$ & \multirow[t]{2}{*}{$84,5 \%^{(8)}$} \\
\hline Sonstige Gase & $2,3 \%$ & Unbekannt & --- & \\
\hline Wasser/Wind & $6,8 \%{ }^{(9)}$ & $\begin{array}{l}\text { Nahezu unbe- } \\
\text { grenzt }\end{array}$ & $\approx 0$ & 0 \\
\hline Kernenergie & $29,0 \%$ & $388^{(10)}$ & $2,58 \cdot 10^{-3}$ & $100 \%$ \\
\hline Gewichtetes Mittel $^{(11)}$ & & & $1,99 \cdot 10^{-3}$ & $57,8 \%$ \\
\hline
\end{tabular}

Anmerkungen: ${ }^{(1)}$ Anteil am Einsatz der Energieträger zur Stromerzeugung in Deutschland im Jahr 2008. ${ }^{(2)}$ Statische Reichweite von Ressourcen und Reserven; Stand: 2007. ${ }^{(3)}$ Hartkohle.

${ }^{(4)}$ Weichbraunkohle ${ }^{(5)}$ Konventionelles Erdöl. ${ }^{(6)}$ Mineralöl ${ }^{(7)}$ Konventionelles Erdgas. ${ }^{\left({ }^{(8)}\right.}$ Einschl. Erdgas, Erdölgas, Grubengas. ${ }^{(9)}$ Berechnet auf Basis des Wirkungsgradansatzes. ${ }^{(10)}$ Uran.

(11) Unter Vernachlässigung der Beiträge „Übrige fester Brennstoffe“ und „Sonstige Gase“.

Quelle: BMWi (2010); Cramer et al. (2009); vgl. auch Abbildung 2.2 und Abbildung 2.3.

Der Herfindahl-Index sowie der Indikator zur Abschätzung der politischen und wirtschaftlichen Risiken in den Förderländern werden auf Basis von BGR (2008), Frondel et al. (2007) und Kaufmann et al. (2007) je Energieträger geschätzt (Tabelle 7.4). Der Herfindahl-Index geht bei gleichmäßiger Verteilung der jährlich geförderten Mengen auf unendlich viele Länder gegen Null. Im Falle der Konzentration der Förderung auf ein einziges Land resultiert ein Wert von Eins. Je gleichmäßiger sich die Förderung über mehrere Länder verteilt, desto geringer sind die mit der Bereitstellung des entsprechenden Energieträgers verbundenen Unsicherheiten. Somit ist eine Technologie umso besser zu bewerten, je geringer der Wert des Herfindahl-Index für den eingesetzten Energieträger ist. Der Risiko-Indikator basiert auf Einzelindikatoren, die durch die Weltbank für einzelne Länder erhoben werden und Aspekte wie politische Korruption, Instabilität und Kriminalität, Effektivität von Behörden, Grad der Bürokratie und Rechtsstaatlichkeit messen (vgl. Kaufmann et al. 2007). Um den Risiko-Indikator je Energieträger zu ermitteln, wird ein gewichteter Mittelwert über die Indikatoren je Land gebildet. Als Gewichtungsfaktoren dienen die Anteile der einzelnen Länder an der weltwei- 
ten Förderung. 17 Höhere Werte des Risiko-Indikators kennzeichnen geringere politische und wirtschaftliche Risiken. Analog der statischen Reichweite wird daher der Kehrwert als Kriterium zugrunde gelegt, so dass den erneuerbaren Energieträgern, für die grundsätzlich keine länderspezifischen Risiken bezüglich der Förderung bestehen, der Wert Null zugeordnet werden kann. Der Biomasse, die im Fallbeispiel entsprechend den Annahmen aus heimischen Quellen stammt, wird der Risiko-Indikator Deutschlands zugeordnet bzw. dessen Kehrwert (Tabelle 7.4).

Tabelle 7.4: $\quad$ Herfindahl-Index und Indikator zur Bewertung politischer und wirtschaftlicher Risiken in den Förderländern

\begin{tabular}{l|c|c|c|c}
\hline Energieträger & Anteil & $\begin{array}{c}\text { (1) } \\
\text { Herfindahl-Index } \\
\mathbf{( 2 )}^{(\mathbf{2})}\end{array}$ & $\begin{array}{c}\text { Kehrwert des Risi- } \\
\text { ko-Indikators }\end{array}$ \\
\hline Steinkohle & $19,5 \%$ & 0,2578 & 0,1488 & 6,72 \\
\hline Braunkohle & $25,3 \%$ & 0,1025 & 0,5907 & 1,69 \\
\hline Übrige feste Brennstoffe & $5,6 \%$ & Unbekannt & Unbekannt & -- \\
\hline Heizöl & $1,6 \%$ & $0,1055^{(3)}$ & $0,0110^{(3)}$ & 90,89 \\
\hline Erdgas & $10,0 \%$ & 0,1669 & 0,3073 & 3,25 \\
\hline Sonstige Gase & $2,3 \%$ & Unbekannt & Unbekannt & -- \\
\hline Wasser/Wind & $6,8 \%{ }^{(4)}$ & 0 & Nicht quantifizierbar & 0 \\
\hline Kernenergie & $29,0 \%$ & 0,1456 & 0,5015 & 1,99 \\
\hline Biomasse & k. A. & 0 & 1,43 & 0,70 \\
\hline Gewichtetes Mittel ${ }^{(5)}$ & & 0,1484 & & 4,44 \\
\hline
\end{tabular}

Anmerkungen: (1) Anteil am Einsatz der Energieträger zur Stromerzeugung in Deutschland im Jahr 2008. (2) Basierend auf Angaben für das Jahr 2007. (3) Erdöl. (4) Berechnet auf Basis des Wirkungsgradansatzes. (5) Unter Vernachlässigung der Beiträge „Übrige fester Brennstoffe“ und "Sonstige Gase“.

Quelle: BGR (2008); BMWi (2010); Frondel et al. (2007); Kaufmann et al. (2007).

Zur Bestimmung des Variationskoeffizienten der Energieträgerpreise werden historische Preiszeitreihen (jährliche Mittelwerte) für Strom, Heizöl und Erdgas (Haushaltspreise) basierend auf BMWi (2010) sowie für Holzhackschnitzel, Holzpellets und Getreide basierend auf Breitschopf et al. (2010) und TFZ (2010) zugrunde gelegt. Da für Hackschnitzel erst ab dem Jahr 2003 Preise zur Verfügung stehen, beschränkt sich die Bestimmung des Koeffizienten auf die Jahre von 2003 bis 2009, um einen vergleichbaren Betrachtungszeitraum zu gewährleisten. Die Bewertung der Volatilität der Preise für Ganzpflanzensilage, die als Input zur Biogas-Erzeugung dient, basiert auf Schwan-

17 Der so konstruierte Risikoindikator dient bei Frondel et al. (2007) zur Identifizierung von Risiken in Verbindung mit der Bereitstellung mineralischer Rohstoffe. 
kungen von Getreidepreisen in der Vergangenheit. Unter Beachtung des Gülle-Anteils wird der so ermittelte Variationskoeffizient um ca. $40 \%$ reduziert, um der Tatsache Rechnung zu tragen, dass die Biogas-Produktion nicht ausschließlich vom Input an Ganzpflanzensilage abhängt.

Die Effizienz des Einsatzes von Energieträgern in unterschiedlichen Technologien wird durch die Nutzungsgrade der betrachteten Alternativen bewertet. Diese werden analog den Annahmen bei der Wirtschaftlichkeitsbewertung und Ökobilanzierung zugrunde gelegt (vgl. Anhang 10.3.2). Für den Strommix wird der mittlere Nutzungsgrad der Stromerzeugung in Deutschland auf Basis von BMWi (2010) geschätzt. Demnach wurden im Jahr 2008 insgesamt ca. 5.604 PJ (entspricht etwa 1.556,7 TWh) an Energieträgern eingesetzt und 637,6 TWh Strom erzeugt (Bruttostromerzeugung). Dies entspricht einem mittleren Wirkungsgrad von ca. 41,0 \%. Für Technologien zur Wärmebereitstellung werden Verteilungs- und Übergabeverluste (Nahwärmenetz, Verteilung im Haus) nach Eikmeier et al. (2006) und Oschatz und Mailach (2007) berücksichtigt. Für die Wärmepumpe wird die Jahresarbeitszahl zugrunde gelegt. Für Windkraft- und PVAnlagen sowie Solarkollektoren werden Annahmen auf Basis von Quaschning (2008) getroffen (Leistungsbeiwert Wind 50 \%; Modulwirkungsgrad PV $15 \%$, Verhältnis vom realen zum Nennwirkungsgrad 0,75; Kollektorwirkungsgrad $64 \%$ ).

Für die Verfügbarkeit werden Annahmen basierend auf Literaturangaben getroffen. Zur Bewertung des Strommixes wird die Verfügbarkeit des deutschen Kraftwerkspark zugrunde gelegt. Diese ist nach CONSENTEC et al. (2008) trotz einiger in der Vergangenheit beobachteter Ausfälle als hoch einzustufen, so dass für das Fallbeispiel eine Verfügbarkeit von $97 \%$ angenommen wird. Für das Biogas-BHKW wird auf Basis der Angaben bei Ruppert et al. (2008), der von einer Stromerzeugungsmenge von 5 Mio. $\mathrm{kWh}_{\mathrm{el}}$ je Jahr bei einer installierten elektrischen Leistung von $716 \mathrm{~kW}_{\mathrm{el}}$ ausgeht, eine Verfügbarkeit von etwa 7.000 h/a (ca. $80 \%$ ) unterstellt. Da es sich bei dem ErdgasBHKW um eine baugleiche Anlage handelt, werden für dieses die gleichen Werte zugrunde gelegt. Die Anlagenverfügbarkeit der SOFC-Brennstoffzelle wird nach Gummert und Suttor (2006) auf 75 \% geschätzt. Für das Heizwerk als Kombination aus Holzhackschnitzel-Heizwerk und Öl-Spitzenlastkessel wird eine hohe Verfügbarkeit von $95 \%$ angenommen, da dieses dafür ausgelegt ist, auch bei Spitzenlasten einsatzbereit zu sein. Ebenso wird für die etablierten Hausheizungssysteme (Heizöl-Kessel und Erdgas-Brennwertkessel) eine hohe Verfügbarkeit von ca. $95 \%$ unterstellt. Für die solar unterstützte Erdgas-Heizung werden die gleichen Werte angenommen, da diese prinzipiell auch ohne den Solarkollektor die nachgefragte Wärme bereit stellen kann und somit die Verfügbarkeit des Erdgas-Brennwertkessels maßgeblich ist. 


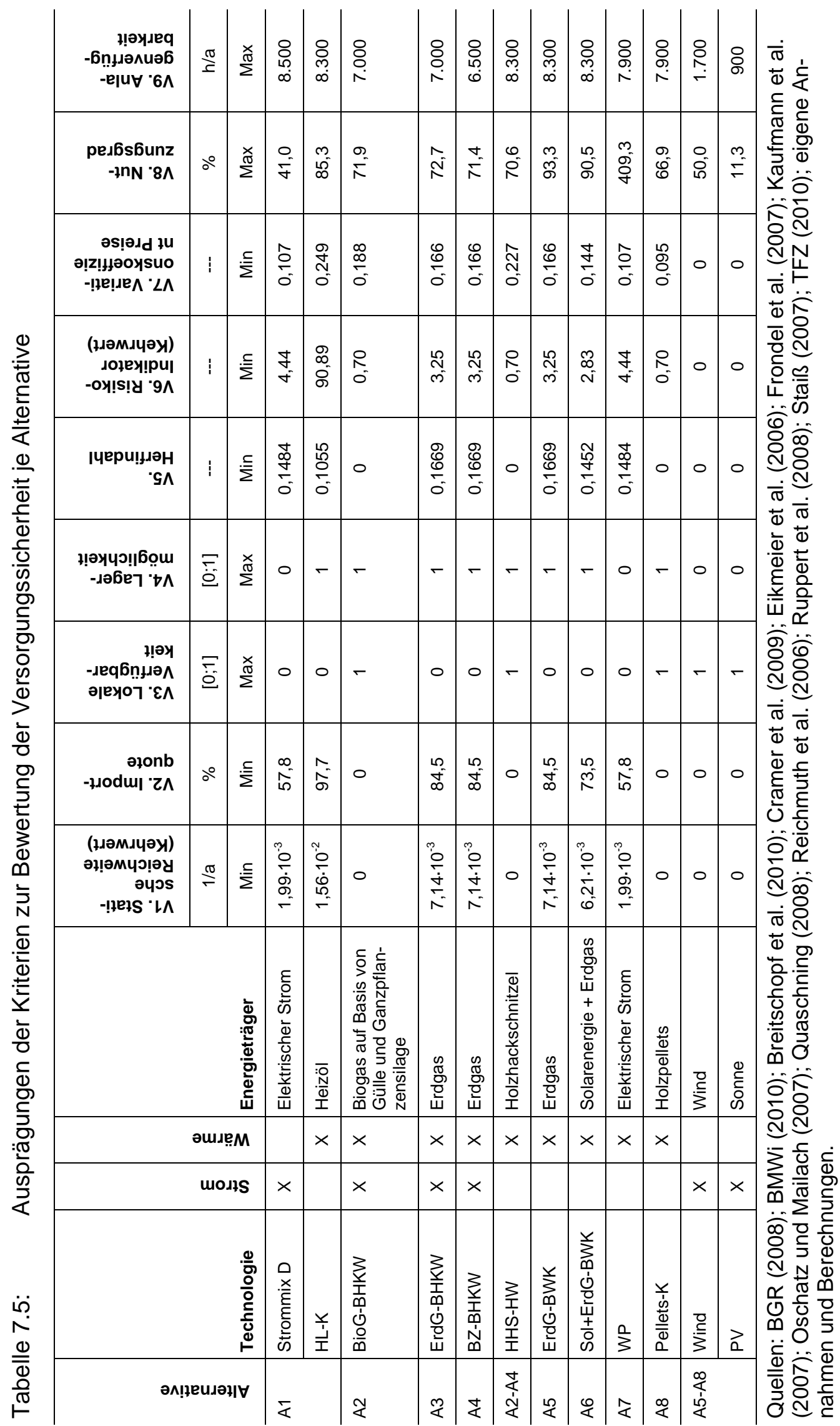


Für die noch weniger weit verbreiteten Heizungssysteme (Wärmepumpe und Holzpellets-Heizung) wird eine etwas geringere Verfügbarkeit von ca. $90 \%$ zugrunde gelegt. Die für die Verfügbarkeit von Wind $(1.700 \mathrm{~h} / \mathrm{a})$ und Photovoltaik (900 h/a) zugrunde gelegten Werte basieren auf Angaben für Deutschland bei Staiß (2007) und Reichmuth et al. (2006). Die Ausprägungen aller Kriterien zur Bewertung des Beitrags zur Versorgungssicherheit sind in Tabelle 7.5 zusammengefasst.

\subsubsection{Soziale Aspekte}

Ähnlich dem bisher nur vage definierten Konzept der Versorgungssicherheit existiert für die Messung sozialer Aspekte bisher keine allgemein anerkannte Theorie (vgl. Gallego Carrera und Mack 2009; Gallego Carrera und Mack 2010). Bis heute beschäftigen sich nur wenige Studien zur Bewertung von Optionen zur Energiebereitstellung mit sozialen Aspekten und deren Operationalisierung. So heben beispielsweise White und Lee (2009) hervor, dass im Bereich Operations Research die soziale Dimension der Nachhaltigkeit im Vergleich zu ökonomischen und ökologischen Aspekten bisher nur wenig Aufmerksamkeit erfahren hat. Im Kontext der multikriteriellen Entscheidungsunterstützung schlägt Munda (2004) das Konzept der „Social Multi-Criteria Evaluation“ (SMCE) vor (vgl. auch Gamboa 2006; Gamboa und Munda 2007; Munda 2006). Der Schwerpunkt dieser Veröffentlichungen liegt auf der Integration (variierender) gesellschaftlicher Sichtweisen in Entscheidungsprozesse als soziale Komponente. Auch wenn dies im Hinblick auf die Umsetzung zukünftiger Bioenergiedorf-Projekte möglicherweise ein interessantes Konzept darstellt, liegt hier jedoch nicht der Schwerpunkt dieser Arbeit. An dieser Stelle ist vielmehr die Auswahl von Kriterien zur Bewertung der Auswirkungen unterschiedlicher Alternativen der Energiebereitstellung auf die Gesellschaft von Interesse.

In Veröffentlichungen zur Anwendung multikriterieller Methoden im Energiebereich kann eine breite Vielfalt an Kriterien zur Einschätzung sozialer Aspekte identifiziert werden. Diese umfassen unterschiedliche Indikatoren zur Einschätzung der sozialen Akzeptanz, von Beschäftigungseffekten, Gesundheits- und Unfallrisiken, Auswirkungen auf die lokale Entwicklung, Gerechtigkeitsaspekte und Verkehrsbelastungen der Anwohner (vgl. Tabelle 7.6). 


\section{Tabelle 7.6: $\quad$ Kriterien zur Bewertung sozialer Aspekte der Energiebereitstellung}

\begin{tabular}{|c|c|}
\hline \multicolumn{2}{|c|}{ Kriterien } \\
\hline $\begin{array}{l}\text { Akzep } \\
\text { ablen }\end{array}$ & $\begin{array}{l}\text { anz auf Basis paarweiser Vergleiche, linguistischer Vari- } \\
\text { der Punkteskalen, z. B. }\end{array}$ \\
\hline . & $\begin{array}{l}\text { Soziale, politische und/oder öffentliche Akzeptanz } \\
\text { Reaktionen der Anwohner } \\
\text { Passfähigkeit mit gesellschaftlichen Wertvorstellungen } \\
\text { Existenz von Interessengruppen } \\
\text { NIMBY-Syndrom („Not In My Backyard“) } \\
\text { Hemmnisse durch lokale Behörden } \\
\text { Image des Betreibers }\end{array}$ \\
\hline
\end{tabular}

Soziale bzw. gesellschaftliche Effekte allgemein (z. B. hinsichtlich Lebensstandard, -qualität, Beschäftigung) auf Basis paarweiser Vergleiche linguistischer Variablen oder Punkteskalen

\section{Quellen}

Capros et al. 1988; Cavallaro und Ciraolo 2005; Chatzimouratidis und Pilavachi 2008; de Almeida et al. 2005; Georgopoulou et al. 1998; Haldi et al. 2002; Hämäläinen 1990; Hämäläinen und Seppäläinen 1986; Kagazyo et al. 1997; Kahraman et al. 2009; Lhendup 2008; Liposcak et al. 2006; Lootsma et al. 1986; Polatidis und Haralambopoulos 2007; Roth et al. 2009; Tsoutsos et al. 2009; Tzeng et al. 1992

Akash et al. 1999; Burton und Hubacek 2007; Gholamnezhad 1983; Gholamnezhad und Saaty 1982; Hämäläinen und Karjalainen 1992; Karger und Hennings 2009; Mamlook 2006; Mamlook et al. 2001b; Mohsen und Akash 1997; Nigim et al. 2004; Önüt et al. 2008; Renn 2003; Ulutas 2005

Beschäftigungseffekte, z. B.

- Anzahl geschaffener Arbeitsplätzen, ggf. bezogen auf installierte Leistung oder Energie

- Arbeitstage/-stunden bzw. Vollzeitäquivalente

- Lohnniveau (GE/a)

- Umsiedlung von Arbeitskräften aufgrund der Konstruktionsarbeiten

- $\quad$ Einschätzung geschaffener Arbeitsplätze auf Basis paarweiser Vergleiche oder linguistischer Variablen

Gesundheits- und Unfallgefahren, z. B.

- $\quad$ Anzahl verlorener Arbeitstage, z.B. aufgrund von Gesundheitsschäden durch Luftverschmutzung, Strahlung, Unfällen

- Anzahl verletzter Personen je kWh

- Gesundheitsschädliche Emissionen z.B. in kg/MWh

- Kosten für das Gesundheitswesen aufgrund lokaler Emissionen

- $\quad$ Morbidität (Krankheitsjahre)

- Mortalität bzw. Risiko von Todesfällen, z. B. Anzahl je 1.000 Arbeiter oder YOLL („Years of Life Lost“)

- Expertenschätzungen auf Basis paarweiser Vergleiche, Punkteskalen oder linguistischer Variablen

Auswirkungen auf die lokale Wertschöpfung, z.B.

- Zunahme des Bruttosozialprodukts der Gemeinde (GE)

- Lokale Steuereinnahmen (GE)

- Anteil des Haushaltseinkommens, der für Brennstoffe ausgegeben wird (\%)

- $\quad$ Einschätzung des Beitrags zur regionalen Entwicklung auf Punkteskala oder durch linguistische Variablen

- Einstufung der Auswirkungen auf verbundene Industriezweige auf Basis linguistischer Variablen

Verteilungswirkungen und Gerechtigkeitsaspekte auf Basis von

Punkteskalen oder linguistischen Variablen, z. B.

- $\quad$ Auswirkungen auf lokale Armut

- Verteilung des Haushaltseinkommens

- $\quad$ soziale Gerechtigkeit

- $\quad$ Schutz der Menschenrechte und -würde

Kompatibilität mit politischen, gesetzlichen und verwaltungstechnischen Rahmenbedingungen auf Basis paarweiser Vergleiche, linguistischer Variablen und Punkteskalen
Afgan et al. 2007a; Afgan et al. 2000; Afgan et al. 2007b; Afgan und Carvalho 2004; Basson und Petrie 2007; Beccali et al. 2003; Begic und Afgan 2007; Chatzimouratidis und Pilavachi 2008; Doukas et al. 2007; Haldi et al. 2002; Hanson et al. 1991; Jovanovic et al. 2009; Kahraman et al. 2009; Keeney und Sicherman 1983; Longden et al. 2007; Madlener und Stagl 2005; Papadaki et al. 2003; Polatidis und Haralambopoulos 2007; Terrados et al. 2009

Chatzimouratidis und Pilavachi 2008; Hämäläinen 1990; Hämäläinen und Karjalainen 1992;

Hämäläinen und Seppäläinen 1986; Jovanovic et al. 2009; Karger und Hennings 2009; Keeney und Sicherman 1983; Lhendup 2008; Liposcak et al. 2006; Longden et al. 2007; Lootsma et al. 1986; Papadaki et al. 2003; Renn 2003; Siskos und Hubert 1983

Afgan et al. 2000; Doukas et al. 2006; Doukas et al. 2007; Jovanovic et al. 2009; Keeney und Sicherman 2009; Tzeng et al. 1992; Wenstöp und Carlsen 1988

Haldi et al. 2002; Kowalski et al. 2009; Madlener et al. 2007; Madlener und Stagl 2005; Renn 2003 1983; Madlener und Stagl 2005; Tsoutsos et al.
Beccali et al. 2003; Kahraman et al. 2009; Terrados et al. 2009 
Tabelle 7.6 (Fortsetzung)

\begin{tabular}{|c|c|}
\hline Kriterien & Quellen \\
\hline $\begin{array}{l}\text { Verkehrsbelastungen, z. B. } \\
\text { - Anzahl der durch die Anlieferung von Brennstoffen be- } \\
\text { lasteten Personen } \\
\text { - } \quad \text { LKW-Verkehr in der lokalen Gemeinde aufgrund von } \\
\text { Anlieferungen je Tag und Anlage } \\
\text { Einschätzung auf Basis linguistischer Variablen } \\
\text { Wenstöp und Carlsen } 1988\end{array}$ & Keeney und Sicherman 1983; Longden et al. 2007 \\
\hline $\begin{array}{l}\text { Sonstige messbare Indikatoren, z. B. } \\
\text { - } \quad \text { Lärmbelästigung in dB oder Einschätzung auf Punkte- } \\
\text { skala } \\
\text { - } \\
\text { Anzahl der Menschen, die die Anlage täglich sehen als } \\
\text { - Undikator für visuelle Auswirkungen } \\
\text { - Bevölkerung der von Konstruktionsarbeiten betroffenen } \\
\text { - Anzahl potenziell zu evakuierender Personen } \\
\text { - Stromgestehungskosten als Maß für die Entwick- } \\
\quad \text { lungsmöglichkeiten der Gesellschaft }\end{array}$ & $\begin{array}{l}\text { Burton und Hubacek 2007; Keeney und Sicherman } \\
\text { 1983; Madlener und Stagl 2005; van Alphen et al. } \\
2007\end{array}$ \\
\hline $\begin{array}{l}\text { Sonstige soziale Aspekte, die auf Basis von Punkteskalen einge- } \\
\text { stuft werden, z. B. } \\
\text { - Politische Stabilität und Legitimität } \\
\text { - Demokratische Kontrolle über Märkte } \\
\text { - Eigentümerschaft in der Gemeinde } \\
\text { - Erhaltung und Erweiterung vorhandener Kompetenzen }\end{array}$ & $\begin{array}{l}\text { Longden et al. 2007; Madlener und Stagl 2005; Renn } \\
2003\end{array}$ \\
\hline
\end{tabular}

Gallego Carrera und Mack (2010) identifizieren neun Kriterien, die nach Einschätzung von Experten die höchste Relevanz hinsichtlich der Bewertung sozialer Aspekte von Energiesystemen in Europa aufweisen:

S1. Flexibilität hinsichtlich der Eingliederung technologischer Innovationen

S2. Verfügbarkeit von Infrastrukturen zur Abfallentsorgung

S3. Potenzial von Konflikten, die durch das Energiesystem induziert werden können

S4. Notwendigkeit einer partizipativen Entscheidungsfindung bei der Standortwahl

S5. Subjektive Wahrnehmung der erwarteten Gesundheitsauswirkungen bei Normalbetrieb

S6. Bekanntheitsgrad von Risiken

S7. Wahrgenommenes Katastrophenpotenzial

S8. Beeinträchtigung anderer Landnutzungsmöglichkeiten durch das Energiesystem

S9. Ästhetische Auswirkungen der Energie-Infrastrukturen auf die Landschaft

Diese Kriterien dienen als Basis der Bewertung der sozialen Dimension im Fallbeispiel. Die Kriterien S4 und S8 werden jedoch aus folgenden Gründen in der vorliegenden Arbeit nicht berücksichtigt: Gallego Carrera und Mack (2009) verstehen die Partizipation (S4) als eine Möglichkeit zur Reduzierung von Konflikten bei der Standortwahl. Notwendigkeit zur Partizipation besteht demnach vor allem dann, wenn Konflikte bei 
der Standortwahl für ein bestimmtes Energiesystem wahrscheinlich sind. Folglich wird dieses Kriterium umso höher bewertet, je höher die Experten die Wahrscheinlichkeit von Konflikten einschätzen. Da jedoch das Konfliktpotenzial bereits durch Kriterium S3 („Potenzial von Konflikten“) bewertet wird, wird S4 für das vorliegende Fallbeispiel nicht berücksichtigt, um Redundanzen zu vermeiden. Kriterium S8 wird bereits durch die Wirkungskategorie „Landschaftsinanspruchnahme“(LAND) im Rahmen der Ökobilanzierung (vgl. Abschnitt 7.1.3.2) berücksichtigt und wird daher ebenfalls von den weiteren Betrachtungen ausgeschlossen. Basierend auf Befragungen von Energieexperten in Europa stufen Gallego Carrera und Mack (2009) Technologien zur Stromerzeugung hinsichtlich dieser Kriterien auf einer Skala von 1 (sehr gering) bis 5 (sehr hoch) ein. Für einige Indikatoren wird die Skala auf 0 (irrelevant) erweitert. Auch Ergebnisse der Befragung deutscher Experten stehen zur Verfügung, die für dieses Fallbeispiel zugrunde gelegt werden (vgl. Tabelle 7.7). Für Technologien zur Wärmebereitstellung stehen keine Bewertungen zur Verfügung (mit Ausnahme von KWK-Anlagen), so dass diese Indikatoren nur zur Bewertung der Optionen zur Stromerzeugung angewendet werden können.

Tabelle 7.7: $\quad$ Bewertung sozialer Aspekte durch Experten in Deutschland

\begin{tabular}{|c|c|c|c|c|c|c|c|c|c|}
\hline \multirow{3}{*}{\multicolumn{2}{|c|}{$\begin{array}{l}\text { Alternative zur Strom- } \\
\text { erzeugung im Fallbei- } \\
\text { spiel }\end{array}$}} & \multirow{3}{*}{$\begin{array}{l}\text { Energiesystem nach } \\
\text { (Gallego Carrera und } \\
\text { Mack 2009) }\end{array}$} & 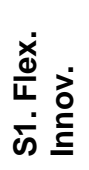 & ふ่ & $\begin{array}{l}\dot{\bar{\theta}} \\
\dot{y} \\
\dot{\mathscr{n}}\end{array}$ & 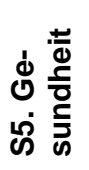 & 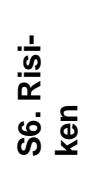 & 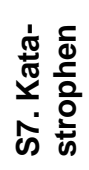 & 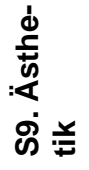 \\
\hline & & & {$[1-5]$} & [0-5] & [1-5] & {$[0-5]$} & [1-5] & {$[1-5]$} & {$[1-5]$} \\
\hline & & & $\operatorname{Max}$ & Min & Min & Min & Min & Min & Min \\
\hline A1 & Strommix & $\begin{array}{l}\text { (basierend auf den } \\
\text { Anteilen der Technolo- } \\
\text { gien an der Stromver- } \\
\text { sorgung) }\end{array}$ & 2,23 & 2,54 & 3,37 & 3,53 & 3,56 & 3,14 & 3,64 \\
\hline $\begin{array}{l}\text { A2, } \\
\text { A3 }\end{array}$ & $\begin{array}{l}\text { BHKW (Biogas, } \\
\text { Erdgas) }\end{array}$ & KWK, kleiner Motor & 3,25 & 1,67 & 1,33 & 1,89 & 2,63 & 1,50 & 1,44 \\
\hline A4 & Brennstoffzelle & KWK, Brennstoffzelle & 4,00 & 1,67 & 1,33 & 2,25 & 2,88 & 2,13 & 1,44 \\
\hline A5- & Wind, Onshore & Wind, Onshore & 3,50 & 1,71 & 2,78 & 1,71 & 3,5 & 2,25 & 3,78 \\
\hline & PV & PV & 4,50 & 1,75 & 1,33 & 1,40 & 2,88 & 1,13 & 2,22 \\
\hline
\end{tabular}

Quelle: Gallego Carrera und Mack (2009).

Zusätzlich wird die soziale Akzeptanz der Technologien berücksichtigt, da dies eines der am häufigsten angewendeten Merkmale zur Bewertung sozialer Aspekte der Energiebereitstellung ist (vgl. Tabelle 7.7). Dabei werden Experteneinschätzungen zugrunde gelegt, die im Rahmen des Projektes „Multidimensionale Technikbewertung“ (vgl. Bartels et al. 2008) erhoben wurden. In der Befragung von Experten im Energiebereich in Deutschland wurde die gesellschaftliche Akzeptanz eines breiten Spektrums an 
Energietechnologien auf einer Skala in Anlehnung an Schulnoten von 1 (= sehr gut) bis 6 (= ungenügend) durch die Experten eingeschätzt. Tabelle 7.8 zeigt die Ergebnisse (Mittelwert über alle gültigen Antworten) für die im Fallbeispiel betrachteten Alternativen. Diese gehen als Ausprägung des Kriteriums „soziale Akzeptanz“ in die multikriterielle Bewertung im Fallbeispiel ein.

Tabelle 7.8: $\quad$ Einschätzung der sozialen Akzeptanz von Energietechnologien durch Experten aus der Energiewirtschaft

\begin{tabular}{|c|c|c|c|c|}
\hline \multirow[b]{2}{*}{ Alternative } & \multirow[b]{2}{*}{ Technologie } & \multirow[b]{2}{*}{ Strom } & \multirow[b]{2}{*}{ Wärme } & S10. Soziale Akzeptanz \\
\hline & & & & [1 (sehr gut) bis 6 (ungenügend)] \\
\hline \multirow[t]{2}{*}{ A1 } & Strommix D & $x$ & & $3,17^{(1)}$ \\
\hline & HL-K & & $x$ & 2,90 \\
\hline $\mathrm{A} 2$ & BioG-BHKW & $x$ & $\mathrm{x}$ & 2,10 \\
\hline A3 & ErdG-BHKW & $x$ & $x$ & 2,73 \\
\hline A4 & BZ-BHKW & $x$ & $x$ & 2,55 \\
\hline A2-A4 & HHS-HW & & $x$ & 2,22 \\
\hline A5 & ErdG-BWK & & $x$ & 2,40 \\
\hline A6 & Sol+ErdG-BWK & & $x$ & $2,28^{(2)}$ \\
\hline A7 & WP & & $x$ & 2,50 \\
\hline A8 & Pellets-K & & $\mathrm{X}$ & 1,89 \\
\hline \multirow[t]{2}{*}{ A5-A8 } & Wind & $x$ & & 3,09 \\
\hline & PV & $x$ & & 2,00 \\
\hline
\end{tabular}

Anmerkungen: (1) Gewichtetes Mittel basierend auf den Anteilen der Technologien an der Stromversorgung in Deutschland. (2) Gewichtetes Mittel aus dem Ergebnis für den Brennwertkessel und den Solar-Flachkollektor.

\subsubsection{Gewichtungen je Technologiezyklus-Phase}

Entsprechend der Analyse in Kapitel 5.2 werden die untersuchten Alternativen den Phasen im Technologiezyklus zugeordnet (Tabelle 7.9). Die meisten der ausgewählten Alternativen befinden sich in der Aufstiegs- oder Reifephase. Lediglich die Brennstoffzelle ist einem frühen Entwicklungsstadium (der Phase der Euphorie) zuzuordnen. Der konventionelle Heizölkessel wird als einzige Alternative der Rückgangs-Phase zugeordnet. Für die Auswertung sind somit Gewichtungen für die Euphorie, die Aufstiegs-, Reife- und Rückgangs-Phase abzuleiten. Da eine Gewichtung aller 30 zugrunde gelegten Kriterien mit Bezug zu mehreren unterschiedlichen Phasen zu komplex erscheint, erfolgt die phasenabhängige Gewichtung lediglich bezogen auf die übergeordneten Bewertungsdimensionen (Wirtschaftlichkeit, Umweltauswirkungen, Versorgungssicher- 
heit und soziale Aspekte). In zukünftigen Anwendungen ist je nach Fall zu prüfen, ob und inwieweit die Gewichtung in Abhängigkeit der Technologiezyklus-Phasen von den Entscheidungsträgern gewünscht ist.

Tabelle 7.9: $\quad$ Zuordnung der Alternativen zu Technologiezyklus-Phasen

\begin{tabular}{|c|c|c|c|c|c|c|c|c|c|c|}
\hline \multirow[b]{2}{*}{$\begin{array}{l}\stackrel{0}{D} \\
\stackrel{\Xi}{ \pm} \\
\frac{5}{ \pm} \\
\frac{ \pm}{\alpha}\end{array}$} & \multirow[b]{2}{*}{ Technologie } & \multirow[b]{2}{*}{ Strom } & \multirow[b]{2}{*}{ Wärme } & \multicolumn{7}{|c|}{ Aktuelle Technologiezyklus-Phase } \\
\hline & & & & 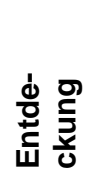 & $\begin{array}{l}\stackrel{0}{\frac{0}{2}} \\
\frac{c}{0} \\
\frac{0}{2}\end{array}$ & 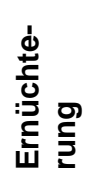 & 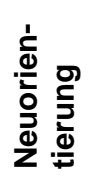 & $\begin{array}{l}\text { O্d } \\
\stackrel{0}{0} \\
\frac{0}{5} \\
\frac{1}{4}\end{array}$ & $\begin{array}{l}\stackrel{\circlearrowright}{\bar{d}} \\
\widetilde{\square}\end{array}$ & 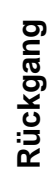 \\
\hline \multirow[t]{2}{*}{ A1 } & Strommix D & $x$ & & & & & & & $x$ & \\
\hline & HL-K & & $x$ & & & & & & & $x$ \\
\hline A2 & BioG-BHKW & $x$ & $x$ & & & & & $\mathrm{x}$ & & \\
\hline A3 & ErdG-BHKW & $x$ & $x$ & & & & & & $x$ & \\
\hline A4 & BZ-BHKW & $x$ & $x$ & & $x$ & & & & & \\
\hline A2-A4 & HHS-HW & & $x$ & & & & & $\mathrm{x}$ & & \\
\hline A5 & ErdG-BWK & & $x$ & & & & & & $x$ & \\
\hline A6 & Sol+ErdG-BWK & & $x$ & & & & & $x$ & & \\
\hline A7 & WP & & $x$ & & & & & $x$ & & \\
\hline A8 & Pellets-K & & $x$ & & & & & $x$ & & \\
\hline \multirow[t]{2}{*}{ A5-A8 } & Wind & $x$ & & & & & & $x$ & & \\
\hline & PV & $x$ & & & & & & $x$ & & \\
\hline
\end{tabular}

Ausgangspunkt der für das Fallbeispiel zugrunde gelegten Gewichtungen ist die in Abbildung 6.2 (S. 124) dargestellte Einschätzung der Relevanz unterschiedlicher Kriterien zur Bewertung von Energietechnologien. Die daraus abgeleiteten Gewichtungen für die Dimensionen Wirtschaftlichkeit, Umwelt, Versorgungssicherheit und soziale Aspekte18 werden für die Reife-Phase zugrunde gelegt. Weiterhin wird angenommen, dass die Bedeutung der Wirtschaftlichkeit umso höher ist, je weiter fortgeschritten das Entwicklungsstadium einer Technologie ist. Dies kann zum einen dadurch begründet werden, dass sich am Markt mittel- bis langfristig nur solche Technologien durchsetzen können,

18 Zur Ableitung der Gewichtung der sozialen Dimension bei der Bewertung der Optionen zur Stromerzeugung werden die Punkte der Kriterien „Akzeptanz“, „Innovationspotenzial“ und „Risiken“ addiert, da sich diese Aspekte auch in den für das Fallbeispiel für die soziale Dimension verwendeten Indikatoren widerspiegeln. Da bei der Bewertung der Optionen zur Wärmebereitstellung lediglich das Kriterium soziale Akzeptanz zur Bewertung der sozialen Dimension dient, werden in diesem Fall ausschließlich die Punkte für das Kriterium „Akzeptanz" zugrunde gelegt. 
die wirtschaftlich betrieben werden können. Zum anderen sind bei weiter entwickelten Technologien, im Vergleich zu innovativen Alternativen, in der Regel nur noch marginale Kostensenkungspotenziale zu erwarten. In früheren Phasen werden folglich höhere Gewichte für die anderen Bewertungsdimensionen zugrunde gelegt. Es wird unterstellt, dass die relative Bedeutung der Bewertungsdimensionen Umwelt, Versorgungssicherheit und soziale Aspekte im Vergleich zu Wirtschaftlichkeit umso höher ist, je früher die Entwicklungsphase ist (vgl. Abbildung 7.10 und Abbildung 7.11). Weiterhin wird angenommen, dass dies vor allem für die Umweltauswirkungen gilt und nur in geringem Maße für die Versorgungssicherheit und soziale Aspekte.

Da die Bewertungsdimensionen (mit Ausnahme der Wirtschaftlichkeit) durch mehrere Kriterien abgebildet werden, sind weitere Betrachtungen zur relativen Bedeutung der untergeordneten Kriterien erforderlich. Zur Bewertung der Wirtschaftlichkeit wird lediglich ein Kriterium (die spezifischen Gesamtkosten der Energiebereitstellung) angewendet. Dessen Bedeutung ist somit mit $100 \%$ hinsichtlich der Dimension Wirtschaftlichkeit zu gewichten. Zur Priorisierung von Umweltauswirkungen wird die vom Umweltbundesamt (UBA) vorgeschlagene Methode zugrunde gelegt (vgl. Schmitz und Paulini 1999). Da diese nicht alle der hier betrachteten Wirkungskategorien abdeckt, wird die Priorisierung durch eigene Annahmen ergänzt (vgl. Tabelle 7.10). Die Einstufung der Umweltwirkungen in Kategorien der "Ökologischen Priorität" erfolgt auf Basis der Relationen der spezifischen Beiträge der betrachteten Alternativen in den einzelnen Wirkungskategorien sowie aufgrund der Einstufungen bei Schmitz und Paulini (1999) hinsichtlich „Ökologische Gefährdung“ und des „Distance-to-Target“.

Abbildung 7.10: Gewichtung der Bewertungsdimensionen in Abhängigkeit der Technologiezyklus-Phasen (Stromerzeugung)

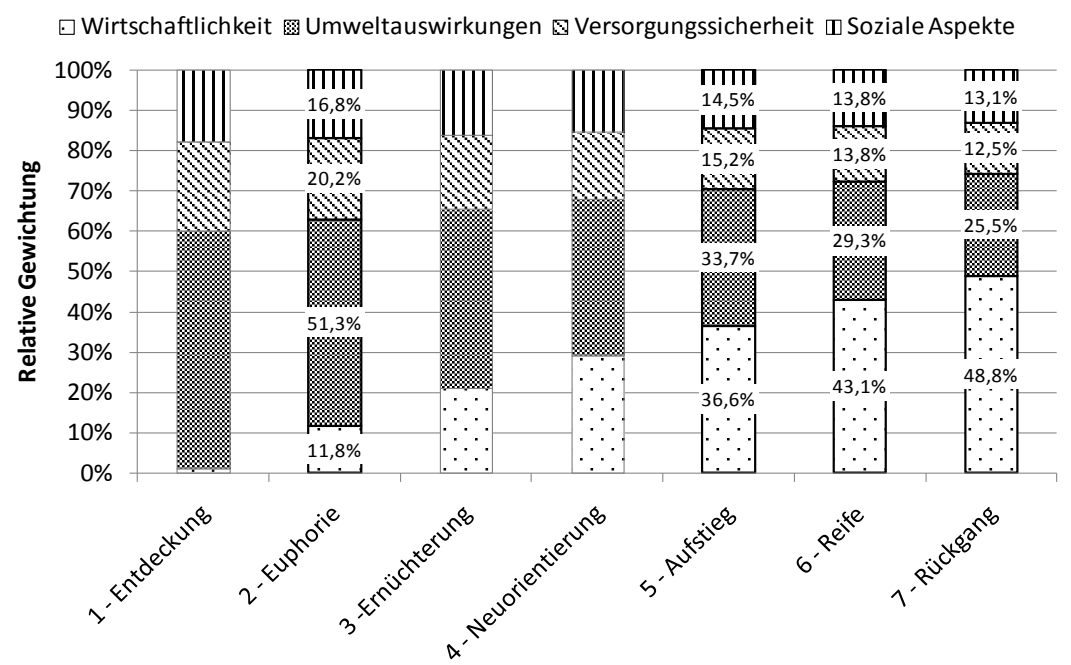

Quellen: Eigene Annahmen basierend auf Bartels et al. (2008). 
Abbildung 7.11: Gewichtung der Bewertungsdimensionen in Abhängigkeit der Technologiezyklus-Phasen (Wärmebereitstellung)

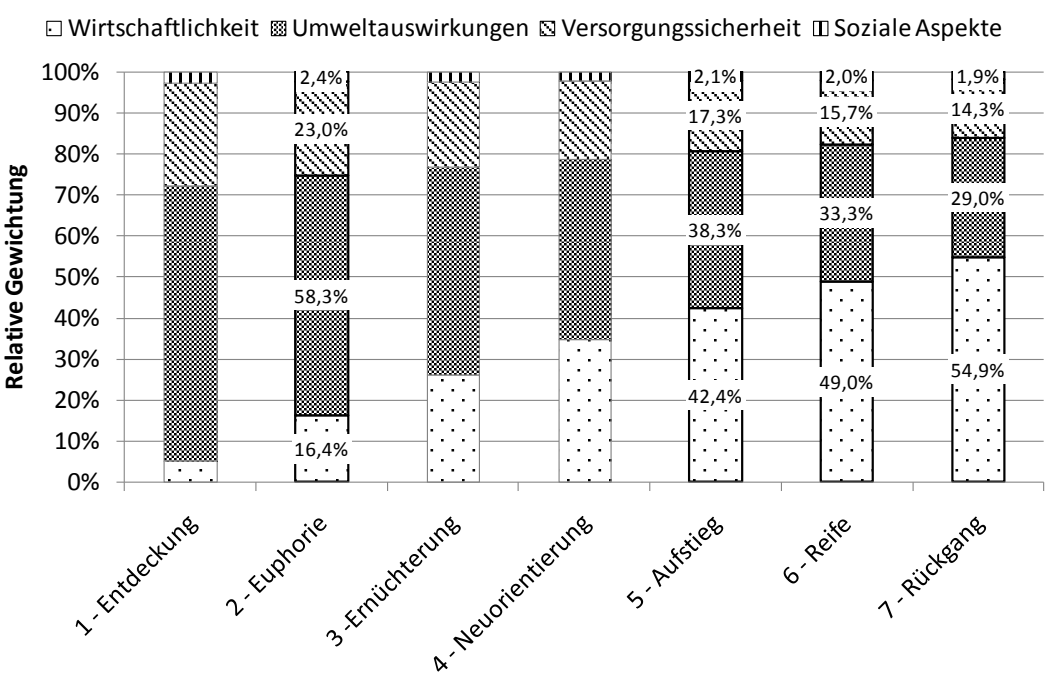

Quellen: Eigene Annahmen basierend auf Bartels et al. (2008).

Tabelle 7.10: $\quad$ Priorisierung von Umweltauswirkungen

\begin{tabular}{|c|c|c|c|c|c|c|c|}
\hline \multicolumn{2}{|c|}{ Kriterium } & $\begin{array}{c}\text { Spezifischer } \\
\text { Beitrag* }\end{array}$ & $\begin{array}{l}\text { Ökologische } \\
\text { Gefährdung }\end{array}$ & $\begin{array}{c}\text { Distance-to- } \\
\text { Target }\end{array}$ & $\begin{array}{l}\text { Ökologische } \\
\text { Priorität }\end{array}$ & Punkte* & $\begin{array}{c}\text { Ge- } \\
\text { wicht* }^{*}\end{array}$ \\
\hline U1 & ADP & $E$ & $\mathrm{C}$ & B & mittel & 3 & $10,0 \%$ \\
\hline U2 & LAND & $A$ & $A$ & B & sehr groß & 5 & $16,7 \%$ \\
\hline U3 & GWP & B & $A$ & $A$ & sehr groß & 5 & $16,7 \%$ \\
\hline U4 & ODP & k. A. & k. A. & k. A. & (sehr gering)* & 1 & $3,3 \%$ \\
\hline U5 & HTP & k. A. & k. A. & k. A. & (sehr gering)* $^{\star}$ & 1 & $3,3 \%$ \\
\hline U6 & ETP & k. A. & k. A. & k. A. & (sehr gering)* & 1 & $3,3 \%$ \\
\hline U7 & POCP & $E$ & $\mathrm{D}$ & B & gering & 2 & $6,7 \%$ \\
\hline U8 & AP & A & B & B & groß & 4 & $13,3 \%$ \\
\hline U9 & EP & A & B & B & groß & 4 & $13,3 \%$ \\
\hline U10 & KEA & A & k. A. & k. A. & (groß)* & 4 & $13,3 \%$ \\
\hline
\end{tabular}

Anmerkungen: *eigene Annahmen bzw. Berechnungen

Quellen: Hillenbrand (2009); Schmitz und Paulini (1999); UBA (2007); eigene Annahmen und Berechnungen.

Die im Fallbeispiel angewendeten Kriterien zur Bewertung der Dimension der Versorgungssicherheit wurden auf Basis unterschiedlicher Quellen zusammengestellt. Anwendungen der Gesamtheit dieser Kriterien zur Bewertung von Energietechnologien in anderen Veröffentlichungen sind bisher nicht bekannt. Folglich liegen keine weiteren Anhaltspunkte zur relativen Bedeutung dieser Kriterien im Vergleich zueinander vor. 
Daher werden diese hinsichtlich ihrer Bedeutung für die Bewertung der Versorgungssicherheit zunächst gleich gewichtet.

Hinsichtlich der Gewichtung der Kriterien zur Bewertung der sozialen Aspekte können aus den Experteneinschätzungen bei Gallego Carrera und Mack (2009) und Bartels et al. (2008) Rückschlüsse auf deren relative Bedeutung gezogen werden. Die Experteneinschätzungen bei Gallego Carrera und Mack (2009) spiegeln nicht nur die Bewertung der Technologien im Vergleich zueinander wider, sondern implizieren auch eine Aussage über die Wichtigkeit eines jeden Indikators: je höher die Experteneinschätzungen im Mittel sind, desto höher wird die Bedeutung dieses Kriteriums eingestuft. Auf dieser Basis werden die Gewichtungen in Tabelle 7.11 abgeleitet. Aus Abbildung 6.2 kann des Weiteren abgeleitet werden, dass aufgrund der Expertenbefragung bei Bartels et al. (2008) das Verhältnis der Relevanz der Kriterien „Akzeptanz" und „Innovationspotenzial" mit 1:2 Punkten bewertet wird. Dementsprechend wird in diesem Fallbeispiel die Gewichtung des Kriteriums S10 (soziale Akzeptanz) aus der Gewichtung des Kriterium S1 (Flexibilität hinsichtlich Innovationen) abgeleitet.

Tabelle 7.11: $\quad$ Gewichtung der Kriterien zur Bewertung sozialer Aspekte

\begin{tabular}{l|c|c}
\hline & $\begin{array}{c}\text { Mittelwert aller } \\
\text { Bewertungen }\end{array}$ & Gewichtung \\
\hline S1. Flexibilität hinsichtlich Innovationen & 1,93 & $10,2 \%$ \\
\hline S2. Abfallinfrastrukturen & 2,27 & $12,1 \%$ \\
\hline S3. Konflikte & 2,52 & $13,4 \%$ \\
\hline S5. Gesundheit & 2,40 & $12,7 \%$ \\
\hline S6. Risiken & 3,32 & $17,7 \%$ \\
\hline S7. Katastrophen & 2,51 & $13,3 \%$ \\
\hline S9. Ästhetik & 2,91 & $15,5 \%$ \\
\hline S10. Soziale Akzeptanz & --- & $5,1 \%$ \\
\hline Summe & 17,84 & $100,0 \%$ \\
\hline
\end{tabular}

Quelle: Gallego Carrera und Mack (2009); eigene Annahmen.

Als Eingabe für das Excel-Tool MAESTRO müssen die Gewichtungen der einzelnen Kriterien mit der Gewichtung der übergeordneten Bewertungsdimension in Abhängigkeit der Technologiezyklus-Phasen verknüpft werden. Web-HIPRE unterstützt die Abbildung einer entsprechenden hierarchischen Problemstruktur (Abbildung 7.12). Die bisher dargestellten Gewichtungen, die sich jeweils auf einzelne Knotenpunkte dieser Hierarchie beziehen, werden in dieses Modell eingegeben. Die Funktionalitäten von Web-HIPRE erlauben dann eine einfache und schnelle Ermittlung der KriterienGewichtungen auf der untersten Hierarchie-Ebene („Criteria 4“) mit Bezug zu unter- 
schiedlichen Technologiezyklus-Phasen. Diese Gewichtungen werden wiederum exportiert und dienen als Input für das Excel-Tool MAESTRO.

Abbildung 7.12: Hierarchische Problemstrukturierung zur Ermittlung der KriterienGewichtungen der untersten Ebene in Abhängigkeit der Technologiezyklus-Phasen (Alternativen zur Stromerzeugung)

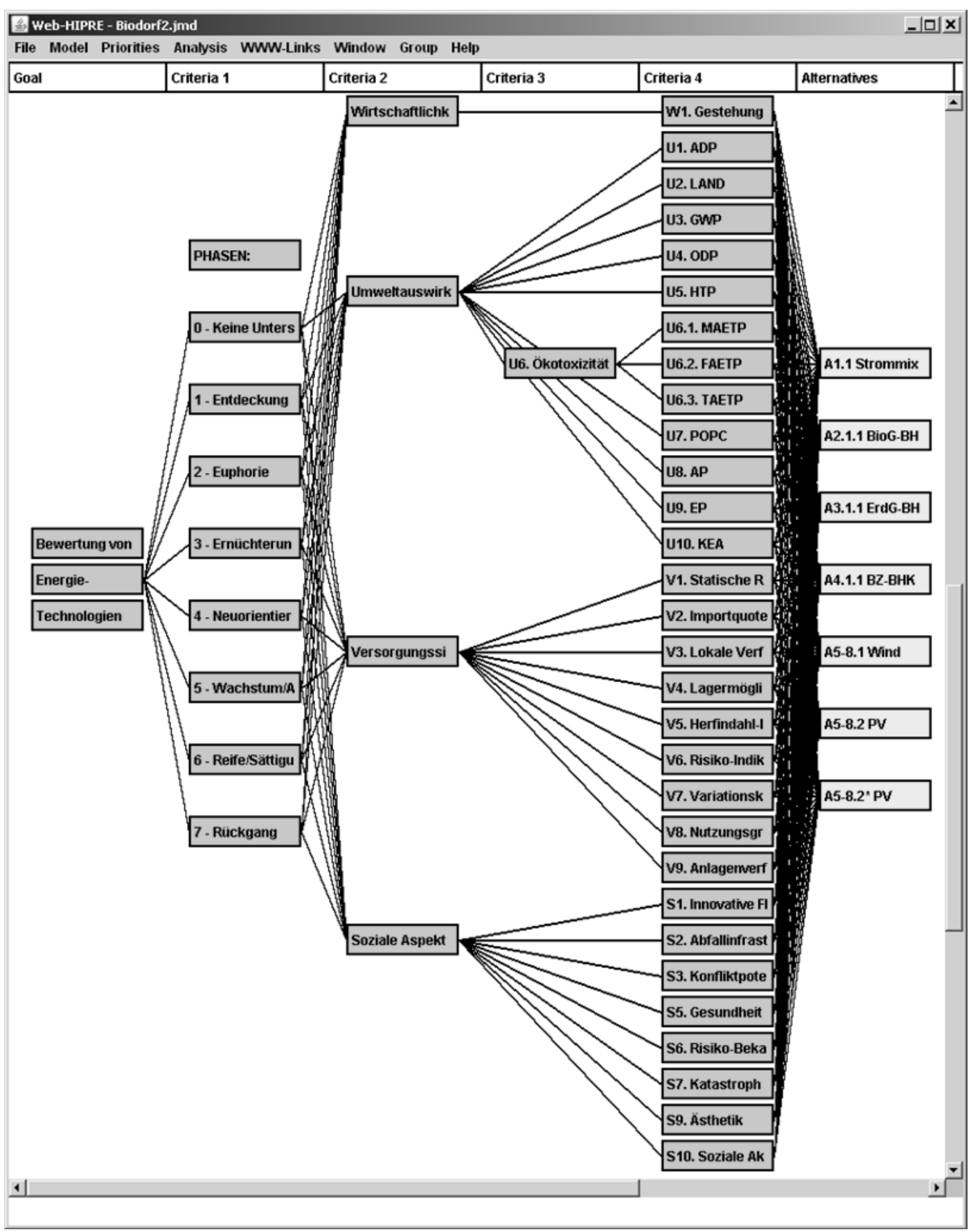


Bevor die Auswertung der Daten durch den modifizierten PROMETHEE-Algorithmus starten kann, sind zusätzlich die Präferenzfunktionen festzulegen (Tabelle 7.12). Für die spezifischen Gesamtkosten (W1), die statische Reichweite (V1), den HerfindahlIndex (V5) und den Risiko-Indikator (V6) wird Typ 6 zugrunde gelegt, da dieser Typ stabile Ergebnisse verspricht (vgl. Brans et al. 1986; Queiruga et al. 2008). Als Schwellenwert s werden $50 \%$ der maximalen Differenz zwischen den jeweiligen KriterienAusprägungen der betrachteten Alternativen festgelegt. Da bei den Umweltkriterien (U1 bis U10), bei den quantitativen Kriterien zur Versorgungssicherheit (V2. Importquote, V7. Variationskoeffizient der Energieträgerpreise, V8. Nutzungsgrad, V9. Anlagenverfügbarkeit) und bei den Kriterien zur Bewertung der sozialen Aspekte (S1 bis S10) von einer linearen Präferenz ausgegangen werden kann, werden für diese Kriterien die Typen 3 bzw. 5 angewendet. Für die Umweltkriterien wird entsprechend den Empfehlungen bei Tsoutsos et al. (2009) ein Schwellenwert für Indifferenz bei $5 \%$ bzw. für strikte Präferenz bei $30 \%$ der maximalen Differenz zwischen den KriterienAusprägungen der im Fallbeispiel betrachteten Alternativen festgelegt (Präferenzfunktion Typ 5). Für die Kriterien zur Versorgungssicherheit und zu sozialen Aspekten mit linearer Präferenz wird lediglich ein Schwellenwert für strikte Präferenz festgelegt, der dem theoretisch erreichbaren Maximum des jeweiligen Kriteriums entspricht (Präferenzfunktion Typ 3). Für die Kriterien Lokale Verfügbarkeit (V3) und Lagermöglichkeit (V4) eignet sich Typ 1 als Präferenzfunktion, da es sich hierbei um „ja/nein“-Variablen handelt, für die keine Abstufungen schwacher Präferenz festgelegt werden können. 
Tabelle 7.12: $\quad$ Präferenzfunktionen und Schwellenwerte

\begin{tabular}{|c|c|c|c|c|c|c|c|c|}
\hline \multirow{3}{*}{$\begin{array}{l}\text { Dimension } \\
\text { Wirtschaft }\end{array}$} & \multirow{2}{*}{\multicolumn{2}{|c|}{ Kriterium }} & \multirow{3}{*}{$\begin{array}{l}\text { Einheit } \\
\mathrm{Ct} / \mathrm{kWh}\end{array}$} & \multirow{3}{*}{ 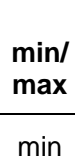 } & \multicolumn{4}{|c|}{ Präferenzfunktion } \\
\hline & & & & & \multirow{2}{*}{\begin{tabular}{|c|} 
Typ \\
6 \\
\end{tabular}} & \multirow[t]{2}{*}{$q$} & \multirow[t]{2}{*}{$\mathrm{p}$} & \multirow{2}{*}{$\frac{s}{32,0}$} \\
\hline & W1.0 & Spez. Gesamtk. & & & & & & \\
\hline \multirow[t]{12}{*}{ Umwelt } & U1 & ADP & kg antimon & $\min$ & 5 & 2,6194E-04 & 1,5716E-03 & \\
\hline & U2 & LAND & $\mathrm{m}^{2} \mathrm{a}$ & $\min$ & 5 & 1,3096E-02 & 7,8577E-02 & \\
\hline & U3 & GWP & $\mathrm{kg} \mathrm{CO}-\mathrm{Eq}$ & $\min$ & 5 & $3,5249 \mathrm{E}-02$ & 2,1149E-01 & \\
\hline & U4 & ODP & kg CFC-11 & $\min$ & 5 & 8,6171E-09 & 5,1702E-08 & \\
\hline & U5 & HTP & kg 1,4-DCB & $\min$ & 5 & $7,7388 \mathrm{E}-03$ & 4,6433E-02 & \\
\hline & U6.1 & FAETP & kg 1,4-DCB & $\min$ & 5 & 1,3359E-03 & 8,0151E-03 & \\
\hline & U6.2 & MAETP & kg 1,4-DCB & $\min$ & 5 & $1,5983 \mathrm{E}+01$ & $9,5897 E+01$ & \\
\hline & U6.3 & TAETP & kg 1,4-DCB & $\min$ & 5 & $4,8518 \mathrm{E}-04$ & 2,9111E-03 & \\
\hline & U7 & POCP & kg Eth & $\min$ & 5 & 4,6677E-06 & 2,8006E-05 & \\
\hline & U8 & AP & $\mathrm{kg} \mathrm{SO}_{2}-\mathrm{Eq}$ & $\min$ & 5 & 1,0093E-04 & 6,0560E-04 & \\
\hline & U9 & EP & $\mathrm{kg} \mathrm{NO}_{x}-\mathrm{Eq}$ & $\min$ & 5 & 1,8347E-05 & 1,1008E-04 & \\
\hline & U10 & KEA & MJ-Eq & $\min$ & 5 & 5,9713E-01 & $3,5828 \mathrm{E}+00$ & \\
\hline \multirow[t]{9}{*}{ Versorgungss. } & V1 & Stat. Rw. (Kehrwert) & $1 / a$ & $\min$ & 6 & & & 7,8000E-03 \\
\hline & V2 & Importquote & $\%$ & $\min$ & 3 & & 100,0 & \\
\hline & V3 & Lokale Verfügbarkeit & {$[0 ; 1]$} & $\max$ & 1 & & & \\
\hline & V4 & Lagermöglichkeit & {$[0 ; 1]$} & $\max$ & 1 & & & \\
\hline & V5 & Herfindahl & --- & $\min$ & 6 & & & 0,08345 \\
\hline & V6 & Risiko-Indikator (Kehrwert) & --- & $\min$ & 6 & & & 45,445 \\
\hline & V7 & Variationskoeff. Preise & --- & $\min$ & 3 & & 1,000 & \\
\hline & V8 & Nutzungsgrad & $\%$ & $\max$ & 3 & & 100,0 & \\
\hline & V9 & Anlagenverfügbarkeit & h/a & $\max$ & 3 & & 8.760 & \\
\hline \multirow[t]{8}{*}{ Soziale Aspekte } & S1 & Flex. Innov. & {$[1-5]$} & $\max$ & 3 & & 4 & \\
\hline & S2 & Abfallinfr. & {$[0-5]$} & $\min$ & 3 & & 5 & \\
\hline & S3 & Konflikte & {$[1-5]$} & $\min$ & 3 & & 4 & \\
\hline & S5 & Gesundheit & {$[0-5]$} & $\min$ & 3 & & 5 & \\
\hline & S6 & Risiken & {$[1-5]$} & $\min$ & 3 & & 4 & \\
\hline & S7 & Katastrophen & {$[1-5]$} & $\min$ & 3 & & 4 & \\
\hline & S9 & Ästhetik & {$[1-5]$} & $\min$ & 3 & & 4 & \\
\hline & S10 & Soziale Akzeptanz & {$[1-6]$} & $\min$ & 3 & & 5 & \\
\hline
\end{tabular}




\subsubsection{Ergebnisse}

Die Auswertung und Aggregation der Daten erfolgt nach dem in Abschnitt 6.6 beschriebenen modifizierten PROMETHEE-Algorithmus mit Hilfe des Excel-Tools MAESTRO. Einen Überblick über die Ergebnisse in Abhängigkeit der Szenarien zeigen Abbildung 7.13 und Abbildung 7.14. Jedes Symbol stellt eine Alternative dar und jeder Punkt ein Bewertungsergebnis je Szenario. Dabei ist auf der $\mathrm{x}$-Achse der positive Outranking-Fluss $\phi^{+}$als Maß für die relative Stärke und auf der $y$-Achse der negative Outranking-Fluss $\phi^{-}$als Maß für die relative Schwäche einer Alternative aufgetragen. Dies bedeutet, dass eine Alternative umso besser einzustufen ist, je weiter rechts und je weiter oben im Diagramm der entsprechende Punkt liegt. 19 Als Nachteil erweist sich, dass in dieser Darstellungsform nicht unmittelbar erkennbar ist, welcher Punkt welchem Szenario zuzuordnen ist. Jedoch werden die Schwankungsbreite der Ergebnisse und die Stabilität der Rangfolge in Abhängigkeit der Szenarien deutlich. Weitere Darstellungsformen ermöglichen dezidierte Schlussfolgerungen mit Bezug zu einzelnen Szenarien.

Abbildung 7.13: Relative Stärke und relative Schwäche der Alternativen zur Stromerzeugung in Abhängigkeit der Szenarien

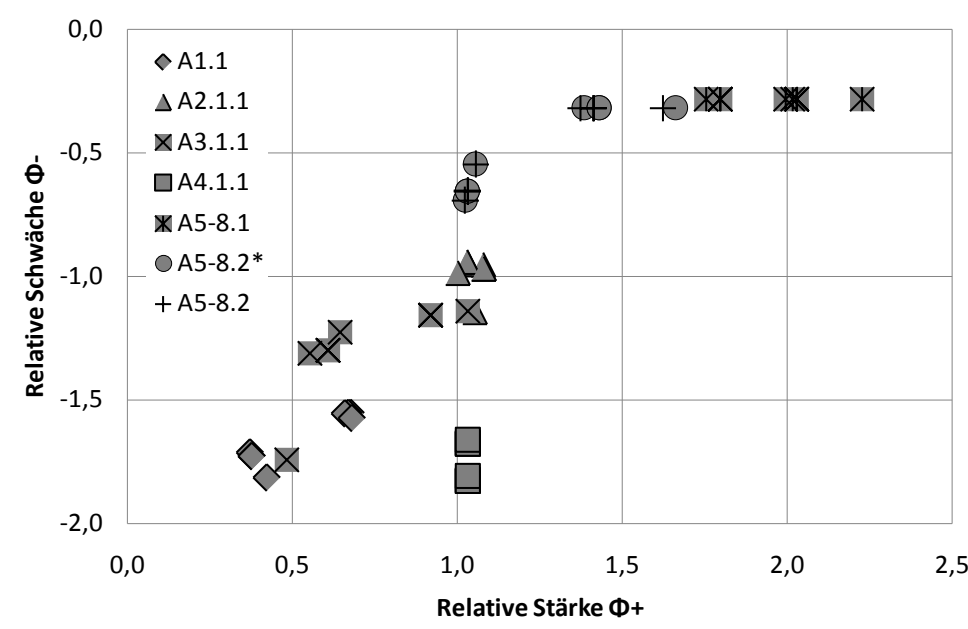

Anmerkungen: A1.1: Strommix D; A2.1.1: Biogas-BHKW, Stromerzeugung; A3.1.1: ErdgasBHKW, Stromerzeugung; A4.1.1: Brennstoffzellen-BHKW, Stromerzeugung; A5-8.1: Windkraftanlage; A5-8.2: PV-Dachanlage; A5-8.2*: PV-Anlage unter Berücksichtigung von Fördersätzen für Eigenverbrauch.

19 Eine ähnliche Darstellungsform nutzen auch Terrados et al. (2009), die jedoch auf eine Abbildung der Ergebnisse in Abhängigkeit von Szenarien in der hier dargestellten Form verzichten. 
Bei den Alternativen zur Stromerzeugung (Abbildung 7.13) wird ein besonders gutes Ergebnis für die Windkraftanlage (A5-8.1) deutlich. Das Maß für die relativen Schwächen ist über alle Szenarien stabil. Das Maß für relative Stärken variiert zwar je Szenario, bleibt aber in allen Szenarien am höchsten im Vergleich zu den anderen Alternativen. Auch für die PV-Anlage (A5-8.2) zeigt sich ein sehr gutes Ergebnis, das in Abhängigkeit der Szenarien stark variiert. Dies ist insbesondere auf die hohen Fördersätze für PV-Strom zurückzuführen, die sich in den Szenarien mit Berücksichtigung aktueller Förderbedingungen positiv auf die Wirtschaftlichkeitsbewertung dieser Alternative auswirken. Die relativen Stärken der PV-Anlage, des Biogas-BHKW (A2.1.1), des Erdgas-BHKW (A3.1.1) und der Brennstoffzelle (A4.1.1) liegen zumindest in einigen Szenarien in einem ähnlichen Bereich. Die relativen Stärken und des Erdgas-BHKW sind jedoch in anderen Szenarien wesentlich geringer. Der Bezug von Strom aus dem öffentlichen Versorgungsnetz (A1.1 Strommix) weist in den meisten Szenarien sowohl weniger Stärken als auch mehr Schwächen im Vergleich zu den anderen Alternativen auf.

Abbildung 7.14: Relative Stärke und relative Schwäche der Alternativen zur Wärmebereitstellung in Abhängigkeit der Szenarien

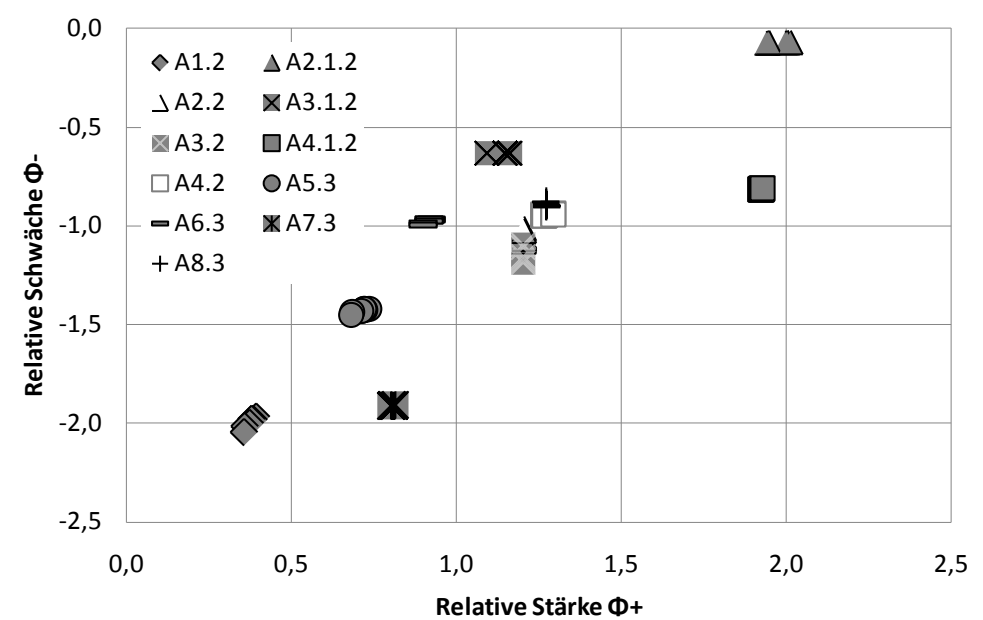

Anmerkungen: A1.2: Konventioneller Heizöl-Kessel; A2.1.2: Biogas-BHKW, Wärmeerzeugung; A2.2 Holzhackschnitzel-Heizwerk (mittlere Auslastung); A3.1.2: Erdgas-BHKW, Wärmeerzeugung; A3.2 Holzhackschnitzel-Heizwerk (geringe Auslastung); A4.1.2: Brennstoffzellen-BHKW, Wärmeerzeugung; A4.2 Holzhackschnitzel-Heizwerk (hohe Auslastung); A5.3: Erdgas-

Brennwertkessel; A6.3: Solar unterstützte Erdgas-Heizung; A7.3: Wärmepumpe; A8.3: Holzpellets-Heizung.

Bei den Alternativen zur Wärmebereitstellung ist generell ein weniger starker Einfluss der Szenarien auf das Endergebnis erkennbar (Abbildung 7.14). Im oberen Bereich der Bewertungsergebnisse liegt das Biogas-BHKW (A2.1.2), wobei die Brennstoffzelle (A4.1.2) ein ähnlich gutes Maß für die relativen Stärken aufweist. Andererseits ist das 
Maß für die relativen Schwächen wesentlich schlechter und liegt zudem unter dem des Erdgas-BHKW (A3.1.2). Letzteres weist wiederum ein deutlich geringeres Maß an Stärken auf. Die Bewertungsergebnisse für die Holzpellets-Heizung (A8.3) sowie die drei Varianten des Holzhackschnitzel-Heizwerks (A2.2, A3.2 und A4.2) liegen sehr nahe beieinander. Auch die relativen Schwächen der solar unterstützten Erdgas-Heizung (A6.3) liegen im gleichen Bereich; dessen relative Stärke wird jedoch deutlich geringer eingestuft. Der Erdgas-Brennwertkessel (A5.2) weist sowohl weniger Stärken als auch mehr Schwächen im Vergleich zur solar unterstützten Erdgas-Heizung auf. Die Wärmepumpe (A7.3) zeigt zwar mehr Stärken, jedoch auch mehr Schwächen als die Erdgas-Heizung. Der konventionelle Heizöl-Kessel (A1.2) liegt schließlich im unteren Bereich des Bewertungsdiagramms.

Eine Analyse nach den Bewertungsdimensionen ermöglicht Rückschlüsse auf die wesentlichen Einflussfaktoren auf das Ergebnis je Alternative. Da sich die diesbezüglichen Bewertungsergebnisse in den Szenarien 0 bis 2 nur in geringem Maße unterscheiden, werden in Abbildung 7.15 (Stromerzeugung) und Abbildung 7.16 (Wärmebereitstellung) stellvertretend die Ergebnisse in den Szenarien 1 und 3 in Abhängigkeit der Bewertungsdimensionen dargestellt. Hinsichtlich der Optionen zur Stromerzeugung wird im Szenario 1 (ohne Förderung; Abbildung 7.15, links oben) für den Strommix (A1.1) deutlich, dass sich insbesondere die Wirtschaftlichkeit positiv auf die relativen Stärken $\left(\phi^{+}\right)$auswirkt, diese im Vergleich zu den anderen Alternativen jedoch insgesamt relativ gering sind. Das Maß für die relativen Schwächen ( $\phi$ ) überwiegt deutlich die relativen Stärken und wird vor allem durch die Umweltauswirkungen bestimmt. Die sozialen Aspekte und die Kriterien zur Bewertung der Versorgungssicherheit haben einen geringeren Einfluss, wirken sich jedoch überwiegend nachteilig auf das Ergebnis für den Strommix auf. Für das Biogas-BHKW (A2.1.1) werden hinsichtlich der Umweltauswirkungen sowohl deutliche Vorteile als auch signifikante Nachteile deutlich. Während der positive Outranking-Fluss neben den Umweltauswirkungen vor allem durch die Aspekte der Versorgungssicherheit positiv beeinflusst wird, hat die Wirtschaftlichkeit einen eher negativen Einfluss auf das Ergebnis. Insgesamt überwiegen zu einem geringen Anteil die relativen Stärken des Biogas-BHKW. Im Gegensatz dazu überwiegen beim Erdgas-BHKW (A3.1.1) insgesamt die relativen Schwächen, die vor allem durch die Umweltauswirkungen und zu einem deutlich geringeren Anteil durch die Bewertungsdimension der Versorgungssicherheit bestimmt werden. Positiv wirkt sich für diese Alternative in erster Linie die relative Vorteilhaftigkeit hinsichtlich der Wirtschaftlichkeit aus. Das Ergebnis des Brennstoffzellen-BHKW (A4.1.1) ist insofern dem des Biogas-BHKW ähnlich, dass sowohl deutliche Vorteile als auch Nachteile hinsichtlich der Umweltauswirkungen zu beobachten sind. Jedoch überwiegen in diesem Fall die relativen Nachteile, die zudem durch die relativen Schwächen hinsichtlich der 
Wirtschaftlichkeit und Versorgungssicherheit verschärft werden. Die Windkraftanlage (A5-8.1) weist das höchste Maß an Stärken auf, was sowohl auf relative Vorteile hinsichtlich der Wirtschaftlichkeit als auch der Umweltauswirkungen im Vergleich zu den anderen Alternativen zurückzuführen ist. Das geringe Maß für relative Schwächen ist durch die Bewertung in den Dimensionen Versorgungssicherheit und soziale Aspekte bestimmt. Für die PV-Anlage (A5-8.2) werden die relativen Vorteile hinsichtlich der Umweltauswirkungen deutlich, wohingegen die relative Schwäche dieser Alternative vor allem in der Wirtschaftlichkeit liegt.

Abbildung 7.15: Analyse der Ergebnisse nach Bewertungsdimensionen (Stromerzeugung)

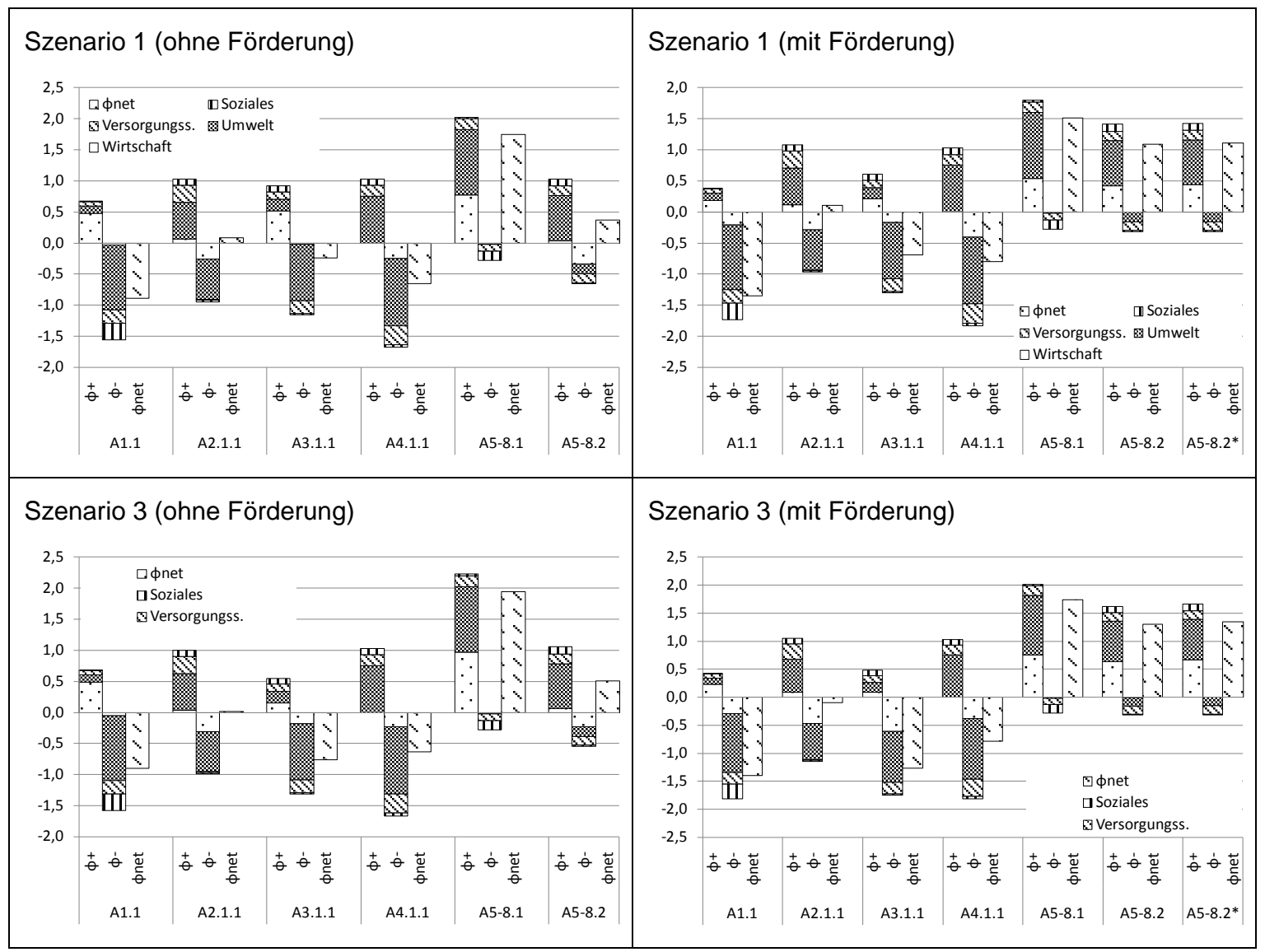

Anmerkungen: A1.1: Strommix D; A2.1.1: Biogas-BHKW, Stromerzeugung; A3.1.1: ErdgasBHKW, Stromerzeugung; A4.1.1: Brennstoffzellen-BHKW, Stromerzeugung; A5-8.1: Windkraftanlage; A5-8.2: PV-Dachanlage; A5-8.2*: PV-Anlage unter Berücksichtigung von Fördersätzen für Eigenverbrauch.

Beim Vergleich der Alternativen unter Berücksichtigung aktueller Förderbedingungen im Szenario 1 (Abbildung 7.15, rechts oben) ändern sich die relativen Vor- und Nachteile der Alternativen hinsichtlich der Wirtschaftlichkeit. Während sich vor allem das Ergebnis der PV-Anlage (A5-8.2 und A5-8.2*) aufgrund der Einbeziehung der Ein- 
speisevergütungen nach EEG in der Wirtschaftlichkeitsdimension deutlich verbessert, wird insbesondere für den Strommix (A1.1) und das Erdgas-BHKW (A3.1.1) eine Reduzierung der relativen Vorteilhaftigkeit in der Wirtschaftlichkeitsbewertung deutlich. Erhöhte Energieträgerpreise im Szenario 3 (Abbildung 7.15, links unten) wirken sich in erster Linie negativ auf die relativen Stärken des Erdgas-BHKW (A3.1.1) aus, während die relativen Schwächen der PV-Anlage (A5-8.2) erkennbar verringert werden. Dies gilt in verstärktem Maße unter Berücksichtigung von Förderbedingungen in Szenario 3 (Abbildung 7.15, rechts unten).

Hinsichtlich des Einflusses der Bewertungsdimensionen auf die Ergebnisse der Optionen zur Wärmebereitstellung (Abbildung 7.16) ist generell eine starke Abhängigkeit der relativen Vorteilhaftigkeit von den Umweltauswirkungen erkennbar. Des Weiteren wirken sich die Bewertungen hinsichtlich der Versorgungssicherheit im Vergleich zu den Optionen zur Stromerzeugung stärker auf das Ergebnis aus. Die Wirtschaftlichkeit ist in diesem Fall von geringerer Bedeutung, obwohl dieser sowohl in der Aufstiegs- als auch in der Reife- und Rückgangs-Phase ein sehr hohes Gewicht beigemessen wird (vgl. Abbildung 7.11). Weiterhin wird deutlich, dass die Gesamtergebnisse für die Optionen zur Wärmebereitstellung nur in geringem Maße von den unterschiedlichen Szenarien abhängen.

Deutlich überwiegende Nachteile weist der Heizöl-Kessel (A1.2) hinsichtlich Umweltauswirkungen und der Versorgungssicherheit auf. Das Ergebnis des Biogas-BHKW (A2.1.2) ist dem entgegengesetzt positiv durch die relativen Vorteile hinsichtlich Umweltauswirkungen und des Beitrags zur Versorgungssicherheit beeinflusst. Relative Nachteile sind für diese Alternative kaum erkennbar. Für das HolzhackschnitzelHeizwerk (A2.2, A3.2 und A4.2) halten sich die Vor- und Nachteile hinsichtlich der Umweltauswirkungen ungefähr die Waage. Hinsichtlich der Versorgungssicherheit sind überwiegend relative Vorteile erkennbar. Bei geringer Auslastung des Holzhackschnitzel-Heizwerks (A3.2) sind zudem deutliche relative Nachteile bezüglich der Wirtschaftlichkeit erkennbar. Die relativen Stärken der Wärmebereitstellung aus dem ErdgasBHKW (A3.1.2) werden in erster Linie durch die Umweltauswirkungen und in geringerem Maße durch die Wirtschaftlichkeit beeinflusst. Relative Schwächen dieser Alternative liegen vor allem beim Beitrag zur Versorgungssicherheit. Das hohe Maß für die relativen Stärken der Brennstoffzelle (A4.1.2) ist in erster Linie auf die relativen Vorteile hinsichtlich der Umweltauswirkungen zurückzuführen. Hinsichtlich des Beitrags zur Versorgungssicherheit wird dieser Alternative eher negativ bewertet, was vor allem auf den eingesetzten Brennstoff Erdgas und die relativ geringe Anlagenverfügbarkeit zurückzuführen ist. Für den Erdgas-Brennwertkessel (A5.3) überwiegen die relativen Schwächen bezüglich der Umweltauswirkungen die relativen Stärken. Die relativen 
Schwächen werden zudem durch die Ergebnisse hinsichtlich der Versorgungssicherheit erhöht.

Abbildung 7.16: Analyse der Ergebnisse nach Bewertungsdimensionen (Wärmebereitstellung)

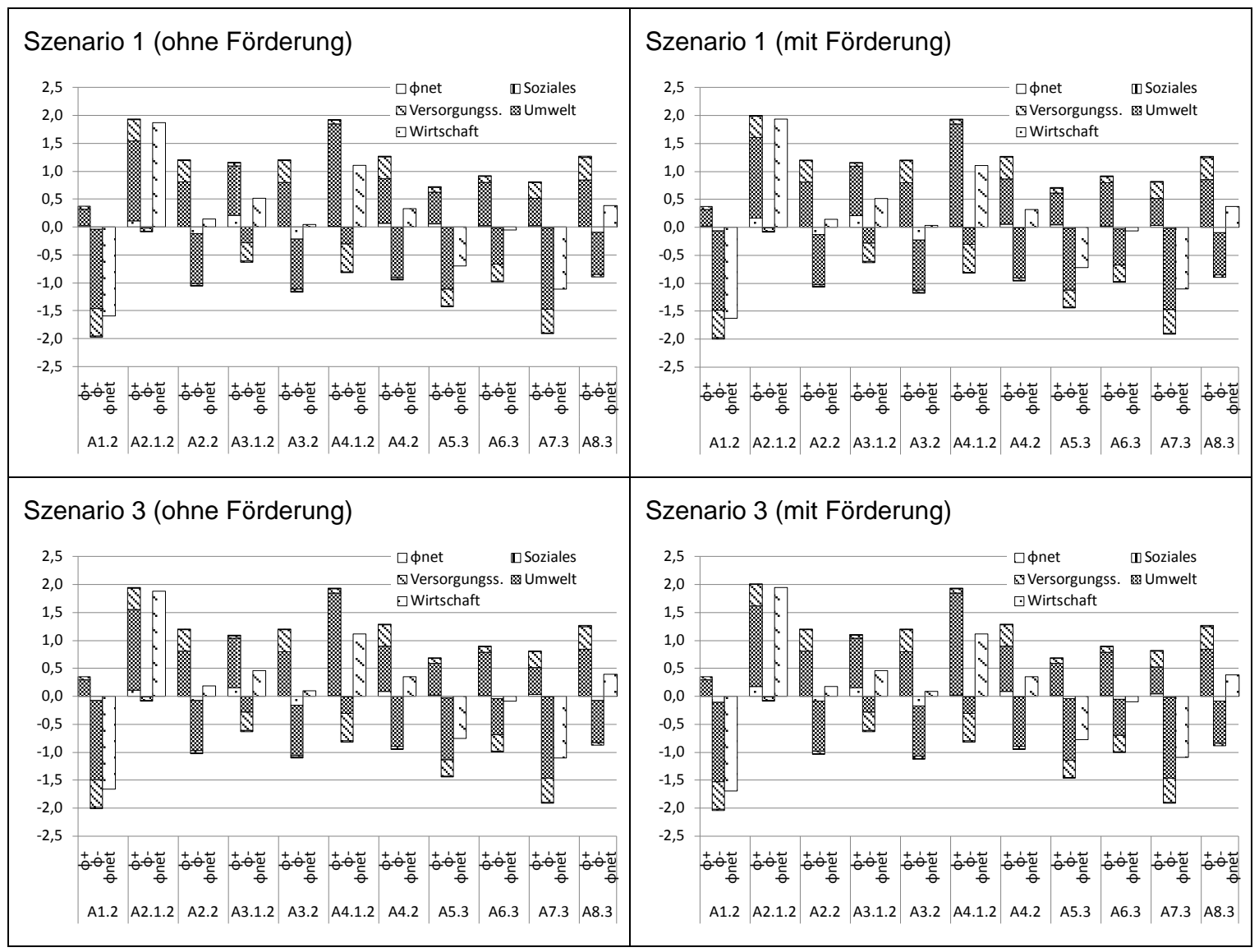

Anmerkungen: A1.2: Konventioneller Heizöl-Kessel; A2.1.2: Biogas-BHKW, Wärmeerzeugung; A2.2 Holzhackschnitzel-Heizwerk (mittlere Auslastung); A3.1.2: Erdgas-BHKW, Wärmeerzeugung; A3.2 Holzhackschnitzel-Heizwerk (geringe Auslastung); A4.1.2: Brennstoffzellen-BHKW, Wärmeerzeugung; A4.2 Holzhackschnitzel-Heizwerk (hohe Auslastung); A5.3: ErdgasBrennwertkessel; A6.3: Solar unterstützte Erdgas-Heizung; A7.3: Wärmepumpe; A8.3: Holzpellets-Heizung.

Bei der solar unterstützten Erdgas-Heizung (A6.3) gleichen sich die relativen Stärken und Schwächen hinsichtlich der Umweltauswirkungen ungefähr aus. Hinsichtlich des Beitrages zur Versorgungssicherheit überwiegen deutlich die relativen Schwächen. Für die Wärmepumpe (A7.3) zeichnet sich für die Schwächen ein dem Heizöl-Kessel (A1.2) ähnliches Profil ab. Es zeigen sich erhebliche relative Schwächen hinsichtlich der Umweltauswirkungen und des Beitrages zur Versorgungssicherheit, die nicht durch die relativen Stärken aufgewogen werden können. Bei der Holzpellets-Heizung (A8.3) gleichen sich die relativen Stärken und Schwächen hinsichtlich der Umweltauswirkun- 
gen in etwa aus. Insgesamt überwiegen die relativen Stärken vor allem aufgrund der guten Bewertung des Beitrags zur Versorgungssicherheit.

\subsubsection{Rangfolgen der Alternativen}

Aus den Bewertungsergebnissen kann die Rangfolge der Alternativen nach PROMETHEE I und II abgeleitet werden. Unter den Optionen zur Stromerzeugung ist nach PROMETHEE I die Windkraftanlage (A5-8.1) allen anderen Alternativen eindeutig vorzuziehen. Dies gilt für alle Szenarien mit und ohne Berücksichtigung von Fördermitteln (vgl. Abbildung 7.17 bis Abbildung 7.19). Nach PROMETHEE I stehen an zweiter Stelle der Rangfolge das Biogas-BHKW (A2.1.1) und die PV-Anlage (A5-8.2). Diese sind im Szenario 0 (ohne Förderung) miteinander unvergleichbar (Abbildung 7.17, oben). In allen anderen Fällen wird die PV-Anlage insgesamt günstiger bewertet als das Biogas-BHKW. Dabei wird die PV-Anlage bei Inanspruchnahme der Fördersätze für Eigenverbrauch $\left(A 5-8.2^{*}\right)$ in allen Szenarien besser bewertet als die FörderAlternative mit vollständiger Einspeisung der erzeugten Strommenge (A5-8.2).

Abbildung 7.17: $\quad$ Rangfolge der Optionen zur Strombereitstellung nach PROMETHEE I ohne Berücksichtigung von Förderbedingungen

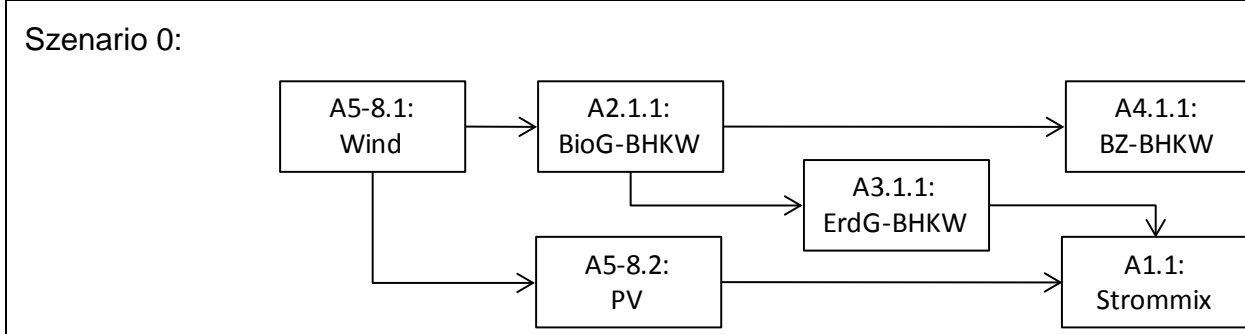

Szenarien 1 und 2:

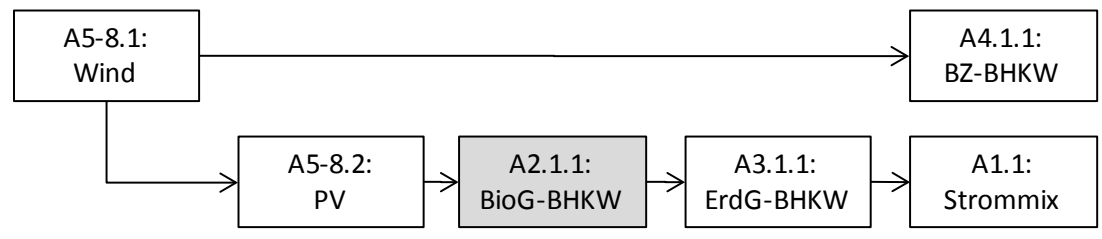

Szenario 3:

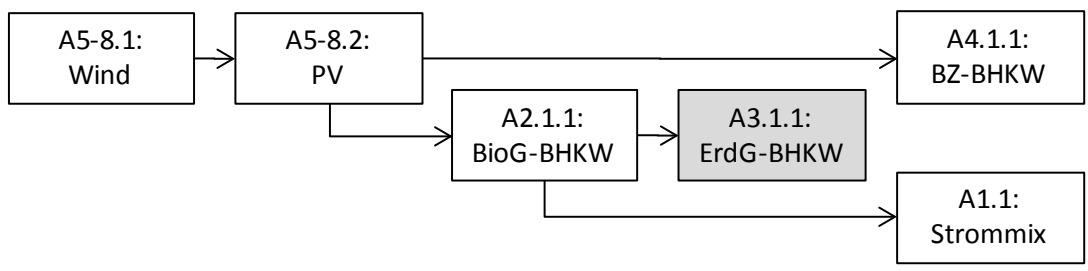


Das Biogas-BHKW (A2.1.1) wird nach PROMETHEE I in allen Szenarien eindeutig besser eingestuft als das Erdgas-BHKW. Für das Brennstoffzellen-BHKW (A4.1.1) sind nach PROMETHEE I Unvergleichbarkeiten mit allen anderen Alternativen außer der Windkraftanlage zu beobachten. Dies deutet darauf hin, dass das BrennstoffzellenBHKW im Vergleich zu den anderen Alternativen sowohl erhebliche Vor- aber auch Nachteile aufweist. Wird keine Förderung berücksichtigt, ist nach PROMETHEE I neben der Windkraftanlage im Szenario 0 nur das Biogas-BHKW der Brennstoffzelle eindeutig vorzuziehen (Abbildung 7.17, oben). Der Strommix (A1.1) und das Brennstoffzellen-BHKW (A4.1.1) sind nach PROMETHEE I nahezu unter allen betrachteten Bedingungen unvergleichbar. Lediglich in Szenario 3 ist die Brennstoffzelle bei Beachtung von Förderbedingungen eindeutig vorzuziehen (Abbildung 7.18, unten). Alle anderen Alternativen werden in den meisten Szenarien eindeutig besser eingestuft als der Strommix, mit Ausnahme des Erdgas-BHKW in Szenario 3 (ohne Förderung; vgl. Abbildung 7.17 , unten).

Abbildung 7.18: Rangfolge der Optionen zur Strombereitstellung nach PROMETHEE I unter Berücksichtigung von Förderbedingungen

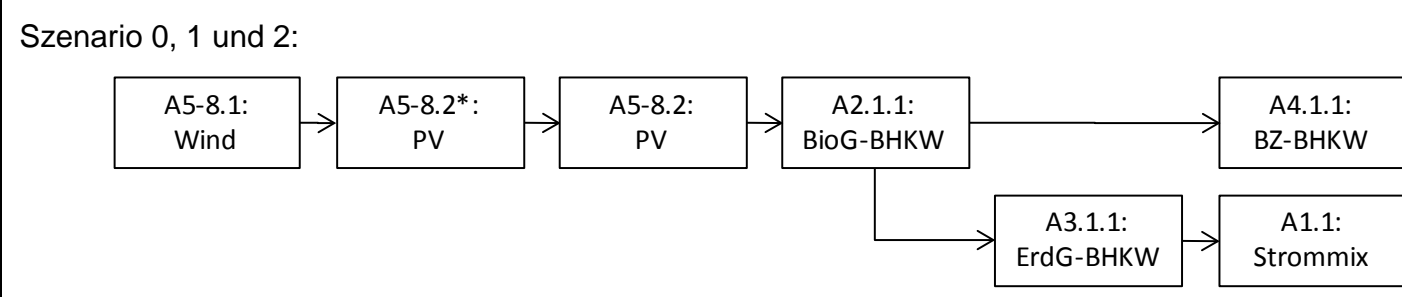

Szenario 3:

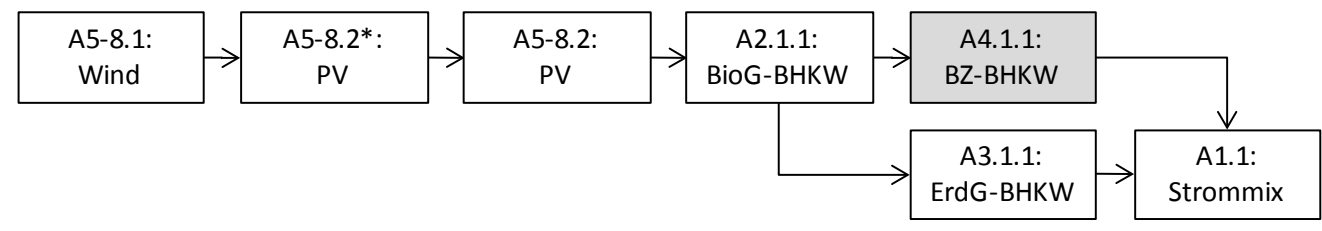

Nach PROMETHEE II (Abbildung 7.19) ist die Rangfolge in den Szenarien 0 bis 2 (mit und ohne Förderung) identisch. Auf den vorderen Rangplätzen liegen die erneuerbaren Alternativen zur Stromerzeugung. An erster Stelle steht die Windkraftanlage, gefolgt von der PV-Anlage an zweiter bzw. dritter und dem Biogas-BHKW an vierter Stelle. Den fünften Rangplatz belegt das Erdgas-BHKW, den sechsten Platz die Brennstoffzelle. Am schlechtesten wird der deutsche Strommix bewertet. Im Szenario 3 sind lediglich die Rangplätze des Erdgas-BHKW und der Brennstoffzelle vertauscht. Dies ist auf den höheren Anteil der Brennstoffkosten an den spezifischen Gesamtkosten beim Erdgas-BHKW in Verbindung mit den starken Preissteigerungen bei fossilen Brennstoffen im Szenario 3 zurückzuführen. 
Abbildung 7.19: Rangfolge der Optionen zur Strombereitstellung nach PROMETHEE II

Szenarien 0 bis 2 (mit und ohne Förderung):

\begin{tabular}{|c|c|c|c|c|c|c|}
\hline $\begin{array}{l}\text { A5-8.1: } \\
\text { Wind }\end{array}$ & $\begin{array}{c}\text { A5-8.2*: } \\
\text { PV }\end{array}$ & $\begin{array}{c}\text { A5-8.2: } \\
\text { PV }\end{array}$ & $\begin{array}{c}\text { A2.1.1: } \\
\text { BioG-BHKW }\end{array}$ & $\begin{array}{c}\text { A3.1.1: } \\
\text { ErdG-BHKW }\end{array}$ & $\begin{array}{c}\text { A4.1.1: } \\
\text { BZ-BHKW }\end{array}$ & $\begin{array}{c}\text { A1.1: } \\
\text { Strommix }\end{array}$ \\
\hline
\end{tabular}

Szenario 3 (mit und ohne Förderung):

\begin{tabular}{|c|c|c|c|c|c|c|}
\hline $\begin{array}{l}\text { A5-8.1: } \\
\text { Wind }\end{array}$ & $\begin{array}{l}\text { A5-8.2*: } \\
\text { PV }\end{array}$ & $\begin{array}{c}\text { A5-8.2: } \\
\text { PV }\end{array}$ & $\begin{array}{c}\text { A2.1.1: } \\
\text { BioG-BHKW }\end{array}$ & $\begin{array}{l}\text { A4.1.1: } \\
\text { BZ-BHKW }\end{array}$ & $\rightarrow \quad \begin{array}{c}\text { A3.1.1: } \\
\text { ErdG-BHKW }\end{array}$ & $\begin{array}{c}\text { A1.1: } \\
\text { Strommix }\end{array}$ \\
\hline
\end{tabular}

Abbildung 7.20: Rangfolge der Optionen zur Wärmebereitstellung nach PROMETHEE I

Szenarien 0 bis 2 (mit und ohne Förderung):

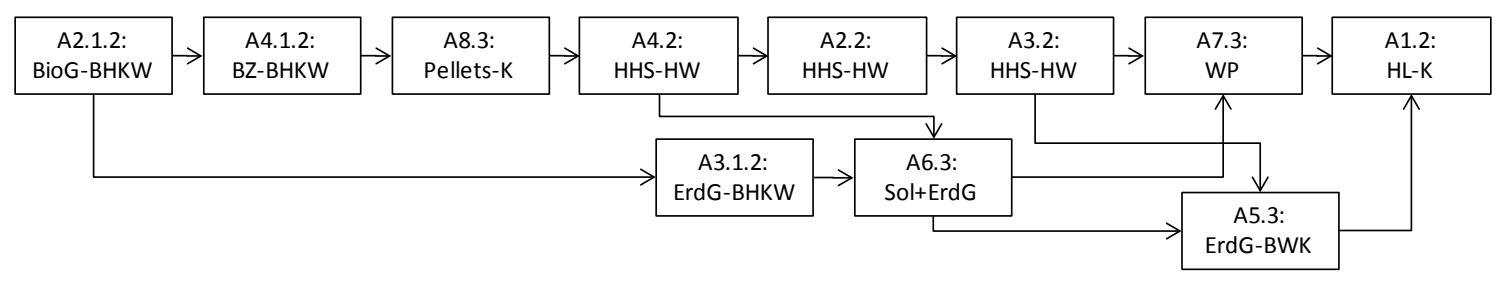

Szenario 3 (mit und ohne Förderung):

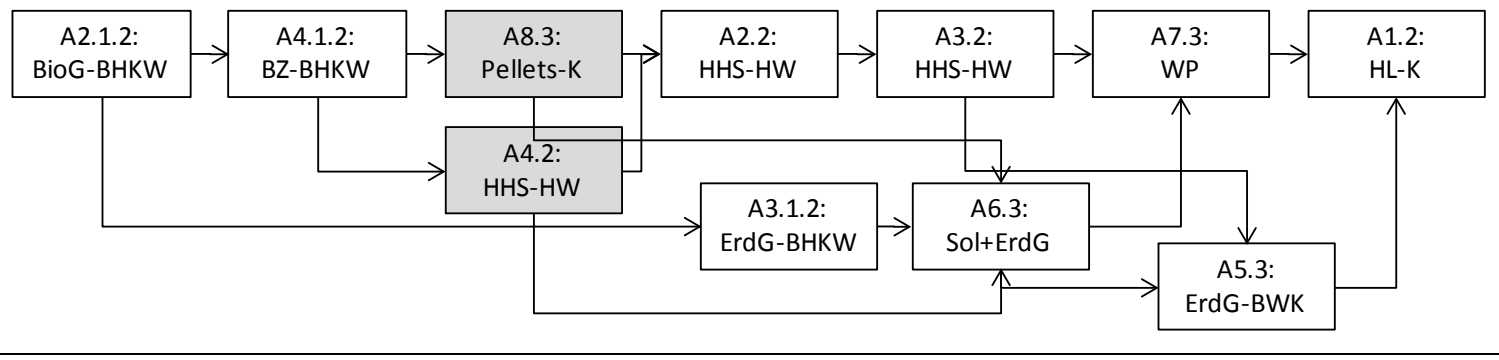

Die Rangfolge der Optionen zur Wärmebereitstellung nach PROMETHEE I und II wird weniger stark als die Optionen zur Stromerzeugung von den Förderbedingungen beeinflusst (vgl. Abbildung 7.20und Abbildung 7.21). Das Biogas-BHKW (A2.1.2) ist unter allen Bedingungen den anderen Alternativen zur Wärmebereitstellung vorzuziehen. Das Brennstoffzellen-BHKW (A4.1.2) wird nach PROMETHEE I besser eingestuft als die Holzpellets-Heizung (A8.3) und diese wiederum besser als das HolzhackschnitzelHeizwerk (A4.2). Die drei zuletzt genannten Alternativen sind nach PROMETHEE I unvergleichbar mit dem Erdgas-BHKW (A3.1.2), dem lediglich das Biogas-BHKW eindeutig vorzuziehen ist. Das Erdgas-BHKW und das Holzhackschnitzel-Heizwerk (bei hoher Auslastung, A4.2) sind wiederum eindeutig der solar unterstützten ErdgasHeizung (A6.3) vorzuziehen. Letztere steht in der Rangfolge vor der Wärmepumpe und dem Erdgas-Brennwertkessel, ist jedoch mit dem Holzhackschnitzel-Heizwerk bei ge- 
ringer Auslastung (A2.2 und A3.2) unvergleichbar. Das Holzhackschnitzel-Heizwerk ist in jedem Fall der Wärmepumpe (A7.3) und dem Erdgas-Brennwertkessel (A5.3) vorzuziehen. Der konventionelle Heizöl-Kessel (A1.2) wird eindeutig schlechter bewertet als alle anderen Alternativen. Im Szenario 3 unterscheidet sich die Rangfolge insofern, dass die Holzpellets-Heizung (A8.3) und das Holzhackschnitzel-Heizwerk mit hoher Auslastung (A4.2) (Abbildung 7.20, unten) unvergleichbar sind.

Die Rangfolge der Optionen zur Wärmebereitstellung nach PROMETHEE II ist in allen Szenarien identisch (Abbildung 7.21). Die KWK-Technologien werden besser eingestuft als alle anderen betrachteten Optionen. Dies könnte auf den zugrunde gelegten exergetischen Allokationsmechanismus zurückgeführt werden, durch den der Wärmebereitstellung im Vergleich zur Stromerzeugung ein wesentlich geringerer Anteil der Kosten bzw. Umweltauswirkungen zugerechnet wird. An erster Stelle steht das BiogasBHKW (A2.1.2), gefolgt von der Brennstoffzelle (A4.1.2) an zweiter und dem ErdgasBHKW (A3.1.2) an dritter Stelle. Auf den folgenden Rangplätzen liegen die Alternativen basierend auf Holzbrennstoffen, wobei die Pellets-Heizung (A8.3) besser als das Holzhackschnitzel-Heizwerk (A4.2, 2.2, 3.2) eingestuft wird. Letzteres wird umso besser bewertet, je höher dessen Auslastung über das Jahr ist. Nach dem HolzhackschnitzelHeizwerk steht in der Rangfolge nach PROMETHEE II die solar unterstützte ErdgasHeizung (A6.2), gefolgt vom Erdgas-Brennwertkessel (A5.3). Auf dem vorletzten Rangplatz liegt die Wärmepumpe (A7.3), gefolgt vom konventionellen Heizöl-Kessel (A1.2) an letzter Stelle.

Abbildung 7.21: Rangfolge der Optionen zur Wärmebereitstellung nach PROMETHEE II (alle Szenarien)

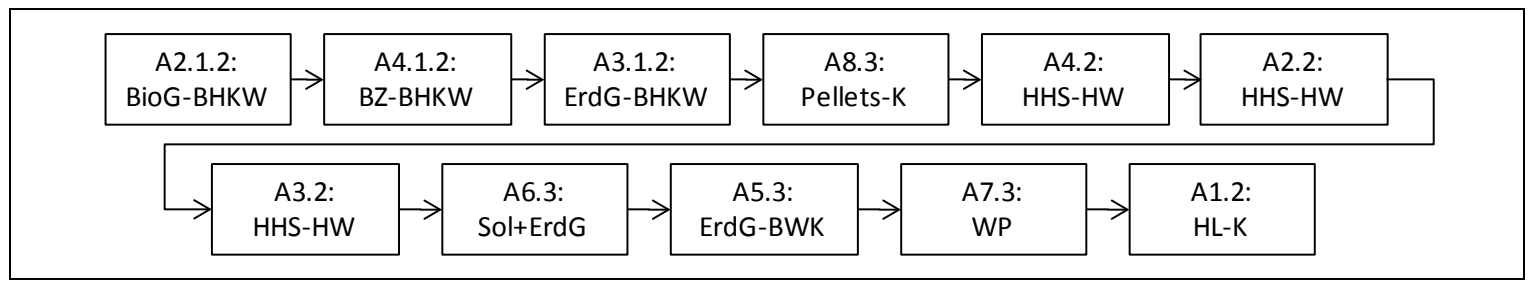

Aus den dargestellten Ergebnissen ist nicht unmittelbar ersichtlich, wie die Gesamtkonzepte zur Strom- und Wärmebereitstellung im Vergleich zueinander zu bewerten sind. Zur Aggregation der Einzelbewertungen je Technologie stehen prinzipiell zwei Vorgehensweisen zur Verfügung. Diese können in Anlehnung an Methoden zur Aggregation in Gruppenentscheidungen unterschieden werden. Einerseits können in Gruppenentscheidungen individuelle Modelle einzelner Entscheider kombiniert und daraus ein Bewertungsergebnis abgeleitet werden. Andererseits können Ergebnisse auf Basis individueller Modelle ermittelt und anschließend aggregiert werden (vgl. Geldermann et al. 2002a; Geldermann et al. 2002b). Analog können entweder einzelne Energietech- 
nologien zunächst zu verschiedenen Gesamtversorgungssystemen kombiniert werden, die dann mit Hilfe multikriterieller Methoden bewertet werden. Oder einzelne Energietechnologien werden zunächst auf Basis multikriterieller Methoden bewertet, und die Ergebnisse der einzelnen Technologien werden anschließend aggregiert (Abbildung 7.22). Implizit wurde erstere Methode zur Bewertung des Strommix angewendet. Im Folgenden wird für diese Arbeit jedoch aus folgenden Gründen letztere Methode gewählt:

- Die Ermittlung von Kriterien-Ausprägungen für Energieversorgungssysteme ist in der Regel aufwändiger im Vergleich zu einzelnen Technologien und zudem weniger leicht nachzuvollziehen.

- Versorgungskonzepte, die sich aus unterschiedlichen Technologien zusammensetzen, können unter Umständen nicht eindeutig einer der Technologiezyklus-Phasen zugeordnet werden.

Abbildung 7.22: Bewertung von Energieversorgungskonzepten

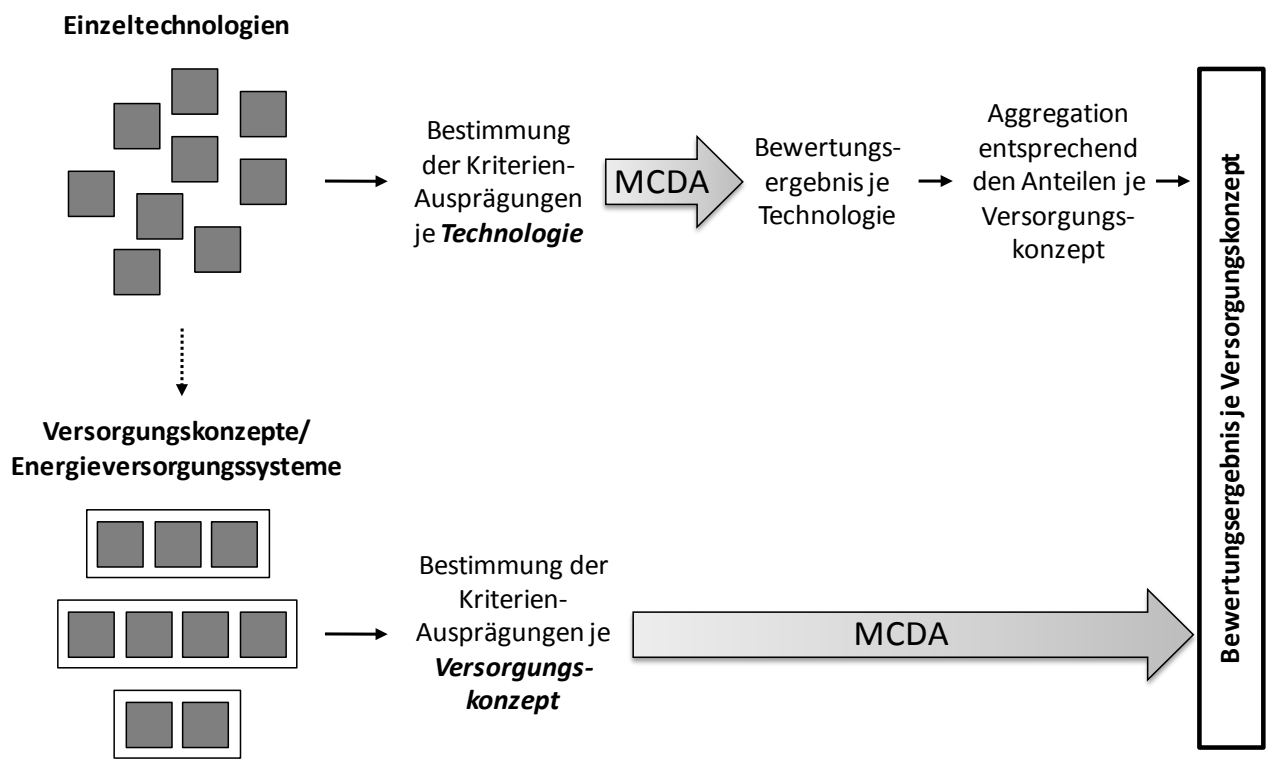

Um die Versorgungskonzepte $\mathrm{A} 1$ bis $\mathrm{A} 8$, die sich teilweise aus mehreren Technologien zusammensetzen, zu bewerten, werden zunächst je Alternative die Teilergebnisse der Technologien zur Strom- bzw. Wärmebereitstellung auf Basis einer gewichteten Summe aggregiert. Die Gewichtungen basieren dabei auf den Anteilen der Technologien an der Strom- bzw. Wärmebereitstellung (vgl. Tabelle 7.13 und Tabelle 7.14). Zur Abschätzung der Anteile der Wind- und PV-Anlage an der Strombereitstellung bei den Alternativen A5, A7 und A8 werden folgende Annahmen getroffen: Es wird eine Windkraftanlage mit $1 \mathrm{MW}_{\mathrm{el}}$ installiert, die über mindestens $1.700 \mathrm{~h} / \mathrm{a}$ läuft. Folglich werden mindestens $1.700 \mathrm{MWh}_{\mathrm{el}} / \mathrm{a}$ erzeugt. 
Tabelle 7.13: Anteile der Technologien am zu bewertenden Versorgungskonzept für die Stromerzeugung

\begin{tabular}{|c|c|c|c|c|c|c|c|}
\hline $\begin{array}{l}\text { Alter- } \\
\text { native }\end{array}$ & Strommix D & $\begin{array}{l}\text { BioG- } \\
\text { BHKW }\end{array}$ & $\begin{array}{l}\text { ErdG- } \\
\text { BHKW }\end{array}$ & BZ-BHKW & Wind & PV & $\mathrm{PV}^{*}$ \\
\hline A1 & $100 \%$ & --- & -- & --- & --- & -- & --- \\
\hline $\mathrm{A} 2$ & --- & $100 \%$ & --- & --- & --- & --- & --- \\
\hline A3 & --- & --- & $100 \%$ & --- & --- & --- & --- \\
\hline A4 & $16 \%$ & --- & --- & $84 \%$ & --- & --- & --- \\
\hline A5/7/8 & $8 \%$ & --- & --- & --- & $85 \%$ & $8 \%$ & --- \\
\hline A5/7/8* & $8 \%$ & --- & --- & --- & $85 \%$ & --- & $8 \%$ \\
\hline A6 & --- & --- & --- & --- & $100 \%$ & --- & --- \\
\hline
\end{tabular}

Tabelle 7.14: $\quad$ Anteile der Technologien am zu bewertenden Versorgungskonzept für die Wärmebereitstellung

\begin{tabular}{|c|c|c|c|c|c|c|c|c|c|}
\hline $\begin{array}{l}\text { Techno- } \\
\text { logie } \\
\text { Alter- } \\
\text { native }\end{array}$ & Heizöl-K & $\begin{array}{l}\text { BioG- } \\
\text { BHKW }\end{array}$ & $\begin{array}{l}\text { ErdG- } \\
\text { BHKW }\end{array}$ & $\begin{array}{c}\text { BZ- } \\
\text { BHKW }\end{array}$ & $\begin{array}{l}\text { HHS- } \\
\text { HW }\end{array}$ & $\begin{array}{l}\text { ErdG- } \\
\text { BWK }\end{array}$ & Sol+ErdG & WP & $\begin{array}{l}\text { Pellets- } \\
\mathrm{K}\end{array}$ \\
\hline A1 & $100 \%$ & --- & --- & --- & -- & --- & --- & --- & -- \\
\hline A2 & --- & $71 \%$ & --- & --- & $29 \%$ & --- & --- & --- & --- \\
\hline A3 & --- & -- & $8 \%$ & -- & $23 \%$ & -- & -- & --- & --- \\
\hline A4 & --- & --- & --- & $29 \%$ & $71 \%$ & --- & --- & --- & --- \\
\hline A5 & --- & --- & --- & --- & --- & $100 \%$ & --- & --- & --- \\
\hline A6 & --- & --- & --- & -- & --- & -- & $100 \%$ & -- & --- \\
\hline A7 & --- & --- & --- & -- & --- & --- & --- & $100 \%$ & --- \\
\hline A8 & --- & --- & --- & --- & --- & --- & --- & --- & $100 \%$ \\
\hline
\end{tabular}

Für PV-Anlagen werden nach Staiß (2007) 7,7 m²/kW an Dachfläche benötigt. Nach IEA (2002) stehen je Einwohner durchschnittlich $9 \mathrm{~m}^{2}$ an Dachfläche zur Verfügung, die für PV-Anlagen genutzt werden können. In Jühnde beteiligten sich an dem Biomasse-Projekt 142 Haushalte (vgl. Ruppert et al. 2008). Wird unterstellt, dass sich die gleiche Anzahl an Haushalten an einem Konzept mit PV-Dachanlagen beteiligen würde, könnte folglich mindestens eine Gesamtleistung von ca. $166 \mathrm{~kW}_{\mathrm{p}}$ installiert werden. Bei 900 Volllaststunden im Jahr entspricht dies einer Stromerzeugungsmenge von ca. $149 \mathrm{MWh}_{\mathrm{e}} / \mathrm{a}$. Zusammen mit der Windkraftanlage können somit über das Jahr ca. 1.849 $\mathrm{MWh}_{\mathrm{e}} / \mathrm{a}$ erzeugt werden. Der Rest des Bedarfs von 2.000 MWhel/a wird über das öffentliche Versorgungsnetz gedeckt. Bei Alternative A6 werden keine PV-Anlagen 
installiert. Stattdessen wird angenommen dass zwei Windkraftanlagen à $1 \mathrm{MW}_{\mathrm{el}}$ mit mindestens 1.700 Betriebsstunden je Jahr installiert werden. Diese erzeugen über das Jahr mehr Strom als in Jühnde benötigt wird.

Nach der Aggregation der Ergebnisse für die Stromerzeugung einerseits und die Wärmebereitstellung andererseits werden die Bewertungsergebnisse der Strom- und Wärmeseite je Alternative addiert. In dieser Weise wird ein Maß für die relativen Stärken und Schwächen des jeweiligen Gesamtkonzeptes zur Strom- und Wärmeversorgung ermittelt. Aus den so ermittelten Kennzahlen können Rangfolgen der Versorgungskonzepte in Abhängigkeit der Szenarien nach PROMETHEE I und II abgeleitet werden. Je nach Szenario sind Unterschiede in den so ermittelten Rangfolgen zu beobachten (vgl. Abbildung 7.23 bis Abbildung 7.25, in denen Änderungen im Vergleich zu den vorherigen Szenarien grau markiert sind). In den Szenarien 0, 2 und 3 (ohne Förderung, Abbildung 7.23) ist die Kombination aus Windkraft, PV-Anlagen und Holzpellets-Kesseln (A8) allen anderen Alternativen außer dem Versorgungskonzept mit Wind und solar unterstützter Erdgas-Heizung (A6) eindeutig vorzuziehen. Im Szenario 1 ist zudem eine Unvergleichbarkeit zwischen der Alternative A8 und dem in Jühnde umgesetzten Biomasse-Konzept (A2) zu beobachten. Letzteres steht in der Rangfolge nach PROMETHEE I eindeutig vor allen anderen Alternativen mit Ausnahme von A8 und A6, unabhängig von den Szenarien. Auf den mittleren Rangplätzen liegen die Versorgungskonzepte basierend auf Wind, PV und Wärmepumpe (A7) sowie basierend auf Wind, PV und Erdgas-Brennwertkessel (A5). Diese beiden Alternativen (A5 und A7) sind in allen Szenarien nach PROMETHEE I miteinander unvergleichbar und in den meisten Fällen dem Versorgungskonzept mit Brennstoffzelle (A4) und demjenigen mit Erdgas-BHKW (A3) vorzuziehen. Nach PROMETHEE I wird als einzige Alternative der betrachtete Referenzfall (A1: Strombezug aus dem öffentlichen Versorgungsnetz und Wärmeversorgung auf Basis konventioneller Heizöl-Kessel) von allen anderen Optionen dominiert. In allen Szenarien (mit und ohne Förderung) ist Alternative A7 (Wind, PV und Wärmepumpe) eindeutig der Alternative A4 (Brennstoffzelle), und die Alternative A5 (Wind, PV und Erdgas-Brennwertkessel) eindeutig der Alternative A3 (Erdgas$B H K W$ ) vorzuziehen. A7 und A4 einerseits sowie A5 und A3 andererseits sind in den meisten Szenarien nach PROMETHEE I miteinander unvergleichbar (Ausnahmen: Szenario 3 mit und ohne Förderung, Szenarien 0 und 1 mit Förderung). 
Abbildung 7.23: Rangfolge der Versorgungskonzepte nach PROMETHEE I ohne Berücksichtigung von Förderbedingungen

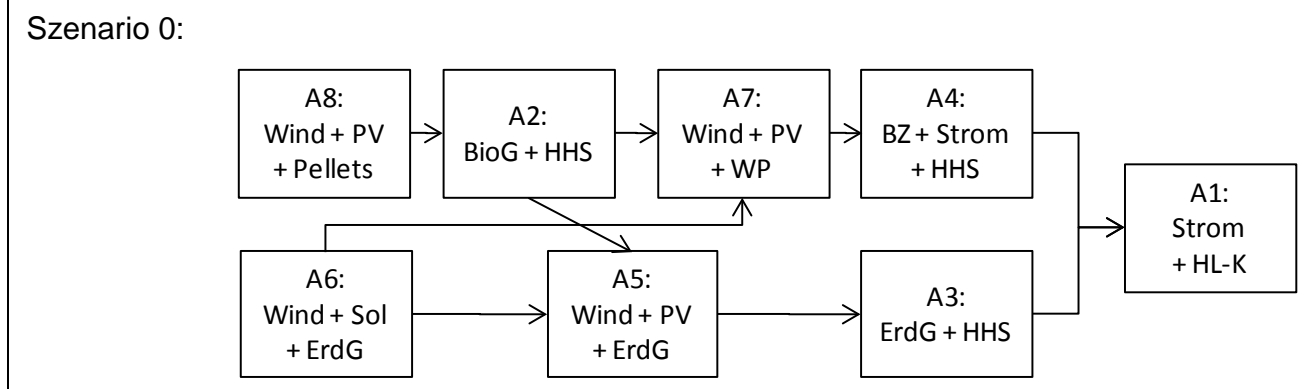

Szenario 1:

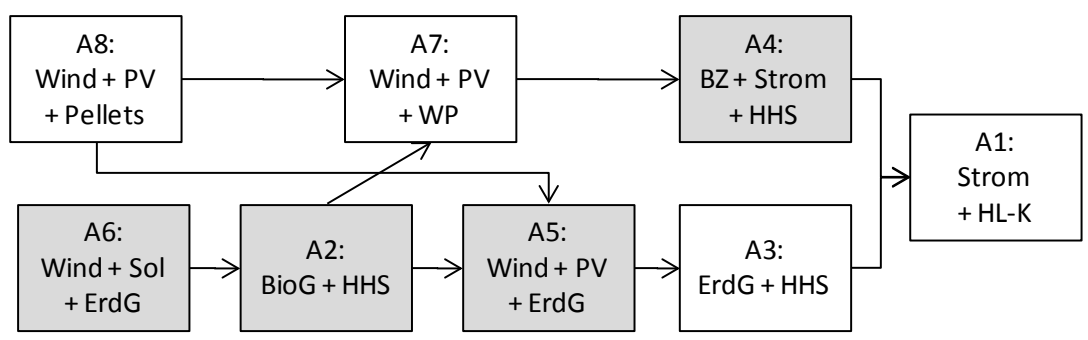

Szenario 2:

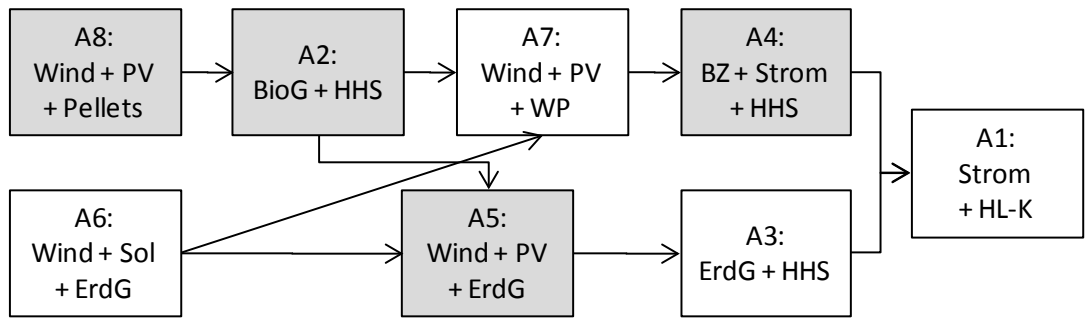

Szenario 3:

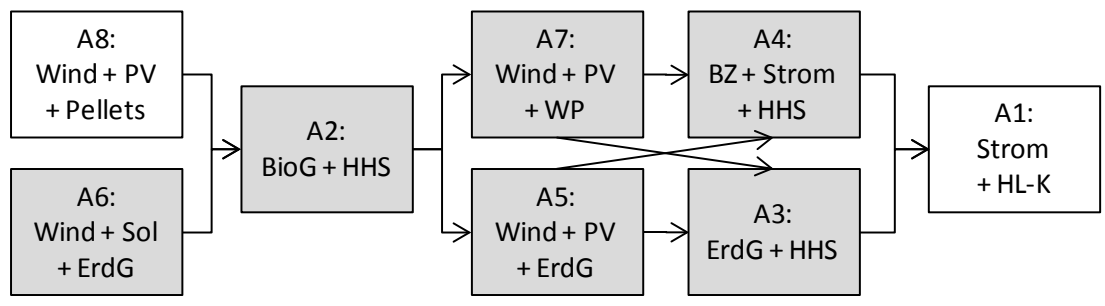


Abbildung 7.24: Rangfolge der Versorgungskonzepte nach PROMETHEE I unter Berücksichtigung von Förderbedingungen

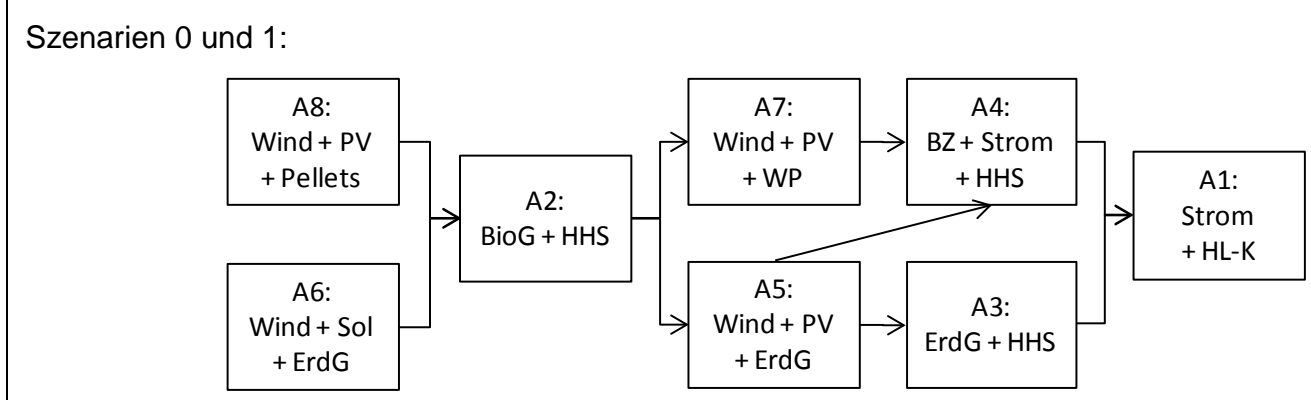

Szenario 2:

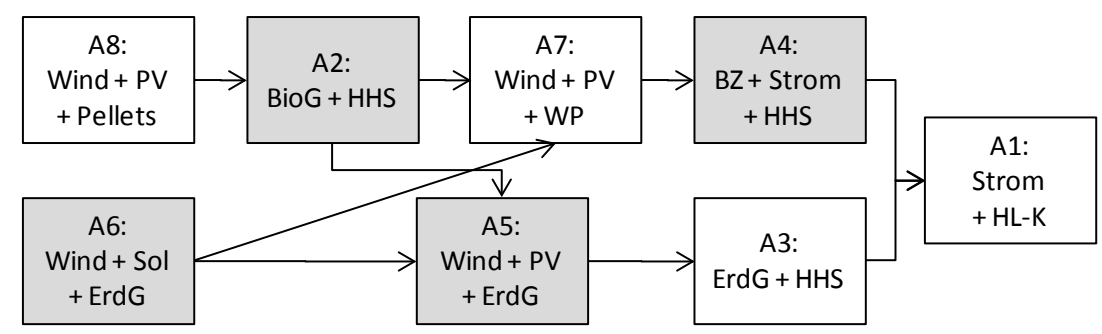

Szenario 3:

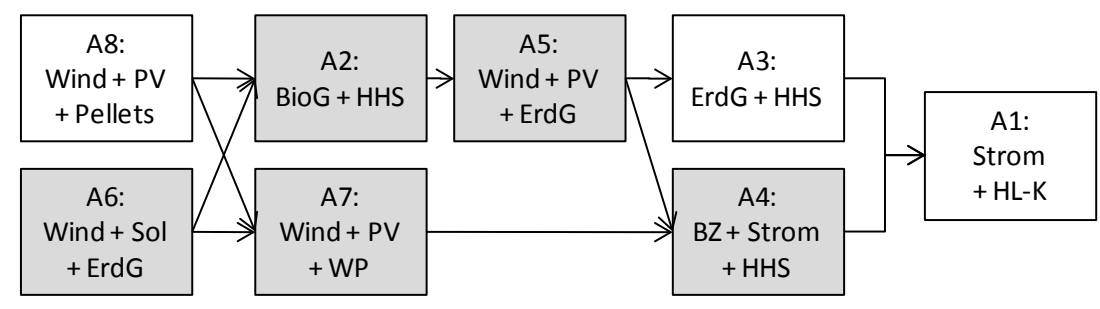

Nach PROMETHEE II schneidet in allen Szenarien die Kombination aus Windkraft, PVAnlagen und Pellets-Heizungen (A8) am besten ab (Abbildung 7.25). Im Szenario 0 (ohne Förderung) sowie im Szenario 2 (mit und ohne Förderung) weist das BiomasseKonzept Jühnde (A2) das zweitbeste Ergebnis auf. In allen anderen Fällen liegt A2 auf dem dritten Rangplatz und das Konzept mit Windkraft, PV-Anlage und solar unterstützter Erdgas-Heizung (A6) auf dem zweiten Rang. An vierter Stelle steht in allen Fällen die Kombination aus Windkraft, PV und Erdgas-Brennwertkessel (A5), gefolgt von dem Konzept mit Windkraft, PV und Wärmepumpe (A7). Den sechsten Rangplatz belegt in den Szenarien 0, 1 und 2 (mit und ohne Förderung) das Versorgungskonzept mit Erdgas-BHKW und Holzhackschnitzel-Heizwerk (A3), gefolgt von der Alternative mit Brennstoffzelle (A4). Die Rangplätze dieser beiden Alternativen sind im Szenario 3 vertauscht. Wie bereits bei der Rangfolge nach PROMETHEE I festgestellt werden konnte, wird die Referenz-Alternative (A1) auch nach PROMETHEE II von allen anderen Optionen dominiert. 
Abbildung 7.25: Rangfolge der Versorgungskonzepte nach PROMETHEE II

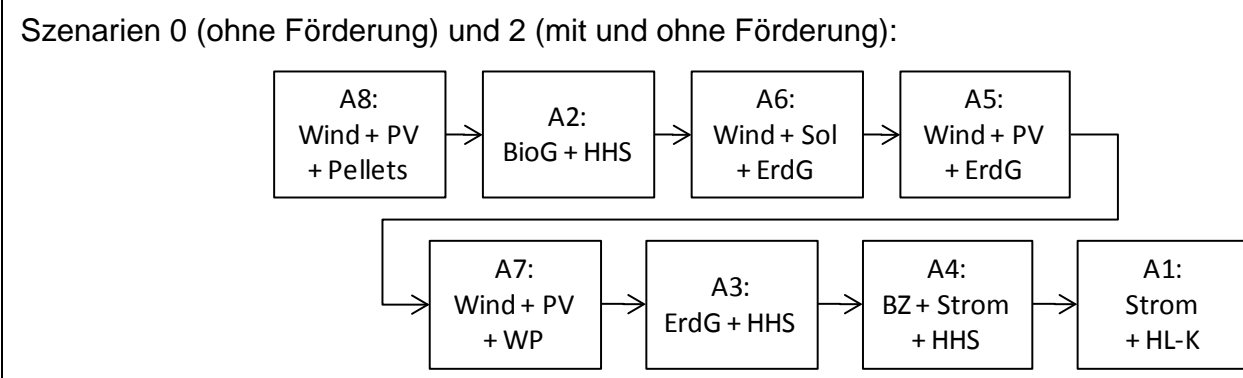

Szenarien 0 (mit Förderung) und 1 (mit und ohne Förderung):

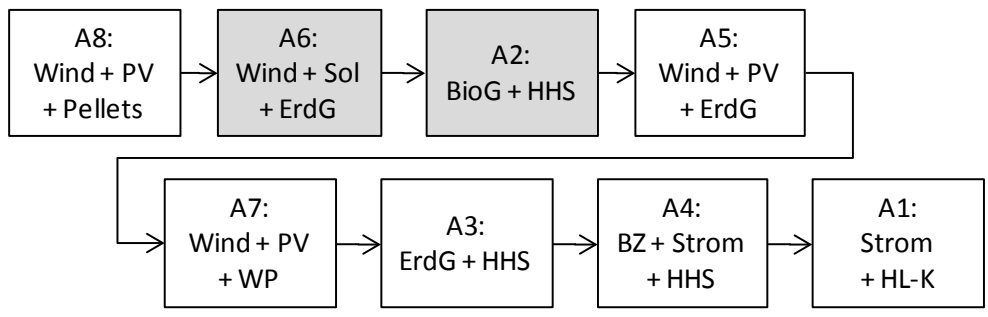

Szenario 3 (mit und ohne Förderung):

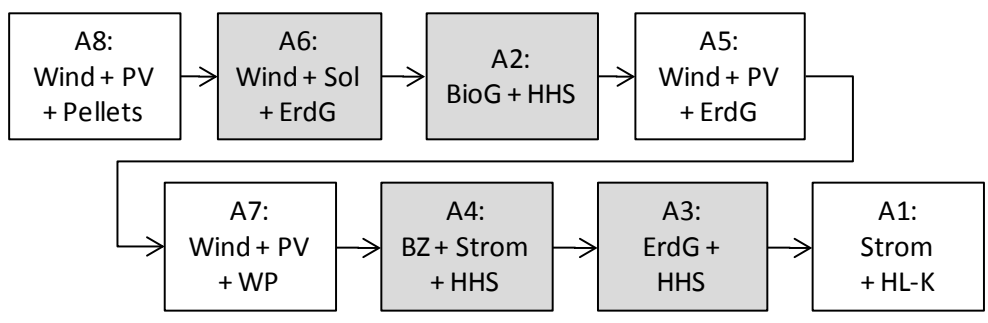

\subsubsection{Sensitivitätsanalyse}

Im Excel-Tool MAESTRO sind folgende Arten der Sensitivitätsanalyse implementiert:

- Die Berechnung von Stabilitätsintervallen unter Beachtung der Technologiezyklusabhängigen Gewichtungen.

- Die grafische Darstellung der Veränderung des Nettoflusses je Alternative, wenn die Gewichtung eines Kriteriums zwischen $0 \%$ und $100 \%$ variiert wird.

- Die Berechnung und Darstellung des Nettoflusses je Alternative, wenn die Zuordnung zu den Technologiezyklus-Phasen geändert wird.

Bei der Bestimmung der Stabilitätsintervalle wird die die Gewichtung jeweils eines Kriterium zwischen $0 \%$ und $100 \%$ variiert, während das Verhältnis aller anderen Kriterien-Gewichtungen zueinander konstant bleibt. Der Netto-Fluss, der sich in Abhängigkeit der so variierenden Gewichtungen ändert, wird als Gerade in einem zweidimensionalen Diagramm dargestellt (x-Achse: Gewichtung des betrachteten Kriteriums, minimal $0 \%$, maximal $100 \%$; y-Achse: Netto-Fluss). Die Schnittpunkte $w^{S}$ der Geraden kenn- 
zeichnen die Grenzen der Stabilitätsintervalle. Für die untere und obere Grenze des Stabilitätsintervalls $\left[w_{\min } ; w_{\max }\right]$, das sich ausgehend von der ursprünglichen Gewichtung $w^{*}$ des betrachteten Kriteriums ergibt, gilt:

$$
\begin{aligned}
& w_{\text {min }}=\max \left\{w^{s} \mid w^{s}<w^{*}\right\} \\
& w_{\text {max }}=\min \left\{w^{s} \mid w^{s}>w^{*}\right\}
\end{aligned}
$$

Bei unterschiedlicher Gewichtung je Technologiezyklus-Phase $T$, ist jedoch der Ausgangspunkt $w^{*}$ nicht zwangsläufig identisch für alle Alternativen. Zudem variiert je nach Phase das Verhältnis der Gewichtungen der anderen Kriterien zueinander. Daher sind zunächst die oberen und unteren Grenzen des Stabilitätsintervalls in Abhängigkeit der Technologiezyklus-Phasen $\left[w_{\min }(T) ; w_{\max }(T)\right] z u$ bestimmen:

$$
\begin{aligned}
& w_{\text {min }}(T)=\max \left\{w^{S} \mid w^{S}<w^{*}(T)\right\} \\
& w_{\text {max }}(T)=\min \left\{w^{S} \mid w^{S}>w^{*}(T)\right\}
\end{aligned}
$$

Die absolute Unter- bzw. Obergrenze des Stabilitätsintervalls kann dann folgendermaßen definiert werden:

$$
\begin{aligned}
& w_{\text {min }}=\max \left\{w_{\text {min }}(T)\right\}=\max \left\{\max \left\{w^{s} \mid w^{s}<w^{*}(T)\right\}\right\} \\
& w_{\text {max }}=\min \left\{w_{\text {max }}(T)\right\}=\min \left\{\min \left\{w^{s} \mid w^{s}>w^{*}(T)\right\}\right\}
\end{aligned}
$$

Die so ermittelten Stabilitätsintervalle sind beispielhaft in Tabelle 7.15 für die Bewertung der Optionen zur Stromerzeugung im Szenario 0 dargestellt. Stabilitätsintervalle für alle weiteren Szenarien sind im Anhang 10.4 zusammengestellt. Zusätzlich sind die jeweils minimalen und maximalen in Abhängigkeit der Technologiezyklus-Phasen angewendeten Gewichtungen dargestellt $\left(w(T)_{\min }\right.$ und $\left.w(T)_{\max }\right)$. Bei Betrachtung der Daten für das Kriterium W1.0 (Spezifische Gesamtkosten) wird deutlich, dass die Gewichtung einiger Technologiezyklus-Phasen außerhalb des so berechneten Stabilitätsintervalls liegt und negative Werte für das Stabilitätsintervall resultieren können. Dieser Sachverhalt kann durch die grafische Darstellung der Sensitivitäten bezüglich der Gewichtung des Kriteriums „Spezifische Gesamtkosten“ verdeutlicht werden (Abbildung 7.26). 
Tabelle 7.15: $\quad$ Stabilitätsintervalle Strom, Szenario 0 (ohne Förderung)

\begin{tabular}{|c|c|c|c|c|c|c|}
\hline & & $\mathbf{w}_{\min }$ & $w(T)_{\min }$ & $w(T)_{\max }$ & $\mathbf{w}_{\max }$ & $\Delta \mathbf{w}$ \\
\hline W1.0 & Spez. Gesamtk. & $37,5 \%$ & $11,8 \%$ & $43,1 \%$ & $19,2 \%$ & $-18,3 \%$ \\
\hline U1 & ADP & $0,7 \%$ & $2,9 \%$ & $5,1 \%$ & $23,0 \%$ & $22,3 \%$ \\
\hline U2 & LAND & $0,0 \%$ & $4,9 \%$ & $8,6 \%$ & $40,1 \%$ & $40,1 \%$ \\
\hline U3 & GWP & $2,1 \%$ & $4,9 \%$ & $8,6 \%$ & $21,7 \%$ & $19,5 \%$ \\
\hline U4 & ODP & $0,0 \%$ & $1,0 \%$ & $1,7 \%$ & $5,7 \%$ & $5,7 \%$ \\
\hline U5 & HTP & $0,0 \%$ & $1,0 \%$ & $1,7 \%$ & $4,0 \%$ & $4,0 \%$ \\
\hline U6.1 & FAETP & $0,0 \%$ & $0,3 \%$ & $0,6 \%$ & $3,8 \%$ & $3,8 \%$ \\
\hline U6.2 & MAETP & $0,0 \%$ & $0,3 \%$ & $0,6 \%$ & $6,0 \%$ & $6,0 \%$ \\
\hline U6.3 & TAETP & $0,0 \%$ & $0,3 \%$ & $0,6 \%$ & $98,3 \%$ & $98,3 \%$ \\
\hline U7 & POCP & $0,0 \%$ & $2,0 \%$ & $3,4 \%$ & $14,1 \%$ & $14,1 \%$ \\
\hline U8 & AP & $1,4 \%$ & $3,9 \%$ & $6,8 \%$ & $7,9 \%$ & $6,6 \%$ \\
\hline U9 & EP & $1,3 \%$ & $3,9 \%$ & $6,8 \%$ & $8,7 \%$ & $7,4 \%$ \\
\hline U10 & KEA & $1,6 \%$ & $3,9 \%$ & $6,8 \%$ & $24,6 \%$ & $23,0 \%$ \\
\hline V1 & Stat. Rw. (Kehrwert) & $0,0 \%$ & $1,5 \%$ & $2,2 \%$ & $12,9 \%$ & $12,9 \%$ \\
\hline V2 & Importquote & $0,0 \%$ & $1,5 \%$ & $2,2 \%$ & $13,3 \%$ & $13,3 \%$ \\
\hline V3 & Lokale Verfügbarkeit & $0,0 \%$ & $1,5 \%$ & $2,2 \%$ & $100,0 \%$ & $100,0 \%$ \\
\hline V4 & Lagermöglichkeit & $0,0 \%$ & $1,5 \%$ & $2,2 \%$ & $5,1 \%$ & $5,1 \%$ \\
\hline V5 & Herfindahl & $0,0 \%$ & $1,5 \%$ & $2,2 \%$ & $42,5 \%$ & $42,5 \%$ \\
\hline V6 & Risiko-Indikator (Kehrwert) & $0,0 \%$ & $1,5 \%$ & $2,2 \%$ & $100,0 \%$ & $100,0 \%$ \\
\hline V7 & Variationskoeff. Preise & $0,0 \%$ & $1,5 \%$ & $2,2 \%$ & $36,8 \%$ & $36,8 \%$ \\
\hline V8 & Nutzungsgrad & $0,0 \%$ & $1,5 \%$ & $2,2 \%$ & $7,2 \%$ & $7,2 \%$ \\
\hline V9 & Anlagenverfügbarkeit & $0,0 \%$ & $1,5 \%$ & $2,2 \%$ & $6,5 \%$ & $6,5 \%$ \\
\hline S1 & Flex. Innov. & $0,0 \%$ & $1,4 \%$ & $1,7 \%$ & $30,1 \%$ & $30,1 \%$ \\
\hline S2 & Abfallinfr. & $0,0 \%$ & $1,7 \%$ & $2,0 \%$ & $69,7 \%$ & $69,7 \%$ \\
\hline S3 & Konflikte & $0,0 \%$ & $1,8 \%$ & $2,2 \%$ & $36,5 \%$ & $36,5 \%$ \\
\hline S5 & Gesundheit & $0,0 \%$ & $1,8 \%$ & $2,1 \%$ & $76,6 \%$ & $76,6 \%$ \\
\hline S6 & Risiken & $0,0 \%$ & $2,4 \%$ & $3,0 \%$ & $38,1 \%$ & $38,1 \%$ \\
\hline S7 & Katastrophen & $0,0 \%$ & $1,8 \%$ & $2,2 \%$ & $42,5 \%$ & $42,5 \%$ \\
\hline S9 & Ästhetik & $0,0 \%$ & $2,1 \%$ & $2,6 \%$ & $17,4 \%$ & $17,4 \%$ \\
\hline S10 & Soziale Akzeptanz & $0,0 \%$ & $0,7 \%$ & $0,9 \%$ & $47,9 \%$ & $47,9 \%$ \\
\hline
\end{tabular}

Anmerkungen: Die Grauabstufungen in der Spalte „ $\Delta \mathrm{w}^{\prime \prime}$ signalisieren besonders große (weiß bzw. hellgrau), mittelgroße (mittleres grau) und besonders kleine oder negative (dunkelgrau) Stabilitätsintervalle. Negative Stabilitätsintervalle sind zudem durch fett kursive Schrift hervorgehoben. 
Abbildung 7.26: Besonderheiten der Sensitivitätsanalyse bei Anwendung des modifizierten PROMETHEE-Ansatzes

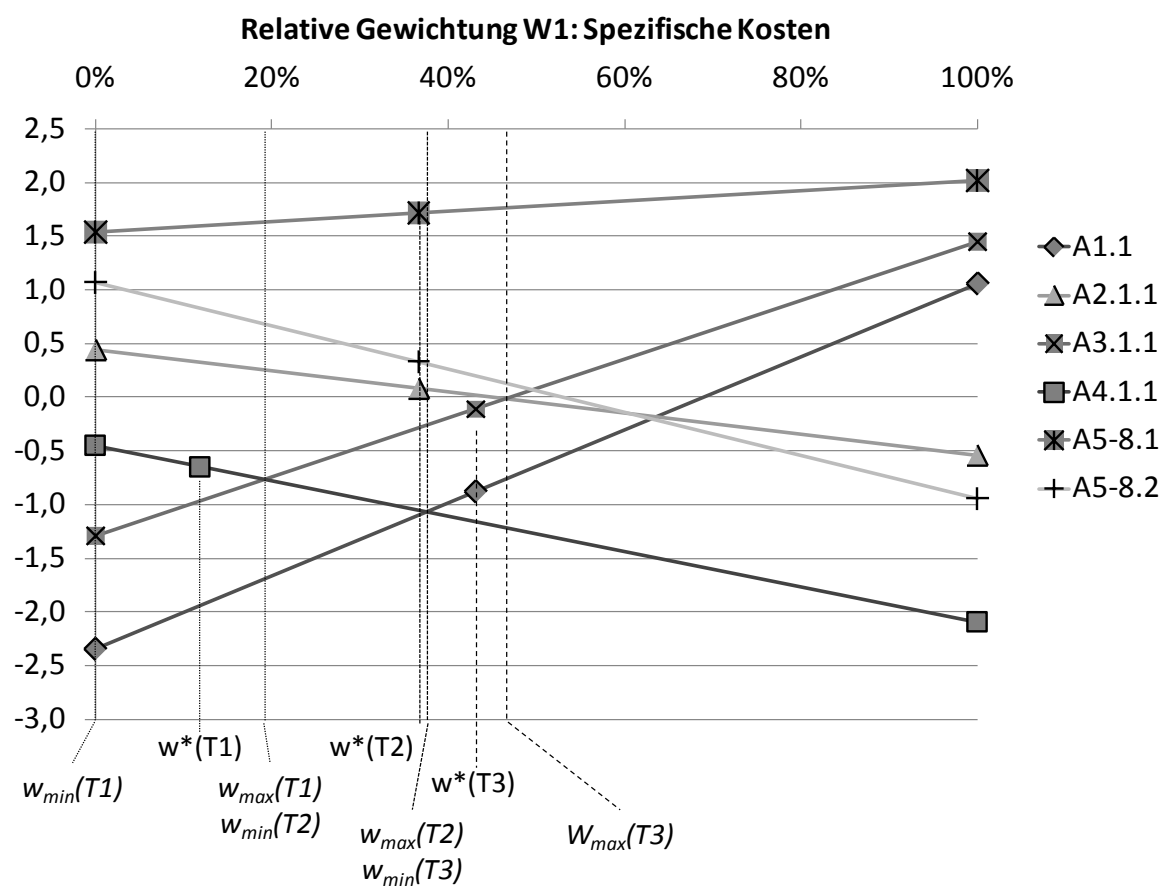

Bei den betrachteten Alternativen zur Stromerzeugung sind drei Entwicklungsphasen voneinander zu unterscheiden, die aus praktischen Gründen an dieser Stelle mit T1, T2 und T3 bezeichnet werden. Die Gewichtung des Kriteriums in Phase T1 liegt bei etwa $11,8 \%$, für T2 bei 36,6 \% und T3 bei 43,1\%. Ausgehend von der Gewichtung nach T1 liegt die obere bzw. untere Grenze des Stabilitätsintervalls bei $0 \%$ bzw. 19,2\%. Die obere Grenze dieses Stabilitätsintervalls stellt zugleich die minimale Obergrenze über alle Phasen dar und entspricht somit in Tabelle $7.15 \mathrm{w}_{\max }$. Dieser Wert liegt jedoch unterhalb der Gewichtung der anderen beiden Phasen. Folglich liegen die Gewichtungen der Phasen T2 und T3 außerhalb des Stabilitätsintervalls. Für T2 liegt die obere bzw. untere Grenze des Stabilitätsintervalls bei $19,2 \%\left(w_{\min }(T 2)=w_{\max }(T 1)\right)$ bzw. 37,5\%. Die Obergrenze dieses Intervalls ist zugleich die Untergrenze des Sensitivitätsintervalls ausgehend von der Gewichtung für T3 $\left(w_{\min }(T 3)=w_{\max }(T 2)\right)$. Diese entspricht zudem der maximalen Untergrenze über alle ermittelten Stabilitätsintervalle und ist somit in Tabelle 7.15 für $w_{\text {min }}$ eingetragen. Da dieser Wert höher liegt im Vergleich zur ermittelten minimalen Obergrenze $\left(w_{\max }=w_{\max }(T 1)\right)$ resultiert ein negativer Wert für das Stabilitätsintervall. Um die Sensitivitätsanalyse in solchen Fällen interpretieren zu können, ist daher eine Betrachtung der Grenzwerte in Abhängigkeit der Technologiezyklus-Phasen und deren grafische Darstellung (wie etwa in Abbildung 7.26) erforderlich. Die entsprechenden Daten werden im Excel-Tool MAESTRO bereit gestellt. Zudem kann die Änderung der Ergebnisse (insbesondere des Netto-Flusses) 
in Abhängigkeit der spezifischen Gewichtungen je Technologiezyklus-Phase dargestellt werden (Abbildung 7.27). Diese Darstellungsform verdeutlicht zudem die Unterschiede im Vergleich zur Anwendung von für alle Alternativen identischen Gewichtungen. Darüber hinaus wird dadurch die Veränderung der Ergebnisse bei der gleichzeitigen Variation der Gewichtungen mehrerer Kriterien veranschaulicht.

Abbildung 7.27: Ergebnisse der Optionen zur Stromerzeugung in Abhängigkeit unterschiedlicher Technologiezyklus-Phasen (Szenario 0, ohne Förderung)

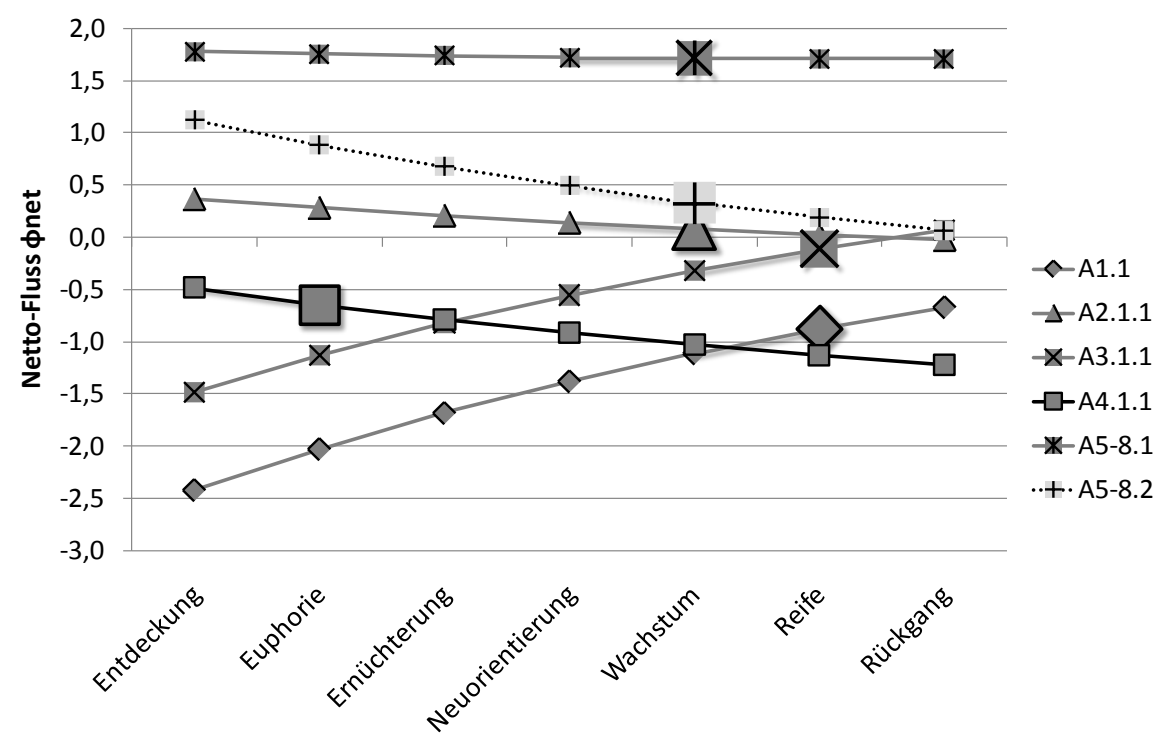

In Abbildung 7.27 ist für die Optionen zur Stromerzeugung der Netto-Fluss in Abhängigkeit der Technologiephasen-spezifischen Gewichtungen dargestellt. Vergrößerte Symbole markieren die jeweils aktuell zutreffende Phase einer Technologie. Zu betonen ist an dieser Stelle, dass durch diese Darstellungsform nicht die technologische Weiterentwicklung über die Zeit abgebildet wird. Vielmehr bleiben die Kriterien-Ausprägungen konstant, während je nach Phasen-Zuordnung unterschiedliche KriterienGewichtungen angewendet werden. Eine vergleichende Betrachtung der vergrößerten Symbole verdeutlicht die Rangfolge nach PROMETHEE II, die sich bei Gewichtung entsprechend der Technologiezyklus-Phase ergibt und auch in Abbildung 7.19 (oben) dargestellt ist. Werden hingegen die Ergebnisse verglichen, die sich bei identischer Gewichtung der Kriterien für alle Alternativen ergeben, werden die Unterschiede der hier vorgeschlagenen Methode im Vergleich zum herkömmlichen PROMETHEE deutlich.

Der erste Rangplatz für die Windkraftanlage (A5-8.1) bleibt über alle betrachteten Gewichtungssets stabil. Bei Gewichtung entsprechende der Phasen Entdeckung, Eupho- 
rie und Ernüchterung ergibt sich als Rangfolge: $A 5.8 .1$ (Wind) $\rightarrow$ A5.8.2 (PV) $\rightarrow$ A2.1.1 $($ BioG-BHKW) $\rightarrow$ A4.1.1 (BZ-BHKW) $\rightarrow$ A3.1.1 (ErdG-BHKW) $\rightarrow$ A1.1 (Strommix). In dieser Rangfolge wird (im Gegensatz zum ursprünglichen Ergebnis, Abbildung 7.19) das Brennstoffzellen-BHKW (A4.1.1) besser bewertet als das Erdgas-BHKW (A3.1.1). Bei Gewichtung entsprechend den Phasen Neuorientierung und Wachstum für alle Alternativen stimmt die Rangfolge mit den Ergebnissen basierend auf den Gewichtungen in Abhängigkeit der zutreffenden Technologiezyklus-Phasen überein. Werden alle Technologien basierend auf Gewichtungen entsprechend der Reife-Phase bewertet, wird hingegen der Strommix (A1.1) besser als das Brennstoffzellen-BHKW (A4.1.1) bewertet. Bei Anwendung der Gewichtungen, die für die Rückgangs-Phase zugrunde gelegt werden, für alle Alternativen, wird zudem das Erdgas-BHKW (A3.1.1) besser bewertet als PV (A5-8.2) und das Biogas-BHKW (A2.1.1). Somit wird deutlich, dass sich die Ergebnisse bei unterschiedlicher relativer Gewichtung mehrere Kriterien unterscheiden und dass eine Gewichtung in Abhängigkeit von Technologiezyklus-Phasen zu anderen Ergebnissen führen kann als eine über alle Technologien identische Gewichtung.

Abbildung 7.28: Ergebnisse der Optionen zur Wärmebereitstellung in Abhängigkeit unterschiedlicher Technologiezyklus-Phasen (Szenario 0, ohne Förderung)

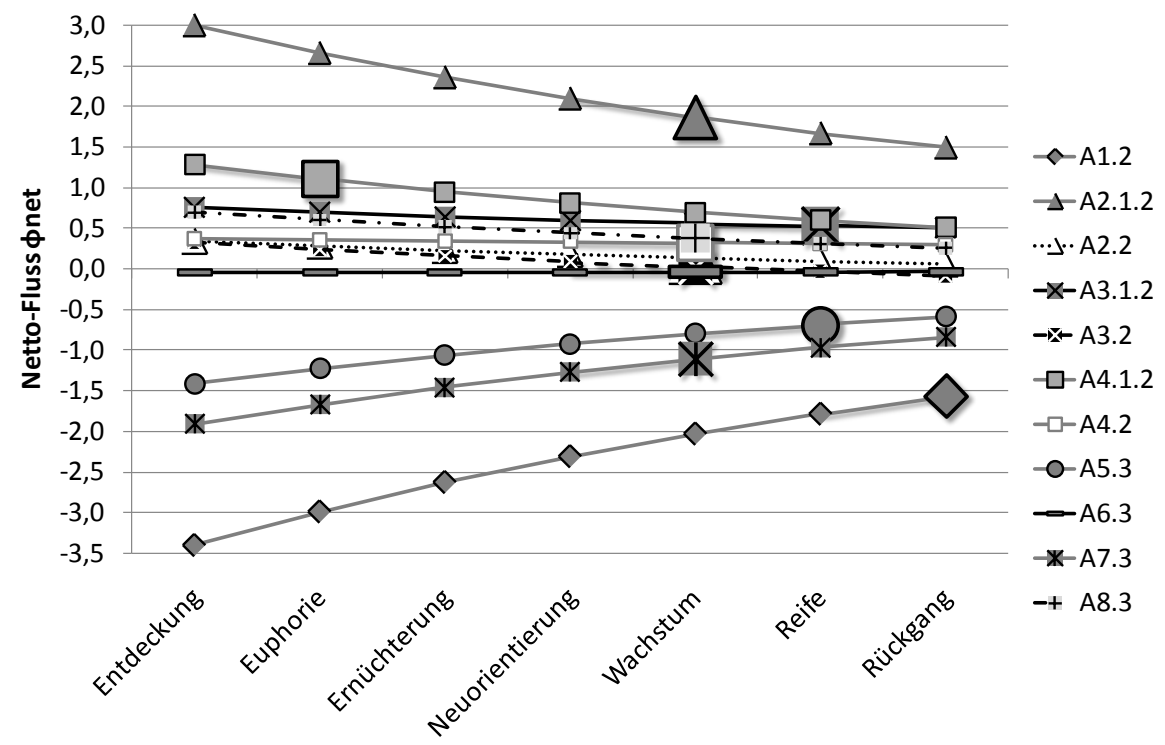

Auch bei Betrachtung der Ergebnisse der Optionen zur Wärmebereitstellung in Abhängigkeit der Phasen-spezifischen Gewichtungssets wird deutlich, dass der erste Rangplatz (A2.1.2: Biogas-BHKW) über den gesamten Bereich konstant bleibt (Abbildung 7.28). Ebenso bleiben die drei letzten Rangplätze konstant (A5.3: ErdgasBrennwertkessel $\rightarrow$ A7.3: Wärmepumpe $\rightarrow$ A1.2: Heizöl-Kessel). In der Detailansicht 
(Abbildung 7.29) wird deutlich, dass auch der zweite Rangplatz des BrennstoffzellenBHKW (A4.1.2) und der dritte Rangplatz des Erdgas-BHKW (A3.1.2) für alle betrachteten Gewichtungssets konstant bleiben. An vierter Stelle steht der Pellets-Kessel (A8.3), der lediglich bei Gewichtung entsprechend der Rückgangs-Phase schlechter abschneidet als das Holzhackschnitzel-Heizwerk mit hoher Auslastung (A4.2). Letzteres zeigt wiederum über alle betrachteten Gewichtungs-Sets ein besseres Ergebnis als die übrigen Alternativen. Je geringer die Auslastung des Holzhackschnitzel-Heizwerkes ist, desto schlechter schneidet dieses ab, was an der über alle zugrunde gelegten Gewichtungs-Sets stabilen Rangfolge von A4.2 $\rightarrow$ A2.2 $\rightarrow$ A3.2 deutlich wird. Die solar unterstützte Erdgas-Heizung (A6.3) wird nur bei Gewichtung entsprechend der RückgangsPhase besser bewertet als das Holzhackschnitzel-Heizwerk mit geringer Auslastung. Insgesamt ist die Rangfolge der Technologien zur Wärmebereitstellung über alle betrachteten Gewichtungs-Sets in Abhängigkeit der Technologiezyklus-Phasen relativ stabil.

Abbildung 7.29: Ergebnisse der Optionen zur Wärmebereitstellung in Abhängigkeit unterschiedlicher Technologiezyklus-Phasen (Detailansicht)

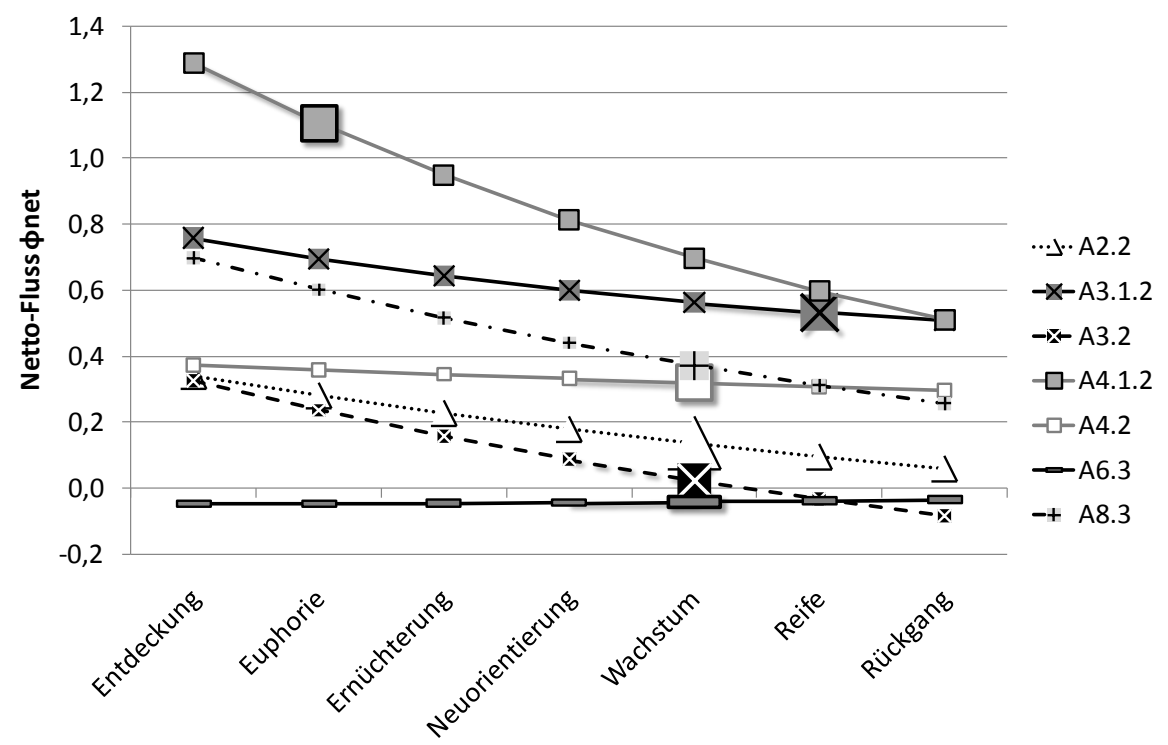

Aufgrund der Fülle an Ergebnissen (30 Kriterien in vier Szenarien, weiterhin unterschieden in Strom und Wärme sowie ohne und mit Berücksichtigung von Fördermitteln) beschränkt sich die weitere Sensitivitätsanalyse auf diejenigen Fälle, in denen Stabilitätsintervalle mit einer Breite von weniger als $3 \%$ ermittelt wurden. Die Stabilitätsintervalle für sämtliche Kriterien in allen Szenarien, die als Ausgangspunkt zur Auswahl der hier betrachteten Sensitivitäten dienen, sind tabellarisch im Anhang 10.4 dargestellt. 
Abbildung 7.30: Sensitivitätsanalyse hinsichtlich der Gewichtung des Kriteriums „Treibhauspotenzial“ (GWP, U3) für Optionen zur Stromerzeugung (Szenario 1, mit Förderung)

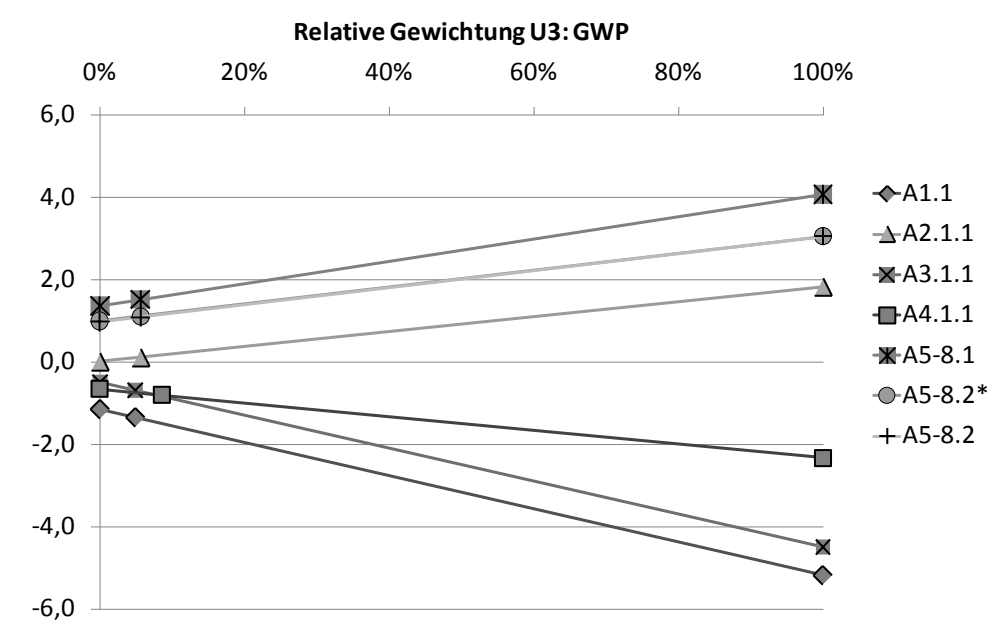

Bei Variation der Gewichtung des Kriteriums Treibhauseffekt (GWP, U3, vgl. Abbildung 7.30) im Szenario 1 (mit Förderung) bleibt die Rangfolge der Alternativen A5-8.1 (Windkraftanlage), der PV-Anlage (A5-8.2) und des Biogas-BHKW (A2.1.1) über den gesamten Gewichtungsbereich stabil. Dabei werden diese Alternativen insgesamt umso besser bewertet, je höher die Gewichtung dieses Kriteriums gewählt wird, sofern die Gewichtung aller anderen Kriterien konstant bleibt. Auch der letzte Rangplatz des Strommix (A1.1) bleibt in diesem Fall über den gesamten Gewichtungsbereich bestehen. Änderungen resultieren lediglich hinsichtlich des Brennstoffzellen-BHKW (A4.1.1) und des Erdgas-BHKW (A3.1.1). Dabei schneidet das Brennstoffzellen-BHKW ab einer Gewichtung des GWP von über $11 \%$ besser ab als das Erdgas-BHKW.

Ähnlich dem Kriterium GWP schneiden die erneuerbaren Alternativen zur Stromerzeugung A5-8.1 (Windkraftanlage), A5-8.2 (PV-Anlage) und A2.1.1 (Biogas-BHKW) umso besser ab, je höher das Kriterium „Kumulierter Energieaufwand“ (KEA, U10) gewichtet wird. Ab einer Gewichtung von über $65,9 \%$ ist hierbei das Biogas-BHKW insgesamt besser zu bewerten als die PV-Anlage. Die Gesamtbewertung der anderen Alternativen sinkt hingegen mit zunehmender Gewichtung des KEA. Für Gewichtungen von mehr als 11,9\% ist das Brennstoffzellen-BHKW (A4.1.1) dem Erdgas-BHKW vorzuziehen. 
Abbildung 7.31: Sensitivitätsanalyse hinsichtlich der Gewichtung des Kriteriums „Kumulierter Energieaufwand“ (KEA, U10) für Optionen zur Stromerzeugung (Szenario 1, mit Förderung)

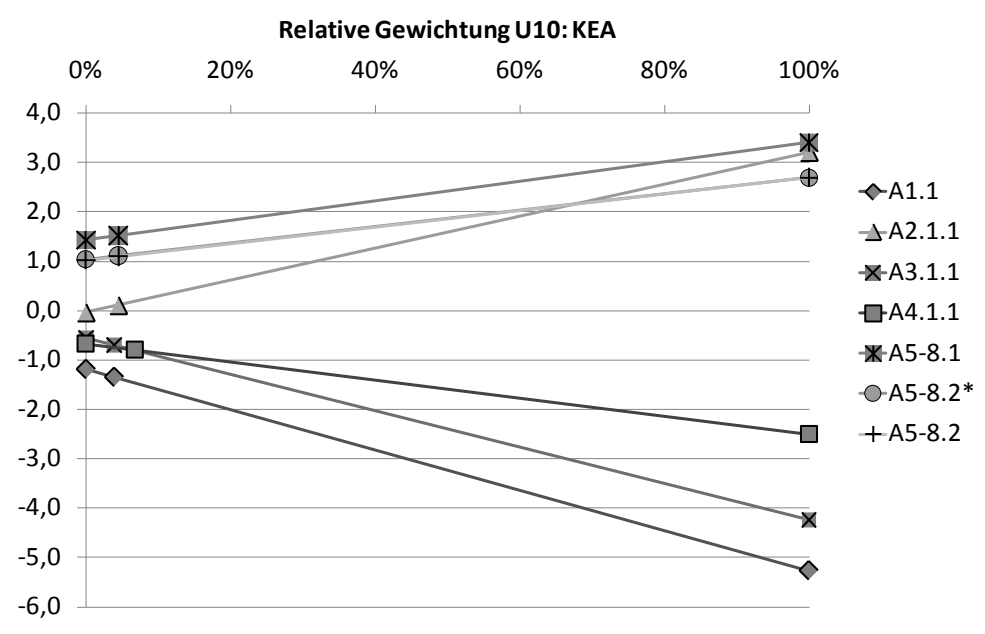

Bei Variation der Gewichtung des Kriteriums „Landschaftsinanspruchnahme“ (LAND, U2) zur Bewertung der Optionen zur Wärmebereitstellung ist eine auffallende Konzentration der Bewertungsergebnisse mit zunehmender Gewichtung zu beobachten (Abbildung 7.32). Dies ist darauf zurückzuführen, dass sich die Ergebnisse in dieser Wirkungskategorie für bestimmte Gruppen von Alternativen stark unterscheiden. Dabei werden die Alternativen auf Basis von Holzbrennstoffen hinsichtlich Landschaftsinanspruchnahme am schlechtesten bewertet mit $\phi^{\text {net }}=-8$ für A2.2, 3.2 und 4.2 (Holzhackschnitzel-Heizwerk) und $\phi^{\text {net }}=-4$ für A8.3 (Holzpellets-Heizung) bei einer Gewichtung $\mathrm{w}(\mathrm{U} 2)=100 \%$. Alle anderen Alternativen werden hinsichtlich Landschaftsinanspruchnahme wesentlich besser bewertet. Daher wird bei ausschließlicher Betrachtung dieses Kriteriums die relative Vorteilhaftigkeit der anderen Alternativen im Vergleich zu den auf Holzbrennstoffen basierenden Technologien auf dem gleichen Niveau eingestuft. Das kleinste Stabilitätsintervall wird für die Alternativen in der Aufstiegs- und Reifephase ermittelt (A2.1.2 bis A3.2; A6.3 bis A8.3), mit einem unteren Grenzwert von $5,2 \%$ und einem oberen Grenzwert von $6,9 \%$.

Da es sich bei dem Kriterium „Lokale Verfügbarkeit“ (V3) um eine einfaches Kriterium (ja =1, nein = 0; Präferenzfunktion Typ 1) handelt, konzentrieren sich bei ausschließlicher Berücksichtigung dieses Kriteriums ( $w=100 \%$ ) die Gesamtbewertungen auf zwei Punkte (Abbildung 7.33). Diejenigen Alternativen, deren Energieträger lokal verfügbar ist werden bei $w(V 3)=10 \%$ mit $\phi^{\text {net }}=6$, diejenigen die nicht lokal verfügbar sind mit $\phi^{\text {net }}=-5$ bewertet. Die im Fallbeispiel zugrunde gelegte Gewichtung dieses Kriteriums ist relativ gering und liegt für alle Phasen des Technologiezyklus innerhalb der Grenzen des Stabilitätsintervalls von 1,3\% und 3,2 \%. 
Abbildung 7.32: Sensitivitätsanalyse hinsichtlich der Gewichtung des Kriteriums "Landschaftsinanspruchnahme" (LAND, U2) für Optionen zur Wärmebereitstellung (Szenario 0, ohne Förderung)

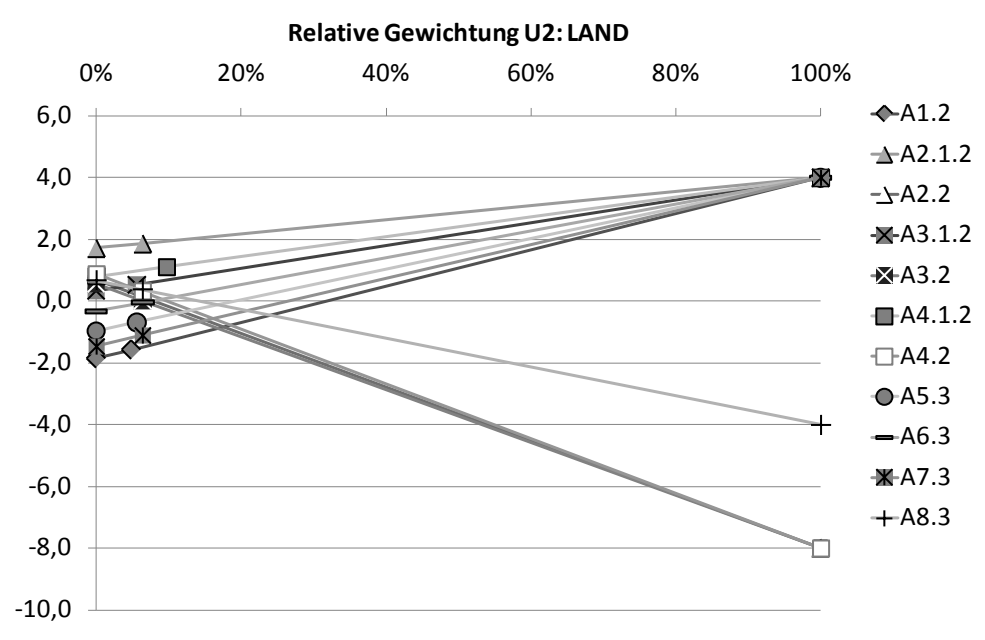

Abbildung 7.33: Sensitivitätsanalyse hinsichtlich der Gewichtung des Kriteriums „Lokale Verfügbarkeit" (V3) für Optionen zur Wärmebereitstellung (Szenario 0, ohne Förderung)

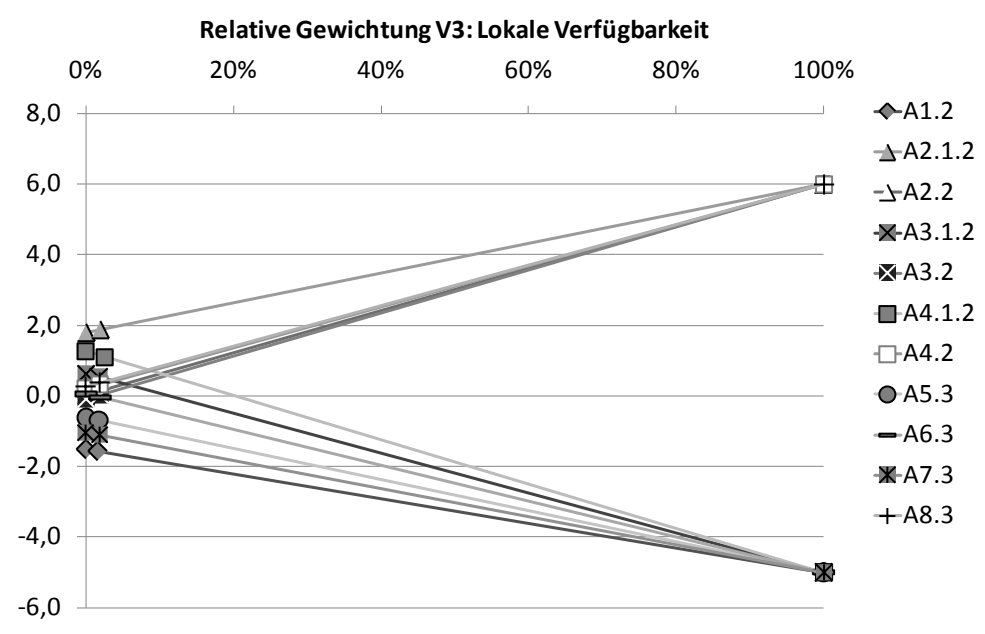

Das Kriterium „Herfindahl-Index“ (V5) dient zur Bewertung der Risiken hinsichtlich der Versorgungssicherheit aufgrund der Konzentration der Förderung (Abbildung 7.34). Am geringsten wird das Ergebnis der Alternative A1.2 (Heizöl-Kessel) von der Gewichtung dieses Kriteriums beeinflusst. Die Bewertung der auf Erdgas basierenden Alternativen (A3.1.2: Erdgas-BHKW, A4.1.2: Brennstoffzelle, A5.3: Erdgas-Brennwertkessel und A6.3: solar unterstützte Erdgas-Heizung) wird umso schlechter, je höher die Konzentration der Förderung gewichtet wird. Für die auf erneuerbaren Energieträgern basierenden Technologien wird das Ergebnis umso besser, je höher die Konzentration der Förderung gewichtet wird. Das Stabilitätsintervall liegt zwischen 1,2 \% und 3,5\%, wo- 
bei die Gewichtungen aller Technologiezyklus-Phasen innerhalb dieser Grenzen liegen.

Abbildung 7.34: Sensitivitätsanalyse hinsichtlich der Gewichtung des Kriteriums „Herfindahl-Index“ (Konzentration der Förderung, V5) für Optionen zur Wärmebereitstellung (Szenario 0, ohne Förderung)

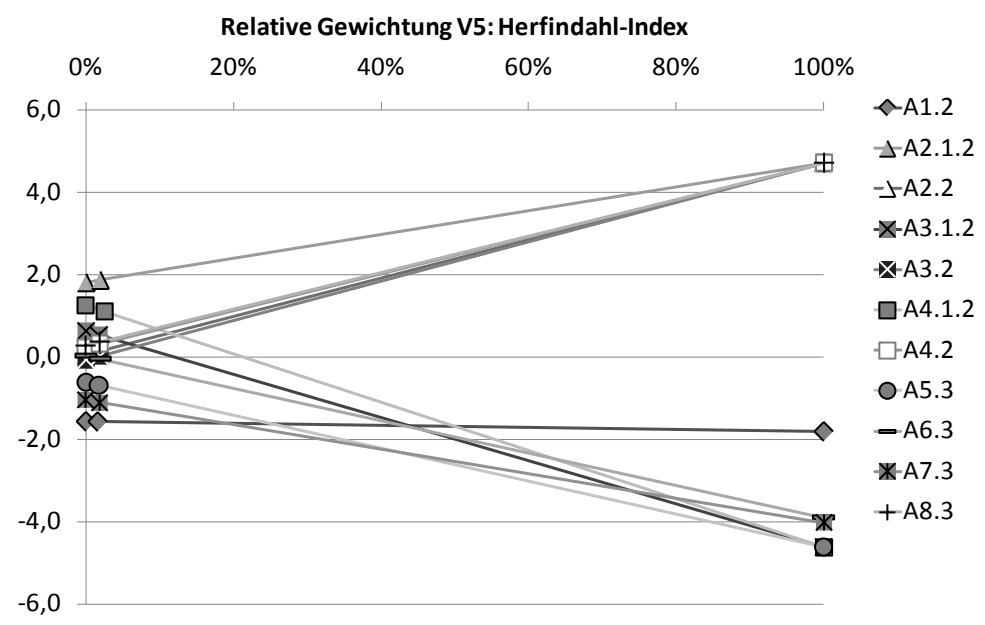

Abbildung 7.35: Sensitivitätsanalyse hinsichtlich der Gewichtung des Kriteriums „Importquote“ (V2) für Optionen zur Wärmebereitstellung (Szenario 0 , mit Förderung)

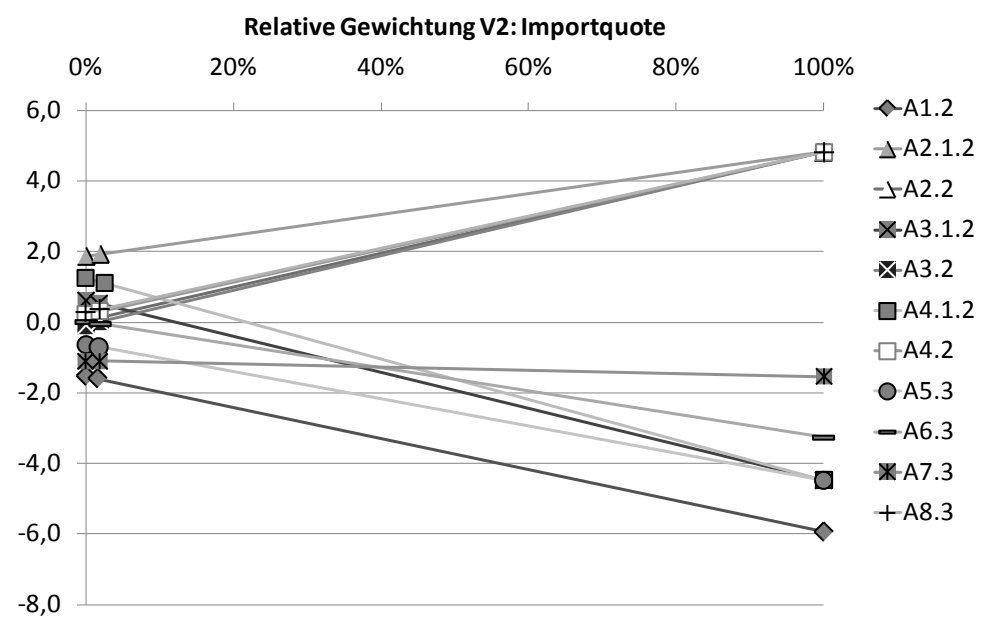

Die unter Grenze des Stabilitätsintervalls für die Gewichtung des Kriteriums „Importquote" (V2) liegt bei 1,2\% und die obere Grenze bei 3,5 \% (Abbildung 7.35). Je höher dieses Kriterium gewichtet wird, desto besser werden wie zu erwarten die Technologien basierend auf erneuerbaren Energieträgern bewertet. Ab einer Gewichtung von ca. $14 \%$ werden generell die erneuerbaren Energien besser eingestuft. Am geringsten wird die Gesamtbewertung der Wärmepumpe (A7.3) von der Gewichtung dieses Krite- 
riums beeinflusst. Der erste und der letzte Rangplatz (A2.1.2: Biogas-BHKW und A1.2: Heizöl-Kessel) bleibt über den gesamten Gewichtungsbereich stabil. Die Erdgasbasierten Technologien schneiden umso schlechter ab, je höher die „Importquote" als Kriterium gewichtet wird.

Das Kriterium „Eutrophierung“ (EP, U9) zeigt einen relativ starken Einfluss auf die Gesamtbewertung der Alternativen zur Wärmeerzeugung und die darauf basierende Rangfolge nach PROMETHEE II (Abbildung 7.36). Die Gewichtungen dieses Kriteriums in Abhängigkeit der Technologiezyklus-Phasen liegen zwischen 3,9 \% und 7,8 \%, während das kleinste ermittelte Stabilitätsintervall zwischen den Grenzwerten 3,2\% und 5,8\% liegt. Folglich liegt die Gewichtung der Euphorie-Phase von 7,8\%, die für das Brennstoffzellen-BHKW (A4.1.2) angewendet wird, außerhalb dieses Intervalls. Das Gesamtergebnis verschlechtert sich für die auf Holzbrennstoffen basierenden Alternativen (A2.2, 3.2, 4.2 und A8.3) mit zunehmender Gewichtung dieses Kriteriums. Ab Gewichtungen von ca. $28 \%$ und mehr ist das Holzhackschnitzel-Heizwerk am schlechtesten bewertet. Die Holzpellets-Heizung belegt den vorletzten Rangplatz ab Gewichtungen der Eutrophierung von ca. $44 \%$ und darüber. Für Technologien basierend auf anderen erneuerbaren Energieträgern sowie fossil- und Strom-basierte Alternativen wird das Bewertungsergebnis umso besser je höher die Eutrophierung gewichtet wir. Ab einer Gewichtung von über 30,5\% übertrifft die Bewertung der Brennstoffzelle (A4.1.2) alle anderen Alternativen. Bei noch höheren Gewichtungen schneiden zudem der Erdgas-Brennwertkessel (A5.3), die solar unterstützte Erdgas-Heizung (A6.3) sowie das Erdgas-BHKW (A3.1.2) besser ab als das Biogas-BHKW (A2.1.2).

Abbildung 7.36: Sensitivitätsanalyse hinsichtlich der Gewichtung des Kriteriums „Eutrophierung“ (EP, U9) für Optionen zur Wärmebereitstellung (Szenario 0, ohne Förderung)

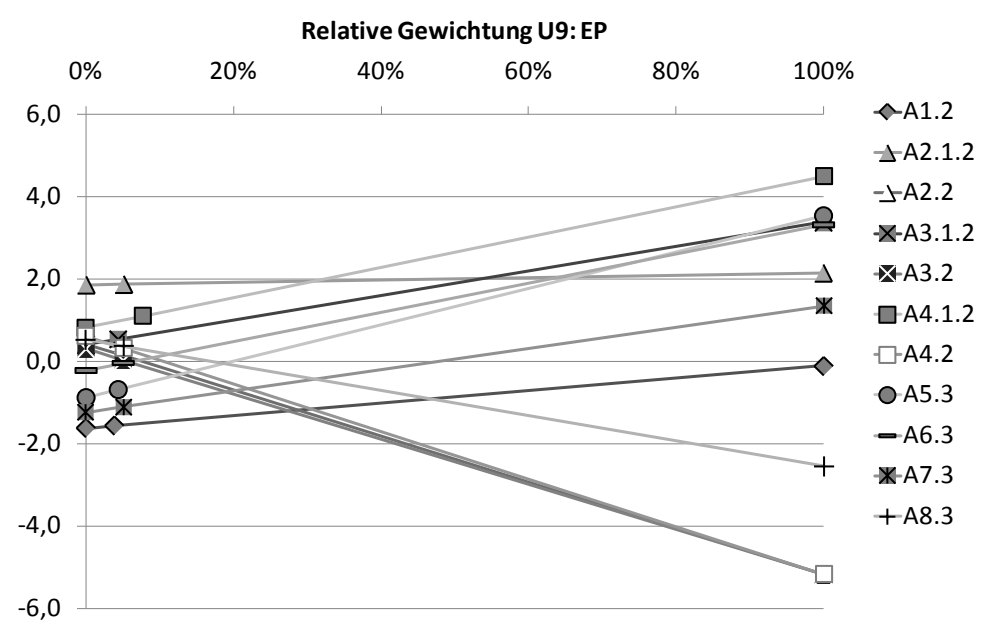


Das Kriterium „Süßwasser-Ökotoxizität“ (FAETP, U6.1) wird in allen Technologiezyklus-Phasen nur sehr gering mit weniger als $1 \%$ gewichtet (Abbildung 7.37). Die untere Grenze des Stabilitätsintervalls liegt bei $0 \%$, die obere Grenze bei 2,8 \%. Eine Erhöhung der Gewichtung dieses Kriteriums wirkt sich insbesondere auf das Gesamtergebnis der solar unterstützen Erdgas-Heizung (A6.3) und der Wärmepumpe (A7.3) sowie in geringerem Maße auf die Holzpellets-Heizung (A8.3) negativ aus. Die anderen Alternativen sind tendenziell umso besser zu bewerten, je höher die Süßwasser-Ökotoxizität gewichtet wird. Da in Zusammenhang mit der Wirkungsabschätzung dieser Kategorie im Rahmen der Ökobilanzierung noch zahlreiche Unsicherheiten bestehen, sollten Bewertungen auf Basis dieses Kriteriums jedoch mit Vorsicht interpretiert werden.

Abbildung 7.37: Sensitivitätsanalyse hinsichtlich der Gewichtung des Kriteriums „Süßwasser-Ökotoxizität" (FAETP, U6.1) für Optionen zur Wärmebereitstellung (Szenario 3, mit Förderung)

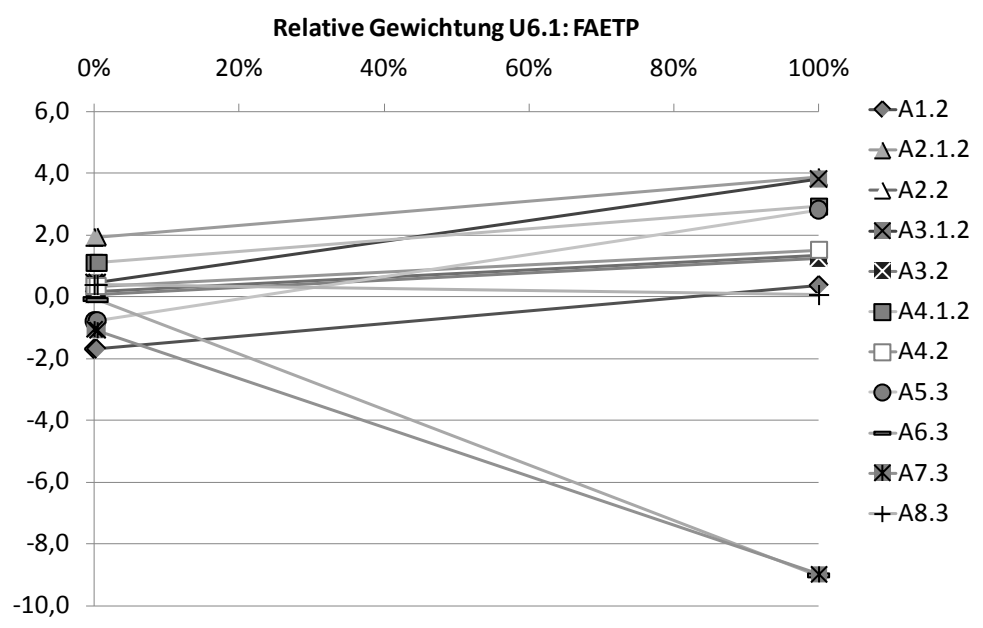

Die Sensitivitätsanalyse zeigt, dass bereits kleine Änderungen in den Gewichtungen der Kriterien Änderungen in den Rangfolgen nach PROMETHEE II hervorrufen können. Andererseits bleibt das Ergebnis der „am besten“ bewerteten Technologien (Windkraftanlagen zur Stromerzeugung und Biogas-BHKW zur Wärmebereitstellung) in vielen Fällen relativ stabil. Für zukünftige konkrete Anwendungsfälle muss insbesondere bei nur kleinen Stabilitätsintervallen sorgfältig abgewogen werden, inwiefern die Entscheidungsträger dazu bereit sind Kompromisse zwischen einzelnen Bewertungskriterien und-dimensionen einzugehen. 


\subsubsection{Diskussion}

Da die dargestellten Ergebnisse nicht auf den „wahren“ Präferenzen der Entscheidungsträger beruhen, sollten daraus keine direkten Handlungsempfehlungen abgeleitet werden. Dennoch können grundsätzliche Schlussfolgerungen sowohl hinsichtlich der Anwendung der vorgeschlagenen Methodik als auch hinsichtlich der Bewertung der betrachteten Optionen zur Strom- und Wärmeerzeugung gezogen werden. Zum einen wurde die prinzipielle Anwendbarkeit des EUS MAESTRO zur Bewertung von Alternativen der Strom- und Wärmebereitstellung aufgezeigt. Bei der Durchführung der Fallstudie zeigt sich, dass die Anwendung des Excel-Tools mit einer variablen Anzahl an Kriterien und Alternativen unproblematisch ist. Herausforderungen liegen vielmehr in der adäquaten Vorauswahl der Alternativen und Kriterien sowie in der Bestimmung der Kriterien-Ausprägungen. Insbesondere die Bestimmung der Kriterien-Ausprägungen erweist sich trotz der in der MTB-Datenbank zur Verfügung stehenden Basisdaten als sehr aufwändig, da die spezifischen Rahmenbedingungen der betrachteten Versorgungsaufgabe nicht außer Acht gelassen werden können. Eine weitgehende Standardisierung der Basisdaten ist wünschenswert, wird jedoch auch für zukünftige Anwendungsfälle aufgrund der Vielzahl möglicher Rahmenbedingungen und technologischer Varianten nicht uneingeschränkt möglich sein. Zudem erfordern fortlaufende technologische Weiterentwicklungen sowie sich ändernde Rahmenbedingungen eine regelmäßige Aktualisierung der Technologie- und Rahmendaten, was mit einem erheblichen Zeitaufwand verbunden ist.

Stehen die benötigten Daten in geeigneter Form bereit, kann mit dem Excel-Tool MAESTRO die Aggregation und Auswertung in effizienter Weise durchgeführt werden, mit oder ohne die Berücksichtigung von Gewichtungen in Abhängigkeit der Technologiezyklus-Phasen. Dabei erweist sich das Java-Tool Web-HIPRE als nützlich, um vorab das Entscheidungsproblem hierarchisch zu strukturieren und auf Basis der entworfenen Problemstruktur Kriterien-Gewichtungen abzuleiten. Der Einsatz von WebHIPRE in Verbindung mit MAESTRO ist jedoch nicht zwingend erforderlich. Auch andere Methoden und Tools können zur Bestimmung der Gewichtungen für die Analyse im Excel-Tool MAESTRO angewendet werden. Flexible Variationen der Input-Daten wie etwa im Fallbeispiel unterschiedlicher Kostendaten in Abhängigkeit von Szenarien, können ohne großen Aufwand mit Hilfe des Excel-Tools analysiert werden. Aufgrund der Anwendung von Gewichtungen in Abhängigkeit der Technologiezyklus-Phasen wird die Sensitivitätsanalyse zwar erschwert. Andererseits entsteht dem Anwender durch die Implementierung der Berechnung von Stabilitätsintervallen im Excel-Tool, die ausgehend von den sich je Phase unterscheidenden Kriterien-Gewichtungen ermittelt werden, kein zusätzlicher Aufwand. Eine grafische Darstellung der Änderung des Netto-Flusses in Abhängigkeit der Gewichtung einzelner Kriterien zur Visualisierung der 
entsprechenden Stabilitätsintervalle ist im Excel-Tool möglich. Zudem können die Ergebnisse basierend auf unterschiedlichen Gewichtungs-Sets je TechnologiezyklusPhase verglichen werden. Dabei weichen Ergebnisse basierend auf Gewichtungen, die den aktuellen Technologiezyklus-Phasen der Alternativen angepasst sind, von Ergebnissen basierend auf identischen Gewichtungs-Sets für alle Alternativen ohne Berücksichtigung unterschiedlicher Entwicklungsphasen ab.

Hinsichtlich der Ergebnisse, die auf Basis eigener Annahmen zu den Kriterien-Gewichtungen berechnet wurden, kann hervorgehoben werden, dass die auf erneuerbaren Energieträgern basierenden Technologien in den meisten Fällen besser abschneiden als fossil befeuerte Alternativen. Insbesondere die Kombination von Windkraft- und PVAnlagen mit Holzpellets-Heizungen oder solar unterstützen Erdgas-Heizungen sowie das in Jühnde bereits umgesetzte Biomasse-Konzept sind unter den meisten Bedingungen bei den zugrunde gelegten Kriterien-Gewichtungen zu bevorzugen. Die Beibehaltung des Status Quo, das heißt der Strombezug aus dem öffentlichen Netz und Heizungssysteme mit konventionellen Heizöl-Kesseln, scheint hingegen keine langfristig tragfähige Option zu sein. Die Sensitivitätsanalyse zeigt, dass bereits kleine Änderungen in den Gewichtungen zu einer anderen Rangfolge der Alternativen führen können. Daher ist eine transparente Darstellung der zugrunde liegenden Annahmen und Abhängigkeiten von wesentlicher Bedeutung. Dabei besteht das Ziel insbesondere darin, den Entscheidungsträgern zu veranschaulichen, welchen Einfluss unterschiedliche Kriterien-Gewichtungen auf das Endergebnis ausüben. Dies wird durch die im ExcelTool implementierten Funktionen unterstützt und kann durch zusätzliche grafische Darstellungsformen der Ergebnisse ergänzt werden.

Für zukünftige Anwendungen sollte eine möglichst weitgehende Einbeziehung der betroffenen Akteure in den Entscheidungsprozess angestrebt werden, insbesondere im Rahmen der Vorauswahl und Gewichtung der zu berücksichtigenden Kriterien. Herausforderungen werden hierbei insbesondere darin gesehen, den Akteuren, die nicht mit Methoden der multikriteriellen Entscheidungsunterstützung vertraut sind, die angewendeten Methoden nahezubringen, und diese zu motivieren, aktiv am Entscheidungsprozess teilzunehmen.

\subsection{Vergleich von Stromspeichertechnologien}

Nachdem der vorherige Anwendungsfalls „Bioenergiedörfer“ ausführlich dargestellt wurde, um die Funktionalitäten des EUS im Detail zu erläutern, wird mit dem folgenden Fallbeispiel eine kompaktere Auswertung zur Bewertung von Stromspeichertechnologien vorgestellt. Das Thema der Speicherung von Elektrizität hat in den letzten Jahren an Bedeutung gewonnen. Aufgrund des fortschreitenden Ausbaus regenerativer Ener- 
giequellen fallen Stromangebot und -nachfrage in zeitlicher wie räumlicher Hinsicht in zunehmendem Maße auseinander. Folglich sind verstärkte Anstrengungen hinsichtlich Netzmanagement und Systemausgleich erforderlich. Dabei können skalierbare, dezentrale Speichertechnologien zur zeitlichen Anpassung von Angebot und Nachfrage beitragen und die Flexibilität im Netzbetrieb erhöhen. Die hierzu benötigten Speicherkapazitäten liegen im Bereich mehrerer hundert Kilowatt bis hin zu einigen Megawatt. Wünschenswert ist eine möglichst verlustfreie Speicherung über mehrere Stunden bis hin zu einigen Wochen. Mit konventionellen Stromspeichertechnologien, wie etwa BleiSäure-Batterien, können höhere Leistungen nur durch Serienschaltung mehrerer Einheiten erreicht werden. Folglich steigen die Kosten proportional zur Erhöhung der Leistung. Andere Technologien, wie zum Beispiel Schwungräder, sind aufgrund permanenter Energieverluste nur in kleinen Anwendungen mit kurzen Speicherzeiten einsetzbar. Das zusätzliche Potenzial zum Ausbau konventioneller Pumpspeicherkraftwerke ist vor allem durch geografische Gegebenheiten stark begrenzt. Folglich besteht Bedarf für eine Weiterentwicklung von Speichertechnologien, welche die geforderten Funktionen erfüllen können. Vor diesem Hintergrund werden im vorliegenden Fallbeispiel drei innovative Stromspeichertechnologien untersucht (vgl. auch Oberschmidt und Klobasa 2009):

A1. Eine Vanadium-Redox-Flow-Batterie (VRFB): Als Speichermedium dient ein Elektrolyt, der in einem externen Tank gespeichert ist und bei Bedarf zur Be- bzw. Entladung durch die elektrochemische Zelle gepumpt wird.

A2. Ein adiabates Druckluftspeichersystem (TACAS - Thermal and Compressed Air Storage): Luft wird komprimiert, gespeichert und in einer Gasturbine zur Stromerzeugung eingesetzt. Die bei der Kompression entstehende Wärme wird in einem thermischen Speicher zwischengespeichert und zur Erwärmung der Luft vor der Gasturbine eingesetzt, um eine Zusatzfeuerung zu vermeiden.

A3. Ein PEM-Elektrolyseur (Proton Exchange Membrane) zur Erzeugung von Wasserstoff mit anschließender Speicherung in Druckbehältern und Rückverstromung in einem Gasmotor.

Diese Speichertechnologien zeichnen sich zum einen dadurch aus, dass Leistung und Speichervolumen unabhängig voneinander skalierbar sind, und können zum anderen weitgehend Standort-unabhängig betrieben werden. Im Rahmen eines Forschungsprojektes (Dötsch et al. 2008) wurden Patent- und Bibliometrieanalysen sowie Analysen der Akteursarena für diese Technologien und deren Komponenten durchgeführt. Auf dieser Grundlage werden die Technologien den Phasen im Technologiezyklus nach Meyer-Krahmer und Dreher (2004) zugeordnet (vgl. auch Abbildung 5.3):

- die VRFB der Phase der Euphorie; 
- das TACAS-System der Neuorientierung oder der Aufstiegsphase;

- das PEM-System der Phase der Ernüchterung.

Ausgehend von diesen Betrachtungen werden im Folgenden die drei Speichertechnologien auf Basis des EUS MAESTRO einander gegenübergestellt.

\subsubsection{Vorbereitung der Entscheidungsmatrix}

Die zugrunde gelegten Basisdaten der untersuchten Stromspeichertechnologien sind in Tabelle 7.16 zusammengefasst. In Zusammenarbeit mit Experten wurden eine Kriterien-Hierarchie zur Bewertung von Speichertechnologien erarbeitet (Abbildung 7.38) und die Kriterien-Ausprägungen ermittelt. 20 Im Rahmen einer Befragung wurden Gewichtungen dieser Kriterien mit Hilfe von Web-HIPRE auf Basis des AHP entsprechend der Präferenzen einzelner Experten ermittelt. Die Befragung bezog sich jedoch nicht auf Unterschiede in der relativen Bedeutung der Kriterien in Abhängigkeit der Technologiezyklus-Phasen. Daher werden für das vorliegende Fallbeispiel folgende Annahmen getroffen, um die Gewichtungen in Abhängigkeit der Phasen abzuleiten (Abbildung 7.39):

- Die Bedeutung der Wirtschaftlichkeit und des Risikopotenzials nimmt mit fortschreitendem Entwicklungsstadium, das heißt mit zunehmender Marktdurchdringung, zu. Die minimale Gewichtung aus den individuellen Befragungen wird für die Phase der Entdeckung, die maximale Gewichtung für die Rückgangs-Phase zugrunde gelegt. Dazwischen steigen die relativen Gewichtungen dieser Kriterien linear an.

- Die Bedeutung des zukünftigen Entwicklungspotenzials nimmt mit fortschreitendem Entwicklungsstadium ab. Die maximale Gewichtung aus den individuellen Befragungen wird für die Phase der Entdeckung, die minimale Gewichtung für die Rückgangs-Phase zugrunde gelegt. Dazwischen nimmt die relative Gewichtung dieses Kriteriums linear $a b$.

- Die Relationen der Gewichtungen der übrigen Kriterien werden über den gesamten Technologiezyklus als konstant angenommen und aus den Mittelwerten der Ergebnisse der Expertenbefragung bestimmt (vgl. Abbildung 7.40).

20 Aus Gründen der Vertraulichkeit können an dieser Stelle nicht die detaillierten Daten zu den einzelnen, in Entwicklung befindlichen Speichertechnologien offengelegt werden. Basisdaten der Speichertechnologien sind in Tabelle 7.16 zusammengefasst. 
Tabelle 7.16: Basisdaten der Speichertechnologien

\begin{tabular}{|c|c|c|c|}
\hline & VRFB & TACAS & PEM \\
\hline Technische Spezifikationen & $\begin{array}{l}\text { Redoxpaar (Elektrolyt) } \\
\mathrm{V}^{4+} / \mathrm{N}^{5+} \text { und } \mathrm{V}^{3+} \mathrm{V}^{2+} \\
\text { Polystyrensulfonsäure- } \\
\text { Membran (Nafion) } \\
\text { Graphit-Elektroden }\end{array}$ & $\begin{array}{l}\text { Speicherung der kom- } \\
\text { primierten Luft in Stahl- } \\
\text { druckrohrbündeln } \\
\text { Druckloser Wärmespei- } \\
\text { cher mit Thermoöl }\end{array}$ & $\begin{array}{l}\text { PEM-Elektrolyse und } \\
\text { Speicherung bei } 30 \\
\text { bar } \\
\text { Gasmotor zur Rück- } \\
\text { verstromung }\end{array}$ \\
\hline Größe & $2 \mathrm{MW}, 20 \mathrm{MWh}$ & $2 \mathrm{MW}, 20 \mathrm{MWh}$ & $2 \mathrm{MW}, 20 \mathrm{MWh}$ \\
\hline Gesamtwirkungsgrad & $70 \%$ & $55 \%$ & $35 \%$ \\
\hline Lebensdauer (Jahre) & 30 & 30 & 30 \\
\hline $\begin{array}{l}\text { Zyklenfestigkeit (max. } \\
\text { Zyklenzahl) }\end{array}$ & 15.000 & 10.000 & 10.500 \\
\hline $\begin{array}{l}\text { Investitionen Wandler } \\
(€ \text { je } k W)\end{array}$ & 1.390 & 1.623 & 2.870 \\
\hline $\begin{array}{l}\text { Investitionen Speicher } \\
\text { (€ je kWh Speichervolumen) }\end{array}$ & 67 & 196 & 10 \\
\hline $\begin{array}{l}\text { Wartung und Instandhaltung } \\
\text { (\% je Jahr bezogen auf Inves- } \\
\text { titionen) }\end{array}$ & $3,2 \%$ & $4,7 \%$ & $3,4 \%$ \\
\hline
\end{tabular}

Quelle: Oberschmidt und Klobasa (2009). 
Abbildung 7.38: Kriterien-Hierarchie zur Bewertung von Technologien zur Stromspeicherung

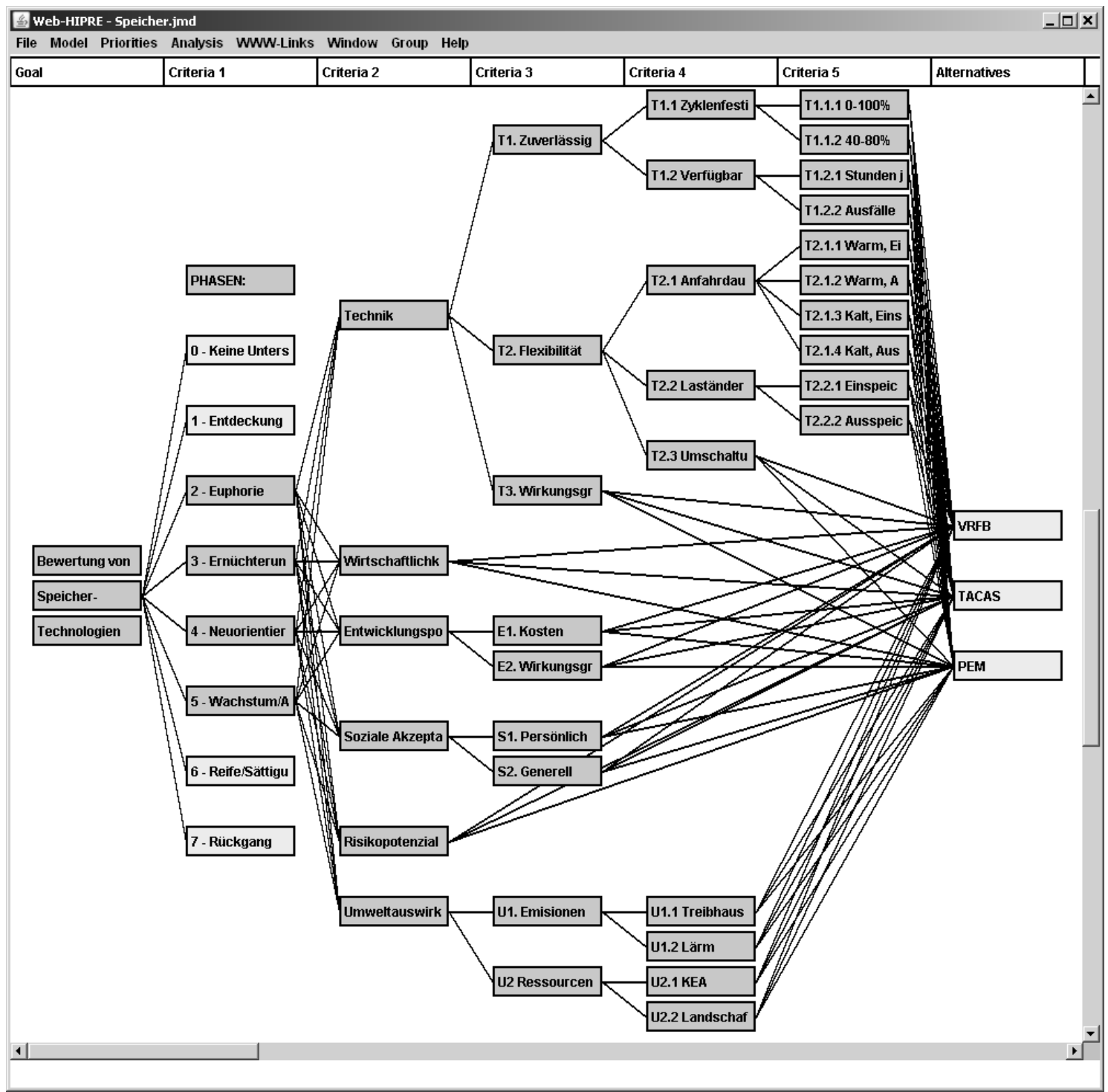

Quelle: Dötsch et al. (2008). 
Abbildung 7.39: Gewichtung der übergeordneten Kriterien zur Bewertung der Speichertechnologien in Abhängigkeit der Technologiezyklusphasen

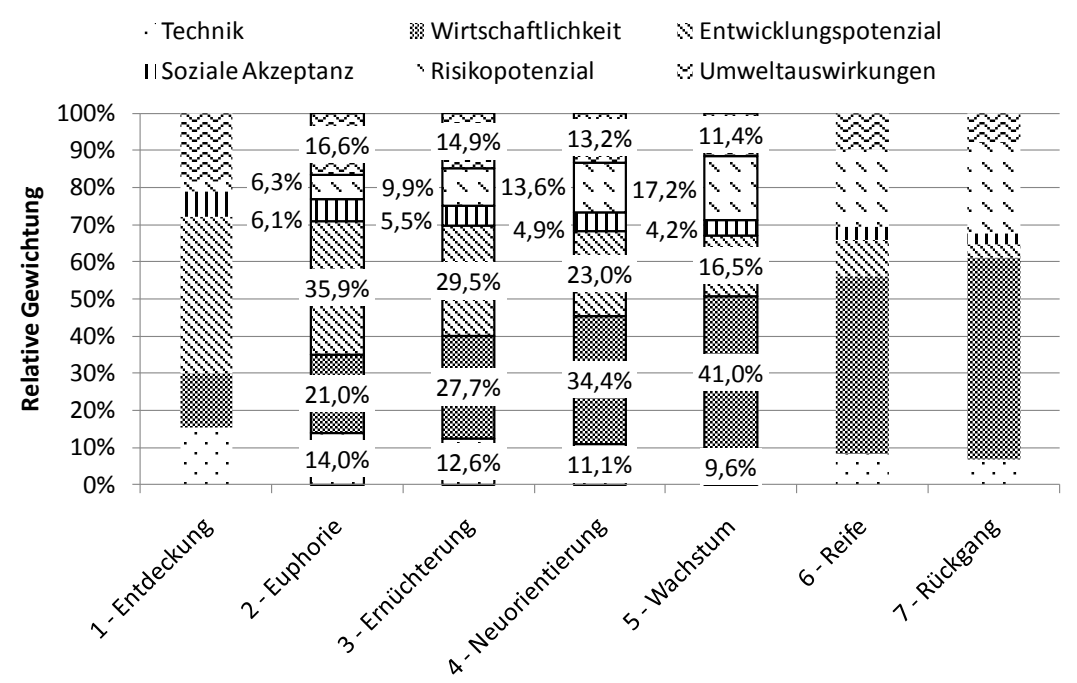

Quelle: Eigene Annahmen basierend auf Dötsch et al. (2008).

Abbildung 7.40: Mittelwerte der aus der Expertenbefragung ermittelten Gewichtungen

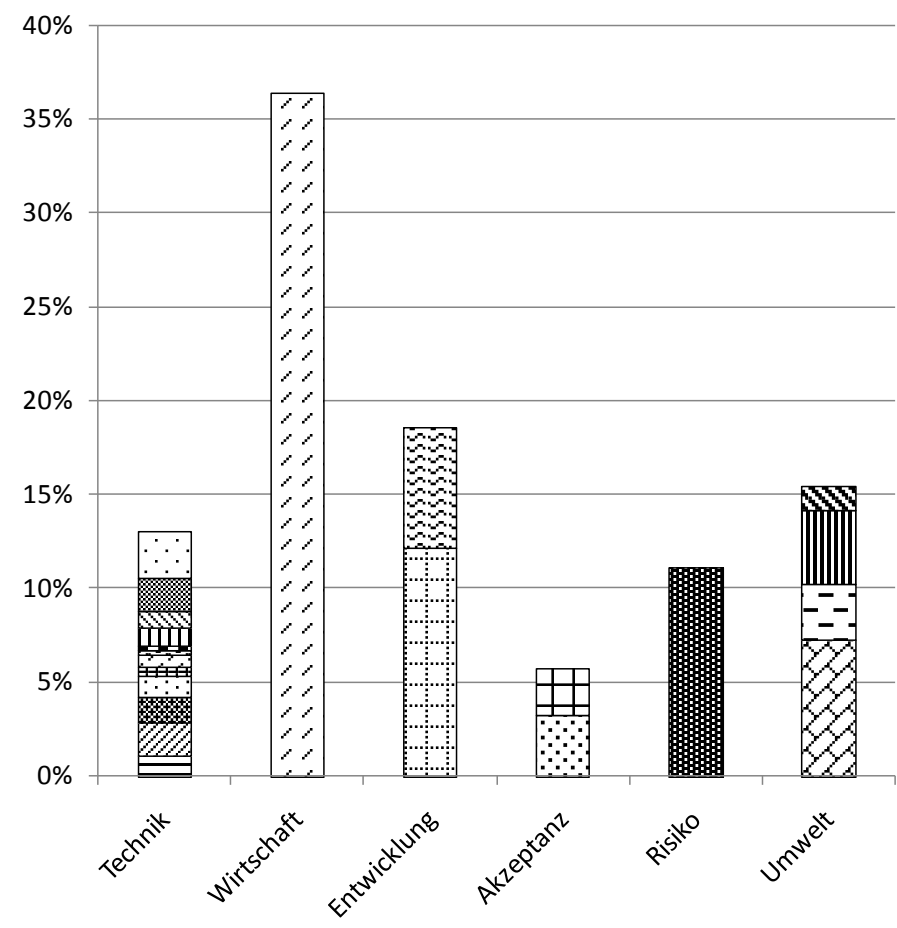

母 2.2 Fläche mU2.1 KEA $\square$ U1.2 Lärm ■U1.1 Treibhausgase 眸 R Risikopotenzial 円S2 Generelle Akzeptanz in der Gesellschaft $\bigoplus$ S1 Akzeptanz bei persönlicher Betroffenheit Đ2 Wirkungsgradsteigerungspotenzial $\boxplus$ E1 Kostensenkungspotenzial $\checkmark$ W Wirtschaftlichkeit (spez. Kosten) $\square T 3$ Gesamtwirkungsgrad T2.3 Zeitdauer Umschaltung Be-/Entladen ⿶T2.2.2 Laständerungsgeschw. (Aussp.) 凹T2.2.1 Laständerungsgeschw. (Einsp.) - T2.1.4 Anfahrdauer, Kaltstart (Aussp.) T2.1.3 Anfahrdauer, Kaltstart (Einsp.) घT2.1.2 Anfahrdauer, Warmstart (Aussp.) 田T2.1.1 Anfahrdauer, Warmstart (Einsp.) ๑T1.2.2 Häufigkeit ungeplanter Ausfälle 圈T1.2.1 Stunden verfügbar je Jahr ÐT1.1.2 Zyklenfestigkeit, Be-/Entladung 0-40\% 曰T1.1.1 Zyklenfestigkeit, Be-/Entladung 0-100\%

Anmerkungen: spez. $=$ spezifisch; Aussp. $=$ Ausspeicherung; Einsp. $=$ Einspeicherung .

Quelle: Dötsch et al. (2008). 
Zur Bewertung der Wirtschaftlichkeit im vorliegenden Fallbeispiel werden zum Einen die Kosten der Stromspeicherung, einschließlich Investitionen sowie Wartung und Instandhaltung, einbezogen (vgl. auch Tabelle 7.16). Zum anderen werden Erlösmöglichkeiten in Abhängigkeit der Strompreisschwankungen je Szenario berücksichtigt (Tabelle 7.17). Vergleichsgrößen sind die auf Basis dieser Daten berechneten Annuitäten der Speichertechnologien.

Tabelle 7.17: $\quad$ Erlösmöglichkeiten durch Stromzwischenspeicherung je Szenario in Euro/Jahr

\begin{tabular}{l|c|c|c|c}
\hline & Jahr & VRFB & TACAS & PEM \\
\hline \multirow{3}{*}{ Szenario 1 (Moderat) } & 2010 & 64.917 & 42.025 & 19.913 \\
\cline { 2 - 5 } & 2020 & 50.641 & 26.742 & 12.323 \\
\cline { 2 - 5 } & 2030 & 120.564 & 81.588 & 56.756 \\
\hline \multirow{2}{*}{ Szenario 2 (Klima) } & 2010 & 65.237 & 42.366 & 20.414 \\
\cline { 2 - 5 } & 2020 & 57.914 & 35.530 & 20.007 \\
\cline { 2 - 5 } & 2030 & 143.909 & 115.338 & 95.001 \\
\hline \multirow{5}{*}{ Szenario 3 (Ressourcen) } & 2010 & 268.388 & 221.893 & 152.343 \\
\cline { 2 - 5 } & 2020 & 430.445 & 389.045 & 322.981 \\
\cline { 2 - 5 } & 2030 & 440.162 & 411.841 & 375.140 \\
\hline
\end{tabular}

Quelle: Modellrechnung Fraunhofer ISI.

Die Parameter zur Festlegung der Präferenzfunktionen sind in Tabelle 7.18 zusammengefasst. Für Kriterien mit definierbarer Unter- und Obergrenze der Ausprägungen werden die Typen 3 bzw. 5 gewählt. Als Schwellenwerte werden jeweils die theoretischen Minimal- bzw. Maximalwerte der Kriterien-Ausprägungen festgelegt. Für alle anderen Kriterien wird Typ 6 als Präferenzfunktion gewählt. Der entsprechende Schwellenwert wird bei der Hälfte der Differenz zwischen der maximalen und der minimalen im Fallbeispiel beobachteten Ausprägung festgelegt. 
Tabelle 7.18: $\quad$ Festlegung der Präferenzfunktionen in PROMETHEE zur Bewertung von Speichertechnologien

\begin{tabular}{|c|c|c|c|c|c|c|c|}
\hline & & Einheit & $\min / \max$ & Typ & $\mathbf{q}$ & $\mathbf{p}$ & s \\
\hline T1.1.1 & Zyklenfestigkeit (0-100\%) & Anzahl & $\max$ & 6 & & & 2.500 \\
\hline T1.1.2 & Zyklenfestigkeit (40-80\%) & Anzahl & $\max$ & 6 & & & 2.500 \\
\hline T1.2.1 & Verfügbarkeit h/a & $\mathrm{h} / \mathrm{a}$ & $\max$ & 3 & & 8.760 & \\
\hline T1.2.2 & Ausfälle & Anzahl/a & $\min$ & 6 & & & 0,75 \\
\hline T2.1.1 & Anfahrdauer (Warmstart, Einsp.) & $s$ & $\min$ & 6 & & & 29,95 \\
\hline T2.1.2 & Anfahrdauer (Kaltstart, Einsp.) & s & $\min$ & 6 & & & 89,95 \\
\hline T2.1.3 & Anfahrdauer (Warmstart, Aussp.) & s & $\min$ & 6 & & & 597 \\
\hline T2.1.4 & Anfahrdauer (Kaltstart, Aussp.) & $\mathrm{s}$ & $\min$ & 6 & & & 147 \\
\hline T2.2.1 & Laständerungsgeschw. (Einsp.) & $\mathrm{kW} / \mathrm{s}$ & $\max$ & 6 & & & 49.825 \\
\hline T2.2.2 & Laständerungsgeschw. (Aussp.) & $\mathrm{kW} / \mathrm{s}$ & $\max$ & 6 & & & 49.825 \\
\hline T2.3 & $\begin{array}{l}\text { Dauer zur Umschaltung Be-/ } \\
\text { Entladung }\end{array}$ & ms & $\min$ & 6 & & & 1 \\
\hline T3 & Gesamtwirkungsgrad & $\%$ & $\max$ & 3 & & 100 & \\
\hline E1 & Kostensenkung & $\%$ & $\max$ & 3 & & 100 & \\
\hline E2 & Wirkungsgradsteigerung & $\%$ & $\max$ & 3 & & 100 & \\
\hline S1 & Persönliche Akzeptanz & $0 \ldots 1$ & $\max$ & 3 & & 1 & \\
\hline $\mathrm{S} 2$ & Gesellschaftliche Akzeptanz & $0 \ldots 1$ & $\max$ & 3 & & 1 & \\
\hline $\mathrm{R}$ & Risikopotenzial & $1 \ldots 5$ & $\max$ & 5 & 1 & 5 & \\
\hline U1.1 & Treibhausgasemissionen & $\mathrm{kg} \mathrm{CO}_{2} / \mathrm{kWh}_{\text {out }}$ & $\min$ & 6 & & & 0,35 \\
\hline $\mathrm{U} 1.2$ & Lärm & dBA & $\min$ & 6 & & & 14 \\
\hline U2.1 & KEA & $\mathrm{MJ} / \mathrm{kWh}_{\text {out }}$ & $\min$ & 6 & & & 3,41 \\
\hline $\mathrm{U} 2.2$ & Landschaftsinanspruchnahme & $\mathrm{m}^{2} \cdot \mathrm{a} / \mathrm{kWh}_{\text {out }}$ & $\min$ & 6 & & & 105,22 \\
\hline W (Sz1) & Wirtschaftlichkeit & Euro/a & $\min$ & 6 & & & 232.200 \\
\hline
\end{tabular}

\subsubsection{Ergebnisse}

Der Vergleich der Speichertechnologien hängt nur zu einem geringen Maße von den unterschiedlichen Szenarien ab (Abbildung 7.41). Dies ist dadurch zu begründen, dass für alle Technologien der gleiche Energieträger (Strom) eingesetzt wird. Daher wirken sich die Unterschiede in den Energieträgerpreisen je Szenario in etwa gleichem Maße auf die drei Alternativen aus, so dass sich die relativen Vor- bzw. Nachteile kaum ändern. Auch die Zuordnung des Druckluft-Systems (TACAS) zur Phase der Neuorientierung einerseits und zur Phase des Aufstiegs andererseits hat nur marginale Auswir- 
kungen auf das Bewertungsergebnis, da in diesen beiden Phasen ähnliche Gewichtungen zugrunde gelegt werden. Insgesamt ist das Ergebnis bei Gewichtung entsprechend der Aufstiegs-Phase etwas günstiger für die Alternative TACAS. Das höchste Maß für relative Stärken und geringste Maß für relative Schwächen weist die betrachtete Redox-Flow-Batterie auf (VRFB). Für das Druckluft-System (TACAS) resultiert ein etwas höheres Maß für relative Schwächen und deutlich geringer bewertete relative Stärken. Für das Wasserstoff-System (PEM) zeigen sich sowohl mehr relative Schwächen als auch weniger relative Stärken im Vergleich zu den beiden anderen Alternativen. Da sich die Ergebnisse je Szenario kaum unterscheiden, wird im Folgenden lediglich Szenario 1 weiter betrachtet.

Abbildung 7.41: Bewertung der Speichertechnologien in Abhängigkeit der Szenarien

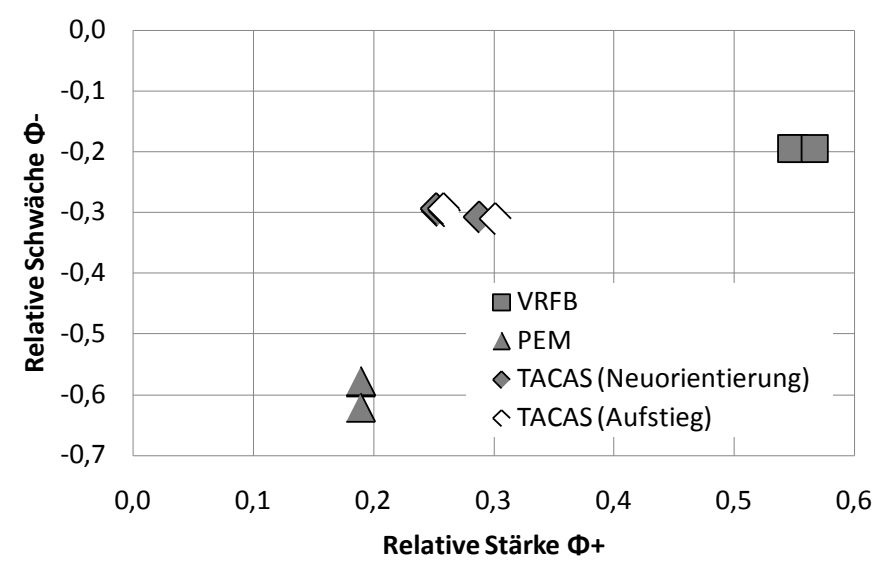

Für die VRFB wirken sich insbesondere die Vorteile hinsichtlich technischer Merkmale, Umweltauswirkungen und Wirtschaftlichkeit positiv auf das Ergebnis aus (Abbildung 7.42). Die Schwächen sind im Wesentlichen durch das im Vergleich zu den beiden anderen Alternativen relativ gering eingeschätzte Entwicklungspotenzial bestimmt. Insgesamt überwiegen deutlich die relativen Stärken der VRFB. Für das WasserstoffSystem (PEM) überwiegen hingegen die relativen Schwächen im Vergleich zu den anderen beiden Alternativen. Entgegengesetzt der VRFB wirken sich für die PEMAlternative insbesondere die Wirtschaftlichkeit, die Umweltauswirkungen und die Bewertungen in der technischen Dimension negativ aus. Positiv wird das WasserstoffSystem vor allem hinsichtlich des Entwicklungspotenzials bewertet. Die relativen Stärken und Schwächen des Druckluft-Systems (TACAS) hinsichtlich der Wirtschaftlichkeit halten sich in etwa die Waage. Dies ist darauf zurückzuführen, dass das DruckluftSystem hinsichtlich dieses Kriteriums einerseits besser als das Wasserstoff-System abschneidet und andererseits schlechter als die VRFB. Auch in den anderen Bewertungsdimensionen sind sowohl relative Vor- als auch Nachteile des Druckluft-Systems erkennbar, die sich jedoch in geringerem Maße auf das Ergebnis auswirken als die 
Wirtschaftlichkeit. Generell kann ein hoher Einfluss des Wirtschaftlichkeits-Kriteriums beobachtet werden, was auf dessen hohe Gewichtung zurückzuführen ist. Das Kriterium soziale Akzeptanz beeinflusst hingegen kaum das Ergebnis, was einerseits auf die geringe Gewichtung und andererseits auf die geringen Unterschiede zwischen den Kriterien-Ausprägungen zurückgeführt werden kann.

Abbildung 7.42: Analyse der Ergebnisse nach Bewertungsdimensionen (Szenario 1)

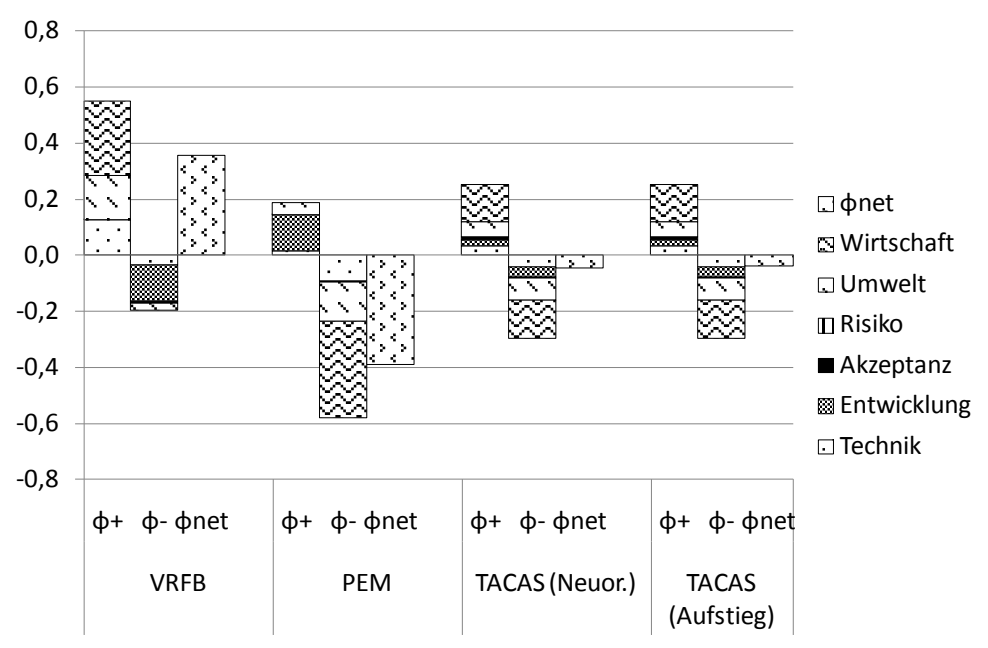

Die Rangfolge der Speichertechnologien nach PROMETHEE I und II ist in allen Szenarien gleich, wobei die Redox-Flow-Batterie an erster Stelle steht, gefolgt vom Drucklufts-System an zweiter und dem Wasserstoff-System an dritter Stelle (Abbildung 7.43). Dies legt die Empfehlung nahe, dass für den betrachteten Anwendungsbereich mit einer Leistung von $2 \mathrm{MW}$ und einer Speicherkapazität von $20 \mathrm{MWh}$ insbesondere diese Alternative eine interessante Option ist, die für zukünftige Weiterentwicklungen und Anwendungen in Betracht gezogen werden sollte. Dabei sollten sich Weiterentwicklungen insbesondere auf eine Reduktion der hohen Anfangsinvestitionen konzentrieren. Zwar wird die Wirtschaftlichkeit der VRFB im Vergleich zu den beiden anderen Technologien besser bewertet, dennoch sind die Kosten derzeit noch zu hoch, um wettbewerbsfähige Produkte bereitstellen zu können. Ob in Zukunft, etwa durch Skaleneffekte oder effizienteren Materialeinsatz, die erforderlichen Kostensenkungen in der Produktion erreicht werden können, bleibt abzuwarten. Ähnliches gilt auch für die beiden anderen betrachteten Optionen.

Abbildung 7.43: Rangfolge der Speichertechnologien nach PROMETHEE I und II (alle Szenarien)

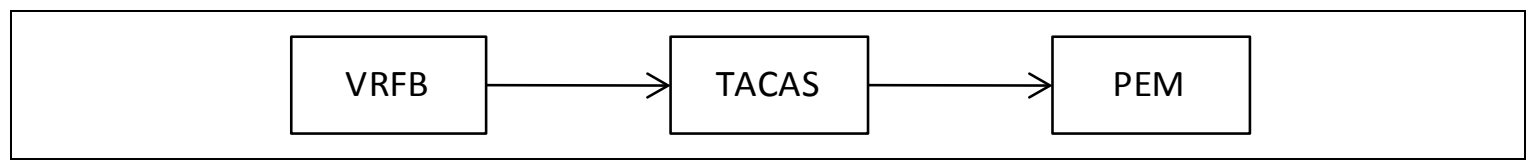


Die Sensitivitätsanalyse zeigt darüber hinaus, dass die Ergebnisse der multikriteriellen Bewertung relativ stabil sind. Alle Stabilitätsintervalle weisen eine Breite von mehr als 14 \%-Punkten auf (Tabelle 7.19). Für viele Kriterien ist das Ergebnis über den gesamten Gewichtungsbereich stabil, und für die Mehrzahl der Kriterien sind die Stabilitätsintervalle größer als 50 \%-Punkte. Die Kriterien mit den kleinsten Stabilitätsintervallen sind in allen Technologiezyklus-Phasen sehr gering gewichtet, so dass die Ergebnisse insgesamt als sehr robust angesehen werden können. Zudem resultiert für alle betrachteten Gewichtungs-Sets je Technologiezyklus-Phase die gleiche Rangfolge (Abbildung 7.4).

Tabelle 7.19: $\quad$ Stabilitätsintervalle der Kriterien-Gewichtungen zur Bewertung von Speichertechnologien

\begin{tabular}{|c|c|c|c|c|c|c|}
\hline & & $w(\min )$ & $w(t, \min )$ & $w(t, \max )$ & $w(\max )$ & $\Delta \mathbf{w}$ \\
\hline $\mathrm{T} 1.1 .1$ & Zyklenfestigkeit (0-100\%) & $0,0 \%$ & $0,9 \%$ & $1,1 \%$ & $77,4 \%$ & $77,4 \%$ \\
\hline T1.1.1 & Zyklenfestigkeit (40-80\%) & $0,0 \%$ & $1,5 \%$ & $1,9 \%$ & $77,5 \%$ & $77,5 \%$ \\
\hline T1.1.2 & Verfügbarkeit h/a & $0,0 \%$ & $1,2 \%$ & $1,5 \%$ & $100,0 \%$ & $100,0 \%$ \\
\hline $\mathrm{T} 1.2 .1$ & Ausfälle & $0,0 \%$ & $0,9 \%$ & $1,2 \%$ & $100,0 \%$ & $100,0 \%$ \\
\hline T1.2.2 & Anfahrdauer (Warmstart, Einsp.) & $0,0 \%$ & $0,4 \%$ & $0,5 \%$ & $100,0 \%$ & $100,0 \%$ \\
\hline T2.1.1 & Anfahrdauer (Kaltstart, Einsp.) & $0,0 \%$ & $0,6 \%$ & $0,7 \%$ & $100,0 \%$ & $100,0 \%$ \\
\hline T2.1.2 & Anfahrdauer (Warmstart, Ausp.) & $0,0 \%$ & $0,2 \%$ & $0,3 \%$ & $95,4 \%$ & $95,4 \%$ \\
\hline $\mathrm{T} 2.1 .3$ & Anfahrdauer (Kaltstart, Ausp.) & $0,0 \%$ & $0,2 \%$ & $0,3 \%$ & $76,0 \%$ & $76,0 \%$ \\
\hline T2.1.4 & Laständerungsgeschw. (Einsp.) & $0,0 \%$ & $0,8 \%$ & $1,0 \%$ & $97,1 \%$ & $97,1 \%$ \\
\hline T2.2.1 & Laständerungsgeschw. (Aussp.) & $0,0 \%$ & $0,7 \%$ & $0,9 \%$ & $97,1 \%$ & $97,1 \%$ \\
\hline $\mathrm{T} 2.2 .2$ & Dauer zur Umschaltung Be-/Entladung & $0,0 \%$ & $1,5 \%$ & $1,9 \%$ & $14,9 \%$ & $14,9 \%$ \\
\hline T2.3 & Gesamtwirkungsgrad & $0,0 \%$ & $2,2 \%$ & $2,7 \%$ & $100,0 \%$ & $100,0 \%$ \\
\hline T3 & Kostensenkung & $0,0 \%$ & $15,0 \%$ & $23,4 \%$ & $48,2 \%$ & $48,2 \%$ \\
\hline E1 & Wirkungsgradsteigerung & $0,0 \%$ & $8,0 \%$ & $12,5 \%$ & $57,9 \%$ & $57,9 \%$ \\
\hline E2 & Persönliche Akzeptanz & $0,0 \%$ & $2,7 \%$ & $3,5 \%$ & $80,0 \%$ & $80,0 \%$ \\
\hline S1 & Gesellschaftliche Akzeptanz & $0,0 \%$ & $2,1 \%$ & $2,7 \%$ & $40,3 \%$ & $40,3 \%$ \\
\hline S2 & Risikopotenzial & $0,0 \%$ & $6,3 \%$ & $13,6 \%$ & $100,0 \%$ & $100,0 \%$ \\
\hline $\mathrm{R}$ & Treibhausgasemissionen & $0,0 \%$ & $6,2 \%$ & $7,8 \%$ & $100,0 \%$ & $100,0 \%$ \\
\hline U1.1 & Lärm & $0,0 \%$ & $2,5 \%$ & $3,2 \%$ & $100,0 \%$ & $100,0 \%$ \\
\hline $\mathrm{U} 1.2$ & KEA & $0,0 \%$ & $3,4 \%$ & $4,3 \%$ & $15,2 \%$ & $15,2 \%$ \\
\hline $\mathrm{U} 2.1$ & Landschaftsinanspruchnahme & $0,0 \%$ & $1,1 \%$ & $1,4 \%$ & $14,7 \%$ & $14,7 \%$ \\
\hline $\mathrm{U} 2.2$ & Wirtschaftlichkeit & $0,0 \%$ & $21,0 \%$ & $34,4 \%$ & $100,0 \%$ & $100,0 \%$ \\
\hline
\end{tabular}


Abbildung 7.44: Ergebnisse Stromspeichertechnologien in Abhängigkeit unterschiedlicher Technologiezyklus-Phasen (Szenario 1)

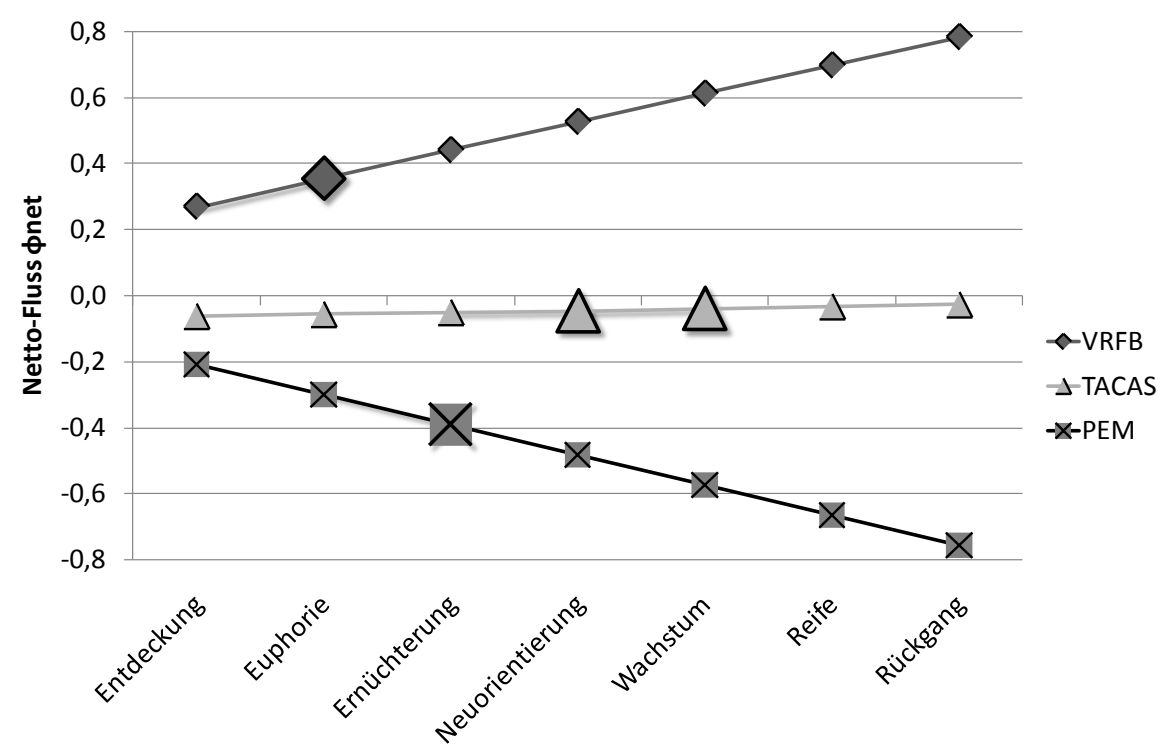

\subsubsection{Diskussion}

Das Fallbeispiel zeigt, dass die Anwendung von MAESTRO einen effizienten und transparenten Vergleich der Alternativen zur Stromspeicherung, die sich in unterschiedlichen Entwicklungsphasen befinden, auf Basis der durch die Experten bestimmten Zielgrößen ermöglicht. Dies gilt umso mehr, wenn die Daten zu den KriterienAusprägungen in vergleichbarer Form bereits vorliegen. Im vorliegenden Fall unterscheiden sich die Ergebnisse, die resultieren, wenn Gewichtungen in Abhängigkeit der aktuellen Technologiezyklus-Phasen angewendet werden, zwar nicht von den Ergebnissen auf Basis identischer Gewichtungen für alle Technologien. Dennoch trägt die in Verbindung mit dem Technologiezyklus-Modell durchgeführte Analyse dazu bei, ein klareres Bild der Entscheidungssituation zu vermitteln. Denn zum Einen wird der unterschiedliche Entwicklungsstand der Optionen verdeutlicht, was wiederum Rückschlüsse auf geeignete Forschungs- und Entwicklungsaktivitäten zulässt. Zum anderen wird die Sensitivitätsanalyse im Sinne einer simultanen Variation der Gewichtungen mehrere Kriterien unterstützt, indem Gewichtungs-Sets in Abhängigkeit der TechnologiezyklusPhasen untersucht werden. Das Modul Web-HIRPE ist im vorliegenden Anwendungsfall von Nutzen, da individuelle Kriterien-Gewichtungen einzelner Entscheider in dem hierarchisch strukturierten Entscheidungsproblem in einfacher Weise zu einem Gruppenmodell aggregiert werden können. Die so ermittelte Gewichtung einer Gruppe von Entscheidungsträgern kann wiederum exportiert und in MAESTRO verwendet werden. Die Ergebnisse, die aus der multikriteriellen Analyse resultieren, können Entscheidun- 
gen im Hinblick auf die Prioritätensetzung für oder wider bestimmte Technologien bei einem begrenzten Forschungsbudget unterstützen.

\subsection{Fazit}

Die durchgeführten Fallstudien demonstrieren die Anwendbarkeit des EUS MAESTRO und die unterschiedlichen Funktionalitäten der einzelnen Module. Die MTB-Datenbank stellt eine Datenbasis für Energietechnologien zur Verfügung, aus der für bestimmte Alternativen und Kriterien eine Entscheidungsmatrix generiert werden kann. Die Daten sind jedoch nicht uneingeschränkt für jeden Anwendungsfall anwendbar, da jeweils die spezifischen Rahmenbedingungen der Entscheidungssituation berücksichtigt werden müssen. Dies wird beispielsweise für das in Jühnde umgesetzte Biomasse-Konzept deutlich, das für eine bestimmte Strom- und Wärmenachfrage mit einer bestimmten Auslastung der Systemkomponenten konzipiert wurde. Bei der hierarchischen Strukturierung der Problemstellung und der Ableitung entsprechender Kriterien-Gewichtungen kann das Modul Web-HIRPE hilfreich sein. Dieses bietet zudem Funktionalitäten, um Gruppen-Entscheidungen zu unterstützen. Die so ermittelten Gewichtungen können exportiert und für die Bewertung im Excel-Tool MAESTRO zugrunde gelegt werden. Mit den Technologie-Daten aus der MTB-Datenbank und den Gewichtungen aus WebHIPRE stehen wesentliche Ausgangsdaten für die Bewertung der Technologien im Excel-Tool MAESTRO zur Verfügung. Zusätzlich müssen die Präferenzfunktionen je Kriterium und die Technologiezyklus-Phasen je Alternative definiert werden. Die im Excel-Tool zur Verfügung stehenden Informationen erleichtern die Festlegung der entsprechenden Parameter.

Die wesentliche Neuerung des Excel-Tools liegt darin, dass die Gewichtungen der Kriterien an die aktuell zutreffende Technologiezyklus-Phase je Alternative angepasst werden können. Dazu werden die bekannten Algorithmen nach PROMETHEE I und II modifiziert und entsprechend angepasste Methoden zur Sensitivitätsanalyse eingeführt. Diese Abhängigkeit der Gewichtungen von den Technologiezyklus-Phasen wird dadurch gerechtfertigt, dass Technologien einem dynamischen Wandel unterliegen, so dass je nach Entwicklungsstand die Bewertungsgrößen von unterschiedlicher Bedeutung sind. Die Anwendung der entsprechend modifizierten PROMETHEE-Methode, die im Excel-Tool MAESTRO implementiert ist, zeigt, dass bei Berücksichtigung Technologiezyklus-abhängiger Gewichtungen in den beiden Fallbeispielen teilweise andere Ergebnisse im Vergleich zur konventionellen PROMETHEE-Methode resultieren. Neben diesen direkten Auswirkungen der methodischen Weiterentwicklungen auf das Ergebnis der Bewertung bietet die Anwendung des modifizierten PROMETHEE-Algorithmus weitere Vorteile. Zum Einen trägt die systematische Einordnung von Energietechnolo- 
gien zu den Phasen im Technologiezyklus zur Strukturierung der Entscheidungssituation bei und ermöglicht Rückschlüsse auf den Entwicklungsstand sowie zukünftigen Forschungs- und Entwicklungsbedarf. Zum anderen wird durch die Berücksichtigung mehrerer Gewichtungs-Sets die Sensitivitätsanalyse bei simultaner Variation der Gewichtungen mehrere Kriterien unterstützt. Für konkrete zukünftige Anwendungen ist im Einzelfall zu überprüfen, inwieweit der zusätzliche Aufwand zur Zuordnung der Technologien zu den Phasen und zur Ableitung phasenabhängiger Gewichtungen gerechtfertigt ist. Im Fall, dass die Entscheidungsträger die konventionellen Methoden PROMETHEE I und II bevorzugen, ist deren Anwendung ebenfalls im Excel-Tool MAESTRO möglich. Zudem kann mit Hilfe der anderen Module des EUS auch eine additive Nutzwertanalyse durchgeführt werden, falls diese Methode von den Anwendern bevorzugt wird oder eine vergleichende Betrachtung der Ergebnisse basierend auf unterschiedlichen Methoden erwünscht ist. Das Excel-Tool MAESTRO kann darüber hinaus auch unabhängig von den Modulen MTB-Datenbank und Web-HIPRE genutzt werden. Auch Anwendungen außerhalb des Energiebereichs sind möglich, wobei die Anzahl der betrachteten Alternativen und Kriterien flexibel variiert werden kann. 


\section{$8 \quad$ Schlussfolgerungen und Ausblick}

Weder eine ideale Technologie noch eine ideale Methode existieren, um die Probleme der zukünftigen Energieversorgung uneingeschränkt lösen zu können. Dies gilt umso mehr, da es in der subjektiven Betrachtungsweise individueller Entscheidungsträger oder Gruppen liegt, welche Option als ideal anzusehen ist. Vor diesem Hintergrund trägt die vorliegende Arbeit dazu bei, Entscheidungsprozesse im Energiebereich zur Technologieauswahl unter Berücksichtigung vielfältiger Zielsetzungen und unterschiedlicher Sichtweisen transparenter zu gestalten. Die Betrachtung der historischen Entwicklung der Energieversorgung in Kapitel 2.1 verdeutlicht, dass die Gestaltung von Versorgungssystemen durch multiple Einflussfaktoren bestimmt wird. Dazu zählen die lokale Verfügbarkeit von Energieträgern, die Kosten in Verbindung mit der Erzeugung und Verwendung von Energie, ökologische Faktoren, technische Entwicklungen, Pfadabhängigkeiten und Aspekte des Nutzer-Komforts. Beeinflusst durch diese Faktoren und weitere Rahmenbedingungen hat sich die heute vorherrschende Struktur der Energiebereitstellung entwickelt, die aus unterschiedlichen Gründen nicht als nachhaltig bezeichnet werden kann. Erstens sind der Abbau und die Verwendung der vorwiegend eingesetzten fossilen und nuklearen Energieträger mit potenziell negativen Umwelt- und Gesundheitsauswirkungen verbunden. Zweitens ist die begrenzte Reichweite dieser Rohstoffe insbesondere vor dem Hintergrund einer weltweit wachsenden Energienachfrage problematisch. Zudem bedroht die zunehmende Importabhängigkeit hinsichtlich fossiler Energieträger die Wettbewerbsposition und Versorgungssicherheit Deutschlands. Aufgrund tendenziell steigender Preise der importierten Energieträger und zunehmender Volatilität der Preisentwicklungen treten darüber hinaus vermehrt Risiken und Unsicherheiten hinsichtlich der Wirtschaftlichkeit der Energieversorgung auf. Diese vielfältigen Aspekte müssen bei den anstehenden Entscheidungen zur Ausund Umgestaltung des Energieversorgungssystems berücksichtigt werden.

Aktuelle politische Rahmenbedingungen auf nationaler, europäischer und internationaler Ebene zielen darauf ab, die zukünftige Energieversorgung kostengünstig, umweltfreundlich, sicher, effizient und verbraucherfreundlich zu gestalten. Entsprechende Zielsetzungen sind zum Beispiel im deutschen Energiewirtschaftsgesetz sowie im Grünbuch der Europäischen Kommission zur Strategie für zukünftige die Energieversorgung Europas festgehalten. Möglichkeiten zur Erreichung dieser Ziele werden vor allem in einer Erhöhung des Anteils erneuerbarer Energien sowie der Kraft-WärmeKopplung gesehen. Dabei eröffnen sich den Akteuren auf den Energiemärkten einerseits neue Chancen, andererseits bestehen neue Risiken aufgrund einer zunehmenden Anzahl an Wettbewerbern in liberalisierten Energiemärkten. Auf internationaler Ebene führen die befürchteten wirtschaftlichen und sozialen Folgen des Klimawandels dazu, dass eine Reduktion der Treibhausgasemissionen angestrebt wird. Dabei kann 
die Erreichung der entsprechenden Zielsetzungen durch neue, umweltfreundlichere Lösungen im Energiebereich unterstützt werden. Jedoch dürfen neben der notwendigen Minderung der Treibhausgasemissionen weitere Zielsetzungen, unter anderem hinsichtlich Wirtschaftlichkeit, anderer Umweltauswirkungen, Versorgungssicherheit und auch sozialer Aspekte nicht vernachlässigt werden. Vor dem Hintergrund der teilweise konfliktären Zielsetzungen und sich wandelnder Rahmenbedingungen ist die Aus- bzw. Umgestaltung der Energieversorgungssysteme insbesondere aufgrund alternder Anlagen im bestehenden System sowie sich ändernder Strukturen in der Strom- und Wärmenachfrage notwendig. Daher stellt sich für Entscheidungsträger in der Energiepolitik und Energiewirtschaft die Frage, welche Energietechnologien zukünftig verstärkt genutzt und daher weiterentwickelt, erforscht und gefördert werden sollten, das heißt auf welche Energietechnologien sich Forschungs- und Entwicklungsanstrengungen konzentrieren sollten.

Eine Vielzahl prinzipiell realisierbarer technologischer Lösungen steht zur Bereitstellung von Strom und Wärme zur Verfügung. Die verfügbaren und in Entwicklung befindlichen Optionen umfassen Technologien auf Basis fossiler, nuklearer oder erneuerbarer Energieträger, zentrale oder dezentral organisierte Versorgungssysteme sowie weit entwickelte, etablierte Technologien einerseits und innovative, neuartige Technologien andererseits, die bisher nicht in breitem Umfang realisiert wurden oder noch in einem Stadium der Ideenfindung sind. Aufgrund der Komplexität der Entscheidungssituation und individuellen Einflussfaktoren in spezifischen Problemstellungen kann nicht unmittelbar ein Urteil über die relative Vorteilhaftigkeit der Optionen gefällt werden. Daher ist ein wissenschaftlicher Analyserahmen erforderlich, um die Stärken und Schwächen der Optionen zur Energiebereitstellung im Kontext konkreter Problemstellungen systematisch einander gegenüberstellen zu können. Aufgrund der weitreichenden gesamtwirtschaftlichen Bedeutung der Energieversorgung ist dabei ein besonders transparenter Entscheidungsprozess wünschenswert, der eine Einbeziehung multipler Zielsetzungen erlaubt. Für derartige Entscheidungsprozesse bieten sich Methoden der multikriteriellen Entscheidungsunterstützung an.

Um die zur Verfügung stehenden Optionen als diskrete Alternativen auf Basis mehrere Zielsetzungen bzw. Kriterien zu bewerten, bieten sich insbesondere Multi-AttributMethoden für die Entscheidungsunterstützung an. Die Literaturanalyse zeigt, dass diese seit den 1970er Jahren vor allem aufgrund der zunehmenden Wahrnehmung von Umweltaspekten in steigendem Maße für unterschiedliche Problemstellungen im Energiebereich eingesetzt werden. Dabei zeigen die analysierten Veröffentlichungen Wege auf, wie mehrere Zielgrößen bei der Bewertung von Optionen zur Energiebereitstellung simultan berücksichtigt werden können. Somit steht eine anerkannte, wissenschaftlich etablierte Methoden-Familie zur Verfügung, die eine Bewertung auf Basis multipler 
Zielsetzungen erlaubt. Eine Schwachstelle in vielen Anwendungen multikriterieller Methoden liegt darin, dass häufig weder dem technologischen Wandel noch den sich ändernden, zukünftig ungewissen Rahmenbedingungen in ausreichendem Maße Rechnung getragen wird. Daher zielen die methodischen Weiterentwicklungen der vorliegenden Arbeit insbesondere darauf ab, ein multikriterielles Entscheidungsunterstützungssystem zu entwickeln, das diese Schwachstellen beheben und für unterschiedliche Problemstellungen im Energiebereich eingesetzt werden kann.

Als Grundlage der Berücksichtigung des technologischen Wandels dienen im Rahmen dieser Arbeit Lebenszyklus-Modelle für Produkte bzw. Technologien, die als Erklärungs- und Prognose-Modelle in den Wirtschaftswissenschaften etabliert sind. Diese Modelle erlauben eine Abgrenzung definierter Phasen mit spezifischen Merkmalen, die ein Produkt bzw. eine Technologie vor und während ihres Bestehens am Markt durchläuft. Ausgangspunkt der Überlegungen ist der Umstand, dass zu einem bestimmten Zeitpunkt, zu dem eine Entscheidung zu treffen ist, in der Regel mehrere Technologien zur Verfügung stehen, die unterschiedlich weit entwickelt sind. Dabei fällt die Entscheidung nicht zwangsläufig zugunsten einer etablierten Technologie mit zu diesem Zeitpunkt besseren Eigenschaften im Vergleich zu einer innovativen Alternative aus. Vielmehr sollten die Bewertungsmaßstäbe vom aktuellen Entwicklungsstand einer Technologie abhängig gemacht werden. Da bisher keine multikriteriellen Entscheidungsunterstützungssysteme bekannt sind, die diesem Umstand Rechnung tragen, wird im Rahmen der vorliegenden Arbeit eine entsprechende Methode entwickelt und implementiert. Bei Anwendung der entwickelten Methode können Kriterien-Gewichtungen in Abhängigkeit der unterschiedlichen Entwicklungsstadien der betrachteten Alternativen zugrunde gelegt. Als Basis hierfür wird in Anlehnung an etablierte Technologiezyklusund Marktdiffusionsmodelle ein integrierter F\&E- und Marktzyklus eingeführt. Dieser unterscheidet in sieben Phasen, die eine Technologie vor und nach der Markteinführung durchläuft. Auf der Grundlage empirischer Daten werden bekannte Technologien zur Bereitstellung von Strom und Wärme diesen Phasen zugeordnet. Weiterhin werden Möglichkeiten aufgezeigt, wie Technologien, die noch nicht am Markt verbreitet sind, frühen Entwicklungsstadien zugeordnet werden können.

Neben der Integration unterschiedlicher Technologiezyklus-Phasen ist das entwickelte Entscheidungsunterstützungssystem (EUS) MAESTRO durch weitere Merkmale gekennzeichnet. Das System besteht aus drei Komponenten: der Datenbank „Multidimensionale Technikbewertung“ (MTB-Datenbank), dem Java-Applet „Web-HIPRE“ sowie dem Excel-Tool MAESTRO. Die MTB-Datenbank dient in erster Linie der Datensammlung und -verwaltung. Diese wurde entwickelt, um den Aufwand bei der Datenverwaltung und -erhebung im Rahmen der multikriteriellen Bewertung von Energietechnologien zu reduzieren und die Reproduzierbarkeit von Ergebnissen zu erleichtern. 
Hierfür wurden für eine Vielzahl an Energietechnologien zur Strom- und Nutzwärmebereitstellung Daten zu technischen Merkmalen, Kosten sowie Umweltauswirkungen aus unterschiedlichen Quellen zusammengetragen und in der Datenbank hinterlegt. Des Weiteren wurden Charakteristika (zum Beispiel $\mathrm{CO}_{2}$-Emissionsfaktoren) der wichtigsten Energieträger wie etwa Braunkohle, Steinkohle, Heizöl oder Holzbrennstoffe erfasst. Zudem sind zur Abschätzung zukünftiger Rahmenbedingungen drei Szenarien hinsichtlich der zukünftigen Entwicklung der Energieträgerpreise implementiert. In dieser Weise können Unsicherheiten bei der Bewertung von Optionen zur Energiebereitstellung bezüglich ökonomischer Zielgrößen berücksichtigt und robuste Alternativen identifiziert werden. Vorteile der Datenbank liegen darin, dass Basisdaten für die Bewertung von Energietechnologien in strukturierter Weise bereitgestellt werden, in der intuitiven Benutzerführung sowie in der Nachvollziehbarkeit und leichten Verständlichkeit der implementierten additiven Nutzwertanalyse. Nachteile betreffen die relativ starre Struktur der Datenbank, die eine Erweiterung der Kriterien, die für die Nutzwertanalyse innerhalb der Datenbank zugrunde gelegt werden, sowie eine Anpassung an spezifische Fragestellungen erschwert. Herausforderungen liegen darüber hinaus in der Datenerhebung, die mit einem hohen Zeitaufwand verbunden ist, sowie in der Gewährleistung der Datenqualität, insbesondere hinsichtlich der Aktualität der sich im Zeitverlauf ändernden Technologie- und Rahmendaten. Daher sollte in zukünftigen Arbeiten die Umstellung auf moderne und leistungsfähige Datenmanagementsysteme und entsprechender Softwareanwendungen überprüft werden.

Um einige der Nachteile der MTB-Datenbank zu überwinden, wurden dem Entscheidungsunterstützungssystem MAESTRO zwei weitere Komponenten hinzugefügt. Das kommerziell verfügbare Java-Applet Web-HIPRE zeichnet sich insbesondere durch eine hohe Nutzerfreundlichkeit und Flexibilität aus. Dabei können hierarchische Problemstrukturen aufgebaut und mit unterschiedlichen nutzentheoretisch basierten Methoden (Multi Attribute Value Theory - MAVT) analysiert werden. Im Kontext des EUS MAESTRO kann es insbesondere dazu dienen, Kriterien-Gewichtungen in Abhängigkeit unterschiedlicher Technologiezyklus-Phasen in hierarchischen Entscheidungsstrukturen zu ermitteln und Ergebnisse auf Basis unterschiedlicher Aggregationsmethoden zu vergleichen. Als Outranking-Methode wurde in einem zusätzlichen ExcelTool ein modifizierter PROMETHEE-Algorithmus implementiert, der Gewichtungen in Abhängigkeit der Technologiezyklus-Phasen der betrachteten Alternativen sowie entsprechende Sensitivitätsanalysen erlaubt. Hiermit wurde erstmals eine Methode implementiert, die multikriterielle Ansätze mit Technologiezyklusmodellen zur Berücksichtigung dynamischer Veränderungen von Technologien im Zeitverlauf integriert. Das Excel-Tool bietet zudem den Vorteil einer hohen Flexibilität hinsichtlich der Erweiterung der Entscheidungsmatrix um zusätzliche Alternativen und Kriterien. Durch zusätzlich 
implementierte Informationen sowohl zu den Technologiezyklus-Phasen wie auch hinsichtlich der Anwendung geeigneter Präferenzfunktionen, kann das Tool auch von Anwendern genutzt werden, die über wenige Erfahrungen mit der Methode verfügen. Aufgrund kurzer Rechenzeiten kann die Analyse ohne großen Aufwand mehrfach variiert und Ergebnisse reproduziert werden. Entsprechend dem modifizierten PROMETHEEAlgorithmus werden Ausgangs- und Eingangsfluss sowie der Netto-Outranking-Fluss je Alternative ermittelt. Die Ergebnisse können hinsichtlich des Beitrags einzelner Kriterien und deren Gewichtung analysiert und grafisch dargestellt werden. Darüber hinaus werden Stabilitätsintervalle unter Berücksichtigung der Gewichtungen in Abhängigkeit der Technologiezyklus-Phasen für alle Kriterien ermittelt. Zur Visualisierung der Stabilitätsintervalle dient die grafische Darstellung der Veränderung des Netto-Flusses je Alternative in Abhängigkeit der Variation der Gewichtung jeweils eines Kriteriums zwischen $0 \%$ und $100 \%$. Darüber hinaus wird die Untersuchung der simultanen Variation der Gewichtungen aller Kriterien unterstützt, indem die Ergebnisse in Abhängigkeit der je Technologiezyklus-Phase definierten Gewichtungs-Sets berechnet und entsprechend grafisch dargestellt werden können.

Mit MAESTRO wurde somit ein Entscheidungsunterstützungssystem geschaffen, das im Gegensatz zu anderen bekannten Modellen im Energiebereich zum einen eine systematische Berücksichtigung des technologischen Wandels in Form von Technologiezyklus-abhängigen Gewichtungen erlaubt. Zum zweiten wird eine Basis von Technologie- und Rahmendaten in Form mehrerer Szenarien zur Verfügung gestellt. Diese umfasst Datensätze, die technische Merkmale, Kosten und Umweltauswirkungen einzelner Energietechnologien beschreiben. Unabhängig von der MTB-Datenbank kann das Excel-Tool MAESTRO zum Einsatz in den unterschiedlichsten multikriteriellen Fragestellungen auch außerhalb des Energiebereichs, mit einer flexiblen Anzahl an Kriterien und Alternativen genutzt werden. Dabei steht es dem Nutzer frei, ob die Alternativen in Abhängigkeit unterschiedlicher Entwicklungs-Phasen bewertet werden oder ob für alle Alternativen die gleiche Gewichtung zugrunde gelegt werden. Erweiterungsmöglichkeiten der derzeit implementieren Analysemöglichkeiten liegen zum Beispiel in der Implementierung von PROMETHEE GAIA, der Methode der "Walking Weights" sowie in der Berechnung der minimal erforderlichen Änderung der Gewichtung, um eine bestimmte Alternative als beste Option zu bewerten. Weiterhin ist in zukünftigen Anwendungen zu untersuchen, wie sich eine den fortschreitenden Entwicklungsphasen entsprechende Änderung der Basisdaten der Technologien in Verbindung mit den phasenabhängigen Gewichtungen der Kriterien auswirkt. Herausforderungen liegen hierbei in der Prognose zukünftiger Entwicklungspotenziale der Technologien, etwa hinsichtlich Kostensenkung oder Wirkungsgradsteigerung. Methodische Ansatzpunkte sind diesbezüglich zum Beispiel in der Theorie der Lernkurven zu sehen. 
Die Anwendbarkeit des Entscheidungsunterstützungssystems MAESTRO wird auf Basis von zwei Fallbeispielen validiert. Der detailliert dargestellte Anwendungsfall „Bioenergiedörfer" verdeutlicht die implementierten Funktionalitäten des Entscheidungsunterstützungssystems. Die kompaktere Fallstudie zur Bewertung von Stromspeichertechnologien zeigt weitere potenzielle Anwendungsbereiche des EUS auf. Für das Bioenergiedorf Jühnde werden auf Basis von 30 Kriterien in vier Bewertungsdimensionen acht Konzepte zur Versorgung mit Strom und Wärme untersucht. Zur Abbildung der Bewertungsdimensionen Wirtschaftlichkeit und Umwelt werden etablierte Methoden der Investitionsrechnung bzw. Ökobilanzierung angewendet. Für die Dimensionen Versorgungsicherheit und soziale Aspekte existiert hingegen kein allgemein anerkannter Bewertungsansatz. Während zur Bewertung sozialer Aspekte auf Erhebungen in anderen Veröffentlichungen zurückgegriffen wird, wird zur Bewertung des Beitrags einzelner Technologien zur Versorgungssicherheit ein neues Kriterien-Set vorgeschlagen. Dieses bildet Technologie-spezifische wie auch Energieträger-spezifische Aspekte der Versorgungssicherheit ab und kann auch auf andere Anwendungen im Energiebereich übertragen werden. Die Bewertung mit Hilfe des EUS MAESTRO zeigt, dass der modifizierte PROMETHEE-Algorithmus teilweise zu anderen Ergebnissen führt als eine Bewertung basierend auf identischen Gewichtungen für alle Alternativen. Unter den getroffenen Annahmen werden zur Strom- und Wärmebereitstellung in Bioenergiedörfern die Alternativen basierend auf erneuerbaren Energieträgern besser bewertet als fossil befeuerte Anlagen. Insbesondere Kombinationen von Windkraft und Solaranlagen und/oder Biomasse zeigen sehr gute Ergebnisse. Die Referenz-Alternative, das heißt die Stromversorgung aus dem öffentlichen Netz und Heizöl-Kessel zur Wärmebereitstellung, scheint hingegen langfristig keine tragfähige Option zu sein. Im zweiten Fallbeispiel ist die ermittelte Rangfolge der Stromspeichertechnologien für alle betrachteten Gewichtungs-Sets und Szenarien konstant. Unter Beachtung der getroffenen Annahmen ist die Redox-Flow-Batterie dem Druckluftspeicher-System und dieses wiederum dem Wasserstoffspeicher-System vorzuziehen. Demnach sollten sich zukünftige Entwicklungsarbeiten für den betrachteten Einsatzbereich insbesondere auf die RedoxFlow-Batterie konzentrieren. Jedoch ist hervorzuheben, dass unter geänderten Randbedingungen andere Optionen die bessere Alternative darstellen können.

In methodischer Hinsicht ist darauf hinzuweisen, dass es in der subjektiven Betrachtungsweise des individuellen Entscheiders liegt, ob die Gewichtungen in Abhängigkeit der Technologiezyklus-Phasen seine Präferenzvorstellungen in besserer Weise abbilden können als konstante Gewichtungen unabhängig vom Entwicklungsstand der betrachteten Alternativen. Vor dem Hintergrund der dynamischen und komplexen Veränderungen, denen Technologien im Zeitverlauf unterliegen, scheint jedoch die Gewichtung in Abhängigkeit unterschiedlicher Technologiezyklus-Phasen gerechtfertigt. Zu- 
künftige Forschungsarbeiten sollten sich diesbezüglich insbesondere damit beschäftigen, mit welchen Methoden die Gewichtungen in Abhängigkeit der Entwicklungsphasen in geeigneter Weise von den Entscheidungsträgern erhoben werden können. Insbesondere bei partizipativen Entscheidungsprozessen in Zusammenarbeit mit lokalen und politischen Akteuren, die unter Umständen weder mit den wissenschaftlichen Methoden der multikriteriellen Analyse noch mit den Theorien zum technologischen Wandel vertraut sind, müssen dabei Komplexität und Kommunizierbarkeit der Methoden und Ergebnisse sorgfältig gegeneinander abgewogen werden.

Die Bewertungsgrößen für Stärken und Schwächen der Alternativen, die mit Hilfe von MAESTRO in den Fallbeispielen für einzelne Technologien ermittelt wurden, können in einfacher Weise zu einem Gesamtergebnis aggregiert werden, wie das Fallbeispiel „Bioenergiedörfer“ zeigt. Hier liegt eine Erweiterungsmöglichkeit darin, Modelle und Methoden zu implementieren, die in automatisierter Weise mögliche Kombinationen von Energietechnologien zu Versorgungssystemen unter Berücksichtigung von Randbedingungen ermitteln, die dann durch den vorgestellten Aggregationsmechanismus einander gegenübergestellt werden können. Damit könnten auch größere Versorgungskonzepte (etwa für größere Regionen oder einzelner Länder) auf Basis der Bewertung einzelner Technologien und deren Anteile an der Strom-und Wärmebereitstellung in effizienter Weise vergleichend einander gegenübergestellt werden. Andererseits ist eine Kopplung des Excel-Tools mit Energiesystemmodellen denkbar, die das Zusammenwirken unterschiedlicher Systemkomponenten in Versorgungssystemen simulieren. Jedoch erschwert diese Vorgehensweise die Berücksichtigung der Technologiezyklus-Phasen einzelner Technologien. Eine weitere Schwierigkeit liegt in dem Aufwand, die Ausprägungen einer Vielzahl an Kriterien-Ausprägungen für Energieversorgungssysteme in einem Modell zu bestimmen und bei Bedarf zusätzliche OutputGrößen als Kriterien zu implementieren. Eine Alternative liegt in der Bewertung von Energieversorgungssystemen hinsichtlich einzelner Kriterien durch ExpertenEinschätzungen, die jedoch mit zunehmender Komplexität der Systeme als unsicherer einzustufen sind.

Die zusammenfassende Betrachtung der vorliegenden Arbeit zeigt, dass das EUS MAESTRO in der vorliegenden Version, die im Rahmen dieser Arbeit entwickelt wurde, eine solide Basis bietet, um Energietechnologien in unterschiedlichen Entwicklungsstadien auf Basis multipler Zielsetzungen einander gegenüberzustellen. Somit kann es als Grundlage dienen, um Entscheidungsträger in Energiepolitik und -wirtschaft insbesondere im Hinblick auf die Priorisierung von Forschungs- und Entwicklungsthemen zu unterstützen. Erweiterungs- und Verbesserungsmöglichkeiten können im Kontext zukünftiger Anwendungen entsprechend den spezifischen Erfordernissen realisiert werden. 


\section{$9 \quad$ Zusammenfassung}

Die vorliegende Arbeit beschäftigt sich mit der Fragestellung, wie Entscheidungen hinsichtlich der zukünftigen Energieversorgung vor dem Hintergrund teilweise konfliktärer Zielsetzungen in effektiver, transparenter und nachvollziehbarer Weise unterstützt werden können. Die Relevanz der Fragestellung wird zunächst vor dem Hintergrund der historischen Entwicklung der Energieversorgung sowie wichtiger aktueller Rahmenbedingungen für Entscheidungen im Energiebereich erläutert. Die aufgezeigten Entwicklungstendenzen verdeutlichen, dass Energiewirtschaft und -politik vor Herausforderungen hinsichtlich der Umstrukturierung der Energiesysteme stehen. Zu beachten sind hierbei insbesondere die zunehmenden Unsicherheiten in Verbindung mit der Bereitstellung fossiler Energieträger, die weltweit steigende Energienachfrage, die Intensivierung des Wettbewerbs aufgrund liberalisierter Energiemärkte und politische Maßnahmen, die den Ausbau von erneuerbaren Energien und Kraft-Wärme-KopplungsAnlagen begünstigen. Nach der Beschreibung der Rahmenbedingungen werden im dritten Kapitel die wichtigsten Technologiepfade, die bereits heute zur Energieversorgung beitragen oder potenzielle zukünftige Optionen darstellen, aufgezeigt. Betrachtet werden etablierte und innovative Technologien zur Erzeugung, Verteilung und Speicherung von Energie, die auf erneuerbaren, fossilen oder nuklearen Energieträgern basieren und zentralisiert oder dezentral zum Einsatz kommen. Dabei wird einerseits deutlich, dass eine Vielzahl an Optionen zur Verfügung steht. Andererseits kann keine der Optionen alleine den Anforderungen an eine wirtschaftliche, umweltfreundliche und zuverlässige Energieversorgung gerecht werden. Vielmehr müssen deren Stärken und Schwächen vor dem Hintergrund der spezifischen Rahmenbedingungen sorgfältig gegeneinander abgewogen werden. Daraus leitet sich der Bedarf einer multikriteriellen Entscheidungsunterstützung ab, um Energietechnologien unter simultaner Berücksichtigung mehrere Zielsetzungen vergleichend bewerten zu können.

Im vierten Kapitel werden daher Grundlagen der multikriteriellen Entscheidungsunterstützung dargestellt und geeignete Methoden für die zugrunde liegende Fragestellung identifiziert. Eine umfassende Analyse bekannter Anwendungen von Multi-AttributMethoden im Energiebereich zeigt, dass diese für energiewirtschaftliche und energiepolitische Fragestellungen bereits etabliert sind. Schwachstellen bekannter Anwendungen liegen unter anderem darin, dass einige Zielgrößen nur in eingeschränktem Maße berücksichtigt werden, nur selten eine systematische Betrachtung möglicher zukünftiger Rahmenbedingungen erfolgt und in vielen Fällen der technologische Wandel nur unzureichend berücksichtigt wird. Jedoch sollte insbesondere dem Umstand, dass sich die heute und perspektivisch zur Verfügung stehenden Energietechnologien in unterschiedlichen Stadien der Entwicklung befinden, bei einer vergleichenden Bewertung in geeigneter Weise Rechnung getragen werden. Der neu entwickelte Ansatz der vorlie- 
genden Arbeit besteht daher darin, eine adäquate Berücksichtigung der Veränderung von Energietechnologien im Zeitverlauf im Rahmen der multikriteriellen Entscheidungsunterstützung zu integrieren. Dazu werden zunächst im fünften Kapitel Methoden zur Abbildung des technologischen Wandels aufgezeigt und deren Anwendung im Rahmen der multikriteriellen Bewertung von Energietechnologien überprüft. Auf Basis etablierter Technologiezyklus- und Marktdiffusionsmodelle wird ein integrierter F\&Eund Marktzyklus mit sieben Phasen eingeführt, der als Grundlage zur Berücksichtigung des technologischen Wandels dient. In diesem Kontext werden Technologiezyklen von Energietechnologien auf Basis empirischer Daten dargestellt und die Technologien den Phasen zugeordnet. Darauf aufbauend wird im sechsten Kapitel eine multikriterielle Methode entwickelt, die einen systematischen Vergleich etablierter und innovativer Technologien in Verbindung mit der Berücksichtigung von Kriterien-Gewichtungen in Abhängigkeit unterschiedlicher Technologiezyklus-Phasen erlaubt. Die entwickelte Methode basiert auf den etablierten Outranking-Verfahren PROMETHEE I und II (Preference Ranking Organisation Method for Enrichment Evaluations) und wird einschließlich angepasster Sensitivitätsanalysen in einem Entscheidungsunterstützungssystem (EUS) implementiert. Das EUS enthält darüber hinaus eine Datenbasis für die multikriterielle Bewertung von Energietechnologien und bietet zudem die Möglichkeit, auch nutzentheoretische Methoden anzuwenden.

Die neu erarbeitete Methode und das entwickelte EUS werden im siebten Kapitel anhand von zwei Fallbeispielen validiert. Zum einen werden alternative Energieversorgungskonzepte für ein Bioenergiedorf einander gegenübergestellt. Dabei wird unter anderem ein Kriterien-Set zur Bewertung des Beitrags von Energietechnologien zur Versorgungssicherheit erarbeitet. Des Weiteren wird ein Konzept zur Aggregation der Bewertung von Einzeltechnologien zu einer Gesamtbewertung für Kombinationen von Einzeltechnologien aufgezeigt. Im zweiten Fallbeispiel werden Technologien zur stationären Stromspeicherung im Hinblick auf zukünftige Anwendungsbereiche in Energiesystemen mit hohem Anteil erneuerbarer Energien einander gegenübergestellt. Durch die Fallbeispiele werden die prinzipielle Anwendbarkeit und die Funktionalitäten des EUS aufgezeigt. Die Resultate zeigen, dass Kriterien-Gewichtungen in Abhängigkeit unterschiedlicher Technologiezyklus-Phasen zu anderen Ergebnissen führen als eine Analyse basierend auf identischen Gewichtungen für alle Alternativen. Weiterhin wird deutlich, dass das EUS eine solide Basis bietet, um Energietechnologien in unterschiedlichen Entwicklungsstadien auf Basis multipler Zielsetzungen einander gegenüberzustellen und somit Entscheidungsprozesse in Energiewirtschaft und -politik zu unterstützen. Abschließend werden im achten Kapitel Vor- und Nachteile des entwickelten Analyserahmens sowie Anknüpfungspunkte für weiterführende Forschungsarbeiten diskutiert. 


\subsection{Datensätze in der Kraftwerksdatenbank}

Tabelle 10.1: $\quad$ Energieträger und deren Nutzung zur Stromerzeugung in Europa (Anzahl der bei Platts (2007b) registrierten Kraftwerkseinheiten)

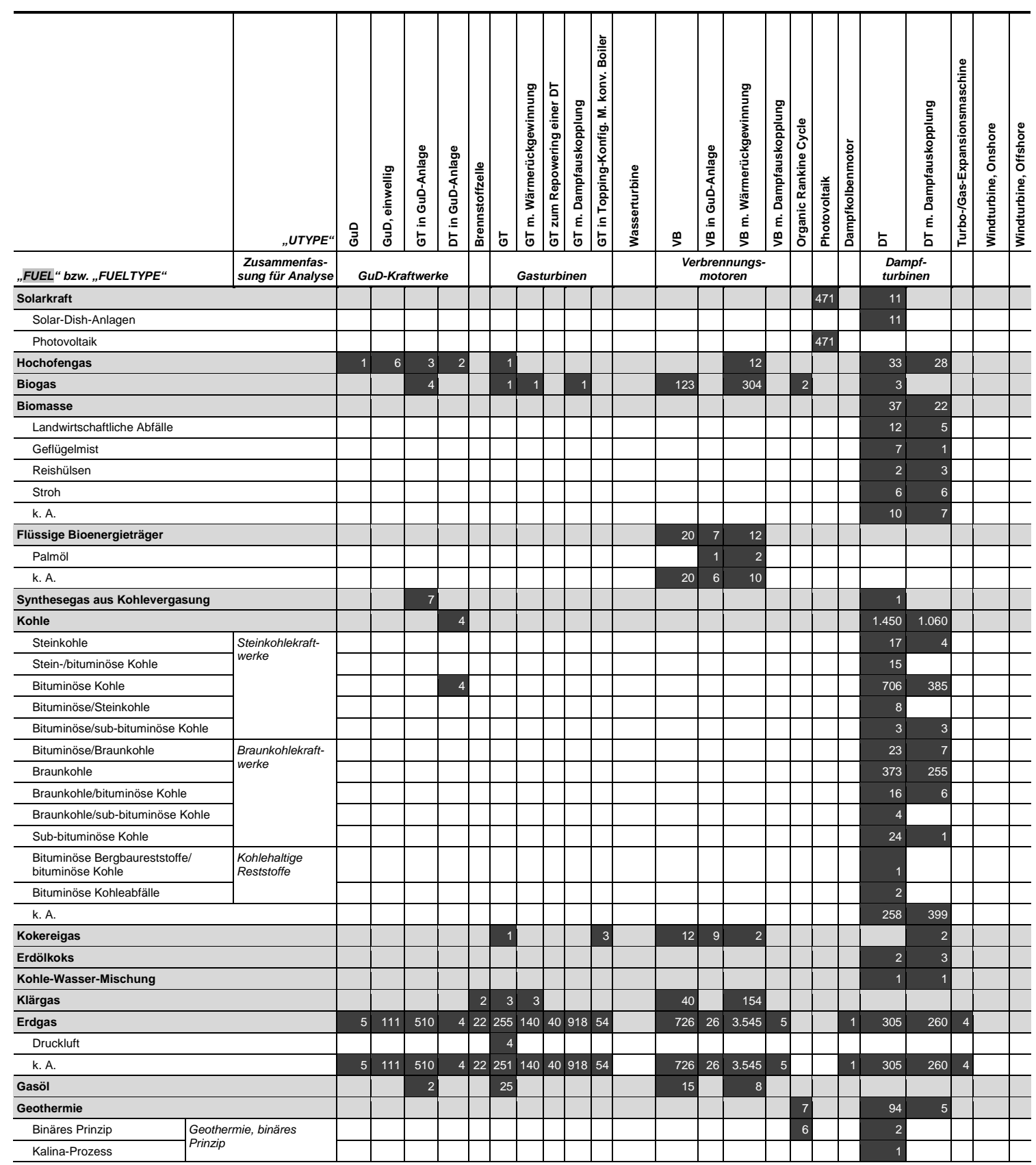

Anmerkungen: DT = Dampfturbine; GT = Gasturbine; GuD = Kombinierter Gas- und Dampfturbinenprozess; $\mathrm{VB}=$ Verbrennungsmotor; $\mathrm{VOC}=$ Volatile Organic Compounds.

Quelle: Basierend auf Platts (2007b). 
Tabelle 10.1 (Fortsetzung)

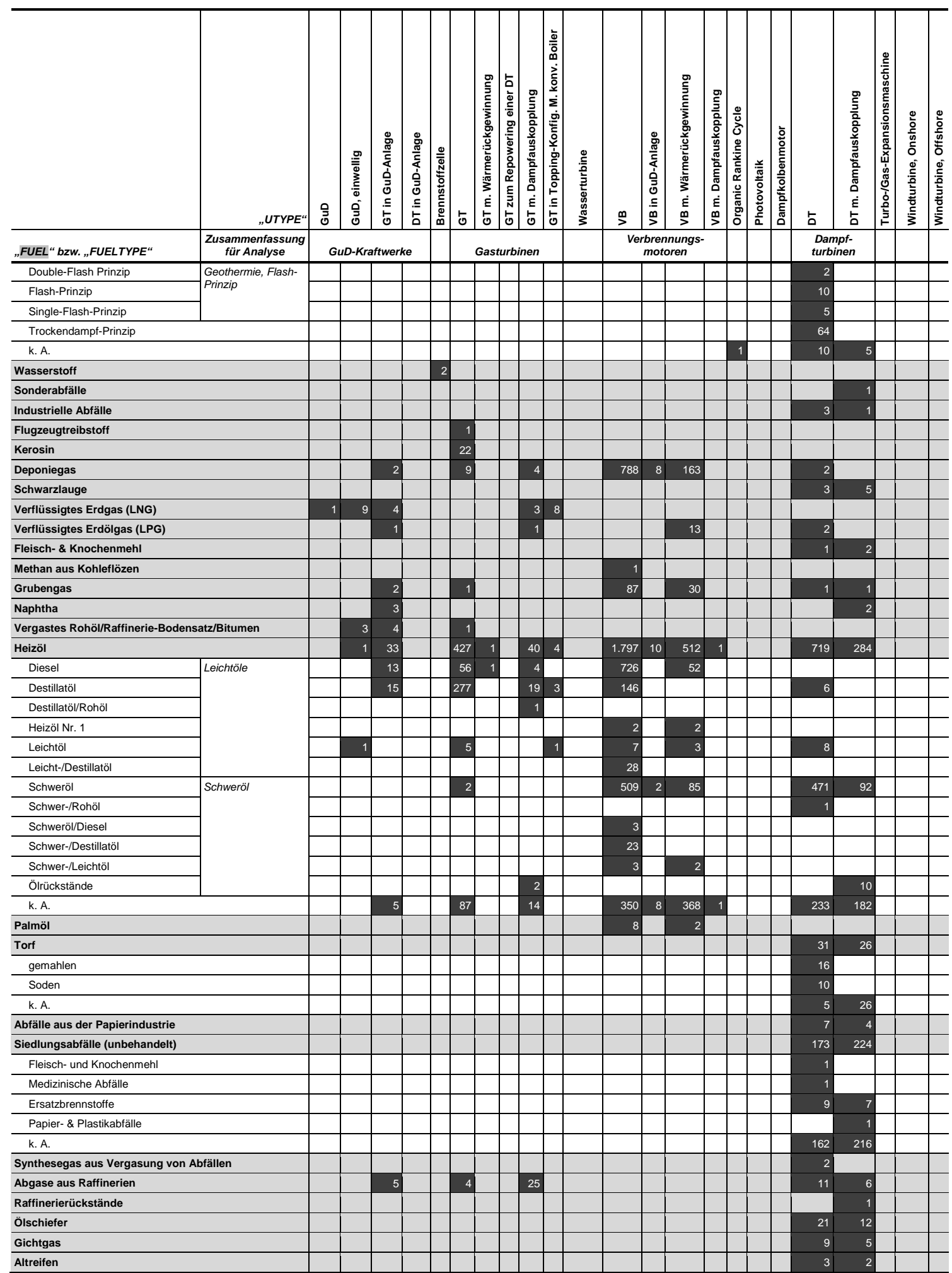

Anmerkungen: DT = Dampfturbine; GT = Gasturbine; GuD = Kombinierter Gas- und Dampfturbinenprozess; VB = Verbrennungsmotor; $\mathrm{VOC}=$ Volatile Organic Compounds.

Quelle: Basierend auf Platts (2007b). 
Tabelle 10.1 (Fortsetzung)

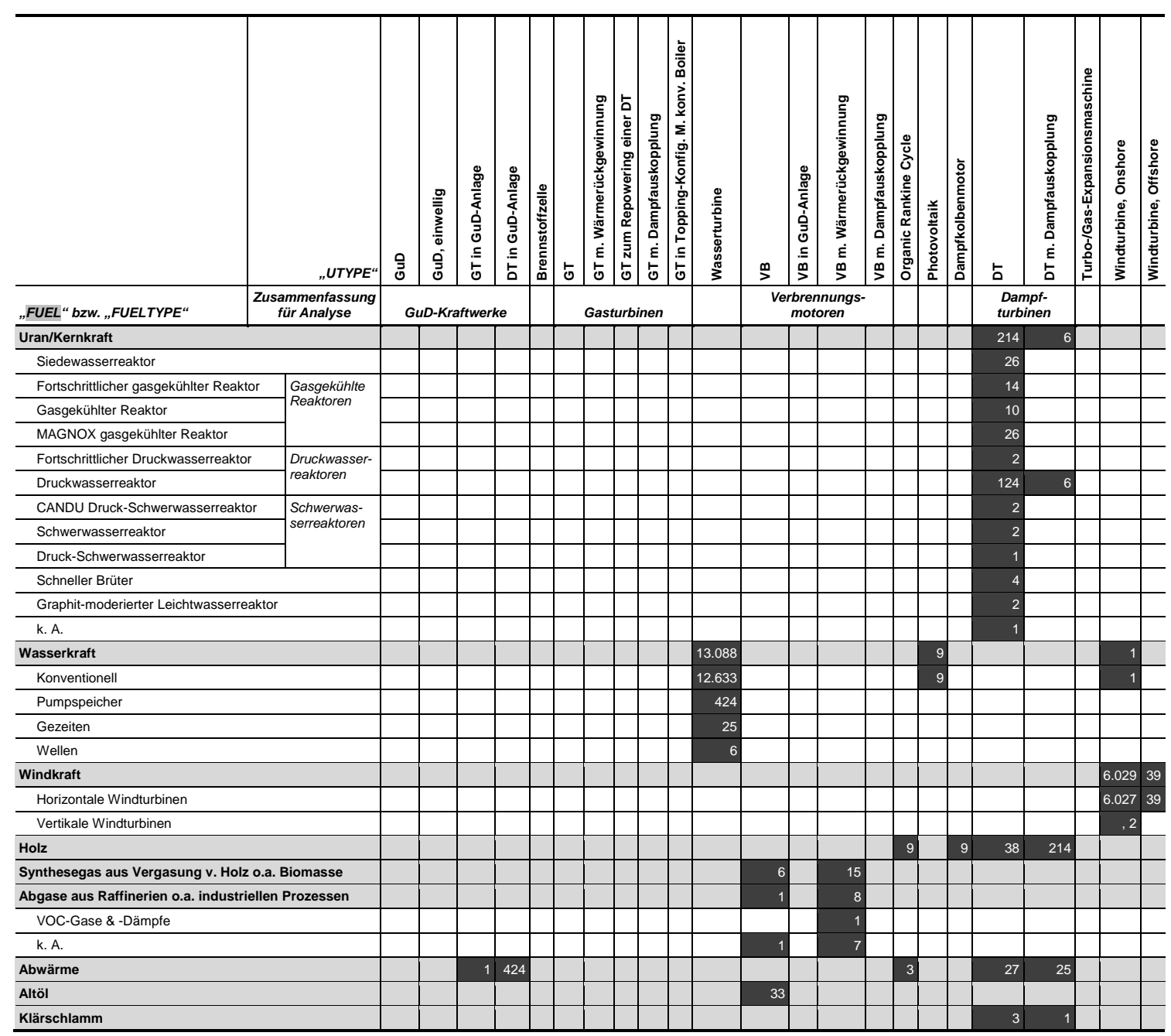

Anmerkungen: DT = Dampfturbine; GT = Gasturbine; GuD = Kombinierter Gas- und Dampfturbinenprozess; $\mathrm{VB}=$ Verbrennungsmotor; $\mathrm{VOC}=$ Volatile Organic Compounds.

Quelle: Basierend auf Platts (2007b). 


\subsection{Darstellung der Entwicklung der Verwendung unter- schiedlicher Energieträgertypen in Europa}

Abbildung 10.1: Nutzung von Braun- und Steinkohle, konventionellen Wasserkraftwerken, Schwerölen und Druckwasserreaktoren zur Stromerzeugung in Europa

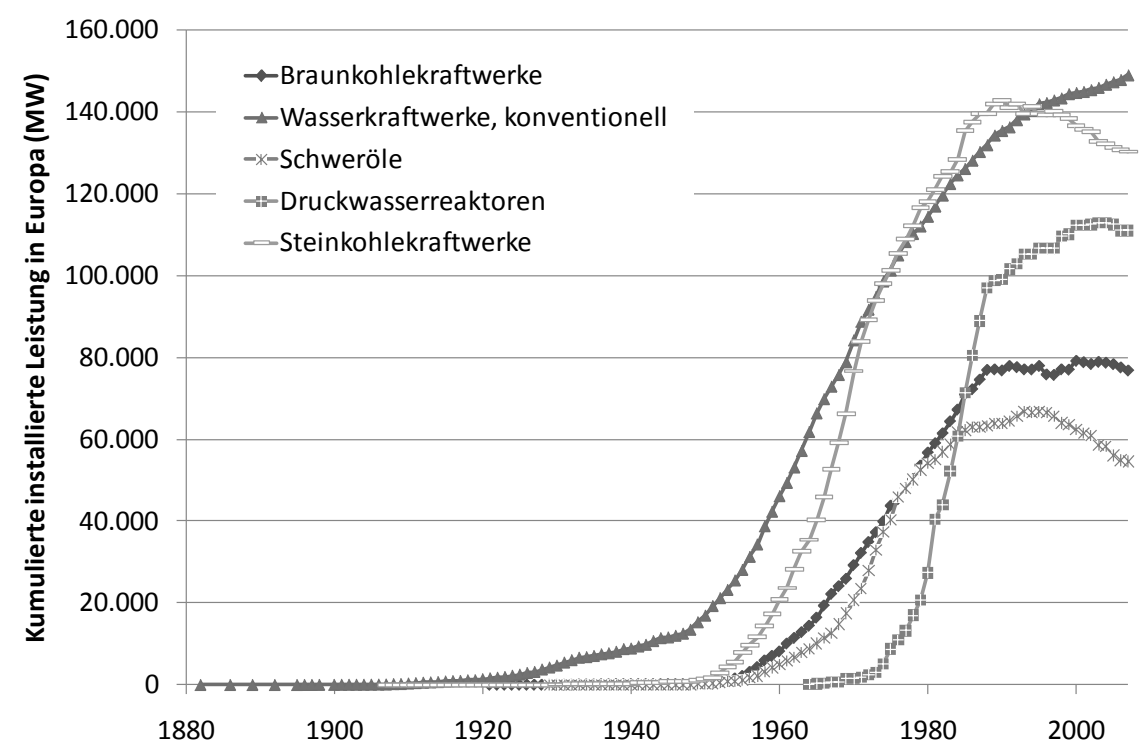

Quelle: Basierend auf Platts (2007b).

Abbildung 10.2: Nutzung von Siedewasserreaktoren, horizontalen Windturbinen, Pumpspeichern, gasgekühlten Reaktoren und Leichtölen zur Stromerzeugung in Europa

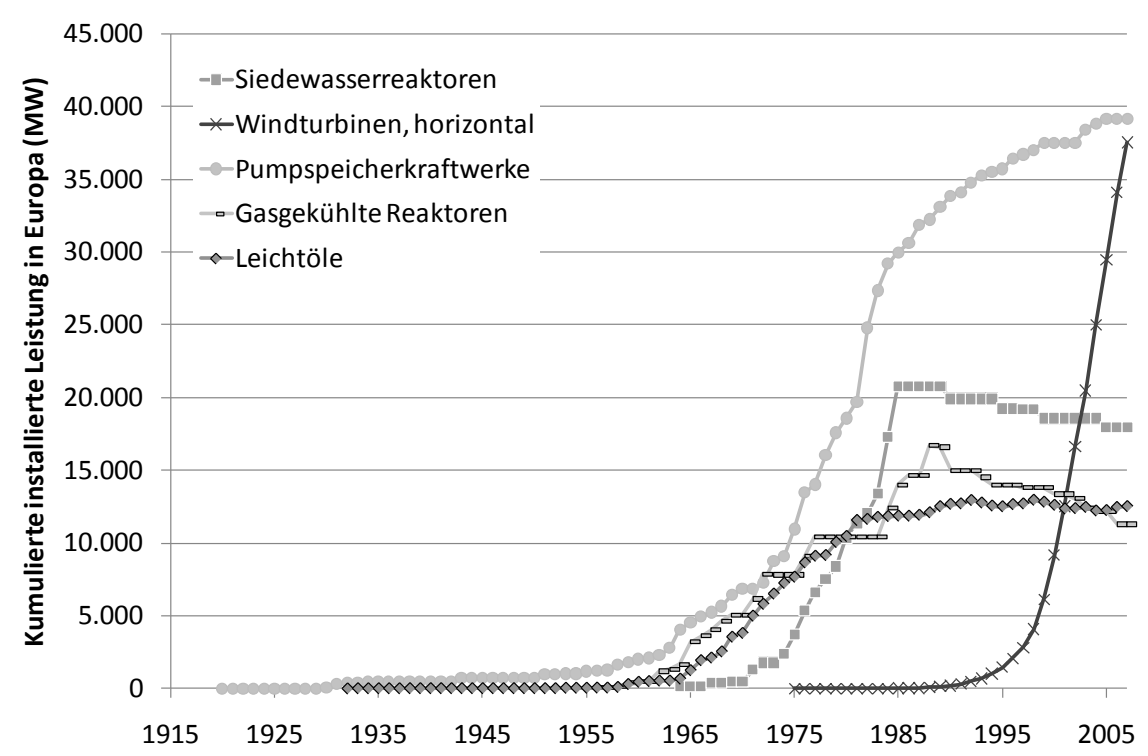

Quelle: Basierend auf Held et al. (2010); Platts (2007b). 
Abbildung 10.3: Nutzung verschiedener Kernkraftwerkstypen sowie der geothermischen Stromerzeugung nach dem Trockendampf-Prinzip, der Photovoltaik und solarthermischer Kraftwerke zur Stromerzeugung in Europa

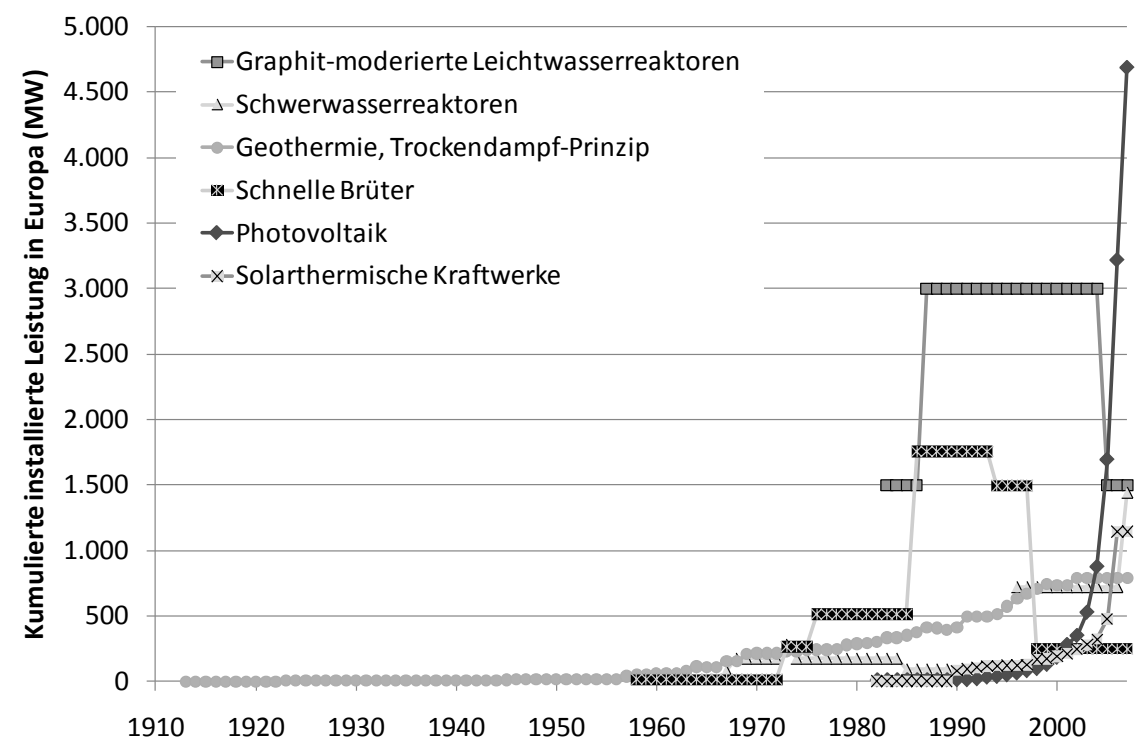

Quelle: Basierend auf Held et al. (2010); Platts (2007b).

Abbildung 10.4: Nutzung von Druckluftspeichern, kohlehaltigen Reststoffen, Stroh, des geothermischen Flash-Prinzip, Geflügelmist, landwirtschaftlicher Abfälle, Ersatzbrennstoffen sowie Wellen- und Gezeitenkraftwerken zur Stromerzeugung in Europa

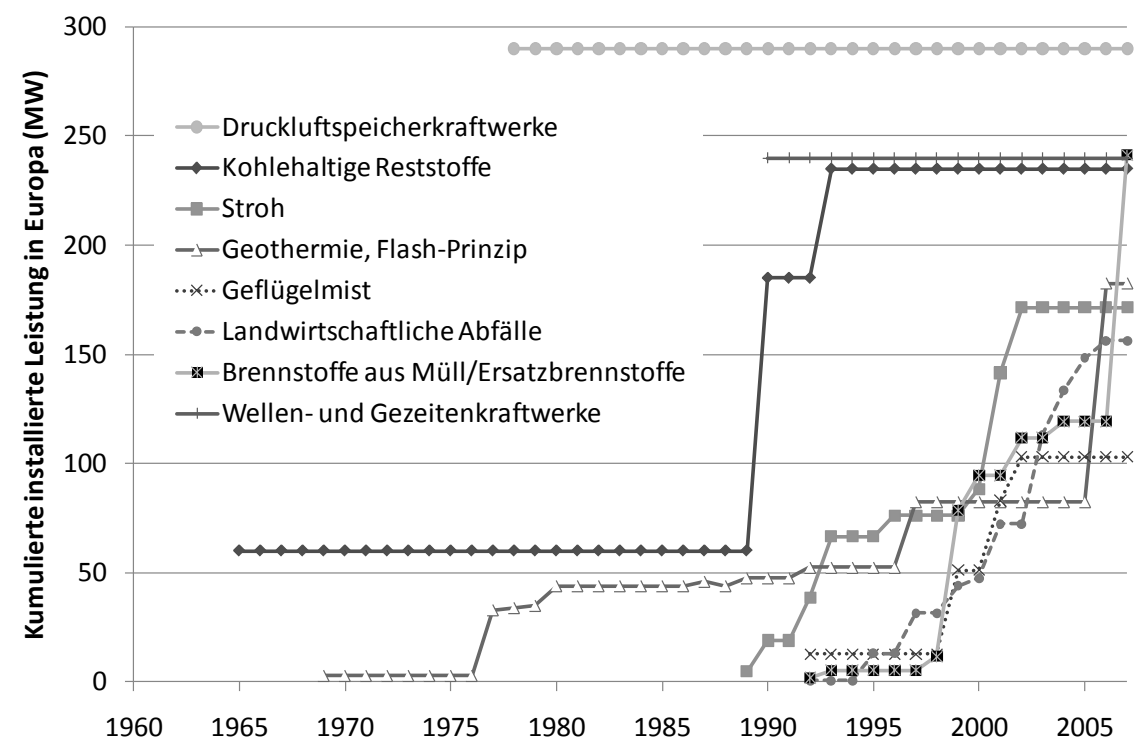

Quelle: Basierend auf Platts (2007b). 
Abbildung 10.5: Nutzung vertikaler Windturbinen, von VOC-Gasen und -Dämpfen, medizinischen Abfällen, Reishülsen, Palmöl, Fleisch- und Knochenmehl sowie der Geothermie nach dem binären Prinzip zur Stromerzeugung in Europa

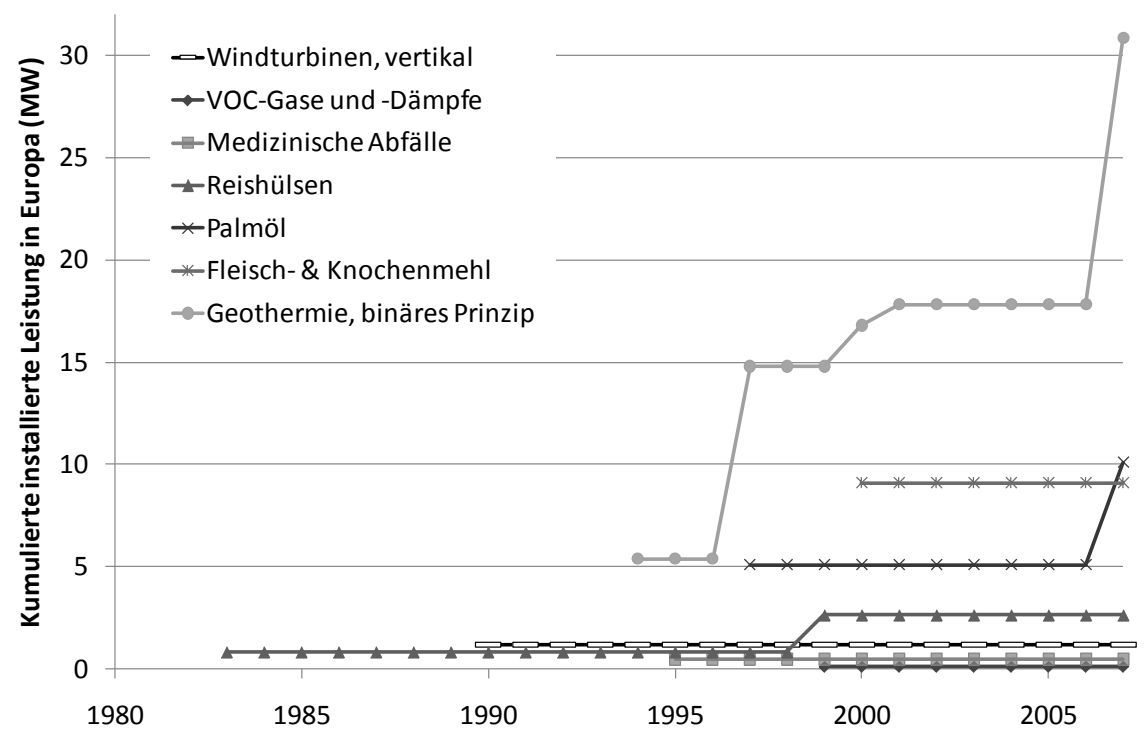

Quelle: Basierend auf Platts (2007b).

Abbildung 10.6: Nutzung sonstiger Energieträger zur Stromerzeugung in Europa

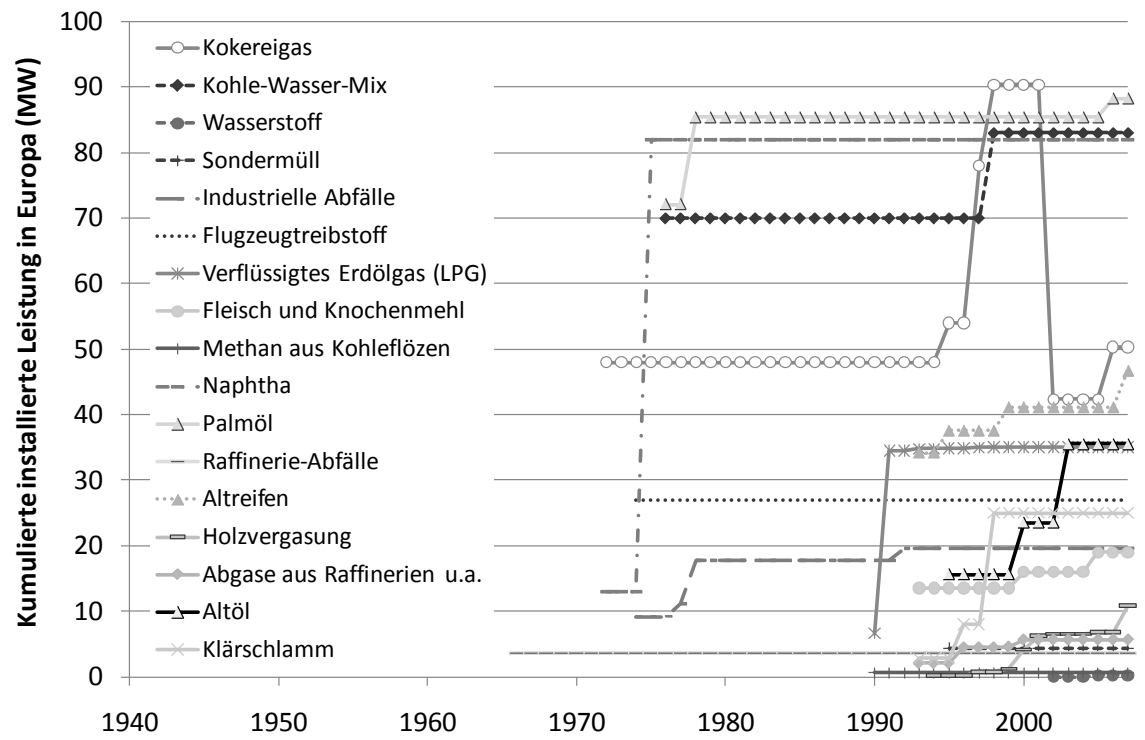

Quelle: Basierend auf Platts (2007b). 


\subsection{Wirtschaftlichkeitsbewertung}

\subsubsection{Generelle Vorgehensweise}

Als Kriterium zur Wirtschaftlichkeitsbewertung werden die mittleren spezifischen Gesamtkosten je bereitgestellter Energieeinheit (Strom- bzw. Wärmegestehungskosten) betrachtet. Grundlage der Wirtschaftlichkeitsbewertung ist die Annuitäten-Methode, die in der Energiewirtschaft aufgrund der hohen Anschaulichkeit häufig zur Anwendung kommt (Götze 2006; Konstantin 2009). Dabei werden Investitionen sowie fixe und variable Betriebskosten berücksichtigt. Investitionen (gesamt und/oder für einzelne Komponenten) sowie typische Fixkosten je Jahr (gesamt und/oder für verschiedene Positionen, z.B. Versicherung, Wartung etc.) sind in der MTB-Datenbank je Technologiedatensatz hinterlegt. Variable Betriebskosten entstehen insbesondere aufgrund des Bedarfs an Primär- bzw. Sekundärenergieträgern. Der Bedarf an Energieträgern je Jahr ergibt sich aus der jährlich erzeugten Nutzenergiemenge (Strom oder Nutzwärme in MWh/a) dividiert durch den Jahresnutzungsgrad der eingesetzten Technologie(n). Die Kosten für Energieträger je Jahr resultieren aus der Multiplikation der Energieträgernachfrage (MWh/a) mit den Energieträgerpreisen ( $€ / M W h)$. Annahmen zur zukünftigen Entwicklung der Energieträgerpreise ab dem Basisjahr 2010 sind in der Datenbank je Szenario als jährliche Durchschnittswerte in €/MWh (bezogen auf den unteren Heizwert der Energieträger) bis zum Jahr 2050 hinterlegt (vgl. Abschnitt 6.7). Weiterhin sind typische Jahresnutzungsgrade sowie typische Volllaststundenäquivalente (h/a) je Technologie hinterlegt. Für einige Technologien sind Nutzungsgrade unterschieden nach mehreren Komponenten in der Bereitstellungskette gegeben (z.B. Verteilung, Erzeugung, etc.). Der Gesamtjahresnutzungsgrad wird dann aus dem Produkt der Nutzungsgrade der einzelnen Komponenten berechnet. Die Volllaststundenzahl muss gegebenenfalls je nach Anwendungsfall angepasst werden (bspw. für einen Spitzenlastkessel, der in Verbindung mit einer KWK-Anlage eingesetzt wird: Je nachdem wie hoch der Wärmebedarf im entsprechenden Anwendungsfall ist und welchen Anteil davon die KWK-Anlage decken kann, kommt der Spitzenlastkessel mehr oder weniger häufig zum Einsatz). Rechnerisch ergibt sich die erzeugte Endenergiemenge aus der Multiplikation der installierten Leistung mit der je Technologie (bezogen auf einen bestimmten Anwendungsfall) gegebenen Volllaststundenäquivalenten.

Im Folgenden werden die Rechenschritte erläutert, die zur Berechnung der Annuitäten bzw. spezifischen Gesamtkosten in der Datenbank implementiert sind. Grundsätzlich basiert die Annuitäten-Methode auf der Bestimmung des Kapitalwertes aller eingehenden und ausgehenden Zahlungsströme: 


$$
\begin{array}{ll}
K_{0}= & -I_{0}+\sum_{t=1}^{n} \frac{E_{t}-A_{t}}{q^{t}} \\
\text { mit } & \\
K_{0} & \text { Kapitalwert } \\
I_{0} & \text { Anfangsinvestition } \\
E_{t} & \text { Einzahlungen in Periode } \mathrm{t} \\
A_{t} & \text { Auszahlungen in Periode } \mathrm{t} \\
q & =1+i \\
i & \text { Kalkulatorischer Zinssatz } \\
n & \text { Kalkulatorische Nutzungsdauer }
\end{array}
$$

Hier wird nur die Kostenseite betrachtet, da dies für einen Vergleich von Alternativen im Rahmen einer Technologiebewertung ausreicht:

$$
-K_{0}=I_{0}+\sum_{t=1}^{n} \frac{A_{t}}{q^{t}}
$$

Bei den jährlichen Auszahlungen wird in fixe und variable Betriebskosten unterschieden:

$$
\begin{aligned}
& -K_{0}=I_{0}+\sum_{t=1}^{n} \frac{K_{f}+K_{v, t}^{S}}{q^{t}}=I_{o}+\sum_{t=1}^{n} \frac{K_{f}}{q^{t}}+\sum_{t=1}^{n} \frac{K_{v, t}^{S}}{q^{t}} \\
& =I_{o}+K_{f} \cdot \frac{q^{n}-1}{q^{n} \cdot(q-1)}+\sum_{t=1}^{n} \frac{K_{v t,}^{s}}{q^{t}}=I_{o}+K_{f} \cdot B W S+\sum_{t=1}^{n} \frac{K_{v, t}^{s}}{q^{t}}
\end{aligned}
$$

mit

$K_{f} \quad$ Fixe Betriebskosten [€/a]

$K_{v, t}^{s} \quad$ Variable Betriebskosten in Abhängigkeit der Szenarien [€/a]

BWSF Barwertsummenfaktor

$t \quad$ Index für Jahr

$S \quad$ Index für Szenario

Die Annuität wird durch Multiplikation des Barwertes mit dem Annuitätsfaktor berechnet. Der Annuitätsfaktor ist der Kehrwert des Barwertsummenfaktors:

$$
a_{n}=\frac{1}{B W S F}=\frac{q^{n} \cdot(q-1)}{q^{n}-1}
$$

mit

$a_{n} \quad$ Annuitätsfaktor 
Die in der Datenbank gegebenen Anfangsinvestitionen teilen sich für einige Technologiedatensätze auf unterschiedliche Komponenten mit voneinander abweichender Nutzungsdauer auf. Da der Annuitätsfaktor von der Nutzungsdauer abhängt, wird in diesen Fällen bei der Bestimmung der Annuität in einzelne Anlagenkomponenten unterschieden. Nach VDI (2000) ist darüber hinaus für gebäudetechnische Anlagen (betrifft im vorliegenden Fallbeispiel Heizungssysteme zur Hausenergieversorgung) ein Instandsetzungsfaktor zu berücksichtigen, der je nach Technologie bzw. Komponente variiert:

$$
\begin{aligned}
& A N\left(I_{o}\right)=\sum_{k} A N\left(I_{0, k}\right) \\
& A N\left(I_{o, k}\right)=\left(a_{n, k}+f_{k}\right) \cdot I_{o, k}=\left(\frac{q^{n(k)} \cdot(q-1)}{q^{n(k)}-1}+f_{k}\right) \cdot I_{o, k} \\
& \text { mit } \\
& \text { AN }\left(I_{0}\right) \quad \text { Annuität der gesamten Anfangsinvestitionen [€/a] } \\
& A N\left(I_{o, k}\right) \quad \text { Annuität der Anfangsinvestitionen für Komponente k [€/a] } \\
& I_{0, k} \quad \text { Anfangsinvestition für Komponente } \mathrm{k}[€] \\
& a_{n, k} \quad \text { Annuitätsfaktor für Komponente } \mathrm{k} \\
& f_{k} \quad \text { Instandsetzungsfaktor für Komponente } \mathrm{k} \\
& n(k) \quad \text { Kalkulatorische Nutzungsdauer der Komponente } \mathrm{k} \text { [a] }
\end{aligned}
$$

Auch bei den fixen Betriebskosten ist gegebenenfalls in einzelne Komponenten zu unterscheiden. Eine Umrechnung der fixen Betriebskosten in Annuitäten erübrigt sich, da diese bereits als mittlere jährliche Angaben vorliegen:

$$
A N\left(K_{f}\right)=K_{f} \text { und } K_{f}=\sum_{k} K_{f, k}
$$

mit

$K_{f, k} \quad$ Fixe Betriebskosten, die für Komponente $\mathrm{k}$ anfallen [€/a]

Die variablen Betriebskosten werden in erster Linie durch die Kosten für die eingesetzten Energieträger in Abhängigkeit der Szenarien bestimmt. Bei einigen Alternativen sind darüber hinaus Kosten für Hilfsenergie (z.B. Pumpenstrom in Heizungssystemen) sowie Zinskosten für gelagerte Brennstoffe (z.B. Heizöl, Holzpellets; vgl. Oschatz und Mailach 2007) zu berücksichtigen:

$$
K_{v, t}^{S}=K_{B, t}^{S}+K_{L t}^{S}+K_{H, t}^{S}
$$


mit

$K_{B, t}^{S} \quad$ Kosten für Brennstoffe bzw. eingesetzte Energieträger [€/a]

$K_{L, t}^{S} \quad$ Zinskosten für Lagerung von Brennstoffen [€/a]

$K_{H, t}^{S} \quad$ Kosten für Hilfsenergie [€/a]

Für die jährlichen Brennstoffkosten gilt:

$$
K_{B, t}^{s}=p_{B, t}^{S} \cdot \dot{m}_{B} \cdot H_{u, B}
$$

mit

$p_{B, t}^{s} \quad$ Preise für eingesetzte Energieträger bezogen auf unteren Heizwert [€/MWh]

$\dot{m}_{B} \quad$ Jährlich eingesetztes Menge des Energieträgers [kg/a]

$H_{u, B} \quad$ Unterer Heizwert des eingesetzten Energieträgers [MWh/kg]

Die jährlich benötigte Menge an Energieträgern wird über die installierte Leistung, die Volllaststundeäquivalente je Jahr und die Jahresnutzungsgrade je Technologiedatensatz bestimmt:

$$
\begin{aligned}
& \dot{m}_{B} \cdot H_{u, B}=\frac{\dot{W}}{\eta_{n, e l}}+\frac{\dot{Q}}{\eta_{n, t h}}=\left(\frac{P_{e l}}{\eta_{n, e l}}+\frac{P_{t h}}{\eta_{n, t h}}\right) d t \\
& \text { mit } \\
& \dot{W} \quad \text { Jährlich erzeugte Strommenge [MWh/a] } \\
& \dot{Q} \quad \text { Jährlich erzeugte Wärmemenge [MWh/a] } \\
& \eta_{n, e l} \quad \text { Jahresnutzungsgrad der Strombereitstellung } \\
& \eta_{n, t h} \quad \text { Jahresnutzungsgrad der Wärmebereitstellung } \\
& P_{e l} \quad \text { Installierte elektrische Leistung [MW] } \\
& P_{t h} \quad \text { Installierte thermische Leistung [MW] } \\
& d t \quad \text { Betriebsstunden in Vollastäquivalenten [h/a] }
\end{aligned}
$$

Für einige Technologiedatensätze sind Nutzungsgrade für einzelne Komponenten in der Bereitstellungskette gegeben (z.B. Erzeugung, Verteilung, etc.). Der GesamtJahresnutzungsgrad wird dann aus dem Produkt der Nutzungsgrade der einzelnen Komponenten berechnet:

$$
\eta_{n, e l}=\prod_{k} \eta_{n, e l, k} \text { bzW. } \eta_{n, t h}=\prod_{k} \eta_{n, t h, k}
$$


mit

$\eta_{n, e l, k} \quad$ Jahresnutzungsgrad der Komponente $\mathrm{k}$ in der Strombereitstellung

$\eta_{n, t h, k} \quad$ Jahresnutzungsgrad der Komponente $\mathrm{k}$ in der Wärmebereitstellung

Des Weiteren wird bei der Wärmebereitstellung in einigen Fällen unterschieden in die Warmwasserbereitstellung und in die Raumbeheizung. In diesen Fällen gilt:

$$
\begin{aligned}
& \dot{Q}=\dot{Q}_{h}+\dot{Q}_{w} \\
& \dot{m}_{B} \cdot H_{u, B}=\frac{\dot{W}}{\eta_{n, e l}}+\frac{\dot{Q}_{h}}{\eta_{n, t h, h}}+\frac{\dot{Q}_{w}}{\eta_{n, t h, w}} \\
& \text { mit } \\
& \dot{Q}_{h} \quad \text { Jährlich bereit gestellte Heizwärme [MWh/a] } \\
& \dot{Q}_{w} \quad \text { Jährliche Warmwasserbereitstellung [MWh/a] } \\
& \eta_{n, t h h} \quad \text { Jahresnutzungsgrad der Heizenergiebereitstellung } \\
& \eta_{n, t h, w} \quad \text { Jahresnutzungsgrad der Warmwasserbereitstellung }
\end{aligned}
$$

Zur Abschätzung der Zinskosten für gelagerte Brennstoffe ist das Verhältnis der mittleren Lagermenge zur insgesamt je Jahr eingesetzten Brennstoffmenge hinterlegt. Die Zinskosten werden wie folgt berechnet:

$$
K_{L, t}^{S}=i \cdot v_{L} \cdot K_{B, t}^{S}
$$

mit

$v_{L} \quad$ Verhältnis der mittleren Lagermenge zur jährlichen Einsatzmenge eines Energieträgers

Zur Abschätzung der Kosten für Hilfsenergie ist das Verhältnis der benötigten Hilfsenergie zur erzeugten Endenergiemenge hinterlegt:

$$
\begin{array}{ll}
K_{H, t}^{S}= & v_{H} \cdot(\dot{W}+\dot{Q}) \cdot p_{H, t}^{S} \\
\text { mit } & \\
v_{H} & \text { Verhältnis von benötigter Hilfsenergie zur erzeugten Endenergie } \\
p_{H, t}^{S} & \text { Preis für den benötigten Hilfsenergieträger im Jahr t (z.B. Strompreise für } \\
& \text { Haushaltskunden) }
\end{array}
$$

Die Annuität der variablen Betriebskosten wird schließlich in Abhängigkeit der Brennstoffpreise je Szenario und der Nutzungsdauer der Technologie berechnet:

$$
A N\left(K_{v}^{s}\right)=a_{n} \cdot \sum_{t=1}^{n} \frac{K_{v t}^{s}}{q^{t}}
$$


Unter Berücksichtigung der Investitionen sowie fixen und variablen Betriebskosten kann dann die Annuität der Gesamtkosten je Technologie berechnet werden:

$$
A N_{g e s}^{s}=\sum_{k} A N\left(I_{0, k}\right)+K_{f}+A N\left(K_{v}^{t}\right)
$$

Bei KWK-Technologien wird die so berechnete Annuität zunächst auf die Produkte Strom und Wärme aufgeteilt. Dazu wird ein Allokationsfaktor basierend auf dem Exergiegehalt der erzeugten Produktmengen angewendet (zur Begründung der Wahl des Allokationsfaktors vgl. Abschnitt 7.1.3.1). Der Exergiegehalt der erzeugten Produktmengen kann auf Basis der erzeugten Endenergiemengen berechnet werden. Dabei ist zu berücksichtigen, dass gegebenenfalls nur ein Teil der erzeugten Wärme verwendet werden kann:

$$
\begin{array}{ll}
E_{W}=\dot{W} \text { bzw. } E_{Q}=\dot{Q}_{n} \cdot \eta_{C} \text { mit } \dot{Q}_{n}=\dot{Q}-\dot{Q}_{a b} \text { und } \eta_{C}=1-\frac{T_{U}}{T_{Q}} \\
\text { mit } \\
E_{W} \quad \text { Exergie der Stromerzeugungsmenge [MWh/a] } \\
E_{Q} \quad \text { Exergie der Wärmeerzeugungsmenge [MWh/a] } \\
\dot{Q}_{n} \quad \text { Genutzter Anteil der erzeugten Wärmemenge [MWh/a] } \\
\dot{Q}_{a b} \quad \text { Anteil der Wärmemenge, der keinem Verwendungszweck zugeführt wird } \\
\eta_{C} \quad \text { Carnot-Faktor } \\
T_{U} \quad \text { Temperatur der Umgebung }[\mathrm{K}] \\
T_{Q} & \text { Temperatur der Wärmeabgabe }[\mathrm{K}]
\end{array}
$$

Um den Anteil der Wärmemenge berechnen zu können, der in stromgeführten KWKAnlagen erzeugt, jedoch keinem Verwendungszweck zugeführt wird, ist je nach Anwendungsfall in der MTB-Datenbank die Stundenzahl (in Volllastäquivalenten) anzugeben, in denen eine KWK-Anlage zwar betrieben wird, die erzeugte Wärmemenge jedoch nicht genutzt werden kann. Die Allokationsfaktoren für Strom bzw. Wärme resultieren aus:

$$
\begin{aligned}
& a l_{e l}=\frac{E_{W}}{E_{w}+E_{Q}} \text { bzw. } a l_{t h}=\frac{E_{Q}}{E_{W}+E_{Q}} \\
& \text { mit } \\
& a l_{e l} \quad \text { Exergetischer Allokationsfaktor Strom } \\
& a l_{t h} \quad \text { Exergetischer Allokationsfaktor Wärme }
\end{aligned}
$$


Die Annuität kann mit diesem Faktor auf die Produkte Strom und Wärme aufgeteilt werden:

$$
A N_{e l}^{s}=a l_{e l} \cdot A N_{\text {ges }}^{s} \text { bzW. } A N_{t h}^{s}=a l_{t h} \cdot A N_{\text {ges }}^{s}
$$

In einigen Fällen fallen weitere Investitionen und Betriebskosten für Komponenten an, die nur der Strom- oder Wärmeseite zuzurechnen sind. Beispielsweise werden Investitionen für ein Nahwärmenetz, in das die Wärme aus einem BHKW eingespeist wird (so zum Beispiel in Jühnde), nur der Wärmeseite des BHKW zugerechnet, da das Nahwärmenetz nicht für die Strombereitstellung benötigt wird. Die Annuität für solche Investitionen bzw. fixen Betriebskosten werden separat berechnet bzw. gegeben und zur zuvor berechneten Annuität addiert:

$$
\begin{aligned}
& A N_{e l}^{s *}=A N_{e l}^{s}+\sum_{k, e l} a_{n, k} \cdot I_{0, k, e l}+\sum_{k, e l} K_{f, k, e l} \text { bzW. } A N_{t h}^{s *}=A N_{t h}^{s}+\sum_{k, h} a_{n, k} \cdot I_{0, k, h}+\sum_{k, t h} K_{f, k, t h} \\
& \text { mit } \\
& I_{0, k, l} \quad \text { Anfangsinvestitionen für Komponenten, die ausschließlich für die Strom- } \\
& \text { bereitstellung erforderlich sind [€] } \\
& I_{0, k \downarrow h} \quad \text { Anfangsinvestitionen für Komponenten, die ausschließlich für die Wärme- } \\
& \text { bereitstellung erforderlich sind }[€] \\
& K_{f k, e l} \quad \text { Fixe Betriebskosten, die aufgrund von Komponenten anfallen, die aus- } \\
& \text { schließlich für die Strombereitstellung erforderlich sind [€/a] } \\
& K_{f, k, h} \quad \text { Fixe Betriebskosten, die aufgrund von Komponenten anfallen, die aus- } \\
& \text { schließlich für die Wärmebereitstellung erforderlich sind [€/a] }
\end{aligned}
$$

Die mittleren spezifischen Kosten der Strom- bzw. Wärmebereitstellung werden schließlich durch Division der Annuitäten durch die bereitgestellten Strom- bzw. Wärmemengen berechnet:

$$
c_{m, e l}^{s}=\frac{A N_{e l}^{S *}}{\dot{W}} \text { bzW. } c_{m \neq h}^{s}=\frac{A N_{t h}^{s} *}{\dot{Q}_{n}}
$$

\subsubsection{Anwendung im Fallbeispiel Bioenergiedörfer}

Im Folgenden werden die Datenquellen und Annahmen erläutert, die der Schätzung der mittleren spezifischen Energiebereitstellung zugrunde liegen, die als Wirtschaftlichkeitskriterium im Fallbeispiel Bioenergiedörfer angewendet werden.

\subsubsection{Alternative 1}

Als Referenz wird im Anwendungsbeispiel Bioenergiedörfer die Stromversorgung aus dem öffentlichen Netz und die Wärmeversorgung auf Basis konventioneller Heizölkessel betrachtet. Für den deutschen Strom-Mix werden zur Bewertung der Wirtschaft- 
lichkeit Verbraucherpreise für Haushalte zugrunde gelegt, da diese für die Stromkunden in Jühnde maßgeblich sind. Dabei ist die Entwicklung der Preise in Abhängigkeit der Szenarien zu beachten. Aus Sicht des Verbrauchers sind keine weiteren Investitionen oder fixen Betriebskosten zu berücksichtigen. Als Referenz der Wärmebereitstellung wird ein konventioneller Heizölkessel für einen Einfamilien-Haushalt betrachtet. Zur Wirtschaftlichkeitsbewertung unterschiedlicher Heizungssysteme für die Einzelobjektversorgung wird der Vergleich bei Oschatz und Mailach (2007) zugrunde gelegt. Dies betrifft neben dem konventionellen Heizöl-Kessel den Erdgas-Brennwertkessel (Alternativen 5 und 6), die Wärmepumpe (Alternative 7) und die Holzpellets-Heizung (Alternative 8). Der bei Oschatz und Mailach (2007) durchgeführte Heizkostenvergleich wird jedoch modifiziert, da sich dieser zum einen auf Neubauten beziehen und zum anderen konstante Brennstoffpreise zugrunde legt. Für Bioenergiedörfer ist hingegen eine Bewertung unterschiedlicher Heizungssysteme für Bestandsbauten mit höherem Wärmebedarf relevant. Zudem werden in der vorliegenden Arbeit die Kosten in Abhängigkeit unterschiedlicher Szenarien zur Entwicklung der Brennstoffpreise untersucht.

Nach Ruppert et al. (2008) wird ein Gesamtwärmebedarf von 24.000 kWh/a für einen typischen 4-Personen-Haushalt zugrunde gelegt. Typische Vollbenutzungsstunden eines Heizungssystems liegen nach Karl (2006) für ein Einfamilienhaus (Altbau) bei $1.600 \mathrm{~h} / \mathrm{a}$. Daher wird eine installierte Heizleistung von $(24.000 \mathrm{kWh} / \mathrm{a}) /(1.600 \mathrm{~h} / \mathrm{a})=$ $15 \mathrm{~kW}_{\text {th }}$ angenommen. Für den spezifischen Wärmebedarf zur Warmwasserbereitstellung wird angenommen, dass dieser weitgehend unabhängig von Dämmstandard des Gebäudes ist und nach Oschatz und Mailach (2007) bei $12,5 \mathrm{kWh} / \mathrm{m}^{2}$ a liegt. Bei Annahme einer Wohnfläche von $150 \mathrm{~m}^{2}$ resultiert ein Warmwasserbedarf von $1.875 \mathrm{kWh} / \mathrm{a}$. Bei Annahme eines Gesamtwärmebedarfs von $24.000 \mathrm{kWh} / \mathrm{a}$ resultiert ein Heizwärmebedarf von 22.125 kWh/a bzw. 147,5 kWh/m²a. Diese Annahmen werden gleichermaßen für alle Alternativen zur Wärmebereitstellung in einzelnen Haushalten unterstellt (Tabelle 10.2). Die in die Datenbank einzutragenden Betriebsstunden als Grundlage zur Berechnung des Endenergiebedarfs entsprechen bei $15 \mathrm{~kW}_{\text {th }} 1.475 \mathrm{~h} / \mathrm{a}$ für die Heizung bzw. $125 \mathrm{~h} / \mathrm{a}$ für die Warmwasserbereitstellung (in Volllastäquivalenten). Die Nutzungsgrade einzelner Komponenten der zu bewertenden Heizungssysteme sind in Tabelle 10.3 zusammengefasst. 
Tabelle 10.2: $\quad$ Basisannahmen zur Bewertung der Wirtschaftlichkeit unterschiedlicher Heizungssysteme

\begin{tabular}{l|l|r|r|r}
\hline & & \multicolumn{1}{|c|}{ Gesamt } & \multicolumn{1}{c}{ Warmwasser } & \multicolumn{1}{c}{ Heizung } \\
\hline Spezifischer Wärmebedarf & $\mathrm{kWh} / \mathrm{m}^{2} \mathrm{a}$ & 160 & 12,5 & 147,5 \\
\hline Wohnfläche & $\mathrm{m}^{2}$ & 150 & 1.875 & 22.125 \\
\hline Jährlicher Wärmebedarf & $\mathrm{kWh} / \mathrm{a}$ & 24.000 & 125 & 1.475 \\
\hline Betriebsstunden (Volllastäquivalente) & $\mathrm{h} / \mathrm{a}$ & 1.600 & & \\
\hline Installierte thermische Leistung & $\mathrm{kW}$ & 15 & & \\
\hline
\end{tabular}

Anmerkungen: Annahmen gelten für Alternativen zur Einzelobjektversorgung, 4-PersonenHaushalt, Bestandsbauten.

Quellen: Oschatz und Mailach (2007); Ruppert et al. (2008).

Tabelle 10.3: Jahresnutzungsgrade der Komponenten von Systemen zur Hausenergieversorgung (Heizung und Warmwasser)

\begin{tabular}{l|c|c|c|c}
\hline & Heizöl-Kessel & $\begin{array}{c}\text { Erdgas- } \\
\text { Brennwertkessel }\end{array}$ & $\begin{array}{c}\text { Sole-Wasser- } \\
\text { Wärmepumpe }\end{array}$ & $\begin{array}{c}\text { Holzpellets- } \\
\text { Heizkessel }\end{array}$ \\
\hline $\begin{array}{l}\text { Heizung } \\
\begin{array}{l}\text { Wärmeüberga- } \\
\text { be/Regelung }\end{array}\end{array} \quad 0,98$ & 0,98 & 0,98 & 0,98 \\
\hline Verteilung & 0,96 & 0,96 & 0,99 & 0,96 \\
\hline Wärmeerzeugung & 0,93 & 1,02 & 4,35 & 0,73 \\
\hline Gesamt & 0,875 & 0,96 & 4,22 & 0,687 \\
\hline Warmwasser & 0,7 & 0,7 & 0,7 & 0,7 \\
\hline Verteilung & 0,84 & 0,88 & 3,7 & 0,65 \\
\hline Wärmeerzeugung & 0,588 & 0,616 & 2,59 & 0,455 \\
\hline Gesamt & \multicolumn{5}{|l|}{}
\end{tabular}

Quelle: Oschatz und Mailach (2007).

Neben den Brennstoffkosten in Abhängigkeit der Brennstoffpreise und des Brennstoffbedarfs entstehen verbrauchsabhängige Kosten aufgrund der Bereitstellung von Hilfsenergie (Strom) sowie Zinskosten für gelagerte Brennstoffe (Heizöl, Holzpellets). Dabei wird angenommen, dass der bei Oschatz und Mailach (2007) gegebene Hilfsenergiebedarf proportional zum Heizwärmebedarf steigt. Darüber hinaus sind steigende Strompreise in Abhängigkeit der Szenarien zu beachten. Zur Berechnung der Zinskosten für die Lagerung von Brennstoffen wird nach Oschatz und Mailach (2007) für Heizöl und Holzpellets eine mittlere Lagermenge von $50 \%$ des jährlichen Brennstoffbedarfs angenommen (Tabelle 10.4). 
Tabelle 10.4: $\quad$ Verhältniszahlen zur Bestimmung des Hilfsenergiebedarfs und der mittleren Lagermenge

\begin{tabular}{l|c|c|c|c}
\hline & Heizöl-Kessel & $\begin{array}{c}\text { Erdgas- } \\
\text { Brennwertkessel }\end{array}$ & $\begin{array}{c}\text { Sole-Wasser- } \\
\text { Wärmepumpe }\end{array}$ & $\begin{array}{c}\text { Holzpellets- } \\
\text { Heizkessel }\end{array}$ \\
\hline $\begin{array}{l}\text { Verhältnis Hilfsenergie zu } \\
\text { erzeugter Energie }\end{array}$ & 0,0358 & 0,0358 & 0,0615 & 0,0537 \\
\hline $\begin{array}{l}\text { Verhältnis Lagermenge zu } \\
\text { eingesetzter Brennstoffmenge }\end{array}$ & 0,5 & -- & --- & 0,5 \\
\hline
\end{tabular}

Quellen: Oschatz und Mailach (2007).

Die Investitionen, die für die Wirtschaftlichkeitsbewertung des konventionellen Heizölkessels zugrunde gelegt werden, sind in Tabelle 10.5 zusammengefasst. Fixe Betriebskosten, die zur Wirtschaftlichkeitsbewertung der betrachteten Heizungsalternativen zur Einzelobjektversorgung zu berücksichtigen sind, sind in Tabelle 10.6 zusammengestellt.

Tabelle 10.5: $\quad$ Annahmen zu Investitionen für konventionelle Heizöl-Kessel

\begin{tabular}{l|c|c|c}
\hline & Investitionen & $\begin{array}{c}\text { Kalkulatorische Nut- } \\
\text { zungsdauer }\end{array}$ & $\begin{array}{c}\text { Aufwand für Instand- } \\
\text { setzung }\end{array}$ \\
\cline { 2 - 4 } & $€$ & $\mathrm{a}$ & $\%$ \\
\hline $\begin{array}{l}\text { Wärmeerzeuger (inkl. Speicher, } \\
\text { Regelung und Montage) }\end{array}$ & 6.200 & 20 & $2,0 \%$ \\
\hline Leitungssystem/Heizflächen & 4.700 & 30 & $1,0 \%$ \\
\hline Schornstein/LAS-Durchführung & 2.100 & 50 & $1,0 \%$ \\
\hline Anschluss/Baukosten & 1.400 & 50 & $1,0 \%$ \\
\hline Gas-/Elektroinstallation & 700 & 50 & $1,0 \%$ \\
\hline Tank & 1.500 & 30 & $1,0 \%$ \\
\hline Gesamt & 16.600 & & \\
\hline
\end{tabular}

Quellen: Oschatz und Mailach (2007); Ruppert et al. (2008).

Tabelle 10.6: $\quad$ Fixe Betriebskosten für Heizenergieanlagen zur Einzelobjektversorgung

\begin{tabular}{l|c|c|c|c|c}
\hline & & Heizöl-Kessel & $\begin{array}{c}\text { Erdgas- } \\
\text { Brennwertkessel }\end{array}$ & $\begin{array}{c}\text { Sole-Wasser- } \\
\text { Wärmepumpe }\end{array}$ & $\begin{array}{c}\text { Holzpellets- } \\
\text { Heizkessel }\end{array}$ \\
\hline Schornsteinfeger & $€ / a$ & 58 & 17 & --- & 158 \\
\hline Wartung & $€ / a$ & 150 & 100 & 50 & 220 \\
\hline $\begin{array}{l}\text { Versicherung/ } \\
\text { Überwachung }\end{array}$ & $€ / a$ & 60 & --- & --- & --- \\
\hline Gesamt & $€ / a$ & 268 & 117 & 50 & 378 \\
\hline
\end{tabular}

Quellen: Oschatz und Mailach (2007). 


\subsubsection{Alternative 2}

Alternative 2 entspricht dem in Jühnde umgesetzten Biomasse-Konzept zur Strom- und Wärmeversorgung. Dort wird ein Biogas-BHKW (Gasmotor) mit einer GeneratorLeistung von $716 \mathrm{~kW}_{\mathrm{el}}$ betrieben, das bei vorgegebener Biogas-Qualität einen elektrischen Wirkungsgrad von 40,3\% und einen thermischen Wirkungsgrad von 39,8 \% aufweist (vgl. Ruppert et al. 2008). Das entsprechende BHKW hat nach ASUE (2005) eine thermische Leistung von $701 \mathrm{~kW}_{\text {th. }}$ Je Jahr können damit nach Ruppert et al. (2008, S.19) ca. $5.000 \mathrm{MWh}_{\mathrm{el}}$ Strom erzeugt werden. Den Angaben im Datenblatt (Bioenergiedorf Jühnde eG 2006) zufolge wurden im Jahr 2006 insgesamt jedoch nur 4.000 $\mathrm{MWh}_{\mathrm{e}} / \mathrm{a}$ erzeugt. Dies wird auf Anlaufschwierigkeiten bei der Inbetriebnahme des Biogas-BHKW zurückgeführt. Es wird jedoch angenommen, dass nach Überwindung dieser Anlaufschwierigkeiten eine mittlere Stromerzeugungsmenge von $5.000 \mathrm{MWh}_{\mathrm{e}} / \mathrm{a}$ erreicht wird. Dies entspricht Betriebsstunden von $6.983 \mathrm{~h} / \mathrm{a}$ (in Volllastäquivalenten). Bei dieser Betriebsstundenzahl werden ca. $4.895 \mathrm{MWh}_{\mathrm{th}} / \mathrm{a}$ Wärme erzeugt.

Zu unterscheiden ist die erzeugte von der genutzten Wärmemenge, da in diesem Fall die Wärme als Nebenprodukt der Stromerzeugung entsteht und nicht (wie etwa bei einem Heizöl-Kessel in einem einzelnen Haushalt) nur bei einem zeitgleichen Bedarf erzeugt wird. Die im Biogas-BHKW Jühnde erzeugte Wärmemenge wird entweder in das Nahwärmenetz eingespeist oder vor Ort für den Vergärungsprozess oder (seit 2007) zur Vortrocknung von Holzhackschnitzeln eingesetzt (Karpenstein-Machan und Schmuck 2007). Für das Jahr 2006 ist für das Biogas-BHKW bei Bioenergiedorf Jühnde eG (2006) ein Output von 4.000 $\mathrm{MWh}_{\mathrm{el}} / \mathrm{a}$ und 2.800 $\mathrm{MWh}_{\mathrm{th}} / \mathrm{a}$ gegeben. Diese im Jahr 2006 erzeugte Strommenge entspricht bei einer installierten elektrischen Leistung von $716 \mathrm{~kW}_{\text {el }}$ Betriebsstunden von $5.587 \mathrm{~h} / \mathrm{a}$ (Volllastäquivalente). Rechnerisch werden demnach bei einer installierten thermischen Leistung von $701 \mathrm{~kW}_{\text {th }}$ ca. $3.916 \mathrm{MWh}_{\mathrm{th}} / \mathrm{a}$ Wärme erzeugt. Es wird angenommen, dass die Differenz zwischen der berechneten und der im Datenblatt genannten erzeugten Wärmemenge darauf zurückzuführen ist, dass im Datenblatt nur die Wärmemenge genannt ist, die ins Nahwärmenetz eingespeist bzw. vor Ort verwendet wird. Um für eine höhere Betriebsstundenzahl bzw. für eine höhere erzeugte Strommenge je Jahr von $5.000 \mathrm{MWh}_{\mathrm{e}} / \mathrm{a}$ die Wärmemengen zu berechnen, die im Biogas-BHKW erzeugt, ins Nahwärmenetz eingespeist und vor Ort eingesetzt werden, sind weitere Annahmen erforderlich:

- Das Verhältnis der thermischen Energiemenge, die jährlich für den Vergärungsprozess eingesetzt wird, zur insgesamt je Jahr durch das Biogas-BHKW erzeugten Energiemenge (Strom + Wärme) bleibt konstant. Dies kann dadurch begründet werden, dass bei einer höheren Strom- und Wärmeerzeugung mehr Biogas und somit auch mehr Biomasse für den Vergärungsprozess eingesetzt werden muss. 
- Zur Vortrocknung der Holzhackschnitzel wird nur überschüssige thermische Energie aus dem Biogas-BHKW eingesetzt. Die in das Nahwärmenetz eingespeiste Energiemenge bleibt davon unbeeinflusst.

- Das Verhältnis der jährlichen Wärmemenge, die im BHKW erzeugt und in das Nahwärmenetz eingespeist wird, zur insgesamt pro Jahr im BHKW erzeugten Wärmemenge bleibt konstant.

- Die Wärmemenge, die in Jühnde von den an das Nahwärmenetz angeschlossenen Haushalten jährlich nachgefragt wird, bleibt konstant. Somit bleibt auch die insgesamt je Jahr in das Nahwärmenetz eingespeiste Wärmemenge konstant bei 3,5 Mio. $k W h_{\text {th }}$ nach Bioenergiedorf Jühnde eG (2006).

Aus den Angaben bei Bioenergiedorf Jühnde eG (2006) kann die im Jahr 2006 für die Vergärung benötigte Wärmemenge ermittelt werden:

$$
\begin{aligned}
& \dot{Q}_{B H K W, \text { eerg }}^{2006}=\dot{Q}_{B H K W, \text { Netz }}^{2006}+\dot{Q}_{H W}^{2006}-\dot{Q}_{\text {Neth , in }} \\
& =2.800 M W h_{t h} / a+1.500 M W h_{t h} / a-3.500 M W h_{t h} / a \\
& =800 \mathrm{MWh}_{\mathrm{th}} / \mathrm{a} \\
& \text { mit }
\end{aligned}
$$

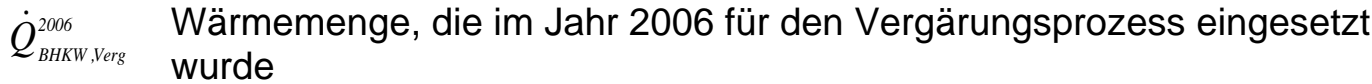

$$
\begin{aligned}
& \dot{Q}_{\text {BHKW Netz }}^{2006} \quad \text { Anteil der Wärmemenge, die im Jahr } 2006 \text { im BHKW erzeugt und ins } \\
& Q_{B н K W, \text { Nez }} \quad \text { Nahwärmenetz eingespeist wurde } \\
& \dot{Q}_{H W}^{2006} \quad \text { Wärmemenge, die im Jahr } 2006 \text { im Heizwerk (Holzhackschnitzel- } \\
& \text { Heizkessel + Öl-Spitzenlastkessel) erzeugt wurde (Annahme: wird voll- } \\
& \text { ständig ins Nahwärmenetz eingespeist) } \\
& \dot{Q}_{\text {Netr, }, \text { na }} \quad \text { Wärmemenge, die in das Nahwärmenetz jährlich eingespeist wird (ent- }
\end{aligned}
$$

Das Verhältnis der für den Vergärungsprozess eingesetzten Energiemenge zur insgesamt im Biogas-BHKW erzeugten Energiemenge kann dann wie folgt berechnet werden:

$$
v_{\text {Verg }}=\frac{\dot{Q}_{B H K W, \text { Verg }}^{2006}}{\dot{W}_{B H K W}^{2006}+\dot{Q}_{B \text { BHW ,erz }}^{2006}}
$$

mit

$$
\begin{array}{ll}
\dot{W}_{B H K W}^{2006} & \text { Strommenge, die im Jahr } 2006 \mathrm{im} \text { BHKW erzeugt wurde (4.000 MWh/a) } \\
\dot{Q}_{B H K W, \text { erz }}^{2006} & \text { Wärmemenge, die im Jahr } 2006 \mathrm{im} \text { BHKW erzeugt wurde }
\end{array}
$$




$$
\dot{Q}_{\text {BHKW, er }}(t)=P_{t h} \cdot d(t)
$$

mit

$P_{t h} \quad$ Thermische Leistung des BHKW (701 kW)

$d(t) \quad$ Betriebsstunden im Jahr $t$

$$
d(2006)=\frac{\dot{W}_{\text {BнКW }}(2006)}{P_{e l}}=\frac{4.000 \mathrm{MWh} / \mathrm{a}}{716 \mathrm{~kW}}=5.587 \mathrm{~h} / \mathrm{a}
$$

mit

$P_{e l} \quad$ Elektrische Leistung des BHKW (716 kW)

$$
\begin{aligned}
& \Rightarrow \dot{Q}_{\text {BHKW } 2017}^{2006}=701 \mathrm{~kW} \cdot 5.587 \mathrm{~h} / a \approx 3.916 \mathrm{kWh} / a \\
& \Rightarrow v_{\text {Verg }}=\frac{800}{4.000+3.916} \approx 0,101
\end{aligned}
$$

Bei einer Stromerzeugung von $5.000 \mathrm{MWh}_{\mathrm{e}} / \mathrm{a}$, die im Fallbeispiel zugrunde gelegt wird, und einer entsprechenden erzeugten Wärmemenge von ca. 4.895 $\mathrm{MWh}_{\text {th }} / \mathrm{a}$ resultiert daraus ein Wärmebedarf für die Vergärung von $999 \mathrm{MWh}_{\mathrm{th}} / \mathrm{a}$. Dementsprechend stehen zur Einspeisung ins Nahwärmenetz noch ca. 3.896 $\mathrm{MWh}_{\mathrm{th}} / \mathrm{a}$ zur Verfügung. Aufgrund der zeitlichen Verteilung von Wärmeangebot und Wärmenachfrage kann diese Wärmemenge jedoch nicht vollständig abgesetzt werden. Folglich wird nur ein Teil davon ins Nahwärmenetz eingespeist. Dabei wird angenommen, dass das Verhältnis der insgesamt über das Jahr eingespeisten zur insgesamt jährlich erzeugten Wärmemenge des BHKW konstant bleibt. Auf Basis der Daten, die für 2006 zur Verfügung stehen, kann dieses Verhältnis berechnet werden:

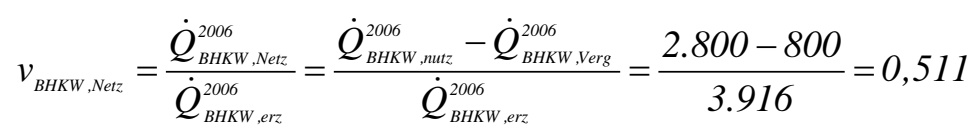

Demnach kann von der im BHKW erzeugten Wärmemenge von $4.895 \mathrm{MWh}_{\text {th }} / \mathrm{a}$ (bei einer Stromerzeugung von $5.000 \mathrm{MWh}_{\mathrm{e}} / \mathrm{a}$ ) ein Anteil von ca. $2.501 \mathrm{MWh}_{\mathrm{th}} / \mathrm{a}$ über das Nahwärmenetz abgesetzt werden. Zusätzlich müssen ca. $1.000 \mathrm{MWh}_{\mathrm{th}} / \mathrm{a}$ ins Nahwärmenetz eingespeist werden, um den Wärmebedarf der Haushalte über das ganze Jahr decken zu können. Dieser Bedarf wird über das Heizwerk (Holzhackschnitzel und ÖlSpitzenlastkessel) gedeckt. Entsprechend den Annahmen können von der erzeugten Wärmemenge insgesamt $3.500 \mathrm{MWh}_{\mathrm{th}} / \mathrm{a}$ zur Einspeisung ins Nahwärmenetz und für den Vergärungsprozess verwendet werden. Ein Anteil von $1.395 \mathrm{MWh}_{\text {th }} / \mathrm{a}$ kann nicht verwendet werden (entspricht bei einer thermischen Leistung von $701 \mathrm{~kW}_{\text {th }} 1.990$ Volllaststunden je Jahr, in denen das Biogas-BHKW läuft, die Wärme jedoch nicht abge- 
setzt werden kann). Diese Abwärme-Menge ist bei der Berechnung des exergetischen Allokationsfaktors und der mittleren spezifischen Kosten zu berücksichtigen.

Die Investitionen für das Bioenergie-Konzept in Jühnde belaufen sich auf 2,9 Mio. € für die Biogas- und Stromproduktion, auf 0,9 Mio. $€$ für die Wärmeerzeugung und 1,6 Mio. € für die Wärmeverteilung (Ruppert et al. 2008). Die Investitionen für „Biogasund Stromproduktion“ von 2,9 Mio. € werden gemäß dem exergetischen Allokationsfaktor den Produkten Strom und Wärme, die im Biogas-BHKW erzeugt werden, zugerechnet. Ebenso wird mit den jährlichen Betriebskosten des BHKW verfahren. Nach ASUE (2005) können die Kosten für einen Instandhaltungsvertrag für ein Biogas-BHKW mit $716 \mathrm{~kW}_{\mathrm{el}}$ einschließlich aller Wartungs- und Reparaturarbeiten, Ersatzteile und Betriebsstoffe (außer Brennstoffe) und Generalüberholung auf 1,149 Ct/kWh $/ \mathrm{k}_{\mathrm{el}}$ geschätzt werden. Bei einer Stromerzeugung von 5 Mio. $\mathrm{kWh}_{\mathrm{el}} / \mathrm{a}$ entspricht dies jährlichen Kosten in Höhe von $57.444 € / a$ (ca. 2 \% der Investitionen für Biogas- und Stromproduktion).

Die Investitionen und fixen Betriebskosten für das Nahwärmenetz werden entsprechend den angenommenen Anteilen an der Nahwärmeversorgung der Wärmeseite des Biogas-BHKW und dem Heizwerk (siehe unten) zugerechnet (vgl. Tabelle 10.7). Als fixe Betriebskosten für Wartung und Instandhaltung des Nahwärmenetzes sind nach FNR (2005) $2 \% / a$ bezogen auf die Investitionen zu berücksichtigen. Die anteiligen Investitionen bzw. fixen Betriebskosten für das Nahwärmenetz werden bei der Wirtschaftlichkeitsbewertung der Wärmebereitstellung aus dem BHKW berücksichtigt.

Werden die aktuellen Förderbedingungen des KWKG beachtet, ist weiterhin zu berücksichtigen, dass der Bau von Nahwärmenetzen nach §7a KWKG2008 mit einem Euro je mm Nenndurchmesser pro Meter Trassenlänge (maximal mit 5 Mio. Euro je Projekt bzw. $20 \%$ der Investitionen) gefördert wird. Die Investitionen in das Nahwärmenetz in Jühnde liegen bei 1,6 Mio. €. Somit liegt die Obergrenze der Förderung bei $320.000 €$. Nach Bioenergiedorf Jühnde eG (2006) wurden in Jühnde 5.500 Meter an Leitungen mit einem Durchmesser von 2 bis $20 \mathrm{~cm}$ verlegt. Die rechnerisch mögliche Fördersumme beläuft sich somit auf einen Betrag von mindestens 110.000 (20 mm Durchmesser) bzw. höchstens $1.100 .000 €$ (200 mm Durchmesser). Für das Fallbeispiel wird die Obergrenze des Förderbetrags von $320.000 €$ angesetzt. 
Tabelle 10.7: $\quad$ Aufteilung der Investitionen und fixen Betriebskosten für das Nahwärmenetz auf Biogas-BHKW und Heizwerk

\begin{tabular}{l|l|r|r|r}
\hline & & Gesamt & $\begin{array}{c}\text { Anteil Bio- } \\
\text { gas-BHKW }\end{array}$ & $\begin{array}{c}\text { Anteil Heiz- } \\
\text { werk }\end{array}$ \\
\hline In das Nahwärmenetz eingespeiste Wärmemenge & \multicolumn{1}{|c|}{$\begin{array}{l}\text { gWh } \\
\text { Anteil (gerundet) }\end{array}$} & $\begin{array}{r}3.500 \\
100\end{array}$ & 2.500 & 1.000 \\
\hline Investitionen Nahwärmenetz (Ruppert et al. 2008) & $€$ & 1.600 .000 & 1.142 .857 & 457.143 \\
\hline Förderung Neubau Nahwärmenetz (BMU 2009e) & $€$ & 320.000 & 228.571 & 91.429 \\
\hline Jährliche Betriebskosten Nahwärmenetz nach (FNR 2005) & $€ / a$ & 32.000 & 22.857 & 9.143 \\
\hline
\end{tabular}

Um die Kosten für die eingesetzte Biomasse je Jahr berechnen zu können, müssen die eingesetzten Mengen an Biomasse in MWh/a ermittelt werden, da in der MTBDatenbank die Kosten der Energieträger in €/MWh gegeben sind. Entsprechend den Angaben zur installierten Leistung bzw. zu den erzeugten Strom-/Wärmemengen und zu den Wirkungsgraden des BHKW kann die eingesetzte Biogasmenge in Energieeinheiten berechnet werden:

$$
\frac{5.000 M W h_{e l} / a}{0,403}+\frac{4.900 M W h_{e l} / a}{0,398} \approx(12.407+12.312) M W h_{\text {Biogas }} / a=24.719 M W h_{\text {Biogas }} / a
$$

Die spezifischen Kosten für das in Jühnde produzierte Biogas resultieren wiederum aus den Preisen für die im Vergärungsprozess eingesetzten Biomassen. Je Jahr werden ca. 9.000 m³/a Gülle und ca. 15.000 t/a Frischmasse Ganzpflanzensilage eingesetzt (Ruppert et al. 2008; S. 19). Es wird unterstellt, dass die Gülle kostenfrei bereitgestellt wird, was in der Praxis häufig der Realität entspricht. Annahmen zur Entwicklung der jährlichen Durchschnittspreise für Ganzpflanzengetreide sind in der Datenbank in €/MWh hinterlegt. Diese stellen jedoch nicht die korrekte Bezugsgröße für die in der Datenbank durchgeführte Kostenrechnung dar, da der im BHKW eingesetzte Energieträger nicht die Ganzpflanzensilage, sondern das daraus (in Verbindung mit der Gülle) erzeugte Biogas ist. Maßgeblich für die Berechnung der Kosten in der Datenbank sind somit die Kosten für eine MWh Biogas.

Gemäß der oben dargestellten Berechnung werden im BHKW je Jahr ca. 24.719 MWh Biogas eingesetzt. Nach Ruppert et al. (2008) müssen dafür 15.000 t Ganzpflanzensilage je Jahr eingesetzt werden (unter Berücksichtigung der zusätzlich eingesetzten Gülle). Nach FNR (2005) liegt der Heizwert von Ganzpflanzengetreide bei 14,1 MJ/kg. Die Energiemenge der in Jühnde jährlich eingesetzten Ganzpflanzensilage kann demnach geschätzt werden:

$$
15.000 \mathrm{t} / \mathrm{a} \cdot 14,1 \mathrm{MJ} / \mathrm{kg}=211.500 .000 \mathrm{MJ} / \mathrm{a}=58.750 \mathrm{MWh}_{\text {Ganpfanzen }} / \mathrm{a}
$$


Als energetisches Verhältnis von Biogas zu Ganzpflanzen resultiert für diesen speziellen Fall:

$$
\frac{24.719 M W h_{\text {Biogas }}}{58.750 M W h_{\text {Ganzpplanzen }}} \approx 0,42 \text { bzW. } \frac{58.750 M W h_{\text {Ganzplanzen }}}{24.719 M W h_{\text {Biogas }}} \approx 2,38
$$

Dies bedeutet, um eine MWh Biogas zu erzeugen müssen etwa 2,38 MWh (entspricht etwa 8.568 MJ bzw. 608 kg) Ganzpflanzen eingesetzt werden. Dieser Wert ist jedoch nicht allgemeingültig anwendbar, da implizit die in Jühnde eingesetzte Menge an Gülle sowie die dort verwendete Anlagentechnik zugrunde liegen. Der in der Datenbank für Ganzpflanzen hinterlegte Preis wird mit einem entsprechenden Faktor beaufschlagt, um den für die Kostenberechnung maßgeblichen Preis für Biogas in Jühnde zu berechnen. Dementsprechend wird eine Preiszeitreihe für den Energieträger "Biogas Jühnde" je Szenario implementiert, die jeweils mit der entsprechenden Preiszeitreihe für Ganzpflanzen je Szenario durch den Multiplikationsfaktor 2,38 gekoppelt ist.

Nach den Bestimmungen des EEG (Bundesregierung 2008b) wird Strom aus Biomasse-Anlagen mit einer installierten elektrischen Leistung von mehr als $500 \mathrm{~kW}$ bis einschließlich $5 \mathrm{MW}$, die vor 2010 in Betrieb gingen, mit 8,25 Ct/kWh über einen Zeitraum von 20 Jahren vergütet (\$27 Abs. 1 Nr. 3 EEG2008). Für eine Inbetriebnahme im Jahr 2010 und den folgenden Jahren ist eine Degression der Vergütungssätze um 1 \%/a zu berücksichtigen. Für eine im Jahr 2010 in Betrieb genommene Anlage (Basisjahr für das betrachtete Fallbeispiel) gilt somit ein Vergütungssatz von $8,17 \mathrm{Ct} / \mathrm{kWh}_{\mathrm{el}}$. Für Biogas-Anlagen auf Basis nachwachsender Rohstoffe und Gülle, die vor dem Jahr 2010 in Betrieb genommen wurden, gilt zudem ein Bonus von $4 \mathrm{Ct} / \mathrm{kWh}$ in der betrachteten Leistungsklasse (§27 Abs. 4 Nr. 2 EEG2008). Weiterhin gilt ein KWK-Bonus in Höhe von $3 \mathrm{Ct} / \mathrm{kWh}$ (\$27 Abs. 4 Nr. 3 EEG2008). Unter Berücksichtigung der Degression beläuft sich die Vergütung für das im Fallbeispiel betrachtete BHKW somit auf insgesamt 15,10 Ct/kWh (bei Annahme einer Inbetriebnahme im Jahr 2010). Bei einem Vergleich der Alternativen unter Berücksichtigung aktueller Förderbedingungen ist für die ersten 20 Jahre der Nutzungsdauer dieser Betrag von den spezifischen Kosten der Stromerzeugung in Abzug zu bringen. Da für das Biogas-BHKW eine Nutzungsdauer von 20 Jahren unterstellt wird, ist davon auszugehen, dass die Vergütung über die gesamte Nutzungsdauer in Anspruch genommen wird.

Um Spitzen in der Wärmenachfrage decken zu können, stehen in Jühnde zusätzlich zum Biogas-BHKW ein Holzhackschnitzel-Heizwerk (HHS-HW, $550 \mathrm{~kW}_{\text {th }}$ ) und ein Spitzenlastkessel $\left(1,6 \mathrm{MW}_{\text {th }}\right)$ zur Verfügung. Das HHS-HW mit einer Vorschubrostfeuerung hat eine Wärmeleistung von $550 \mathrm{~kW}_{\text {th }}$ (Ruppert et al. 2008). Nach obigen Angaben und Berechnungen zum Biogas-BHKW werden im Heizwerk je Jahr ca. 
1.000 $\mathrm{MWh}_{\text {th }}$ erzeugt. Die Betriebsstunden des HHS-HW belaufen sich in diesem Fall auf (in Volllaststundenäquivalenten):

$$
\frac{1.000 \mathrm{MWh}_{\mathrm{th}} / \mathrm{a}}{550 \mathrm{~kW}} \approx 1.818 \mathrm{~h} / \mathrm{a}
$$

Nach Ruppert et al. (2008) belaufen sich die Investitionen für die Wärmeerzeugung auf 0,9 Mio. €, einschließlich HHS-HW und Spitzenlastkessel (im Folgenden Heizwerk HW). Gemäß dem Anteil an der Wärmbereitstellung (vgl. Tabelle 10.7) sind dem HW darüber hinaus ca. $29 \%$ der Investitionen für das Nahwärmenetz zuzurechnen. Des Weiteren können Anlagen zur thermischen Nutzung von Biomasse mit einer Leistung von mehr als $100 \mathrm{~kW}$ nach BMU (2009e) mit $20 € / \mathrm{kW}$, maximal $100.000 € /$ Anlage gefördert werden. Für das HHS-Heizwerk mit $550 \mathrm{~kW}$ ist somit ein Investitionszuschuss in Höhe von $11.000 €$ möglich. Bei der Wirtschaftlichkeitsbewertung unter Berücksichtigung aktueller Förderbedingungen reduzieren sich die Anfangsinvestitionen um den entsprechenden Betrag.

Nach FNR (2005) entstehen für eine Bioenergieanlage jährliche Betriebskosten für Wartungs-, Reparatur- oder Instandhaltungsmaßnahmen (ohne Personalkosten des eigenen Betriebspersonals), die mit insgesamt 4,5\%/a der Investitionen angesetzt werden können. Des Weiteren können Kosten für Steuern und Versicherungen einer Bioenergieanlage mit 0,5 bis $1 \%$ /a der Investitionen geschätzt werden. Zudem entstehen Betriebskosten in Höhe von schätzungsweise 0,5 bis $1 \% / a$ der Investitionen für Betriebsmittel und zur Ascheentsorgung. Darüber hinaus sind $2 \% / a$ der (anteiligen) Investitionen für Wartung und Instandhaltung des Nahwärmenetzes anzusetzen. Personalkosten werden nach FNR (2005) mit 30.000 bis $50.000 €$ je Mannjahr angegeben, wobei der Personalbedarf für eine Anlage $<1 \mathrm{MW}_{\text {th }}$ auf 0,2 bis 0,4 Mannjahre beziffert wird. Davon ausgehend werden für das Heizwerk im Fallbeispiel basierend auf mittleren Werten die fixen Betriebskosten in Tabelle 10.8 berücksichtigt.

Tabelle 10.8: $\quad$ Fixe Betriebskosten für das Heizwerk

\begin{tabular}{l|c|c}
\hline & \%/a der Investitionen & $\boldsymbol{\epsilon} / \mathbf{a}$ \\
\hline Wartung, Reparaturen, Instandhaltung & 4,5 & 40.500 \\
\hline Steuern, Versicherung & 0,75 & 6.750 \\
\hline Ascheentsorgung & 0,75 & 6.750 \\
\hline Personal (Personalbedarf 0,3 Mannjahre, 40.000 €/Mannjahr) & -- & 12.000 \\
\hline Anteilige Kosten für Instandhaltung des Nahwärmenetzes (vgl. Tabelle 10.7) & $2\left(^{*}\right)$ & 9.143 \\
\hline Gesamt & & 75.143 \\
\hline
\end{tabular}

Anmerkungen: $\left(^{\star}\right)$ bezogen auf Investitionen für das Nahwärmenetz.

Quelle: FNR (2005). 
Brennstoffpreise für Holzhackschnitzel sind als Preiszeitreihe in der Datenbank hinterlegt. Die Brennstoffkosten werden davon ausgehend in Verbindung mit dem Jahresnutzungsgrad des HHS-HW berechnet. Bei (Bauer 2007; S. 59) werden für Holzhackschnitzelfeuerungen Jahresnutzungsgrade von 85 bis $90 \%$ genannt. Bei (Staiß 2007) wird für Holzhackschnitzelheizwerke ein Wirkungsgrad von $88 \%$ gegeben. Der Jahresnutzungsgrad des HHS-HW in Jühnde kann auf Basis der Angaben bei Bioenergiedorf Jühnde eG (2006) auf ca. 89 \% geschätzt werden (Tabelle 10.9).

Tabelle 10.9: $\quad$ Energieinhalt von Holzhackschnitzeln (HHS) (Basisdaten 2006)

\begin{tabular}{|c|c|c|c|c|c|}
\hline & & Fichte & Buche & Summe & Quelle \\
\hline Wassergehalt bei Lieferung & $\%$ & $35 \%$ & $35 \%$ & & (FNR 2005, S. 207) \\
\hline Schüttdichte bei Lieferung & $\mathrm{kg} / \mathrm{m}^{3}$ & 232 & 341 & & (FNR 2005, S. 207) \\
\hline Liefermenge (Volumen) & $\mathrm{SRM} / \mathrm{a}$ & 900 & 900 & 1.800 & $\begin{array}{l}\text { (Bioenergiedorf Jühnde eG 2006; } \\
\text { eigene Annahmen) }\end{array}$ \\
\hline Liefermenge (Gewicht) & $\mathrm{kg} / \mathrm{a}$ & 208.800 & 306.900 & & \\
\hline $\begin{array}{l}\text { Wassergehalt der getrock- } \\
\text { neten HHS }\end{array}$ & $\%$ & $15 \%$ & $15 \%$ & & \\
\hline $\begin{array}{l}\text { Gewicht der getrockneten } \\
\text { Liefermenge }\end{array}$ & $\mathrm{kg} / \mathrm{a}$ & 159.671 & 234.688 & & \\
\hline $\begin{array}{l}\text { Heizwert getrockneter HHS } \\
\text { (Wassergehalt } 15 \% \text { ) }\end{array}$ & $\mathrm{MJ} / \mathrm{kg}$ & 15,5 & 15,3 & & (FNR 2005, S. 90) \\
\hline $\begin{array}{l}\text { Energieinhalt der getrockne- } \\
\text { ten Liefermenge }\end{array}$ & MWh/a & 687 & 997 & 1.685 & \\
\hline Erzeugte Nutzwärme & MWh/a & & & 1.500 & (Bioenergiedorf Jühnde eG 2006) \\
\hline Jahresnutzungsgrad & $\%$ & & & $89 \%$ & \\
\hline
\end{tabular}

Da der Spitzenlastkessel in der Praxis kaum zum Einsatz kommt sind die Kosten für den darin eingesetzten Brennstoff (Heizöl oder Rapsölmethylester) im Vergleich zu den Holzhackschnitzeln vernachlässigbar. Die Investitionen und fixen Betriebskosten für den Spitzenlastkessel dürfen jedoch nicht vernachlässigt werden und sind in den für das HHS-HW berücksichtigten Daten enthalten (siehe oben).

\subsubsection{Alternative 3}

Bei Alternative 3 wird anstelle des Biogas-BHKW ein Erdgas-BHKW in Verbindung mit dem gleichen Heizwerk und Nahwärmenetz wie bei Alternative 2 installiert. Für den Vergleich wird ein dem Biogas-BHKW baugleiches Erdgas-BHKW mit $774 \mathrm{~kW}_{\mathrm{el}}$ und $752 \mathrm{~kW}_{\text {th }}$ zugrunde gelegt (vgl. ASUE 2005). Der elektrische Wirkungsgrad beträgt ca. $41 \%$ und der thermische Wirkungsgrad ca. $40 \%$. Die Investitionen für das BHKWModul werden nach ASUE (2005) auf ca. 485,63 €/kW el bzw. 375.874 € geschätzt. Die Kosten für einen Instandhaltungsvertrag einschließlich aller Wartungs- und Reparaturarbeiten, Ersatzteile und Betriebsstoffe (außer Brennstoffe) und Generalüberholung 
belaufen sich nach ASUE (2005) auf ca. 0,9759 Ct/kWh $\mathrm{h}_{\mathrm{el}}$. Da es sich um eine dem Biogas-BHKW sehr ähnliche Anlagentechnik handelt, werden die gleiche Verfügbarkeit und somit die gleichen Betriebsstunden (in Volllastäquivalenten) von $6.983 \mathrm{~h} / \mathrm{a}$ unterstellt. Daraus resultieren eine Stromerzeugungsmenge von $5.405 \mathrm{MWh}_{\mathrm{el}} / \mathrm{a}$ und eine erzeugte Wärmemenge von $5.251 \mathrm{MWh}_{\text {th }} / \mathrm{a}$. Dies entspricht Betriebskosten von $52.746 € / a$.

Die jährlichen Betriebskosten und Investitionen für das Erdgas-BHKW werden entsprechend dem exergetischen Allokationsfaktor auf die Produkte Strom und Wärme aufgeteilt. Es wird angenommen, dass das Verhältnis von der Wärmemenge, die ins Nahwärmenetz eingespeist wird, zu der Wärmemenge, die erzeugt wird, dem Verhältnis beim Biogas-BHKW (Alternative 2) von 0,511 entspricht. Dann werden $2.683 \mathrm{MWh}_{\mathrm{th}} / \mathrm{a}$ der im Erdgas-BHKW erzeugten Wärmemenge in das Nahwärmenetz eingespeist (Abwärme 2.568 $\mathrm{MWh}_{\mathrm{th}} / \mathrm{a}$ entspricht 3.415 Volllaststunden). Wenn insgesamt, wie auch beim Biogas-BHKW, $3.500 \mathrm{MWh}_{\mathrm{th}} / \mathrm{a}$ ins Nahwärmenetz eingespeist werden, müssen folglich weitere $817 \mathrm{MWh}_{\mathrm{th}} / \mathrm{a}$ aus dem Heizwerk bereitgestellt werden. Die so berechneten Anteile an der Wärmebereitstellung werden analog dem Biomasse-Konzept zur Aufteilung der Investitionen und Kosten für das Nahwärmenetz zugrunde gelegt (Tabelle 10.10).

Tabelle 10.10: $\quad$ Aufteilung der Investitionen und fixen Betriebskosten für das Nahwärmenetz auf Erdgas-BHKW und Heizwerk

\begin{tabular}{l|l|r|r|r}
\hline & & Gesamt & \multicolumn{1}{c|}{$\begin{array}{c}\text { Anteil Erd- } \\
\text { gas-BHKW }\end{array}$} & $\begin{array}{c}\text { Anteil Heiz- } \\
\text { werk }\end{array}$ \\
\hline Wärmemenge Nahwärmeversorgung & & 3.500 & 2.683 & 817 \\
$\quad$ Anteil (gerundet) & \multicolumn{1}{|l|}{$\mathrm{MWh}_{\mathrm{th}} / \mathrm{a}$} & 100 & 76,7 & 23,3 \\
\hline Investitionen Nahwärmenetz (Ruppert et al. 2008) & $€$ & 1.600 .000 & 1.226 .514 & 373.486 \\
\hline Förderung Neubau Nahwärmenetz (BMU 2009e) & $€$ & 320.000 & 245.303 & 74.697 \\
\hline Jährliche Betriebskosten Nahwärmenetz nach (FNR 2005) & $€ / \mathrm{a}$ & 32.000 & 24.530 & 7.470 \\
\hline
\end{tabular}

Nach den Bestimmungen des KWKG (Bundesregierung 2002a; Bundesregierung 2008a) wird eingespeister Strom aus KWK-Anlagen, (einschließlich Erdgas-betriebener BHKWs) mit einer installierten elektrischen Leistung von über $50 \mathrm{~kW}$ bis einschließlich $2 \mathrm{MW}$ mit 5,11 Ct/kWh für den Leistungsanteil bis $50 \mathrm{~kW}$ und 2,1 Ct/kWh für den Leistungsanteil über $50 \mathrm{~kW}$ vergütet ( $\$ 7$ Abs. 5 KWKG2002). Die Vergütung wird für einen Zeitraum von maximal 6 Jahren ab Inbetriebnahme bzw. über maximal $30.000 \mathrm{Be}-$ triebsstunden gewährt. Für das Erdgas-BHKW im Fallbeispiel resultiert ein Vergütungssatz von:

$$
\left(\frac{50}{500} \cdot 5,11+\frac{500-50}{500} \cdot 2,1\right) C t / k W h=2,40 C t / k W h
$$


Die Grenze von 30.000 Betriebsstunden wird bei den unterstellten Volllaststunden je Jahr (6.983 h/a) im fünften Jahr der Nutzung überschritten. Darüber hinaus wird keine weitere Förderung berücksichtigt.

Um die zusätzlich benötigte Wärmemenge je Jahr bereitzustellen, muss das Heizwerk mindestens folgende Betriebsstunden (Volllastäquivalente) aufweisen:

$$
\frac{817 M W h_{t h} / a}{550 k W_{t h}} \approx 1.485 h / a
$$

Zur Berechnung des entsprechenden Brennstoffbedarfs wird der im Rahmen des Biomasse-Konzeptes ermittelte Jahresnutzungsgrad von $89 \%$ zugrunde gelegt. Für Investitionen und Betriebskosten werden die gleichen Werte wie beim Biomasse-Konzept zugrunde gelegt. Jedoch wird hier dem Heizwerk ein etwas geringerer Anteil an den Investitionen und Kosten der Nahwärmeverteilung zugerechnet (vgl. Tabelle 10.10).

\subsubsection{Alternative 4}

Bei Alternative 4 wird anstelle des Biogas-BHKW ein Brennstoffzellen-BHKW in Verbindung mit dem gleichen Heizwerk und Nahwärmnetz wie bei Alternative 2 installiert. Blesl et al. (2006) fasst Daten zu verschiedene Brennstoffzellentypen zusammen. Demnach stehen SOFCs mit einer installierten elektrischen Leistung von $109 \mathrm{~kW}_{\mathrm{el}}$ und einer thermischen Leistung von $130 \mathrm{~kW}_{\text {th }}$ zur Verfügung, deren spezifischen Investitionen bei $11.000 € / \mathrm{kW}_{\text {el }}$ liegen. Der Gesamtwirkungsgrad einer solchen Brennstoffzelle liegt bei $78 \%$ und der elektrische Wirkungsgrad bei $46 \%$. Der thermische Wirkungsgrad beträgt somit $32 \%$. Zu Wartung und Instandhaltung ist bei Roads2HyCom (2008) ein Betrag von 0,024 \$/kWh für eine SOFC mit $100 \mathrm{~kW}$ gegeben. Dies entspricht beim aktuellen Wechselkurs (13.04.2010):

$$
0,7356 € / \$ \cdot 0,024 \$ / k W h=1,76544 C t / k W h
$$

Basierend auf Angaben bei Gummert und Suttor (2006) wird die Anlagenverfügbarkeit auf $75 \%$ geschätzt. Dies entspricht 6.570 Betriebsstunden je Jahr. Bei einer StackLebensdauer von $22.000 \mathrm{~h}$ (vgl. Blesl et al. 2006) entspricht dies einer Lebensdauer von etwas mehr als 3 Jahren. Für dieses Fallbeispiel wird eine Nutzungsdauer von 4 Jahren bei einer Verfügbarkeit von $75 \%$ zugrunde gelegt. Derzeitig verfügbare SOFCSysteme sind in der Regel für den Betrieb mit Erdgas bzw. Biogas in Erdgas-Qualität ausgelegt (Primas 2007). Daher wird für das Fallbeispiel angenommen, dass die Brennstoffzelle mit Erdgas betrieben wird.

Bei einer installierten elektrischen Leistung von 109 kWel, die den bei Blesl et al. (2006) gegebenen Wirtschaftlichkeitsdaten zugrunde liegt, und $6.570 \mathrm{~h} / \mathrm{a}$ werden an Strom 
$716.130 \mathrm{kWh} / \mathrm{e} / \mathrm{a}$ erzeugt. Nach Ruppert et al. (2008) liegt der Strombedarf in Jühnde bei etwa 2 Mio. kWh $\mathrm{el}_{\mathrm{el}}$ (vgl. S. 68), so dass entweder eine größere oder mehrere Anlagen installiert werden müssen oder der zusätzliche Strombedarf aus anderen Quellen gedeckt werden muss. Nach Primas (2007; S. 30) sind SOFC-Systeme mit einer installierten elektrischen Leistung von bis zu $250 \mathrm{~kW}_{\text {el }}$ verfügbar. Diese Anlagengröße wird für das Fallbeispiel unterstellt, um einen möglichst hohen Anteil des Strombedarfs in Jühnde decken zu können. Bei Temming (2005) werden für eine (zukünftige) 300 kWel SOFC spezifische Investitionen in Höhe von ca. $10.000 € / \mathrm{kW}_{\mathrm{el}}$ ausgewiesen. Für die $250 \mathrm{~kW}_{\mathrm{el}}$ im Fallbeispiel resultieren Anfangsinvestitionen in Höhe von $250 \mathrm{~kW}$ el $10.000 € / \mathrm{kW}_{\mathrm{el}}=2,5 \mathrm{Mio}$. $€$. Basierend auf der Anlagenverfügbarkeit von $6.570 \mathrm{~h} / \mathrm{a}$, einer installierten Leistung von $250 \mathrm{~kW}_{\mathrm{el}}$ und spezifischen Kosten für Wartung und Instandhaltung in Höhe von 1,76544 Ct/kWh werden die fixen Betriebskosten je Jahr auf $28.997 € / a$ geschätzt.

Hinsichtlich des elektrischen und thermischen Nutzungsgrades werden die bei Blesl et al. (2006) genannten $46 \%$ bzw. $32 \%$ angenommen. Als thermische Leistung werden basierend auf den Angaben bei Blesl et al. (2006) ca. $300 \mathrm{~kW}_{\text {th }}$ angenommen. Unter Annahme der Betriebsstunden von 6.570 h/a resultieren die jährlich erzeugten Stromund Wärmemengen zu:

$$
\begin{aligned}
& 250 \mathrm{~kW}_{e l} \cdot 6.570 \mathrm{~h} / \mathrm{a}=1.643 \mathrm{MWh}_{\mathrm{el}} / \mathrm{a} \\
& 300 \mathrm{~kW}_{\mathrm{th}} \cdot 6.570 \mathrm{~h} / \mathrm{a}=1.971 \mathrm{MWh}_{\mathrm{th}} / \mathrm{a}
\end{aligned}
$$

Folglich müssen zusätzlich etwa $357 \mathrm{MWh}_{\mathrm{el}} / \mathrm{a}$ Strom aus dem öffentlichen Versorgungsnetz bereitgestellt werden. Wird ebenso wie für das Biogas- und ErdgasGasmotoren-BHKW unterstellt, dass aufgrund der zeitlichen Verteilung von Wärmeangebot und-nachfrage nur ein Anteil von 0,511 der erzeugten Wärme über das Nahwärmenetz abgesetzt werden kann, können lediglich $1.007 \mathrm{MWh}_{\text {th }}$ der im Brennstoffzellen-BHKW erzeugten Abwärme abgesetzt werden (Abwärme $964 \mathrm{MWth} / \mathrm{a}$ entspricht 3.213 Volllaststunden). Folglich müssen aus dem Heizwerk ca. $2.500 \mathrm{MWh}_{\text {th }}$ zusätzlich bereitgestellt werden. Die Investitionen und jährlichen Betriebskosten der Brennstoffzelle werden entsprechend dem exergetischen Allokationsfaktor auf die Strom- und Wärmeproduktion aufgeteilt.

Zusätzlich müssen 3.500 $\mathrm{MWh}_{\mathrm{th}} / \mathrm{a}-1.007 \mathrm{MW}_{\mathrm{th}} / \mathrm{a}=2.493 \mathrm{MWh}_{\mathrm{th}} / \mathrm{a}$ zur Einspeisung ins Nahwärmenetz aus dem Heizwerk bereitgestellt werden (vgl. Alternative 1). Entsprechend den Anteilen an der Wärmeversorgung werden die Investitionen und fixen Betriebskosten für die Nahwärmeverteilung aufgeteilt (Tabelle 10.11). 
Tabelle 10.11: $\quad$ Aufteilung der Investitionen und fixen Betriebskosten für das Nahwärmenetz auf Erdgas-BHKW und Heizwerk

\begin{tabular}{|c|c|c|c|c|}
\hline & & Gesamt & $\begin{array}{c}\text { Anteil } \\
\text { Brennstoff- } \\
\text { zelle }\end{array}$ & $\begin{array}{l}\text { Anteil Heiz- } \\
\text { werk }\end{array}$ \\
\hline Wärmemenge Nahwärmeversorgung & $\mathrm{MWh}_{\mathrm{th}} / \mathrm{a}$ & 3.500 & 1.007 & 2.493 \\
\hline Anteil (gerundet) & $\%$ & 100 & 28,8 & 71,2 \\
\hline Investitionen Nahwärmenetz (Ruppert et al. 2008) & $€$ & 1.600 .000 & 460.343 & 1.139 .657 \\
\hline Förderung Neubau Nahwärmenetz (BMU 2009e) & $€$ & 320.000 & 92.069 & 227.931 \\
\hline Jährliche Betriebskosten Nahwärmenetz nach (FNR 2005) & $€ / a$ & 32.000 & 9.207 & 22.793 \\
\hline
\end{tabular}

Nach den Bestimmungen des KWKG (Bundesregierung 2002a; Bundesregierung 2008a) wird eingespeister Strom aus Brennstoffzellen-KWK-Anlagen mit 5,11 Ct/kWh für eine Dauer von 10 Jahren ab Inbetriebnahme der entsprechenden Anlage vergütet (§7 Abs. 5 KWKG2002). Da eine Nutzungsdauer von 4 Jahren unterstellt wird, ist davon auszugehen, dass die Vergütung über die gesamte Nutzungsdauer gewährt wird.

Um die zusätzlich benötigte Wärmemenge bereitzustellen, muss das Heizwerk mindestens folgende Betriebsstunden (Volllastäquivalente) aufweisen:

$[s t d]=\frac{2.493 M W h_{t h} / a}{550 k W_{t h}} \approx 4.533 h / a$

Zur Berechnung des entsprechend höheren Brennstoffbedarfs wird der im Rahmen des Biomasse-Konzeptes ermittelte Jahresnutzungsgrad von $89 \%$ zugrunde gelegt. Für Investitionen und Betriebskosten werden die gleichen Werte wie beim BiomasseKonzept zugrunde gelegt. Jedoch wird hier dem Heizwerk ein wesentlich höherer Anteil an den Investitionen und Kosten der Nahwärmeverteilung zugerechnet (vgl. Tabelle 10.11).

\subsubsection{Alternative 5}

Für Alternative 5 wird angenommen, dass die Gemeinde in eine Windkraftanlage und Photovoltaik-Dachanlagen investiert. Abweichungen von Angebot und Nachfrage aufgrund der fluktuierenden erneuerbaren Energieträger werden durch Strombezug aus dem Netz ausgeglichen. Für die Wärmeversorgung werden in den einzelnen Haushalten moderne Erdgas-Brennwertkessel installiert. Für Windkraftanlagen stehen Daten zu Investitionen und Betriebskosten bei Reichmuth et al. (2006) zur Verfügung (Tabelle 10.12). Weiterhin wird für dieses Fallbeispiel eine Nutzungsdauer von 20 Jahren und 1.700 Betriebsstunden je Jahr zugrunde gelegt (durchschnittlicher Wert für einen Standort in Deutschland). Brennstoffkosten entstehen nicht. 
Tabelle 10.12: Investitionen und fixe Betriebskosten für Windkraftanlagen

\begin{tabular}{|c|c|c|c|c|}
\hline \multicolumn{2}{|c|}{ Investitionen } & \multicolumn{3}{|c|}{ Fixe Betriebskosten } \\
\hline & $€$ & & $\% / a$ & $€ / a$ \\
\hline Anlagen & 587.000 & Wartung, Instandhaltung & 1 & 8.670 \\
\hline Nebenkosten & 280.000 & Versicherung & 0,6 & 5.202 \\
\hline \multirow[t]{5}{*}{ Gesamt } & 867.000 & Verwaltung & 1 & 8.670 \\
\hline & & Unvorhergesehenes & 2,5 & 21.675 \\
\hline & & Strom/Telekom & 0,17 & 1.500 \\
\hline & & Pacht & 0,89 & 7.700 \\
\hline & & Gesamt & 6,2 & 53.417 \\
\hline
\end{tabular}

Quelle: Reichmuth et al. (2006).

Für Windenergieanlagen, die vor dem Jahr 2014 in Betrieb genommen werden, gilt gemäß EEG für die ersten 5 Jahre ab Inbetriebnahme eine Anfangsvergütung von 9,7 Ct/kWh (\$29 Abs. 2 EEG2008). Darüber hinaus wird für die folgenden 15 Jahre ein Vergütungssatz von mindestens 5,02 Ct/kWh gewährt. Die Anwendung des Anfangsvergütungssatzes verlängert sich unter bestimmten Bedingungen. Diese sind für die hier betrachtete Windenergieanlage über deren gesamten Nutzungsdauer erfüllt. Daher gilt unter Berücksichtigung der Degression (\$20 Abs. 2 Nr. 7b) ein Vergütungssatz von 9,60 Ct/kWh über die gesamte Nutzungsdauer von 20 Jahren.

Die Investitionen für eine typische PV-Dachanlage mit $3 \mathrm{~kW}_{\mathrm{p}}$ liegen nach Reichmuth et al. (2006) bei $13.200 €$, die fixen Betriebskosten werden bezogen auf die Investitionen mit 0,02 \%/a (entspricht 2,64 €/a) geschätzt. Als Nutzungsdauer werden 20 Jahre und als Betriebsstunden mit $900 \mathrm{~h} / \mathrm{a}$ zugrunde gelegt. Bei der Bewertung der Alternativen unter Beachtung aktueller Förderbedingungen sind für PV-Anlagen bis zu $30 \mathrm{~kW}$ zwei alternative Möglichkeiten zu unterscheiden. Die Stromeinspeisung aus PV-Anlagen mit einer installierten Leistung von bis zu 30 kW, die vor 2010 an oder auf baulichen Anlagen installiert wurden, wird nach EEG mit 43,01 Ct/kWh vergütet (§33 Abs. 1 Nr. 1 EEG2008). Unter Berücksichtigung der Degression von 8 \% im Jahr 2010 für die betrachtete Leistungsklasse (\$20 Abs. 2 Nr. 8 b) aa) aaa) EEG2008) resultiert für bis Juni 2010 installierte Anlagen ein Vergütungssatz von 39,57 Ct/kWh.

Alternativ besteht bei PV-Anlagen von bis zu $30 \mathrm{~kW}$ die Möglichkeit, den erzeugten Strom selbst zu verbrauchen. Für die selbst verbrauchten Strommengen liegt die Vergütung bei 23,01 Ct/kWh für im Jahr 2010 installierte Anlagen ( $\$ 33$ Abs. 2 i. V. m. $\S 22$ Abs. 2 Nr. 8 b) aa) aaa) EEG2008). Der geringere Vergütungssatz wird dadurch kompensiert, dass die entsprechende Strommenge nicht aus dem Netz bezogen wird und somit Stromkosten eingespart werden. Wird der Strom zum Zeitpunkt der Erzeugung vor Ort nicht benötigt, wird dieser eingespeist und zum entsprechenden Vergütungs- 
satz $(39,57 \mathrm{Ct} / \mathrm{kWh})$ vergütet. Bei derzeitigen Anlagen kann der Anteil des selbst verbrauchten Stroms nach Wenzel und Nitsch (2008) auf $20 \%$ geschätzt werden. Für diesen Anteil wird bei der Bewertung unter Berücksichtigung derzeitiger Förderbedingungen der Vergütungssatz von 23,01 Ct/kWh zuzüglich des je nach Szenario und Jahr variierenden Strompreises zugrunde gelegt.

Die Investitionen für eine durchschnittliche Erdgas-Brennwertheizung sind in Tabelle 10.13 zusammengefasst. Die fixen Betriebskosten sind in Tabelle 10.6 dargestellt. Als Brennstoffpreise werden die Erdgaspreise für Haushalte je Szenario zugrunde gelegt. Nutzungsgrade und Angaben zum Hilfsenergiebedarf sind in Tabelle 10.3 bzw. Tabelle $10.4 \mathrm{zu}$ finden. Für Hilfsenergie werden Preise für Strom für Haushaltskunden zugrunde gelegt.

Tabelle 10.13: $\quad$ Annahmen zu Investitionen für Erdgas-Brennwertkessel

\begin{tabular}{l|c|c|c}
\hline & Investitionen & Kalkulatorische Nutzungsdauer & Aufwand für Instandsetzung \\
\hline & $€$ & $\mathrm{a}$ & $\%$ \\
\hline Wärmeerzeuger (inkl. Regelung) & 2.900 & 18 & $1,5 \%$ \\
\hline Warmwasserspeicher & 1.400 & 25 & $1,0 \%$ \\
\hline Leitungssystem/Heizflächen & 4.700 & 30 & $1,0 \%$ \\
\hline Schornstein/LAS-Durchführung & 500 & 50 & $1,0 \%$ \\
\hline Anschluss/Baukosten & 1.700 & 50 & $1,0 \%$ \\
\hline Gas-/Elektroinstallation & 800 & 50 & $1,0 \%$ \\
\hline Gesamt & 12.000 & & \\
\hline
\end{tabular}

Quellen: Oschatz und Mailach (2007).

\subsubsection{Alternative 6}

Für Alternative 6 wird unterstellt, dass die Gemeinde in die gleiche Windkraftanlage wie in Alternative 5 investiert. Zudem werden solar unterstützte Erdgas-Heizungen in den einzelnen Haushalten installiert, um die konventionellen Öl-Heizkessel zu ersetzen. Die Investitionen und fixen Betriebskosten für den Erdgas-Brennwertkessel werden analog Alternative 5 gemäß den Angaben in Tabelle 10.13 und Tabelle 10.6 zugrunde gelegt. Zusätzlich wird in eine Solarkollektoranlage (Flachkollektor) investiert mit Anfangsinvestitionen von $7.530 €$, einem Faktor für die Instandsetzung von 1,5\% und einer Nutzungsdauer von 20 Jahren nach Staiß (2007). Der Brennstoffbedarf bei dieser Alternative reduziert sich um den Deckungsanteil der Solarkollektoren. Dieser wird nach Quaschning (2008) für eine typische Installation in Deutschland mit $10 \%$ für die Heizenergieversorgung und $50 \%$ für die Warmwasserbereitstellung angenommen. Dementsprechend werden die für den entsprechenden Technologiedatensatz eingetragenen Volllaststundenäquivalente für den Erdgas-Brennwertkessel auf 1.329,5 h/a für die 
Heizung und 62,5 h/a für die Trinkwassererwärmung reduziert (vgl. auch Tabelle 10.2). Die Nutzungsgrade für den Erdgas-Brennwertkessel und der Hilfsenergiebedarf entsprechen den Angaben in Tabelle 10.3 und Tabelle 10.4.

Mit der Solarkollektoranlage werden $2.183 \mathrm{kWh} / \mathrm{a}$ des Heizenergiebedarfs und $938 \mathrm{kWh} / \mathrm{a}$ der Trinkwassererwärmung bereit gestellt. Bei Annahme eines spezifischen Ertrages von $300 \mathrm{kWh} / \mathrm{m}^{2}$ (vgl. Quaschning 2008) liegt der Flächenbedarf einer entsprechenden Flachkollektoranlage bei 10,4 $\mathrm{m}^{2}$. Nach BMU (2009e) kann eine solche Anlage mit einem Investitionszuschuss von $105 € / \mathrm{m}^{2}$ angefangener Bruttokollektorfläche gefördert werden. Im vorliegenden Fall ist somit ein Investitionszuschuss von $1.155 €$ möglich.

\subsubsection{Alternative 7}

Die Stromversorgung wird wie bei Alternative 5 gewährleistet. Zur Wärmeversorgung werden Wärmepumpen in den einzelnen Haushalten installiert. Die zugrunde gelegten Investitionen für eine Sole-Wasser-Wärmepumpe sind in Tabelle 10.14 zusammengefasst. Die fixen Betriebskosten sind in Tabelle 10.6 dargestellt. Zur Berechnung der Brennstoffkosten ist je Szenario eine Preiszeitreihe "Strom, Wärmepumpentarif“ in der MTB-Datenbank implementiert. Die Entwicklung des Wärmepumpentarifs je Szenario ist dabei durch einen festen Faktor an die Entwicklung der Haushaltspreise für Strom gekoppelt (Faktor nach Oschatz und Mailach (2007): 0,128/0,185=0,692). Nutzungsgrade und Angaben zum Hilfsenergiebedarf sind in Tabelle 10.3 bzw. Tabelle $10.4 \mathrm{zu}$ finden. Für Hilfsenergie werden Preise für Strom für Haushaltskunden zugrunde gelegt.

Tabelle 10.14: $\quad$ Annahmen zu Investitionen für Sole-Wasser-Wärmepumpe

\begin{tabular}{l|c|c|c}
\hline & Investitionen & Kalkulatorische Nutzungsdauer & Aufwand für Instandsetzung \\
\hline & $€$ & $\mathrm{a}$ & $\%$ \\
\hline Wärmeerzeuger (inkl. Regelung) & 9.300 & 20 & $3,0 \%$ \\
\hline Warmwasserspeicher & 2.200 & 25 & $1,0 \%$ \\
\hline Leitungssystem/Heizflächen & 6.600 & 30 & $1,0 \%$ \\
\hline Anschluss/Baukosten & 6.200 & 50 & $1,0 \%$ \\
\hline Gas-/Elektroinstallation & 600 & 50 & $1,0 \%$ \\
\hline Gesamt & 24.900 & & \\
\hline
\end{tabular}

Quellen: Oschatz und Mailach (2007).

Nach BMU (2009e) können Sole-Wasser-Wärmepumpen in Wohngebäuden mit $20 €$ je $\mathrm{m}^{2}$ Wohnfläche, maximal $3.000 €$ je Wohneinheit, gefördert werden. Bei Annahme einer Wohnfläche von $150 \mathrm{~m}^{2}$ resultiert ein Investitionszuschuss von $3.000 €$. 


\subsubsection{Alternative 8}

Die Stromversorgung wird wie bei Alternative 5 gewährleistet. Zur Wärmeversorgung werden Holzpellets-Heizungen in den einzelnen Haushalten installiert. Die zugrunde gelegten Investitionen für einen Holzpellets-Heizkessel sind in Tabelle 10.15 zusammengefasst. Die fixen Betriebskosten sind in Tabelle 10.6 dargestellt. Die Preise für Holzpellets in der Datenbank sind durch einen festen Faktor an die Preiszeitreihen für Holzhackschnitzel gekoppelt. Nutzungsgrade und Angaben zum Hilfsenergiebedarf sowie zur Lagermenge sind in Tabelle 10.3 bzw. Tabelle 10.4 zusammengefasst. Für die entstehenden Kosten aufgrund des Hilfsenergiebedarfs werden Strompreise für Haushaltskunden zugrunde gelegt.

Tabelle 10.15: $\quad$ Annahmen zu Investitionen für Sole-Wasser-Wärmepumpe

\begin{tabular}{l|c|c|c}
\hline & Investitionen & Kalkulatorische Nutzungsdauer & Aufwand für Instandsetzung \\
\hline & $€$ & $\mathrm{a}$ & $\%$ \\
\hline Wärmeerzeuger (inkl. Regelung) & 11.200 & 20 & $2,0 \%$ \\
\hline Warmwasserspeicher & 1.500 & 25 & $1,0 \%$ \\
\hline Leitungssystem/Heizflächen & 4.700 & 30 & $1,0 \%$ \\
\hline Schornstein/LAS-Durchführung & 2.100 & 50 & $1,0 \%$ \\
\hline Anschluss/Baukosten & 1.400 & 50 & $1,0 \%$ \\
\hline Gas-/Elektroinstallation & 700 & 50 & $1,0 \%$ \\
\hline Tank/Brennstofflager & 4.100 & 30 & $1,0 \%$ \\
\hline Gesamt & 24.700 & & \\
\hline
\end{tabular}

Quellen: Oschatz und Mailach (2007).

Nach BMU (2009e) kann für die „Errichtung automatisch beschickter Anlagen mit Leistungs- und Feuerungsregelung sowie automatischer Zündung zur Verfeuerung fester Biomasse (mit Ausnahme von Holzhackschnitzeln) zur Wärmeerzeugung mit einer installierten Nennwärmeleitsung von $5 \mathrm{~kW}$ bis $100 \mathrm{~kW}$ “ eine Basisförderung von $36 € / \mathrm{kW}$ gewährt werden. Dies entspricht bei einer Anlage von $15 \mathrm{~kW}$ einem Investitionszuschuss von $540 €$. Die Förderung beträgt jedoch mindestens $2.000 €$ für PelletsKessel, welche für die vorliegenden Betrachtungen zugrunde gelegt werden (Der Heizkostenvergleich der bei Oschatz und Mailach (2007) geht von einem Speichervolumen von $200 \mathrm{I}$ aus, was im vorliegenden Fallbeispiel ca. 13 l/kW entspricht. Daher wird hier nicht die für Anlagen mit größeren Speichern mit mindesten $30 \mathrm{l} / \mathrm{kW}$ gewährten $2.500 €$ ausgegangen). 


\subsection{Stabilitätsintervalle der Kriterien-Gewichtungen}

In den folgenden Tabellen sind die Stabilitätsintervalle der Kriterien-Gewichtungen im Fallbeispiel Bioenergiedörfer dargestellt, die im Excel-Tool MAESTRO berechnet werden. Dabei werden folgende Bezeichnungen zugrunde gelegt:

- $\mathrm{w}_{\text {min }}$ : untere Grenze des Stabilitätsintervalls (Maximum der unteren Grenzwerte, die in Abhängigkeit der Gewichtungen je Technologiezyklus-Phasen ermittelt werden);

- $w(T)_{\min }$ : in Abhängigkeit der Technologiezyklus-Phasen minimale angewendete Gewichtung je Kriterium;

- $\mathrm{w}(\mathrm{T})_{\max }$ : in Abhängigkeit der Technologiezyklus-Phasen maximale angewendete Gewichtung je Kriterium;

- $W_{\text {max }}$ : obere Grenze des Stabilitätsintervalls (Minimum der oberen Grenzwerte, die in Abhängigkeit der Gewichtungen je Technologiezyklus-Phasen ermittelt werden);

- $\Delta \mathrm{w}=\mathrm{w}_{\max }-\mathrm{w}_{\min }$ : Breite des Stabilitätsintervalls.

Die Grauabstufungen in der Spalte " $\Delta \mathrm{w}$ “ signalisieren besonders große (weiß bzw. hellgrau), mittelgroße (mittleres grau) und besonders kleine oder negative (dunkelgrau) Stabilitätsintervalle. Negative Stabilitätsintervalle sind zudem durch fett kursive Schrift hervorgehoben:

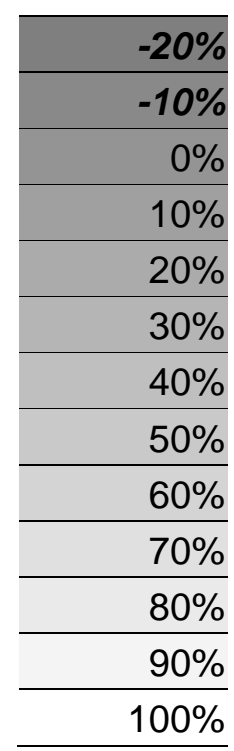


Tabelle 10.16: Stabilitätsintervalle Strom, Szenario 0 (mit Förderung)

\begin{tabular}{|c|c|c|c|c|c|c|}
\hline & & $\mathbf{w}_{\min }$ & $w(T)_{\min }$ & $w(T)_{\max }$ & $\mathbf{w}_{\max }$ & $\Delta \mathbf{w}$ \\
\hline W1.0F & Spez. Gesamtk. & $36,1 \%$ & $11,8 \%$ & $43,1 \%$ & $18,5 \%$ & $-17,65 \%$ \\
\hline U1 & ADP & $0,0 \%$ & $2,9 \%$ & $5,1 \%$ & $10,9 \%$ & $10,86 \%$ \\
\hline U2 & LAND & $0,0 \%$ & $4,9 \%$ & $8,6 \%$ & $69,9 \%$ & $69,89 \%$ \\
\hline U3 & GWP & $0,0 \%$ & $4,9 \%$ & $8,6 \%$ & $11,0 \%$ & $10,99 \%$ \\
\hline U4 & ODP & $0,0 \%$ & $1,0 \%$ & $1,7 \%$ & $10,9 \%$ & $10,85 \%$ \\
\hline U5 & HTP & $0,0 \%$ & $1,0 \%$ & $1,7 \%$ & $11,0 \%$ & $11,00 \%$ \\
\hline U6.1 & FAETP & $0,0 \%$ & $0,3 \%$ & $0,6 \%$ & $12,1 \%$ & $12,12 \%$ \\
\hline U6.2 & MAETP & $0,0 \%$ & $0,3 \%$ & $0,6 \%$ & $18,4 \%$ & $18,37 \%$ \\
\hline U6.3 & TAETP & $0,0 \%$ & $0,3 \%$ & $0,6 \%$ & $99,5 \%$ & $99,54 \%$ \\
\hline U7 & POCP & $0,0 \%$ & $2,0 \%$ & $3,4 \%$ & $6,8 \%$ & $6,77 \%$ \\
\hline U8 & AP & $0,0 \%$ & $3,9 \%$ & $6,8 \%$ & $13,4 \%$ & $13,36 \%$ \\
\hline U9 & EP & $0,0 \%$ & $3,9 \%$ & $6,8 \%$ & $10,9 \%$ & $10,91 \%$ \\
\hline U10 & KEA & $0,0 \%$ & $3,9 \%$ & $6,8 \%$ & $11,9 \%$ & $11,88 \%$ \\
\hline V1 & Stat. Rw. (Kehrwert) & $0,0 \%$ & $1,5 \%$ & $2,2 \%$ & $24,4 \%$ & $24,41 \%$ \\
\hline V2 & Importquote & $0,0 \%$ & $1,5 \%$ & $2,2 \%$ & $24,5 \%$ & $24,50 \%$ \\
\hline V3 & Lokale Verfügbarkeit & $0,0 \%$ & $1,5 \%$ & $2,2 \%$ & $100,0 \%$ & $100,00 \%$ \\
\hline V4 & Lagermöglichkeit & $0,0 \%$ & $1,5 \%$ & $2,2 \%$ & $13,2 \%$ & $13,22 \%$ \\
\hline V5 & Herfindahl & $0,0 \%$ & $1,5 \%$ & $2,2 \%$ & $62,1 \%$ & $62,14 \%$ \\
\hline V6 & Risiko-Indikator (Kehrwert) & $0,0 \%$ & $1,5 \%$ & $2,2 \%$ & $100,0 \%$ & $100,00 \%$ \\
\hline V7 & Variationskoeff. Preise & $0,0 \%$ & $1,5 \%$ & $2,2 \%$ & $57,6 \%$ & $57,59 \%$ \\
\hline V8 & Nutzungsgrad & $0,0 \%$ & $1,5 \%$ & $2,2 \%$ & $19,4 \%$ & $19,37 \%$ \\
\hline v9 & Anlagenverfügbarkeit & $0,0 \%$ & $1,5 \%$ & $2,2 \%$ & $17,4 \%$ & $17,44 \%$ \\
\hline S1 & Flex. Innov. & $0,0 \%$ & $1,4 \%$ & $1,7 \%$ & $15,3 \%$ & $15,28 \%$ \\
\hline S2 & Abfallinfr. & $0,0 \%$ & $1,7 \%$ & $2,0 \%$ & $89,4 \%$ & $89,44 \%$ \\
\hline S3 & Konflikte & $0,0 \%$ & $1,8 \%$ & $2,2 \%$ & $15,5 \%$ & $15,52 \%$ \\
\hline S5 & Gesundheit & $0,0 \%$ & $1,8 \%$ & $2,1 \%$ & $49,4 \%$ & $49,40 \%$ \\
\hline S6 & Risiken & $0,0 \%$ & $2,4 \%$ & $3,0 \%$ & $29,2 \%$ & $29,19 \%$ \\
\hline S7 & Katastrophen & $0,0 \%$ & $1,8 \%$ & $2,2 \%$ & $18,8 \%$ & $18,83 \%$ \\
\hline S9 & Ästhetik & $0,0 \%$ & $2,1 \%$ & $2,6 \%$ & $15,0 \%$ & $14,96 \%$ \\
\hline S10 & Soziale Akzeptanz & $0,0 \%$ & $0,7 \%$ & $0,9 \%$ & $21,7 \%$ & $21,68 \%$ \\
\hline
\end{tabular}


Tabelle 10.17: $\quad$ Stabilitätsintervalle Strom, Szenario 1 (ohne Förderung)

\begin{tabular}{|c|c|c|c|c|c|c|}
\hline & & $\mathbf{w}_{\min }$ & $w(T)_{\min }$ & $w(T)_{\max }$ & $\mathbf{w}_{\max }$ & $\Delta \mathbf{w}$ \\
\hline W1.1 & Spez. Gesamtk. & $37,4 \%$ & $11,8 \%$ & $43,1 \%$ & $20,5 \%$ & $-16,9 \%$ \\
\hline U1 & ADP & $0,0 \%$ & $2,9 \%$ & $5,1 \%$ & $18,7 \%$ & $18,7 \%$ \\
\hline U2 & LAND & $0,0 \%$ & $4,9 \%$ & $8,6 \%$ & $52,3 \%$ & $52,3 \%$ \\
\hline U3 & GWP & $0,0 \%$ & $4,9 \%$ & $8,6 \%$ & $17,8 \%$ & $17,8 \%$ \\
\hline U4 & ODP & $0,0 \%$ & $1,0 \%$ & $1,7 \%$ & $5,8 \%$ & $5,8 \%$ \\
\hline U5 & HTP & $0,0 \%$ & $1,0 \%$ & $1,7 \%$ & $4,4 \%$ & $4,4 \%$ \\
\hline U6.1 & FAETP & $0,0 \%$ & $0,3 \%$ & $0,6 \%$ & $4,3 \%$ & $4,3 \%$ \\
\hline U6.2 & MAETP & $0,0 \%$ & $0,3 \%$ & $0,6 \%$ & $6,8 \%$ & $6,8 \%$ \\
\hline U6.3 & TAETP & $0,0 \%$ & $0,3 \%$ & $0,6 \%$ & $98,5 \%$ & $98,5 \%$ \\
\hline U7 & POCP & $0,0 \%$ & $2,0 \%$ & $3,4 \%$ & $11,4 \%$ & $11,4 \%$ \\
\hline U8 & AP & $1,1 \%$ & $3,9 \%$ & $6,8 \%$ & $10,1 \%$ & $9,1 \%$ \\
\hline U9 & EP & $0,8 \%$ & $3,9 \%$ & $6,8 \%$ & $11,4 \%$ & $10,5 \%$ \\
\hline U10 & KEA & $0,0 \%$ & $3,9 \%$ & $6,8 \%$ & $20,1 \%$ & $20,1 \%$ \\
\hline V1 & Stat. Rw. (Kehrwert) & $0,0 \%$ & $1,5 \%$ & $2,2 \%$ & $13,1 \%$ & $13,1 \%$ \\
\hline V2 & Importquote & $0,0 \%$ & $1,5 \%$ & $2,2 \%$ & $13,5 \%$ & $13,5 \%$ \\
\hline V3 & Lokale Verfügbarkeit & $0,0 \%$ & $1,5 \%$ & $2,2 \%$ & $100,0 \%$ & $100,0 \%$ \\
\hline V4 & Lagermöglichkeit & $0,0 \%$ & $1,5 \%$ & $2,2 \%$ & $5,6 \%$ & $5,6 \%$ \\
\hline V5 & Herfindahl & $0,0 \%$ & $1,5 \%$ & $2,2 \%$ & $42,9 \%$ & $42,9 \%$ \\
\hline V6 & Risiko-Indikator (Kehrwert) & $0,0 \%$ & $1,5 \%$ & $2,2 \%$ & $100,0 \%$ & $100,0 \%$ \\
\hline V7 & Variationskoeff. Preise & $0,0 \%$ & $1,5 \%$ & $2,2 \%$ & $37,3 \%$ & $37,3 \%$ \\
\hline V8 & Nutzungsgrad & $0,0 \%$ & $1,5 \%$ & $2,2 \%$ & $8,0 \%$ & $8,0 \%$ \\
\hline V9 & Anlagenverfügbarkeit & $0,0 \%$ & $1,5 \%$ & $2,2 \%$ & $7,2 \%$ & $7,2 \%$ \\
\hline S1 & Flex. Innov. & $0,0 \%$ & $1,4 \%$ & $1,7 \%$ & $25,0 \%$ & $25,0 \%$ \\
\hline S2 & Abfallinfr. & $0,0 \%$ & $1,7 \%$ & $2,0 \%$ & $72,5 \%$ & $72,5 \%$ \\
\hline S3 & Konflikte & $0,0 \%$ & $1,8 \%$ & $2,2 \%$ & $36,4 \%$ & $36,4 \%$ \\
\hline S5 & Gesundheit & $0,0 \%$ & $1,8 \%$ & $2,1 \%$ & $76,4 \%$ & $76,4 \%$ \\
\hline S6 & Risiken & $0,0 \%$ & $2,4 \%$ & $3,0 \%$ & $41,3 \%$ & $41,3 \%$ \\
\hline S7 & Katastrophen & $0,0 \%$ & $1,8 \%$ & $2,2 \%$ & $42,3 \%$ & $42,3 \%$ \\
\hline S9 & Ästhetik & $0,0 \%$ & $2,1 \%$ & $2,6 \%$ & $19,3 \%$ & $19,3 \%$ \\
\hline S10 & Soziale Akzeptanz & $0,0 \%$ & $0,7 \%$ & $0,9 \%$ & $47,8 \%$ & $47,8 \%$ \\
\hline
\end{tabular}


Tabelle 10.18: Stabilitätsintervalle Strom, Szenario 1 (mit Förderung)

\begin{tabular}{|c|c|c|c|c|c|c|}
\hline & & $\mathbf{w}_{\min }$ & $w(T)_{\min }$ & $w(T)_{\max }$ & $\mathbf{w}_{\max }$ & $\Delta \mathbf{w}$ \\
\hline W1.1F & Spez. Gesamtk. & $36,0 \%$ & $11,8 \%$ & $43,1 \%$ & $19,4 \%$ & $-16,6 \%$ \\
\hline U1 & ADP & $0,0 \%$ & $2,9 \%$ & $5,1 \%$ & $6,0 \%$ & $6,0 \%$ \\
\hline U2 & LAND & $0,0 \%$ & $4,9 \%$ & $8,6 \%$ & $72,4 \%$ & $72,4 \%$ \\
\hline U3 & GWP & $6,8 \%$ & $4,9 \%$ & $8,6 \%$ & $6,8 \%$ & $0,0 \%$ \\
\hline U4 & ODP & $0,0 \%$ & $1,0 \%$ & $1,7 \%$ & $10,1 \%$ & $10,1 \%$ \\
\hline U5 & HTP & $0,0 \%$ & $1,0 \%$ & $1,7 \%$ & $11,5 \%$ & $11,5 \%$ \\
\hline U6.1 & FAETP & $0,0 \%$ & $0,3 \%$ & $0,6 \%$ & $12,7 \%$ & $12,7 \%$ \\
\hline U6.2 & MAETP & $0,0 \%$ & $0,3 \%$ & $0,6 \%$ & $19,2 \%$ & $19,2 \%$ \\
\hline U6.3 & TAETP & $0,0 \%$ & $0,3 \%$ & $0,6 \%$ & $99,6 \%$ & $99,6 \%$ \\
\hline U7 & POCP & $0,0 \%$ & $2,0 \%$ & $3,4 \%$ & $4,1 \%$ & $4,1 \%$ \\
\hline U8 & AP & $0,0 \%$ & $3,9 \%$ & $6,8 \%$ & $10,3 \%$ & $10,3 \%$ \\
\hline U9 & EP & $0,0 \%$ & $3,9 \%$ & $6,8 \%$ & $8,6 \%$ & $8,6 \%$ \\
\hline U10 & KEA & $6,7 \%$ & $3,9 \%$ & $6,8 \%$ & $6,7 \%$ & $0,0 \%$ \\
\hline V1 & Stat. Rw. (Kehrwert) & $0,0 \%$ & $1,5 \%$ & $2,2 \%$ & $24,6 \%$ & $24,6 \%$ \\
\hline V2 & Importquote & $0,0 \%$ & $1,5 \%$ & $2,2 \%$ & $24,7 \%$ & $24,7 \%$ \\
\hline V3 & Lokale Verfügbarkeit & $0,0 \%$ & $1,5 \%$ & $2,2 \%$ & $100,0 \%$ & $100,0 \%$ \\
\hline V4 & Lagermöglichkeit & $0,0 \%$ & $1,5 \%$ & $2,2 \%$ & $13,8 \%$ & $13,8 \%$ \\
\hline V5 & Herfindahl & $0,0 \%$ & $1,5 \%$ & $2,2 \%$ & $62,4 \%$ & $62,4 \%$ \\
\hline V6 & Risiko-Indikator (Kehrwert) & $0,0 \%$ & $1,5 \%$ & $2,2 \%$ & $100,0 \%$ & $100,0 \%$ \\
\hline V7 & Variationskoeff. Preise & $0,0 \%$ & $1,5 \%$ & $2,2 \%$ & $57,8 \%$ & $57,8 \%$ \\
\hline V8 & Nutzungsgrad & $0,0 \%$ & $1,5 \%$ & $2,2 \%$ & $20,2 \%$ & $20,2 \%$ \\
\hline v9 & Anlagenverfügbarkeit & $0,0 \%$ & $1,5 \%$ & $2,2 \%$ & $18,2 \%$ & $18,2 \%$ \\
\hline S1 & Flex. Innov. & $0,0 \%$ & $1,4 \%$ & $1,7 \%$ & $9,1 \%$ & $9,1 \%$ \\
\hline S2 & Abfallinfr. & $0,0 \%$ & $1,7 \%$ & $2,0 \%$ & $90,0 \%$ & $90,0 \%$ \\
\hline S3 & Konflikte & $0,0 \%$ & $1,8 \%$ & $2,2 \%$ & $15,5 \%$ & $15,5 \%$ \\
\hline S5 & Gesundheit & $0,0 \%$ & $1,8 \%$ & $2,1 \%$ & $49,3 \%$ & $49,3 \%$ \\
\hline S6 & Risiken & $0,0 \%$ & $2,4 \%$ & $3,0 \%$ & $29,1 \%$ & $29,1 \%$ \\
\hline S7 & Katastrophen & $0,0 \%$ & $1,8 \%$ & $2,2 \%$ & $18,8 \%$ & $18,8 \%$ \\
\hline S9 & Ästhetik & $0,0 \%$ & $2,1 \%$ & $2,6 \%$ & $14,9 \%$ & $14,9 \%$ \\
\hline S10 & Soziale Akzeptanz & $0,0 \%$ & $0,7 \%$ & $0,9 \%$ & $21,6 \%$ & $21,6 \%$ \\
\hline
\end{tabular}


Tabelle 10.19: $\quad$ Stabilitätsintervalle Strom, Szenario 2 (ohne Förderung)

\begin{tabular}{|c|c|c|c|c|c|c|}
\hline & & $\mathbf{w}_{\min }$ & $w(T)_{\min }$ & $w(T)_{\max }$ & $\mathbf{w}_{\max }$ & $\Delta \mathbf{w}$ \\
\hline W1.2 & Spez. Gesamtk. & $37,7 \%$ & $11,8 \%$ & $43,1 \%$ & $20,5 \%$ & $-17,2 \%$ \\
\hline U1 & ADP & $0,0 \%$ & $2,9 \%$ & $5,1 \%$ & $18,7 \%$ & $18,7 \%$ \\
\hline U2 & LAND & $0,0 \%$ & $4,9 \%$ & $8,6 \%$ & $52,4 \%$ & $52,4 \%$ \\
\hline U3 & GWP & $0,0 \%$ & $4,9 \%$ & $8,6 \%$ & $17,8 \%$ & $17,8 \%$ \\
\hline U4 & ODP & $0,0 \%$ & $1,0 \%$ & $1,7 \%$ & $6,0 \%$ & $6,0 \%$ \\
\hline U5 & HTP & $0,0 \%$ & $1,0 \%$ & $1,7 \%$ & $4,4 \%$ & $4,4 \%$ \\
\hline U6.1 & FAETP & $0,0 \%$ & $0,3 \%$ & $0,6 \%$ & $4,3 \%$ & $4,3 \%$ \\
\hline U6.2 & MAETP & $0,0 \%$ & $0,3 \%$ & $0,6 \%$ & $6,8 \%$ & $6,8 \%$ \\
\hline U6.3 & TAETP & $0,0 \%$ & $0,3 \%$ & $0,6 \%$ & $98,5 \%$ & $98,5 \%$ \\
\hline U7 & POCP & $0,0 \%$ & $2,0 \%$ & $3,4 \%$ & $11,4 \%$ & $11,4 \%$ \\
\hline U8 & AP & $0,9 \%$ & $3,9 \%$ & $6,8 \%$ & $10,2 \%$ & $9,3 \%$ \\
\hline U9 & EP & $0,8 \%$ & $3,9 \%$ & $6,8 \%$ & $11,4 \%$ & $10,6 \%$ \\
\hline U10 & KEA & $0,0 \%$ & $3,9 \%$ & $6,8 \%$ & $20,1 \%$ & $20,1 \%$ \\
\hline V1 & Stat. Rw. (Kehrwert) & $0,0 \%$ & $1,5 \%$ & $2,2 \%$ & $13,6 \%$ & $13,6 \%$ \\
\hline V2 & Importquote & $0,0 \%$ & $1,5 \%$ & $2,2 \%$ & $14,0 \%$ & $14,0 \%$ \\
\hline V3 & Lokale Verfügbarkeit & $0,0 \%$ & $1,5 \%$ & $2,2 \%$ & $100,0 \%$ & $100,0 \%$ \\
\hline V4 & Lagermöglichkeit & $0,0 \%$ & $1,5 \%$ & $2,2 \%$ & $5,6 \%$ & $5,6 \%$ \\
\hline V5 & Herfindahl & $0,0 \%$ & $1,5 \%$ & $2,2 \%$ & $44,1 \%$ & $44,1 \%$ \\
\hline V6 & Risiko-Indikator (Kehrwert) & $0,0 \%$ & $1,5 \%$ & $2,2 \%$ & $100,0 \%$ & $100,0 \%$ \\
\hline V7 & Variationskoeff. Preise & $0,0 \%$ & $1,5 \%$ & $2,2 \%$ & $38,5 \%$ & $38,5 \%$ \\
\hline V8 & Nutzungsgrad & $0,0 \%$ & $1,5 \%$ & $2,2 \%$ & $8,0 \%$ & $8,0 \%$ \\
\hline v9 & Anlagenverfügbarkeit & $0,0 \%$ & $1,5 \%$ & $2,2 \%$ & $7,2 \%$ & $7,2 \%$ \\
\hline S1 & Flex. Innov. & $0,0 \%$ & $1,4 \%$ & $1,7 \%$ & $25,0 \%$ & $25,0 \%$ \\
\hline S2 & Abfallinfr. & $0,0 \%$ & $1,7 \%$ & $2,0 \%$ & $72,6 \%$ & $72,6 \%$ \\
\hline S3 & Konflikte & $0,0 \%$ & $1,8 \%$ & $2,2 \%$ & $36,4 \%$ & $36,4 \%$ \\
\hline S5 & Gesundheit & $0,0 \%$ & $1,8 \%$ & $2,1 \%$ & $76,4 \%$ & $76,4 \%$ \\
\hline S6 & Risiken & $0,0 \%$ & $2,4 \%$ & $3,0 \%$ & $41,3 \%$ & $41,3 \%$ \\
\hline S7 & Katastrophen & $0,0 \%$ & $1,8 \%$ & $2,2 \%$ & $42,3 \%$ & $42,3 \%$ \\
\hline S9 & Ästhetik & $0,0 \%$ & $2,1 \%$ & $2,6 \%$ & $19,3 \%$ & $19,3 \%$ \\
\hline S10 & Soziale Akzeptanz & $0,0 \%$ & $0,7 \%$ & $0,9 \%$ & $47,7 \%$ & $47,7 \%$ \\
\hline
\end{tabular}


Tabelle 10.20: $\quad$ Stabilitätsintervalle Strom, Szenario 2 (mit Förderung)

\begin{tabular}{|c|c|c|c|c|c|c|}
\hline & & $\mathbf{w}_{\min }$ & $w(T)_{\min }$ & $w(T)_{\max }$ & $\mathbf{w}_{\max }$ & $\Delta \mathbf{w}$ \\
\hline W1.2F & Spez. Gesamtk. & $36,0 \%$ & $11,8 \%$ & $43,1 \%$ & $19,4 \%$ & $-16,6 \%$ \\
\hline U1 & ADP & $0,0 \%$ & $2,9 \%$ & $5,1 \%$ & $6,0 \%$ & $6,0 \%$ \\
\hline U2 & LAND & $0,0 \%$ & $4,9 \%$ & $8,6 \%$ & $72,4 \%$ & $72,4 \%$ \\
\hline U3 & GWP & $6,8 \%$ & $4,9 \%$ & $8,6 \%$ & $6,8 \%$ & $0,0 \%$ \\
\hline U4 & ODP & $0,0 \%$ & $1,0 \%$ & $1,7 \%$ & $10,1 \%$ & $10,1 \%$ \\
\hline U5 & HTP & $0,0 \%$ & $1,0 \%$ & $1,7 \%$ & $11,5 \%$ & $11,5 \%$ \\
\hline U6.1 & FAETP & $0,0 \%$ & $0,3 \%$ & $0,6 \%$ & $12,7 \%$ & $12,7 \%$ \\
\hline U6.2 & MAETP & $0,0 \%$ & $0,3 \%$ & $0,6 \%$ & $19,2 \%$ & $19,2 \%$ \\
\hline U6.3 & TAETP & $0,0 \%$ & $0,3 \%$ & $0,6 \%$ & $99,6 \%$ & $99,6 \%$ \\
\hline U7 & POCP & $0,0 \%$ & $2,0 \%$ & $3,4 \%$ & $4,1 \%$ & $4,1 \%$ \\
\hline U8 & AP & $0,0 \%$ & $3,9 \%$ & $6,8 \%$ & $10,3 \%$ & $10,3 \%$ \\
\hline U9 & EP & $0,0 \%$ & $3,9 \%$ & $6,8 \%$ & $8,6 \%$ & $8,6 \%$ \\
\hline U10 & KEA & $6,7 \%$ & $3,9 \%$ & $6,8 \%$ & $6,7 \%$ & $0,0 \%$ \\
\hline V1 & Stat. Rw. (Kehrwert) & $0,0 \%$ & $1,5 \%$ & $2,2 \%$ & $24,6 \%$ & $24,6 \%$ \\
\hline V2 & Importquote & $0,0 \%$ & $1,5 \%$ & $2,2 \%$ & $24,7 \%$ & $24,7 \%$ \\
\hline V3 & Lokale Verfügbarkeit & $0,0 \%$ & $1,5 \%$ & $2,2 \%$ & $100,0 \%$ & $100,0 \%$ \\
\hline V4 & Lagermöglichkeit & $0,0 \%$ & $1,5 \%$ & $2,2 \%$ & $13,8 \%$ & $13,8 \%$ \\
\hline V5 & Herfindahl & $0,0 \%$ & $1,5 \%$ & $2,2 \%$ & $62,4 \%$ & $62,4 \%$ \\
\hline V6 & Risiko-Indikator (Kehrwert) & $0,0 \%$ & $1,5 \%$ & $2,2 \%$ & $100,0 \%$ & $100,0 \%$ \\
\hline V7 & Variationskoeff. Preise & $0,0 \%$ & $1,5 \%$ & $2,2 \%$ & $57,8 \%$ & $57,8 \%$ \\
\hline V8 & Nutzungsgrad & $0,0 \%$ & $1,5 \%$ & $2,2 \%$ & $20,2 \%$ & $20,2 \%$ \\
\hline v9 & Anlagenverfügbarkeit & $0,0 \%$ & $1,5 \%$ & $2,2 \%$ & $18,2 \%$ & $18,2 \%$ \\
\hline S1 & Flex. Innov. & $0,0 \%$ & $1,4 \%$ & $1,7 \%$ & $9,1 \%$ & $9,1 \%$ \\
\hline S2 & Abfallinfr. & $0,0 \%$ & $1,7 \%$ & $2,0 \%$ & $90,0 \%$ & $90,0 \%$ \\
\hline S3 & Konflikte & $0,0 \%$ & $1,8 \%$ & $2,2 \%$ & $15,5 \%$ & $15,5 \%$ \\
\hline S5 & Gesundheit & $0,0 \%$ & $1,8 \%$ & $2,1 \%$ & $49,3 \%$ & $49,3 \%$ \\
\hline S6 & Risiken & $0,0 \%$ & $2,4 \%$ & $3,0 \%$ & $29,1 \%$ & $29,1 \%$ \\
\hline S7 & Katastrophen & $0,0 \%$ & $1,8 \%$ & $2,2 \%$ & $18,8 \%$ & $18,8 \%$ \\
\hline S9 & Ästhetik & $0,0 \%$ & $2,1 \%$ & $2,6 \%$ & $14,9 \%$ & $14,9 \%$ \\
\hline S10 & Soziale Akzeptanz & $0,0 \%$ & $0,7 \%$ & $0,9 \%$ & $21,6 \%$ & $21,6 \%$ \\
\hline
\end{tabular}


Tabelle 10.21: $\quad$ Stabilitätsintervalle Strom, Szenario 3 (ohne Förderung)

\begin{tabular}{|c|c|c|c|c|c|c|}
\hline & & $\mathbf{w}_{\min }$ & $w(T)_{\min }$ & $w(T)_{\max }$ & $\mathbf{w}_{\max }$ & $\Delta \mathbf{w}$ \\
\hline W1.3 & Spez. Gesamtk. & $38,6 \%$ & $11,8 \%$ & $43,1 \%$ & $30,4 \%$ & $-8,3 \%$ \\
\hline U1 & ADP & $0,0 \%$ & $2,9 \%$ & $5,1 \%$ & $43,5 \%$ & $43,5 \%$ \\
\hline U2 & LAND & $0,0 \%$ & $4,9 \%$ & $8,6 \%$ & $69,1 \%$ & $69,1 \%$ \\
\hline U3 & GWP & $0,0 \%$ & $4,9 \%$ & $8,6 \%$ & $100,0 \%$ & $100,0 \%$ \\
\hline U4 & ODP & $0,0 \%$ & $1,0 \%$ & $1,7 \%$ & $3,2 \%$ & $3,2 \%$ \\
\hline U5 & HTP & $0,0 \%$ & $1,0 \%$ & $1,7 \%$ & $6,6 \%$ & $6,6 \%$ \\
\hline U6.1 & FAETP & $0,0 \%$ & $0,3 \%$ & $0,6 \%$ & $5,5 \%$ & $5,5 \%$ \\
\hline U6.2 & MAETP & $0,0 \%$ & $0,3 \%$ & $0,6 \%$ & $10,9 \%$ & $10,9 \%$ \\
\hline U6.3 & TAETP & $0,0 \%$ & $0,3 \%$ & $0,6 \%$ & $99,1 \%$ & $99,1 \%$ \\
\hline U7 & POCP & $0,0 \%$ & $2,0 \%$ & $3,4 \%$ & $5,8 \%$ & $5,8 \%$ \\
\hline U8 & AP & $2,2 \%$ & $3,9 \%$ & $6,8 \%$ & $12,3 \%$ & $10,1 \%$ \\
\hline U9 & EP & $2,9 \%$ & $3,9 \%$ & $6,8 \%$ & $12,2 \%$ & $9,3 \%$ \\
\hline U10 & KEA & $0,0 \%$ & $3,9 \%$ & $6,8 \%$ & $51,2 \%$ & $51,2 \%$ \\
\hline V1 & Stat. Rw. (Kehrwert) & $0,0 \%$ & $1,5 \%$ & $2,2 \%$ & $8,3 \%$ & $8,3 \%$ \\
\hline V2 & Importquote & $0,0 \%$ & $1,5 \%$ & $2,2 \%$ & $8,2 \%$ & $8,2 \%$ \\
\hline V3 & Lokale Verfügbarkeit & $0,0 \%$ & $1,5 \%$ & $2,2 \%$ & $100,0 \%$ & $100,0 \%$ \\
\hline V4 & Lagermöglichkeit & $0,0 \%$ & $1,5 \%$ & $2,2 \%$ & $8,2 \%$ & $8,2 \%$ \\
\hline V5 & Herfindahl & $0,0 \%$ & $1,5 \%$ & $2,2 \%$ & $28,7 \%$ & $28,7 \%$ \\
\hline V6 & Risiko-Indikator (Kehrwert) & $0,0 \%$ & $1,5 \%$ & $2,2 \%$ & $100,0 \%$ & $100,0 \%$ \\
\hline V7 & Variationskoeff. Preise & $0,0 \%$ & $1,5 \%$ & $2,2 \%$ & $25,8 \%$ & $25,8 \%$ \\
\hline V8 & Nutzungsgrad & $0,0 \%$ & $1,5 \%$ & $2,2 \%$ & $12,0 \%$ & $12,0 \%$ \\
\hline V9 & Anlagenverfügbarkeit & $0,0 \%$ & $1,5 \%$ & $2,2 \%$ & $10,8 \%$ & $10,8 \%$ \\
\hline S1 & Flex. Innov. & $0,0 \%$ & $1,4 \%$ & $1,7 \%$ & $34,1 \%$ & $34,1 \%$ \\
\hline S2 & Abfallinfr. & $0,0 \%$ & $1,7 \%$ & $2,0 \%$ & $81,9 \%$ & $81,9 \%$ \\
\hline S3 & Konflikte & $0,0 \%$ & $1,8 \%$ & $2,2 \%$ & $37,4 \%$ & $37,4 \%$ \\
\hline S5 & Gesundheit & $0,0 \%$ & $1,8 \%$ & $2,1 \%$ & $21,5 \%$ & $21,5 \%$ \\
\hline S6 & Risiken & $0,0 \%$ & $2,4 \%$ & $3,0 \%$ & $23,9 \%$ & $23,9 \%$ \\
\hline S7 & Katastrophen & $0,0 \%$ & $1,8 \%$ & $2,2 \%$ & $12,0 \%$ & $12,0 \%$ \\
\hline S9 & Ästhetik & $0,0 \%$ & $2,1 \%$ & $2,6 \%$ & $28,3 \%$ & $28,3 \%$ \\
\hline S10 & Soziale Akzeptanz & $0,0 \%$ & $0,7 \%$ & $0,9 \%$ & $48,8 \%$ & $48,8 \%$ \\
\hline
\end{tabular}


Tabelle 10.22: $\quad$ Stabilitätsintervalle Strom, Szenario 3 (mit Förderung)

\begin{tabular}{|c|c|c|c|c|c|c|}
\hline & & $\mathbf{w}_{\min }$ & $w(T)_{\min }$ & $w(T)_{\max }$ & $\mathbf{w}_{\max }$ & $\Delta \mathbf{w}$ \\
\hline W1.3F & Spez. Gesamtk. & $37,9 \%$ & $11,8 \%$ & $43,1 \%$ & $29,5 \%$ & $-8,5 \%$ \\
\hline U1 & ADP & $0,0 \%$ & $2,9 \%$ & $5,1 \%$ & $67,8 \%$ & $67,8 \%$ \\
\hline U2 & LAND & $0,0 \%$ & $4,9 \%$ & $8,6 \%$ & $70,5 \%$ & $70,5 \%$ \\
\hline U3 & GWP & $0,0 \%$ & $4,9 \%$ & $8,6 \%$ & $100,0 \%$ & $100,0 \%$ \\
\hline U4 & ODP & $0,0 \%$ & $1,0 \%$ & $1,7 \%$ & $3,1 \%$ & $3,1 \%$ \\
\hline U5 & HTP & $0,0 \%$ & $1,0 \%$ & $1,7 \%$ & $15,3 \%$ & $15,3 \%$ \\
\hline U6.1 & FAETP & $0,0 \%$ & $0,3 \%$ & $0,6 \%$ & $17,0 \%$ & $17,0 \%$ \\
\hline U6.2 & MAETP & $0,0 \%$ & $0,3 \%$ & $0,6 \%$ & $25,2 \%$ & $25,2 \%$ \\
\hline U6.3 & TAETP & $0,0 \%$ & $0,3 \%$ & $0,6 \%$ & $99,7 \%$ & $99,7 \%$ \\
\hline U7 & POCP & $0,0 \%$ & $2,0 \%$ & $3,4 \%$ & $5,7 \%$ & $5,7 \%$ \\
\hline U8 & AP & $0,0 \%$ & $3,9 \%$ & $6,8 \%$ & $12,8 \%$ & $12,8 \%$ \\
\hline U9 & EP & $0,0 \%$ & $3,9 \%$ & $6,8 \%$ & $12,6 \%$ & $12,6 \%$ \\
\hline U10 & KEA & $0,0 \%$ & $3,9 \%$ & $6,8 \%$ & $74,2 \%$ & $74,2 \%$ \\
\hline V1 & Stat. Rw. (Kehrwert) & $0,0 \%$ & $1,5 \%$ & $2,2 \%$ & $8,1 \%$ & $8,1 \%$ \\
\hline V2 & Importquote & $0,0 \%$ & $1,5 \%$ & $2,2 \%$ & $8,0 \%$ & $8,0 \%$ \\
\hline V3 & Lokale Verfügbarkeit & $0,0 \%$ & $1,5 \%$ & $2,2 \%$ & $100,0 \%$ & $100,0 \%$ \\
\hline V4 & Lagermöglichkeit & $0,0 \%$ & $1,5 \%$ & $2,2 \%$ & $18,0 \%$ & $18,0 \%$ \\
\hline V5 & Herfindahl & $0,0 \%$ & $1,5 \%$ & $2,2 \%$ & $28,2 \%$ & $28,2 \%$ \\
\hline V6 & Risiko-Indikator (Kehrwert) & $0,0 \%$ & $1,5 \%$ & $2,2 \%$ & $100,0 \%$ & $100,0 \%$ \\
\hline V7 & Variationskoeff. Preise & $0,0 \%$ & $1,5 \%$ & $2,2 \%$ & $25,3 \%$ & $25,3 \%$ \\
\hline V8 & Nutzungsgrad & $0,0 \%$ & $1,5 \%$ & $2,2 \%$ & $26,0 \%$ & $26,0 \%$ \\
\hline v9 & Anlagenverfügbarkeit & $0,0 \%$ & $1,5 \%$ & $2,2 \%$ & $11,3 \%$ & $11,3 \%$ \\
\hline S1 & Flex. Innov. & $0,0 \%$ & $1,4 \%$ & $1,7 \%$ & $19,5 \%$ & $19,5 \%$ \\
\hline S2 & Abfallinfr. & $0,0 \%$ & $1,7 \%$ & $2,0 \%$ & $92,7 \%$ & $92,7 \%$ \\
\hline S3 & Konflikte & $0,0 \%$ & $1,8 \%$ & $2,2 \%$ & $15,1 \%$ & $15,1 \%$ \\
\hline S5 & Gesundheit & $0,0 \%$ & $1,8 \%$ & $2,1 \%$ & $48,5 \%$ & $48,5 \%$ \\
\hline S6 & Risiken & $0,0 \%$ & $2,4 \%$ & $3,0 \%$ & $28,5 \%$ & $28,5 \%$ \\
\hline S7 & Katastrophen & $0,0 \%$ & $1,8 \%$ & $2,2 \%$ & $18,3 \%$ & $18,3 \%$ \\
\hline S9 & Ästhetik & $0,0 \%$ & $2,1 \%$ & $2,6 \%$ & $14,5 \%$ & $14,5 \%$ \\
\hline S10 & Soziale Akzeptanz & $0,0 \%$ & $0,7 \%$ & $0,9 \%$ & $21,1 \%$ & $21,1 \%$ \\
\hline
\end{tabular}


Tabelle 10.23: Stabilitätsintervalle Wärme, Szenario 0 (ohne Förderung)

\begin{tabular}{|c|c|c|c|c|c|c|}
\hline & & $\mathbf{w}_{\min }$ & $w(T)_{\min }$ & $w(T)_{\max }$ & $\mathbf{w}_{\max }$ & $\Delta \mathbf{w}$ \\
\hline W1.0 & Spez. Gesamtk. & $24,0 \%$ & $16,4 \%$ & $54,9 \%$ & $48,0 \%$ & $24,0 \%$ \\
\hline U1 & ADP & $2,8 \%$ & $2,9 \%$ & $5,8 \%$ & $7,2 \%$ & $4,5 \%$ \\
\hline U2 & LAND & $5,2 \%$ & $4,8 \%$ & $9,7 \%$ & $6,9 \%$ & $1,7 \%$ \\
\hline U3 & GWP & $5,1 \%$ & $4,8 \%$ & $9,7 \%$ & $10,7 \%$ & $5,6 \%$ \\
\hline U4 & ODP & $0,0 \%$ & $1,0 \%$ & $1,9 \%$ & $10,0 \%$ & $10,0 \%$ \\
\hline U5 & HTP & $0,3 \%$ & $1,0 \%$ & $1,9 \%$ & $6,6 \%$ & $6,2 \%$ \\
\hline U6.1 & FAETP & $0,0 \%$ & $0,3 \%$ & $0,6 \%$ & $3,9 \%$ & $3,9 \%$ \\
\hline U6.2 & MAETP & $0,0 \%$ & $0,3 \%$ & $0,6 \%$ & $4,6 \%$ & $4,6 \%$ \\
\hline U6.3 & TAETP & $0,0 \%$ & $0,3 \%$ & $0,6 \%$ & $5,3 \%$ & $5,3 \%$ \\
\hline U7 & POCP & $0,9 \%$ & $1,9 \%$ & $3,9 \%$ & $6,4 \%$ & $5,4 \%$ \\
\hline U8 & AP & $0,6 \%$ & $3,9 \%$ & $7,8 \%$ & $7,7 \%$ & $7,1 \%$ \\
\hline U9 & EP & $3,2 \%$ & $3,9 \%$ & $7,8 \%$ & $5,8 \%$ & $2,7 \%$ \\
\hline U10 & KEA & $4,0 \%$ & $3,9 \%$ & $7,8 \%$ & $9,0 \%$ & $5,0 \%$ \\
\hline V1 & Stat. Rw. (Kehrwert) & $0,0 \%$ & $1,6 \%$ & $2,6 \%$ & $5,9 \%$ & $5,9 \%$ \\
\hline V2 & Importquote & $1,1 \%$ & $1,6 \%$ & $2,6 \%$ & $3,5 \%$ & $2,4 \%$ \\
\hline V3 & Lokale Verfügbarkeit & $1,3 \%$ & $1,6 \%$ & $2,6 \%$ & $3,2 \%$ & $1,9 \%$ \\
\hline V4 & Lagermöglichkeit & $0,0 \%$ & $1,6 \%$ & $2,6 \%$ & $5,8 \%$ & $5,8 \%$ \\
\hline V5 & Herfindahl & $1,2 \%$ & $1,6 \%$ & $2,6 \%$ & $3,5 \%$ & $2,3 \%$ \\
\hline V6 & Risiko-Indikator (Kehrwert) & $0,0 \%$ & $1,6 \%$ & $2,6 \%$ & $83,3 \%$ & $83,3 \%$ \\
\hline V7 & Variationskoeff. Preise & $0,0 \%$ & $1,6 \%$ & $2,6 \%$ & $8,4 \%$ & $8,4 \%$ \\
\hline V8 & Nutzungsgrad & $0,0 \%$ & $1,6 \%$ & $2,6 \%$ & $5,0 \%$ & $5,0 \%$ \\
\hline V9 & Anlagenverfügbarkeit & $0,0 \%$ & $1,6 \%$ & $2,6 \%$ & $11,2 \%$ & $11,2 \%$ \\
\hline S10 & Soziale Akzeptanz & $0,0 \%$ & $1,9 \%$ & $2,4 \%$ & $9,8 \%$ & $9,8 \%$ \\
\hline
\end{tabular}


Tabelle 10.24: Stabilitätsintervalle Wärme, Szenario 0 (mit Förderung)

\begin{tabular}{|c|c|c|c|c|c|c|}
\hline & & $\mathbf{w}_{\min }$ & $w(T)_{\min }$ & $w(T)_{\max }$ & $\mathbf{w}_{\max }$ & $\Delta \mathbf{w}$ \\
\hline W1.0F & Spez. Gesamtk. & $24,0 \%$ & $16,4 \%$ & $54,9 \%$ & $47,6 \%$ & $23,6 \%$ \\
\hline U1 & ADP & $2,8 \%$ & $2,9 \%$ & $5,8 \%$ & $7,3 \%$ & $4,4 \%$ \\
\hline U2 & LAND & $5,1 \%$ & $4,8 \%$ & $9,7 \%$ & $6,9 \%$ & $1,8 \%$ \\
\hline U3 & GWP & $5,2 \%$ & $4,8 \%$ & $9,7 \%$ & $10,8 \%$ & $5,5 \%$ \\
\hline U4 & ODP & $0,0 \%$ & $1,0 \%$ & $1,9 \%$ & $10,6 \%$ & $10,6 \%$ \\
\hline U5 & HTP & $0,4 \%$ & $1,0 \%$ & $1,9 \%$ & $6,7 \%$ & $6,3 \%$ \\
\hline U6.1 & FAETP & $0,0 \%$ & $0,3 \%$ & $0,6 \%$ & $4,0 \%$ & $4,0 \%$ \\
\hline U6.2 & MAETP & $0,0 \%$ & $0,3 \%$ & $0,6 \%$ & $4,8 \%$ & $4,8 \%$ \\
\hline U6.3 & TAETP & $0,0 \%$ & $0,3 \%$ & $0,6 \%$ & $5,2 \%$ & $5,2 \%$ \\
\hline U7 & POCP & $1,0 \%$ & $1,9 \%$ & $3,9 \%$ & $6,5 \%$ & $5,4 \%$ \\
\hline U8 & AP & $0,9 \%$ & $3,9 \%$ & $7,8 \%$ & $7,5 \%$ & $6,6 \%$ \\
\hline U9 & EP & $3,1 \%$ & $3,9 \%$ & $7,8 \%$ & $5,8 \%$ & $2,7 \%$ \\
\hline U10 & KEA & $4,1 \%$ & $3,9 \%$ & $7,8 \%$ & $9,1 \%$ & $5,0 \%$ \\
\hline V1 & Stat. Rw. (Kehrwert) & $0,0 \%$ & $1,6 \%$ & $2,6 \%$ & $6,0 \%$ & $6,0 \%$ \\
\hline V2 & Importquote & $1,2 \%$ & $1,6 \%$ & $2,6 \%$ & $3,5 \%$ & $2,3 \%$ \\
\hline V3 & Lokale Verfügbarkeit & $1,4 \%$ & $1,6 \%$ & $2,6 \%$ & $3,2 \%$ & $1,9 \%$ \\
\hline V4 & Lagermöglichkeit & $0,0 \%$ & $1,6 \%$ & $2,6 \%$ & $6,1 \%$ & $6,1 \%$ \\
\hline V5 & Herfindahl & $1,2 \%$ & $1,6 \%$ & $2,6 \%$ & $3,5 \%$ & $2,3 \%$ \\
\hline V6 & Risiko-Indikator (Kehrwert) & $0,0 \%$ & $1,6 \%$ & $2,6 \%$ & $83,4 \%$ & $83,4 \%$ \\
\hline V7 & Variationskoeff. Preise & $0,0 \%$ & $1,6 \%$ & $2,6 \%$ & $8,0 \%$ & $8,0 \%$ \\
\hline V8 & Nutzungsgrad & $0,0 \%$ & $1,6 \%$ & $2,6 \%$ & $4,8 \%$ & $4,8 \%$ \\
\hline V9 & Anlagenverfügbarkeit & $0,0 \%$ & $1,6 \%$ & $2,6 \%$ & $11,5 \%$ & $11,5 \%$ \\
\hline S10 & Soziale Akzeptanz & $0,0 \%$ & $1,9 \%$ & $2,4 \%$ & $9,8 \%$ & $9,8 \%$ \\
\hline
\end{tabular}


Tabelle 10.25: Stabilitätsintervalle Wärme, Szenario 1 (ohne Förderung)

\begin{tabular}{|c|c|c|c|c|c|c|}
\hline & & $\mathbf{w}_{\min }$ & $w(T)_{\min }$ & $w(T)_{\max }$ & $\mathbf{w}_{\max }$ & $\Delta \mathbf{w}$ \\
\hline W1.1 & Spez. Gesamtk. & $25,4 \%$ & $16,4 \%$ & $54,9 \%$ & $50,3 \%$ & $24,9 \%$ \\
\hline U1 & ADP & $2,3 \%$ & $2,9 \%$ & $5,8 \%$ & $6,7 \%$ & $4,4 \%$ \\
\hline U2 & LAND & $5,1 \%$ & $4,8 \%$ & $9,7 \%$ & $7,1 \%$ & $2,0 \%$ \\
\hline U3 & GWP & $4,6 \%$ & $4,8 \%$ & $9,7 \%$ & $10,1 \%$ & $5,5 \%$ \\
\hline U4 & ODP & $0,0 \%$ & $1,0 \%$ & $1,9 \%$ & $9,5 \%$ & $9,5 \%$ \\
\hline U5 & HTP & $0,0 \%$ & $1,0 \%$ & $1,9 \%$ & $6,6 \%$ & $6,6 \%$ \\
\hline U6.1 & FAETP & $0,0 \%$ & $0,3 \%$ & $0,6 \%$ & $4,1 \%$ & $4,1 \%$ \\
\hline U6.2 & MAETP & $0,0 \%$ & $0,3 \%$ & $0,6 \%$ & $4,8 \%$ & $4,8 \%$ \\
\hline U6.3 & TAETP & $0,0 \%$ & $0,3 \%$ & $0,6 \%$ & $5,6 \%$ & $5,6 \%$ \\
\hline U7 & POCP & $0,2 \%$ & $1,9 \%$ & $3,9 \%$ & $6,6 \%$ & $6,4 \%$ \\
\hline U8 & AP & $1,0 \%$ & $3,9 \%$ & $7,8 \%$ & $8,8 \%$ & $7,8 \%$ \\
\hline U9 & EP & $3,0 \%$ & $3,9 \%$ & $7,8 \%$ & $6,1 \%$ & $3,1 \%$ \\
\hline U10 & KEA & $3,5 \%$ & $3,9 \%$ & $7,8 \%$ & $9,2 \%$ & $5,7 \%$ \\
\hline V1 & Stat. Rw. (Kehrwert) & $0,0 \%$ & $1,6 \%$ & $2,6 \%$ & $5,3 \%$ & $5,3 \%$ \\
\hline V2 & Importquote & $0,8 \%$ & $1,6 \%$ & $2,6 \%$ & $3,2 \%$ & $2,5 \%$ \\
\hline V3 & Lokale Verfügbarkeit & $1,1 \%$ & $1,6 \%$ & $2,6 \%$ & $3,0 \%$ & $2,0 \%$ \\
\hline V4 & Lagermöglichkeit & $0,0 \%$ & $1,6 \%$ & $2,6 \%$ & $6,0 \%$ & $6,0 \%$ \\
\hline V5 & Herfindahl & $0,8 \%$ & $1,6 \%$ & $2,6 \%$ & $3,2 \%$ & $2,4 \%$ \\
\hline V6 & Risiko-Indikator (Kehrwert) & $0,0 \%$ & $1,6 \%$ & $2,6 \%$ & $81,0 \%$ & $81,0 \%$ \\
\hline V7 & Variationskoeff. Preise & $0,0 \%$ & $1,6 \%$ & $2,6 \%$ & $11,1 \%$ & $11,1 \%$ \\
\hline V8 & Nutzungsgrad & $0,0 \%$ & $1,6 \%$ & $2,6 \%$ & $6,1 \%$ & $6,1 \%$ \\
\hline V9 & Anlagenverfügbarkeit & $0,0 \%$ & $1,6 \%$ & $2,6 \%$ & $11,7 \%$ & $11,7 \%$ \\
\hline S10 & Soziale Akzeptanz & $0,0 \%$ & $1,9 \%$ & $2,4 \%$ & $8,7 \%$ & $8,7 \%$ \\
\hline
\end{tabular}


Tabelle 10.26: Stabilitätsintervalle Wärme, Szenario 1 (mit Förderung)

\begin{tabular}{|c|c|c|c|c|c|c|}
\hline & & $\mathbf{w}_{\min }$ & $w(T)_{\min }$ & $w(T)_{\max }$ & $\mathbf{w}_{\max }$ & $\Delta \mathbf{w}$ \\
\hline W1.1F & Spez. Gesamtk. & $25,2 \%$ & $16,4 \%$ & $54,9 \%$ & $50,3 \%$ & $25,1 \%$ \\
\hline U1 & ADP & $2,4 \%$ & $2,9 \%$ & $5,8 \%$ & $6,8 \%$ & $4,4 \%$ \\
\hline U2 & LAND & $5,1 \%$ & $4,8 \%$ & $9,7 \%$ & $7,1 \%$ & $2,0 \%$ \\
\hline U3 & GWP & $4,6 \%$ & $4,8 \%$ & $9,7 \%$ & $10,2 \%$ & $5,6 \%$ \\
\hline U4 & ODP & $0,0 \%$ & $1,0 \%$ & $1,9 \%$ & $9,7 \%$ & $9,7 \%$ \\
\hline U5 & HTP & $0,0 \%$ & $1,0 \%$ & $1,9 \%$ & $6,7 \%$ & $6,7 \%$ \\
\hline U6.1 & FAETP & $0,0 \%$ & $0,3 \%$ & $0,6 \%$ & $4,1 \%$ & $4,1 \%$ \\
\hline U6.2 & MAETP & $0,0 \%$ & $0,3 \%$ & $0,6 \%$ & $5,1 \%$ & $5,1 \%$ \\
\hline U6.3 & TAETP & $0,0 \%$ & $0,3 \%$ & $0,6 \%$ & $5,9 \%$ & $5,9 \%$ \\
\hline U7 & POCP & $0,3 \%$ & $1,9 \%$ & $3,9 \%$ & $6,6 \%$ & $6,3 \%$ \\
\hline U8 & AP & $1,0 \%$ & $3,9 \%$ & $7,8 \%$ & $8,6 \%$ & $7,6 \%$ \\
\hline U9 & EP & $3,0 \%$ & $3,9 \%$ & $7,8 \%$ & $6,1 \%$ & $3,1 \%$ \\
\hline U10 & KEA & $3,6 \%$ & $3,9 \%$ & $7,8 \%$ & $9,3 \%$ & $5,7 \%$ \\
\hline V1 & Stat. Rw. (Kehrwert) & $0,0 \%$ & $1,6 \%$ & $2,6 \%$ & $5,4 \%$ & $5,4 \%$ \\
\hline V2 & Importquote & $0,8 \%$ & $1,6 \%$ & $2,6 \%$ & $3,3 \%$ & $2,5 \%$ \\
\hline V3 & Lokale Verfügbarkeit & $1,1 \%$ & $1,6 \%$ & $2,6 \%$ & $3,1 \%$ & $2,0 \%$ \\
\hline V4 & Lagermöglichkeit & $0,0 \%$ & $1,6 \%$ & $2,6 \%$ & $6,3 \%$ & $6,3 \%$ \\
\hline V5 & Herfindahl & $0,9 \%$ & $1,6 \%$ & $2,6 \%$ & $3,3 \%$ & $2,4 \%$ \\
\hline V6 & Risiko-Indikator (Kehrwert) & $0,0 \%$ & $1,6 \%$ & $2,6 \%$ & $81,3 \%$ & $81,3 \%$ \\
\hline V7 & Variationskoeff. Preise & $0,0 \%$ & $1,6 \%$ & $2,6 \%$ & $10,8 \%$ & $10,8 \%$ \\
\hline V8 & Nutzungsgrad & $0,0 \%$ & $1,6 \%$ & $2,6 \%$ & $5,8 \%$ & $5,8 \%$ \\
\hline V9 & Anlagenverfügbarkeit & $0,0 \%$ & $1,6 \%$ & $2,6 \%$ & $11,7 \%$ & $11,7 \%$ \\
\hline S10 & Soziale Akzeptanz & $0,0 \%$ & $1,9 \%$ & $2,4 \%$ & $8,9 \%$ & $8,9 \%$ \\
\hline
\end{tabular}


Tabelle 10.27: Stabilitätsintervalle Wärme, Szenario 2 (ohne Förderung)

\begin{tabular}{|c|c|c|c|c|c|c|}
\hline & & $\mathbf{w}_{\min }$ & $w(T)_{\min }$ & $w(T)_{\max }$ & $\mathbf{w}_{\max }$ & $\Delta \mathbf{w}$ \\
\hline W1.2 & Spez. Gesamtk. & $25,4 \%$ & $16,4 \%$ & $54,9 \%$ & $50,2 \%$ & $24,8 \%$ \\
\hline U1 & ADP & $2,3 \%$ & $2,9 \%$ & $5,8 \%$ & $6,7 \%$ & $4,5 \%$ \\
\hline U2 & LAND & $5,1 \%$ & $4,8 \%$ & $9,7 \%$ & $7,1 \%$ & $2,0 \%$ \\
\hline U3 & GWP & $4,5 \%$ & $4,8 \%$ & $9,7 \%$ & $10,1 \%$ & $5,6 \%$ \\
\hline U4 & ODP & $0,0 \%$ & $1,0 \%$ & $1,9 \%$ & $9,6 \%$ & $9,6 \%$ \\
\hline U5 & HTP & $0,0 \%$ & $1,0 \%$ & $1,9 \%$ & $6,6 \%$ & $6,6 \%$ \\
\hline U6.1 & FAETP & $0,0 \%$ & $0,3 \%$ & $0,6 \%$ & $4,1 \%$ & $4,1 \%$ \\
\hline U6.2 & MAETP & $0,0 \%$ & $0,3 \%$ & $0,6 \%$ & $4,8 \%$ & $4,8 \%$ \\
\hline U6.3 & TAETP & $0,0 \%$ & $0,3 \%$ & $0,6 \%$ & $5,6 \%$ & $5,6 \%$ \\
\hline U7 & POCP & $0,2 \%$ & $1,9 \%$ & $3,9 \%$ & $6,6 \%$ & $6,4 \%$ \\
\hline U8 & AP & $0,9 \%$ & $3,9 \%$ & $7,8 \%$ & $8,8 \%$ & $7,9 \%$ \\
\hline U9 & EP & $3,1 \%$ & $3,9 \%$ & $7,8 \%$ & $6,1 \%$ & $3,1 \%$ \\
\hline U10 & KEA & $3,5 \%$ & $3,9 \%$ & $7,8 \%$ & $9,2 \%$ & $5,7 \%$ \\
\hline V1 & Stat. Rw. (Kehrwert) & $0,0 \%$ & $1,6 \%$ & $2,6 \%$ & $5,4 \%$ & $5,4 \%$ \\
\hline V2 & Importquote & $0,8 \%$ & $1,6 \%$ & $2,6 \%$ & $3,3 \%$ & $2,5 \%$ \\
\hline V3 & Lokale Verfügbarkeit & $1,1 \%$ & $1,6 \%$ & $2,6 \%$ & $3,0 \%$ & $2,0 \%$ \\
\hline V4 & Lagermöglichkeit & $0,0 \%$ & $1,6 \%$ & $2,6 \%$ & $6,0 \%$ & $6,0 \%$ \\
\hline V5 & Herfindahl & $0,8 \%$ & $1,6 \%$ & $2,6 \%$ & $3,2 \%$ & $2,4 \%$ \\
\hline V6 & Risiko-Indikator (Kehrwert) & $0,0 \%$ & $1,6 \%$ & $2,6 \%$ & $81,0 \%$ & $81,0 \%$ \\
\hline V7 & Variationskoeff. Preise & $0,0 \%$ & $1,6 \%$ & $2,6 \%$ & $11,2 \%$ & $11,2 \%$ \\
\hline V8 & Nutzungsgrad & $0,0 \%$ & $1,6 \%$ & $2,6 \%$ & $6,1 \%$ & $6,1 \%$ \\
\hline V9 & Anlagenverfügbarkeit & $0,0 \%$ & $1,6 \%$ & $2,6 \%$ & $11,6 \%$ & $11,6 \%$ \\
\hline S10 & Soziale Akzeptanz & $0,0 \%$ & $1,9 \%$ & $2,4 \%$ & $8,8 \%$ & $8,8 \%$ \\
\hline
\end{tabular}


Tabelle 10.28: $\quad$ Stabilitätsintervalle Wärme, Szenario 2 (mit Förderung)

\begin{tabular}{|c|c|c|c|c|c|c|}
\hline & & $\mathbf{w}_{\min }$ & $w(T)_{\min }$ & $w(T)_{\max }$ & $\mathbf{w}_{\max }$ & $\Delta \mathbf{w}$ \\
\hline W1.2F & Spez. Gesamtk. & $25,2 \%$ & $16,4 \%$ & $54,9 \%$ & $50,3 \%$ & $25,1 \%$ \\
\hline U1 & ADP & $2,3 \%$ & $2,9 \%$ & $5,8 \%$ & $6,8 \%$ & $4,5 \%$ \\
\hline U2 & LAND & $5,1 \%$ & $4,8 \%$ & $9,7 \%$ & $7,1 \%$ & $2,0 \%$ \\
\hline U3 & GWP & $4,6 \%$ & $4,8 \%$ & $9,7 \%$ & $10,2 \%$ & $5,6 \%$ \\
\hline U4 & ODP & $0,0 \%$ & $1,0 \%$ & $1,9 \%$ & $9,7 \%$ & $9,7 \%$ \\
\hline U5 & HTP & $0,0 \%$ & $1,0 \%$ & $1,9 \%$ & $6,7 \%$ & $6,7 \%$ \\
\hline U6.1 & FAETP & $0,0 \%$ & $0,3 \%$ & $0,6 \%$ & $4,1 \%$ & $4,1 \%$ \\
\hline U6.2 & MAETP & $0,0 \%$ & $0,3 \%$ & $0,6 \%$ & $5,1 \%$ & $5,1 \%$ \\
\hline U6.3 & TAETP & $0,0 \%$ & $0,3 \%$ & $0,6 \%$ & $5,9 \%$ & $5,9 \%$ \\
\hline U7 & POCP & $0,3 \%$ & $1,9 \%$ & $3,9 \%$ & $6,6 \%$ & $6,3 \%$ \\
\hline U8 & AP & $1,0 \%$ & $3,9 \%$ & $7,8 \%$ & $8,7 \%$ & $7,6 \%$ \\
\hline U9 & EP & $3,1 \%$ & $3,9 \%$ & $7,8 \%$ & $6,1 \%$ & $3,1 \%$ \\
\hline U10 & KEA & $3,6 \%$ & $3,9 \%$ & $7,8 \%$ & $9,2 \%$ & $5,6 \%$ \\
\hline V1 & Stat. Rw. (Kehrwert) & $0,0 \%$ & $1,6 \%$ & $2,6 \%$ & $5,4 \%$ & $5,4 \%$ \\
\hline V2 & Importquote & $0,8 \%$ & $1,6 \%$ & $2,6 \%$ & $3,3 \%$ & $2,5 \%$ \\
\hline V3 & Lokale Verfügbarkeit & $1,1 \%$ & $1,6 \%$ & $2,6 \%$ & $3,1 \%$ & $2,0 \%$ \\
\hline V4 & Lagermöglichkeit & $0,0 \%$ & $1,6 \%$ & $2,6 \%$ & $6,3 \%$ & $6,3 \%$ \\
\hline V5 & Herfindahl & $0,9 \%$ & $1,6 \%$ & $2,6 \%$ & $3,3 \%$ & $2,4 \%$ \\
\hline V6 & Risiko-Indikator (Kehrwert) & $0,0 \%$ & $1,6 \%$ & $2,6 \%$ & $81,4 \%$ & $81,4 \%$ \\
\hline V7 & Variationskoeff. Preise & $0,0 \%$ & $1,6 \%$ & $2,6 \%$ & $10,8 \%$ & $10,8 \%$ \\
\hline V8 & Nutzungsgrad & $0,0 \%$ & $1,6 \%$ & $2,6 \%$ & $5,8 \%$ & $5,8 \%$ \\
\hline V9 & Anlagenverfügbarkeit & $0,0 \%$ & $1,6 \%$ & $2,6 \%$ & $11,7 \%$ & $11,7 \%$ \\
\hline S10 & Soziale Akzeptanz & $0,0 \%$ & $1,9 \%$ & $2,4 \%$ & $8,9 \%$ & $8,9 \%$ \\
\hline
\end{tabular}


Tabelle 10.29: $\quad$ Stabilitätsintervalle Wärme, Szenario 3 (ohne Förderung)

\begin{tabular}{|c|c|c|c|c|c|c|}
\hline & & $w_{\min }$ & $w(T)_{\min }$ & $w(T)_{\max }$ & $\mathbf{w}_{\max }$ & $\Delta \mathbf{w}$ \\
\hline W1.3 & Spez. Gesamtk. & $30,8 \%$ & $16,4 \%$ & $54,9 \%$ & $47,9 \%$ & $17,2 \%$ \\
\hline U1 & ADP & $0,7 \%$ & $2,9 \%$ & $5,8 \%$ & $5,2 \%$ & $4,5 \%$ \\
\hline U2 & LAND & $5,4 \%$ & $4,8 \%$ & $9,7 \%$ & $7,9 \%$ & $2,4 \%$ \\
\hline U3 & GWP & $2,6 \%$ & $4,8 \%$ & $9,7 \%$ & $8,1 \%$ & $5,5 \%$ \\
\hline U4 & ODP & $0,0 \%$ & $1,0 \%$ & $1,9 \%$ & $5,4 \%$ & $5,4 \%$ \\
\hline U5 & HTP & $0,5 \%$ & $1,0 \%$ & $1,9 \%$ & $6,7 \%$ & $6,2 \%$ \\
\hline U6.1 & FAETP & $0,0 \%$ & $0,3 \%$ & $0,6 \%$ & $3,1 \%$ & $3,1 \%$ \\
\hline U6.2 & MAETP & $0,0 \%$ & $0,3 \%$ & $0,6 \%$ & $5,2 \%$ & $5,2 \%$ \\
\hline U6.3 & TAETP & $0,0 \%$ & $0,3 \%$ & $0,6 \%$ & $6,2 \%$ & $6,2 \%$ \\
\hline U7 & POCP & $0,0 \%$ & $1,9 \%$ & $3,9 \%$ & $5,6 \%$ & $5,6 \%$ \\
\hline U8 & AP & $2,9 \%$ & $3,9 \%$ & $7,8 \%$ & $12,2 \%$ & $9,3 \%$ \\
\hline U9 & EP & $3,7 \%$ & $3,9 \%$ & $7,8 \%$ & $7,2 \%$ & $3,5 \%$ \\
\hline U10 & KEA & $1,9 \%$ & $3,9 \%$ & $7,8 \%$ & $7,2 \%$ & $5,3 \%$ \\
\hline V1 & Stat. Rw. (Kehrwert) & $0,0 \%$ & $1,6 \%$ & $2,6 \%$ & $3,5 \%$ & $3,5 \%$ \\
\hline V2 & Importquote & $0,0 \%$ & $1,6 \%$ & $2,6 \%$ & $2,5 \%$ & $2,5 \%$ \\
\hline V3 & Lokale Verfügbarkeit & $0,2 \%$ & $1,6 \%$ & $2,6 \%$ & $2,4 \%$ & $2,2 \%$ \\
\hline V4 & Lagermöglichkeit & $0,0 \%$ & $1,6 \%$ & $2,6 \%$ & $6,5 \%$ & $6,5 \%$ \\
\hline V5 & Herfindahl & $0,0 \%$ & $1,6 \%$ & $2,6 \%$ & $2,5 \%$ & $2,5 \%$ \\
\hline V6 & Risiko-Indikator (Kehrwert) & $0,0 \%$ & $1,6 \%$ & $2,6 \%$ & $67,2 \%$ & $67,2 \%$ \\
\hline V7 & Variationskoeff. Preise & $0,0 \%$ & $1,6 \%$ & $2,6 \%$ & $9,4 \%$ & $9,4 \%$ \\
\hline V8 & Nutzungsgrad & $0,0 \%$ & $1,6 \%$ & $2,6 \%$ & $5,5 \%$ & $5,5 \%$ \\
\hline v9 & Anlagenverfügbarkeit & $0,0 \%$ & $1,6 \%$ & $2,6 \%$ & $7,0 \%$ & $7,0 \%$ \\
\hline S10 & Soziale Akzeptanz & $0,0 \%$ & $1,9 \%$ & $2,4 \%$ & $5,3 \%$ & $5,3 \%$ \\
\hline
\end{tabular}


Tabelle 10.30: $\quad$ Stabilitätsintervalle Wärme, Szenario 3 (mit Förderung)

\begin{tabular}{|c|c|c|c|c|c|c|}
\hline & & $\mathbf{w}_{\min }$ & $w(T)_{\min }$ & $w(T)_{\max }$ & $\mathbf{w}_{\max }$ & $\Delta \mathbf{w}$ \\
\hline W1.3F & Spez. Gesamtk. & $29,9 \%$ & $16,4 \%$ & $54,9 \%$ & $47,2 \%$ & $17,2 \%$ \\
\hline U1 & ADP & $0,7 \%$ & $2,9 \%$ & $5,8 \%$ & $5,4 \%$ & $4,6 \%$ \\
\hline U2 & LAND & $5,6 \%$ & $4,8 \%$ & $9,7 \%$ & $7,8 \%$ & $2,3 \%$ \\
\hline U3 & GWP & $2,7 \%$ & $4,8 \%$ & $9,7 \%$ & $8,4 \%$ & $5,7 \%$ \\
\hline U4 & ODP & $0,0 \%$ & $1,0 \%$ & $1,9 \%$ & $5,9 \%$ & $5,9 \%$ \\
\hline U5 & HTP & $0,4 \%$ & $1,0 \%$ & $1,9 \%$ & $6,8 \%$ & $6,4 \%$ \\
\hline U6.1 & FAETP & $0,0 \%$ & $0,3 \%$ & $0,6 \%$ & $2,8 \%$ & $2,8 \%$ \\
\hline U6.2 & MAETP & $0,0 \%$ & $0,3 \%$ & $0,6 \%$ & $4,7 \%$ & $4,7 \%$ \\
\hline U6.3 & TAETP & $0,0 \%$ & $0,3 \%$ & $0,6 \%$ & $6,6 \%$ & $6,6 \%$ \\
\hline U7 & POCP & $0,0 \%$ & $1,9 \%$ & $3,9 \%$ & $5,2 \%$ & $5,2 \%$ \\
\hline U8 & AP & $2,7 \%$ & $3,9 \%$ & $7,8 \%$ & $12,1 \%$ & $9,5 \%$ \\
\hline U9 & EP & $3,8 \%$ & $3,9 \%$ & $7,8 \%$ & $7,2 \%$ & $3,4 \%$ \\
\hline U10 & KEA & $2,0 \%$ & $3,9 \%$ & $7,8 \%$ & $7,5 \%$ & $5,5 \%$ \\
\hline V1 & Stat. Rw. (Kehrwert) & $0,0 \%$ & $1,6 \%$ & $2,6 \%$ & $3,8 \%$ & $3,8 \%$ \\
\hline V2 & Importquote & $0,0 \%$ & $1,6 \%$ & $2,6 \%$ & $2,6 \%$ & $2,6 \%$ \\
\hline V3 & Lokale Verfügbarkeit & $0,2 \%$ & $1,6 \%$ & $2,6 \%$ & $2,5 \%$ & $2,3 \%$ \\
\hline V4 & Lagermöglichkeit & $0,0 \%$ & $1,6 \%$ & $2,6 \%$ & $6,9 \%$ & $6,9 \%$ \\
\hline V5 & Herfindahl & $0,0 \%$ & $1,6 \%$ & $2,6 \%$ & $2,6 \%$ & $2,6 \%$ \\
\hline V6 & Risiko-Indikator (Kehrwert) & $0,0 \%$ & $1,6 \%$ & $2,6 \%$ & $69,9 \%$ & $69,9 \%$ \\
\hline V7 & Variationskoeff. Preise & $0,0 \%$ & $1,6 \%$ & $2,6 \%$ & $10,3 \%$ & $10,3 \%$ \\
\hline V8 & Nutzungsgrad & $0,0 \%$ & $1,6 \%$ & $2,6 \%$ & $5,2 \%$ & $5,2 \%$ \\
\hline V9 & Anlagenverfügbarkeit & $0,0 \%$ & $1,6 \%$ & $2,6 \%$ & $7,7 \%$ & $7,7 \%$ \\
\hline S10 & Soziale Akzeptanz & $0,0 \%$ & $1,9 \%$ & $2,4 \%$ & $5,8 \%$ & $5,8 \%$ \\
\hline
\end{tabular}




\section{Literatur}

Afgan, N.H.; Begic, F.; Kazagic, A. (2007a): Multi-criteria sustainability assessment - A tool for evaluation of new energy system. In: Thermal Science, 11 (3), S. 43-53.

Afgan, N.H.; Carvalho, M.G. (2002): Multi-criteria assessment of new and renewable energy power plants. In: Energy, 27 (8), S. 739-755.

Afgan, N.H.; Carvalho, M.G. (2004): Sustainability assessment of hydrogen energy systems. In: International Journal of Hydrogen Energy, 29 (13), S. 1327-1342.

Afgan, N.H.; Carvalho, M.G.; Hovanov, N.V. (2000): Energy system assessment with sustainability indicators. In: Energy Policy, 28 (9), S. 603-612.

Afgan, N.H.; Pilavachi, P.A.; Carvalho, M.G. (2007b): Multi-criteria evaluation of natural gas resources. In: Energy Policy, 35 (1), S. 704-713.

Afgan, N.H.; Veziroglu, A.; Carvalho, M.G. (2007c): Multi-criteria evaluation of hydrogen system options. In: International Journal of Hydrogen Energy, 32 (15), S. 3183-3193.

Afgan, N.H.; Carvalho, M.G. (2008): Sustainability assessment of a hybrid energy system. In: Energy Policy, 36 (8), S. 2903-2910.

AGEB (2010): Primärenergieverbrauch - Bundesgebiet insgesamt - Auswertungstabellen September 2009. Stand: 28.02.2010. Arbeitsgemeinschaft Energiebilanzen.

Ahl, C.; Eigner-Thiel, S.; Girschner, W.; Karpenstein-Machan, M.; Roland, F.; Ruppert, H.; Ruwisch, V.; Sauer, B.; Scheffer, K.; Schmuck, P. (2007): Bioenergiedörfer Dörfer mit Zukunft, Projektgruppe Bioenergiedörfer der Georg-AugustUniversität Göttingen; Interdisziplinäres Zentrum für Nachhaltige Entwicklung (IZNE) der Universität Göttingen (Hrsg.).

Akash, B.A.; Mamlook, R.; Mohsen, M.S. (1999): Multi-criteria selection of electric power plants using analytical hierarchy process. In: Electric Power Systems Research, 52 (1), S. 29-35.

Al Shemmeri, T.; Al Kloub, B.; Pearman, A. (1997): Model choice in multicriteria decision aid. In: European Journal of Operational Research, 97 (3), S. 550-560.

Alanne, K.; Salo, A.; Saari, A.; Gustafsson, S.-I. (2007): Multi-criteria evaluation of residential energy supply systems. In: Energy and Buildings, 39 (12), S. 1218-1226.

Albadvi, A.; Chaharsooghi, S.K.; Esfahanipour, A. (2007): Decision making in stock trading: An application of PROMETHEE. In: European Journal of Operational Research, 177 (2), S. 673-683.

Anand, G.; Kodali, R. (2008): Selection of lean manufacturing systems using the PROMETHEE. In: Journal of Modelling in Management, 3 (1), S. 40-70. 
Andrews, C.J. (2003): Spurring inventiveness by analyzing tradeoffs: A public look at New England's electricity alternatives. In: Environmental Impact Assessment Review, 12 (1-2), S. 185-210.

Araz, C.; Mizrak Özfirat, P.; Ozkarahan, I. (2007): An integrated multicriteria decisionmaking methodology for outsourcing management. In: Computers \& Operations Research, 34 (12), S. 3738-3756.

Araz, C.; Ozkarahan, I. (2007): Supplier evaluation and management system for strategic sourcing based on a new multicriteria sorting procedure. In: International Journal of Production Economics, 106 (2), S. 585-606.

Assimakopoulos, V.; Charalambopoulos, C.; Samouilidis, J.E. (1991): Combining decision support tools and knowledge-based approach for the development of an integrated system for regional energy planning. In: Energy Systems and Policy, 15 (4), S. 245-255.

ASUE (2005): BHKW-Kenndaten 2005: Module, Anbieter, Kosten, Arbeitsgemeinschaft für sparsamen und umweltfreundlichen Energieverbrauch e.V. (Hrsg.), Kaiserslautern: Verlag Rationeller Erdgaseinsatz.

Ayoko, G.A.; Singh, K.; Balerea, S.; Kokot, S. (2007): Exploratory multivariate modeling and prediction of the physico-chemical properties of surface water and groundwater. In: Journal of Hydrology, 336 (1-2), S. 115-124.

BAFA (2009a): Amtliche Mineralöldaten. Bundesamt für Wirtschaft und Ausfuhrkontrolle (BAFA). Online:

http://www.bafa.de/bafa/de/energie/mineraloel/amtliche mineraloeldaten/index. html (Stand: 11.12.2009a).

BAFA (2009b): Drittlandssteinkohlepreise frei deutsche Grenze. Online: http://www.bafa.de/bafa/de/energie/steinkohle/statistiken/index.html (Stand: 30.07.2009b).

Baker, J. (2008): New technology and possible advances in energy storage. In: Energy Policy, 36 (12), S. 4368-4373.

Bana e Costa, C.A. (2005): On the mathematical foundations of MACBETH. In: Figueira, J.; Greco, S.; Ehrgott, M. (Hrsg.): Multiple criteria decision analysis: State of the art surveys. New York: Springer, S. 409-442.

Bana e Costa, C.; Stewart, T.J.; Vansnick, J.C. (1997): Multicriteria decision analysis: Some thoughts based on the tutorial and discussion sessions of the ESIGMA meetings. In: European Journal of Operational Research, 99 (1), S. 28-37.

Bartels, M.; Dittmann, L.; Huther, H.; Kocyba, H.; Lindenberger, D.; Lutsch, W.; Münch, W.; Neckel, S.; Oberschmidt, J.; Radgen, P.; Witterhold, F.-G. (2008): Schwerpunkte und Effizienzstrategien in der Energieforschung: Abschlussbericht zum Forschungsvorhaben "Multidimensionale Technikbewertung" im Programm EduaR\&D des BMWi, Arbeitsgemeinschaft für Wärme und Heizkraftwirtschaft (AGFW) e.V. bei dem VDEW (Hrsg.), Frankfurt am Main. 
Bartels, M.; Gatzen, C.; Lindenberger, D.; Müsgens, F.; Peek, M.; Seeliger, A.; Steuber, D.; Wissen, R.; Hofer, P.; Kirchner, A.; Scheelhaase, M.; Schlesinger, M. (2005): Energiereport IV: Die Entwicklung der Energiemärkte bis zum Jahr 2030. Energiewirtschaftliche Referenzprognose. Untersuchung im Auftrag des Bundesministeriums für Wirtschaft und Arbeit. Schlussbericht, Energiewirtschaftliches Institut an der Universität zu Köln (EWI); Prognos AG Basel (Hrsg.), Berlin: Bundesministerium für Wirtschaft und Arbeit.

Basson, L.; Petrie, J.G. (2007): An integrated approach for the consideration of uncertainty in decision making supported by Life Cycle Assessment. In: Environmental Modelling and Software, 22 (2), S. 167-176.

Bauer, C. (2007): Holzenergie. In: Dones, R.e.al. (Hrsg.): Sachbilanzen von Energiesystemen: Grundlagen für den ökologischen Vergleich von Energiesystemen und den Einbezug von Energiesystemen in Ökobilanzen für die Schweiz. Final report ecoinvent No. 6-IX. Dübendorf: Paul Scherrer Institut Villigen; Swiss Centre for Life Cycle Inventories.

Bauer, C.; Bolliger, R.; Tuchschmid, M.; Faist-Emmenegger, M. (2007): Wasserkraft. In: Dones, R.e.al. (Hrsg.): Sachbilanzen von Energiesystemen: Grundlagen für den ökologischen Vergleich von Energiesystemen und den Einbezug von Energiesystemen in Ökobilanzen für die Schweiz. Final report ecoinvent No. 6. Dübendorf: Paul Scherrer Institut Villigen; Swiss Centre for Life Cycle Inventories.

BDEW (2009): Energiemarkt Deutschland. Zahlen und Fakten zur Gas-, Strom- und Fernwärmeversorgung. Sommer 2009, BDEW Bundesverband der Energieund Wasserwirtschaft e.V. (Hrsg.), Frankfurt a.M.: VWEW Energieverlag $\mathrm{GmbH}$.

Beccali, M.; Cellura, M.; Ardente, D. (1998): Decision making in energy planning. The ELECTRE multicriteria analysis approach compared to a fuzzy-sets methodology. In: Energy Conversion Management, 39 (16), S. 1869-1881.

Beccali, M.; Cellura, M.; Mistretta, M. (2003): Decision-making in energy planning. Application of the Electre method at regional level for the diffusion of renewable energy technology. In: Renewable Energy, 28 (13), S. 2063-2087.

Begic, F.; Afgan, N.H. (2007): Sustainability assessment tool for the decision making in selection of energy system--Bosnian case. In: Energy, 32 (10), S. 1979-1985.

Behzadian, M.; Kazemzadeh, R.B.; Albadvi, A.; Aghdasi, M. (2010): PROMETHEE: A comprehensive literature review on methodologies and applications. In: European Journal of Operational Research, 200 (1), S. 198-215.

Belton, V.; Stewart, T.J. (2002): Multiple criteria decision analysis: an integrated approach, Boston, Dordrecht, London: Kluwer Academic Publishers.

Bertsch, V. (2008): Uncertainty handling in multi-attribute decision support for industrial risk management, Karlsruhe: Universitätsverlag. 
Beynon, M.J.; Wells, P. (2008): The lean improvement of the chemical emissions of motor vehicles based on preference ranking: A PROMETHEE uncertainty analysis. In: Omega, 36 (3), S. 384-394.

BGR (2008): Bundesrepublik Deutschland Rohstoffsituation 2007, Bundesanstalt für Geowissenschaften (BGR) (Hrsg.), Rohstoffwirtschaftliche Länderstudien, Band XXXVII, Hannover.

Bickel, P.; Friedrich, R.H. (2005): ExternE. Externalities of Energy. Methodology 2005 Update, Bickel, P.; Friedrich, R. (Hrsg.), Luxembourg: European Communities.

Bioenergiedorf Jühnde eG (2006): Datenblatt Bioenergieanlage Jühnde (Stand: 15.06.2006). Online: http://www.bioenergiedorf.de/con/cms/front content.php?idcat=75 (Stand: 13.04.2010).

Blesl, M.; Ohl, M.; Leipnitz, T. (2006): Entwicklungsstand und Entwicklungsbedarf stationärer Brennstoffzellen, Institut für Energiewirtschaft und Rationelle Energieanwendung (IER) (Hrsg.), Stuttgart.

Blunden, L.S.; Bahaj, A.S. (2006): Initial evaluation of tidal stream energy resources at Portland Bill, UK. In: Renewable Energy, 31 (2), S. 121-132.

BMBF (2010): Innovationsallianz "Lithium Ionen Batterie LIB 2015". Bundesministerium für Bildung und Forschung (BMBF). Online: http://www.bmbf.de/de/11828.php (Stand: 27.05.2010).

BMU (2002): Erste Allgemeine Verwaltungsvorschrift zum BundesImmissionsschutzgesetz (Technische Anleitung zur Reinhaltung der Luft - TA Luft) Vom 24. Juli 2002, Berlin: Bundesministerium für Umwelt, Naturschutz und Reaktorsicherheit (BMU).

BMU (2006): Nationaler Allokationsplan 2008-2012 für die Bundesrepublik Deutschland, Berlin: Bundesministerium für Umwelt, Naturschutz und Reaktorsicherheit (BMU).

BMU (2009a): Erneuerbare Energien 2008 in Deutschland. Aktueller Sachstand Mai 2009, Berlin: Bundesministerium für Umwelt, Naturschutz und Reaktorsicherheit (BMU).

BMU (2009b): Hindernis Atomkraft. Die Auswirkungen einer Laufzeitverlängerung der Atomkraftwerke auf erneuerbare Energien, Berlin: Bundesministerium für Umwelt, Naturschutz und Reaktorsicherheit (BMU).

BMU (2009c): Nachhaltige Wärmekonzepte, Berlin: Bundesministerium für Umwelt, Naturschutz und Reaktorsicherheit (BMU).

BMU (2009d): Neues Denken - neue Energie: Roadmap Energiepolitik 2020, Bonn: Bundesministerium für Umwelt, Naturschutz und Reaktorsicherheit (BMU). 
BMU (2009e): Richtlinien zur Förderung von Maßnahmen zur Nutzung erneuerbarer Energien im Wärmemarkt Vom 20. Februar 2009, Berlin: Bundesministerium für Umwelt, Naturschutz und Reaktorsicherheit (BMU).

BMWi (2008): Sichere, bezahlbare und umweltverträgliche Stromversorgung in Deutschland - Geht es ohne Kernenergie?, Berlin: Bundesministerium für Wirtschaft und Technologie (BMWi).

BMWi (2009a): Energie in Deutschland. Trends und Hintergründe zur Energieversorgung in Deutschland, Berlin: Bundesministerium für Wirtschaft und Technologie (BMWi).

BMWi (2009b): Kernenergie in Deutschland. Bundesministerium für Wirtschaft und Technologie (BMWi). Online:

http://www.bmwi.de/BMWi/Navigation/Energie/kernenergie,did=156022.html (Stand: 16.12.2009b).

BMWi (2010): Zahlen und Fakten. Energiedaten. Nationale und internationale Entwicklung (Stand: 22.01.2010). Bundesministerium für Wirtschaft und Technologie. Online: http://www.bmwi.de/BMWi/Navigation/Energie/energiestatistiken.html.

BMWi; BAFA (2009): Monatliche Erdgasbilanz und Entwicklung der Grenzübergangspreise ab 1991. Bundesministerium für Wirtschaft und Technologie (BMWi); Bundesamt für Wirtschaft und Ausfuhrkontrolle (BAFA). Online: http://www.bmwi.de/BMWi/Navigation/Service/publikationen,did=53736.html (Stand: 11.12.2009).

BMWi; BMU (2007): Bericht zur Umsetzung der in der Kabinettsklausur am 23./24.08.2007 in Meseberg beschlossenen Eckpunkte für ein Integriertes Energie- und Klimaprogramm, Berlin: Bundesministerium für Wirtschaft und Technologie (BMWi); Bundesministerium für Umwelt, Naturschutz und Reaktorsicherheit (BMU).

Botzian, R.; Glaunsinger, W.; Kleimaier, M.; Leonhard, W.; Pokojski, M.; Pyc, I.; Raphael, T.; Schwarz, J.; Schröppel, W.; Westermann, D. (2009): Die deutschen Energie- und Klimaziele in Gefahr - lassen sich die Vorgaben im Stromsektor erreichen?, Frankfurt: Verband der Elektrotechnik, Elektronik, Informationstechnik (VDE) e.V.

Bouffard, F.; Kirschen, D.S. (2008): Centralised and distributed electricity systems. In: Energy Policy, 36 (12), S. 4504-4508.

Brans, J.P.; Vincke, P.; Mareschal, B. (1986): How to select and how to rank projects: The PROMETHEE method. In: European Journal of Operational Research, 24 (2), S. 228-238.

Brans, J.-P.; Mareschal, B. (2005): PROMETHEE Methods. In: Figueira, J.; Greco, S.; Ehrgott, M. (Hrsg.): Multiple Criteria Decision Analysis: State of the Art Surveys. New York: Springer, S. 163-195. 
Breitschopf, B.; Klobasa, M.; Sensfuß, F.; Steinbach, J.; Ragwitz, M.; Lehr, U.; Horst, J.; Leprich, U.; Diekmann, J.; Braun, F.; Horn, M. (2010): Einzel- und gesamtwirtschaftliche Analyse von Kosten- und Nutzenwirkungen des Ausbaus Erneuerbarer Energien im deutschen Strom- und Wärmemarkt. Bestandsaufnahme und Bewertung vorliegender Ansätze zur Quantifizierung der Kosten-NutzenWirkungen des Ausbaus Erneuerbarer Energien im Strom- und Wärmebereich Arbeitspaket 1 -, Fraunhofer-Institut für System- und Innovationsforschung (ISI); Gesellschaft für Wirtschaftliche Strukturforschung mbH (GWS); Institut für ZukunftsEnergieSysteme (IZES); Deutsches Institut für Wirtschaftsforschung (DIW) (Hrsg.), Karlsruhe; Osnabrück; Saarbrücken; Berlin.

Briem, S.; Blesl, M.; Fahl, U.; Ohl, M.; Moerschner, J.; Eltrop, L.; Voß, A.; Viebahn, P.; Krewitt, W.; Gürzenich, D.; Tryfonidou, R.; Wagner, H.-J.; Corradini, R.; Richter, S. (2004): Lebenszyklusanalysen ausgewählter zukünftiger Stromerzeugungstechniken. Ein Forschungsvorhaben mit finanzieller Unterstützung des Bundesministeriums für Wirtschaft und Arbeit, Institut für Energiewirtschaft und Rationelle Energieverwendung (IER) Universität Stuttgart; Deutsches Zentrum für Luft- und Raumfahrt (DLR) Stuttgart; Lehrstuhl für Energiesysteme und Energiewirtschaft (LEE) Ruhr-Universität Bochum; Forschungsstelle für Energiewirtschaft (FfE) München (Hrsg.), Düsseldorf: Verein Deutscher Ingenieure (VDI) e.V.

Browne, D.; O'Regan, B.; Moles, R. (2010): Use of multi-criteria decision analysis to explore alternative domestic energy and electricity policy scenarios in an Irish city-region. In: Energy, 35 (2), S. 518-528.

Buchanan, S.C. (1990): Estimating environmental costs of energy resources. In: The Electricity Journal, 3 (6), S. 36-41.

Buchholz, T.; Rametsteiner, E.; Volk, T.A.; Luzadis, V.A. (2009): Multi criteria analysis for bioenergy systems assessments. In: Energy Policy, 37 (2), S. 484-495.

Buehring, W.A.; Foell, W.K.; Keeney, R.L. (1978): Examining energy/environment policy using decision analysis. In: Energy Systems and Policy, 2 (3), S. 341-367.

Bundesregierung (1990): Gesetz über die Einspeisung von Strom aus erneuerbaren Energien in das öffentliche Netz (Stromeinspeisungsgesetz), Bundesgesetzblatt Ausg. 1990 Teil I S. 2633.

Bundesregierung (2000): Gesetz über den Vorrang Erneuerbarer Energien (Erneuerbare-Energien-Gesetz - EEG), Bonn: Bundesgesetzblatt Jahrgang 2000 Teil I, S. 305-309.

Bundesregierung (2002a): Gesetz für die Erhaltung, die Modernisierung und den Ausbau der Kraft-Wärme-Kopplung (Kraft-Wärme-Kopplungsgesetz - KWKG): Bundesgesetzblatt Jahrgang 2002 Teil I Nr. 19, S. 1092-1096.

Bundesregierung (2002b): Gesetz zur geordneten Beendigung der Kernenergienutzung zur gewerblichen Erzeugung von Elektrizität vom 22. April 2002, Bundesgesetzblatt Jahrgang 2002 Teil I Nr. 26, ausgegeben zu Bonn am 26. April 2002, Bonn. 
Bundesregierung (2004a): Dreizehnte Verordnung zur Durchführung des BundesImmissionsschutzgesetzes (Verordnung über Großfeuerungs- und Gasturbinenanlagen) vom 20. Juli 2004 (BGBI. I S. 1717 (2847)), die zuletzt durch Artikel 1 der Verordnung vom 27. Januar 2009 (BGBI. I S. 129) geändert worden ist.

Bundesregierung (2004b): Gesetz zur Neuregelung des Rechts der Erneuerbaren Energien im Strombereich Vom 31. Juli 2004, Bonn: Bundesgesetzblatt Jahrgang 2004 Teil I Nr. 40, S. 1918-1930.

Bundesregierung (2005): Zweites Gesetz zur Neuregelung des Energiewirtschaftsrechts Vom 7. Juli 2005: Bundesgesetzblatt Jahrgang 2005 Teil I, S. 1970 ff.

Bundesregierung (2007): Eckpunkte für ein integriertes Energie- und Klimaprogramm, Berlin.

Bundesregierung (2008a): Gesetz zur Förderung der Kraft-Wärme-Kopplung Vom 25. Oktober 2008, Bundesgesetzblatt Jahrgang 2008 Teil I Nr. 49, S. 2101-2108: Bundesanzeiger.

Bundesregierung (2008b): Gesetz zur Förderung Erneuerbarer Energien im Wärmebereich (Erneuerbare-Energien-Wärmegesetz - EEWärmeG), Bonn: Bundesgesetzblatt Jahrgang 2008 Teil I Nr. 36, S. 1658.

Bundesregierung (2008c): Gesetz zur Neuregelung des Rechts der Erneuerbaren Energien im Strombereich und zur Änderung der damit zusammenhängenden Vorschriften Vom 25. Oktober 2008, Bonn: Bundesgesetzblatt Jahrgang 2008 Teil I Nr. 49, S. 2074-2100.

Bundesregierung (2009a): Energiesteuergesetz (EnergieStG), Bundesgesetzblatt Jahrgang 2009 Teil I, S. 1979.

Bundesregierung (2009b): Nationaler Entwicklungsplan Elektromobilität der Bundesregierung. Online: http://www.elektromobilitaet2008.de/ (Stand: 27.05.2010b).

Bundesregierung (2009c): Wachstum. Bildung. Zusammenhalt. Koalitionsvertrag zwischen CDU, CSU und FDP vom 26.10.2009 (17. Legislaturperiode), Berlin.

Bundesregierung (2010): Energie: Bezahlbar und verlässlich. Presse- und Informationsamt der Bundesregierung. Online:

http://www.bundesregierung.de/nn 774/Content/DE/Artikel/2010/02/2010-0223-Energiekonzept-wird-erarbeitet.html (Stand: 21.05.2010).

Bundesregierung; Energieversorgungsunternehmen (2000): Vereinbarung zwischen der Bundesregierung und den Energieversorgungsunternehmen vom 14. Juni 2000. Online:

http://www.bmwi.de/BMWi/Navigation/Energie/kernenergie,did=156020.html (Stand: 16.12.2009).

Bünger, U.; Crotogino, F.; Donadai, S.; Gatzen, C.; Glaunsinger, W.; Kleinmaier, M.; Könemund, M.; Landinger, H.; Lebioda, T.J.; Leonhard, W.; Sauer, D.; Weber, 
H.; Wenzel, A.; Wolf, E.; Woyke, W.; Zunft, S. (2009): Energiespeicher in Stromversorgungssystemen mit hohem Anteil erneuerbarer Energieträger. Bedeutung, Stand der Technik und Handlungsbedarf, Energietechnische Gesellschaft im VDE (ETG) (Hrsg.), Frankfurt.

Burton, J.; Hubacek, K. (2007): Is small beautiful? A multicriteria assessment of smallscale energy technology applications in local governments. In: Energy Policy, 35 (12), S. 6402-6412.

BWE (2007): Wind Energy Market 2007/2008. 18. Auflage der BWE-Marktübersicht, Bundesverband WindEnergie e.V. (Hrsg.), Berlin.

Capros, P.; Papathanassiou, S.; Samouilidis, J.E. (1988): Multicriteria analysis of energy supply decisions in an uncertain future. In: Omega, 16 (2), S. 107-115.

Carapellucci, R. (2009): A unified approach to assess performance of different techniques for recovering exhaust heat from gas turbines. In: Energy Conversion and Management, 50 (5), S. 1218-1226.

Carmody, O.; Frost, R.; Xi, Y.; Kokot, S. (2007): Adsorption of hydrocarbons on organo-clays--Implications for oil spill remediation. In: Journal of Colloid and Interface Science, 305 (1), S. 17-24.

Cavalcante, C.A.V.; de Almeida, A.T. (2007): A multi-criteria decision-aiding model using PROMETHEE III for preventive maintenance planning under uncertain conditions. In: Journal of Quality in Maintenance Engineering, 13 (4), S. 385397.

Cavallaro, F. (2005): An integrated multi-criteria system to assess sustainable energy options: An application of the PROMETHEE method. In: Fondazione Eni Enrico Mattei, Nota di Lavaro.

Cavallaro, F. (2009): Multi-criteria decision aid to assess concentrated solar thermal technologies. In: Renewable Energy, 34 (7), S. 1678-1685.

Cavallaro, F.; Ciraolo, L. (2005): A multicriteria approach to evaluate wind energy plants on an Italian island. In: Energy Policy, 33 (2), S. 235-244.

Cavelius, R.; Altgeld, H.; Storch, G.; Hauer, A. (2007): Mobile Speicher zur Abwärmenutzung. Wirtschaftlichkeit und Ökobilanz. In: BWK BrennstoffWärme-Kraft, 59 (5), S. 67-71.

Chatzimouratidis, A.I.; Pilavachi, P.A. (2007): Objective and subjective evaluation of power plants and their non-radioactive emissions using the analytic hierarchy process. In: Energy Policy, 35 (8), S. 4027-4038.

Chatzimouratidis, A.I.; Pilavachi, P.A. (2008): Multicriteria evaluation of power plants impact on the living standard using the analytic hierarchy process. In: Energy Policy, 36 (3), S. 1074-1089. 
Chatzimouratidis, A.I.; Pilavachi, P.A. (2009): Technological, economic and sustainability evaluation of power plants using the Analytic Hierarchy Process. In: Energy Policy, 37 (3), S. 778-787.

Cherni, J.A.; Dyner, I.; Henao, F.; Jaramillo, P.; Smith, R.; Font, R.I.O. (2007): Energy supply for sustainable rural livelihoods. A multi-criteria decision-support system. In: Energy Policy, 35 (3), S. 1493-1504.

Chester, L. (2010): Conceptualising energy security and making explicit its polysemic nature. In: Energy Policy, 38 (2), S. 887-895.

Chou, W.C.; Lin, W.T.; Lin, C.Y. (2007): Application of fuzzy theory and PROMETHEE technique to evaluate suitable ecotechnology method: A case study in Shihmen Reservoir Watershed, Taiwan. In: Ecological Engineering, 31 (4), S. 269-280.

Cloquell-Ballester, V.A.; Monterde-Díaz, R.; Cloquell-Ballester, V.A.; SantamarinaSiurana, M.C. (2007): Systematic comparative and sensitivity analyses of additive and outranking techniques for supporting impact significance assessments. In: Environmental Impact Assessment Review, 27 (1), S. 62-83.

CONSENTEC; EWI; IAEW (2008): Analyse und Bewertung der Versorgungssicherheit in der Elektrizitätsversorgung, Untersuchung im Auftrag des Bundesministeriums für Wirtschaft und Technologie (BMWi). Abschlussbericht vom 30.05.2008: CONSenTEC Consulting für Energiewirtschaft und -technik $\mathrm{GmbH}$; Energiewirtschaftliches Institut an der Universität zu Köln (ewi); Institut für Elektrische Anlagen und Energiewirtschaft der RWTH Aachen (IAEW).

Cornelissen, T.; Jans, M.; Stals, M.; Kuppens, T.; Thewys, T.; Janssens, G.K.; Pastijn, H.; Yperman, J.; Reggers, G.; Schreurs, S.; Carleer, R. (2009): Flash copyrolysis of biomass: The influence of biopolymers. In: Journal of Analytical and Applied Pyrolysis, 85 (1-2), S. 87-97.

Cramer, B.; Andruleit, H.; Rempel, H.; Babies, H.-G.; Schlömer, S.; Schmidt, S.; Schwarz-Schampera, U.; Ochmann, N.; Meßner, J.; Rehder, S. (2009): Energierohstoffe 2009: Reserven, Ressourcen, Verfügbarkeit. Erdöl, Erdgas, Kohle, Kernbrennstoffe, Geothermische Energie, Bundesanstalt für Geowissenschaften und Rohstoffe (BGR) (Hrsg.), Hannover.

Crastan, V. (2007): Elektrische Energieversorgung 1: Netzelemente, Modellierung, stationäres Verhalten, Bemessung, Schalt- und Schutztechnik, 2, Berlin; Heidelberg; New York: Springer.

Crastan, V. (2009): Elektrische Energieversorgung 2: Energie- und Elektrizitätswirtschaft, Kraftwerktechnik, alternative Stromversorgung, Dynamik, Regelung und Stabilität, Betriebsplanung und -führung, 2, Berlin; Heidelberg: Springer.

Czisch, G. (2005): Szenarien zur zukünftigen Stromversorgung. Kostenoptimierte Variationen zur Versorgung Europas und seiner Nachbarn mit Strom aus erneuerbaren Energien, Institut für Elektrische Energietechnik / Rationelle Energieumwandlung (Hrsg.): Universität Kassel. 
Dagdeviren, M. (2008): Decision making in equipment selection: An integrated approach with AHP and PROMETHEE. In: Journal of Intelligent Manufacturing, 19 (4), S. 397-406.

Daim, T.; Yates, D.; Peng, Y.; Jimenez, B. (2009): Technology assessment for clean energy technologies: The case of the Pacific Northwest. In: Technology in Society, 31 (3), S. 232-243.

de Almeida, A.T.; Moura, P.S.; Marques, A.S.; de Almeida, J.L. (2005): Multi-impact evaluation of new medium and large hydropower plants in Portugal centre region. In: Renewable and Sustainable Energy Reviews, 9 (2), S. 149-167.

Deane, J.P.; Ó Gallachóir, B.P.; McKeogh, E.J. (2010): Techno-economic review of existing and new pumped hydro energy storage plant. In: Renewable and Sustainable Energy Reviews, 14 (4), S. 1293-1302.

den Elzen, M.; Meinshausen, M. (2006): Meeting the EU $2{ }^{\circ} \mathrm{C}$ climate target: Global and regional emission implications. In: Climate Policy, 6 (5), S. 545-564.

Denkena, B.; Tönshoff, H.K.; Apitz, R.; Granthien, M. (2002): Wissensmanagement im integrierten Produktlebenszyklus. In: Zeitschrift für wirtschaftlichen Fabrikbetrieb, 97 (9), S. 428-431.

DESERTEC Foundation (2009): Red Paper. Das DESERTEC Konzept im Überblick (3. Auflage). Online: http://www.desertec.org/de/presse/mediathek/zusammenfassungen/ (Stand: 26.05.2010).

Diakoulaki, D.; Georgiou, P.; Tourkolias, C.; Georgopoulou, E.; Lalas, D.; Mirasgedis, S.; Sarafidis, Y. (2007): A multicriteria approach to identify investment opportunities for the exploitation of the clean development mechanism. In: Energy Policy, 35 (2), S. 1088-1099.

Diakoulaki, D.; Henggeler Antunes, C.; Gomes Martins, A. (2005): MCDA and energy planning. In: Figueira, J.; Greco, S.; Ehrgott, M. (Hrsg.): Multiple Criteria Decision Analysis: State of the Art Surveys. New York: Springer, S. 859-897.

Diakoulaki, D.; Karangelis, F. (2007): Multi-criteria decision analysis and cost-benefit analysis of alternative scenarios for the power generation sector in Greece. In: Renewable and Sustainable Energy Reviews, 11 (4), S. 716-727.

Dinca, C.; Badea, A.; Rousseaux, P.; Apostol, T. (2007): A multi-criteria approach to evaluate the natural gas energy systems. In: Energy Policy, 35 (11), S. 57545765.

Dittmann, F. (2009): Zum 125. Geburtstag der Bewag. 125 Jahre öffentliche Elektrizitätsversorgung. In: ew - das magazin für die energie wirtschaft, 108 (10), S. 5861.

Dötsch, C.; Nicolai, S.; Arnold, C.; Hochloff, P.; Oberschmidt, J.; Tübke, J.; Noack, J.; Bertold, S.; Wolf, D.; Lenz, B.; Luttermann, H.; Smolinka, T.; Klobasa, M.; 
Marscheider-Weidemann, F.; Radgen, P. (2008): MAVO "Advanced Energy Storage" Endbericht Phase A (unveröffentlicht): Fraunhofer Gesellschaft.

Doukas, H.; Patlitzianas, K.D.; Psarras, J. (2006): Supporting sustainable electricity technologies in Greece using MCDM. In: Resources Policy, 31 (2), S. 129-136.

Doukas, H.C.; Andreas, B.M.; Psarras, J.E. (2007): Multi-criteria decision aid for the formulation of sustainable technological energy priorities using linguistic variables. In: European Journal of Operational Research, 182 (2), S. 844-855.

Dyer, J.S. (2005): MAUT - Multi Attribute Utility Theory. In: Figueira, J.; Greco, S.; Ehrgott, M. (Hrsg.): Multiple Criteria Decision Analysis: State of the Art Surveys. New York: Springer, S. 265-295.

ecoinvent Centre (2007): ecoinvent data v2.0. ecoinvent reports No. 1-25, Swiss Centre for Life Cycle Inventories (Hrsg.), Dübendorf: www.ecoinvent.org.

Edwards, P.P.; Kuznetsov, V.L.; David, W.I.F.; Brandon, N.P. (2008): Hydrogen and fuel cells: Towards a sustainable energy future. In: Energy Policy, 36 (12), S. 4356-4362.

Eigner, S.; Schmuck, P. (2002): Motivating collective action: converting to sustainable energy sources in a German community. In: Schmuck, P.; Schultz, W.P. (Hrsg.): Psychology of sustainable development. Boston, Dordrecht, London: Kluwer Academic Publishers, S. 241-256.

Eikmeier, B.; Gabriel, J.; Schulz, W.; Krewitt, W.; Nast, M. (2006): Analyse des nationalen Potenzials für den Einsatz hocheffizienter KWK, einschließlich hocheffizienter Kleinst-KWK, unter Berücksichtigung der sich aus der EU-KWK-RL ergebenden Aspekte, Bremer Energie Institut; Deutsches Zentrum für Luft- und Raumfahrt (DLR) (Hrsg.), Bremen, Stuttgart.

Ender, C. (2010): Windenergienutzung in Deutschland - Status 31.12.2009 -. In: DEWI Magazin (32), S. 28-41.

Erdmenger, C. (2006): Plädoyer für ein Einheitsbenchmark. In: Energie \& Management, Sonderteil Power-Gen (10), S. 18-19.

Erdogmus, S.; Aras, H.; Koç, E. (2006): Evaluation of alternative fuels for residential heating in Turkey using analytic network process (ANP) with group decisionmaking. In: Renewable and Sustainable Energy Reviews, 10 (3), S. 269-279.

Eurobserv'er (2009): Wind Energy Barometer. In: Systèmes Solaires Le Journal de l'Éolien (4).

Europäische Kommission (2006): Grünbuch: Eine europäische Strategie für nachhaltige, wettbewerbsfähige und sichere Energie KOM(2006) 105 endgültig, Brüssel.

Europäische Kommission (2007): Mitteilung der Kommission an den Rat und das Europäische Parlament: Fahrplan für erneuerbare Energien Erneuerbare Energien 
im 21. Jahrhundert: Größere Nachhaltigkeit in der Zukunft KOM(2006) 848 endgültig, Brüssel.

Europäische Kommission (2009): Mitteilung der Kommission an das Europäische Parlament, den Rat, den Europäischen Wirtschafts- und Sozialausschuss und den Ausschuss der Regionen: Ein umfassendes Klimaschutzübereinkommen als Ziel für Kopenhagen. KOM(2009) 39 endgültig, Brüssel.

Europäischer Rat (2007): Übermittlungsvermerk des Vorsitzes für die Delegationen: Schlussfolgerungen des Vorsitzes, Tagung vom 8./9. März 2007, Brüssel.

Europäisches Parlament; Rat der Europäischen Union (2003a): Richtlinie 2003/54/EG des Europäischen Parlaments und des Rates vom 26. Juni 2003 über gemeinsame Vorschriften für den Elektrizitätsbinnenmarkt und zur Aufhebung der Richtlinie 96/92/EG: Amtsblatt der Europäischen Union L 176/37-55.

Europäisches Parlament; Rat der Europäischen Union (2003b): Richtlinie 2003/55/EG des Europäischen Parlaments und des Rates vom 26. Juni 2003 über gemeinsame Vorschriften für den Erdgasbinnenmarkt und zur Aufhebung der Richtlinie 98/30/EG.

Europäisches Parlament; Rat der Europäischen Union (2003c): Richtlinie 2003/87/EG des Europäischen Parlaments und des Rates vom 13. Oktober 2003 über ein System für den Handel mit Treibhausgasemissionszertifikaten in der Gemeinschaft und zur Änderung der Richtlinie 96/61/EG des Rates: Amtsblatt der Europäischen Union L 257/32-46.

Europäisches Parlament; Rat der Europäischen Union (2004): Richtlinie 2004/8/EG des Europäischen Parlaments und des Rates vom 11. Februar 2004 über die Förderung einer am Nutzwärmebedarf orientierten Kraft-Wärme-Kopplung im Energiebinnenmarkt und zur Änderung der Richtlinie 92/42/EWG: Amtsblatt der Europäischen Union L 52/50-60.

Europäisches Parlament; Rat der Europäischen Union (2009): Richtlinie 2009/29/EG des Europäischen Parlaments und des Rates vom 23. April 2009 zur Änderung der Richtlinie 2003/87/EG zwecks Verbesserung und Ausweitung des Gemeinschaftssystems für den Handel mit Treibhausgasemissionszertifikaten: Amtsblatt der Europäischen Union L 140/63-87.

EWI; EEFA (2007): Energiewirtschaftliches Gesamtkonzept 2030. Erweiterte Szenariendokumentation, Energiewirtschaftliches Institut an der Universität zu Köln; Energy Environment Forecast Analysis GmbH Münster (Hrsg.), Köln; Münster.

Figueira, J.; Greco, S.; Ehrgott, M. (2005a): Introduction. In: Figueira, J.; Greco, S.; Ehrgott, M. (Hrsg.): Multiple Criteria Decision Analysis: State of the Art Reviews. New York: Springer, S. xxi-xxxvi.

Figueira, J.; Greco, S.; Ehrgott, M. (2005b): Multiple Criteria Decision Analysis: State of the Art Surveys, New York: Springer. 
Figueira, J.; Roy, B. (2002): Determining the weights of criteria in the ELECTRE type methods with a revised Simos' procedure. In: European Journal of Operational Research, 139 (2), S. 317-326.

FIZ (2005): Wärmespeicher, 7, FIZ Karlsruhe; BINE Informationsdienst (Hrsg.), Berlin: Solarpraxis AG.

FNR (2005): Leitfaden Bioenergie. Planung, Betrieb und Wirtschaftlichkeit von Bioenergieanlagen, Fachagentur Nachwachsende Rohstoffe e.V.(FNR) (Hrsg.), Gülzow.

French, S.; Maule, J.; Papamichail, N. (2009): Decision Behaviour, Analysis and Support, Cambridge, New York, Melbourne, Madrid, Cape Town, Singapore, São Paulo, Delhi: Cambridge University Press.

Friedrich, R.; Voss, A. (1993): External costs of electricity generation. In: Energy Policy, 21 (2), S. 114-122.

Frischknecht, R.; Jungbluth, N.; Althaus, H.-J.; Bauer, C.; Doka, G.; Dones, R.; Hischier, R.; Hellweg, S.; Humbert, S.; Köllner, T.; Loerincik, Y.; Margni, M.; Nemecek, T. (2007a): Implementation of life cycle impact assessment methods. ecoinvent report No. 3, Swiss Centre for Life Cycle Inventories (Hrsg.), Dübendorf.

Frischknecht, R.; Jungbluth, N.; Althaus, H.-J.; Doka, G.; Heck, T.; Hellweg, S.; Hischier, R.; Nemecek, T.; Rebitzer, K.; Spielmann, M.; Wernet, G. (2007b): Overview and methodology. ecoinvent report No. 1, Swiss Centre for Life Cycle Inventories (Hrsg.), Dübendorf.

Fritsche, U.R.; Schmidt, K. (2008): Global Emission Model of Integrated Systems (GEMIS) Manual, Öko-Institut e.V. (Hrsg.), Darmstadt.

Frondel, M.; Grösche, P.; Huchtemann, D.; Oberheitmann, A.; Peters, J.; Angerer, G.; Sartorius, C.; Buchholz, P.; Wagner, M. (2007): Trends der Angebots- und Nachfragesituation bei mineralischen Rohstoffen: Endbericht zum Forschungsprojekt Nr. 09/05 des Bundesministeriums für Wirtschaft und Technologie (BMWi), Rheinisch-Westfälisches Institut für Wirtschaftsforschung (RWI Essen); Fraunhofer Institut für System- und Innovationsforschung (ISI); Bundesanstalt für Geowissenschaften (BGR) (Hrsg.).

Frondel, M.; Schmidt, C.M. (2008): Measuring energy security. A conceptual note. In: Ruhr Economic Papers (52).

Frost \& Sullivan (2005): Developments in gas turbine technologies (D602), San Antonio: Frost \& Sullivan.

G8 (2009): G8 Leaders Declaration: Responsible leadership for a sustainable future, G8 Summit, 8.-10. Juli 2009, L'Aquila, http://www.g8italia2009.it/G8/Home/Summit/G8-G8 Layout locale1199882116809 Atti.htm (03.04.2010). 
Gallego Carrera, D.; Mack, A. (2009): Quantification of social indicators for the assessment of energy system related effects. In: Stuttgart contributions to risk and sustainability research, 12 .

Gallego Carrera, D.; Mack, A. (2010): Sustainability assessment of energy technologies via social indicators: Results of a survey among European energy experts. In: Energy Policy, 38 (2), S. 1030-1039.

Gamboa, G. (2006): Social multi-criteria evaluation of different development scenarios of the Aysén region, Chile. In: Ecological Economics, 59 (1), S. 157-170.

Gamboa, G.; Munda, G. (2007): The problem of windfarm location: A social multicriteria evaluation framework. In: Energy Policy, 35 (3), S. 1564-1583.

Gatzen, C. (2008): The economics of power storage: theory and empirical analysis for Central Europe - Theory and Empirical Analysis for Central Europe, Energiewirtschaftliches Institut an der Universität zu Köln (Hrsg.), Schriften des Energiewirtschaftlichen Instituts, München: Oldenburg Industrieverlag.

Geldermann, J. (2005): Mehrzielentscheidungen in der industriellen Produktion, Karlsruhe: Universitätsverlag.

Geldermann, J.; Rentz, O. (2000): Bridging the gap between American and European MADM-approaches?, Presented at the 51st Meeting of the European Working Group "Multicriteria Aid for Decisions", Madrid: 30.-31.03.2000.

Geldermann, J.; Rentz, O. (2001): Integrated technique assessment with imprecise information as a support for the identification of best available techniques (BAT). In: OR Spektrum, 23 (1), S. 137-157.

Geldermann, J.; Spengler, T.; Rentz, O. (2000): Fuzzy outranking for environmental assessment. Case study: Iron and steel making industry. In: Fuzzy Sets and Systems, 115 (1), S. 45-65.

Geldermann, J.; Zhang, K.; Rentz, O. (2002a): Development of an integrated multicriteria group decision support system (MGDSS), 12th Mini EURO Conference, Brüssel: 2.-5.04.2002.

Geldermann, J.; Zhang, K.; Rentz, O. (2002b): Entwicklung eines integrierten multikriteriellen Gruppenentscheidungsunterstützungssystems. In: Fichtner, W.; Geldermann, G. (Hrsg.): Einsatz von OR-Verfahren zur techno-ökonomischen Analyse von Produktionssystemen. Frankfurt am Main, Berlin, Bern, Brüssel, New York, Oxford, Wien: Peter Lang, S. 169-186.

Geldermann, J.; Zhang, K.; Rentz, O. (2003): Sensitivitätsanalysen für das OutrankingVerfahren PROMETHEE. In: Habenicht, W.; Scheubrein, B.; Scheubrein, R. (Hrsg.): Multi-Criteria- und Fuzzy-Systeme in Theorie und Praxis. Lösungsansätze für Entscheidungsprobleme mit komplexen Zielsystemen. Wiesbaden: Deutscher Universitäts-Verlag, S. 127. 
Georgopoulou, E.; Lalas, D.; Papagiannakis, L. (1997): A multicriteria decision aid approach for energy planning problems: The case of renewable energy option. In: European Journal of Operational Research, 103 (1), S. 38-54.

Georgopoulou, E.; Sarafidis, Y.; Diakoulaki, D. (1998): Design and implementation of a group DSS for sustaining renewable energies exploitation. In: European Journal of Operational Research, 109 (2), S. 483-500.

Georgopoulou, E.; Sarafidis, Y.; Mirasgedis, S.; Zaimi, S.; Lalas, D.P. (2003): A multiple criteria decision-aid approach in defining national priorities for greenhouse gases emissions reduction in the energy sector. In: European Journal of Operational Research, 146 (1), S. 199-215.

Ghafghazi, S.; Sowlati, T.; Sokhansanj, S.; Melin, S. (2009): A multicriteria approach to evaluate district heating system options. In: Applied Energy, 87 (4), S. 11341140.

Ghazinoory, S.; Divsalar, A.; Soofi, A.S. (2009): A new definition and framework for the development of a national technology strategy: The case of nanotechnology for Iran. In: Technological Forecasting and Social Change, 76 (6), S. 835-848.

Gholamnezhad, A.H. (1983): A new model in the energy policy planning. In: Mathematics and Computers in Simulation, 25 (2), S. 125-134.

Gholamnezhad, A.H.; Saaty, T.L. (1982): A desired energy mix for the United States in the year 2000: an analytic hierarchy approach. In: International Journal of Policy Analysis and Information Systems, 6 (1), S. 47-64.

Goletsis, Y.; Psarras, J.; Samouilidis, J.E. (2003): Project ranking in the Armenian energy sector using a multicriteria method for groups. In: Annals of Operations Research, 120 (1-4), S. 135-157.

Gombert, A.; Hakenjos, A.; Heile, I.; Wüllner, J.; Gerstmaier, T.; van Riesen, S. (2009): Flatcon CPV systems - field data and new developments, 24th PVSEC, 22. September 2009, Hamburg.

Götze, U.; Rudolph, F. (1994): Instrumente der strategischen Planung. In: Bloech, J.; Götze, U.; Huch, B.; Lücke, W.; Rudolph, F. (Hrsg.): Strategische Planung. Instrumente, Vorgehensweisen und Informationssysteme. Heidelberg: PhysicaVerlag, S. 1-56.

Götze, U. (2006): Investitionsrechnung. Modelle und Analysen zur Beurteilung von Investitionsvorhaben, Berlin, Heidelberg, New York: Springer.

Goumas, M.; Lygerou, V. (2000): An extension of the PROMETHEE method for decision making in fuzzy environment: Ranking of alternative energy exploitation projects. In: European Journal of Operational Research, 123 (3), S. 606-613.

Goumas, M.G.; Lygerou, V.A.; Papayannakis, L.E. (1999): Computational methods for planning and evaluating geothermal energy projects. In: Energy Policy, 27 (3), S. 147-154. 
Greening, L.A.; Bernow, S. (2004): Design of coordinated energy and environmental policies. Use of multicriteria decision making. In: Energy Policy, 32 (6), S. 721735.

Grupp, H. (1997): Messung und Erklärung des Technischen Wandels. Grundzüge einer empirischen Innovationsökonomik, Berlin, Heidelberg, New York: Springer.

Guinée, J.B.H.; de Bruijn, H.; van Duin, R.; Huijbregts, M.A.J.; Lindejer, E.; Roorda, A.A.H.; van der Ven, B.L.; Weidema, B.P. (2002): Handbook of life cycle assessment. Operational guide to the ISO standards, Dordrecht, Boston, London: Kluwer Academic Publishers.

Gummert, G.; Suttor, W. (2006): Stationäre Brennstoffzellen, Heidelberg: C. F. Müller Verlag.

Güngör, Z.; Arikan, F. (2000): A fuzzy outranking method in energy policy planning. In: Fuzzy Sets and Systems, 114 (1), S. 115-122.

Haldi, P.A.; Frei, C.; Beurskens, L.; Zhuikova, N. (2002): Multicriteria/multi-stakeholders comparative assessment of electricity generation scenarios in the sustainability context: A Swiss case study. In: International Journal of Sustainable Development, 5 (1-2), S. 102-124.

Hämäläinen, R.P. (1990): A decision aid in the public debate on nuclear power. In: European Journal of Operational Research, 48 (1), S. 66-76.

Hämäläinen, R.P.; Karjalainen, R. (1992): Decision support for risk analysis in energy policy. In: European Journal of Operational Research, 56 (2), S. 172-183.

Hämäläinen, R.P.; Seppäläinen, T.O. (1986): The analytic network process in energy policy planning. In: Socio-Economic Planning Sciences, 20 (6), S. 399-405.

Hanegraaf, M.; Biewinga, E.; Van der Bijl, G. (1998): Assessing the ecological and economic sustainability of energy crops. In: Biomass and Bioenergy, 15 (4/5), S. $345-355$.

Hanson, M.; Kidwell, S.; Ray, D.; Stevenson, R. (1991): Electric utility least-cost planning: making it work within a multiattribute decision-making framework. In: Journal - American Planning Association, 57 (1), S. 34-43.

Haralambopoulos, D.A.; Polatidis, H. (2003): Renewable energy projects: Structuring a multi-criteria group decision-making framework. In: Renewable Energy, 28 (6), S. 961-973.

Härdtlein, M.; Eltrop, L.T.D.H. (2004): Voraussetzungen zur Standardisierung biogener Festbrennstoffe, Nachwachsende Rohstoffe, Band 23, Münster: Landwirtschaftsverlag $\mathrm{GmbH}$.

Hartmann, F.; Schwarzburger, H. (2009): Systemtechnik für Wärmepumpen. Solar- und Umweltwärme für Wohngebäude, München; Heidelberg: Hüthig \& Pflaum. 
Hartz, T.; Koll, G. (2008): Solarturm Jülich - Das erste solarthermische Versuchskraftwerk in Deutschland wird gebaut, 11. Kölner Sonnenkolloquium, 24. Juni 2008, Deutsches Zentrum für Luft- und Raumfahrt (DLR) e.V., http://www.dlr.de/tt/Portaldata/41/Resources/dokumente/soko2008/3 Solarturm Juelich Erstes solartherm VersuchsKW in D.pdf (26.05.2010).

Heinrich, G.; Basson, L.; Cohen, B.; Howells, M.; Petrie, J. (2007): Ranking and selection of power expansion alternatives for multiple objectives under uncertainty. In: Energy, 32 (12), S. 2350-2369.

Held, A.; Breitschopf, B.; Lürich, L. (2010): Renewables development database - electricity. Karlsruhe: Fraunhofer ISI.

Hermans, C.; Erickson, J.; Noordewier, T.; Sheldon, A.; Kline, M. (2007): Collaborative environmental planning in river management: An application of multicriteria decision analysis in the White River Watershed in Vermont. In: Journal of Environmental Management, 84 (4), S. 534-546.

Heuck, K.; Dettmann, K.-D.; Schulz, D. (2007): Elektrische Energieversorgung. Erzeugung, Übertragung und Verteilung elektrischer Energie für Studium und Praxis, 7, Wiesbaden: Vieweg \& Sohn.

Hillenbrand, T. (2009): Analyse und Bewertung neuer urbaner Wasserinfrastruktursysteme, Schriftenreihe SWW, Band 134, Karlsruhe: Verlag Siedlungswasserwirtschaft.

Hobbs, B.; Meier, P. (2003): Energy decisions and the environment: a guide to the use of multicriteria methods, International series in operations research \& management sciences, Boston, Dordrecht, London: Kluwer Academic Publishers.

Hoffmann, K. (1972): Der Produktlebenszyklus. Eine kritische Analyse, Absatzwirtschaft und Konsumforschung, Meyer-Dohm, P., Freiburg: Rombach.

Hohmeyer, O. (1992): Renewables and the full costs of energy. In: Energy Policy, 20 (4), S. 365-375.

Hyde, K.M.; Maier, H.R.; Colby, C.B. (2003): Incorporating uncertainty in the PROMETHEE MCDA method. In: Journal of Multi-Criteria Decision Analysis, 12 (4-5), S. 245-259.

Ibrahim, H.; llinca, A.; Perron, J. (2008): Energy storage systems--Characteristics and comparisons. In: Renewable and Sustainable Energy Reviews, 12 (5), S. 12211250.

IEA (2002): Potential for Building Integrated Photovoltaics. Report IEA - PVPS T7-4, International Energy Agency (Hrsg.), Paris.

IEA (2009a): Energy technology roadmaps: charting a low-carbon energy revolution, Paris: International Energy Agency (IEA). 
IEA (2009b): How the energy sector can deliver on a climate agreement in Copenhagen: Special early excerpt of the World Energy Outlook 2009 for the Bangkok UNFCCC meeting, Paris: Organisation for Economic Co-operation and Development (OECD); International Energy Agency (IEA).

IEA (2009c): World Energy Outlook 2009, Paris: Organisation for Economic Cooperation and Development (OECD); International Energy Agency (IEA).

ifu; Ifeu (2008): Umberto. Version 5.5. Online: www.umberto.de.

IPCC (2007a): Climate Change 2007: Mitigation. Contribution of Working Group III to the Fourth Assessment Report of the International Panel on Climate Change, Metz, B.; Davidson, O.R.; Bosch, P.R.; Dave, R.; Meyer, L.A. (Hrsg.), Cambridge; New York: Cambridge University Press.

IPCC (2007b): Climate change 2007: synthesis report: contribution of working groups I, II and III to the fourth assessment report of the Intergovernmental Panel on Climate Change, Core Writing Team, I.; Pachauri, R.K.; Reisinger, A. (Hrsg.), Genf.

ISO (2006): ISO 14040: Environmental management - life cycle assessment - principles and framework, International Organization for Standardization (Hrsg.), Genf: Beuth.

Jaber, J.O.; Jaber, Q.M.; Sawalha, S.A.; Mohsen, M.S. (2008): Evaluation of conventional and renewable energy sources for space heating in the household sector. In: Renewable and Sustainable Energy Reviews, 12 (1), S. 278-289.

Jakiel, C.; Zunft, S.; Nowi, A. (2007): Adiabatic compressed air energy storage plants for efficient peak load power supply from wind energy: The European project AA-CAES. In: International Journal of Energy Technology and Policy, 5 (3), S. 296-306.

Jansen, A.; Molly, J.P.; Neddermann, B.; Bachmann, U.; Gerch, H.-P.; Grebe, E.; Gröninger, S.; König, M.; Könnemann, A.; Lösing, M.; Saßnick, Y.; Seifert, G.; Siebels, C.; Winter, W.; Bartels, M.; Gatzen, C.; Peek, M.; Schulz, W.; Wissen, R. (2005): Energiewirtschaftliche Planung für die Netzintegration von Windenergie in Deutschland an Land und Offshore bis zum Jahr 2020. Studie im Auftrag der Deutschen Energie-Agentur GmbH (dena), DEWl; E.ON Netz; EWI; RWE Transportnetz Strom; VE Transmission (Hrsg.), Köln.

Jansen, J.C.; Seebregts, A.J. (2010): Long-term energy services security: What is it and how can it be measured and valued? In: Energy Policy, 38 (4), S. 16541664.

Jiang-Jiang, W.; Chun-Fa, Z.; You-Yin, J. (2010): Multi-criteria analysis of combined cooling, heating and power systems in different climate zones in China. In: Applied Energy, 87 (4), S. 1247-1259.

Jochem, E.; Bradke, H.; Marscheider-Weidemann, F.; Som, O.; Mannsbart, W.; Cremer, C.; Dreher, C.; Edler, J.; Ebersberger, B.; Radgen, P.; Ruhland, S.; Krebs, 
A. (2009): Improving the efficiency of R\&D and the market diffusion of energy technologies, Jochem, E. (Hrsg.), Heidelberg: Physica.

Jochem, E.; Jaeger, C.; Battaglini, A.; Bradke, H.; Cremer, C.; Eichhammer, W.; Förster, H.; Haas, A.; Henning, E.; Idrissova, F.; Kasper, B.; Köhler, J.; Köwener, D.; Krause, J.; Lass, W.; Lilliestam, J.; Mannsbart, W.; Müller, M.; Meißner, F.; Pfluger, B.; Radgen, P.; Ragwitz, M.; Rauschen, M.; Reitze, F.; Riffeser, L.; Saure, K.; Schade, W.; Sensfuß, F.; Toro, F.; Walz, W.; Wietschel, M. (2008): Investitionen für ein klimafreundliches Deutschland, BSR-Sustainability; European Climate Forum (ECF); Fraunhofer Institut System- und Innovationsforschung (ISI); Öko-Zentrum NRW; Potsdam-Institut für Klimafolgenforschung (PIK) (Hrsg.), Berlin: Bundesministerium für Umwelt, Naturschutz und Reaktorsicherheit (BMU).

Joest, S.; Fichtner, M.; Wietschel, M.; Bünger, U.; Stiller, C.; Schmidt, P.; Merten, F.; Parker, N.; Bonhoff, K. (2009): GermanHy Studie zur Frage "Woher kommt der Wasserstoff in Deutschland bis 2050?", Deutsche Energie-Agentur; LudwigBölkow-Systemtechnik; Forschungszentrum Karlsruhe; Fraunhofer ISI; Wuppertal Institut (Hrsg.), Berlin: Bundesministerium für Verkehr, Bau und Stadtentwicklung.

Jones, M.; Hope, C.; Hughes, R. (1990): A multi-attribute value model for the study of UK energy policy. In: Journal of the Operational Research Society, 41 (10), S. 919-929.

Jovanovic, M.; Afgan, N.; Radovanovic, P.; Stevanovic, V. (2009): Sustainable development of the Belgrade energy system. In: Energy, 34 (5), S. 532-539.

Kablan, M. (1997): Prioritization of decentralized electricity options available for rural areas in Jordan. In: Energy Conversion and Management, 38 (14), S. 15151521.

Kagazyo, T.; Kaneko, K.; Akai, M.; Hijikata, K. (1997): Methodology and evaluation of priorities for energy and environmental research projects. In: Energy, 22 (2-3), S. 121-129.

Kahle, C. (2009): Die Elektrizitätsversorgung zwischen Versorgungssicherheit und Umweltverträglichkeit, Schriften zum Umweltrecht, Schulze-Fielitz, H.; Müller, T., Baden-Baden: Nomos.

Kahraman, C.; Kaya, I.; Cebi, S. (2009): A comparative analysis for multiattribute selection among renewable energy alternatives using fuzzy axiomatic design and fuzzy analytic hierarchy process. In: Energy, 34 (10), S. 1603-1616.

Kaltschmitt, M.; Hartmann, H.; Hofbauer, H.H. (2009): Energie aus Biomasse. Grundlagen, Techniken und Verfahren, 2, Dordrecht; Heidelberg; London; New York: Springer.

Kaltschmitt, M.; Thrän, D. (2007): Bioenergie. In: Bullinger, H.-J. (Hrsg.): Technologieführer. Grundlagen - Anwendungen - Trends. Berlin; Heidelberg: Springer, S. 346-349. 
Kaminaris, S.D.; Tsoutsos, T.D.; Agoris, D.; Machias, A.V. (2006): Assessing renewables-to-electricity systems: A fuzzy expert system model. In: Energy Policy, 34 (12), S. 1357-1366.

Kapepula, K.M.; Colson, G.; Sabri, K.; Thonart, P. (2007): A multiple criteria analysis for household solid waste management in the urban community of Dakar. In: Waste Management, 27 (11), S. 1690-1705.

Karakosta, C.; Doukas, H.; Psarras, J. (2009): Directing clean development mechanism towards developing countries' sustainable development priorities. In: Energy for Sustainable Development, 13 (2), S. 77-84.

Karger, C.R.; Hennings, W. (2009): Sustainability evaluation of decentralized electricity generation. In: Renewable and Sustainable Energy Reviews, 13 (3), S. 583593.

Karl, J. (2006): Dezentrale Energiesysteme: Neue Technologien im liberalisierten Energiemarkt, München: Oldenbourg Wissenschaftsverlag GmbH.

Karpenstein-Machan, M.; Schmuck, P. (2007): Bioenergy village - ecological and social aspects in the implementation of a sustainability project. In: Journal of Biobased Materials and Bioenergy, 1, S. 148-154.

Kaufmann, D.; Kraay, A.; Mastruzzi, M. (2007): Governance Matters VI: Aggregate and Individual Governance Indicators 1996-2006. In: World Bank Policy Research, Working Paper 4280.

Keeney, R.L.; Sicherman, A. (1983): Illustrative comparison of one utility's coal and nuclear choices. In: Operations Research, 31 (1), S. 50-83.

Keller, G. (2008): Statistics for management and economics, Mason: Cengage Learning.

Kim, S.H. (2007): Evaluation of negative environmental impacts of electricity generation: Neoclassical and institutional approaches. In: Energy Policy, 35 (1), S. 413-423.

Klobasa, M. (2009): Dynamische Simulation eines Lastmanagements und Integration von Windenergie in ein Elektrizitätsnetz, Fraunhofer Institut für System- und Innovationsforschung ISI (Hrsg.), ISI-Schriftenreihe "Innovationspotenziale", Karlsruhe: Fraunhofer IRB.

Köne, A.Ç.; Büke, T. (2007): An Analytical Network Process (ANP) evaluation of alternative fuels for electricity generation in Turkey. In: Energy Policy, 35 (10), S. $5220-5228$.

Konstantin, P. (2009): Praxisbuch Energiewirtschaft: Energieumwandlung, -transport und -beschaffung im liberalisierten Markt, 2, Berlin; Heidelberg: Springer.

Köpke, R. (2009): Mini-Mühlen in der Offensive. In: Energie \& Management, 15. Februar 2009, S. 27. 
Kowalski, K.; Madlener, R.; Stagl, S. (2006): Neue Wege der integrierten Bewertung von nationalen Energieszenarien für Österreich, 9. Symposium Energieinnovation, Graz: TU Graz.

Kowalski, K.; Stagl, S.; Madlener, R.; Omann, I. (2009): Sustainable energy futures: Methodological challenges in combining scenarios and participatory multicriteria analysis. In: European Journal of Operational Research, 197 (3), S. 1063-1074.

Krebs, A. (2006): Technologische Innovation: Entwicklungsverläufe und Messung - Am Fallbeispiel der PEM-Brennstoffzellentechnologie -, Diplomarbeit, Karlsruhe: Universität Karlsruhe (TH); Fraunhofer ISI.

Krewitt, W.; Teske, S.; Simon, S.; Pregger, T.; Graus, W.; Blomen, E.; Schmid, S.; Schäfer, O. (2009): Energy [R]evolution 2008--a sustainable world energy perspective. In: Energy Policy, 37 (12), S. 5764-5775.

Kumar, A.; Sokhansanj, S.; Flynn, P.C. (2006): Development of a multicriteria assessment model for ranking biomass feedstock collection and transportation systems. In: Applied Biochemistry and Biotechnology, 129 (1-3), S. 71-87.

Lee, D.J.; Hwang, J. (2010): Decision support for selecting exportable nuclear technology using the analytic hierarchy process: A Korean case. In: Energy Policy, 38 (1), S. 161-167.

Lefèvre, N. (2010): Measuring the energy security implications of fossil fuel resource concentration. In: Energy Policy, 38 (4), S. 1635-1644.

Lhendup, T. (2008): Rural electrification in Bhutan and a methodology for evaluation of distributed generation system as an alternative option for rural electrification. In: Energy for Sustainable Development, 12 (3), S. 13-24.

Liang, Z.; Yang, K.; Sun, Y.; Yuan, J.; Zhang, H.; Zhang, Z. (2006): Decision support for choice optimal power generation projects: Fuzzy comprehensive evaluation model based on the electricity market. In: Energy Policy, 34 (17), S. 3359-3364.

Lindenberger, D.; Wissen, R.; Bartels, M.; Hillebrand, B.; Buttermann, H.G. (2005): Ökonomische Auswirkungen alternativer Laufzeiten von Kernkraftwerken in Deutschland, Energiewirtschaftliches Institut an der Universität zu Köln (EWI); Energy Environment Forecast Analysis GmbH (EEFA) (Hrsg.), Köln, Berlin: Bundesverband der deutschen Industrie e.V. (BDI).

Liposcak, M.; Afgan, N.H.; Duic, N.; da Graça Carvalho, M. (2006): Sustainability assessment of cogeneration sector development in Croatia. In: Energy, 31 (13), S. 2276-2284.

Løken, E. (2007): Use of multicriteria decision analysis methods for energy planning problems. In: Renewable and Sustainable Energy Reviews, 11 (7), S. 15841595. 
Løken, E.; Botterud, A.; Holen, A.T. (2009): Use of the equivalent attribute technique in multi-criteria planning of local energy systems. In: European Journal of Operational Research, 197 (3), S. 1075-1083.

Longden, D.; Brammer, J.; Bastin, L.; Cooper, N. (2007): Distributed or centralised energy-from-waste policy? Implications of technology and scale at municipal level. In: Energy Policy, 35 (4), S. 2622-2634.

Lootsma, F.A. (1980): Saaty's priority theory and the nomination of a senior professor in operations research. In: European Journal of Operational Research, 4 (6), S. 380-388.

Lootsma, F.A. (1996): A model for the relative importance of the criteria in the Multiplicative AHP and SMART. In: European Journal of Operational Research, 94 (3), S. 467-476.

Lootsma, F.A.; Boonekamp, P.G.M.; Cooke, R.M.; Van Oostvoorn, F. (1990a): Choice of a long-term strategy for the national electricity supply via scenario analysis and multi-criteria analysis. In: European Journal of Operational Research, 48 (2), S. 189-203.

Lootsma, F.A.; Meisner, J.; Schellemans, F. (1986): Multi-criteria decision analysis as an aid to the strategic planning of Energy R\&D. In: European Journal of Operational Research, 25 (2), S. 216-234.

Lootsma, F.A.; Mensch, T.C.A.; Vos, F.A. (1990b): Multi-criteria analysis and budget reallocation in long-term research planning. In: European Journal of Operational Research, 47 (3), S. 293-305.

Löschel, A.; Moslener, U.; Rübbelke, D.T.G. (2010): Indicators of energy security in industrialised countries. In: Energy Policy, 38 (4), S. 1665-1671.

Lutsch, W.; Neuffer, H.; Witterhold, F.-G.; Pfaffenberger, W. (2004): Strategien und Technologien einer pluralistischen Fern- und Nahwärmeversorgung in einem liberalisierten Energiemarkt unter besonderer Berücksichtigung der KraftWärme-Kopplung und regenerativer Energien, Frankfurt: Arbeitsgemeinschaft Fernwärme e. V. (AGFW).

Madlener, R.; Kowalski, K.; Stagl, S. (2007): New ways for the integrated appraisal of national energy scenarios: The case of renewable energy use in Austria. In: Energy Policy, 35 (12), S. 6060-6074.

Madlener, R.; Stagl, S. (2001): Sozio-ökologisch-ökonomische Beurteilung handelbarer Zertifikate und garantierter Einspeisetarife für Ökostrom, 2. Internationale Energiewirtschaftstagung (IEWT): Strategien und Instrumente für den Wettbewerb in der Energiewirtschaft, 21.-23. Februar 2001, Technische Universität Wien.

Madlener, R.; Stagl, S. (2005): Sustainability-guided promotion of renewable electricity generation. In: Ecological Economics, 53 (2), S. 147-167. 
Madlener, R.; Antunes, C.H.; Dias, L.C. (2009): Assessing the performance of biogas plants with multi-criteria and data envelopment analysis. In: European Journal of Operational Research, 197 (3), S. 1084-1094.

Madsen, R.W.; Moeschberger, M.L. (1983): Introductory statistics for business and economics, London; Sydney; Rio de Janeiro; Toronto, New Delhi; Tokyo; Singapore; Wellington: Prentice-Hall.

Mamlook, R. (2006): Fuzzy set methodology for evaluating alternatives to compare between different power production systems. In: Journal of Applied Sciences, 6 (9), S. 2117-2125.

Mamlook, R.; Akash, B.A.; Mohsen, M.S. (2001a): A neuro-fuzzy program approach for evaluating electric power generation systems. In: Energy, 26 (6), S. 619-632.

Mamlook, R.; Akash, B.A.; Nijmeh, S. (2001b): Fuzzy sets programming to perform evaluation of solar systems in Jordan. In: Energy Conversion and Management, 42 (14), S. 1717-1726.

Mareschal, B. (1988): Weight stability intervals in multicriteria decision aid. In: European Journal of Operational Research, 33, S. 54-64.

Markandya, A.; Pemberton, M. (2010): Energy security, energy modelling and uncertainty. In: Energy Policy, 38 (4), S. 1609-1613.

Markovska, N.; Taseska, V.; Pop-Jordanov, J. (2009): SWOT analyses of the national energy sector for sustainable energy development. In: Energy, 34 (6), S. 752756.

Meinshausen, M. (2006): What does a $2{ }^{\circ} \mathrm{C}$ target mean for greenhouse gas concentrations? A brief analysis based on multi-gas emission pathways and several climate sensitivity uncertainty estimates. In: Schellnhuber, H.J.; Cramer, W.; Nakicenovic, N.; Wigley, T.; Yohe, G. (Hrsg.): Avoiding dangerous climate change. Cambridge: Cambridge University Press, S. 265-279.

Mergias, I.; Moustakas, K.; Papadopoulos, A.; Loizidou, M. (2007): Multi-criteria decision aid approach for the selection of the best compromise management scheme for ELVs: The case of Cyprus. In: Journal of Hazardous Materials, 147 (3), S. 706-717.

Meyer-Krahmer, F.; Dreher, C. (2004): Neuere Betrachtungen zu Technikzyklen und Implikationen für die Fraunhofer-Gesellschaft. In: Spath, D. (Hrsg.): Forschungs- und Technologiemanagement. Potenziale nutzen - Zukunft gestalten. München, Wien: Carl Hanser, S. 27-35.

Mirasgedis, S.; Diakoulaki, D. (1997): Multicriteria analysis vs. externalities assessment for the comparative evaluation of electricity generation systems. In: European Journal of Operational Research, 102 (2), S. 364-379. 
Mladineo, N.; Margeta, J.; Brans, J.P.; Mareschal, B. (1987): Multicriteria ranking of alternative locations for small scale hydro plants. In: European Journal of Operational Research, 31 (2), S. 215-222.

Mohsen, M.S.; Akash, B.A. (1997): Evaluation of domestic solar water heating system in Jordan using analytic hierarchy process. In: Energy Conversion and Management, 38 (18), S. 1815-1822.

Morais, D.C.; de Almeida, A.T. (2007): Group decision-making for leakage management strategy of water network. In: Resources, Conservation and Recycling, 52 (2), S. 441-459.

Moriarty, P.; Honnery, D. (2009): What energy levels can the Earth sustain? In: Energy Policy, 37 (7), S. 2469-2474.

Mróz, T.M.; Thiel, T. (2005): Evaluation of a heating system for buildings using multiple criteria decision analysis. In: Archives of Civil Engineering, 51 (2), S. 281-298.

Mueller, M.; Wallace, R. (2008): Enabling science and technology for marine renewable energy. In: Energy Policy, 36 (12), S. 4376-4382.

Mühlstein, J.; Schirmer, C. (2008): Kraftwerkstechnik + Innovation. Zukünftige Anforderungen an fossil befeuerte Kraftwerke, Bayern Innovativ GmbH (Hrsg.), Herrschning: Energie- \& Management Verlagsgesellschaft mbH.

Müller-Lyda, I.; Sailer, M.; Brasser, T.; Droste, J.; Neles, J.; Schmidt, G.; Steinhoff, M. (2008): Endlagerung wärmeentwickelnder radioaktiver Abfälle in Deutschland, Öko-Institut e.V.; Gesellschaft für Anlagen- und Reaktorsicherheit (GRS) mbH (Hrsg.), Braunschweig, Darmstadt.

Munda, G. (2004): Social multi-criteria evaluation: Methodological foundations and operational consequences. In: European Journal of Operational Research, 158 (3), S. 662-677.

Munda, G. (2006): Social multi-criteria evaluation for urban sustainability policies. In: Land Use Policy, 23 (1), S. 86-94.

Mustajoki, J. (2006): Interactive multi-criteria decision support - new tools and processes for practical applications, Dissertation: Helsinki University of Technology, Department of Engineering Physics and Mathematics, Systems Analysis Laboratory.

Mustajoki, J.; Hämäläinen, R.P. (2000): Web-HIPRE: Global decision support by value tree and AHP analysis. In: INFOR, 38 (3), S. 217-220.

Neupert, U. (2007): Wasserkraft, Windenergie und Geothermie. In: Bullinger, H.-J. (Hrsg.): Technologieführer. Grundlagen - Anwendungen - Trends. Berlin; Heidelberg: Springer, S. 340-345. 
Ni, Y.; Zhang, L.; Churchill, J.; Kokot, S. (2007): Application of high performance liquid chromatography for the profiling of complex chemical mixtures with the aid of chemometrics. In: Talanta, 72 (4), S. 1533-1539.

Nigim, K.; Munier, N.; Green, J. (2004): Pre-feasibility MCDM tools to aid communities in prioritizing local viable renewable energy sources. In: Renewable Energy, 29 (11), S. 1775-1791.

Nwofor, O.K.; Chineke, T.C.; Akujor, C.E.; Okoro, U.O. (2007): Energy diversification choices for Nigeria: Optimisation by the criteria ranking method. In: Energy and Environment, 18 (5), S. 577-589.

O.V. (2009): World's first osmotic power plant opened. In: Renewable Energy Focus, 10 (6), S. 23.

Oberschmidt, J.; Klobasa, M. (2009): Ökonomische und technische Bewertung stationärer Stromspeicherung. In: Solarzeitalter - Politik, Kultur und Ökonomie Erneuerbarer Energien, 21 (1), S. 46-53.

Oeding, D.; Oswald, B.R. (2004): Elektrische Kraftwerke und Netze, 6, Berlin, Heidelberg, New York, Hongkong, London, Mailand, Paris, Tokio: Springer.

Oertel, D. (1997): Monitoring "Zum Entwicklungsstand der BrennstoffzellenTechnologie". Sachstandsbericht, Arbeitsbericht Nr. 51: Büro für TechnikfolgenAbschätzung beim Deutschen Bundestag (TAB).

Oertel, D. (2008): Energiespeicher - Stand und Perspektiven. Sachstansbericht zum Monitoring "Nachhaltige Energieversorgung"'", Arbeitsbericht Nr. 123: Büro für Technikfolgen-Abschätzung beim Deutschen Bundestag (TAB).

Önüt, S.; Tuzkaya, U.R.; Saadet, N. (2008): Multiple criteria evaluation of current energy resources for Turkish manufacturing industry. In: Energy Conversion and Management, 49 (6), S. 1480-1492.

Oschatz, B.; Mailach, B. (2007): ASUE Heizkostenvergleich Neubau 2007. Überarbeitung der Kostenansätze und Preise der Broschüre Wärmeversorgung im Neubau: Ratgeber für Bauherren und Planung: Arbeitsgemeinschaft für sparsamen und umweltfreundlichen Energieverbrauch (ASUE) e.V. Kaiserslautern; Institut für Technische Gebäudeausrüstung (ITG) Dresden.

Palma, J.; Graves, A.R.; Burgess, P.J.; van der Werf, W.; Herzog, F. (2007): Integrating environmental and economic performance to assess modern silvoarable agroforestry in Europe. In: Ecological Economics, 63 (4), S. 759-767.

Papadaki, M.; Tsoutsos, T.; Maria, E.; Antonidakis, E. (2003): A multicriteria decision making methodology for sustainable energy development. In: Fresenius Environmental Bulletin, 12 (5), S. 426-430.

Papadopoulos, A.; Karagiannidis, A. (2008): Application of the multi-criteria analysis method Electre III for the optimisation of decentralised energy systems. In: Omega, 36 (5), S. 766-776. 
Pehnt, M. (2002): Energierevolution Brennstoffzelle? Perspektiven, Fakten, Anwendungen, Weinheim: Wiley $\mathrm{VCH}$.

PEPP (2009): 10 langfristige Handlungslinien für die künftige Energieversorgung in Deutschland. Projektgruppe Energiepolitisches Programm (PEPP), Bundesministerium für Wirtschaft und Technologie (BMWi). Online: http://www.bmwi.de/BMWi/Navigation/Service/publikationen, did=288636.html (Stand: 21.05.2010).

Perl, E. (2007): Grundlagen des Innovations- und Technologiemanagements. In: Strebel, H. (Hrsg.): Innovations- und Technologiemanagement. Wien: Facultas Verlags- und Buchhandels AG, S. 17-52.

Pfeiffer, W.; Bischof, P. (1981): Produktlebenszyklen - Instrument jeder strategischen Produktplanung. In: Planung und Kontrolle - Probleme der strategischen Unternehmensführung. München: Verlag Vahlen, S. 133-165.

Phdungsilp, A. (2010): Integrated energy and carbon modeling with a decision support system: Policy scenarios for low-carbon city development in Bangkok. In: Energy Policy, In Press, Corrected Proof.

Pictet, J.; Bollinger, D. (2005): The silent negotiation or how to elicit collective information for group MCDA without excessive discussion. In: Journal of Multi-Criteria Decision Analysis, 13, S. 199-211.

Pilavachi, P.A.; Roumpeas, C.P.; Minett, S.; Afgan, N.H. (2006): Multi-criteria evaluation for CHP system options. In: Energy Conversion and Management, 47 (20), S. 3519-3529.

Pilavachi, P.A.; Stephanidis, S.D.; Pappas, V.A.; Afgan, N.H. (2009): Multi-criteria evaluation of hydrogen and natural gas fuelled power plant technologies. In: Applied Thermal Engineering, 29 (11-12), S. 2228-2234.

Pires Neves, L.; Gomes Martins, A.; Henggeler Antunes, C.; Cândido Dias, L. (2008): A multi-criteria decision approach to sorting actions for promoting energy efficiency. In: Energy Policy, 36 (7), S. 2351-2363.

Platts (2007a): Data base description and research methodology UDI World Electric Power Plants data base, Platts, U.P.G. (Hrsg.), Washington: The McGraw-Hill Companies Inc.

Platts (2007b): UDI World Electric Power Plants Data Base (WEPP). The McGraw-Hill Companies, Inc.

Pohekar, S.D.; Ramachandran, M. (2004): Application of multi-criteria decision making to sustainable energy planning - A review. In: Renewable and Sustainable Energy Reviews, 8 (4), S. 365-381.

Polatidis, H.; Haralambopoulos, D.A. (2007): Renewable energy systems: A societal and technological platform. In: Renewable Energy, 32 (2), S. 329-341. 
Polatidis, H.; Haralambopoulos, D.A. (2008): Renewable energy sources planning and design: a multi-criteria approach. In: van den Bergh, J.C.J.M.; Bruinsma, F.R. (Hrsg.): Managing the transition to renewable energy. Theory and practice from local, regional and macro perspectives. Cheltenham; Northampton: Edward Elgar Publishing Limited, S. 295-322.

Polatidis, H.; Haralambopoulos, D.A.; Kemp, R.; Rothman, D. (2003): Creating an energy system that we want but don't know yet, using integrated assessment, transition management and multi-criteria analysis. In: Integrated Assessment, 4 (3), S. 205-213.

Polatidis, H.; Haralambopoulos, D.A.; Munda, G.; Vreeker, R. (2006): Selecting an appropriate multi-criteria decision analysis technique for renewable energy planning. In: Energy Sources, Part B: Economics, Planning and Policy, 1 (2), S. 181-193.

Powell, C.; Bender, T.; Lawryshyn, Y. (2009): A model to determine financial indicators for organic solar cells. In: Solar Energy, 83 (11), S. 1977-1984.

Praetorius, B.; Bauknecht, D.; Cames, M.; Fischer, C.; Pehnt, M.; Schumacher, K.; Voß, J.-P. (2009): Innovation for Sustainable Electricity Systems - Exploring the dynamics of Energy Transitions, Heidelberg: Physica-Verlag.

Praetorius, B.; Schumacher, K. (2009): Greenhouse gas mitigation in a carbon constrained world: The role of carbon capture and storage. In: Energy Policy, 37 (12), S. 5081-5093.

Primas, A. (2007): Life cycle inventories of new CHP systems. ecoinvent report No. 20, Paul Scherrer Institut Villigen; Swiss Centre for Life Cycle Inventories (Hrsg.), Dübendorf.

Purcell, D.E.; O'Shea, M.G.; Kokot, S. (2007): Role of chemometrics for at-field application of NIR spectroscopy to predict sugarcane clonal performance. In: Chemometrics and Intelligent Laboratory Systems, 87 (1), S. 139-150.

Quaschning, V. (2007): Regenerative Energiesysteme: Technologie - Berechnung Simulation, München: Carl Hanser.

Quaschning, V. (2008): Erneuerbare Energien und Klimaschutz: Hintergründe, Techniken, Anlagenplanung, Wirtschaftlichkeit, München: Carl Hanser.

Queiruga, D.; Walther, G.; González-Benito, J.; Spengler, T. (2008): Evaluation of sites for the location of WEEE recycling plants in Spain. In: Waste Management, 28 (1), S. 181-190.

Rahman, S.; Frair, L.C. (1984): A hierarchical approach to electric utility planning. In: Energy Research, 8, S. 185-196.

Ramanathan, R.; Ganesh, L.S. (1995): Energy resource allocation incorporating qualitative and quantitative criteria: An integrated model using goal programming and AHP. In: Socio-Economic Planning Sciences, 29 (3), S. 197-218. 
Recchia, L.; Cini, E.; Corsi, S. (2010): Multicriteria analysis to evaluate the energetic reuse of riparian vegetation. In: Applied Energy, 87 (1), S. 310-319.

Reichmuth, M.; Bohnenschäfer, W.; Daniel, J.; Fröhlich, N.; Lindner, K.; Müller, M.; Weber, A.; Witt, J.; Seefeldt, F.; Kirchner, A.; Michelsen, C. (2006): Auswirkungen der Änderungen des Erneuerbare-Energien-Gesetzes hinsichtlich des Gesamtvolumens der Förderung, der Belastung der Stromverbraucher sowie der Lenkungswirkung der Fördersätze für die einzelnen Energiearten. Endbericht, Institut für Energetik und Umwelt (IE) gGmbH Leipzig; Prognos AG Basel (Hrsg.), Berlin: Bundesministerium für Wirtschaft und Technologie (BMWi).

Rempel, H.; Schmidt, S.; Schwarz-Schampera, U. (2007): Reserven, Ressourcen und Verfügbarkeit von Energierohstoffen 2006. Jahresbericht 2006, Bundesanstalt für Geowissenschaften und Rohstoffe (BGR) (Hrsg.).

Ren, H.; Gao, W.; Zhou, W.; Nakagami, K. (2009): Multi-criteria evaluation for the optimal adoption of distributed residential energy systems in Japan. In: Energy Policy, 37 (12), S. 5484-5493.

Renn, O. (2003): Social assessment of waste energy utilization scenarios. In: Energy, 28 (13), S. 1345-1357.

Roads2HyCom (2008): Hydrogen Pathway: Cost Analysis. Online: http://www.ika.rwthaachen.de/r2h/index.php/Hydrogen Pathway: Cost Analysis (Stand: 13.04.2010).

Rogers, E.M. (2003): Diffusion of innovations, 5, New York: The Free Press.

Rogers, M.; Bruen, M. (1998): Choosing realistic values of indifference, preference and veto thresholds for use with environmental criteria within ELECTRE. In: European Journal of Operational Research, 107 (3), S. 542-551.

Roth, S.; Hirschberg, S.; Bauer, C.; Burgherr, P.; Dones, R.; Heck, T.; Schenler, W. (2009): Sustainability of electricity supply technology portfolio. In: Annals of Nuclear Energy, 36 (3), S. 409-416.

Rousis, K.; Moustakas, K.; Malamis, S.; Papadopoulos, A.; Loizidou, M. (2008): Multicriteria analysis for the determination of the best WEEE management scenario in Cyprus. In: Waste Management, 28 (10), S. 1941-1954.

Routroy, S.; Kodali, R. (2007): Promethee II for selection of carrier in supply chain. In: The Icfai Journal of Supply Chain Management, IV (1), S. 29-39.

Roux, O.; Duvivier, D.; Dhaevers, V.; Meskens, N.; Artiba, A. (2008): Multicriteria approach to rank scheduling strategies. In: International Journal of Production Economics, 112 (1), S. 192-201.

Roy, B. (1980): Selektieren, Sortieren und Ordnen mit Hilfe von Prävalenzrelationen: Neue Ansätze auf dem Gebiet der Entscheidungshilfe für MultikriteriaProbleme. In: Schmalenbachs Zeitschrift für Betriebswirtschaftliche Forschung, 32, S. 465-497. 
Roy, B. (1996): Multicriteria Methodology for Decision Aiding, Dordrecht: Kluwer Academic Publishers.

Roy, B. (2005): Paradigms and Challenges. In: Figueira, J.; Greco, S.; Ehrgott, M. (Hrsg.): Multiple Criteria Decision Aid: State of the Art Surveys. New York: Springer, S. 3-24.

Roy, B.; Bouyssou, D. (1993): Aide multicritère d'aide á la décision: Méthodes et Cas. In: Economica.

Ruppert, H.; Eigner-Thiel, S.; Girschner, W.; Karpenstein-Machan, M.; Roland, F.; Ruwisch, V.; Sauer, B.; Schmuck, P. (2008): Wege zum Bioenergiedorf. Leitfaden für eine eigenständige Wärme- und Stromversorgung auf Basis von Biomasse im ländlichen Raum, Gülzow: Fachagentur für nachwachsende Rohstoffe FNR e.V.

Ruttan, V.W. (2001): Technology, growth and development. An induced innovation perspective, New York, Oxford: Oxford University Press.

Saaty, T.L. (1980): The Analytic Hierarchy Process, New York: McGraw-Hill.

Saaty, T.L. (2005): The Analytic Hierarchy and Analytic Network Process for the Measurement of Intangible Criteria and for Decision Making. In: Figueira, J.; Greco, S.; Ehrgott, M. (Hrsg.): Multiple Criteria Decision Analysis: State of the Art Surveys. New York: Springer, S. 345-407.

Saaty, T.L.; Vargas, L.G. (2006): Decision Making with the Analytic Network Process: Economic, Political, Social and Technological Applications with benefits, Opportunities, Costs and Risks, International Series in Operations Research \& Management Science, Hillier, F.S., New York: Springer.

Safaei Mohamadabadi, H.; Tichkowsky, G.; Kumar, A. (2009): Development of a multicriteria assessment model for ranking of renewable and non-renewable transportation fuel vehicles. In: Energy, 34 (1), S. 112-125.

Salo, A.A.; Hämäläinen, R.P. (1995): Preference programming through approximate ratio comparisons. In: European Journal of Operational Research, 82 (3), S. 458-475.

Schiffer, H.-W. (2008): Energiemarkt Deutschland, Praxiswissen Energie und Umwelt, Köln: TÜV Media GmbH, TÜV Rheinland Group.

Schmitz, S.; Paulini, I. (1999): Bewertung in Ökobilanzen. Methode des Umweltbundesamtes zur Normierung von Wirkungsindikatoren, Ordnung (Rangbildung) von Wirkungskategorien und zur Auswertung nach ISO 14042 und 14043 (Version '99), Umweltbundesamt (Hrsg.), Berlin.

Schneider, M.; Thomas, S.; Froggatt, A.; Koplow, D. (2009): Der Welt-Statusreport der Atomindustrie 2009. Unter besonderer Berücksichtigung wirtschaftlicher Fragen, Paris; Berlin: Bundesministerium für Umwelt, Naturschutz und Reaktorsicherheit (BMU). 
Scholvin, S. (2009): Desertec: Wirtschaftliche Dynamik und politische Stabilität durch Solarkraft? In: GIGA Focus Nahost (11).

Schulz, V.; Stehfest, H. (1984): Regional energy supply optimization with multiple objectives. In: European Journal of Operational Research, 17 (3), S. 302-312.

Schwab, A.J. (2009): Elektroenergiesysteme. Erzeugung, Transport, Übertragung und Verteilung elektrischer Energie, 2, Dordrecht, Heidelberg, London, New York: Springer.

Shackley, S.; McLachlan, C. (2006): Trade-offs in assessing different energy futures: a regional multi-criteria assessment of the role of carbon dioxide capture and storage. In: Environmental Science \& Policy, 9 (4), S. 376-391.

Siegwart, H.; Senti, R. (1995): Product Life Cycle Management. Die Gestaltung eines integrierten Produktlebenszyklus, Stuttgart: Schäffer-Poeschel.

Siskos, J.; Hubert, P. (1983): Multi-criteria analysis of the impacts of energy alternatives: A survey and a new comparative approach. In: European Journal of Operational Research, 13 (3), S. 278-299.

Siskos, Y. (2005): UTA Methods. In: Figueira, J.; Greco, S.; Ehrgott, M. (Hrsg.): Multiple Criteria Decision Analysis: State of the Art Surveys. New York: Springer, S. 297-343.

Skilhagen, S.E.; Dugstad, J.E.; Aaberg, R.J. (2008): Osmotic power -- power production based on the osmotic pressure difference between waters with varying salt gradients. In: Desalination, 220 (1-3), S. 476-482.

Son, H.J.; Min, K.J. (1998): Capital budgeting process for electric power utilities - an analytic hierarchy process approach. In: International Journal of Energy Research, 22 (7), S. 671-681.

Stadtwerke Jülich GmbH (2007): Solarturm Jülich. Online: http://www.solarturmjuelich.de/de/node/31 (Stand: 26.05.2010).

Stadtwerke Walldorf GmbH (2010): Solarpark Walldorf. Online: http://www.stadtwerkewalldorf.de/servlet/PB/menu/1273742 I1/index.html (Stand: 28.05.2010).

Stagl, S. (2006): Multicriteria evaluation and public participation: the case of UK energy policy. In: Land Use Policy, 23 (1), S. 53-62.

Staiß, F. (2007): Jahrbuch Erneuerbare Energien 2007 (Ausgabe auf CD-ROM), Stiftung Energieforschung Baden-Württemberg (SEf) (Hrsg.), Radebeul: Bieberstein.

Staiß, F.; Kratzat, M.; Nitsch, J.; Lehr, U.; Edler, D.; Lutz, C. (2006): Erneuerbare Energien: Arbeitsplatzeffekte. Wirkungen des Ausbaus erneuerbarer Energien auf den deutschen Arbeitsmarkt, Zentrum für Sonnenenergie- und WasserstoffForschung Baden-Württemberg (ZSW); Deutsches Zentrum für Luft- und Raumfahrt (DLR); Deutsches Institut für Wirtschaftsforschung (DIW); Gesell- 
schaft für wirtschaftliche Strukturforschung (GWS) (Hrsg.), Berlin: Bundesministerium für Umwelt, Naturschutz und Reaktorsicherheit (BMU).

Stirling, A. (2010): Multicriteria diversity analysis: A novel heuristic framework for appraising energy portfolios. In: Energy Policy, In Press, Corrected Proof (38), S. 1622-1634.

Strebel, H. (2009): Innovation und Nachhaltigkeit. In: UmweltWirtschaftsForum (uwf), 17, S. 299-304.

Strohschein, J.; Erdmenger, C.; Albert, R.; Bade, M.; Behnke, A.; Böhme, S.; Hannig, A.; Hülsmann, W.; Johnke, B.; Kaschenz, H.; Lohse, C.; Miehe, A.; Pichl, P.; Saupe, S.; Schubert, A.; Wachsmann, U.; Werner, K.; Westermann, B. (2007): Nachhaltige Wärmeversorgung: Sachstandsbericht, 03/07, Umweltbundesamt (UBA) (Hrsg.), Climate Change, Dessau.

Suttor, W. (2009): Blockheizkraftwerke. Ein Leitfaden für Anwender, 7, FIZ Karlsruhe; BINE Informationsdienst (Hrsg.), Berlin: Solarpraxis AG.

Szargut, J.; Morris, D.R.; Steward, F.R. (1988): Exergy analysis of thermal, chemical, and metallurgical processes, Berlin u.a.: Springer.

Temming, V.H. (2005): Analysen und Simulationen zur Bewertung der Einsatzmöglichkeiten und Marktpotenziale von Brennstoffzellen in der Industrie, Schriftenreihe des Lehrstuhls für Energiesysteme und Energiewirtschaft (LEE) 11, Bochum: Ruhr-Universität Bochum.

Terrados, J.; Almonacid, G.; Pérez-Higueras, P. (2009): Proposal for a combined methodology for renewable energy planning. Application to a Spanish region. In: Renewable and Sustainable Energy Reviews, 13 (8), S. 2022-2030.

Tester, J.W.; Drake, E.M.; Driscoll, M.J.; Golay, M.W.; Peters, W.A. (2005): Sustainable energy: choosing among options, Cambridge: MIT Press.

TFZ (2010): Entwicklung der Brennstoffpreise. Technologie- und Förderzentrum Straubing. Online: http://www.tfz.bayern.de/festbrennstoffe/17387/ (Stand: 06.05.2010).

Thiel, T.; Mróz, T. (2001): Application of multi-criterion decision aid method in designing heating systems for museum buildings. In: Informatica, 12 (1), S. 133-146.

Tidd, J.; Bessant, J. (2009): Managing Innovation. Integrating technological, market and organizational change, 4: John Wiley \& Sons.

Topcu, Y.I.; Ulengin, F. (2004): Energy for the future: An integrated decision aid for the case of Turkey. In: Energy, 29 (1), S. 137-154.

Treitz, M. (2006): Production process design using multi-criteria analysis, Karlsruhe: Universitätsverlag. 
Tromm, W.; Bahm, W. (2007): Kernenergie. In: Bullinger, H.-J. (Hrsg.): Technologieführer. Grundlagen - Anwendungen - Trends. Berlin; Heidelberg: Springer, S. 334339.

Tsoutsos, T.; Drandaki, M.; Frantzeskaki, N.; Iosifidis, E.; Kiosses, I. (2009): Sustainable energy planning by using multi-criteria analysis application in the island of Crete. In: Energy Policy, 37 (5), S. 1587-1600.

Tuzkaya, G.; Gülsün, B.; Kahraman, C.; Ízgen, D. (2009): An integrated fuzzy multicriteria decision making methodology for material handling equipment selection problem and an application. In: Expert Systems with Applications, In Press, Uncorrected Proof.

Tzeng, G.-H.; Shiau, T.-A.; Lin, C.-Y. (1992): Application of multicriteria decision making to the evaluation of new energy system development in Taiwan. In: Energy, 17 (10), S. 983-992.

UBA (2007): Umweltdaten Deutschland. Nachhaltig wirtschaften - Natürliche Ressourcen und Umwelt schonen, Umweltbundesamt (Hrsg.), Dessau.

Ulutas, B.H. (2005): Determination of the appropriate energy policy for Turkey. In: Energy, 30 (7), S. 1146-1161.

UN (1987): Report of the World Commission on Environment and Development: "Our Common Future" (Brundtland-Report).

UN (1992): United Nations framework convention on climate change, United Nations (UN) (Hrsg.), Kyoto.

UN (1998): Kyoto Protocol to the United Nations framework convention on climate change, United Nations (UN) (Hrsg.), Kyoto.

UN (2009): Copenhagen Accord of 18 December 2009 (Decision-/CP.15), Copenhagen: United Nations.

UNPD (2009): World population prospects: the 2008 revision population database. United Nations Population Division (UNDP) (Stand: 10.12.2009).

Utterback, J.M.; Abernathy, W.J. (1975): A dynamic model of process and product innovation. In: Omega, 3 (6), S. 639-656.

van Alphen, K.; van Sark, W.G.J.H.; Hekkert, M.P. (2007): Renewable energy technologies in the Maldives--determining the potential. In: Renewable and Sustainable Energy Reviews, 11 (8), S. 1650-1674.

VDI (1997): VDI 4600: Kumulierter Energieaufwand - Begriffe, Definitionen, Berechnungsmethoden, Verein Deutscher Ingenieure (Hrsg.), Berlin: Beuth.

VDI (2000): VDI 2067 Blatt 1: Wirtschaftlichkeit gebäudetechnischer Anlagen - Grundlagen und Kostenberechnung, Verein Deutscher Ingenieure (Hrsg.), Berlin: Beuth. 
Vego, G.; Kucar-Dragicevic, S.; Koprivanac, N. (2008): Application of multi-criteria decision-making on strategic municipal solid waste management in Dalmatia, Croatia. In: Waste Management, 28 (11), S. 2192-2201.

Vetschera, R. (1994): Visualisierungstechniken in Entscheidungsproblemen bei mehrfacher Zielsetzung. In: OR Spektrum, 16 (4), S. 227-241.

Voropai, N.I.; Ivanova, E.Yu. (2002): Multi-criteria decision analysis techniques in electric power system expansion planning. In: International Journal of Electrical Power \& Energy Systems, 24 (1), S. 71-78.

Wang, J.J.; Yang, D.L. (2007): Using a hybrid multi-criteria decision aid method for information systems outsourcing. In: Computers and Operations Research, 34 (12), S. 3691-3700.

Wang, J.-J.; Jing, Y.-Y.; Zhang, C.-F. (2009a): Weighting methodologies in multicriteria evaluations of combined heat and power systems. In: International Journal of Energy Research, 33, S. 1023-1039.

Wang, J.J.; Jing, Y.Y.; Zhang, C.F.; Zhao, J.H. (2009b): Review on multi-criteria decision analysis aid in sustainable energy decision-making. In: Renewable and Sustainable Energy Reviews, 13 (9), S. 2263-2278.

Watter, H. (2009): Nachhaltige Energiesysteme - Grundlagen, Systemtechnik und Anwendungsbeispiele aus der Praxis, Wiesbaden: Vieweg+Teubner.

WBGU (2009): Welt im Wandel: Zukunftsfähige Bioenergie und nachhaltige Landnutzung, Wissenschaftlicher Beirat der Bundesregierung Globale Umweltveränderungen (WBGU) (Hrsg.), Berlin.

WEC (1992): Energiewörterbuch. Jouve Systèmes d'information, World Energy Council (WEC) (Hrsg.).

Weistroffer, H.R.; Smith, C.H.; Narula, S.C. (2005): Multiple criteria decision support software. In: Figueira, J.; Greco, S.; Ehrgott, M. (Hrsg.): Multiple criteria decision analysis: State of the art surveys. New York: Springer, S. 989-1033.

Wenstöp, F.E.; Carlsen, A.J. (1988): Ranking hydroelectric power projects with multicriteria decision analysis. In: Interfaces, 18 (4), S. 36-48.

Wenzel, B.; Nitsch, J. (2008): Ausbau erneuerbarer Energien im Strombereich. EEGVergütungen, -Differenzkosten und -Umlage sowie ausgewählte Nutzeneffekte bis zum Jahr 2030, Berlin; Teltow; Stuttgart: Bundesministerium für Umwelt, Naturschutz und Reaktorsicherheit (BMU).

Weule, H. (2002): Integriertes Forschungs- und Entwicklungsmanagement. Grundlagen, Strategien, Umsetzung, München, Wien: Carl Hanser.

White, L.; Lee, G.J. (2009): Operational research and sustainable development: Tackling the social dimension. In: European Journal of Operational Research, 193 (3), S. 683-692. 
Wietschel, M.; Arens, M.; Dötsch, C.; Herkel, S.; Krewitt, W.; Markewitz, P.; Möst, D.; Scheufen, M.; Oberschmidt, J. et al. (2010): Energietechnologien 2050 Schwerpunkte für Forschung und Entwicklung. Technologiebericht, Stuttgart: Fraunhofer Verlag.

Winkler, W. (2002): Brennstoffzellenanlagen, Berlin; Heidelberg: Springer.

Wittwer, V. (2007): Solarenergie. In: Bullinger, H.-J. (Hrsg.): Technologieführer. Grundlagen - Anwendungen - Trends. Berlin; Heidelberg: Springer, S. 350-355.

Wöhe, G.; Döring, U. (1996): Einführung in die Betriebswirtschaftslehre, 19, München: Franz Vahlen.

Wolters, W.T.M.; Mareschal, B. (1995): Novel types of sensitivity analysis for additive MCDM methods. In: European Journal of Operational Research, 81 (2), S. 281290.

Xu, Z. (2005): Deviation measures of linguistic preference relations in group decision making. In: Omega, 33 (3), S. 249-254.

Zangeneh, A.; Jadid, S.; Rahimi-Kian, A. (2009): A hierarchical decision making model for the prioritization of distributed generation technologies: A case study for Iran. In: Energy Policy, 37 (12), S. 5752-5763.

Zhou, P.; Ang, B.W.; Poh, K.L. (2006): Decision analysis in energy and environmental modeling: An update. In: Energy, 31 (14), S. 2268-2286.

Zhu, Z.; Xu, L.; Chen, G.; Li, Y. (2010): Optimization on tribological properties of aramid fibre and CaSO4 whisker reinforced non-metallic friction material with analytic hierarchy process and preference ranking organization method for enrichment evaluations. In: Materials \& Design, 31 (1), S. 551-555.

Zimmermann, H.-J.; Gutsche, L. (1991): Multi-Criteria Analyse: Einführung in die Theorie der Entscheidungen bei Mehrfachzielsetzungen, Heidelberger Lehrtexte Wirtschaftswissenschaften, Berlin, Heidelberg: Springer.

Zotter, K.-A. (2007): Modelle des Innovations- und Technologiemanagements - Innovations- und Technologiemanagement, Strebel, H.H. (Hrsg.), Wien: Facultas Verlags- und Buchhandels AG, S. 53-93.

Zunft, S.; Jakiel, C.; Koller, M.; Bullough, C. (2006): Adiabatic compressed air energy storage for the grid integration of wind power, Sixth International Workshop on Large-Scale Integration of Wind Power and Transmission Networks for Offshore Windfarms, Delft: 26.-28. Oktober 2006. 